\section{OAK RIDGE}

NATIONAL LABORATORY

MANAGED BY UT-BATTELLE

FOR THE DEPARTMENT OF ENERGY
Scoping Study on the Safety Impact of Valve Spacing in Natural Gas Pipelines

\author{
C. D. Sulfredge
}

UT-BATTELLE 


\section{DOCUMENT AVAILABILITY}

Reports produced after January 1, 1996, are generally available free via the U.S. Department of Energy (DOE) Information Bridge:

Web site: http://www.osti.gov/bridge

Reports produced before January 1, 1996, may be purchased by members of the public from the following source:

National Technical Information Service

5285 Port Royal Road

Springfield, VA 22161

Telephone: 703-605-6000 (1-800-553-6847)

TDD: 703-487-4639

Fax: 703-605-6900

E-mail: info@ntis.fedworld.gov

Web site: http://www.ntis.gov/support/ordernowabout.htm

Reports are available to DOE employees, DOE contractors, Energy Technology Data Exchange (ETDE) representatives, and International Nuclear Information System (INIS) representatives from the following source:

Office of Scientific and Technical Information

P.O. Box 62

Oak Ridge, TN 37831

Telephone: 865-576-8401

Fax: 865-576-5728

E-mail: reports@adonis.osti.gov

Web site: http://www.osti.gov/contact.html

This report was prepared as an account of work sponsored by an agency of the United States Government. Neither the United States government nor any agency thereof, nor any of their employees, makes any warranty, express or implied, or assumes any legal liability or responsibility for the accuracy, completeness, or usefulness of any information, apparatus, product, or process disclosed, or represents that its use would not infringe privately owned rights. Reference herein to any specific commercial product, process, or service by trade name, trademark, manufacturer, or otherwise, does not imply its endorsement, recommendation, or favoring by the United States Government or any agency thereof. The views and opinions of authors expressed herein do not necessarily state or reflect those of the United States Government or any agency thereof. 
Computational Sciences and Engineering Division

\title{
SCOPING STUDY ON THE SAFETY IMPACT OF VALVE SPACING IN NATURAL GAS PIPELINES
}

\author{
C. D. Sulfredge
}

May 2007

\author{
Prepared by \\ OAK RIDGE NATIONAL LABORATORY \\ Oak Ridge, Tennessee 37831-6283 \\ managed by \\ UT-BATTELLE, LLC \\ for the \\ U.S. DEPARTMENT OF ENERGY \\ under contract DE-AC05-00OR22725
}




\section{CONTENTS}

Page

LIST OF TABLES $\mathrm{V}$

EXECUTIVE SUMMARY

vii

1 INTRODUCTION

1

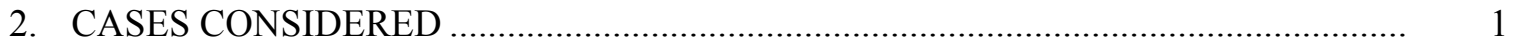

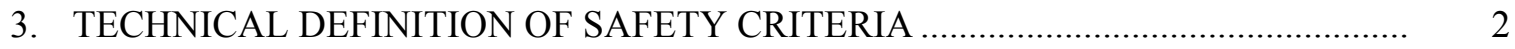

4. IDEAL GAS ANALYSIS OF CASES ….......................................................................

5. REAL GAS AND DEPRESSURIZATION PIPE COOLING EFFECTS ........................ 5

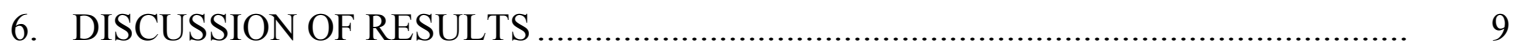

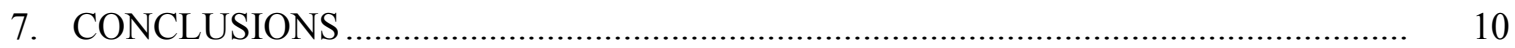

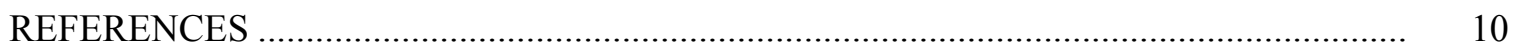

Appendix A. IDEAL GAS RUNGE-KUTTA FORTRAN CODE LISTING ...................... 11

Appendix B. COLLECTED IDEAL GAS FORTRAN CODE OUTPUT ................................ 14

Appendix C. REAL GAS RUNGE-KUTTA FORTRAN CODE LISTING ........................ 26

Appendix D. COLLECTED REAL GAS FORTRAN CODE OUTPUT ............................. 40 


\section{LIST OF TABLES}

Table

1 Average $\lambda$ Values over the First 30 Seconds as Calculated by the FORTRAN Program............................................................................................ 4

2 Hazard Area Radius for Each Case ….................................................................... 5

3 Saturation Line Properties for Methane …............................................................. 6

$4 \quad$ Real Gas and Pipe Wall Cooling Results ...................................................... 8 


\section{EXECUTIVE SUMMARY}

The U.S. Department of Transportation's Pipeline and Hazardous Materials Safety Administration (PHMSA) is responsible for ensuring the safe, reliable, and environmentally sound operation of the nation's natural gas and hazardous liquid pipelines. Regulations adopted by PHMSA for gas pipelines are provided in 49 CFR 192, and spacing requirements for valves in gas transmission pipelines are presented in 49 CFR 192.179.

The present report describes the findings of a scoping study conducted by Oak Ridge National Laboratory (ORNL) to assist PHMSA in assessing the safety impact of system valve spacing. Calculations of the pressures, temperatures, and flow velocities during a set of representative pipe depressurization transients were carried out using a one-dimensional numerical model with either ideal gas or real gas properties for the fluid. With both ideal gas and real gas properties, the high-consequence area radius for any resulting fire as defined by Stevens in GRI-00/0189 was evaluated as one measure of the pipeline safety. In the real gas case, a model for convective heat transfer from the pipe wall is included to assess the potential for shut-off valve failures due to excessively low temperatures resulting from depressurization cooling of the pipe. A discussion is also provided of some additional factors by which system valve spacing could affect overall pipeline safety.

The following conclusions can be drawn from this work:

- Using an adaptation of the Stephens hazard radius criteria, valve spacing has a negligible influence on natural gas pipeline safety for the pipeline diameter, pressure range, and valve spacings considered in this study.

- Over the first $30 \mathrm{~s}$ of the transient, pipeline pressure has a far greater effect on the hazard radius calculated with the Stephens criteria than any variations in the transient flow decay profile and the average discharge rate.

- Other factors besides the Stephens criteria, such as the longer burn time for an accidental fire, greater period of danger to emergency personnel, increased unavoidable loss of gas, and possible depressurization cooling of the shut-off valves may also be important when deciding whether a change in the required valve spacing would be beneficial from a safety standpoint.

- The average normalized discharge rate of $\lambda_{\text {avg }}=0.33$ assumed by Stephens in developing his safety criteria is an excellent conservative value for natural gas discharge at the pressures, valve spacings, and pipe diameter used in this study. This conclusion remains valid even when real rather than ideal gas properties are considered in the analysis.

- Significant pipe wall cooling effects $\left(T_{w}<-50{ }^{\circ} \mathrm{F}\right.$ or $\left.228 \mathrm{~K}\right)$ can extend for a mile or more upstream from the rupture point within $30 \mathrm{~s}$ of a break. These conditions are colder than the temperature range specifications for many valve lubricants. The length of the low-temperature zone due to this cooling effect is also essentially independent of the system shut-off valve spacing or the distance between the break and a compressor station.

- Having more redundant shut-off valves available would reduce the probability that pipe cooling effects could interfere with isolating the broken area following a pipeline rupture accident. 


\section{INTRODUCTION}

The U.S. Department of Transportation's Pipeline and Hazardous Materials Safety Administration (PHMSA) is responsible for ensuring the safe, reliable, and environmentally sound operation of the nation's 2.3 million miles of natural gas and hazardous liquid pipelines. To accomplish this mission, PHMSA regulates pipeline safety and spill response planning by developing regulations and other approaches to risk management to assure safety in design, construction, testing, operation, maintenance, and emergency response of pipeline facilities. Regulations adopted by PHMSA for gas pipelines are provided in 49 CFR 192. Spacing requirements for valves in gas transmission pipelines are presented in 49 CFR 192.179. The minimum spacing between valves in gas transmission pipelines is a function of their class location. Class 1 areas include rural locations where few people or buildings are located. In general, Class 4 areas include cities and urban locations with higher population densities.

On April 3, 2006, the Interstate Natural Gas Association of America (INGAA) submitted a letter to PHMSA concerning a "Request for Clarification-Valve Proximity and Class Location Changes". This letter included an attachment titled: "White Paper on Equivalent Safety for Alternative Valve Spacing" 2 which provides a technical basis for the request.

The present report describes the findings of a scoping study conducted by Oak Ridge National Laboratory (ORNL) to assist PHMSA in addressing the INGAA request. Results are presented for several combinations of valve spacing and pipeline pressure to determine the potential safety impact. The safety assessment criteria for each case are based on an adaptation of the Stephens hazard radius. ${ }^{3}$ The potential for valve failures due to depressurization cooling of the pipe was also investigated.

\section{CASES CONSIDERED}

The pipeline used in the analysis has the following characteristics:

- Material: API Specification 5L, Grade X70 [Specified Minimum Yield Strength (SMYS) of 70,000 psi]

- Diameter (D): 36 in.

- Wall thickness $(\mathrm{t}): 0.75$ in.

- $\quad$ Maximum Allowable Operating Pressure (MAOP) $=2 * \mathrm{t} * \mathrm{SMYS} * \mathrm{~F} / \mathrm{D}$

- Where design factor $\mathrm{F}=0.72$ for Class 1 locations

0.60 for Class 2 locations

0.50 for Class 3 locations

0.40 for Class 4 locations

A total of four cases were examined, consisting of two different valve spacings at two distinct pressure levels:

1. Maximum 5 mile valve spacing (Class 4), pressure corresponding to a stress of 0.4 SMYS

2. Maximum 5 mile valve spacing (Class 4), pressure corresponding to a stress of 0.72 SMYS

3. Maximum 20 mile valve spacing (Class 1), pressure corresponding to a stress of 0.4 SMYS

4. Maximum 20 mile valve spacing (Class 1), pressure corresponding to a stress of 0.72 SMYS

The maximum credible event in each case assumed failure from a guillotine break at a valve location, so that a pipe segment equal to the full valve spacing in length would be discharging from each side of the opening. 
At 0.40 SMYS:

Pressure $=2(70000 \mathrm{psi})(0.75$ in. $)(0.4) /(36$ in. $)=1167 \mathrm{psi}$

And at 0.72 SMYS:

Pressure $=2(70000 \mathrm{psi})(0.75$ in. $)(0.72) /(36$ in. $)=2100 \mathrm{psi}$

These four combinations of valve spacing and operating pressure provide a good picture of how each factor influences natural gas pipeline safety.

\section{TECHNICAL DEFINITION OF SAFETY CRITERIA}

Based on PHMSA guidance, the standard used to assess the safety for each combination of pipeline parameters in this study is the high consequence hazard area radius defined in 49 CFR 192.903 and derived in the report by Stephens. ${ }^{3}$ Stephens defined the potential hazard area from a jet or trench fire as the radius inside which people and structures could be exposed to radiant heat fluxes exceeding 5000

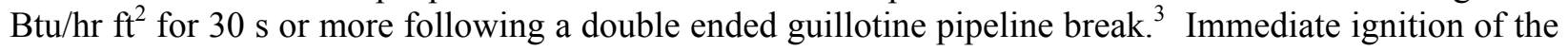
escaping gas is assumed. This safety criterion does not take into account such factors as total burn time, total quantity of gas released, area of service disrupted, or any interference with emergency response. Further discussion of some of these issues are given in Sect. 6.

Carrying through his calculations, Stephens obtained the following expression for the hazard radius, $r$, in feet:

$$
r=0.685\left(P d^{2}\right)^{1 / 2}
$$

where $P$ is the pipeline pressure (in psi) and $d$ is the diameter (in in.). ${ }^{3}$

In order to assess the safety impact of valve spacing, it is necessary to develop a modified version of Eq. (1) adapted to the conditions for each case. The only factor in Stephens' analysis that potentially depends on valve spacing (or the length of the pipe segment undergoing blowdown) is $\lambda(t)$, which measures how rapidly the mass flow rate from the opening decays as a function of time compared to its initial value at time $t=0$.

$$
\text { Thus, } \lambda(t)=\text { Mass flow rate }(\mathrm{t}) / \text { Mass flow rate }(\mathrm{t}=0) \text {. }
$$

Because Stephens based his calculations on the first $30 \mathrm{~s}$ of the transient when the discharge from the broken line is most rapid, the really significant parameter is $\lambda_{\text {avg, }}$ which is defined as the average value of $\lambda(t)$ over the first $30 \mathrm{~s}$ following the break. Stephens was trying to account for a range of pipeline sizes, and he assumed a uniform value of $\lambda_{\text {avg }}=0.33$ when developing Eq. (1). ${ }^{3}$ Furthermore, $\lambda_{\text {avg }}$ enters Eq. (1) inside the parentheses raised to the one-half power, so that a change in $\lambda_{\text {avg }}$ would modify the Stephens formula as follows:

$$
r=0.685\left(P d^{2}\right)^{1 / 2}\left(\lambda_{\text {avg }} / 0.33\right)^{1 / 2}
$$

Thus it is necessary to calculate $\lambda_{\text {avg }}$ values for each of the four valve spacing and pressure combinations in order to find the impact of each factor on pipeline hazard area radius. 


\section{IDEAL GAS ANALYSIS OF CASES}

Determining $\lambda_{\text {avg }}$ for each case requires a transient analysis of the pipe blowdown to find the exit mass flow rate as a function of time. The most straightforward approach is to do this numerically by discretizing the pipe into a set of finite elements. Figure 1 illustrates the nodalization scheme selected, with Node 1 at the exit plane and consecutively numbered axial nodes of equal spacing extending back to the closed end of the pipe. Each nodal point $n$ has an associated pressure, $P_{n}$, temperature, $T_{n}$, flow velocity, $V_{n}$, cell mass, $m_{n}$, and cell density, $\rho_{n}$. Conditions at Node 1 are governed by the requirement that the exit plane Mach number must be equal to 1 (choked discharge). Conditions for the other nodes are governed by the conservation equations and ideal gas equation of state.

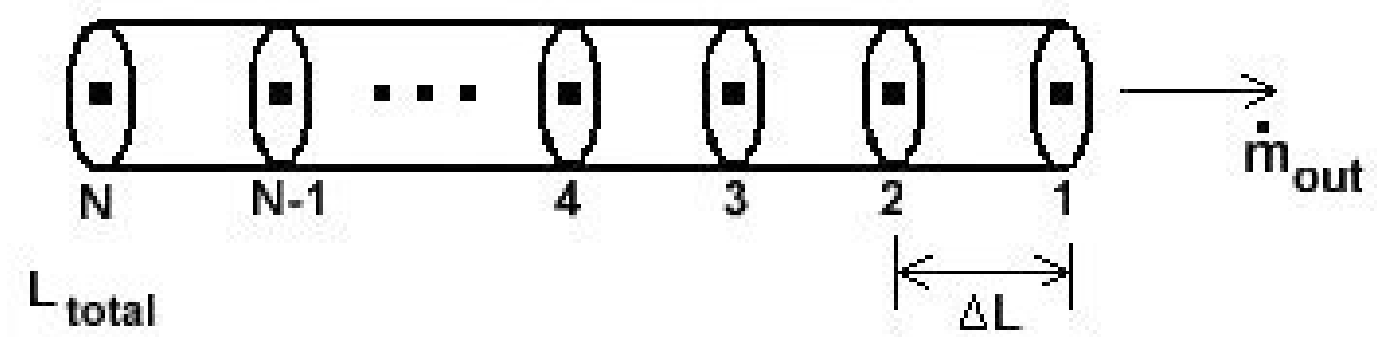

Fig. 1. Sketch of pipeline showing the axial nodalization scheme.

From the conservation of mass

$$
d m_{n} / d t=A_{c}\left(\rho_{n} V_{n}-\rho_{n-1} V_{n-1}\right),
$$

where $A_{c}=(1 / 4) \pi D^{2}$ is the pipe cross-sectional area.

From the conservation of momentum

$$
m_{n} d V_{n} / d t=A_{c}\left[P_{n+1}-P_{n}-f(L / D) \rho_{n} V_{n}^{2} / 2\right],
$$

where $f$ is the pipe flow friction factor (assumed $=0.013$ as an average value) and $L / D$ is the length-todiameter ratio for a nodal element.

From the conservation of energy

$$
m_{n} c_{v} d T_{n} / d t=A_{c}\left[\rho_{n} V_{n}\left(V_{n}^{2} / 2+c_{p} T_{n}\right)-\rho_{n-1} V_{n-1}\left(V_{n-1}^{2} / 2+c_{p} T_{n-1}\right)\right]
$$

in which $c_{v}$ is the gas specific heat at constant volume and $c_{p}$ is the specific heat at constant pressure.

From the definition of density

$$
\rho_{n}=m_{n} /\left[A_{c}(\Delta L)\right]
$$

where $\Delta L$ is the node spacing.

And from the ideal gas equation of state

$$
P_{n}=\rho_{n} R T_{n},
$$

in which $R$ is the gas constant for methane. 
Initially, conditions are at uniform temperature and pressure everywhere except on the exit plane (Node 1), with the nodal velocities equal to zero. Flow at the pipe exit is always choked with a velocity equal to the speed of sound in the gas. The nodal parameters then evolve in time throughout the discharge transient according to the coupled system of differential equations given by Eqs. (4)-(8). The initial gas temperature was taken to be $288 \mathrm{~K}$ for purposes of these calculations.

A FORTRAN computer program was written to solve this system of equations for the discharge transient using fourth order Runge-Kutta integration. The program prints out the discharge mass flow rate and $\lambda(t)$ at each time step, as well as computing an overall value of $\lambda_{\text {avg }}$ for the total transient. A listing of the FORTRAN code is presented in Appendix A.

The FORTRAN Runge-Kutta routine was executed a total of 12 times so that each of the four pressure and valve spacing cases could be processed with three progressively finer node spacings of 1 node/mile, 5 nodes $/$ mile, and 20 nodes/mile of pipe. On each run, $\lambda_{\text {avg }}$ was calculated for the first $30 \mathrm{~s}$ of the transient and the results are summarized in Table 1. A complete record of all the code output from these runs has been collected in Appendix B.

Table 1. Average $\lambda$ values over the first $30 \mathrm{~s}$ as calculated by the FORTRAN program

\begin{tabular}{|c|c|c|c|c|}
\hline $\begin{array}{c}\text { Valve spacing } \\
\text { length }\end{array}$ & Pressure & $\begin{array}{c}\text { Nodes/mile } \\
\text { of pipe }\end{array}$ & $\begin{array}{l}\text { Total number } \\
\text { of axial nodes }\end{array}$ & $\lambda_{\text {avg }}$ \\
\hline \multirow{6}{*}{5 miles } & \multirow{3}{*}{1167 psi } & 1 & 5 & 0.333059520 \\
\hline & & 5 & 25 & 0.313054979 \\
\hline & & 20 & 100 & 0.297197551 \\
\hline & \multirow{3}{*}{$2100 \mathrm{psi}$} & 1 & 5 & 0.332404822 \\
\hline & & 5 & 25 & 0.310781360 \\
\hline & & 20 & 100 & 0.284179538 \\
\hline \multirow{6}{*}{20 miles } & \multirow{3}{*}{$1167 \mathrm{psi}$} & 1 & 20 & 0.333737999 \\
\hline & & 5 & 100 & 0.313054979 \\
\hline & & 20 & 400 & 0.297197551 \\
\hline & \multirow{3}{*}{$2100 \mathrm{psi}$} & 1 & 20 & 0.333029151 \\
\hline & & 5 & 100 & 0.310781360 \\
\hline & & 20 & 400 & 0.284179538 \\
\hline
\end{tabular}

As can be seen in Table 1, the value of $\lambda_{\text {avg }}$ decreased slightly as the number of axial nodes along the pipe was increased for each combination of valve spacing and pressure. This effect tapered off for the finest mesh of 20 nodes/mile, indicating that further refinement of the mesh was probably not necessary. The higher line pressure of 2100 psi was associated with somewhat lower $\lambda_{\text {avg }}$ values than calculated for the corresponding $1167 \mathrm{psi}$ cases. However the difference in $\lambda_{\text {avg }}$ due to line pressure is probably too small to be important from a pipeline safety perspective. From the results in Table 1, the assumption of $\lambda_{\text {avg }}=0.33$ as used by Stephens ${ }^{3}$ is considered to be an excellent bounding value for this size of pipeline and pressure range.

Finally, it is clear from comparing the 5 mile and 20 mile cases in Table 1 that valve spacing has no significant effect on $\lambda_{\text {avg }}$ for the range of parameters being studied. Even with the coarse mesh of 1 node/mile, differences in the calculated $\lambda_{\text {avg }}$ between the two spacings do not appear until the third or fourth decimal place. For the two finer mesh runs, there was absolutely no influence of 5-mile vs 20-mile spacing on $\lambda$ when averaged over the first $30 \mathrm{~s}$ of the discharge. This result occurred because $30 \mathrm{~s}$ did not allow enough time for the transient depressurization effects to propagate 5 miles back into the pipe with a fine node spacing, so that the numerical simulations of the 5-mile and 20-mile discharge cases were 
identical throughout this initial period. Two special check cases run with the Runge-Kutta FORTRAN program that averaged $\lambda$ out to $60 \mathrm{~s}$ instead of $30 \mathrm{~s}$ were able to detect slight differences in $\lambda_{\text {avg }}$ between the 5-mile and 20 -mile valve spacings with a fine node mesh.

Table 2 illustrates the effect of using the $\lambda_{\text {avg }}$ values from Table 1 to modify the Stephens formula for hazard area radius from a natural gas pipeline accident. This table also indicates a minimal influence of valve spacing on the hazard area radius. Comparing the 5 and 20-mile valve spacing cases in Table 2 shows that the greatest difference calculated for the hazard radius is about $1 \mathrm{ft}$ for the coarsest mesh, and most of the corresponding hazard radius values for the two valve spacings are identical.

Table 2. Hazard area radius for each case

\begin{tabular}{|c|c|c|c|c|}
\hline $\begin{array}{l}\text { Valve spacing } \\
\text { length }\end{array}$ & Pressure & $\begin{array}{c}\text { Nodes/mile } \\
\text { of pipe }\end{array}$ & Modified Stephens formula & $\begin{array}{l}\text { Hazard area } \\
\text { radius }\end{array}$ \\
\hline \multirow{6}{*}{5 miles } & \multirow{3}{*}{1167 psi } & 1 & $r=0.685\left(P d^{2}\right)^{1 / 2}(0.333059520 / 0.33)^{1 / 2}$ & $846.316 \mathrm{ft}$ \\
\hline & & 5 & $r=0.685\left(P d^{2}\right)^{1 / 2}(0.313054979 / 0.33)^{1 / 2}$ & $820.506 \mathrm{ft}$ \\
\hline & & 20 & $r=0.685\left(P d^{2}\right)^{1 / 2}(0.297197551 / 0.33)^{1 / 2}$ & $799.455 \mathrm{ft}$ \\
\hline & \multirow{3}{*}{$2100 \mathrm{psi}$} & 1 & $r=0.685\left(P d^{2}\right)^{1 / 2}(0.332404822 / 0.33)^{1 / 2}$ & $1134.173 \mathrm{ft}$ \\
\hline & & 5 & $r=0.685\left(P d^{2}\right)^{1 / 2}(0.310781360 / 0.33)^{1 / 2}$ & $1096.663 \mathrm{ft}$ \\
\hline & & 20 & $r=0.685\left(P d^{2}\right)^{1 / 2}(0.284179538 / 0.33)^{1 / 2}$ & $1048.678 \mathrm{ft}$ \\
\hline \multirow{6}{*}{20 miles } & \multirow{3}{*}{1167 psi } & 1 & $r=0.685\left(P d^{2}\right)^{1 / 2}(0.333737999 / 0.33)^{1 / 2}$ & $847.177 \mathrm{ft}$ \\
\hline & & 5 & $r=0.685\left(P d^{2}\right)^{1 / 2}(0.313054979 / 0.33)^{1 / 2}$ & $820.506 \mathrm{ft}$ \\
\hline & & 20 & $r=0.685\left(P d^{2}\right)^{1 / 2}(0.297197551 / 0.33)^{1 / 2}$ & $799.455 \mathrm{ft}$ \\
\hline & \multirow{3}{*}{2100 psi } & 1 & $r=0.685\left(P d^{2}\right)^{1 / 2}(0.333029151 / 0.33)^{1 / 2}$ & $1135.238 \mathrm{ft}$ \\
\hline & & 5 & $r=0.685\left(P d^{2}\right)^{1 / 2}(0.310781360 / 0.33)^{1 / 2}$ & $1096.663 \mathrm{ft}$ \\
\hline & & 20 & $r=0.685\left(P d^{2}\right)^{1 / 2}(0.284179538 / 0.33)^{1 / 2}$ & $1048.678 \mathrm{ft}$ \\
\hline
\end{tabular}

\section{REAL GAS AND DEPRESSURIZATION PIPE COOLING EFFECTS}

A break in a natural gas pipeline normally leads to significant cooling of the gas as it depressurizes. There is some concern that this cooling could affect the operating characteristics of shut-off valves and make it more difficult to isolate the break. As shown by the Texas Eastern Transmission Corporation (TETCO) pipeline explosion at Edison, New Jersey on March 23, 1994, the inability to shut off the gas flow quickly because of problems with the closest upstream valve can substantially increase property damage from the ensuing fire. ${ }^{4}$ In this accident, the break was not isolated until a valve further upstream was closed $2.5 \mathrm{~h}$ after the pipeline ruptured. A specific cause for TETCO's difficulty with the closer valve was never identified. ${ }^{4}$ However PHMSA is interested in determining whether pipe cooling effects could become a contributing factor in such situations.

An analysis of pipe cooling must be based on real gas properties and a more sophisticated approach than the ideal gas model used in Sect. 4. For an ideal gas, the Joule-Thomson coefficient, $\mu_{J}=(\partial T / \partial P)_{h}$, is zero because enthalpy is only a function of temperature, and thus there is no change in temperature with depressurization at constant enthalpy. ${ }^{5}$ In the methane pressure and temperature region of interest, the Joule-Thomson coefficient is predominantly positive, so that the gas temperature typically decreases with depressurization, even for isenthalpic processes. For a pipeline failure, there will also be a drop in enthalpy because the flow velocity increases as it approaches the break. This conversion of enthalpy to kinetic energy as the flow accelerates contributes an additional cooling effect. The analysis also must take into account that real gases can condense to form liquid droplets if sufficiently cold temperatures occur during the depressurization event. Pressure and temperature combinations where such phase changes occur are defined by the saturation line conditions, as summarized in Table 3 for methane. ${ }^{5}$ Table 3 also includes the vapor enthalpy, $h_{g}$, and enthalpy difference accompanying the phase change, $h_{f g}$, 
that correspond to each point on the saturation line. All this information has been incorporated into a modified version of the Runge-Kutta FORTRAN program using tabular interpolation functions.

Table 3. Saturation line properties for methane

\begin{tabular}{|l|l|l|l|l|l|l|l|}
\hline $\mathrm{P}_{\text {sat }}$ & $0.1 \mathrm{MPa}$ & $0.5 \mathrm{MPa}$ & $1.0 \mathrm{MPa}$ & $2.0 \mathrm{MPa}$ & $3.0 \mathrm{MPa}$ & $4.0 \mathrm{MPa}$ & $4.64 \mathrm{MPa}$ \\
\hline $\mathrm{T}_{\text {sat }}(\mathrm{K})$ & 114.47 & 135.32 & 149.11 & 165.84 & 177.25 & 186.09 & 191.1 \\
\hline $\mathrm{h}_{\mathrm{fg}}(\mathrm{kJ} / \mathrm{kg})$ & 511.49 & 458.63 & 415.32 & 341.92 & 268.55 & 175.04 & 0 \\
\hline $\mathrm{h}_{\mathrm{g}}(\mathrm{kJ} / \mathrm{kg})$ & 796.71 & 830.23 & 841.11 & 840.58 & 825.21 & 791.11 & 791.11 \\
\hline
\end{tabular}

To include real gas effects and wall heat flow, the ideal gas conservation of energy requirement from Eq. (6) must be reformulated in terms of flow enthalpy and the convective heat transfer at the pipe wall.

$$
m_{n} c_{v} d T_{n} / d t=A_{c}\left[\rho_{n+1} V_{n+1}\left(V_{n+1}^{2} / 2+h_{n+1}\right)-\rho_{n} V_{n}\left(V_{n}^{2} / 2+h_{n}\right)\right]+h_{c o n v}(\pi D(\Delta L))\left(T_{w, n}-T_{n}\right),
$$

in which $h_{n}$ and $h_{n+1}$ are the flow enthalpies, $h_{c o n v}$ is the convective heat transfer coefficient, and $T_{w, n}$ is the wall temperature at the node.

A similar differential equation can be written for the wall temperature at the node as a function of time by using an energy balance on the pipe element.

$$
m_{\text {pipe }} c_{\text {pipe }} d T_{w} / d t=-h_{\text {conv }}(\pi D(\Delta L))\left(T_{w, n}-T_{n}\right)
$$

where $m_{\text {pipe }}$ is the mass of the steel pipe annulus and $c_{\text {pipe }}$ is its specific heat. No attempt is made in this analysis to account for the greater thermal mass that is likely to be present in the neighborhood of a valve, conduction to the pipe from any surrounding soil, or internal heat transfer cooling of the valve lubricant.

The convective heat transfer coefficient, $h_{\text {conv }}$, can be evaluated from a correlation in terms of the Nusselt number $N u_{D}:^{6}$

$$
\text { For } R e \leq 2300, N u_{D}=h_{\text {conv }} D / k_{f}=3.66
$$

and

$$
\text { For } R e>2300, \quad N u_{D}=h_{\text {conv }} D / k_{f}=0.023 \operatorname{Re}^{0.8} \operatorname{Pr}^{0.4},
$$

where $R e=\rho V D / \mu$ is the Reynolds number, $\mu$ is the gas viscosity, $k_{f}$ is its thermal conductivity, and $\operatorname{Pr}$ is the Prandtl number. The 0.4-power dependence of $h_{\text {conv }}$ on $P r$ is rather weak, and a constant average value of $\operatorname{Pr}=0.714$ for methane will be assumed throughout this analysis. Vargaftik, et al., ${ }^{7}$ provides thermophysical properties for methane including the enthalpy, viscosity, and thermal conductivity tabulated as functions of temperature and pressure. These thermophysical property tables were also coded in FORTRAN so they are available to the Runge-Kutta equation solver.

When the gas in a cell volume is at saturation conditions, heat transfer leads to changes in the vapor mass fraction or quality, $X_{n}$, rather than to a change in the sensible temperature $T_{n}$. Thus Eq. (9) describing the conservation of energy for these cells is replaced by: 


$$
\begin{aligned}
h_{f g} d X_{n} / d t & =A_{c}\left[\rho_{n+1} V_{n+1}\left(V_{n+1}^{2} / 2+h_{g}\left(P_{n+1}\right)-\left(1-X_{n+1}\right) h_{f g}\left(P_{n+1}\right)\right)\right. \\
& \left.-\rho_{n} V_{n}\left(V_{n}^{2} / 2+h_{g}\left(P_{n}\right)-\left(1-X_{n}\right) h_{f g}\left(P_{n}\right)\right)\right]+h_{\text {conv }}(\pi D(\Delta L))\left(T_{w, n}-T_{n}\right),
\end{aligned}
$$

in which $h_{g}\left(P_{n+1}\right)$ and $h_{f g}\left(P_{n+1}\right)$ are the saturated vapor enthalpy and latent enthalpy of vaporization at pressure $P_{n+1}$ while $h_{g}\left(P_{n}\right)$ and $h_{f g}\left(P_{n}\right)$ are the corresponding properties at pressure $P_{n}$. An enhanced Runge-Kutta FORTRAN routine has been written to solve Eqs. (9)-(13) together with Eqs. (4)-(5) and (7)-(8) from Sect. 4 for a pipeline discharge transient. Initially, conditions are assumed to be at uniform pressure and a temperature of $288 \mathrm{~K}$ for both the gas and the pipe wall. The program prints out the gas temperature, gas pressure, and pipe wall temperature for all the axial nodes at each time step, as well as computing an overall $\lambda_{\text {avg }}$ value that can be compared to that obtained in Sect. 4 with an ideal gas analysis for the transient. A listing of the updated FORTRAN code incorporating real gas effects is given in Appendix C.

For specific cases to test the updated FORTRAN code, it will be assumed that the pipeline compressor stations are located 60 miles apart and are set to trip on abnormal flow conditions. Thus the length of pipe between a compressor station and the failure zone from which gas is discharging may be anything up to 60 miles depending on the break location. Cases to be analyzed will consist of three different compressor station standoff distances ( 5 miles, 20 miles, and 60 miles) at each of the two initial line pressures of interest (1167 psi and 2100 psi). The updated FORTRAN Runge-Kutta routine was executed a total of 15 times involving progressively finer nodalizations for each of the six combinations of pressure and compressor station standoff distance. The cases with an initial pressure of 1167 psi were run with three different node spacings and the 2100 psi cases with only two because the finest nodalization led to some numerical instabilities which produced unreliable code output. On each run, a value of $\lambda_{\text {avg }}$ was calculated for comparison with the ideal gas analysis results from Sect. 4. In addition, results for outlet gas temperature and pipe wall cooling were obtained. Complete code outputs for the 5-mile compressor station distance cases have been collected in Appendix D. Since the fine-mesh code printouts from a calculation with 20-mile or 60-mile compressor station standoff are quite voluminous, only the 1-node/mile output is included in Appendix D to keep this report to a manageable size. Key features for all 15 of the FORTRAN code runs are presented in Table 4 on the following page. Numerical results for the 20-mile and 60-mile standoff cases proved to be identical, so there was no need to run any calculations for intermediate distances. 
Table 4. Real gas and pipe wall cooling results

\begin{tabular}{|c|c|c|c|c|c|c|c|}
\hline \multirow{2}{*}{$\begin{array}{l}\text { Dist. to } \\
\text { compressor } \\
\text { station }\end{array}$} & \multirow{2}{*}{ Pressure } & \multirow{2}{*}{$\begin{array}{l}\text { Nodes/mile } \\
\text { of pipe }\end{array}$} & \multirow{2}{*}{$\begin{array}{c}\text { Total } \\
\text { number of } \\
\text { axial nodes }\end{array}$} & \multicolumn{3}{|c|}{ Temperature results at $t=30$ seconds $s^{1}$} & \multirow{2}{*}{$\lambda_{\text {avg }}$} \\
\hline & & & & $\begin{array}{c}\text { Outlet } \\
\mathrm{T}\end{array}$ & $\begin{array}{l}\text { Outlet } \\
\mathrm{T}_{\mathrm{w}}\end{array}$ & $\begin{array}{c}\text { Dist. upstream } \\
\mathrm{T}_{\mathrm{w}}<228 \mathrm{~K} \\
\left(-50^{\circ} \mathrm{F}\right)\end{array}$ & \\
\hline \multirow{5}{*}{5 miles } & \multirow{3}{*}{1167 psi } & 1 & 5 & $124.808 \mathrm{~K}$ & $166.411 \mathrm{~K}$ & 2 miles & 0.314291954 \\
\hline & & 5 & 25 & $128.466 \mathrm{~K}$ & $164.178 \mathrm{~K}$ & 1.40 miles & 0.248135760 \\
\hline & & 10 & 50 & $129.150 \mathrm{~K}$ & $163.138 \mathrm{~K}$ & 0.90 miles & 0.245157793 \\
\hline & \multirow[b]{2}{*}{$2100 \mathrm{psi}$} & 1 & 5 & $136.327 \mathrm{~K}$ & $155.239 \mathrm{~K}$ & 3 miles & 0.315512955 \\
\hline & & 5 & 25 & $139.954 \mathrm{~K}$ & $154.777 \mathrm{~K}$ & 1.20 miles & 0.248590544 \\
\hline \multirow{5}{*}{20 miles } & \multirow{3}{*}{1167 psi } & 1 & 20 & $126.836 \mathrm{~K}$ & $165.555 \mathrm{~K}$ & 2 miles & 0.319210589 \\
\hline & & 5 & 100 & $128.311 \mathrm{~K}$ & $164.201 \mathrm{~K}$ & 1.40 miles & 0.248050004 \\
\hline & & 10 & 200 & $129.579 \mathrm{~K}$ & $163.103 \mathrm{~K}$ & 1.0 miles & 0.245297492 \\
\hline & \multirow[b]{2}{*}{$2100 \mathrm{psi}$} & 1 & 20 & $138.837 \mathrm{~K}$ & $155.608 \mathrm{~K}$ & 2 miles & 0.320041120 \\
\hline & & 5 & 100 & $140.009 \mathrm{~K}$ & $154.778 \mathrm{~K}$ & 1.20 miles & 0.248610169 \\
\hline \multirow{5}{*}{60 miles } & \multirow{3}{*}{1167 psi } & 1 & 60 & $126.836 \mathrm{~K}$ & $165.555 \mathrm{~K}$ & 2 miles & 0.319210589 \\
\hline & & 5 & 300 & $128.311 \mathrm{~K}$ & $164.201 \mathrm{~K}$ & 1.40 miles & 0.248050004 \\
\hline & & 10 & 600 & $129.579 \mathrm{~K}$ & $163.103 \mathrm{~K}$ & 1.0 miles & 0.245297492 \\
\hline & \multirow[b]{2}{*}{$2100 \mathrm{psi}$} & 1 & 60 & $138.837 \mathrm{~K}$ & $155.608 \mathrm{~K}$ & 2 miles & 0.320041120 \\
\hline & & 5 & 300 & $140.009 \mathrm{~K}$ & $154.778 \mathrm{~K}$ & 1.20 miles & 0.248610169 \\
\hline
\end{tabular}

${ }^{\mathrm{l}}$ Note: $124.808 \mathrm{~K}=-235^{\circ} \mathrm{F}, 166.411 \mathrm{~K}=-160^{\circ} \mathrm{F}$

Table 4 and the code printouts from Appendix D show that the gas temperatures at the pipe outlet do not drop below the atmospheric boiling point of about $111.4 \mathrm{~K}$ for methane during the initial $30 \mathrm{~s}$ modeled for any of the transients. At the higher pressures present back inside the pipe, gas temperatures cold enough to reach saturation conditions do occur. In Appendix D, all runs achieved saturation conditions in one or more nodes near the exit, with vapor qualities generally in the $90 \%$ or greater range. This fact highlights the need to consider real gas properties in such a blowdown analysis to obtain rigorous results. However comparison of the $\lambda_{\text {avg }}$ values between Table 1 and Table 4 indicates only a small decrease in $\lambda_{\text {avg }}$ for each case when real gas effects are included. The Stephens assumption of $\lambda_{\text {avg }}=0.33$ remains an excellent bounding value over the first $30 \mathrm{~s}$ of a pipeline depressurization transient for calculating the hazard area radius of the resulting fire.

The pipe wall temperature results in Table 4 indicate the presence of significant cooling extending some distance back from the break point within $30 \mathrm{~s}$ of the break. Some valve lubricants and sealants for low temperature service are certified down to operating temperature of $-70^{\circ} \mathrm{F}(216 \mathrm{~K})$, although $-40^{\circ} \mathrm{F}$ $(233 \mathrm{~K})$ seems to be a more common minimum. ${ }^{8-10}$ In Table $4,-50{ }^{\circ} \mathrm{F}(228 \mathrm{~K})$ is selected as an average minimum, and the distance from the break for which $T_{w}<228 \mathrm{~K}$ at $t=30 \mathrm{~s}$ is noted. This distance is always in the neighborhood of 1 mile back along the pipe, even for the finest mesh runs. Clearly, any shut-off valves within this zone might experience problems closing due to excessively low lubricant temperatures. Some conduction of heat from the surrounding soil and the resistance to internal heat transfer within the valve would both limit cooling of the lubricant to some extent, but a sophisticated heat transport analysis would be needed to quantify these effects. There is no particular change in the upstream distance calculated for the low-temperature zone in Table 4 whether the pipe discharging length back upstream to a compressor pumping station is 5 miles or 60 miles. Reducing the shut-off valve spacing between pumping stations from 20 miles to 5 miles would actually increase the probability that the first upstream shut-off valve will be in the low-temperature zone associated with depressurization cooling from a break. However a shorter valve spacing would also increase the number of redundant valves available outside the low temperature zone for isolating the leak. 
It is not believed that having moderate levels of impurities, such as propane or mercaptan, in addition to methane in the pipeline would appreciably change these results for depressurization cooling. The cooling effect is governed by the overall thermophysical properties of the mixture, which will not differ significantly from those of pure methane unless impurities constitute a sizable mole fraction of the material. In addition, most impurities like ethane or propane have heavier molecular weights and higher boiling points than methane, so they would tend to condense out of the mixture preferentially as they approached the break point. Nitrogen impurities (normal boiling point $77 \mathrm{~K}$ ) are noncombustible and even more difficult to condense than methane, but if present in sufficient concentrations they might interfere somewhat with the condensation of other gases in the mixture.

\section{DISCUSSION OF RESULTS}

Based solely on adaptation of the Stephens safety criteria, valve spacing appears to have a negligible influence on pipeline safety for the pipeline diameter, pressure range, and valve spacings considered in this scoping study. However, it should be emphasized that factors besides the Stephens criteria can also have a bearing on safety and potential economic losses in a pipeline accident. Longer valve spacings will certainly increase the amount of gas that would be unavoidably vented during a line rupture, although the gas flow rate would be constantly decreasing with time compared to the average value over the first $30 \mathrm{~s}$. The duration of any resulting fire and the period of danger to emergency response personnel would also increase correspondingly. The total integrated heat flux from such a fire, even at reduced gas burn rates, could contribute to additional property damage or hinder emergency response to the accident. In addition, a longer valve spacing would likely lead to service being disrupted over a larger area during repairs following an accident. Economic considerations associated with losing a greater quantity of gas should also be taken into account.

Pipe cooling effects potentially sufficient to interfere with shut-off valve function can extend over a mile upstream from the rupture point. Because valve operation could be adversely impacted by the cooling effect of the flowing gas, installation of additional valves located outside the low-temperature zone may be beneficial from a safety viewpoint. These factors, as well as the Stephens safety criteria, should all be considered to determine the optimum valve spacing for a natural gas pipeline. 


\section{CONCLUSIONS}

Using an adaptation of the Stephens hazard radius criteria, valve spacing has a negligible influence on natural gas pipeline safety for the pipeline diameter, pressure range, and valve spacings considered in this study.

Over the first $30 \mathrm{~s}$ of the transient, pipeline pressure has a far greater effect on the hazard radius calculated with the Stephens criteria than any variations in the transient flow decay profile and the average discharge rate.

Other factors besides the Stephens criteria, such as the longer burn time for an accidental fire, greater period of danger to emergency personnel, increased unavoidable loss of gas, and possible depressurization cooling of the shut-off valves may also be important when deciding whether a change in the required valve spacing would be beneficial from a safety standpoint.

The average normalized discharge rate of $\lambda_{\text {avg }}=0.33$ assumed by Stephens in developing his safety criteria is an excellent conservative value for natural gas discharge at the pressures, valve spacings, and pipe diameter used in this study. This conclusion remains valid even when real rather than ideal gas properties are considered in the analysis.

Significant pipe wall cooling effects $\left(T_{w}<-50^{\circ} \mathrm{F}\right.$ or $\left.228 \mathrm{~K}\right)$ can extend for a mile or more upstream from the rupture point within $30 \mathrm{~s}$ of a break. These conditions are colder than the temperature range specifications for many valve lubricants. The length of the low-temperature zone due to this cooling effect is also essentially independent of the system shut-off valve spacing or the distance between the break and a compressor station.

Having more redundant shut-off valves available would reduce the probability that pipe cooling effects could interfere with isolating the broken area following a pipeline rupture accident.

\section{REFERENCES}

1. T. D. Boss, Request for Clarification-Valve Proximity and Class Location Changes, INGAA, April 3, 2006.

2. INGAA Pipeline Safety Committee, White Paper on Equivalent Safety for Alternative Valve Spacing, Prepared by Process Performance Improvement Consultants, LLC, November 15, 2005.

3. M. J. Stephens, A Model for Sizing High Consequence Areas Associated with Natural Gas Pipelines, Topical Report prepared for the Gas Research Institute by C-FER Technologies, GRI-00/0189, October 2000.

4. National Transportation Safety Board, Texas Eastern Transmission Corporation Natural Gas Pipeline Explosion and Fire, Edison, New Jersey, March 23, 1994, PB95-916501, Washington, DC, January 18, 1995.

5. G. J. Van Wylen and R. E. Sonntag, Fundamentals of Classical Thermodynamics, $3^{\text {rd }}$ Ed., John Wiley \& Sons, Inc., New York, NY, 1985.

6. F. P. Incropera and D. P. DeWitt, Fundamentals of Heat and Mass Transfer, $3^{\text {rd }}$ Ed., John Wiley \& Sons, Inc., New York, NY, 1990.

7. N. B. Vargaftik, Y. K. Vinogradov, and V. S. Yargin, Handbook of Physical Properties of Liquids and Gases, Begell House, Inc., New York, NY, 1996.

8. Lubchem Inc. website, http://www.lubchem.com/sc formasil_sealants.html.

9. Huskey Specialty Lubricants website, $h \mathrm{htp}: / / \mathrm{www}$.huskey.com/h valve.html.

10. Flowserve Corporation website, http://www.flowserve.com/eim/v/index.jsp?vgnextoid=c6d81cc6159f8010VgnVCM100000 5e191eacRCRD\&vgnextfmt=default. 


\section{Appendix A. IDEAL GAS RUNGE-KUTTA FORTRAN CODE LISTING}

FORTRAN program to solve the differential equations

for blowdown of a methane pipeline

using the fourth order Runge-Kutta method.

Integer $i, n$, nsteps, kcount, lsteps

real h, k1m, k2m, k3m, k4m, tbegin, tn, tend, Pini

real k1V, k2V, k3V, k4V

real k1T, k2T, k3T, k4T

real k1rh, k2rh, k3rh, k4rh

real k1P, k2P, k3P, k4P

real Ltot, 1, LoverD, mdot, mdot $\odot$, msum, lambda, lambavg

real $\mathrm{m}(2000), \mathrm{V}(2000), \mathrm{T}(2000)$, $\mathrm{rho}(2000), \mathrm{P}(2000)$

real mprev(2000)

real gamma, machn2, Ptot2, Ttot2, Tinit

write $\left({ }^{*},{ }^{*}\right)$ 'Enter tbegin, tend, nsteps'

1 read $(*, *)$ tbegin, tend, nsteps

write $\left(*,{ }^{*}\right)$ 'Enter Pinit(psi), Ltot(miles), lsteps'

$2 \operatorname{read}\left(*,{ }^{*}\right)$ Pinit, Ltot, lsteps

open $(2, \mathrm{file}=$ 'rungeout. $\mathrm{t} x \mathrm{t}$ ', status='new' $)$

msum $=0.0$

$\mathrm{h}=$ (tend - tbegin)/nsteps

$1=$ Ltot* $^{*}(5280 / 3.2808) /$ lsteps

LoverD $=($ Ltot $* 5280 * 12.0 /$ lsteps $) / 36.0$

tn = tbegin

pi $=3.14159$

$f=0.013$

$\mathrm{R}=518.2$

Tinit $=288.0$

gamma $=1.32$

$\mathrm{cp}=2223.0$

$\mathrm{cV}=1704.8$

$A C=0.25^{*} \mathrm{pi}^{*}(36 / 39.37) * * 2$

Pini $=$ Pinit* $(101325 / 14.7)$

rhoinit $=$ Pini/ $\left(R^{*}\right.$ Tinit $)$

$P(1)=$ Pini*0.5421

$T(1)=$ Tinit $^{*} 0.8621$

$V(1)=\left(1.32{ }^{*} R^{*} T(1)\right) * * 0.5$

$\mathrm{rho}(1)=\mathrm{P}(1) /\left(\mathrm{R}^{*} \mathrm{~T}(1)\right)$

$\mathrm{m}(1)=r h o(1) * A c^{*} 1$

do $5 i=2$, lsteps

$P(i)=$ Pini

$T(i)=$ Tinit

$V(i)=0.0$

$\operatorname{rho}(i)=P(i) /\left(R^{*} T(i)\right)$

$m(i)=r h o(i) * A c^{*} l$

5 continue

$\operatorname{mprev}(i)=\operatorname{rho}(i){ }^{*} A c^{*} 1$

$i=0$

mdot $=r h o(1) * V(1) * A c$

msum $=$ msum + mdot

$\operatorname{mdot} \odot=\operatorname{mdot}$

lambda $=\mathrm{mdot} / \mathrm{mdot} \odot$

write (*,701)'i', 'time (sec)', 'P1 (Pa)', 'T1 (K)',

\&

'V1 (m/sec)',' 'mdot (kg/sec)', 'lambda'

write $(2,701)$ 'i', 'time (sec)', 'P1 (Pa)', 'T1 (K)', 
$\&$

'V1 (m/sec)', 'mdot (kg/sec)', 'lambda'

701 format $(4 x, A 1,6 x, A 10,4 x, A 7,8 x, A 6,7 x, A 10,4 x, A 13,6 x, A 6, /)$ write $(*, 601) i, \mathrm{tn}, \mathrm{P}(1), \mathrm{T}(1), \mathrm{V}(1), \mathrm{mdot}, \mathrm{lambda}$ write $(2,601) i, t n, P(1), T(1), V(1)$, mdot, lambda

601 format $(1 \mathrm{x}, \mathrm{i} 6,5 \mathrm{x}, \mathrm{f} 6.2,4 \mathrm{e} 15.4,6 \mathrm{x}, \mathrm{f} 11.9)$

602 format $(20 x, 3 e 12.4)$

kcount $=0$

do 10 i $=1$, nsteps

machn2 $=\mathrm{V}(2) /\left(\right.$ gamma $\left.{ }^{*} \mathrm{R}^{*} \mathrm{~T}(2)\right)$

Ptot2 $=\mathrm{P}(2) *(1+($ gamma -1$) *($ machn $2 * * 2) / 2) * *($ gamma $/($ gamma -1$))$

Ttot2 $=\mathrm{T}(2) *(1+($ gamma -1$) *(\operatorname{machn} 2 * * 2) / 2)$

$\mathrm{P}(1)=\mathrm{P}(2) /((1+($ gamma -1$) / 2) * *($ gamma $/($ gamma -1$)))$

$\mathrm{T}(1)=\mathrm{T}(2) /(1+($ gamma -1$) / 2)$

$\mathrm{V}(1)=\left(\right.$ gamma $\left.{ }^{*} \mathrm{~T}(1)\right) * * 0.5$

$\operatorname{rho}(1)=\mathrm{P}(1) /\left(\mathrm{R}^{*} \mathrm{~T}(1)\right)$

do $7 \mathrm{n}=2$, lsteps

$\mathrm{k} 1 \mathrm{~m}=h^{*} \operatorname{dmass}(\mathrm{rho}(\mathrm{n}-1), \mathrm{V}(\mathrm{n}-1), \quad r h o(n), V(n), A c)$

If $(n$. ne.lsteps $) k 1 V=h{ }^{*} \operatorname{vel}(m(n), P(n+1), P(n), \operatorname{rho}(n), V(n)$, $f$, LoverD, Ac )

$\mathrm{k} 1 \mathrm{~T}=h^{*} \operatorname{temp}(m(n), T(n-1), T(n), r h o(n-1), r h o(n), V(n-1), V(n)$, $A c, c p, c v)$

$\mathrm{k} 2 \mathrm{~m}=\mathrm{h}^{*} \operatorname{dmass}(\mathrm{rho}(\mathrm{n}-1), \mathrm{V}(\mathrm{n}-1), \mathrm{rho}(\mathrm{n})+\mathrm{k} 1 \mathrm{rh} / 2, \mathrm{~V}(\mathrm{n})+\mathrm{k} 1 \mathrm{~V} / 2, A c)$

If $\left(n\right.$.ne.lsteps) $k 2 V=h{ }^{*} \operatorname{vel}(m(n)+k 1 m / 2, P(n+1), P(n)+k 1 P / 2$, rho $(\mathrm{n})+\mathrm{k} 1 \mathrm{rh} / 2$, $\mathrm{V}(\mathrm{n})+\mathrm{k} 1 \mathrm{~V} / 2, \mathrm{f}$, LoverD, $A \mathrm{c})$

$\&$

\&

$\mathrm{k} 2 \mathrm{~T}=\mathrm{h}{ }^{*} \operatorname{temp}(\mathrm{m}(\mathrm{n})+\mathrm{k} 1 \mathrm{~m} / 2, \mathrm{~T}(\mathrm{n}-1), \mathrm{T}(\mathrm{n})+\mathrm{k} 1 \mathrm{~T} / 2, \mathrm{rho}(\mathrm{n}-1)$, $r h o(n)+k 1 r h / 2, V(n-1), V(n)+k 1 V / 2, A c, c p, c v)$

$\mathrm{k} 3 \mathrm{~m}=\mathrm{h} * \operatorname{dmass}(r h o(n-1), \mathrm{V}(\mathrm{n}-1), \operatorname{rho}(\mathrm{n})+\mathrm{k} 2 \mathrm{rh} / 2, \mathrm{~V}(\mathrm{n})+\mathrm{k} 2 \mathrm{~V} / 2, A c)$

If $\left(n\right.$. ne.lsteps) $k 3 V=h{ }^{*} \operatorname{vel}(m(n)+k 2 m / 2, P(n+1), P(n)+k 2 P / 2$, rho $(\mathrm{n})+\mathrm{k} 2 \mathrm{rh} / 2$, $\mathrm{V}(\mathrm{n})+\mathrm{k} 2 \mathrm{~V} / 2, \mathrm{f}$, LoverD, Ac )

$\mathrm{k} 3 \mathrm{~T}=\mathrm{h}^{*} \operatorname{temp}(\mathrm{m}(\mathrm{n})+\mathrm{k} 2 \mathrm{~m} / 2, \mathrm{~T}(\mathrm{n}-1), \mathrm{T}(\mathrm{n})+\mathrm{k} 2 \mathrm{~T} / 2, \mathrm{rho}(\mathrm{n}-1)$, $r h o(n)+k 2 r h / 2, V(n-1), V(n)+k 2 V / 2, A c, c p, c v)$

$k 4 m=h * d m a s s(r h o(n-1), V(n-1), r h o(n)+k 3 r h, V(n)+k 3 V, A c)$

If $\left(n\right.$. ne.lsteps)k4V $=h^{*} \operatorname{vel}(m(n)+k 3 m, P(n+1), P(n)+k 3 P$, rho $(n)+k 3 r h$, $\mathrm{V}(\mathrm{n})+\mathrm{k} 3 \mathrm{~V}, \mathrm{f}$, LoverD, Ac )

$\&$

$\&$

$k 4 T=h * t e m p(m(n)+k 3 m, T(n-1), T(n)+k 3 T, r h o(n-1), r h o(n)+k 3 r h$, $\mathrm{V}(\mathrm{n}-1), \mathrm{V}(\mathrm{n})+\mathrm{k} 3 \mathrm{~V}, \mathrm{Ac}, \mathrm{cp}, \mathrm{cv})$

$m(n)=m(n)+(k 1 m+2 * k 2 m+2 * k 3 m+k 4 m) / 6.0$

If $(n \cdot g t .2)$ Then

If $(m(n) \cdot g t \cdot m p r e v(n)) m(n)=\operatorname{mprev}(n)$

If $(m(n) \cdot l t \cdot m(n-1)) m(n)=\operatorname{mprev}(n)$

Endif

If (n.ne.lsteps) $V(n)=V(n)+\left(k 1 V+2{ }^{*} k 2 V+2{ }^{*} k 3 V+k 4 V\right) / 6.0$

If $(V(n) \cdot g t \cdot V(n-1)) V(n)=V(n-1)$

If $(V(n) . l t . \Theta) V(n)=-V(n)$

$T(n)=T(n)+\left(k 1 T+2{ }^{*} k 2 T+2^{*} k 3 T+k 4 T\right) / 6.0$

If $(T(n)$.gt. Tinit) $T(n)=$ Tinit

If $(T(n) \cdot$ lt. $T(n-1)) T(n)=T(n-1)$

$\operatorname{rho}(n)=m(n) /\left(A c^{*} l\right)$

If $(r h o(n) \cdot g t$.rhoinit) $r h o(n)=$ rhoinit

$P(n)=r h o(n){ }^{*} R^{\star} T(n)$

If $(P(n) \cdot g t . P i n i) P(n)=$ Pini

If $(P(n) \cdot l t \cdot P(n-1)) P(n)=P(n-1)$

$\operatorname{mprev}(n)=m(n)$

7

continue 


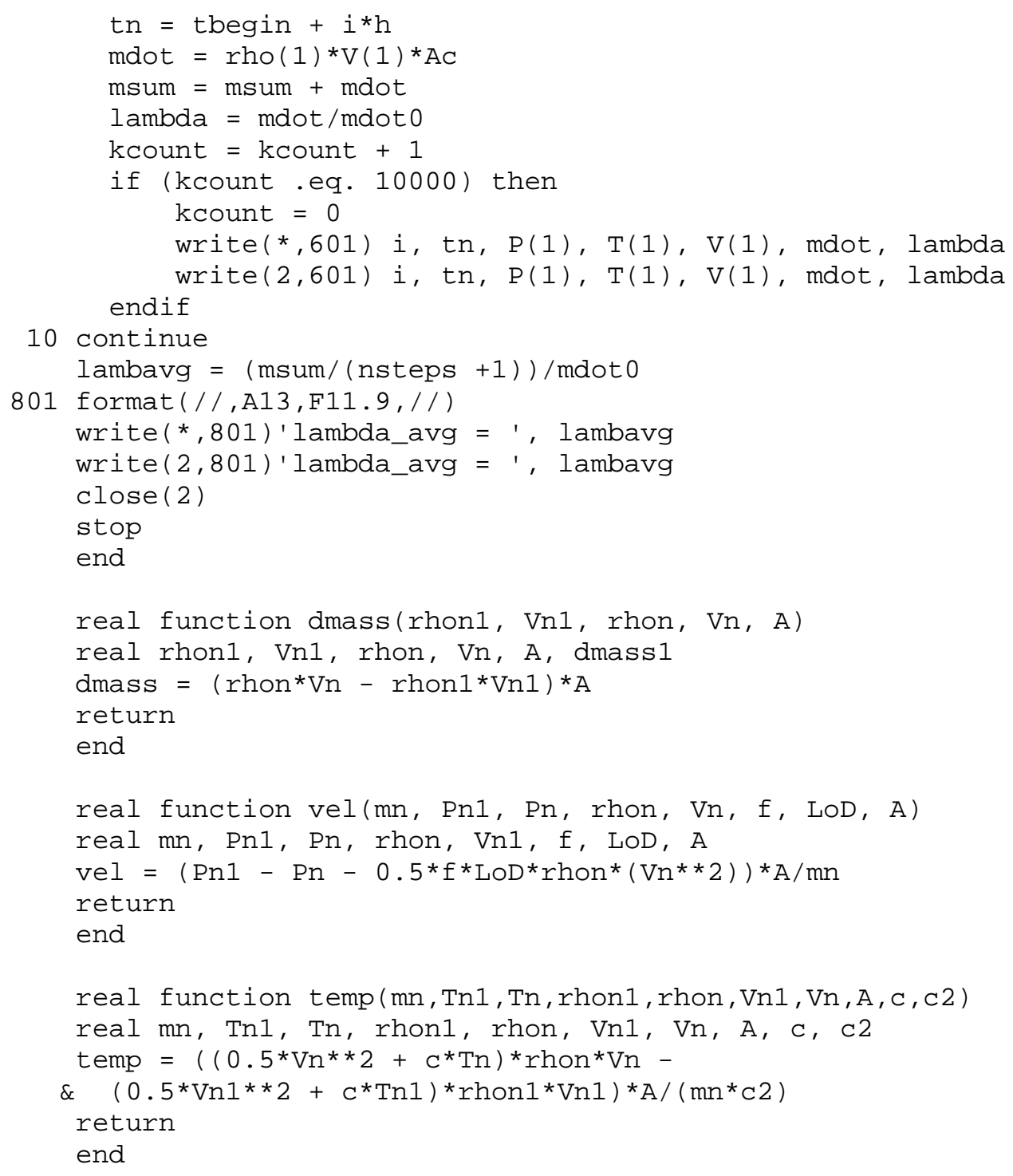




\section{Appendix B. COLLECTED IDEAL GAS FORTRAN CODE OUTPUT}

Valve spacing $=5$ miles, Pressure $=1167$ psi, 5 axial node elements

\begin{tabular}{|c|c|c|c|c|c|c|}
\hline$i$ & time (sec) & $\mathrm{P} 1(\mathrm{~Pa})$ & T1 (K) & V1 $(\mathrm{m} / \mathrm{sec})$ & mdot $(\mathrm{kg} / \mathrm{sec})$ & lambda \\
\hline$\odot$ & $\odot .0 \odot$ & $0.4361 \mathrm{E}+07$ & $\odot .2483 E+03$ & $0.4121 \mathrm{E}+03$ & $\odot .9172 \mathrm{E}+04$ & 1. $00000000 \odot$ \\
\hline 10000 & $1.0 \odot$ & $0.3091 \mathrm{E}+07$ & $\odot .2044 \mathrm{E}+03$ & $0.3739 E+03$ & $\odot .7165 E+04$ & 0.781171799 \\
\hline 20000 & $2 . \odot \odot$ & $\odot .2346 \mathrm{E}+\odot 7$ & $\odot .1749 E+\odot 3$ & $\odot .3459 \mathrm{E}+03$ & $\odot .5878 \mathrm{E}+\odot 4$ & $\odot .640846074$ \\
\hline 30000 & $3.0 \odot$ & $\odot .1906 \mathrm{E}+07$ & $\odot .1556 \mathrm{E}+03$ & $0.3263 E+03$ & $\odot .5064 \mathrm{E}+04$ & $\odot .552084267$ \\
\hline $4 \odot \odot \odot \odot$ & $4.0 \odot$ & $0.1622 \mathrm{E}+07$ & $\odot .1421 E+03$ & $0.3118 \mathrm{E}+03$ & $\odot .45 \odot 8 E+\odot 4$ & $\odot .491497636$ \\
\hline 50000 & 5.00 & $0.1418 \mathrm{E}+07$ & $\odot .1318 \mathrm{E}+03$ & $0.3003 \mathrm{E}+03$ & $\odot .4092 \mathrm{E}+04$ & 0.446139783 \\
\hline 60000 & 6.00 & $0.1262 \mathrm{E}+07$ & $\odot .1235 E+03$ & $0.2907 \mathrm{E}+03$ & $0.3763 E+04$ & 0.410249263 \\
\hline 70000 & $7 . \odot \odot$ & $\odot .1140 \mathrm{E}+\odot 7$ & $\odot .1167 E+03$ & $\odot .2826 \mathrm{E}+\odot 3$ & $\odot .3496 \mathrm{E}+\odot 4$ & ๑. 381132454 \\
\hline 80000 & 8.00 & $0.1042 \mathrm{E}+07$ & $\odot .1111 \mathrm{E}+03$ & $\odot .2756 \mathrm{E}+03$ & $\odot .3276 E+\odot 4$ & $\odot .357208848$ \\
\hline $900 \odot \odot$ & 9.00 & $0.9629 E+06$ & $\odot .1064 \mathrm{E}+03$ & $\odot .2697 \mathrm{E}+03$ & $\odot .3094 \mathrm{E}+04$ & 0.337364435 \\
\hline 100000 & 10.00 & $0.8982 E+06$ & $\odot .1024 \mathrm{E}+03$ & $0.2646 \mathrm{E}+03$ & $0.2942 \mathrm{E}+04$ & $\odot .320735306$ \\
\hline $11000 \odot$ & 11.00 & $\odot .8445 \mathrm{E}+\odot 6$ & $\odot .9903 \mathrm{E}+\odot 2$ & $\odot .2603 \mathrm{E}+\odot 3$ & $\odot .2813 \mathrm{E}+04$ & ๑. 306670129 \\
\hline $120 \odot \odot \odot$ & $12 . \odot \odot$ & $\odot .7997 \mathrm{E}+\odot 6$ & $\odot .9616 \mathrm{E}+\odot 2$ & $\odot .2565 \mathrm{E}+03$ & $\odot .27 \odot 3 E+\odot 4$ & ๑.294675648 \\
\hline 130000 & $13 . \odot \odot$ & $0.7619 \mathrm{E}+06$ & $\odot .9372 \mathrm{E}+02$ & $0.2532 \mathrm{E}+03$ & $\odot .2609 \mathrm{E}+\odot 4$ & $\odot .284393340$ \\
\hline 140000 & 14.00 & $0.7299 \mathrm{E}+06$ & $\odot .9163 \mathrm{E}+02$ & $0.2504 \mathrm{E}+03$ & $\odot .2527 \mathrm{E}+04$ & $\odot .275550008$ \\
\hline $15 \odot \odot \odot \odot$ & $15 . \odot \odot$ & $\odot .7029 E+\odot 6$ & $\odot .8985 E+\odot 2$ & $\odot .2479 \mathrm{E}+03$ & $\odot .2458 \mathrm{E}+\odot 4$ & $\odot .267945677$ \\
\hline $16000 \odot$ & $16 . \odot \odot$ & $\odot .6799 \mathrm{E}+\odot 6$ & $\odot .8835 E+\odot 2$ & $\odot .2458 \mathrm{E}+03$ & $\odot .2398 \mathrm{E}+04$ & ๑.261397749 \\
\hline $1700 \odot \odot$ & $17 . \odot \odot$ & $\odot .6604 \mathrm{E}+\odot 6$ & $\odot .87 \odot 7 E+\odot 2$ & $\odot .2441 \mathrm{E}+03$ & $\odot .2346 \mathrm{E}+\odot 4$ & $\odot .255737931$ \\
\hline 180000 & 18.00 & $0.6431 \mathrm{E}+06$ & $\odot .8594 \mathrm{E}+02$ & $0.2425 E+03$ & $\odot .2299 \mathrm{E}+04$ & $\odot .250648320$ \\
\hline 190000 & $19 . \odot \odot$ & $0.6271 \mathrm{E}+06$ & $\odot .8490 \mathrm{E}+02$ & $0.2410 \mathrm{E}+03$ & $\odot .2256 E+04$ & $\odot .245919734$ \\
\hline $20 \odot \odot \odot \odot$ & $20 . \odot \odot$ & $\odot .6123 E+06$ & $\odot .8393 \mathrm{E}+\odot 2$ & $\odot .2396 \mathrm{E}+03$ & $\odot .2215 E+04$ & $\odot .2414990 \odot 7$ \\
\hline $2100 \odot \odot$ & $21 . \odot \odot$ & $\odot .5984 \mathrm{E}+\odot 6$ & $\odot .8301 \mathrm{E}+\odot 2$ & $\odot .2383 E+03$ & $\odot .2177 \mathrm{E}+\odot 4$ & $\odot .237309173$ \\
\hline $22000 \odot$ & $22 . \odot \odot$ & $\odot .5853 E+\odot 6$ & $\odot .8216 \mathrm{E}+\odot 2$ & $\odot .2371 \mathrm{E}+03$ & $\odot .214 \odot E+\odot 4$ & $\odot .233322337$ \\
\hline 230000 & 23.00 & $\odot .5730 E+06$ & $\odot .8134 \mathrm{E}+\odot 2$ & $\odot .2359 \mathrm{E}+03$ & $\odot .2106 \mathrm{E}+04$ & $\odot .229584709$ \\
\hline 240000 & 24.00 & $\odot .5612 \mathrm{E}+06$ & $\odot .8055 \mathrm{E}+02$ & $\odot .2347 \mathrm{E}+03$ & $\odot .2072 \mathrm{E}+\odot 4$ & 0.225929037 \\
\hline $250 \odot \odot \odot$ & $25 . \odot \odot$ & $\odot .5502 \mathrm{E}+\odot 6$ & $\odot .7983 E+\odot 2$ & $0.2337 \mathrm{E}+03$ & $\odot .2041 \mathrm{E}+04$ & $\odot .222521335$ \\
\hline 260000 & 26.00 & $\odot .5395 \mathrm{E}+06$ & $\odot .7910 \mathrm{E}+02$ & $0.2326 \mathrm{E}+03$ & $\odot .2010 \mathrm{E}+\odot 4$ & ๑.219188049 \\
\hline $270 \odot \odot \odot$ & $27 . \odot \odot$ & $\odot .5293 E+\odot 6$ & $\odot .7843 \mathrm{E}+\odot 2$ & $\odot .2316 \mathrm{E}+03$ & $\odot .1981 E+\odot 4$ & $\odot .215970695$ \\
\hline $2800 \odot \odot$ & $28 . \odot \odot$ & $\odot .5197 \mathrm{E}+06$ & $\odot .7778 \mathrm{E}+\odot 2$ & $\odot .2307 \mathrm{E}+03$ & $\odot .1953 \mathrm{E}+04$ & $\odot .212952137$ \\
\hline 290000 & $29.0 \odot$ & $0.5103 \mathrm{E}+06$ & $\odot .7712 \mathrm{E}+02$ & $0.2297 \mathrm{E}+03$ & $0.1926 \mathrm{E}+04$ & 0.209954888 \\
\hline $30000 \odot$ & $3 \odot . \odot \odot$ & $0.5013 \mathrm{E}+06$ & $\odot .7653 \mathrm{E}+02$ & $0.2288 \mathrm{E}+03$ & $\odot .1899 \mathrm{E}+04$ & $\odot .207053393$ \\
\hline
\end{tabular}

lambda_avg $=0.333059520$ 


\section{Valve spacing $=5$ miles, Pressure $=1167$ psi, 25 axial node elements}

\begin{tabular}{|c|c|c|c|c|c|c|}
\hline$i$ & time ( $\mathrm{sec}$ ) & $\mathrm{P} 1(\mathrm{~Pa})$ & T1 (K) & V1 $(\mathrm{m} / \mathrm{sec})$ & mdot $(\mathrm{kg} / \mathrm{sec})$ & lambda \\
\hline 0 & $\odot . \odot \odot$ & $\odot .4361 \mathrm{E}+07$ & $\odot .2483 \mathrm{E}+03$ & $\odot .4121 \mathrm{E}+03$ & $\odot .9172 \mathrm{E}+\odot 4$ & $1.0000 \odot \odot \odot \odot \odot$ \\
\hline 10000 & 1.00 & $\odot .1650 \mathrm{E}+07$ & $\odot .1439 \mathrm{E}+03$ & $0.3138 E+03$ & $\odot .4559 \mathrm{E}+\odot 4$ & $\odot .497011364$ \\
\hline 20000 & 2.00 & $\odot .1449 \mathrm{E}+\odot 7$ & $\odot .1356 \mathrm{E}+03$ & $\odot .3046 \mathrm{E}+03$ & $\odot .4124 \mathrm{E}+04$ & $\odot .449665874$ \\
\hline $3000 \odot$ & $3.0 \odot$ & $0.1294 \mathrm{E}+07$ & $\odot .129 \odot E+\odot 3$ & $\odot .2970 E+03$ & $\odot .3777 \mathrm{E}+04$ & $\odot .411795527$ \\
\hline 40000 & 4.00 & $0.1251 \mathrm{E}+07$ & $\odot .1286 \mathrm{E}+03$ & $0.2966 \mathrm{E}+03$ & $\odot .3657 \mathrm{E}+04$ & $\odot .398712456$ \\
\hline 50000 & 5.00 & $\odot .1236 \mathrm{E}+07$ & $\odot .1299 \mathrm{E}+03$ & $\odot .2980 E+03$ & $\odot .3595 E+\odot 4$ & $\odot .391921490$ \\
\hline 60000 & 6.00 & $\odot .1197 \mathrm{E}+\odot 7$ & $\odot .1296 E+03$ & $\odot .2978 E+03$ & $\odot .3486 E+\odot 4$ & $\odot .380039185$ \\
\hline 70000 & $7 . \odot \odot$ & $0.1161 \mathrm{E}+07$ & $\odot .1295 E+\odot 3$ & $\odot .2976 \mathrm{E}+03$ & $\odot .3382 \mathrm{E}+04$ & 0.368720829 \\
\hline 80000 & $8.0 \odot$ & $\odot .1127 \mathrm{E}+07$ & $\odot .1294 \mathrm{E}+03$ & $\odot .2975 \mathrm{E}+03$ & $\odot .3285 E+\odot 4$ & $\odot .358121336$ \\
\hline $9000 \odot$ & 9.00 & $0.1096 \mathrm{E}+07$ & $\odot .1294 \mathrm{E}+03$ & $\odot .2975 \mathrm{E}+03$ & $\odot .3192 \mathrm{E}+04$ & $\odot .347986102$ \\
\hline 100000 & 10.00 & $0.1065 E+07$ & $\odot .1294 \mathrm{E}+03$ & $\odot .2975 E+03$ & $\odot .31 \odot 2 E+04$ & $\odot .338183552$ \\
\hline 110000 & 11.00 & $\odot .1035 \mathrm{E}+\odot 7$ & $\odot .1294 \mathrm{E}+03$ & $\odot .2975 \mathrm{E}+03$ & $\odot .3015 \mathrm{E}+04$ & ๑. 328689784 \\
\hline 120000 & 12.00 & $0.1006 \mathrm{E}+07$ & $\odot .1294 \mathrm{E}+03$ & $0.2975 \mathrm{E}+03$ & $\odot .2930 \mathrm{E}+04$ & $\odot .319494337$ \\
\hline 130000 & 13.00 & $\odot .9778 \mathrm{E}+06$ & $\odot .1294 \mathrm{E}+03$ & $\odot .2975 \mathrm{E}+03$ & $\odot .2849 E+\odot 4$ & $\odot .310587078$ \\
\hline 140000 & 14.00 & $0.9507 \mathrm{E}+06$ & $\odot .1294 \mathrm{E}+03$ & $0.2976 \mathrm{E}+03$ & $\odot .2770 \mathrm{E}+04$ & 0.301947236 \\
\hline 150000 & 15.00 & $0.9245 E+06$ & $\odot .1295 E+03$ & $0.2976 \mathrm{E}+03$ & $\odot .2693 \mathrm{E}+04$ & 0.293614388 \\
\hline $16 \odot \odot \odot \odot$ & $16 . \odot \odot$ & $\odot .8990 \mathrm{E}+\odot 6$ & $\odot .1295 E+\odot 3$ & $\odot .2976 \mathrm{E}+\odot 3$ & $\odot .2619 \mathrm{E}+\odot 4$ & ๑.285501331 \\
\hline 170000 & $17 . \odot \odot$ & $\odot .8742 \mathrm{E}+06$ & $\odot .1295 E+03$ & $\odot .2976 \mathrm{E}+03$ & $\odot .2546 \mathrm{E}+\odot 4$ & $\odot .277592838$ \\
\hline 180000 & $18 . \odot \odot$ & $\odot .850 \odot E+\odot 6$ & $\odot .1295 E+03$ & $\odot .2977 \mathrm{E}+03$ & $\odot .2475 E+\odot 4$ & $\odot .269878656$ \\
\hline 190000 & 19.00 & $0.8267 \mathrm{E}+06$ & $\odot .1296 \mathrm{E}+03$ & $0.2977 \mathrm{E}+03$ & $\odot .2407 \mathrm{E}+04$ & $\odot .262466729$ \\
\hline $2000 \odot \odot$ & $20 . \odot \odot$ & $0.8039 \mathrm{E}+06$ & $\odot .1296 E+03$ & $0.2977 \mathrm{E}+03$ & $0.2341 E+04$ & $\odot .255183071$ \\
\hline $21000 \odot$ & $21 . \odot \odot$ & $\odot .7818 \mathrm{E}+\odot 6$ & $\odot .1296 \mathrm{E}+\odot 3$ & $\odot .2977 \mathrm{E}+03$ & $\odot .2276 \mathrm{E}+\odot 4$ & $\odot .248148233$ \\
\hline 220000 & 22.00 & $0.7604 \mathrm{E}+06$ & $\odot .1297 \mathrm{E}+03$ & $0.2978 \mathrm{E}+03$ & $\odot .2213 \mathrm{E}+04$ & 0.241323218 \\
\hline 230000 & $23 . \odot \odot$ & $0.7411 \mathrm{E}+06$ & $\odot .1299 E+03$ & $\odot .2980 \mathrm{E}+03$ & $\odot .2155 E+\odot 4$ & $\odot .234994456$ \\
\hline 240000 & 24.00 & $0.7268 \mathrm{E}+06$ & $\odot .1306 \mathrm{E}+03$ & $0.2988 \mathrm{E}+03$ & $0.2108 \mathrm{E}+04$ & $\odot .229848012$ \\
\hline 250000 & $25 . \odot \odot$ & $0.7161 \mathrm{E}+06$ & $\odot .1316 E+03$ & $0.3001 \mathrm{E}+03$ & $0.2069 \mathrm{E}+04$ & $\odot .225538746$ \\
\hline $260 \odot \odot \odot$ & $26 . \odot \odot$ & $\odot .7075 \mathrm{E}+\odot 6$ & $\odot .1329 E+\odot 3$ & $\odot .3015 \mathrm{E}+\odot 3$ & $\odot .2034 \mathrm{E}+\odot 4$ & $\odot .221731156$ \\
\hline 270000 & 27.00 & $0.7006 \mathrm{E}+06$ & $\odot .1344 \mathrm{E}+03$ & $0.3033 \mathrm{E}+03$ & $0.2003 \mathrm{E}+04$ & 0.218328044 \\
\hline 280000 & $28 . \odot \odot$ & $0.6947 \mathrm{E}+06$ & $\odot .1361 E+03$ & $0.3051 \mathrm{E}+03$ & $\odot .1974 \mathrm{E}+04$ & $\odot .215184450$ \\
\hline $290 \odot \odot \odot$ & $29 . \odot \odot$ & $0.6898 \mathrm{E}+06$ & $\odot .1379 \mathrm{E}+\odot 3$ & $\odot .3071 \mathrm{E}+03$ & $\odot .1947 \mathrm{E}+04$ & $\odot .212259546$ \\
\hline 300000 & $30 . \odot \odot$ & $0.6858 \mathrm{E}+06$ & $0.1398 \mathrm{E}+03$ & $0.3093 \mathrm{E}+03$ & $\odot .1922 \mathrm{E}+04$ & $\odot .209561229$ \\
\hline
\end{tabular}

lambda_avg $=0.313054979$ 


\section{Valve spacing $=5$ miles, Pressure $=1167$ psi, 100 axial node elements}

\begin{tabular}{|c|c|c|c|c|c|c|}
\hline$i$ & time ( $\mathrm{sec}$ ) & $\mathrm{P} 1(\mathrm{~Pa})$ & T1 (K) & V1 $(\mathrm{m} / \mathrm{sec})$ & mdot $(\mathrm{kg} / \mathrm{sec})$ & lambda \\
\hline 0 & $\odot . \odot \odot$ & $\odot .4361 \mathrm{E}+07$ & $\odot .2483 \mathrm{E}+03$ & $\odot .4121 \mathrm{E}+03$ & $\odot .9172 \mathrm{E}+\odot 4$ & $1.0000 \odot \odot \odot \odot \odot$ \\
\hline 10000 & 1.00 & $0.1659 \mathrm{E}+07$ & $0.1528 \mathrm{E}+03$ & $0.3233 E+03$ & $\odot .4448 \mathrm{E}+\odot 4$ & ๑.484976888 \\
\hline 20000 & 2.00 & $\odot .1749 \mathrm{E}+07$ & $\odot .1703 E+03$ & $0.3413 E+03$ & $\odot .4442 \mathrm{E}+04$ & $\odot .484325945$ \\
\hline $3000 \odot$ & $3.0 \odot$ & $\odot .1687 \mathrm{E}+07$ & $\odot .1803 E+\odot 3$ & $\odot .3512 \mathrm{E}+03$ & $\odot .4163 \mathrm{E}+04$ & $\odot .453902245$ \\
\hline 40000 & 4.00 & $0.1704 \mathrm{E}+07$ & $\odot .1969 \mathrm{E}+03$ & $0.3670 \mathrm{E}+03$ & $\odot .4025 \mathrm{E}+04$ & 0.438862771 \\
\hline 50000 & 5.00 & $\odot .1556 \mathrm{E}+\odot 7$ & $\odot .2030 \mathrm{E}+03$ & $\odot .3726 \mathrm{E}+03$ & $\odot .3621 E+04$ & $\odot .394726157$ \\
\hline 60000 & 6.00 & $\odot .1414 \mathrm{E}+07$ & $\odot .2089 E+03$ & $\odot .3780 E+03$ & $\odot .3243 E+\odot 4$ & $\odot .353602171$ \\
\hline 70000 & $7 . \odot \odot$ & $\odot .1359 \mathrm{E}+07$ & $\odot .2227 \mathrm{E}+\odot 3$ & $\odot .3903 E+03$ & $\odot .3019 \mathrm{E}+04$ & 0.329111844 \\
\hline 80000 & $8.0 \odot$ & $\odot .1339 \mathrm{E}+07$ & $\odot .2417 \mathrm{E}+03$ & $\odot .4066 \mathrm{E}+03$ & $\odot .2854 \mathrm{E}+\odot 4$ & $\odot .311163187$ \\
\hline 90000 & 9.00 & $\odot .1295 \mathrm{E}+07$ & $\odot .2483 \mathrm{E}+03$ & $0.4121 \mathrm{E}+03$ & $\odot .2724 \mathrm{E}+04$ & $\odot .296958029$ \\
\hline 100000 & 10.00 & $\odot .1243 E+07$ & $\odot .2483 E+03$ & $0.4121 \mathrm{E}+03$ & $\odot .2615 E+04$ & $\odot .285044909$ \\
\hline 110000 & 11.00 & $\odot .1208 \mathrm{E}+07$ & $\odot .2483 E+03$ & $\odot .4121 \mathrm{E}+03$ & $\odot .2541 \mathrm{E}+\odot 4$ & ๑.276996851 \\
\hline 120000 & 12.00 & $0.1175 \mathrm{E}+07$ & $\odot .2483 \mathrm{E}+03$ & $0.4121 \mathrm{E}+03$ & $\odot .2471 \mathrm{E}+04$ & $\odot .269425094$ \\
\hline 130000 & 13.00 & $\odot .1169 \mathrm{E}+07$ & $\odot .2483 E+03$ & $\odot .4121 \mathrm{E}+03$ & $\odot .2458 \mathrm{E}+\odot 4$ & $\odot .268029869$ \\
\hline 140000 & 14.00 & $0.1151 \mathrm{E}+07$ & $\odot .2483 E+03$ & $0.4121 \mathrm{E}+03$ & $\odot .2420 E+\odot 4$ & $\odot .263870388$ \\
\hline 150000 & 15.00 & $\odot .1135 E+07$ & $\odot .2483 E+03$ & $0.4121 \mathrm{E}+03$ & $0.2388 E+04$ & 0.260385484 \\
\hline $16 \odot \odot \odot \odot$ & $16 . \odot \odot$ & $\odot .1123 \mathrm{E}+\odot 7$ & $\odot .2483 E+\odot 3$ & $\odot .4121 \mathrm{E}+03$ & $\odot .2362 \mathrm{E}+\odot 4$ & $\odot .257469326$ \\
\hline 170000 & $17 . \odot \odot$ & $\odot .1111 \mathrm{E}+07$ & $\odot .2483 E+03$ & $\odot .4121 \mathrm{E}+03$ & $\odot .2336 \mathrm{E}+\odot 4$ & $\odot .254673272$ \\
\hline 180000 & $18 . \odot \odot$ & $\odot .1095 \mathrm{E}+07$ & $\odot .2483 E+03$ & $0.4121 \mathrm{E}+03$ & $\odot .23 \odot 2 \mathrm{E}+\odot 4$ & $\odot .251007944$ \\
\hline 190000 & 19.00 & $\odot .1080 E+07$ & $\odot .2483 E+03$ & $0.4121 \mathrm{E}+03$ & $\odot .2273 \mathrm{E}+04$ & 0.247787222 \\
\hline $2000 \odot \odot$ & $20 . \odot \odot$ & $\odot .1071 \mathrm{E}+07$ & $\odot .2483 E+03$ & $0.4121 \mathrm{E}+03$ & $\odot .2253 E+04$ & $\odot .245658770$ \\
\hline $21000 \odot$ & $21 . \odot \odot$ & $\odot .106 \odot \mathrm{E}+\odot 7$ & $\odot .2483 E+\odot 3$ & $\odot .4121 \mathrm{E}+03$ & $\odot .2231 \mathrm{E}+\odot 4$ & $\odot .243201420$ \\
\hline 220000 & 22.00 & $0.1044 \mathrm{E}+07$ & $\odot .2483 \mathrm{E}+03$ & $0.4121 \mathrm{E}+03$ & $\odot .2196 \mathrm{E}+04$ & $\odot .239462331$ \\
\hline 230000 & $23 . \odot \odot$ & $\odot .1033 \mathrm{E}+07$ & $\odot .2483 E+03$ & $\odot .4121 \mathrm{E}+03$ & $\odot .2173 E+04$ & $\odot .236885637$ \\
\hline 240000 & 24.00 & $\odot .1024 \mathrm{E}+07$ & $\odot .2483 E+03$ & $0.4121 \mathrm{E}+03$ & $\odot .2154 \mathrm{E}+04$ & 0.234883487 \\
\hline 250000 & $25 . \odot \odot$ & $0.1018 \mathrm{E}+07$ & $\odot .2483 E+03$ & $0.4121 \mathrm{E}+03$ & $0.2142 \mathrm{E}+04$ & 0.233495265 \\
\hline $260 \odot \odot \odot$ & $26 . \odot \odot$ & $\odot .1013 \mathrm{E}+\odot 7$ & $\odot .2483 E+\odot 3$ & $\odot .4121 \mathrm{E}+03$ & $\odot .2130 E+\odot 4$ & $\odot .232199878$ \\
\hline 270000 & 27.00 & $0.1007 \mathrm{E}+07$ & $\odot .2483 E+03$ & $0.4121 \mathrm{E}+03$ & $0.2119 \mathrm{E}+04$ & 0.230973512 \\
\hline 280000 & $28 . \odot \odot$ & $\odot .9995 E+06$ & $\odot .2483 E+03$ & $0.4121 \mathrm{E}+03$ & $\odot .2102 \mathrm{E}+04$ & 0.229218483 \\
\hline $290 \odot \odot \odot$ & $29 . \odot \odot$ & $\odot .9911 \mathrm{E}+\odot 6$ & $\odot .2483 E+\odot 3$ & $0.4121 \mathrm{E}+03$ & $\odot .2085 E+04$ & $\odot .227286011$ \\
\hline 300000 & $30 . \odot \odot$ & $0.9846 \mathrm{E}+06$ & $\odot .2483 E+03$ & $0.4121 \mathrm{E}+03$ & $\odot .2071 \mathrm{E}+04$ & $\odot .225793824$ \\
\hline
\end{tabular}

lambda_avg $=0.297197551$ 
Valve spacing $=5$ miles, Pressure $=2100$ psi, 5 axial node elements

\begin{tabular}{|c|c|c|c|c|c|c|}
\hline$i$ & time (sec) & $\mathrm{P} 1 \quad \mathrm{~Pa})$ & $\mathrm{T} 1 （ \mathrm{~K})$ & V1 (m/sec $)$ & mdot $(\mathrm{kg} / \mathrm{sec})$ & lambda \\
\hline$\odot$ & 0.00 & $\odot .7847 \mathrm{E}+07$ & $\odot .2483 E+\odot 3$ & $\odot .4121 \mathrm{E}+03$ & $\odot .1651 \mathrm{E}+05$ & 1. $0000 \odot \odot \odot \odot \odot$ \\
\hline 10000 & $1.0 \odot$ & $\odot .5562 \mathrm{E}+\odot 7$ & $\odot .2044 \mathrm{E}+03$ & $\odot .3739 E+03$ & $\odot .1289 E+05$ & 0.781159282 \\
\hline 20000 & $2.0 \odot$ & $\odot .422 \odot E+\odot 7$ & $\odot .1749 \mathrm{E}+03$ & $\odot .3459 E+03$ & $\odot .1058 \mathrm{E}+05$ & $\odot .640771747$ \\
\hline 30000 & 3.00 & $\odot .3429 \mathrm{E}+07$ & $\odot .1556 \mathrm{E}+03$ & $\odot .3262 \mathrm{E}+03$ & $\odot .9110 \mathrm{E}+04$ & $\odot .551925600$ \\
\hline 40000 & 4.00 & $\odot .2916 \mathrm{E}+07$ & $\odot .1421 \mathrm{E}+03$ & $\odot .3117 \mathrm{E}+03$ & $\odot .8108 \mathrm{E}+04$ & $\odot .491264135$ \\
\hline 50000 & 5.00 & $\odot .2548 \mathrm{E}+07$ & $\odot .1317 \mathrm{E}+\odot 3$ & $\odot .30 \odot 2 \mathrm{E}+03$ & $\odot .7359 \mathrm{E}+04$ & $\odot .445841342$ \\
\hline 60000 & 6.00 & $\odot .2268 \mathrm{E}+07$ & $0.1234 \mathrm{E}+03$ & $\odot .2906 \mathrm{E}+03$ & $\odot .6765 \mathrm{E}+\odot 4$ & ๑.409896642 \\
\hline 70000 & 7.00 & $\odot .2 \odot 48 \mathrm{E}+\odot 7$ & $\odot .1166 \mathrm{E}+03$ & $\odot .2824 \mathrm{E}+03$ & $\odot .6284 \mathrm{E}+\odot 4$ & ๑. 380729765 \\
\hline 80000 & 8.00 & $0.1872 \mathrm{E}+07$ & $\odot .1110 \mathrm{E}+03$ & $\odot .2755 \mathrm{E}+\odot 3$ & $\odot .5888 \mathrm{E}+04$ & $\odot .356759250$ \\
\hline $9000 \odot$ & 9.00 & $\odot .1729 \mathrm{E}+07$ & $0.1062 \mathrm{E}+03$ & $\odot .2696 \mathrm{E}+03$ & $\odot .5560 \mathrm{E}+\odot 4$ & ๑.336863756 \\
\hline $1000 \odot \odot$ & $10.0 \odot$ & $\odot .1612 \mathrm{E}+\odot 7$ & $\odot .1023 \mathrm{E}+\odot 3$ & $\odot .2645 \mathrm{E}+\odot 3$ & $\odot .5285 E+\odot 4$ & $\odot .320193619$ \\
\hline 110000 & 11.00 & $0.1516 \mathrm{E}+07$ & $0.9888 \mathrm{E}+02$ & $\odot .2601 \mathrm{E}+03$ & $\odot .5052 \mathrm{E}+\odot 4$ & ๑.306082577 \\
\hline 120000 & $12 . \odot \odot$ & $\odot .1435 E+07$ & $\odot .960 \odot \mathrm{E}+\odot 2$ & $\odot .2563 E+03$ & $\odot .4853 \mathrm{E}+\odot 4$ & $\odot .294039816$ \\
\hline 130000 & 13.00 & $0.1366 \mathrm{E}+07$ & $0.9354 \mathrm{E}+02$ & $\odot .2530 E+03$ & $\odot .4683 \mathrm{E}+04$ & 0.283713222 \\
\hline 140000 & 14.00 & $\odot .1309 \mathrm{E}+07$ & $\odot .9144 \mathrm{E}+02$ & $\odot .2501 \mathrm{E}+03$ & $\odot .4536 \mathrm{E}+\odot 4$ & $\odot .274833560$ \\
\hline $1500 \odot \odot$ & $15 . \odot \odot$ & $\odot .126 \odot E+\odot 7$ & $\odot .8966 \mathrm{E}+\odot 2$ & $\odot .2476 \mathrm{E}+\odot 3$ & $\odot .4410 E+\odot 4$ & $\odot .267186582$ \\
\hline 160000 & 16.00 & $\odot .1218 \mathrm{E}+07$ & $0.8814 \mathrm{E}+02$ & $0.2455 E+03$ & $\odot .4301 \mathrm{E}+04$ & ๑.260599941 \\
\hline 170000 & 17.00 & $\odot .1183 E+07$ & $0.8686 \mathrm{E}+02$ & $0.2437 E+03$ & $\odot .4207 \mathrm{E}+\odot 4$ & 0.254914224 \\
\hline 180000 & 18.00 & $\odot .1152 \mathrm{E}+07$ & $\odot .8572 \mathrm{E}+02$ & $0.2421 \mathrm{E}+03$ & $\odot .4123 \mathrm{E}+04$ & $\odot .249799609$ \\
\hline 190000 & 19.00 & $\odot .1123 \mathrm{E}+07$ & $\odot .8467 \mathrm{E}+02$ & $\odot .2407 \mathrm{E}+03$ & $\odot .4045 \mathrm{E}+04$ & $\odot .245061770$ \\
\hline $200 \odot \odot \odot$ & $2 \odot . \odot \odot$ & $\odot .1096 \mathrm{E}+\odot 7$ & $\odot .8369 \mathrm{E}+\odot 2$ & $\odot .2393 E+03$ & $\odot .3971 E+\odot 4$ & $\odot .240574658$ \\
\hline 210000 & 21.00 & $\odot .1071 \mathrm{E}+07$ & $0.8277 \mathrm{E}+02$ & $0.2379 \mathrm{E}+03$ & $\odot .3902 \mathrm{E}+\odot 4$ & 0.236386925 \\
\hline 220000 & $22.0 \odot$ & $\odot .1047 \mathrm{E}+07$ & $\odot .8191 \mathrm{E}+02$ & $\odot .2367 \mathrm{E}+03$ & $\odot .3836 E+\odot 4$ & 0.232395172 \\
\hline $23000 \odot$ & 23.00 & $\odot .1025 E+\odot 7$ & $\odot .8109 \mathrm{E}+02$ & $\odot .2355 E+03$ & $\odot .3773 \mathrm{E}+\odot 4$ & $\odot .228608295$ \\
\hline 240000 & 24.00 & $\odot .1004 \mathrm{E}+07$ & $\odot .8030 \mathrm{E}+02$ & $\odot .2344 \mathrm{E}+03$ & $\odot .3713 \mathrm{E}+04$ & $\odot .224932477$ \\
\hline 250000 & 25.00 & $\odot .9840 E+06$ & $0.7956 \mathrm{E}+02$ & $0.2333 \mathrm{E}+03$ & $\odot .3656 \mathrm{E}+\odot 4$ & 0.221520543 \\
\hline 260000 & 26.00 & $0.9646 \mathrm{E}+06$ & $0.7883 \mathrm{E}+02$ & $0.2322 \mathrm{E}+03$ & $\odot .3601 \mathrm{E}+04$ & 0.218148723 \\
\hline $270 \odot \odot \odot$ & 27.00 & $\odot .9463 E+\odot 6$ & $\odot .7815 \mathrm{E}+02$ & $\odot .2312 \mathrm{E}+03$ & $\odot .3548 \mathrm{E}+\odot 4$ & 0.214938357 \\
\hline $2800 \odot \odot$ & 28.00 & $\odot .9290 E+\odot 6$ & $\odot .7749 \mathrm{E}+02$ & $\odot .2302 \mathrm{E}+03$ & $\odot .3498 \mathrm{E}+\odot 4$ & $\odot .2119 \odot 2782$ \\
\hline 290000 & 29.00 & $\odot .9118 \mathrm{E}+06$ & $\odot .7684 \mathrm{E}+02$ & $\odot .2293 \mathrm{E}+03$ & $\odot .3448 \mathrm{E}+04$ & ๑.208881468 \\
\hline $30 \odot \odot \odot \odot$ & $3 \odot . \odot \odot$ & $\odot .8955 E+\odot 6$ & $\odot .7624 \mathrm{E}+\odot 2$ & $\odot .2284 \mathrm{E}+\odot 3$ & $\odot .3399 \mathrm{E}+\odot 4$ & $\odot .205955774$ \\
\hline
\end{tabular}

lambda_avg $=0.332404822$ 
Valve spacing $=5$ miles, Pressure $=2100$ psi, 25 axial node elements

\begin{tabular}{|c|c|c|c|c|c|c|}
\hline$i$ & time (sec) & $\mathrm{P} 1 \quad \mathrm{~Pa})$ & $\mathrm{T} 1 （ \mathrm{~K})$ & V1 (m/sec $)$ & mdot $(\mathrm{kg} / \mathrm{sec})$ & lambda \\
\hline 0 & 0.00 & $\odot .7847 \mathrm{E}+07$ & $\odot .2483 E+\odot 3$ & $\odot .4121 \mathrm{E}+03$ & $\odot .1651 \mathrm{E}+05$ & 1. $0000 \odot \odot \odot \odot \odot$ \\
\hline 10000 & $1.0 \odot$ & $\odot .2965 E+\odot 7$ & $0.1438 E+03$ & $\odot .3136 E+03$ & $\odot .8194 \mathrm{E}+\odot 4$ & ๑. 496471971 \\
\hline 20000 & $2.0 \odot$ & $\odot .260 \odot \mathrm{E}+\odot 7$ & $\odot .1354 \mathrm{E}+03$ & $\odot .3043 E+03$ & $\odot .7406 \mathrm{E}+\odot 4$ & 0.448689342 \\
\hline 30000 & 3.00 & $\odot .2318 \mathrm{E}+07$ & $0.1287 \mathrm{E}+03$ & $\odot .2967 \mathrm{E}+03$ & $\odot .6773 \mathrm{E}+04$ & 0.410372823 \\
\hline 40000 & 4.00 & $\odot .2236 \mathrm{E}+07$ & $\odot .1281 \mathrm{E}+03$ & $\odot .2960 \mathrm{E}+\odot 3$ & $\odot .6549 \mathrm{E}+04$ & $\odot .396752626$ \\
\hline 50000 & 5.00 & $\odot .2210 E+07$ & $\odot .1294 \mathrm{E}+\odot 3$ & $\odot .2975 E+03$ & $\odot .6439 \mathrm{E}+04$ & ๑. 390126139 \\
\hline 60000 & 6.00 & $0.2144 \mathrm{E}+07$ & $0.1293 E+03$ & $\odot .2974 \mathrm{E}+03$ & $\odot .6249 \mathrm{E}+\odot 4$ & $\odot .378604472$ \\
\hline 70000 & 7.00 & $\odot .2079 \mathrm{E}+\odot 7$ & $\odot .1292 \mathrm{E}+03$ & $\odot .2972 \mathrm{E}+03$ & $\odot .6063 \mathrm{E}+04$ & $\odot .367336422$ \\
\hline 80000 & 8.00 & $\odot .2019 E+07$ & $\odot .1291 \mathrm{E}+03$ & $\odot .2972 \mathrm{E}+03$ & $\odot .5888 \mathrm{E}+04$ & $\odot .356724888$ \\
\hline 90000 & 9.00 & $\odot .1961 \mathrm{E}+07$ & $\odot .1291 \mathrm{E}+03$ & ๑. 2972E+03 & $\odot .5719 \mathrm{E}+\odot 4$ & ๑.346506178 \\
\hline $1000 \odot \odot$ & $10.0 \odot$ & $\odot .1905 \mathrm{E}+\odot 7$ & $\odot .1291 \mathrm{E}+\odot 3$ & $\odot .2972 \mathrm{E}+\odot 3$ & $\odot .5556 \mathrm{E}+\odot 4$ & $\odot .336615354$ \\
\hline 110000 & 11.00 & $\odot .1851 \mathrm{E}+07$ & $0.1291 \mathrm{E}+03$ & $\odot .2972 \mathrm{E}+03$ & $\odot .5398 \mathrm{E}+\odot 4$ & $\odot .327030718$ \\
\hline 120000 & $12 . \odot \odot$ & $\odot .1798 \mathrm{E}+07$ & $\odot .1291 \mathrm{E}+03$ & $\odot .2972 \mathrm{E}+03$ & $\odot .5245 E+\odot 4$ & 0.317754477 \\
\hline 130000 & 13.00 & $\odot .1747 \mathrm{E}+07$ & $0.1291 \mathrm{E}+03$ & $\odot .2972 \mathrm{E}+03$ & $\odot .5097 \mathrm{E}+\odot 4$ & ๑. 308788717 \\
\hline 140000 & 14.00 & $\odot .1698 \mathrm{E}+07$ & $\odot .1291 \mathrm{E}+03$ & $\odot .2972 \mathrm{E}+03$ & $\odot .4953 \mathrm{E}+04$ & $\odot .300082654$ \\
\hline $1500 \odot \odot$ & $15 . \odot \odot$ & $\odot .165 \odot E+\odot 7$ & $\odot .1291 \mathrm{E}+\odot 3$ & $\odot .2972 \mathrm{E}+\odot 3$ & $\odot .4813 E+\odot 4$ & $\odot .291619062$ \\
\hline $1600 \odot \odot$ & 16.00 & $\odot .1604 \mathrm{E}+07$ & $\odot .1291 \mathrm{E}+03$ & $\odot .2972 \mathrm{E}+03$ & $\odot .4678 \mathrm{E}+\odot 4$ & 0.283434302 \\
\hline 170000 & 17.00 & $\odot .1559 E+07$ & $0.1291 \mathrm{E}+03$ & $\odot .2972 \mathrm{E}+\odot 3$ & $\odot .4547 \mathrm{E}+\odot 4$ & 0.275477469 \\
\hline 180000 & 18.00 & $\odot .1515 E+07$ & $0.1291 \mathrm{E}+03$ & $\odot .2972 \mathrm{E}+03$ & $\odot .4420 \mathrm{E}+\odot 4$ & ๑.267781764 \\
\hline 190000 & 19.00 & $\odot .1473 \mathrm{E}+07$ & $0.1292 \mathrm{E}+03$ & ๑. 2972E+03 & $\odot .4296 \mathrm{E}+\odot 4$ & $\odot .260302752$ \\
\hline $200 \odot \odot \odot$ & $2 \odot . \odot \odot$ & $\odot .1432 \mathrm{E}+\odot 7$ & $\odot .1292 \mathrm{E}+\odot 3$ & $\odot .2973 E+03$ & $\odot .4176 \mathrm{E}+\odot 4$ & $\odot .253028154$ \\
\hline 210000 & 21.00 & $\odot .1392 \mathrm{E}+07$ & $0.1292 E+03$ & ๑. $2973 \mathrm{E}+03$ & $\odot .4060 \mathrm{E}+\odot 4$ & ๑.245954573 \\
\hline 220000 & $22.0 \odot$ & $\odot .1353 \mathrm{E}+07$ & $0.1292 \mathrm{E}+03$ & $\odot .2973 \mathrm{E}+03$ & $\odot .3946 \mathrm{E}+\odot 4$ & ๑.239083961 \\
\hline $23000 \odot$ & 23.00 & $\odot .1316 E+07$ & $\odot .1293 E+03$ & $\odot .2973 E+03$ & $\odot .3837 E+\odot 4$ & 0.232451767 \\
\hline 240000 & 24.00 & $\odot .1283 \mathrm{E}+07$ & $0.1295 \mathrm{E}+03$ & ๑. 2976E+03 & $\odot .3737 \mathrm{E}+04$ & $\odot .226404816$ \\
\hline 250000 & 25.00 & $\odot .1259 \mathrm{E}+07$ & $0.1303 E+03$ & $\odot .2985 E+03$ & $\odot .3656 \mathrm{E}+\odot 4$ & 0.221496955 \\
\hline 260000 & 26.00 & $\odot .1241 \mathrm{E}+07$ & $0.1314 \mathrm{E}+03$ & $0.2998 \mathrm{E}+03$ & $\odot .3587 \mathrm{E}+04$ & 0.217343241 \\
\hline $270 \odot \odot \odot$ & 27.00 & $\odot .1226 E+\odot 7$ & $\odot .1327 \mathrm{E}+03$ & $\odot .3013 \mathrm{E}+03$ & $\odot .3526 \mathrm{E}+\odot 4$ & 0.213645428 \\
\hline $2800 \odot \odot$ & 28.00 & $\odot .1213 E+07$ & $\odot .1342 \mathrm{E}+03$ & $\odot .3030 E+03$ & $\odot .3471 \mathrm{E}+\odot 4$ & ๑.210272953 \\
\hline 290000 & 29.00 & $\odot .1203 \mathrm{E}+\odot 7$ & $\odot .1359 \mathrm{E}+03$ & $\odot .3 \odot 49 E+\odot 3$ & $\odot .3420 E+\odot 4$ & ๑.207185417 \\
\hline $30 \odot \odot \odot \odot$ & $3 \odot . \odot \odot$ & $\odot .1194 \mathrm{E}+\odot 7$ & $\odot .1377 \mathrm{E}+03$ & $\odot .3069 \mathrm{E}+\odot 3$ & $\odot .3372 \mathrm{E}+\odot 4$ & $\odot .2 \odot 4322457$ \\
\hline
\end{tabular}

lambda_avg $=0.310781360$ 


\section{Valve spacing $=5$ miles, Pressure $=2100$ psi, 100 axial node elements}

\begin{tabular}{|c|c|c|c|c|c|c|}
\hline$i$ & time (sec) & $\mathrm{P} 1 \quad \mathrm{~Pa})$ & T1 (K) & V1 $(\mathrm{m} / \mathrm{sec})$ & mdot $(\mathrm{kg} / \mathrm{sec})$ & lambda \\
\hline 0 & $0.0 \odot$ & $\odot .7847 \mathrm{E}+\odot 7$ & $\odot .2483 E+03$ & $\odot .4121 \mathrm{E}+03$ & $\odot .1651 \mathrm{E}+05$ & 1. $0000 \odot \odot \odot \odot \odot$ \\
\hline $1000 \odot$ & $1 . \odot \odot$ & $\odot .296 \odot E+07$ & $\odot .1521 \mathrm{E}+03$ & $\odot .3226 \mathrm{E}+03$ & $\odot .7955 E+04$ & $\odot .481966555$ \\
\hline $2000 \odot$ & $2.0 \odot$ & $\odot .3101 \mathrm{E}+07$ & $\odot .1689 E+03$ & $\odot .3399 \mathrm{E}+03$ & $\odot .7909 \mathrm{E}+\odot 4$ & $\odot .479150385$ \\
\hline 30000 & 3.00 & $\odot .2994 \mathrm{E}+07$ & $\odot .1790 \mathrm{E}+03$ & $\odot .3499 \mathrm{E}+03$ & $\odot .7417 \mathrm{E}+\odot 4$ & $\odot .449388027$ \\
\hline 40000 & 4.00 & $\odot .2981 \mathrm{E}+07$ & $\odot .1938 \mathrm{E}+03$ & $\odot .3641 \mathrm{E}+03$ & $\odot .7097 \mathrm{E}+04$ & $\odot .429985255$ \\
\hline 50000 & 5.00 & $\odot .2812 \mathrm{E}+\odot 7$ & $\odot .2038 \mathrm{E}+03$ & $\odot .3734 \mathrm{E}+03$ & $\odot .6528 \mathrm{E}+\odot 4$ & ๑.395517766 \\
\hline 60000 & 6.00 & $0.2492 \mathrm{E}+07$ & $\odot .2067 \mathrm{E}+03$ & $0.3761 \mathrm{E}+03$ & $\odot .5745 \mathrm{E}+04$ & ๑.348092884 \\
\hline 70000 & $7.0 \odot$ & $\odot .2353 \mathrm{E}+07$ & $\odot .2182 \mathrm{E}+03$ & $\odot .3863 E+03$ & $\odot .5280 E+\odot 4$ & $\odot .319873244$ \\
\hline 80000 & 8.00 & $\odot .2310 E+\odot 7$ & $\odot .2364 \mathrm{E}+03$ & $\odot .4021 \mathrm{E}+03$ & $\odot .4979 \mathrm{E}+\odot 4$ & ๑.301686347 \\
\hline 90000 & $9.0 \odot$ & $0.2241 \mathrm{E}+07$ & $\odot .2483 E+03$ & $0.4121 \mathrm{E}+03$ & $0.4714 \mathrm{E}+04$ & $\odot .285630912$ \\
\hline $1000 \odot \odot$ & 10.00 & $\odot .2142 \mathrm{E}+\odot 7$ & $\odot .2483 E+\odot 3$ & $\odot .4121 \mathrm{E}+03$ & $\odot .45 \odot 7 \mathrm{E}+\odot 4$ & $\odot .273034990$ \\
\hline 110000 & 11.00 & $0.2103 \mathrm{E}+07$ & $\odot .2483 \mathrm{E}+03$ & $0.4121 \mathrm{E}+03$ & $0.4424 \mathrm{E}+04$ & $\odot .268013239$ \\
\hline 120000 & $12 . \odot \odot$ & $0.2058 \mathrm{E}+07$ & $\odot .2483 E+03$ & $0.4121 \mathrm{E}+03$ & $\odot .4328 E+04$ & $\odot .262243897$ \\
\hline 130000 & 13.00 & $0.2007 \mathrm{E}+07$ & $\odot .2483 E+03$ & $0.4121 \mathrm{E}+03$ & $0.4221 \mathrm{E}+04$ & $\odot .255753368$ \\
\hline 140000 & 14.00 & $\odot .1965 \mathrm{E}+07$ & $\odot .2483 \mathrm{E}+03$ & $0.4121 \mathrm{E}+03$ & $\odot .4134 \mathrm{E}+04$ & 0.250464708 \\
\hline $15000 \odot$ & $15 . \odot \odot$ & $\odot .1931 \mathrm{E}+\odot 7$ & $\odot .2483 E+\odot 3$ & $\odot .4121 \mathrm{E}+03$ & $\odot .4061 \mathrm{E}+\odot 4$ & $\odot .246045515$ \\
\hline 160000 & 16.00 & $0.1898 \mathrm{E}+07$ & $\odot .2483 E+03$ & $0.4121 \mathrm{E}+03$ & $\odot .3993 \mathrm{E}+04$ & $\odot .241893858$ \\
\hline 170000 & 17.00 & $\odot .1870 E+\odot 7$ & $\odot .2483 \mathrm{E}+03$ & $0.4121 \mathrm{E}+03$ & $\odot .3933 \mathrm{E}+04$ & $\odot .238275796$ \\
\hline 180000 & 18.00 & $0.1842 \mathrm{E}+07$ & $\odot .2483 E+03$ & $0.4121 \mathrm{E}+03$ & $\odot .3875 E+04$ & 0.234772876 \\
\hline 190000 & 19.00 & $\odot .1820 \mathrm{E}+07$ & $\odot .2483 \mathrm{E}+03$ & $0.4121 \mathrm{E}+03$ & $\odot .3829 \mathrm{E}+04$ & ๑.231998548 \\
\hline $20 \odot \odot \odot \odot$ & $2 \odot . \odot \odot$ & $\odot .1797 \mathrm{E}+\odot 7$ & $\odot .2483 E+\odot 3$ & $\odot .4121 \mathrm{E}+03$ & $\odot .3781 \mathrm{E}+\odot 4$ & ๑.229064181 \\
\hline 210000 & 21.00 & $0.1771 \mathrm{E}+07$ & $\odot .2483 E+03$ & $0.4121 \mathrm{E}+03$ & $0.3725 E+04$ & $\odot .225681230$ \\
\hline 220000 & $22 . \odot \odot$ & $0.1745 E+07$ & $\odot .2483 E+03$ & $0.4121 \mathrm{E}+03$ & $\odot .3671 E+04$ & $\odot .222416759$ \\
\hline $230 \odot \odot \odot$ & 23.00 & $0.1724 \mathrm{E}+07$ & $\odot .2483 E+03$ & $0.4121 \mathrm{E}+03$ & $\odot .3626 E+04$ & $\odot .219700471$ \\
\hline 240000 & 24.00 & $\odot .1708 \mathrm{E}+07$ & $\odot .2483 \mathrm{E}+03$ & $0.4121 \mathrm{E}+03$ & $\odot .3593 \mathrm{E}+\odot 4$ & 0.217660293 \\
\hline $250 \odot \odot \odot$ & $25 . \odot \odot$ & $\odot .1696 \mathrm{E}+\odot 7$ & $\odot .2483 E+\odot 3$ & $\odot .4121 \mathrm{E}+03$ & $\odot .3568 E+\odot 4$ & $\odot .216191307$ \\
\hline 260000 & 26.00 & $0.1685 \mathrm{E}+07$ & $\odot .2483 E+03$ & $0.4121 \mathrm{E}+03$ & $0.3543 \mathrm{E}+04$ & $\odot .214686975$ \\
\hline $270 \odot \odot \odot$ & $27.0 \odot$ & $\odot .1670 \mathrm{E}+07$ & $\odot .2483 E+\odot 3$ & $0.4121 \mathrm{E}+03$ & $\odot .3513 E+04$ & $\odot .212830275$ \\
\hline $2800 \odot \odot$ & $28 . \odot \odot$ & $0.1652 \mathrm{E}+07$ & $\odot .2483 E+\odot 3$ & $0.4121 \mathrm{E}+03$ & $\odot .3475 E+04$ & $\odot .210537940$ \\
\hline 290000 & 29.00 & $\odot .1636 \mathrm{E}+07$ & $\odot .2483 \mathrm{E}+03$ & $0.4121 \mathrm{E}+03$ & $\odot .3442 \mathrm{E}+\odot 4$ & ๑.208533257 \\
\hline $30 \odot \odot \odot \odot$ & $3 \odot . \odot \odot$ & $\odot .1624 \mathrm{E}+\odot 7$ & $\odot .2483 E+\odot 3$ & $\odot .4121 \mathrm{E}+03$ & $\odot .3415 E+\odot 4$ & $\odot .206920236$ \\
\hline
\end{tabular}

lambda_avg $=0.284179538$ 


\section{Valve spacing $=20$ miles, Pressure $=1167$ psi, 20 axial node elements}

\begin{tabular}{|c|c|c|c|c|c|c|}
\hline$i$ & time ( $\mathrm{sec}$ ) & $\mathrm{P} 1(\mathrm{~Pa})$ & T1 (K) & V1 $(\mathrm{m} / \mathrm{sec})$ & mdot $(\mathrm{kg} / \mathrm{sec})$ & lambda \\
\hline 0 & $\odot . \odot \odot$ & $\odot .4361 \mathrm{E}+07$ & $\odot .2483 \mathrm{E}+03$ & $\odot .4121 \mathrm{E}+03$ & $\odot .9172 \mathrm{E}+\odot 4$ & $1.0000 \odot \odot \odot \odot \odot$ \\
\hline 10000 & 1.00 & $\odot .3091 \mathrm{E}+07$ & $\odot .2044 \mathrm{E}+03$ & $0.3739 E+03$ & $\odot .7165 \mathrm{E}+\odot 4$ & $\odot .781171799$ \\
\hline 20000 & 2.00 & $\odot .2346 \mathrm{E}+\odot 7$ & $\odot .1749 E+03$ & $\odot .3459 E+03$ & $\odot .5878 \mathrm{E}+04$ & $\odot .640846074$ \\
\hline $3000 \odot$ & $3.0 \odot$ & $0.1906 \mathrm{E}+07$ & $\odot .1556 \mathrm{E}+\odot 3$ & $0.3263 E+03$ & $\odot .5064 \mathrm{E}+04$ & $\odot .552084267$ \\
\hline 40000 & 4.00 & $0.1622 \mathrm{E}+07$ & $\odot .1421 \mathrm{E}+03$ & $0.3118 \mathrm{E}+03$ & $\odot .4508 \mathrm{E}+04$ & $\odot .491497636$ \\
\hline 50000 & $5.0 \odot$ & $\odot .1418 \mathrm{E}+07$ & $\odot .1318 \mathrm{E}+03$ & $\odot .3003 \mathrm{E}+03$ & $\odot .4 \odot 92 \mathrm{E}+\odot 4$ & $\odot .446139783$ \\
\hline 60000 & 6.00 & $\odot .1262 \mathrm{E}+\odot 7$ & $\odot .1235 E+03$ & $\odot .2907 \mathrm{E}+03$ & $\odot .3763 E+\odot 4$ & 0.410249263 \\
\hline $7000 \odot$ & $7 . \odot \odot$ & $0.1140 \mathrm{E}+07$ & $\odot .1167 \mathrm{E}+03$ & $\odot .2826 \mathrm{E}+03$ & $\odot .3496 E+04$ & 0.381132573 \\
\hline 80000 & $8.0 \odot$ & $0.1042 \mathrm{E}+07$ & $\odot .1111 \mathrm{E}+03$ & $\odot .2756 \mathrm{E}+\odot 3$ & $\odot .3276 E+\odot 4$ & $\odot .357210010$ \\
\hline $9000 \odot$ & 9.00 & $0.9629 \mathrm{E}+06$ & $\odot .1064 \mathrm{E}+03$ & $\odot .2697 \mathrm{E}+03$ & $\odot .3094 \mathrm{E}+04$ & $\odot .337368637$ \\
\hline 100000 & 10.00 & $0.8982 E+06$ & $\odot .1024 \mathrm{E}+03$ & $\odot .2646 \mathrm{E}+03$ & $\odot .2942 \mathrm{E}+04$ & $\odot .320748001$ \\
\hline 110000 & 11.00 & $\odot .8446 \mathrm{E}+\odot 6$ & $\odot .9903 \mathrm{E}+\odot 2$ & $\odot .2603 \mathrm{E}+03$ & $\odot .2813 E+04$ & $\odot .306693286$ \\
\hline 120000 & 12.00 & $0.7999 \mathrm{E}+06$ & $\odot .9617 \mathrm{E}+02$ & $0.2565 \mathrm{E}+03$ & $\odot .2703 \mathrm{E}+04$ & $\odot .294717491$ \\
\hline 130000 & 13.00 & $\odot .7622 \mathrm{E}+06$ & $\odot .9374 \mathrm{E}+\odot 2$ & $\odot .2532 \mathrm{E}+03$ & $\odot .2609 \mathrm{E}+\odot 4$ & $\odot .284464300$ \\
\hline 140000 & 14.00 & $0.7304 \mathrm{E}+06$ & $\odot .9166 \mathrm{E}+02$ & $0.2504 \mathrm{E}+03$ & $\odot .2529 \mathrm{E}+04$ & $\odot .275674641$ \\
\hline 150000 & 15.00 & $0.7036 \mathrm{E}+06$ & $\odot .8991 \mathrm{E}+02$ & $0.2480 E+03$ & $\odot .2459 E+04$ & $\odot .268142819$ \\
\hline $16 \odot \odot \odot \odot$ & $16 . \odot \odot$ & $\odot .6811 \mathrm{E}+\odot 6$ & $\odot .8843 \mathrm{E}+\odot 2$ & $\odot .2459 \mathrm{E}+\odot 3$ & $\odot .24 \odot \odot E+\odot 4$ & $\odot .261702001$ \\
\hline 170000 & $17 . \odot \odot$ & $\odot .6619 \mathrm{E}+06$ & $\odot .8719 \mathrm{E}+\odot 2$ & $\odot .2442 \mathrm{E}+03$ & $\odot .2349 \mathrm{E}+\odot 4$ & $\odot .256152272$ \\
\hline 180000 & $18 . \odot \odot$ & $0.6449 \mathrm{E}+06$ & $\odot .86 \odot 9 E+\odot 2$ & $\odot .2427 \mathrm{E}+03$ & $\odot .2304 \mathrm{E}+\odot 4$ & $\odot .251147628$ \\
\hline 190000 & 19.00 & $0.6292 \mathrm{E}+06$ & $\odot .8507 \mathrm{E}+02$ & $0.2412 \mathrm{E}+03$ & $\odot .2261 \mathrm{E}+04$ & $\odot .246502310$ \\
\hline $2000 \odot \odot$ & $20 . \odot \odot$ & $0.6147 \mathrm{E}+06$ & $\odot .8413 E+02$ & $0.2399 \mathrm{E}+03$ & $\odot .2221 E+04$ & $\odot .242159858$ \\
\hline $21000 \odot$ & $21 . \odot \odot$ & $\odot .6013 \mathrm{E}+\odot 6$ & $\odot .8326 \mathrm{E}+\odot 2$ & $\odot .2386 \mathrm{E}+03$ & $\odot .2184 \mathrm{E}+\odot 4$ & $\odot .238126889$ \\
\hline 220000 & 22.00 & $0.5890 \mathrm{E}+06$ & $\odot .8246 \mathrm{E}+02$ & $0.2375 \mathrm{E}+03$ & $\odot .2150 \mathrm{E}+04$ & $\odot .234362856$ \\
\hline 230000 & $23 . \odot \odot$ & $0.5773 E+06$ & $\odot .8171 \mathrm{E}+02$ & $\odot .2364 \mathrm{E}+03$ & $\odot .2117 \mathrm{E}+04$ & $\odot .230785593$ \\
\hline 240000 & 24.00 & $0.5665 E+06$ & $\odot .8101 \mathrm{E}+02$ & $0.2354 \mathrm{E}+03$ & $\odot .2086 \mathrm{E}+04$ & $\odot .227441058$ \\
\hline 250000 & $25 . \odot \odot$ & $0.5563 E+06$ & $\odot .8035 \mathrm{E}+02$ & $0.2344 \mathrm{E}+03$ & $0.2057 \mathrm{E}+04$ & $\odot .224249408$ \\
\hline $260 \odot \odot \odot$ & $26 . \odot \odot$ & $\odot .5468 \mathrm{E}+\odot 6$ & $\odot .7975 E+\odot 2$ & $\odot .2336 \mathrm{E}+\odot 3$ & $\odot .2 \odot 29 E+\odot 4$ & $\odot .221263528$ \\
\hline 270000 & 27.00 & $0.5376 \mathrm{E}+06$ & $\odot .7916 \mathrm{E}+02$ & $0.2327 \mathrm{E}+03$ & $0.2003 \mathrm{E}+04$ & 0.218353227 \\
\hline 280000 & $28 . \odot \odot$ & $0.5293 E+06$ & $\odot .7863 \mathrm{E}+02$ & $\odot .2319 \mathrm{E}+03$ & $\odot .1978 \mathrm{E}+04$ & 0.215696573 \\
\hline $290 \odot \odot \odot$ & $29 . \odot \odot$ & $0.5211 \mathrm{E}+06$ & $\odot .7810 \mathrm{E}+\odot 2$ & $0.2311 \mathrm{E}+03$ & $\odot .1954 \mathrm{E}+04$ & $\odot .213050380$ \\
\hline 300000 & $30 . \odot \odot$ & $0.5135 \mathrm{E}+06$ & $0.7764 \mathrm{E}+02$ & $0.2305 \mathrm{E}+03$ & $\odot .1932 \mathrm{E}+04$ & $\odot .210597456$ \\
\hline
\end{tabular}

lambda_avg $=0.333737999$ 


\section{Valve spacing $=20$ miles, Pressure $=1167$ psi, 100 axial node elements}

\begin{tabular}{|c|c|c|c|c|c|c|}
\hline$i$ & time (sec) & $\mathrm{P} 1 \quad \mathrm{~Pa})$ & T1 (K) & V1 $(\mathrm{m} / \mathrm{sec})$ & mdot $(\mathrm{kg} / \mathrm{sec})$ & lambda \\
\hline 0 & $0.0 \odot$ & $\odot .4361 \mathrm{E}+07$ & $\odot .2483 E+03$ & $\odot .4121 \mathrm{E}+03$ & $\odot .9172 \mathrm{E}+\odot 4$ & 1. $0000 \odot \odot \odot \odot \odot$ \\
\hline $1000 \odot$ & $1 . \odot \odot$ & $\odot .1650 E+07$ & $\odot .1439 \mathrm{E}+03$ & $0.3138 \mathrm{E}+03$ & $\odot .4559 \mathrm{E}+\odot 4$ & $\odot .497011364$ \\
\hline $2000 \odot$ & $2.0 \odot$ & $0.1449 \mathrm{E}+07$ & $\odot .1356 \mathrm{E}+03$ & $\odot .3 \odot 46 \mathrm{E}+\odot 3$ & $\odot .4124 \mathrm{E}+\odot 4$ & $\odot .449665874$ \\
\hline 30000 & 3.00 & $\odot .1294 \mathrm{E}+07$ & $\odot .1290 \mathrm{E}+03$ & $\odot .2970 \mathrm{E}+03$ & $\odot .3777 \mathrm{E}+\odot 4$ & $\odot .411795527$ \\
\hline 40000 & 4.00 & $\odot .1251 \mathrm{E}+\odot 7$ & $\odot .1286 \mathrm{E}+03$ & $\odot .2966 \mathrm{E}+03$ & $\odot .3657 \mathrm{E}+\odot 4$ & ๑. 398712456 \\
\hline 50000 & 5.00 & $\odot .1236 \mathrm{E}+\odot 7$ & $\odot .1299 \mathrm{E}+\odot 3$ & $\odot .2980 E+03$ & $\odot .3595 \mathrm{E}+\odot 4$ & ๑. 391921490 \\
\hline 60000 & 6.00 & $0.1197 \mathrm{E}+07$ & $\odot .1296 \mathrm{E}+03$ & $0.2978 \mathrm{E}+03$ & $0.3486 \mathrm{E}+04$ & $\odot .380039185$ \\
\hline 70000 & $7.0 \odot$ & $\odot .1161 \mathrm{E}+07$ & $\odot .1295 E+03$ & $\odot .2976 \mathrm{E}+\odot 3$ & $\odot .3382 E+04$ & $\odot .368720829$ \\
\hline 80000 & 8.00 & $\odot .1127 \mathrm{E}+\odot 7$ & $\odot .1294 \mathrm{E}+03$ & $\odot .2975 \mathrm{E}+\odot 3$ & $\odot .3285 E+04$ & $\odot .358121336$ \\
\hline 90000 & $9.0 \odot$ & $0.1096 \mathrm{E}+07$ & $\odot .1294 \mathrm{E}+03$ & $\odot .2975 \mathrm{E}+03$ & $\odot .3192 \mathrm{E}+04$ & $\odot .347986102$ \\
\hline $1000 \odot \odot$ & 10.00 & $\odot .1065 \mathrm{E}+\odot 7$ & $\odot .1294 \mathrm{E}+\odot 3$ & $\odot .2975 \mathrm{E}+\odot 3$ & $\odot .3102 \mathrm{E}+\odot 4$ & ๑.338183552 \\
\hline 110000 & 11.00 & $0.1035 \mathrm{E}+07$ & $\odot .1294 \mathrm{E}+03$ & $0.2975 \mathrm{E}+03$ & $\odot .3015 \mathrm{E}+04$ & $\odot .328689784$ \\
\hline 120000 & $12 . \odot \odot$ & $0.1006 \mathrm{E}+07$ & $\odot .1294 \mathrm{E}+03$ & $\odot .2975 \mathrm{E}+03$ & $\odot .2930 E+\odot 4$ & $\odot .319494337$ \\
\hline 130000 & 13.00 & $0.9778 \mathrm{E}+06$ & $\odot .1294 \mathrm{E}+03$ & $0.2975 E+03$ & $\odot .2849 \mathrm{E}+04$ & $\odot .310587078$ \\
\hline 140000 & 14.00 & $\odot .9507 \mathrm{E}+06$ & $\odot .1294 \mathrm{E}+03$ & $0.2976 \mathrm{E}+03$ & $\odot .2770 \mathrm{E}+\odot 4$ & ๑. 301947236 \\
\hline $15000 \odot$ & $15 . \odot \odot$ & $\odot .9245 \mathrm{E}+\odot 6$ & $\odot .1295 E+\odot 3$ & $\odot .2976 \mathrm{E}+\odot 3$ & $\odot .2693 \mathrm{E}+\odot 4$ & ๑. 293614388 \\
\hline 160000 & 16.00 & $0.8990 E+06$ & $\odot .1295 E+03$ & $0.2976 \mathrm{E}+03$ & $\odot .2619 \mathrm{E}+04$ & $\odot .285501331$ \\
\hline 170000 & 17.00 & $\odot .8742 E+06$ & $\odot .1295 \mathrm{E}+03$ & $\odot .2976 \mathrm{E}+03$ & $\odot .2546 \mathrm{E}+\odot 4$ & 0.277592838 \\
\hline 180000 & 18.00 & $0.8500 \mathrm{E}+06$ & $\odot .1295 E+03$ & $0.2977 \mathrm{E}+03$ & $\odot .2475 E+04$ & $\odot .269878656$ \\
\hline 190000 & 19.00 & $\odot .8267 \mathrm{E}+06$ & $\odot .1296 \mathrm{E}+03$ & $0.2977 \mathrm{E}+03$ & $\odot .24 \odot 7 \mathrm{E}+\odot 4$ & $\odot .262466729$ \\
\hline $20 \odot \odot \odot \odot$ & $2 \odot . \odot \odot$ & $\odot .8039 \mathrm{E}+\odot 6$ & $\odot .1296 \mathrm{E}+\odot 3$ & $\odot .2977 \mathrm{E}+03$ & $\odot .2341 \mathrm{E}+\odot 4$ & $\odot .255183071$ \\
\hline 210000 & 21.00 & $0.7818 \mathrm{E}+06$ & $\odot .1296 \mathrm{E}+03$ & $0.2977 \mathrm{E}+03$ & $\odot .2276 \mathrm{E}+04$ & 0.248148233 \\
\hline 220000 & $22 . \odot \odot$ & $0.7604 \mathrm{E}+06$ & $\odot .1297 \mathrm{E}+03$ & $0.2978 \mathrm{E}+03$ & $\odot .2213 E+04$ & 0.241323218 \\
\hline $230 \odot \odot \odot$ & 23.00 & $0.7411 \mathrm{E}+06$ & $\odot .1299 \mathrm{E}+03$ & $\odot .2980 E+03$ & $\odot .2155 E+04$ & $\odot .234994456$ \\
\hline 240000 & 24.00 & $\odot .7268 \mathrm{E}+06$ & $0.1306 \mathrm{E}+03$ & $0.2988 \mathrm{E}+03$ & $\odot .2108 \mathrm{E}+04$ & ๑.229848012 \\
\hline $250 \odot \odot \odot$ & $25 . \odot \odot$ & $\odot .7161 \mathrm{E}+\odot 6$ & $\odot .1316 E+\odot 3$ & $\odot .30 \odot 1 \mathrm{E}+\odot 3$ & $\odot .2069 \mathrm{E}+\odot 4$ & $\odot .225538746$ \\
\hline 260000 & 26.00 & $0.7075 \mathrm{E}+06$ & $\odot .1329 \mathrm{E}+03$ & $0.3015 E+03$ & $0.2034 \mathrm{E}+04$ & $\odot .221731156$ \\
\hline $270 \odot \odot \odot$ & $27.0 \odot$ & $0.7006 \mathrm{E}+06$ & $\odot .1344 \mathrm{E}+03$ & $\odot .3033 E+03$ & $\odot .20 \odot 3 E+\odot 4$ & $\odot .218328044$ \\
\hline $2800 \odot \odot$ & $28 . \odot \odot$ & $0.6947 \mathrm{E}+06$ & $\odot .1361 \mathrm{E}+03$ & $\odot .3051 \mathrm{E}+03$ & $\odot .1974 \mathrm{E}+04$ & $\odot .215184450$ \\
\hline 290000 & 29.00 & $\odot .6898 \mathrm{E}+06$ & $\odot .1379 \mathrm{E}+03$ & $0.3071 \mathrm{E}+03$ & $\odot .1947 \mathrm{E}+04$ & $\odot .212259546$ \\
\hline $30 \odot \odot \odot \odot$ & $3 \odot . \odot \odot$ & $\odot .6858 \mathrm{E}+\odot 6$ & $\odot .1398 E+\odot 3$ & $\odot .3093 \mathrm{E}+03$ & $\odot .1922 \mathrm{E}+\odot 4$ & $\odot .209561229$ \\
\hline
\end{tabular}

lambda_avg $=0.313054979$ 


\section{Valve spacing $=20$ miles, Pressure $=1167$ psi, 400 axial node elements}

\begin{tabular}{|c|c|c|c|c|c|c|}
\hline$i$ & time ( $\mathrm{sec}$ ) & $\mathrm{P} 1(\mathrm{~Pa})$ & T1 (K) & V1 $(\mathrm{m} / \mathrm{sec})$ & mdot $(\mathrm{kg} / \mathrm{sec})$ & lambda \\
\hline 0 & $\odot . \odot \odot$ & $\odot .4361 \mathrm{E}+07$ & $\odot .2483 \mathrm{E}+03$ & $\odot .4121 \mathrm{E}+03$ & $\odot .9172 \mathrm{E}+\odot 4$ & $1.0000 \odot \odot \odot \odot \odot$ \\
\hline 10000 & 1.00 & $0.1659 \mathrm{E}+07$ & $0.1528 \mathrm{E}+03$ & $0.3233 E+03$ & $\odot .4448 \mathrm{E}+\odot 4$ & ๑.484976888 \\
\hline 20000 & 2.00 & $\odot .1749 \mathrm{E}+07$ & $\odot .1703 E+03$ & $0.3413 E+03$ & $\odot .4442 \mathrm{E}+04$ & $\odot .484325945$ \\
\hline $3000 \odot$ & $3.0 \odot$ & $\odot .1687 \mathrm{E}+07$ & $\odot .1803 E+\odot 3$ & $\odot .3512 \mathrm{E}+03$ & $\odot .4163 \mathrm{E}+04$ & $\odot .453902245$ \\
\hline 40000 & 4.00 & $0.1704 \mathrm{E}+07$ & $\odot .1969 \mathrm{E}+03$ & $0.3670 \mathrm{E}+03$ & $\odot .4025 \mathrm{E}+04$ & 0.438862771 \\
\hline 50000 & 5.00 & $\odot .1556 \mathrm{E}+\odot 7$ & $\odot .2030 \mathrm{E}+03$ & $\odot .3726 \mathrm{E}+03$ & $\odot .3621 E+04$ & $\odot .394726157$ \\
\hline 60000 & 6.00 & $\odot .1414 \mathrm{E}+07$ & $\odot .2089 E+03$ & $\odot .3780 E+03$ & $\odot .3243 E+\odot 4$ & $\odot .353602171$ \\
\hline 70000 & $7 . \odot \odot$ & $\odot .1359 \mathrm{E}+07$ & $\odot .2227 \mathrm{E}+\odot 3$ & $\odot .3903 E+03$ & $\odot .3019 \mathrm{E}+04$ & 0.329111844 \\
\hline 80000 & $8.0 \odot$ & $\odot .1339 \mathrm{E}+07$ & $\odot .2417 \mathrm{E}+03$ & $\odot .4066 \mathrm{E}+03$ & $\odot .2854 \mathrm{E}+\odot 4$ & $\odot .311163187$ \\
\hline 90000 & 9.00 & $\odot .1295 \mathrm{E}+07$ & $\odot .2483 \mathrm{E}+03$ & $0.4121 \mathrm{E}+03$ & $\odot .2724 \mathrm{E}+04$ & $\odot .296958029$ \\
\hline 100000 & 10.00 & $\odot .1243 E+07$ & $\odot .2483 E+03$ & $0.4121 \mathrm{E}+03$ & $\odot .2615 E+04$ & $\odot .285044909$ \\
\hline 110000 & 11.00 & $\odot .1208 \mathrm{E}+07$ & $\odot .2483 E+03$ & $\odot .4121 \mathrm{E}+03$ & $\odot .2541 \mathrm{E}+\odot 4$ & ๑.276996851 \\
\hline 120000 & 12.00 & $0.1175 \mathrm{E}+07$ & $\odot .2483 \mathrm{E}+03$ & $0.4121 \mathrm{E}+03$ & $\odot .2471 \mathrm{E}+04$ & $\odot .269425094$ \\
\hline 130000 & 13.00 & $\odot .1169 \mathrm{E}+07$ & $\odot .2483 E+03$ & $\odot .4121 \mathrm{E}+03$ & $\odot .2458 \mathrm{E}+\odot 4$ & $\odot .268029869$ \\
\hline 140000 & 14.00 & $0.1151 \mathrm{E}+07$ & $\odot .2483 E+03$ & $0.4121 \mathrm{E}+03$ & $\odot .2420 E+\odot 4$ & $\odot .263870388$ \\
\hline 150000 & 15.00 & $\odot .1135 E+07$ & $\odot .2483 E+03$ & $0.4121 \mathrm{E}+03$ & $0.2388 E+04$ & 0.260385484 \\
\hline $16 \odot \odot \odot \odot$ & $16 . \odot \odot$ & $\odot .1123 \mathrm{E}+\odot 7$ & $\odot .2483 E+\odot 3$ & $\odot .4121 \mathrm{E}+03$ & $\odot .2362 \mathrm{E}+\odot 4$ & $\odot .257469326$ \\
\hline 170000 & $17 . \odot \odot$ & $\odot .1111 \mathrm{E}+07$ & $\odot .2483 E+03$ & $\odot .4121 \mathrm{E}+03$ & $\odot .2336 \mathrm{E}+\odot 4$ & $\odot .254673272$ \\
\hline 180000 & $18 . \odot \odot$ & $\odot .1095 \mathrm{E}+07$ & $\odot .2483 E+03$ & $0.4121 \mathrm{E}+03$ & $\odot .23 \odot 2 \mathrm{E}+\odot 4$ & $\odot .251007944$ \\
\hline 190000 & 19.00 & $\odot .1080 E+07$ & $\odot .2483 E+03$ & $0.4121 \mathrm{E}+03$ & $\odot .2273 \mathrm{E}+04$ & 0.247787222 \\
\hline $2000 \odot \odot$ & $20 . \odot \odot$ & $\odot .1071 \mathrm{E}+07$ & $\odot .2483 E+03$ & $0.4121 \mathrm{E}+03$ & $\odot .2253 E+04$ & $\odot .245658770$ \\
\hline $21000 \odot$ & $21 . \odot \odot$ & $\odot .106 \odot \mathrm{E}+\odot 7$ & $\odot .2483 E+\odot 3$ & $\odot .4121 \mathrm{E}+03$ & $\odot .2231 \mathrm{E}+\odot 4$ & $\odot .243201420$ \\
\hline 220000 & 22.00 & $0.1044 \mathrm{E}+07$ & $\odot .2483 \mathrm{E}+03$ & $0.4121 \mathrm{E}+03$ & $\odot .2196 \mathrm{E}+04$ & $\odot .239462331$ \\
\hline 230000 & $23 . \odot \odot$ & $\odot .1033 \mathrm{E}+07$ & $\odot .2483 E+03$ & $\odot .4121 \mathrm{E}+03$ & $\odot .2173 E+04$ & $\odot .236885637$ \\
\hline 240000 & 24.00 & $\odot .1024 \mathrm{E}+07$ & $\odot .2483 E+03$ & $0.4121 \mathrm{E}+03$ & $\odot .2154 \mathrm{E}+04$ & 0.234883487 \\
\hline 250000 & $25 . \odot \odot$ & $0.1018 \mathrm{E}+07$ & $\odot .2483 E+03$ & $0.4121 \mathrm{E}+03$ & $0.2142 \mathrm{E}+04$ & 0.233495265 \\
\hline $260 \odot \odot \odot$ & $26 . \odot \odot$ & $\odot .1013 \mathrm{E}+\odot 7$ & $\odot .2483 E+\odot 3$ & $\odot .4121 \mathrm{E}+03$ & $\odot .2130 E+\odot 4$ & $\odot .232199878$ \\
\hline 270000 & 27.00 & $0.1007 \mathrm{E}+07$ & $\odot .2483 E+03$ & $0.4121 \mathrm{E}+03$ & $0.2119 \mathrm{E}+04$ & 0.230973512 \\
\hline 280000 & $28 . \odot \odot$ & $\odot .9995 E+06$ & $\odot .2483 E+03$ & $0.4121 \mathrm{E}+03$ & $\odot .2102 \mathrm{E}+04$ & 0.229218483 \\
\hline $290 \odot \odot \odot$ & $29 . \odot \odot$ & $\odot .9911 \mathrm{E}+\odot 6$ & $\odot .2483 E+\odot 3$ & $0.4121 \mathrm{E}+03$ & $\odot .2085 E+04$ & $\odot .227286011$ \\
\hline 300000 & $30 . \odot \odot$ & $0.9846 \mathrm{E}+06$ & $\odot .2483 E+03$ & $0.4121 \mathrm{E}+03$ & $\odot .2071 \mathrm{E}+04$ & $\odot .225793824$ \\
\hline
\end{tabular}

lambda_avg $=0.297197551$ 


\section{Valve spacing $=20$ miles, Pressure $=2100$ psi, 20 axial node elements}

\begin{tabular}{|c|c|c|c|c|c|c|}
\hline i & time (sec) & $\mathrm{P} 1 \quad \mathrm{~Pa})$ & T1 (K) & V1 $(\mathrm{m} / \mathrm{sec})$ & mdot $(\mathrm{kg} / \mathrm{sec})$ & lambda \\
\hline 0 & $\odot . \odot \odot$ & $\odot .7847 E+07$ & $\odot .2483 E+03$ & $\odot .4121 E+03$ & $\odot .1651 E+05$ & 1. .0000000000 \\
\hline 10000 & 1.00 & $0.5562 \mathrm{E}+07$ & $0.2044 \mathrm{E}+03$ & $0.3739 \mathrm{E}+03$ & $0.1289 \mathrm{E}+05$ & 0.781159282 \\
\hline $200 \odot \odot$ & $2 . \odot \odot$ & $\odot .422 \odot E+\odot 7$ & $\odot .1749 E+\odot 3$ & $\odot .3459 \mathrm{E}+03$ & $\odot .1058 \mathrm{E}+05$ & $\odot .640771747$ \\
\hline 30000 & 3.00 & $0.3429 \mathrm{E}+07$ & $0.1556 \mathrm{E}+03$ & $0.3262 \mathrm{E}+03$ & $\odot .9110 \mathrm{E}+04$ & $\odot .551925600$ \\
\hline 40000 & $4.0 \odot$ & $\odot .2916 \mathrm{E}+07$ & $0.1421 \mathrm{E}+03$ & $0.3117 \mathrm{E}+03$ & $0.8108 \mathrm{E}+04$ & 0.491264135 \\
\hline 50000 & 5.00 & $0.2548 \mathrm{E}+07$ & $\odot .1317 \mathrm{E}+03$ & $0.3002 \mathrm{E}+03$ & $0.7359 \mathrm{E}+04$ & 0.445841342 \\
\hline 60000 & 6.00 & $0.2268 \mathrm{E}+07$ & $0.1234 \mathrm{E}+03$ & $0.2906 \mathrm{E}+03$ & $0.6765 \mathrm{E}+04$ & 0.409896642 \\
\hline $70 \odot \odot \odot$ & $7 . \odot \odot$ & $\odot .2 \odot 48 \mathrm{E}+\odot 7$ & $\odot .1166 \mathrm{E}+\odot 3$ & $\odot .2824 \mathrm{E}+03$ & $\odot .6284 \mathrm{E}+\odot 4$ & ๑. 380729824 \\
\hline 80000 & 8.00 & $0.1872 \mathrm{E}+07$ & $0.1110 \mathrm{E}+03$ & $0.2755 \mathrm{E}+03$ & $0.5888 \mathrm{E}+04$ & ๑. 356759816 \\
\hline 90000 & 9.00 & $\odot .1729 \mathrm{E}+07$ & $0.1062 \mathrm{E}+03$ & $0.2696 \mathrm{E}+03$ & $\odot .5560 E+04$ & $\odot .336866200$ \\
\hline 100000 & 10.00 & $\odot .1612 \mathrm{E}+07$ & $\odot .1023 \mathrm{E}+03$ & $\odot .2645 \mathrm{E}+03$ & $\odot .5285 E+\odot 4$ & $\odot .320200086$ \\
\hline 110000 & 11.00 & $0.1516 \mathrm{E}+07$ & $\odot .9888 \mathrm{E}+02$ & $0.2601 \mathrm{E}+03$ & $0.5052 \mathrm{E}+04$ & 0.306096762 \\
\hline 120000 & $12 . \odot \odot$ & $\odot .1435 \mathrm{E}+07$ & $\odot .9601 \mathrm{E}+02$ & $\odot .2563 \mathrm{E}+03$ & $\odot .4854 \mathrm{E}+\odot 4$ & $\odot .294068575$ \\
\hline 130000 & 13.00 & $0.1367 \mathrm{E}+07$ & $0.9356 \mathrm{E}+02$ & $0.2530 \mathrm{E}+03$ & $0.4684 \mathrm{E}+04$ & $\odot .283772022$ \\
\hline 140000 & 14.00 & $0.1310 \mathrm{E}+07$ & $\odot .9147 \mathrm{E}+02$ & $0.2501 \mathrm{E}+03$ & $0.4538 \mathrm{E}+04$ & 0.274939537 \\
\hline 150000 & $15 . \odot \odot$ & $\odot .1261 \mathrm{E}+07$ & $\odot .8971 \mathrm{E}+\odot 2$ & $\odot .2477 \mathrm{E}+03$ & $\odot .4413 E+04$ & $\odot .267368466$ \\
\hline 160000 & 16.00 & $0.1220 \mathrm{E}+07$ & $\Theta .8822 \mathrm{E}+02$ & $0.2457 \mathrm{E}+03$ & $0.4306 \mathrm{E}+04$ & $\odot .260879278$ \\
\hline 170000 & $17 . \odot \odot$ & $\odot .1186 \mathrm{E}+07$ & $\odot .8697 \mathrm{E}+\odot 2$ & $\odot .2439 \mathrm{E}+03$ & $\odot .4214 \mathrm{E}+\odot 4$ & $\odot .255307525$ \\
\hline 180000 & 18.00 & $0.1155 \mathrm{E}+07$ & $0.8586 \mathrm{E}+02$ & $0.2423 \mathrm{E}+03$ & $0.4131 \mathrm{E}+04$ & 0.250266880 \\
\hline 190000 & 19.00 & $0.1127 \mathrm{E}+07$ & $0.8484 \mathrm{E}+02$ & $0.2409 \mathrm{E}+03$ & $0.4054 \mathrm{E}+04$ & $\odot .245619953$ \\
\hline $200 \odot \odot \odot$ & $2 \odot . \odot \odot$ & $0.1101 \mathrm{E}+07$ & $\odot .8389 \mathrm{E}+\odot 2$ & $\odot .2396 \mathrm{E}+03$ & $\odot .3982 E+\odot 4$ & $\odot .241277769$ \\
\hline 210000 & 21.00 & $0.1076 \mathrm{E}+07$ & $\odot .8302 \mathrm{E}+02$ & $0.2383 E+03$ & $0.3915 \mathrm{E}+04$ & 0.237191856 \\
\hline 220000 & 22.00 & $0.1054 \mathrm{E}+07$ & $\odot .8221 \mathrm{E}+02$ & $0.2371 \mathrm{E}+03$ & $\odot .3852 \mathrm{E}+04$ & 0.233401224 \\
\hline 230000 & 23.00 & $0.1033 \mathrm{E}+07$ & $0.8146 \mathrm{E}+02$ & $0.2360 \mathrm{E}+03$ & $0.3793 \mathrm{E}+04$ & 0.229831710 \\
\hline 240000 & 24.00 & $0.1013 \mathrm{E}+07$ & $\odot .8075 \mathrm{E}+02$ & $0.2350 \mathrm{E}+03$ & $0.3737 \mathrm{E}+04$ & 0.226436630 \\
\hline 250000 & $25 . \odot \odot$ & $0.9949 \mathrm{E}+06$ & $\odot .8009 E+\odot 2$ & $0.2341 \mathrm{E}+03$ & $\odot .3685 E+04$ & $\odot .223250866$ \\
\hline 260000 & 26.00 & $0.9777 \mathrm{E}+06$ & $0.7947 \mathrm{E}+02$ & $0.2332 \mathrm{E}+03$ & $0.3635 \mathrm{E}+04$ & 0.220232353 \\
\hline 270000 & $27 . \odot \odot$ & $0.9611 \mathrm{E}+06$ & $\odot .7888 \mathrm{E}+02$ & $0.2323 E+03$ & $\odot .3586 \mathrm{E}+04$ & 0.217292562 \\
\hline 280000 & 28.00 & $0.9460 \mathrm{E}+06$ & $\odot .7834 \mathrm{E}+02$ & $0.2315 \mathrm{E}+03$ & $0.3542 \mathrm{E}+04$ & 0.214626968 \\
\hline 290000 & 29.00 & $0.9312 \mathrm{E}+06$ & $\Theta .7782 \mathrm{E}+02$ & $0.2307 \mathrm{E}+03$ & $0.3499 \mathrm{E}+04$ & 0.211984739 \\
\hline 300000 & $3 \odot . \odot \odot$ & $\odot .9172 \mathrm{E}+06$ & $\odot .7734 \mathrm{E}+02$ & $\odot .2300 \mathrm{E}+03$ & $\odot .3457 E+04$ & $\odot .209443063$ \\
\hline
\end{tabular}

lambda_avg $=0.333029151$ 


\section{Valve spacing $=20$ miles, Pressure $=2100$ psi, 100 axial node elements}

\begin{tabular}{|c|c|c|c|c|c|c|}
\hline$i$ & time ( $\mathrm{sec}$ ) & $\mathrm{P} 1(\mathrm{~Pa})$ & T1 (K) & V1 $(\mathrm{m} / \mathrm{sec})$ & mdot $(\mathrm{kg} / \mathrm{sec})$ & lambda \\
\hline 0 & $\odot . \odot \odot$ & $\odot .7847 \mathrm{E}+07$ & $\odot .2483 \mathrm{E}+03$ & $\odot .4121 \mathrm{E}+03$ & $\odot .1651 \mathrm{E}+05$ & $1.0000 \odot \odot \odot \odot \odot$ \\
\hline 10000 & 1.00 & $\odot .2965 \mathrm{E}+07$ & $0.1438 \mathrm{E}+03$ & $0.3136 \mathrm{E}+03$ & $\odot .8194 \mathrm{E}+04$ & ๑.496471971 \\
\hline 20000 & 2.00 & $\odot .2600 \mathrm{E}+\odot 7$ & $\odot .1354 \mathrm{E}+03$ & $0.3043 E+03$ & $\odot .7406 \mathrm{E}+04$ & 0.448689342 \\
\hline $3000 \odot$ & $3.0 \odot$ & $\odot .2318 \mathrm{E}+07$ & $\odot .1287 E+03$ & $\odot .2967 \mathrm{E}+03$ & $\odot .6773 \mathrm{E}+04$ & $\odot .410372823$ \\
\hline 40000 & 4.00 & $\odot .2236 \mathrm{E}+07$ & $\odot .1281 \mathrm{E}+03$ & $0.2960 \mathrm{E}+03$ & $\odot .6549 \mathrm{E}+04$ & $\odot .396752626$ \\
\hline 50000 & 5.00 & $\odot .2210 \mathrm{E}+\odot 7$ & $\odot .1294 \mathrm{E}+03$ & $\odot .2975 E+\odot 3$ & $\odot .6439 E+\odot 4$ & $\odot .390126139$ \\
\hline 60000 & 6.00 & $\odot .2144 \mathrm{E}+07$ & $\odot .1293 E+03$ & $\odot .2974 \mathrm{E}+03$ & $\odot .6249 \mathrm{E}+\odot 4$ & $\odot .378604472$ \\
\hline $7000 \odot$ & $7 . \odot \odot$ & $\odot .2079 \mathrm{E}+\odot 7$ & $\odot .1292 \mathrm{E}+03$ & $\odot .2972 \mathrm{E}+03$ & $\odot .6063 \mathrm{E}+04$ & 0.367336422 \\
\hline 80000 & $8.0 \odot$ & $\odot .2019 \mathrm{E}+\odot 7$ & $\odot .1291 \mathrm{E}+03$ & $\odot .2972 \mathrm{E}+03$ & $\odot .5888 E+04$ & $\odot .356724888$ \\
\hline 90000 & 9.00 & $\odot .1961 \mathrm{E}+07$ & $\odot .1291 \mathrm{E}+03$ & $\odot .2972 \mathrm{E}+03$ & $\odot .5719 \mathrm{E}+04$ & $\odot .346506178$ \\
\hline 100000 & 10.00 & $\odot .1905 E+07$ & $\odot .1291 \mathrm{E}+03$ & $\odot .2972 \mathrm{E}+03$ & $\odot .5556 E+\odot 4$ & $\odot .336615354$ \\
\hline 110000 & 11.00 & $\odot .1851 \mathrm{E}+07$ & $\odot .1291 \mathrm{E}+03$ & $\odot .2972 \mathrm{E}+03$ & $\odot .5398 \mathrm{E}+04$ & $\odot .327030718$ \\
\hline 120000 & 12.00 & $0.1798 \mathrm{E}+07$ & $\odot .1291 \mathrm{E}+03$ & $0.2972 \mathrm{E}+03$ & $\odot .5245 \mathrm{E}+04$ & $\odot .317754477$ \\
\hline 130000 & 13.00 & $\odot .1747 \mathrm{E}+07$ & $\odot .1291 \mathrm{E}+03$ & $\odot .2972 \mathrm{E}+03$ & $\odot .5 \odot 97 \mathrm{E}+\odot 4$ & $\odot .308788717$ \\
\hline 140000 & 14.00 & $\odot .1698 \mathrm{E}+07$ & $\odot .1291 \mathrm{E}+03$ & $0.2972 \mathrm{E}+03$ & $0.4953 \mathrm{E}+04$ & $\odot .300082654$ \\
\hline 150000 & 15.00 & $\odot .1650 \mathrm{E}+07$ & $\odot .1291 \mathrm{E}+03$ & $0.2972 \mathrm{E}+03$ & $\odot .4813 E+04$ & $\odot .291619062$ \\
\hline $16 \odot \odot \odot \odot$ & $16 . \odot \odot$ & $\odot .1604 \mathrm{E}+\odot 7$ & $\odot .1291 \mathrm{E}+\odot 3$ & $\odot .2972 \mathrm{E}+\odot 3$ & $\odot .4678 \mathrm{E}+\odot 4$ & $\odot .283434302$ \\
\hline 170000 & $17 . \odot \odot$ & $\odot .1559 \mathrm{E}+\odot 7$ & $\odot .1291 \mathrm{E}+03$ & $\odot .2972 \mathrm{E}+03$ & $\odot .4547 \mathrm{E}+\odot 4$ & $\odot .275477469$ \\
\hline 180000 & $18 . \odot \odot$ & $\odot .1515 \mathrm{E}+\odot 7$ & $\odot .1291 \mathrm{E}+03$ & $\odot .2972 \mathrm{E}+03$ & $\odot .442 \odot E+\odot 4$ & $\odot .267781764$ \\
\hline 190000 & 19.00 & $\odot .1473 E+07$ & $\odot .1292 \mathrm{E}+03$ & $0.2972 \mathrm{E}+03$ & $0.4296 \mathrm{E}+04$ & $\odot .260302752$ \\
\hline $2000 \odot \odot$ & $20 . \odot \odot$ & $\odot .1432 \mathrm{E}+07$ & $\odot .1292 \mathrm{E}+03$ & $0.2973 \mathrm{E}+03$ & $0.4176 \mathrm{E}+04$ & 0.253028154 \\
\hline $21000 \odot$ & $21 . \odot \odot$ & $\odot .1392 \mathrm{E}+\odot 7$ & $\odot .1292 \mathrm{E}+\odot 3$ & $\odot .2973 \mathrm{E}+03$ & $\odot .406 \odot E+\odot 4$ & $\odot .245954573$ \\
\hline 220000 & 22.00 & $0.1353 \mathrm{E}+07$ & $\odot .1292 \mathrm{E}+03$ & $0.2973 \mathrm{E}+03$ & $\odot .3946 \mathrm{E}+04$ & $\odot .239083961$ \\
\hline 230000 & $23 . \odot \odot$ & $\odot .1316 \mathrm{E}+07$ & $\odot .1293 E+03$ & $\odot .2973 E+03$ & $\odot .3837 E+04$ & $\odot .232451767$ \\
\hline 240000 & 24.00 & $\odot .1283 E+07$ & $\odot .1295 E+03$ & $0.2976 \mathrm{E}+03$ & $0.3737 \mathrm{E}+04$ & $\odot .226404816$ \\
\hline 250000 & $25 . \odot \odot$ & $\odot .1259 \mathrm{E}+07$ & $0.1303 E+03$ & $\odot .2985 \mathrm{E}+03$ & $\odot .3656 E+04$ & $\odot .221496955$ \\
\hline $260 \odot \odot \odot$ & $26 . \odot \odot$ & $\odot .1241 \mathrm{E}+\odot 7$ & $\odot .1314 \mathrm{E}+03$ & $\odot .2998 \mathrm{E}+\odot 3$ & $\odot .3587 E+\odot 4$ & $\odot .217343241$ \\
\hline 270000 & 27.00 & $0.1226 \mathrm{E}+07$ & $\odot .1327 \mathrm{E}+03$ & $0.3013 \mathrm{E}+03$ & $0.3526 \mathrm{E}+04$ & 0.213645428 \\
\hline 280000 & $28 . \odot \odot$ & $\odot .1213 E+07$ & $\odot .1342 \mathrm{E}+03$ & $\odot .3030 \mathrm{E}+03$ & $\odot .3471 \mathrm{E}+04$ & 0.210272953 \\
\hline $290 \odot \odot \odot$ & $29 . \odot \odot$ & $\odot .1203 \mathrm{E}+07$ & $\odot .1359 \mathrm{E}+\odot 3$ & $\odot .3049 \mathrm{E}+03$ & $\odot .3420 E+\odot 4$ & $\odot .207185417$ \\
\hline 300000 & $30 . \odot \odot$ & $0.1194 \mathrm{E}+07$ & $0.1377 \mathrm{E}+03$ & $0.3069 \mathrm{E}+03$ & $\odot .3372 \mathrm{E}+04$ & 0.204322457 \\
\hline
\end{tabular}

lambda_avg $=0.310781360$ 


\section{Valve spacing $=20$ miles, Pressure $=2100$ psi, 400 axial node elements}

\begin{tabular}{|c|c|c|c|c|c|c|}
\hline$i$ & time (sec) & $\mathrm{P} 1 \quad \mathrm{~Pa})$ & T1 (K) & V1 $(\mathrm{m} / \mathrm{sec})$ & mdot $(\mathrm{kg} / \mathrm{sec})$ & lambda \\
\hline 0 & $0.0 \odot$ & $\odot .7847 \mathrm{E}+\odot 7$ & $\odot .2483 E+03$ & $\odot .4121 \mathrm{E}+03$ & $\odot .1651 \mathrm{E}+05$ & 1. $0000 \odot \odot \odot \odot \odot$ \\
\hline $1000 \odot$ & $1 . \odot \odot$ & $\odot .296 \odot E+07$ & $\odot .1521 \mathrm{E}+03$ & $\odot .3226 \mathrm{E}+03$ & $\odot .7955 E+04$ & $\odot .481966555$ \\
\hline $2000 \odot$ & $2.0 \odot$ & $\odot .3101 \mathrm{E}+07$ & $\odot .1689 E+03$ & $\odot .3399 \mathrm{E}+03$ & $\odot .7909 \mathrm{E}+\odot 4$ & $\odot .479150385$ \\
\hline 30000 & 3.00 & $\odot .2994 \mathrm{E}+07$ & $\odot .1790 \mathrm{E}+03$ & $\odot .3499 \mathrm{E}+03$ & $\odot .7417 \mathrm{E}+\odot 4$ & $\odot .449388027$ \\
\hline 40000 & 4.00 & $\odot .2981 \mathrm{E}+07$ & $\odot .1938 \mathrm{E}+03$ & $\odot .3641 \mathrm{E}+03$ & $\odot .7097 \mathrm{E}+04$ & $\odot .429985255$ \\
\hline 50000 & 5.00 & $\odot .2812 \mathrm{E}+\odot 7$ & $\odot .2038 \mathrm{E}+03$ & $\odot .3734 \mathrm{E}+03$ & $\odot .6528 \mathrm{E}+\odot 4$ & ๑.395517766 \\
\hline 60000 & 6.00 & $0.2492 \mathrm{E}+07$ & $\odot .2067 \mathrm{E}+03$ & $0.3761 \mathrm{E}+03$ & $\odot .5745 \mathrm{E}+04$ & ๑.348092884 \\
\hline 70000 & $7.0 \odot$ & $\odot .2353 \mathrm{E}+07$ & $\odot .2182 \mathrm{E}+03$ & $\odot .3863 E+03$ & $\odot .5280 E+\odot 4$ & $\odot .319873244$ \\
\hline 80000 & 8.00 & $\odot .2310 E+\odot 7$ & $\odot .2364 \mathrm{E}+03$ & $\odot .4021 \mathrm{E}+03$ & $\odot .4979 \mathrm{E}+\odot 4$ & ๑.301686347 \\
\hline 90000 & $9.0 \odot$ & $0.2241 \mathrm{E}+07$ & $\odot .2483 E+03$ & $0.4121 \mathrm{E}+03$ & $0.4714 \mathrm{E}+04$ & $\odot .285630912$ \\
\hline $1000 \odot \odot$ & 10.00 & $\odot .2142 \mathrm{E}+\odot 7$ & $\odot .2483 E+\odot 3$ & $\odot .4121 \mathrm{E}+03$ & $\odot .45 \odot 7 \mathrm{E}+\odot 4$ & $\odot .273034990$ \\
\hline 110000 & 11.00 & $0.2103 \mathrm{E}+07$ & $\odot .2483 \mathrm{E}+03$ & $0.4121 \mathrm{E}+03$ & $0.4424 \mathrm{E}+04$ & $\odot .268013239$ \\
\hline 120000 & $12 . \odot \odot$ & $0.2058 \mathrm{E}+07$ & $\odot .2483 E+03$ & $0.4121 \mathrm{E}+03$ & $\odot .4328 E+04$ & $\odot .262243897$ \\
\hline 130000 & 13.00 & $0.2007 \mathrm{E}+07$ & $\odot .2483 E+03$ & $0.4121 \mathrm{E}+03$ & $0.4221 \mathrm{E}+04$ & $\odot .255753368$ \\
\hline 140000 & 14.00 & $\odot .1965 \mathrm{E}+07$ & $\odot .2483 \mathrm{E}+03$ & $0.4121 \mathrm{E}+03$ & $\odot .4134 \mathrm{E}+04$ & 0.250464708 \\
\hline $15000 \odot$ & $15 . \odot \odot$ & $\odot .1931 \mathrm{E}+\odot 7$ & $\odot .2483 E+\odot 3$ & $\odot .4121 \mathrm{E}+03$ & $\odot .4061 \mathrm{E}+\odot 4$ & $\odot .246045515$ \\
\hline 160000 & 16.00 & $0.1898 \mathrm{E}+07$ & $\odot .2483 E+03$ & $0.4121 \mathrm{E}+03$ & $\odot .3993 \mathrm{E}+04$ & $\odot .241893858$ \\
\hline 170000 & 17.00 & $\odot .1870 E+\odot 7$ & $\odot .2483 \mathrm{E}+03$ & $0.4121 \mathrm{E}+03$ & $\odot .3933 \mathrm{E}+04$ & $\odot .238275796$ \\
\hline 180000 & 18.00 & $0.1842 \mathrm{E}+07$ & $\odot .2483 E+03$ & $0.4121 \mathrm{E}+03$ & $\odot .3875 E+04$ & 0.234772876 \\
\hline 190000 & 19.00 & $\odot .1820 \mathrm{E}+07$ & $\odot .2483 \mathrm{E}+03$ & $0.4121 \mathrm{E}+03$ & $\odot .3829 \mathrm{E}+04$ & ๑.231998548 \\
\hline $20 \odot \odot \odot \odot$ & $2 \odot . \odot \odot$ & $\odot .1797 \mathrm{E}+\odot 7$ & $\odot .2483 E+\odot 3$ & $\odot .4121 \mathrm{E}+03$ & $\odot .3781 \mathrm{E}+\odot 4$ & ๑.229064181 \\
\hline 210000 & 21.00 & $0.1771 \mathrm{E}+07$ & $\odot .2483 E+03$ & $0.4121 \mathrm{E}+03$ & $0.3725 E+04$ & $\odot .225681230$ \\
\hline 220000 & $22 . \odot \odot$ & $0.1745 E+07$ & $\odot .2483 E+03$ & $0.4121 \mathrm{E}+03$ & $\odot .3671 E+04$ & $\odot .222416759$ \\
\hline $230 \odot \odot \odot$ & 23.00 & $0.1724 \mathrm{E}+07$ & $\odot .2483 E+03$ & $0.4121 \mathrm{E}+03$ & $\odot .3626 E+04$ & $\odot .219700471$ \\
\hline 240000 & 24.00 & $\odot .1708 \mathrm{E}+07$ & $\odot .2483 \mathrm{E}+03$ & $0.4121 \mathrm{E}+03$ & $\odot .3593 \mathrm{E}+\odot 4$ & 0.217660293 \\
\hline $250 \odot \odot \odot$ & $25 . \odot \odot$ & $\odot .1696 \mathrm{E}+\odot 7$ & $\odot .2483 E+\odot 3$ & $\odot .4121 \mathrm{E}+03$ & $\odot .3568 E+\odot 4$ & $\odot .216191307$ \\
\hline 260000 & 26.00 & $0.1685 \mathrm{E}+07$ & $\odot .2483 E+03$ & $0.4121 \mathrm{E}+03$ & $0.3543 \mathrm{E}+04$ & $\odot .214686975$ \\
\hline $270 \odot \odot \odot$ & $27.0 \odot$ & $\odot .1670 \mathrm{E}+07$ & $\odot .2483 E+\odot 3$ & $0.4121 \mathrm{E}+03$ & $\odot .3513 E+04$ & $\odot .212830275$ \\
\hline $2800 \odot \odot$ & $28 . \odot \odot$ & $0.1652 \mathrm{E}+07$ & $\odot .2483 E+\odot 3$ & $0.4121 \mathrm{E}+03$ & $\odot .3475 E+04$ & $\odot .210537940$ \\
\hline 290000 & 29.00 & $\odot .1636 \mathrm{E}+07$ & $\odot .2483 \mathrm{E}+03$ & $0.4121 \mathrm{E}+03$ & $\odot .3442 \mathrm{E}+\odot 4$ & ๑.208533257 \\
\hline $30 \odot \odot \odot \odot$ & $3 \odot . \odot \odot$ & $\odot .1624 \mathrm{E}+\odot 7$ & $\odot .2483 E+\odot 3$ & $\odot .4121 \mathrm{E}+03$ & $\odot .3415 E+\odot 4$ & $\odot .206920236$ \\
\hline
\end{tabular}

lambda_avg $=0.284179538$ 


\section{Appendix C. REAL GAS RUNGE-KUTTA FORTRAN CODE LISTING}

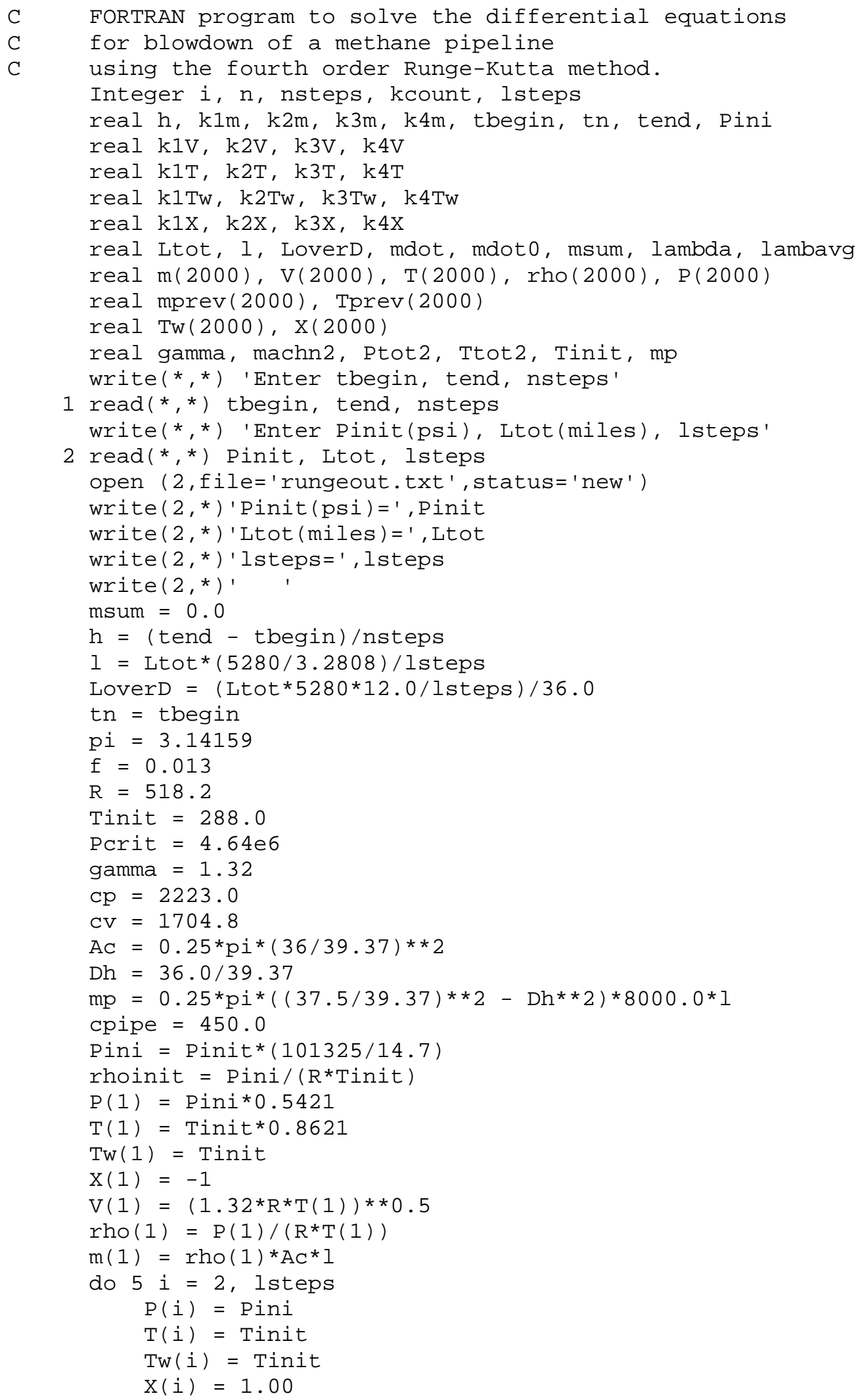




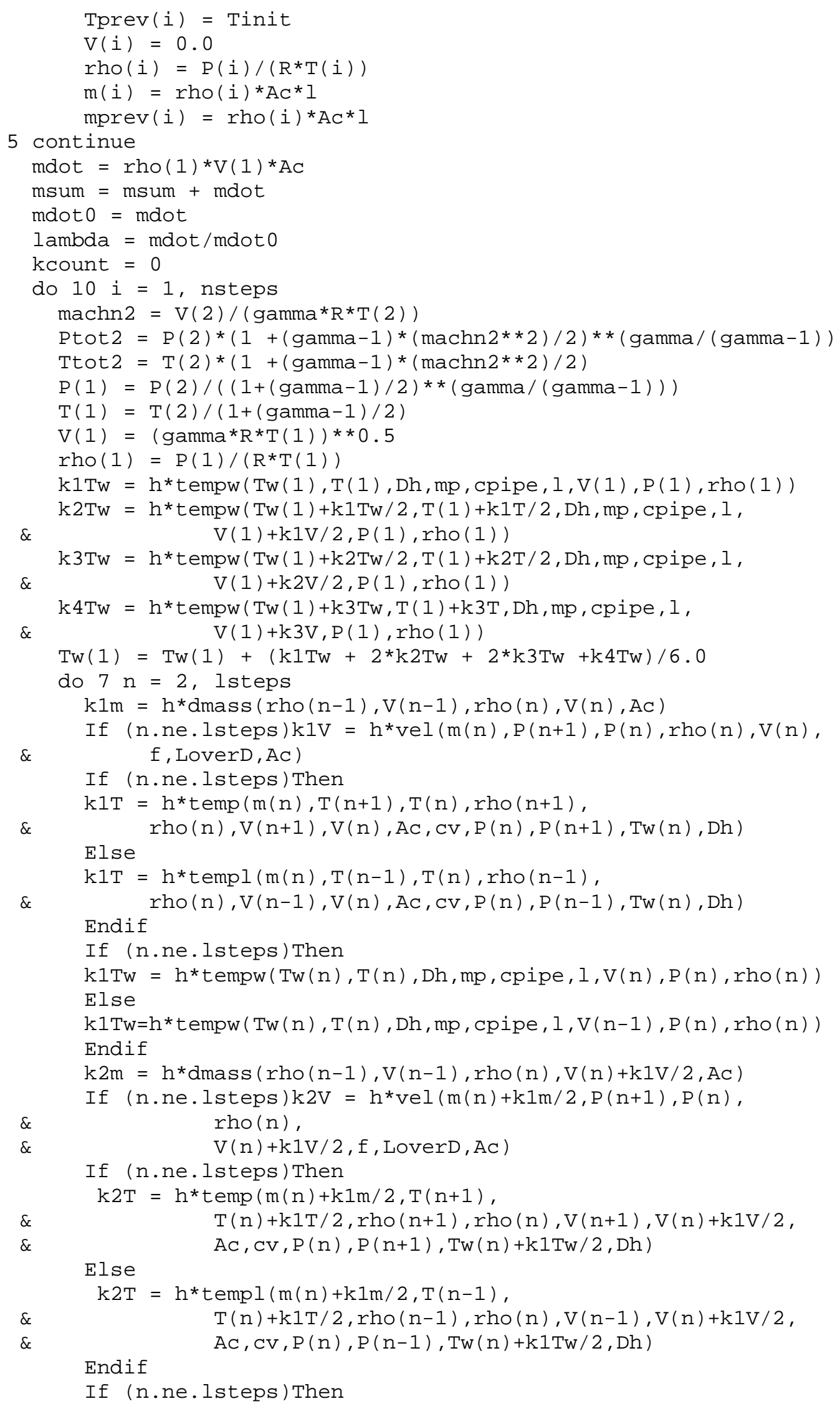


$\&$

$\&$

$\&$

$\&$

$\&$

$\&$

$\&$

$\&$

\&

$\&$

$\&$

$\&$

$\&$

$\&$

$\&$

\&

$\&$

$\mathrm{k} 2 \mathrm{Tw}=\mathrm{h} * \operatorname{tempw}(\mathrm{Tw}(\mathrm{n})+\mathrm{k} 1 \mathrm{Tw} / 2, \mathrm{~T}(\mathrm{n})+\mathrm{k} 1 \mathrm{~T} / 2, \mathrm{Dh}, \mathrm{mp}, \mathrm{cpipe}, 1$, $\mathrm{V}(\mathrm{n})+\mathrm{k} 1 \mathrm{~V} / 2, \mathrm{P}(\mathrm{n}), \mathrm{rho}(\mathrm{n}))$

Else

$\mathrm{k} 2 \mathrm{Tw}=\mathrm{h} * \operatorname{tempw}(\mathrm{Tw}(\mathrm{n})+\mathrm{k} 1 \mathrm{Tw} / 2, \mathrm{~T}(\mathrm{n})+\mathrm{k} 1 \mathrm{~T} / 2, \mathrm{Dh}, \mathrm{mp}, \mathrm{cpipe}, 1$, $V(n-1), P(n)$, rho $(n))$

Endif

$\mathrm{k} 3 \mathrm{~m}=\mathrm{h} * \operatorname{dmass}(\mathrm{rho}(\mathrm{n}-1), \mathrm{v}(\mathrm{n}-1), r h o(n), \mathrm{V}(\mathrm{n})+\mathrm{k} 2 \mathrm{~V} / 2, A c)$

If $(n$. ne.lsteps $) k 3 V=h{ }^{*} \operatorname{vel}(m(n)+k 2 m / 2, P(n+1), P(n)$, rho $(n)$, $\mathrm{V}(\mathrm{n})+\mathrm{k} 2 \mathrm{~V} / 2, \mathrm{f}$, LoverD, Ac )

If (n.ne.lsteps) Then $\mathrm{k} 3 \mathrm{~T}=\mathrm{h} * \operatorname{temp}(\mathrm{m}(\mathrm{n})+\mathrm{k} 2 \mathrm{~m} / 2, \mathrm{~T}(\mathrm{n}+1)$, $T(n)+k 2 T / 2, r h o(n+1), r h o(n), V(n+1), V(n)+k 2 V / 2$,

\section{Else} $A c, c v, P(n), P(n+1), \operatorname{Tw}(n)+k 2 T w / 2, D h)$

$$
\mathrm{k} 3 \mathrm{~T}=\mathrm{h} \text { *templ }(\mathrm{m}(\mathrm{n})+\mathrm{k} 2 \mathrm{~m} / 2, \mathrm{~T}(\mathrm{n}-1) \text {, }
$$
$T(n)+k 2 T / 2, r h o(n-1), r h o(n), V(n-1), V(n)+k 2 V / 2$,

Endif $A c, c v, P(n), P(n-1), T w(n)+k 2 T w / 2, D h)$

If ( $n$. ne.lsteps) Then

$\mathrm{k} 3 \mathrm{Tw}=\mathrm{h} * \operatorname{tempw}(\mathrm{Tw}(\mathrm{n})+\mathrm{k} 2 \mathrm{Tw} / 2, \mathrm{~T}(\mathrm{n})+\mathrm{k} 2 \mathrm{~T} / 2, \mathrm{Dh}, \mathrm{mp}, \mathrm{cpipe}, 1$, $V(n)+k 2 V / 2, P(n), r h o(n))$

Else

$\mathrm{k} 3 \mathrm{Tw}=\mathrm{h} * \operatorname{tempw}(\mathrm{Tw}(\mathrm{n})+\mathrm{k} 2 \mathrm{Tw} / 2, \mathrm{~T}(\mathrm{n})+\mathrm{k} 2 \mathrm{~T} / 2, \mathrm{Dh}, \mathrm{mp}, \mathrm{cpipe}, 1$,

Endif $V(n-1), P(n), r h o(n))$

$\mathrm{k} 4 \mathrm{~m}=h^{*} \operatorname{dmass}(\mathrm{rho}(\mathrm{n}-1), \mathrm{V}(\mathrm{n}-1), \mathrm{rho}(\mathrm{n}), \mathrm{V}(\mathrm{n})+\mathrm{k} 3 \mathrm{~V}, \mathrm{Ac})$

If $\left(n\right.$. ne.lsteps) $k 4 V=h{ }^{*} \operatorname{vel}(m(n)+k 3 m, P(n+1), P(n)$, rho $(n)$, $\mathrm{V}(\mathrm{n})+\mathrm{k} 3 \mathrm{~V}, \mathrm{f}$, LoverD, Ac )

If ( $n$. ne.lsteps) Then $\mathrm{k} 4 \mathrm{~T}=\mathrm{h} * \operatorname{temp}(\mathrm{m}(\mathrm{n})+\mathrm{k} 3 m, T(n+1), T(n)+k 3 T$, rho $(n+1), r h o(n), V(n+1), V(n)+k 3 V, A c, c v, P(n)$, $P(n+1), \operatorname{Tw}(n)+k 3 T w, D h)$

Else

$k 4 T=h * t e m p l(m(n)+k 3 m, T(n-1), T(n)+k 3 T$, rho $(n-1), \operatorname{rho}(n), V(n-1), V(n)+k 3 V, A c, c v, P(n)$, $P(n-1), \operatorname{Tw}(n)+k 3 T w, D h)$

If ( $n$. ne. lsteps) Then

$\mathrm{k} 4 \mathrm{Tw}=\mathrm{h} * \operatorname{tempw}(\mathrm{Tw}(\mathrm{n})+\mathrm{k} 3 \mathrm{Tw}, \mathrm{T}(\mathrm{n})+\mathrm{k} 3 \mathrm{~T}, \mathrm{Dh}, \mathrm{mp}, \mathrm{cpipe}, 1$,

Else $V(n)+k 3 V, P(n), r h o(n))$

$\mathrm{k} 4 \mathrm{Tw}=\mathrm{h}$ *tempw $(\mathrm{Tw}(\mathrm{n})+\mathrm{k} 3 \mathrm{Tw}, \mathrm{T}(\mathrm{n})+\mathrm{k} 3 \mathrm{~T}, \mathrm{Dh}, \mathrm{mp}$, cpipe, 1 ,

$m(n)=m(n)+(k 1 m+2 * k 2 m+2 * k 3 m+k 4 m) / 6.0$

If $(n \cdot g t .2)$ Then

If $(m(n) \cdot g t \cdot \operatorname{mprev}(n)) m(n)=\operatorname{mprev}(n)$

If $(m(n) \cdot l t . m(n-1)) m(n)=\operatorname{mprev}(n)$

Endif

If $(n$. ne. lsteps $) V(n)=V(n)+(k 1 V+2 * k 2 V+2 * k 3 V+k 4 V) / 6.0$

If $(V(n) \cdot g t \cdot V(n-1)) V(n)=V(n-1)$

If $(V(n) . l t . \odot) V(n)=-V(n)$

$T(n)=T(n)+(k 1 T+2 * k 2 T+2 * k 3 T+k 4 T) / 6.0$

$\operatorname{Tw}(n)=\operatorname{Tw}(n)+(k 1 T w+2 * k 2 T w+2 * k 3 T w+k 4 T w) / 6.0$

If $(T(n) \cdot g t$. Tinit) $T(n)=$ Tinit 


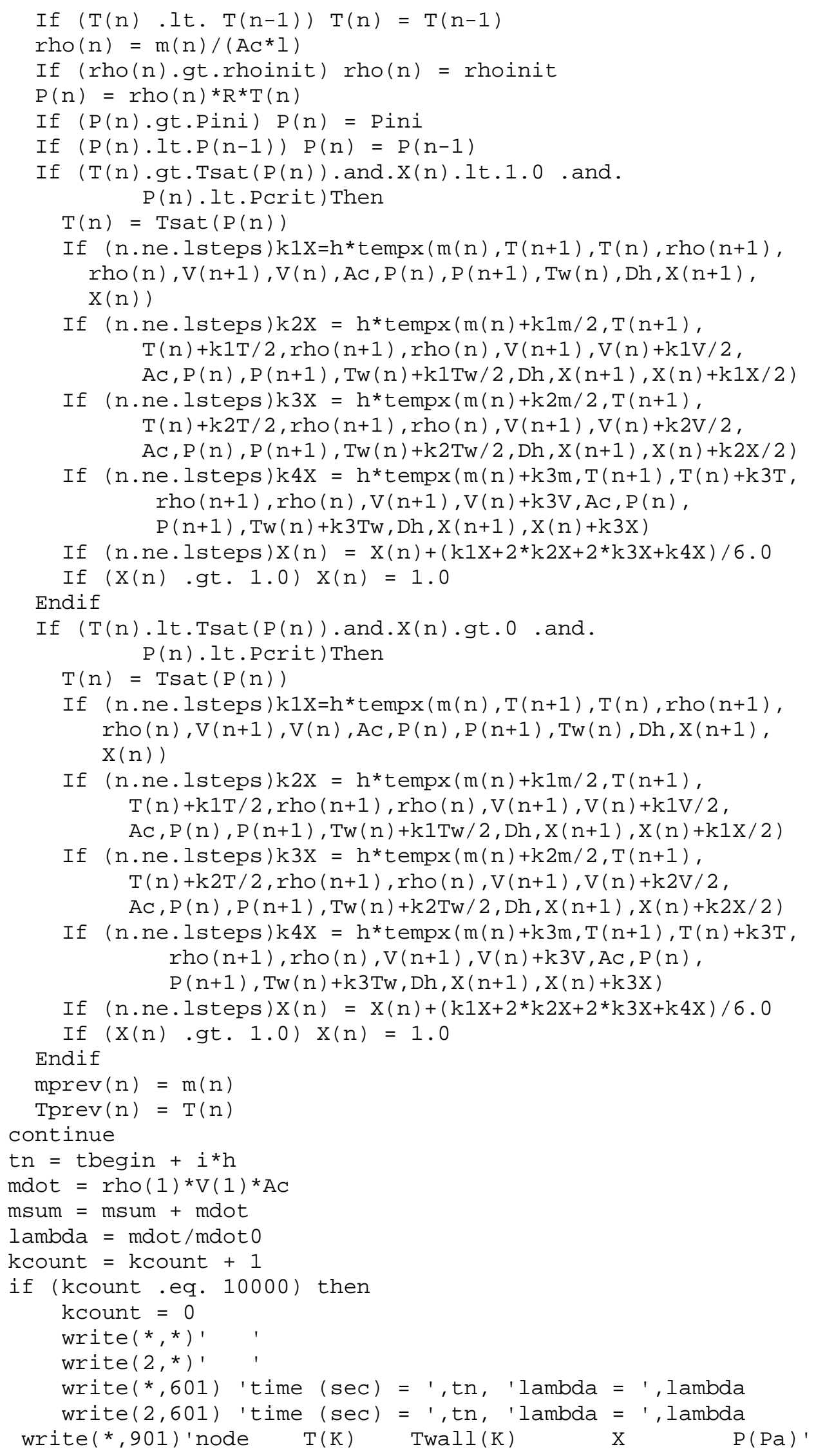




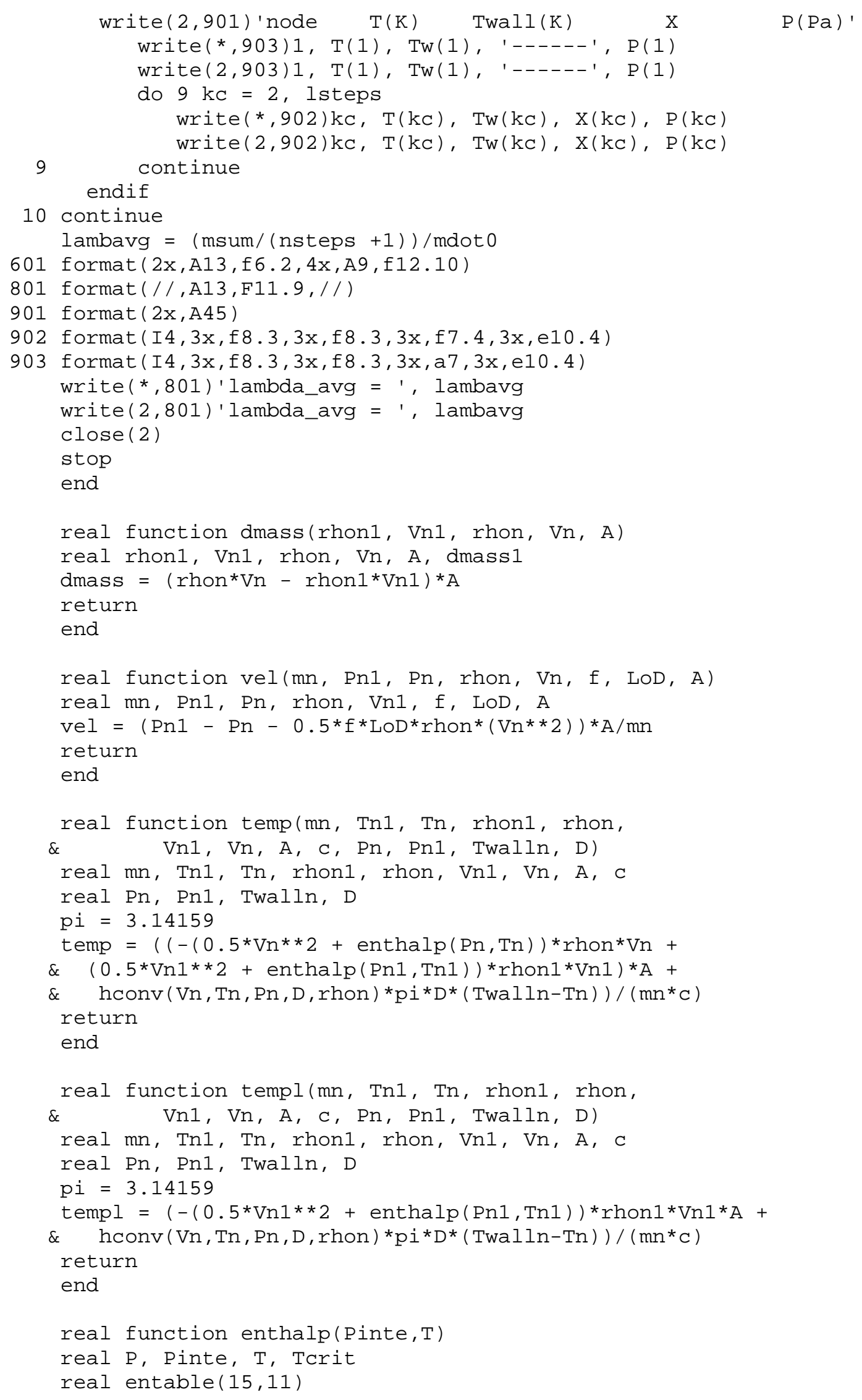




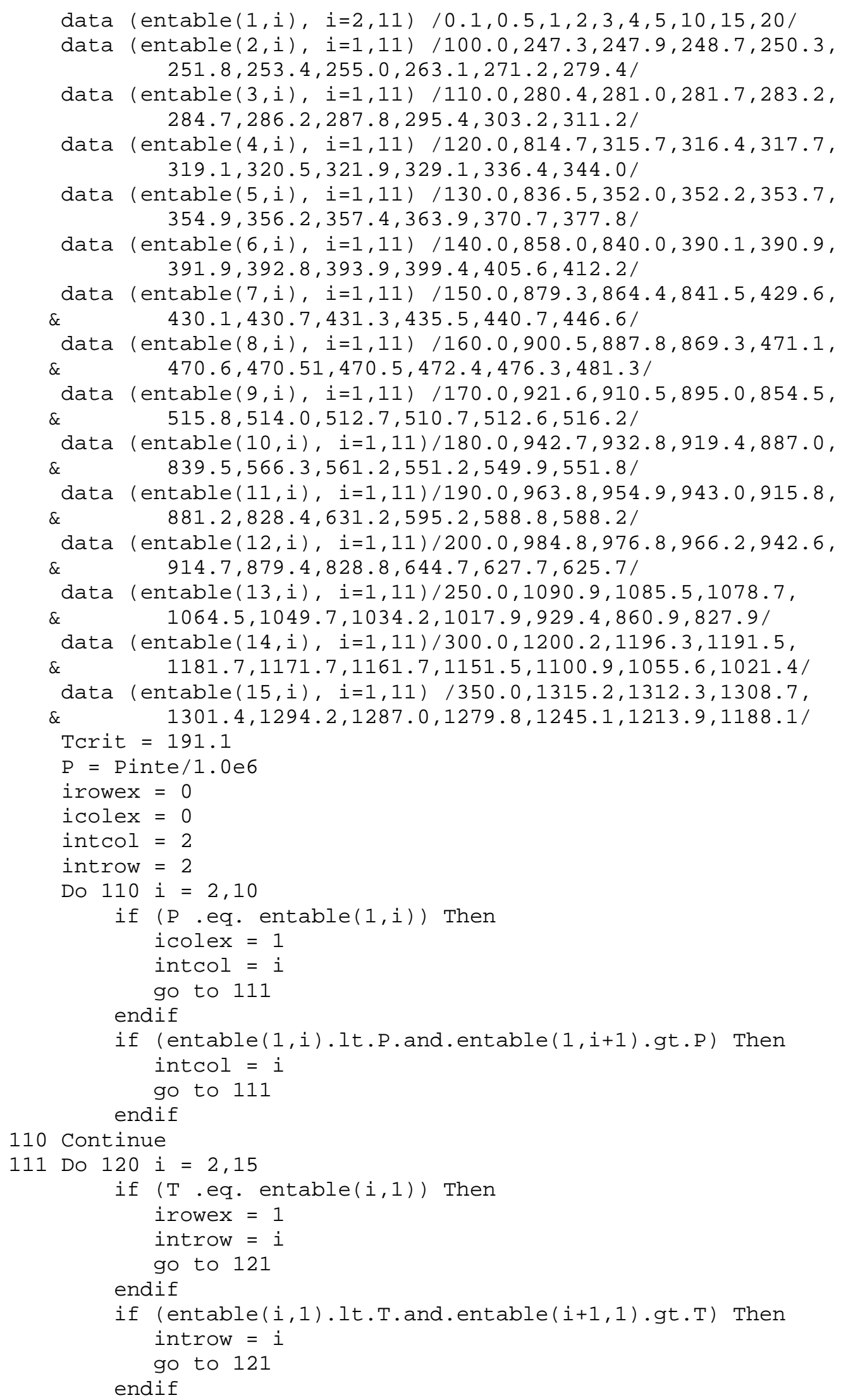


121 Continue

if (T.gt.Tsat (P).and.T.lt.Tcrit) Then

if (irowex .eq. 1 .and. icolex .eq. 1) Then

enthalp $=$ entable $($ irowex, icolex $) * 1000.0$

return

else if (irowex .eq. 1 . and. icolex .eq. $\odot$ ) Then

if (entable (1, intcol+1).gt.Psat (entable (irowex, 1)))Then enthalp $=($ entable $($ irowex, intcol $)+(\mathrm{hg}(\mathrm{P})$ - entable $($ irowex,

$\&$

$\&$ intcol $))^{*}(\mathrm{P}$-entable $(1$, intcol $)) /($ Psat $(T)-$

return entable $(1$, intcol $)){ }^{*} 1000.0$

else

enthalp $=($ entable $($ irowex, intcol $)+($ entable $($ irowex, intcol +1$)$

$\&$-entable (irowex, intcol $)) *(P-\operatorname{entable}(1$, intcol $)) /($ entable $(1$,

\& intcol+1)-entable $(1$, intcol $))) * 1000.0$

return

endif

else if (irowex .eq. $\odot$.and. icolex .eq. 1)Then

if (entable(introw, 1$)$. lt. Tsat (entable (1, icolex)) ) Then enthalp $=(h g(P)+($ entable $($ introw +1 , icolex $)-h g(P))$ *

\& return $(T-T s a t(P)) /($ entable $($ introw $+1,1)-T \operatorname{sat}(P))) * 1000.0$

else

enthalp $=($ entable $($ introw, icolex $)+($ entable $($ introw +1, icolex $)$

\& -entable (introw, icolex $))^{*}($ T-entable (introw, 1$\left.)\right) /($ entable (

\& introw $+1,1)$-entable $($ introw, 1)) )*1000.0

return

endif

else

if (entable(1, intcol+1).gt.Psat (entable(introw, 1)) )Then

Tinterp1=entable (introw, intcol) +(entable (introw +1 , intcol)

\& -entable(introw, intcol $))^{*}(\mathrm{~T}$-entable $($ introw, 1$)) /($ entable

\& $\quad($ introw $+1,1)$-entable $($ introw, 1))

\&

enthalp $=($ Tinterp1 $+($ hg $($ Psat $(T))-$ Tinterp 1$) *(P$-entable $(1$,

return intcol $)) /($ Psat $(T)$-entable $(1$, intcol $)){ }^{*} 1000.0$

else

Tinterp1=entable (introw, intcol $)+($ entable (introw +1 , intcol)

\& -entable (introw, intcol) $) *(T$-entable $($ introw, 1$)) /($ entable

\& (introw 1,1$)$-entable (introw, 1))

Tinterp2=entable (introw, intcol+1)+(entable(introw+1,

\& intcol+1) - entable (introw, intcol +1$)) *(T-e n t a b l e($ introw, 1))

\& $/($ entable $($ introw $+1,1)$-entable $($ introw, 1$))$ enthalp $=($ Tinterp1 $+($ Tinterp $2-$ Tinterp 1$) *(P-$ entable $(1$,

$\&$ intcol $)) /(\operatorname{entable}(1$, intcol +1$)-\operatorname{entable}(1$, intcol $))) * 1000.0$ return

endif

endif

else

if (irowex .eq. 1 .and. icolex .eq. 1) Then

enthalp $=$ entable $($ irowex, icolex $) * 1000.0$

return

else if (irowex .eq. 1 .and. icolex .eq. 0 ) Then

enthalp $=($ entable $($ irowex, intcol $)+($ entable $($ irowex, intcol +1$)$

\& -entable (irowex, intcol $))^{*}(\mathrm{P}$-entable $(1$, intcol $)) /($ entable $(1$,

$\&$ intcol+1)-entable $(1$, intcol $))) * 1000.0$

return 


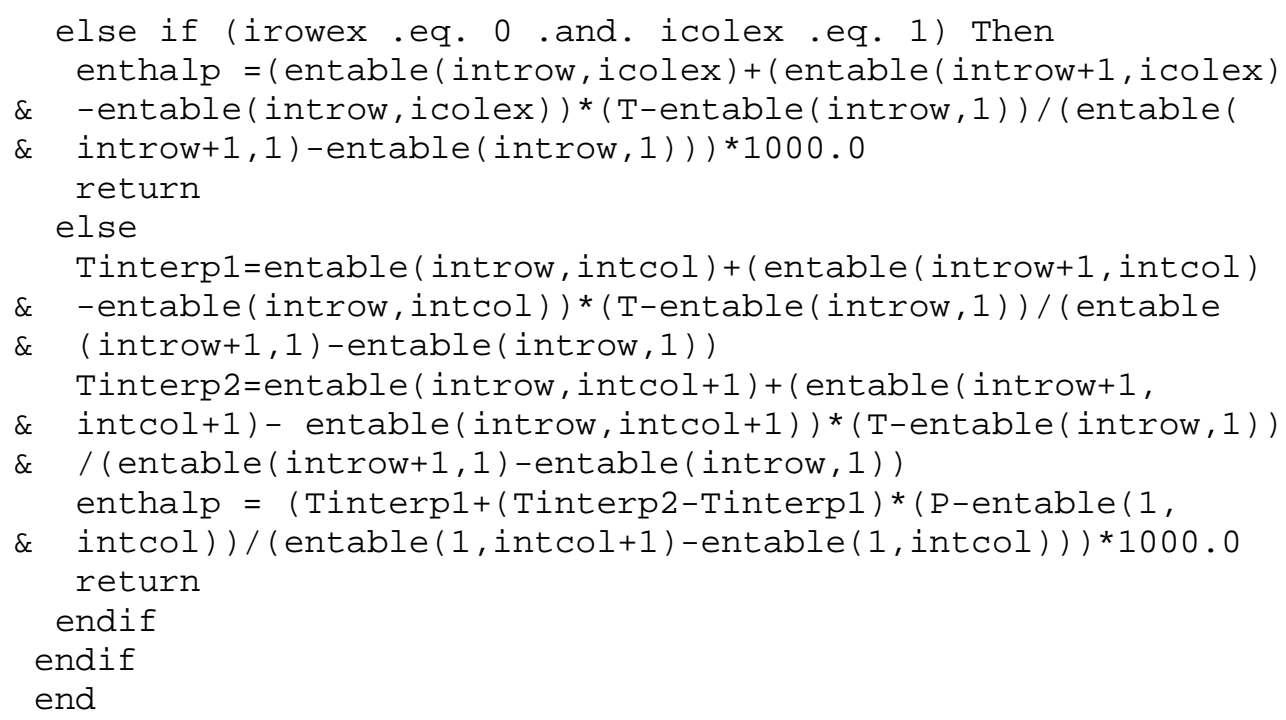




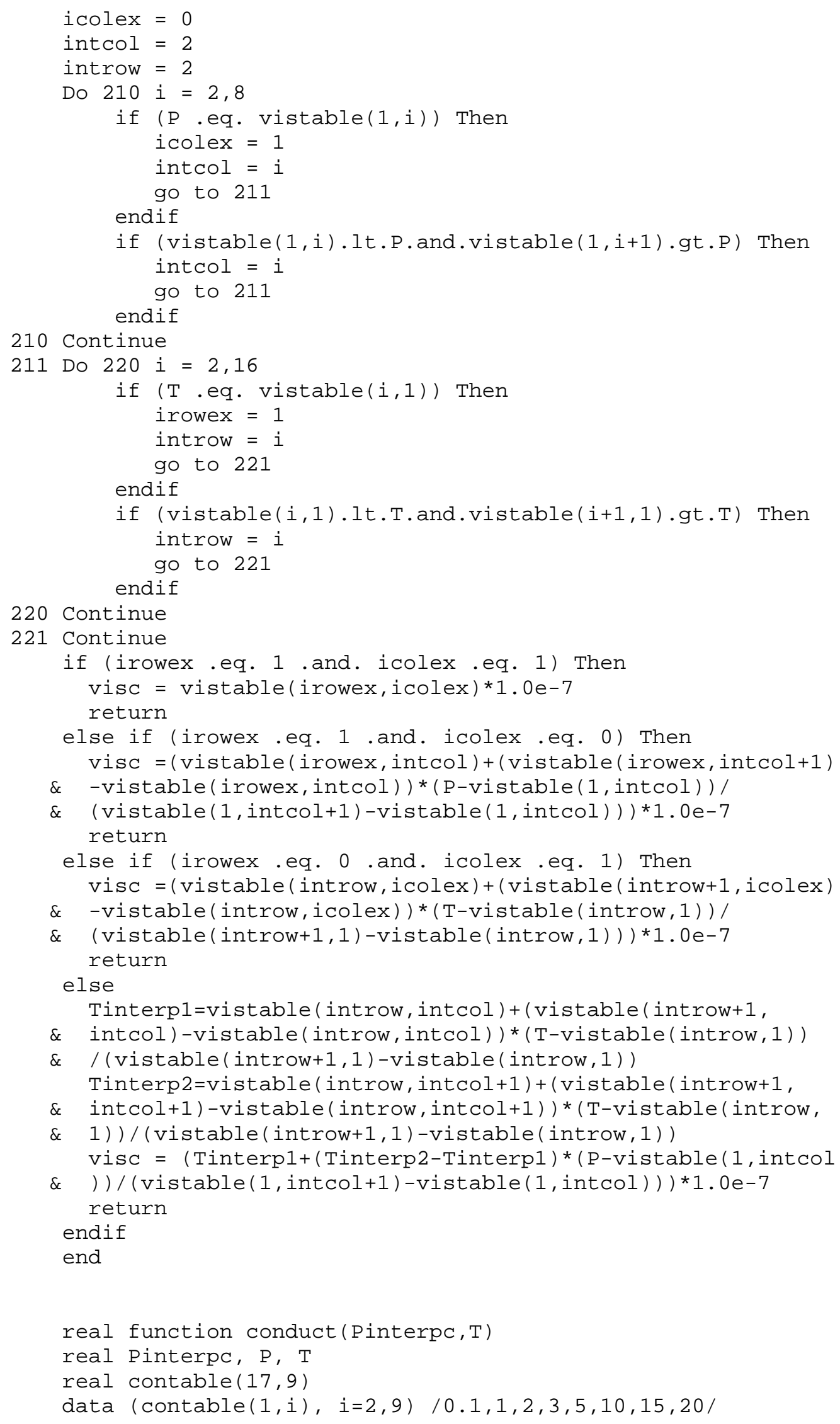




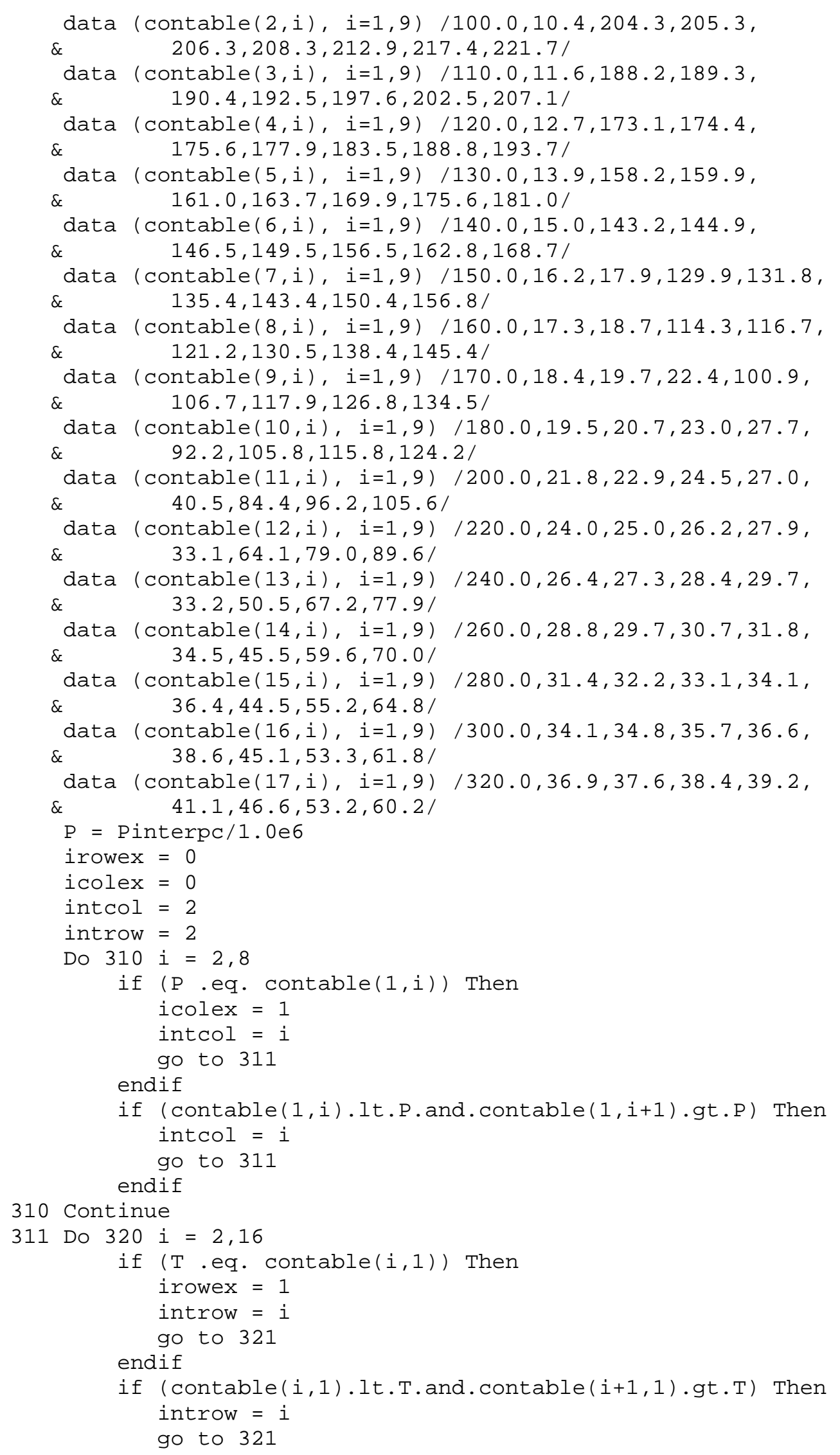




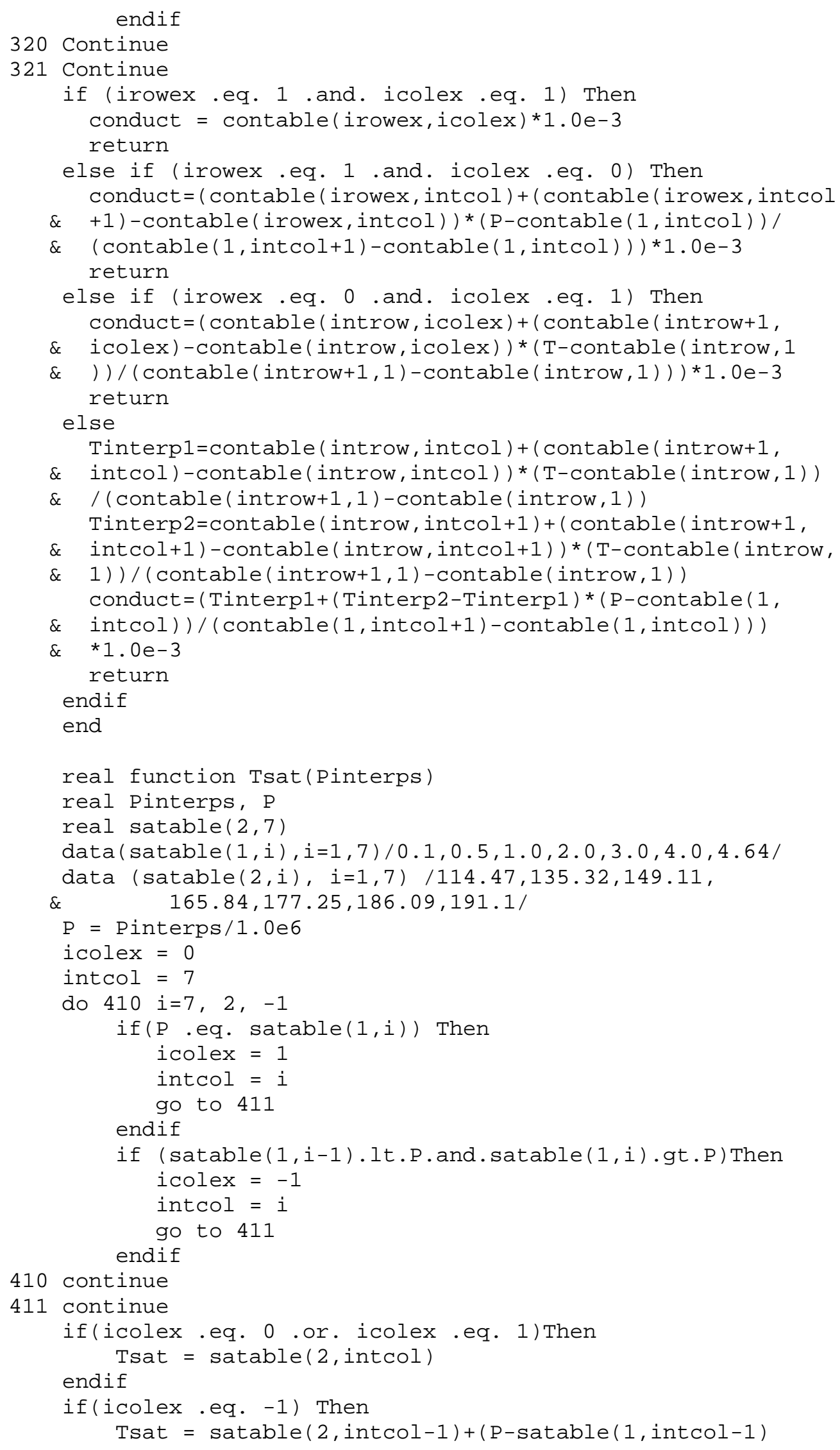




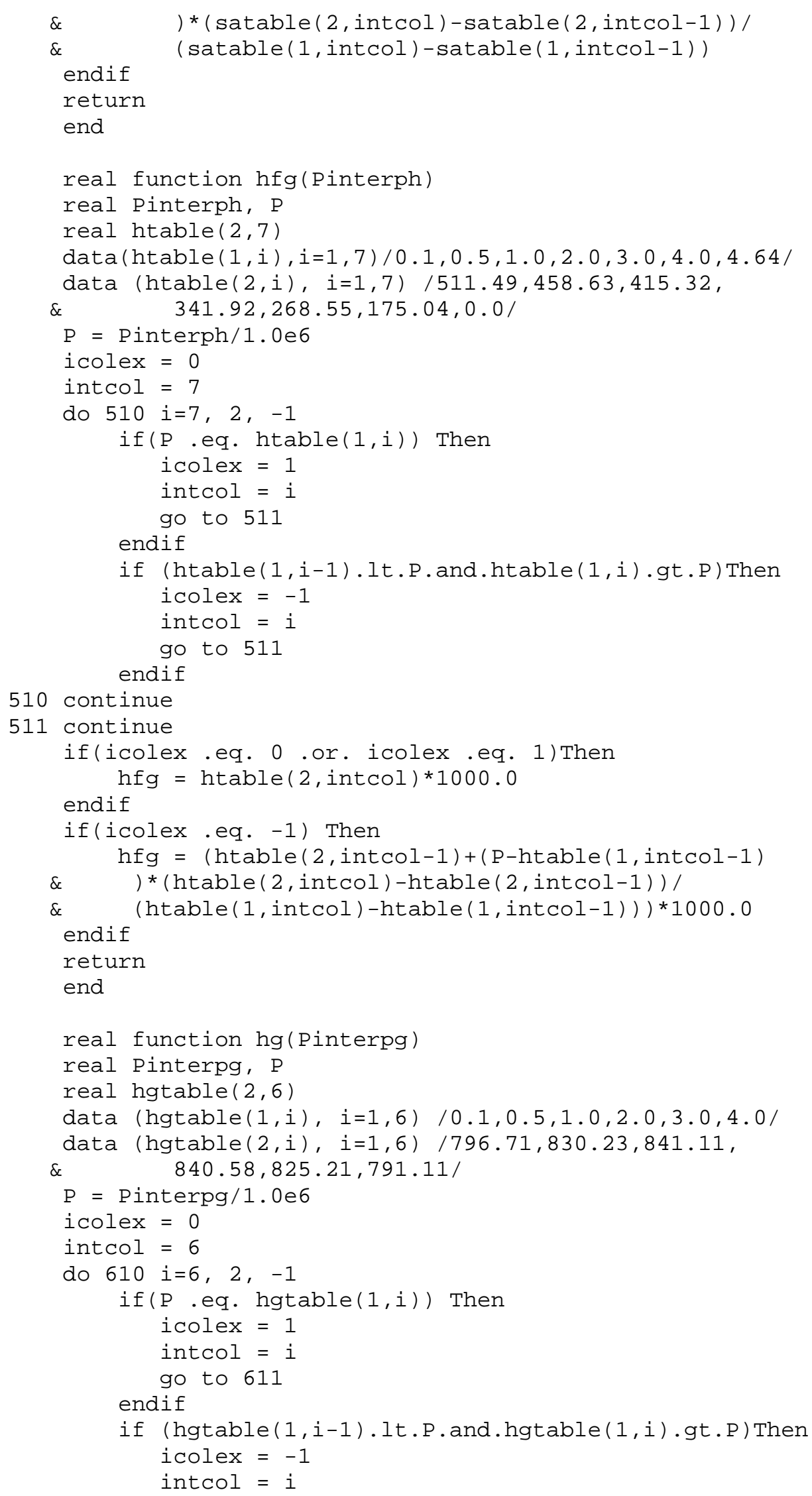




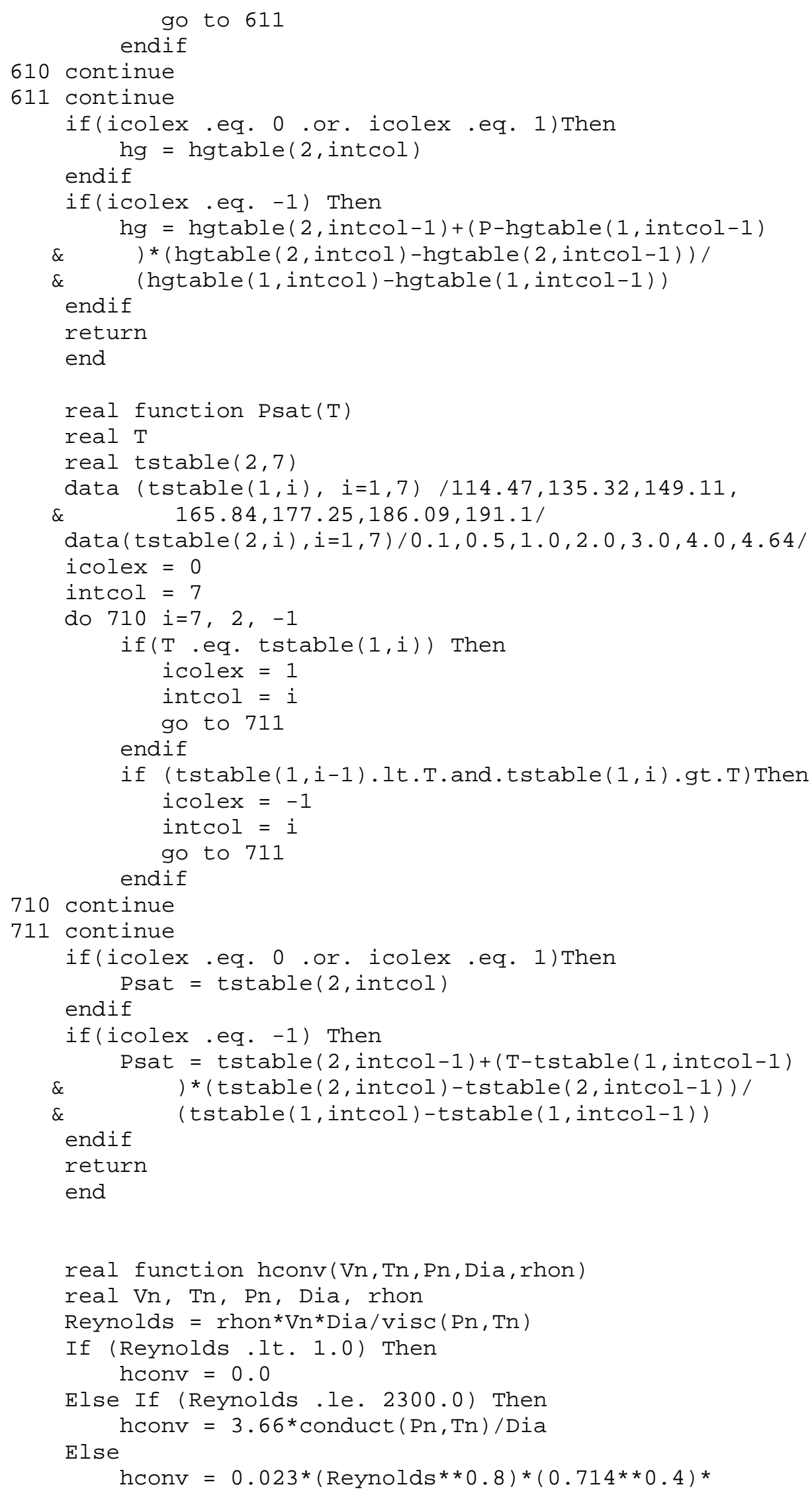




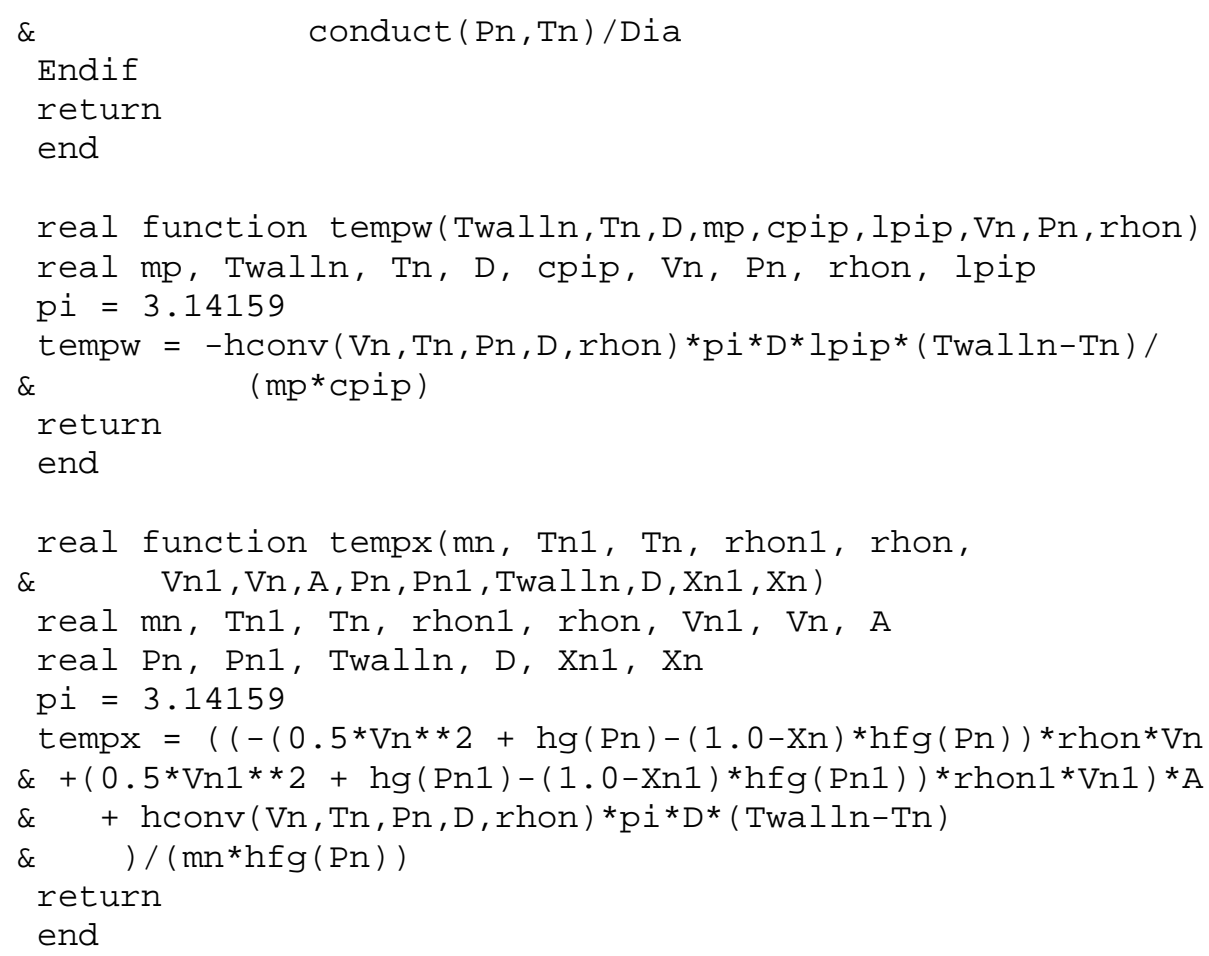




\section{Appendix D. COLLECTED REAL GAS FORTRAN CODE OUTPUT}

Compressor station distance $=5$ miles, Pressure $=1167$ psi, 5 axial node elements

\begin{tabular}{|c|c|c|c|c|}
\hline time & $(\sec )=$ & $1.00 \quad l$ & \multicolumn{2}{|c|}{ lambda $=0.8512680531$} \\
\hline node & $\mathrm{T}(\mathrm{K})$ & Twall(K) & ) $\quad x$ & $\mathrm{P}(\mathrm{Pa})$ \\
\hline 1 & 247.398 & 281.591 & $-\ldots$ & $\odot .3705 \mathrm{E}+07$ \\
\hline 2 & 286.982 & 288.000 & 1.0000 & $\odot .6835 \mathrm{E}+07$ \\
\hline 3 & $288 . \odot \odot \odot$ & 288.000 & $1.000 \odot$ & $\odot .8033 \mathrm{E}+\odot 7$ \\
\hline 4 & 288.000 & 288.000 & 1.0000 & $\odot .8044 \mathrm{E}+07$ \\
\hline 5 & 288.000 & 288.000 & 1.0000 & $\odot .8044 \mathrm{E}+07$ \\
\hline time & $(\mathrm{sec})=$ & \multirow{2}{*}{\multicolumn{2}{|c|}{$\begin{array}{c}2.00 \quad \text { lambda }=0.7244948745 \\
\text { Twall }(K)\end{array}$}} & \\
\hline node & $\mathrm{T}(\mathrm{K})$ & & & $\mathrm{P}(\mathrm{Pa})$ \\
\hline 1 & 241.273 & 276.471 & $-\ldots$ & $\odot .3114 \mathrm{E}+07$ \\
\hline 2 & 279.876 & 287.945 & 1.0000 & $\odot .5744 \mathrm{E}+07$ \\
\hline 3 & 287.963 & $288.00 \odot$ & 1.0000 & $\odot .7962 \mathrm{E}+\odot 7$ \\
\hline 4 & $288 . \odot \odot \odot$ & \multirow{2}{*}{$\begin{array}{l}288 . \odot \odot \odot ~ \\
288.0 \odot \odot\end{array}$} & $1.000 \odot$ & $\odot .8044 \mathrm{E}+\odot 7$ \\
\hline 5 & 288.000 & & 1.0000 & $0.8044 \mathrm{E}+07$ \\
\hline time & $(\sec )=$ & \multirow{2}{*}{$\begin{array}{cc}3.00 \quad 1 \\
\text { Twall(K) }\end{array}$} & \multicolumn{2}{|c|}{ lambda $=0.6175189018$} \\
\hline node & $\mathrm{T}(\mathrm{K})$ & & ) $\quad x$ & $\mathrm{P}(\mathrm{Pa})$ \\
\hline 1 & 226.629 & 271. 401 & - - - - - & $\odot .2573 \mathrm{E}+07$ \\
\hline 2 & 262.888 & 287.379 & 1.0000 & $\odot .4745 \mathrm{E}+\odot 7$ \\
\hline 3 & 287.470 & 288.000 & $1.000 \odot$ & $\odot .7800 \mathrm{E}+\odot 7$ \\
\hline 4 & 288.000 & $288.00 \odot$ & 1.0000 & $\odot .8038 \mathrm{E}+07$ \\
\hline 5 & 288.000 & 288.000 & 1.0000 & $\odot .8044 \mathrm{E}+\odot 7$ \\
\hline time & $(\mathrm{sec})=$ & \multirow{2}{*}{$4.00 \quad 1$} & \multicolumn{2}{|c|}{ lambda $=0.5290004611$} \\
\hline node & $\mathrm{T}(\mathrm{K})$ & & ) $\quad x$ & $P(P a)$ \\
\hline 1 & 205.044 & $\begin{array}{l}\text { I waLI (K) } \\
265.588\end{array}$ & $-\ldots$ & $\odot .2096 \mathrm{E}+\odot 7$ \\
\hline 2 & 237.849 & 285.711 & $1.000 \odot$ & $\odot .3867 \mathrm{E}+\odot 7$ \\
\hline 3 & 285.901 & 288.000 & 1.0000 & $\odot .7555 \mathrm{E}+07$ \\
\hline 4 & $288.00 \odot$ & \multirow{2}{*}{$\begin{array}{l}288.000 \\
288.000\end{array}$} & 1.0000 & $\odot .8019 \mathrm{E}+\odot 7$ \\
\hline 5 & $288 . \odot \odot \odot$ & & $1.000 \odot$ & $\odot .8044 \mathrm{E}+07$ \\
\hline time & $(\sec )=$ & 5.00 & lambda $=0$. & 30084682 \\
\hline node & $\mathrm{T}(\mathrm{K})$ & Twall(K) & $\mathrm{x}$ & $\mathrm{P}(\mathrm{Pa})$ \\
\hline 1 & 181.553 & 258.902 & - - - - - & $\odot .1708 \mathrm{E}+07$ \\
\hline 2 & 210.599 & 282.639 & 1.0000 & $\odot .3150 \mathrm{E}+\odot 7$ \\
\hline 3 & 282.362 & $288.00 \odot$ & 1.0000 & $\odot .7246 \mathrm{E}+07$ \\
\hline 4 & 287.846 & 288.000 & 1.0000 & $\odot .7972 \mathrm{E}+07$ \\
\hline 5 & 287.848 & 288.000 & 1.0000 & $\odot .8039 \mathrm{E}+\odot 7$ \\
\hline time & $(\sec )=$ & $6.00 \quad 1$ & lambda $=0.4$ & 31243324 \\
\hline node & $\mathrm{T}(\mathrm{K})$ & Twall(K) & $\mathrm{X}$ & $P(P a)$ \\
\hline 1 & 160.576 & 251.608 & $-\ldots$ & $0.1414 \mathrm{E}+07$ \\
\hline 2 & 186.266 & 278.269 & $1.000 \odot$ & $\odot .2608 \mathrm{E}+\odot 7$ \\
\hline 3 & 276.147 & 287.806 & $1.00 \odot \odot$ & $\odot .6893 \mathrm{E}+\odot 7$ \\
\hline 4 & 287.387 & 288.000 & 1.0000 & $\odot .7888 \mathrm{E}+07$ \\
\hline 5 & 287.392 & $288.00 \odot$ & 1.0000 & $\odot .8020 \mathrm{E}+\odot 7$ \\
\hline
\end{tabular}




\begin{tabular}{|c|c|c|c|c|}
\hline time & $(\sec )=$ & $7.00 \quad$ la & \multicolumn{2}{|c|}{ lambda $=0.3645296395$} \\
\hline node & $\mathrm{T}(\mathrm{K})$ & Twall(K) & $\mathrm{x}$ & $P(P a)$ \\
\hline 1 & 146.298 & 243.971 & $--_{-}-$ & $\odot .1220 \mathrm{E}+\Theta 7$ \\
\hline 2 & 169.704 & 273.023 & 1.0000 & $\odot .2251 \mathrm{E}+\Theta 7$ \\
\hline 3 & 267.160 & 287.347 & 1.0000 & $\odot .6515 \mathrm{E}+\odot 7$ \\
\hline 4 & 286.299 & 288.000 & 1.0000 & $\odot .7755 \mathrm{E}+\odot 7$ \\
\hline 5 & 286.332 & $288 . \odot \odot \odot$ & $1.000 \odot$ & $\odot .7977 \mathrm{E}+07$ \\
\hline time & $(\sec )=$ & $8.00 \quad l a$ & \multicolumn{2}{|c|}{ lambda $=0.3445668817$} \\
\hline node & $\mathrm{T}(\mathrm{K})$ & Twall(K) & $x$ & $P(P a)$ \\
\hline 1 & 144.058 & 236.554 & ----- & $0.1145 \mathrm{E}+07$ \\
\hline 2 & 167.107 & 267.635 & $\odot .9989$ & $\odot .2111 \mathrm{E}+07$ \\
\hline 3 & 256.035 & 286.428 & 1.0000 & $\odot .6135 \mathrm{E}+07$ \\
\hline 4 & 284.221 & 288.000 & 1.0000 & $\odot .7570 \mathrm{E}+\odot 7$ \\
\hline 5 & 284.333 & 288.000 & $1.000 \odot$ & $\odot .7896 \mathrm{E}+07$ \\
\hline time & $(\sec )=$ & $9.00 \quad$ la & \multicolumn{2}{|c|}{ lambda $=0.3271503747$} \\
\hline node & $\mathrm{T}(\mathrm{K})$ & Twall(K) & $\mathrm{X}$ & $\mathrm{P}(\mathrm{Pa})$ \\
\hline 1 & 142.914 & 229.787 & $--_{-}^{-}-$ & $0.1082 \mathrm{E}+07$ \\
\hline 2 & 165.780 & 262.610 & 0.9977 & $\odot .1996 \mathrm{E}+07$ \\
\hline 3 & 243.967 & 284.938 & $1.000 \odot$ & $\odot .5779 \mathrm{E}+\odot 7$ \\
\hline 4 & 280.757 & $288.0 \odot \odot$ & $1.000 \odot$ & $\odot .7337 \mathrm{E}+07$ \\
\hline 5 & 281.028 & 288.000 & $1.000 \odot$ & $\odot .7763 \mathrm{E}+07$ \\
\hline time & $(\sec )=$ & $10.0 \odot$ & \multicolumn{2}{|c|}{ lambda $=0.3101192117$} \\
\hline node & $\mathrm{T}(\mathrm{K})$ & Twall(K) & $\mathrm{X}$ & $\mathrm{P}(\mathrm{Pa})$ \\
\hline 1 & 141.256 & 223.549 & $--_{-}-$ & $\odot .1020 \mathrm{E}+\odot 7$ \\
\hline 2 & 163.857 & 257.878 & 0.9965 & $\odot .1881 \mathrm{E}+07$ \\
\hline 3 & 232.361 & 282.865 & 1.0000 & $\odot .5469 \mathrm{E}+07$ \\
\hline 4 & 275.637 & 287.810 & 1.0000 & $\odot .7062 \mathrm{E}+07$ \\
\hline 5 & 276.215 & 287.805 & 1.0000 & $\odot .7572 \mathrm{E}+\odot 7$ \\
\hline time & $(\sec )=$ & $11.00 \quad$ la & \multicolumn{2}{|c|}{ lambda $=0.2942265868$} \\
\hline node & $\mathrm{T}(\mathrm{K})$ & Twall(K) & $\mathrm{x}$ & $\mathrm{P}(\mathrm{Pa})$ \\
\hline 1 & 139.725 & 217.768 & $-\ldots$ & $\odot .9625 E+06$ \\
\hline 2 & 162.081 & 253.389 & 0.9958 & $\odot .1775 \mathrm{E}+07$ \\
\hline 3 & 222.098 & 280.275 & 1.0000 & $\odot .5213 \mathrm{E}+07$ \\
\hline 4 & 268.803 & 287.505 & $1.000 \odot$ & $\odot .6757 \mathrm{E}+07$ \\
\hline 5 & 269.858 & 287.500 & $1.000 \odot$ & $\odot .7322 \mathrm{E}+\odot 7$ \\
\hline time & $(\mathrm{sec})=$ & 12.00 & \multicolumn{2}{|c|}{ lambda $=0.2794909179$} \\
\hline node & $\mathrm{T}(\mathrm{K})$ & Twall(K) & $\mathrm{x}$ & $\mathrm{P}(\mathrm{Pa})$ \\
\hline 1 & 138.321 & 212.535 & 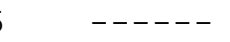 & $\odot .9097 \mathrm{E}+06$ \\
\hline 2 & 160.452 & 249.172 & 0.9952 & $\odot .1678 \mathrm{E}+07$ \\
\hline 3 & 213.544 & 277.317 & 1.0000 & $\odot .5010 \mathrm{E}+07$ \\
\hline 4 & 260.469 & 286.911 & 1.0000 & $\odot .6434 \mathrm{E}+07$ \\
\hline 5 & 262.150 & 286.898 & $1.000 \odot$ & $\odot .7023 \mathrm{E}+07$ \\
\hline time & $(\sec )=$ & $13.00 \quad l a$ & \multicolumn{2}{|c|}{ lambda $=0.2658726871$} \\
\hline node & $\mathrm{T}(\mathrm{K})$ & Twall(K) & $\mathrm{x}$ & $\mathrm{P}(\mathrm{Pa})$ \\
\hline 1 & 137.034 & 207.836 & $-\cdots-$ & $\odot .8613 E+06$ \\
\hline 2 & 158.959 & 245.203 & 0.9947 & $\odot .1589 \mathrm{E}+07$ \\
\hline 3 & 206.690 & 273.975 & $1.000 \odot$ & $\odot .4849 \mathrm{E}+07$ \\
\hline 4 & 250.983 & 286.049 & 1.0000 & $\odot .6105 \mathrm{E}+07$ \\
\hline 5 & 253.413 & 286.028 & $1.000 \odot$ & $\odot .6690 \mathrm{E}+07$ \\
\hline
\end{tabular}




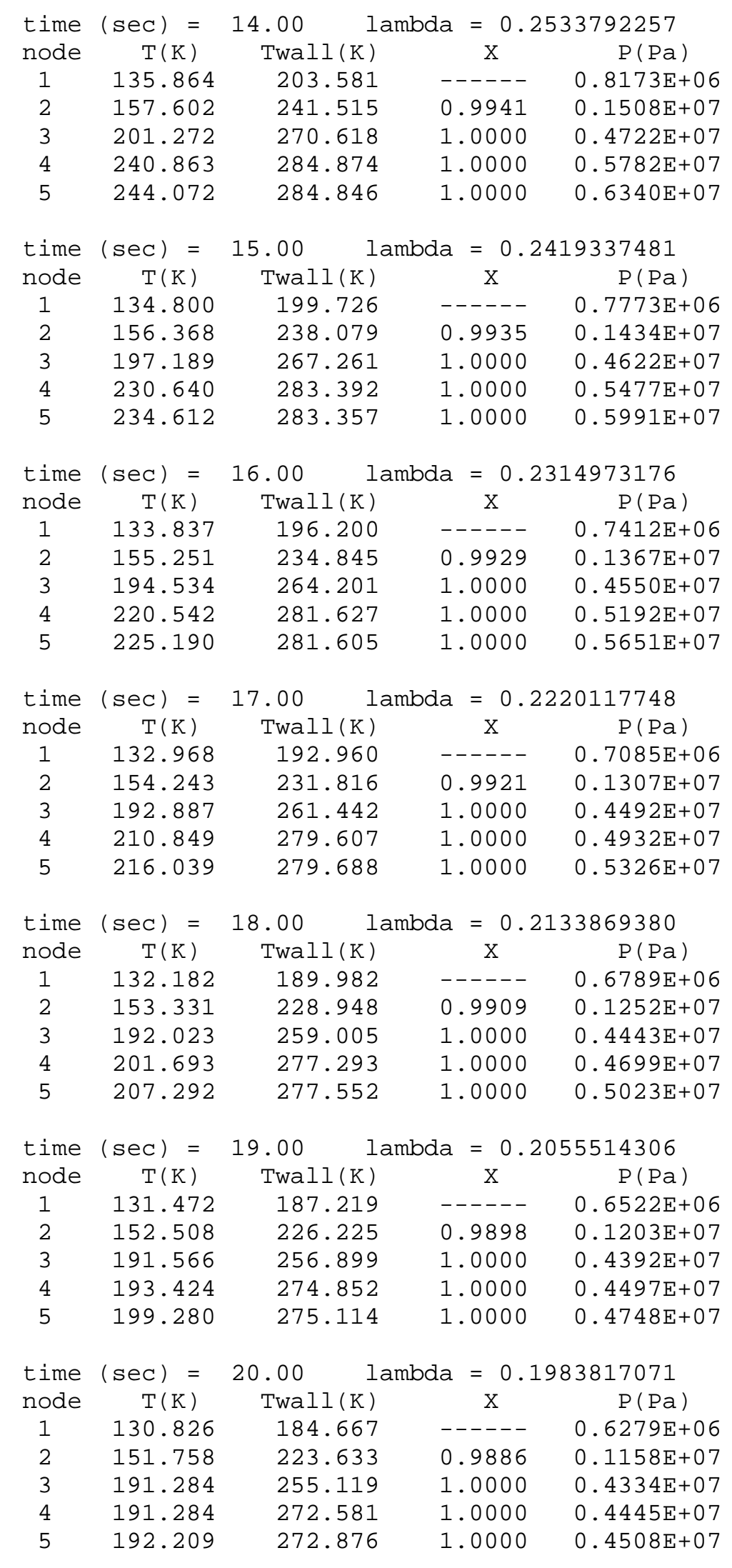




\begin{tabular}{|c|c|c|c|c|}
\hline time & $(\sec )=$ & & \multicolumn{2}{|c|}{ lambda $=0.1917922348$} \\
\hline node & $\mathrm{T}(\mathrm{K})$ & Twall(K) & ) $\quad x$ & $\mathrm{P}(\mathrm{Pa})$ \\
\hline 1 & 130.234 & 182.285 & 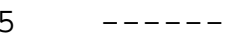 & $0.6057 \mathrm{E}+06$ \\
\hline 2 & 151.072 & 221.192 & $\odot .9874$ & $\odot .1117 \mathrm{E}+07$ \\
\hline 3 & 190.928 & 253.536 & 1.0000 & $\odot .4268 \mathrm{E}+\odot 7$ \\
\hline 4 & 190.928 & $27 \odot .587$ & 1.0000 & $\odot .4436 \mathrm{E}+07$ \\
\hline 5 & 190.928 & 270.872 & 1.0000 & $\odot .4436 \mathrm{E}+07$ \\
\hline time & $(\mathrm{sec})=$ & 22.00 & \multicolumn{2}{|c|}{ lambda $=0.1856861711$} \\
\hline node & $\mathrm{T}(\mathrm{K})$ & Twall(K) & ) $\quad x$ & $P(P a)$ \\
\hline 1 & 129.689 & 180.059 & $--_{-}^{-}$ & $\odot .5852 \mathrm{E}+06$ \\
\hline 2 & 150.439 & 218.864 & $\odot .9865$ & $\odot .1079 \mathrm{E}+07$ \\
\hline 3 & 190.060 & 252.069 & $1.000 \odot$ & $\odot .4188 \mathrm{E}+07$ \\
\hline 4 & 190.060 & 268.825 & 1.0000 & $0.4410 \mathrm{E}+07$ \\
\hline 5 & 190.060 & 269.102 & $1.000 \odot$ & $0.4410 \mathrm{E}+07$ \\
\hline time & $(\sec )=$ & $23.00 \quad l$ & \multicolumn{2}{|c|}{ lambda $=0.1799831539$} \\
\hline node & $\mathrm{T}(\mathrm{K})$ & Twall(K) & ) $\quad x$ & $P(P a)$ \\
\hline 1 & 129.181 & 177.976 & $--_{-}^{-}$ & $0.5661 \mathrm{E}+06$ \\
\hline 2 & 149.850 & 216.616 & $\odot .9860$ & $\odot .1044 \mathrm{E}+07$ \\
\hline 3 & 188.609 & 250.696 & $1.000 \odot$ & $\odot .4098 \mathrm{E}+07$ \\
\hline 4 & 189.036 & 267.300 & 1.0000 & $\odot .4376 \mathrm{E}+\odot 7$ \\
\hline 5 & 189.036 & 267.576 & 1.0000 & $0.4376 \mathrm{E}+07$ \\
\hline time & $(\sec )=$ & $24.00 \quad l$ & \multicolumn{2}{|c|}{ lambda $=0.1745966077$} \\
\hline node & $\mathrm{T}(\mathrm{K})$ & Twall(K) & ) $\quad x$ & $\mathrm{P}(\mathrm{Pa})$ \\
\hline 1 & 128.703 & 176.016 & 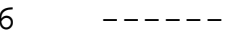 & $0.5482 E+06$ \\
\hline 2 & 149.296 & 214.483 & 0.9854 & $\odot .1011 \mathrm{E}+07$ \\
\hline 3 & 186.695 & 249.323 & 1.0000 & $\odot .4002 \mathrm{E}+07$ \\
\hline 4 & 188.881 & 265.903 & 1.0000 & $\odot .4356 \mathrm{E}+07$ \\
\hline 5 & 188.881 & 266.169 & 1.0000 & $\odot .4357 \mathrm{E}+07$ \\
\hline time & $(\sec )=$ & $25.00 \quad l$ & \multicolumn{2}{|c|}{ lambda $=0.1693378389$} \\
\hline node & $\mathrm{T}(\mathrm{K})$ & Twall(K) & ) $\quad x$ & $\mathrm{P}(\mathrm{Pa})$ \\
\hline 1 & 128.021 & 174.185 & 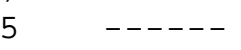 & $\odot .5302 \mathrm{E}+06$ \\
\hline 2 & 148.504 & 212.500 & 0.9848 & $\odot .9780 \mathrm{E}+06$ \\
\hline 3 & 185.429 & 247.809 & 1.0000 & $\odot .3925 \mathrm{E}+07$ \\
\hline 4 & 188.660 & 264.682 & 1.0000 & $\odot .4328 \mathrm{E}+07$ \\
\hline 5 & 188.660 & 264.949 & $1.000 \odot$ & $0.4328 \mathrm{E}+07$ \\
\hline time & $(\sec )=$ & $26.00 \quad 1$ & \multicolumn{2}{|c|}{ lambda $=0.1643495113$} \\
\hline node & $\mathrm{T}(\mathrm{K})$ & Twall(K) & ) $\quad x$ & $\mathrm{P}(\mathrm{Pa})$ \\
\hline 1 & 127.270 & 172.471 & 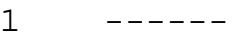 & $0.5131 \mathrm{E}+06$ \\
\hline 2 & 147.633 & 210.516 & 0.9842 & $0.9464 \mathrm{E}+06$ \\
\hline 3 & 184.951 & 246.283 & 1.0000 & $\odot .3871 \mathrm{E}+07$ \\
\hline 4 & 188.372 & 263.461 & 1.0000 & $\odot .4292 \mathrm{E}+07$ \\
\hline 5 & 188.372 & 263.728 & $1 . \odot \odot \odot \odot$ & $\odot .4292 \mathrm{E}+\odot 7$ \\
\hline time & $(\sec )=$ & $27.00 \quad 1$ & \multicolumn{2}{|c|}{ lambda $=0.1597325951$} \\
\hline node & $\mathrm{T}(\mathrm{K})$ & Twall(K) & $\mathrm{X}$ & $\mathrm{P}(\mathrm{Pa})$ \\
\hline 1 & 126.578 & 170.810 & $-\ldots-$ & $0.4973 \mathrm{E}+06$ \\
\hline 2 & 146.831 & 208.532 & 0.9836 & $\odot .9174 \mathrm{E}+06$ \\
\hline 3 & 184.524 & 244.757 & 1.0000 & $\odot .3823 \mathrm{E}+07$ \\
\hline 4 & 188.034 & 262.484 & 1.0000 & $\odot .4248 \mathrm{E}+07$ \\
\hline 5 & 188.080 & 262.738 & $1.000 \odot$ & $0.4254 \mathrm{E}+07$ \\
\hline
\end{tabular}




\begin{tabular}{|c|c|c|c|c|}
\hline time & $\left(\begin{array}{c}(\sec ) \\
T(K)\end{array}\right.$ & $8.00 \quad 1$ & \multicolumn{2}{|c|}{ lambda $=0.1554499865$} \\
\hline node & $\mathrm{T}(\mathrm{K})$ & Twall(K) & ) $\quad x$ & $\mathrm{P}(\mathrm{Pa})$ \\
\hline 1 & 125.940 & 169.285 & 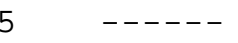 & $0.4828 \mathrm{E}+06$ \\
\hline 2 & 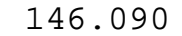 & 206.631 & 0.9830 & $\odot .8905 E+06$ \\
\hline 3 & 184.141 & 243.231 & 1.0000 & $\odot .3780 \mathrm{E}+07$ \\
\hline 4 & 187.656 & 261.568 & 1.0000 & $0.4200 \mathrm{E}+07$ \\
\hline 5 & 187.806 & 261 & $1.000 \odot$ & 0.4219 \\
\hline time & $(\sec )=$ & $29 . \odot \odot$ & \multicolumn{2}{|c|}{ lambda $=0.1514820457$} \\
\hline node & $\mathrm{T}(\mathrm{K})$ & Twall(K) & ) $\quad x$ & $\mathrm{P}(\mathrm{Pa})$ \\
\hline 1 & 125.351 & 167.785 & 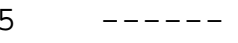 & $0.4694 \mathrm{E}+06$ \\
\hline 2 & 145.407 & 204.800 & $\odot .9824$ & $\odot .8657 \mathrm{E}+\odot 6$ \\
\hline 3 & 183.789 & 241.705 & 1.0000 & $\odot .3740 \mathrm{E}+07$ \\
\hline 4 & 187.247 & 260.653 & 1.0000 & $\odot .4148 \mathrm{E}+07$ \\
\hline 5 & 187.558 & 260.907 & $1.000 \odot$ & $0.4187 \mathrm{E}+07$ \\
\hline time & $(\sec )=$ & $30.0 \odot$ & \multicolumn{2}{|c|}{ lambda $=0.1478069276$} \\
\hline node & $\mathrm{T}(\mathrm{K})$ & Twall(K) & ) $\quad x$ & $\mathrm{P}(\mathrm{Pa})$ \\
\hline 1 & 124.808 & 166.411 & $-\ldots$ & $\odot .457 \odot E+\odot 6$ \\
\hline 2 & 144.777 & 202.969 & 0.9818 & $\odot .8429 \mathrm{E}+06$ \\
\hline 3 & 183.448 & 240.180 & $1.000 \odot$ & $\odot .3701 \mathrm{E}+07$ \\
\hline 4 & 186.841 & 259.737 & 1.0000 & $0.4096 \mathrm{E}+07$ \\
\hline 5 & 187.319 & 259.992 & $1.000 \odot$ & $\odot .4157 \mathrm{E}+07$ \\
\hline
\end{tabular}

lambda_avg $=0.314291954$ 
Compressor station distance $=5$ miles, Pressure $=1167$ psi, 25 axial node elements

\begin{tabular}{|c|c|c|c|c|}
\hline time & $(\sec )=$ & $1.00 \quad 1$ & $=0$ & $\begin{array}{r}2404881 \\
P(P a)\end{array}$ \\
\hline node & $\mathrm{T}(\mathrm{K})$ & Twall(K) & $x$ & $\mathrm{P}(\mathrm{Pa})$ \\
\hline 1 & 159.131 & 280.447 & - - - - - - & $0.1589 \mathrm{E}+\mathrm{C}$ \\
\hline 2 & 184.570 & 286.109 & 1.0000 & $\odot .2931 \mathrm{E}+$ \\
\hline 3 & 281.308 & $288 . \odot \odot \odot$ & 1.0000 & $\odot .6973 \mathrm{E}+$ \\
\hline 4 & 287.772 & $288.0 \odot \odot$ & 1.0000 & $\odot .7959 \mathrm{E}+$ \\
\hline 5 & $288.0 \odot \odot$ & $288 . \odot \odot \odot$ & 1.0000 & $0.8041 \mathrm{E}+\mathrm{c}$ \\
\hline 6 & $288 . \odot \odot \odot$ & $288 . \odot \odot \odot$ & 1.0000 & $0.8044 \mathrm{E}+\mathrm{C}$ \\
\hline 7 & $288.00 \odot$ & $288.0 \odot \odot$ & 1.0000 & $0.8044 \mathrm{E}+\mathrm{C}$ \\
\hline 8 & $288.0 \odot \odot$ & $288 . \odot \odot \odot$ & 1.0000 & $\odot .8044 \mathrm{E}+$ \\
\hline 9 & $288.0 \odot \odot$ & $288 . \odot \odot \odot$ & $1.000 \odot$ & $\odot .8044 \mathrm{E}+$ \\
\hline 10 & 288.000 & $288.0 \odot \odot$ & 1.0000 & $0.8044 \mathrm{E}+$ \\
\hline 11 & $288.00 \odot$ & $288.0 \odot \odot$ & 1.0000 & $0.8044 \mathrm{E}+\mathrm{C}$ \\
\hline 12 & $288.0 \odot \odot$ & $288 . \odot \odot \odot$ & $1.000 \odot$ & $0.8044 \mathrm{E}+\mathrm{C}$ \\
\hline 13 & $288.0 \odot \odot$ & $288 . \odot \odot \odot$ & 1.0000 & $\odot .8 \odot 44 \mathrm{E}+$ \\
\hline 14 & $288.00 \odot$ & $288.0 \odot \odot$ & 1.0000 & $0.8044 \mathrm{E}+$ \\
\hline 15 & $288.0 \odot \odot$ & $288 . \odot \odot \odot$ & $1.000 \odot$ & $\odot .8044 \mathrm{E}+$ \\
\hline 16 & $288 . \odot \odot \odot$ & $288.0 \odot \odot$ & 1.0000 & $\odot .8044 \mathrm{E}+\mathrm{C}$ \\
\hline 17 & 288.000 & $288.0 \odot \odot$ & 1.0000 & $0.8044 \mathrm{E}+\mathrm{C}$ \\
\hline 18 & $288.00 \odot$ & $288 . \odot \odot \odot$ & 1.0000 & $\odot .8044 \mathrm{E}+$ \\
\hline 19 & $288.0 \odot \odot$ & $288 . \odot \odot \odot$ & 1.0000 & $\odot .8044 \mathrm{E}+$ \\
\hline 20 & $288.0 \odot \odot$ & $288.0 \odot \odot$ & 1.0000 & $\odot .8044 \mathrm{E}+$ \\
\hline 21 & $288.0 \odot \odot$ & $288 . \odot \odot \odot$ & 1.0000 & $\odot .8044 \mathrm{E}+$ \\
\hline 22 & 288.000 & 288.000 & 1.0000 & $0.8044 \mathrm{E}+\mathrm{C}$ \\
\hline 23 & 288.000 & $288.0 \odot \odot$ & 1.0000 & $\odot .8044 \mathrm{E}+$ \\
\hline 24 & $288.0 \odot \odot$ & $288 . \odot \odot \odot$ & 1.0000 & $\odot .8044 \mathrm{E}+$ \\
\hline 25 & 288.000 & $288.00 \odot$ & $1.000 \odot$ & $0.8044 \mathrm{E}+$ \\
\hline
\end{tabular}

\begin{tabular}{|c|c|c|c|c|}
\hline \multicolumn{5}{|c|}{ lambda $=0.4292460084$} \\
\hline node & $\mathrm{T}(\mathrm{K})$ & Twall(K) & $\mathrm{x}$ & $\mathrm{P}(\mathrm{Pa})$ \\
\hline 1 & 149.659 & 268.979 & - . - _ & $\odot .1453 \mathrm{E}+07$ \\
\hline 2 & 173.605 & 276.159 & 0.9757 & $\odot .2681 \mathrm{E}+\odot 7$ \\
\hline 3 & 186.451 & 285.065 & $1.000 \odot$ & $\odot .3721 \mathrm{E}+\odot 7$ \\
\hline 4 & 268.124 & 287.882 & $1.000 \odot$ & $\odot .6425 \mathrm{E}+\odot 7$ \\
\hline 5 & 286.110 & $288.00 \odot$ & 1.0000 & $\odot .7760 \mathrm{E}+\odot 7$ \\
\hline 6 & 287.917 & $288.00 \odot$ & 1.0000 & $\odot .8018 \mathrm{E}+\odot 7$ \\
\hline 7 & 288.000 & 288.000 & $1.000 \odot$ & $\odot .8 \odot 43 E+\odot 7$ \\
\hline 8 & $288.0 \odot \odot$ & $288 . \odot \odot \odot$ & $1.0 \odot \odot \odot$ & $\odot .8 \odot 44 \mathrm{E}+\odot 7$ \\
\hline 9 & 288.000 & $288.00 \odot$ & 1.0000 & $\odot .8044 \mathrm{E}+\odot 7$ \\
\hline 10 & 288.000 & $288.00 \odot$ & $1.000 \odot$ & $\odot .8044 \mathrm{E}+07$ \\
\hline 11 & 288.000 & 288.000 & 1.0000 & $\odot .8044 \mathrm{E}+07$ \\
\hline 12 & 288.000 & 288.000 & 1.0000 & $\odot .8044 \mathrm{E}+07$ \\
\hline 13 & 288.000 & $288.00 \odot$ & 1.0000 & $\odot .8044 \mathrm{E}+07$ \\
\hline 14 & $288.00 \odot$ & $288 . \odot \odot \odot$ & $1.000 \odot$ & $\odot .8 \odot 44 \mathrm{E}+\odot 7$ \\
\hline 15 & $288 . \odot \odot \odot$ & $288 . \odot \odot \odot$ & $1.00 \odot \odot$ & $\odot .8 \odot 44 \mathrm{E}+\odot 7$ \\
\hline 16 & 288.000 & $288.00 \odot$ & 1.0000 & $\odot .8044 \mathrm{E}+\odot 7$ \\
\hline 17 & 288.000 & 288.000 & 1.0000 & $\odot .8044 \mathrm{E}+07$ \\
\hline 18 & 288.000 & $288.00 \odot$ & 1.0000 & $\odot .8044 \mathrm{E}+07$ \\
\hline 19 & 288.000 & $288.0 \odot \odot$ & 1.0000 & $\odot .8044 \mathrm{E}+07$ \\
\hline 20 & $288.00 \odot$ & $288 . \odot \odot \odot$ & $1.00 \odot \odot$ & $\odot .8044 \mathrm{E}+\odot 7$ \\
\hline 21 & $288.00 \odot$ & $288 . \odot \odot \odot$ & $1.0 \odot \odot \odot$ & $\odot .8 \odot 44 \mathrm{E}+\odot 7$ \\
\hline 22 & 288.000 & $288.00 \odot$ & 1.0000 & $\odot .8044 \mathrm{E}+07$ \\
\hline 23 & $288.00 \odot$ & $288 . \odot \odot \odot$ & $1.00 \odot \odot$ & $\odot .8044 \mathrm{E}+\odot 7$ \\
\hline 24 & 288.000 & $288.00 \odot$ & $1.000 \odot$ & $\odot .8044 \mathrm{E}+07$ \\
\hline 25 & $288.0 \odot \odot$ & $288 . \odot \odot \odot$ & $1.000 \odot$ & $\odot .8044 \mathrm{E}+\odot 7$ \\
\hline
\end{tabular}




\begin{tabular}{|c|c|c|c|c|}
\hline time & $(\sec )=$ & 3.00 & \multicolumn{2}{|c|}{ lambda $=0.3599389195$} \\
\hline node & $\mathrm{T}(\mathrm{K})$ & Twall(K) & ) $\quad x$ & $\mathrm{P}(\mathrm{Pa})$ \\
\hline 1 & 145.060 & 259.176 & $-\ldots$ & $\odot .1200 \mathrm{E}+07$ \\
\hline 2 & 168.269 & 269.150 & $\odot .9839$ & $\odot .2213 \mathrm{E}+07$ \\
\hline 3 & 183.015 & 276.633 & 0.9912 & $\odot .3652 \mathrm{E}+\odot 7$ \\
\hline 4 & 185.363 & 283.614 & $\odot .9993$ & $\odot .3918 \mathrm{E}+07$ \\
\hline 5 & 250.241 & 287.541 & 1.0000 & $\odot .5833 \mathrm{E}+07$ \\
\hline 6 & 281.683 & 288.000 & 1.0000 & $\odot .7433 \mathrm{E}+07$ \\
\hline 7 & 287.411 & 288.000 & 1.0000 & $\odot .7950 \mathrm{E}+07$ \\
\hline 8 & 288.000 & 288.000 & 1.0000 & $\odot .8037 \mathrm{E}+\odot 7$ \\
\hline 9 & 288.000 & 288.000 & 1.0000 & $0.8044 \mathrm{E}+07$ \\
\hline 10 & 288.000 & 288.000 & 1.0000 & $0.8044 \mathrm{E}+07$ \\
\hline 11 & $288.00 \odot$ & $288.00 \odot$ & $1.000 \odot$ & $0.8044 \mathrm{E}+\odot 7$ \\
\hline 12 & 288.000 & 288.000 & 1.0000 & $\odot .8044 \mathrm{E}+07$ \\
\hline 13 & 288.000 & 288.000 & 1.0000 & $\odot .8044 \mathrm{E}+07$ \\
\hline 14 & 288.000 & 288.000 & 1.0000 & $0.8044 \mathrm{E}+07$ \\
\hline 15 & $288.00 \odot$ & $288.00 \odot$ & $1.000 \odot$ & $\odot .8044 \mathrm{E}+07$ \\
\hline 16 & 288.000 & 288.000 & 1.0000 & $0.8044 \mathrm{E}+07$ \\
\hline 17 & $288 . \odot \odot \odot$ & $288.00 \odot$ & $1.000 \odot$ & $\odot .8044 \mathrm{E}+\odot 7$ \\
\hline 18 & $288.00 \odot$ & $288.00 \odot$ & 1.0000 & $0.8044 \mathrm{E}+07$ \\
\hline 19 & 288.000 & 288.000 & 1.0000 & $0.8044 \mathrm{E}+07$ \\
\hline 20 & 288.000 & 288.000 & 1.0000 & $0.8044 \mathrm{E}+07$ \\
\hline 21 & $288.00 \odot$ & 288.000 & 1.0000 & $\odot .8044 \mathrm{E}+07$ \\
\hline 22 & 288.000 & $288.00 \odot$ & $1.000 \odot$ & $0.8044 \mathrm{E}+07$ \\
\hline 23 & $288.00 \odot$ & 288.000 & 1.0000 & $0.8044 \mathrm{E}+07$ \\
\hline 24 & $288.00 \odot$ & $288.00 \odot$ & $1.000 \odot$ & $\odot .8044 \mathrm{E}+\odot 7$ \\
\hline 25 & $288.00 \odot$ & $288.00 \odot$ & 1.0000 & $\odot .8044 \mathrm{E}+07$ \\
\hline time & $(\sec )=$ & 4.00 & lambda = & 70187473 \\
\hline node & $\mathrm{T}(\mathrm{K})$ & Twall(K) & $\mathrm{X}$ & $\mathrm{P}(\mathrm{Pa})$ \\
\hline 1 & 141.925 & 250.752 & - - - - - & $0.1045 \mathrm{E}+07$ \\
\hline 2 & 164.633 & 262.741 & 0.9782 & $\odot .1928 \mathrm{E}+\odot 7$ \\
\hline 3 & 181.634 & 271.026 & $\odot .9940$ & $\odot .3496 \mathrm{E}+\odot 7$ \\
\hline 4 & 185.365 & 277.336 & $\odot .9989$ & $\odot .3918 \mathrm{E}+07$ \\
\hline 5 & 197.559 & 282.754 & 1.0000 & $\odot .4497 \mathrm{E}+07$ \\
\hline 6 & 230.679 & 286.817 & $1.000 \odot$ & $\odot .5464 \mathrm{E}+07$ \\
\hline 7 & 272.981 & 287.929 & $1.000 \odot$ & $0.6963 \mathrm{E}+07$ \\
\hline 8 & 285.807 & 288.000 & 1.0000 & $0.7801 \mathrm{E}+07$ \\
\hline 9 & 287.844 & 288.000 & 1.0000 & $\odot .8012 \mathrm{E}+07$ \\
\hline 10 & $288.00 \odot$ & $288.00 \odot$ & $1.000 \odot$ & $\odot .8042 \mathrm{E}+\odot 7$ \\
\hline 11 & 288.000 & 288.000 & 1.0000 & $0.8044 \mathrm{E}+07$ \\
\hline 12 & 288.000 & 288.000 & 1.0000 & $0.8044 \mathrm{E}+07$ \\
\hline 13 & $288.0 \odot \odot$ & $288.00 \odot$ & $1.000 \odot$ & $\odot .8044 \mathrm{E}+\odot 7$ \\
\hline 14 & 288.000 & 288.000 & 1.0000 & $0.8044 \mathrm{E}+07$ \\
\hline 15 & 288.000 & 288.000 & 1.0000 & $0.8044 \mathrm{E}+07$ \\
\hline 16 & 288.000 & 288.000 & 1.0000 & $0.8044 \mathrm{E}+07$ \\
\hline 17 & 288.000 & $288.00 \odot$ & 1.0000 & $0.8044 \mathrm{E}+07$ \\
\hline 18 & 288.000 & 288.000 & 1.0000 & $0.8044 \mathrm{E}+07$ \\
\hline 19 & 288.000 & 288.000 & 1.0000 & $\odot .8044 \mathrm{E}+07$ \\
\hline 20 & 288.000 & 288.000 & $1.000 \odot$ & $\odot .8044 \mathrm{E}+07$ \\
\hline 21 & $288.0 \odot \odot$ & $288.00 \odot$ & $1.000 \odot$ & $\odot .8044 \mathrm{E}+07$ \\
\hline 22 & 288.000 & 288.000 & 1.0000 & $0.8044 \mathrm{E}+07$ \\
\hline 23 & $288.00 \odot$ & $288.00 \odot$ & 1.0000 & $\odot .8044 \mathrm{E}+07$ \\
\hline 24 & 288.000 & 288.000 & 1.0000 & $0.8044 \mathrm{E}+07$ \\
\hline 25 & $288.0 \odot \odot$ & 288.000 & 1.0000 & $0.8044 \mathrm{E}+07$ \\
\hline
\end{tabular}




\begin{tabular}{|c|c|c|c|c|}
\hline time & $(\sec )=$ & 5.00 & \multicolumn{2}{|c|}{ lambda $=0.2923625410$} \\
\hline node & $\mathrm{T}(\mathrm{K})$ & Twall(K) & ) $\quad x$ & $\mathrm{P}(\mathrm{Pa})$ \\
\hline 1 & 139.547 & 243.074 & 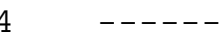 & $\odot .9558 \mathrm{E}+06$ \\
\hline 2 & 161.874 & 256.468 & 0.9722 & $\odot .1763 \mathrm{E}+07$ \\
\hline 3 & 178.825 & 266.422 & 0.9961 & $\odot .3178 \mathrm{E}+07$ \\
\hline 4 & 185.364 & 271.991 & 0.9991 & $\odot .3918 \mathrm{E}+07$ \\
\hline 5 & 204.535 & 277.639 & 1.0000 & $\odot .4616 \mathrm{E}+07$ \\
\hline 6 & 208.061 & 282.023 & 1.0000 & $\odot .4927 \mathrm{E}+\odot 7$ \\
\hline 7 & 218.275 & 285.779 & 1.0000 & $0.5246 \mathrm{E}+07$ \\
\hline 8 & 259.125 & 287.697 & 1.0000 & $0.6433 \mathrm{E}+07$ \\
\hline 9 & 281.958 & $288.00 \odot$ & 1.0000 & $\odot .7532 \mathrm{E}+07$ \\
\hline 10 & 287.281 & 288.000 & 1.0000 & $\odot .7949 \mathrm{E}+07$ \\
\hline 11 & 287.981 & 288.000 & 1.0000 & $\odot .8035 \mathrm{E}+07$ \\
\hline 12 & 288.000 & 288.000 & 1.0000 & $\odot .8044 \mathrm{E}+07$ \\
\hline 13 & $288.00 \odot$ & $288.00 \odot$ & $1.000 \odot$ & $\odot .8044 \mathrm{E}+07$ \\
\hline 14 & 288.000 & 288.000 & 1.0000 & $\odot .8044 \mathrm{E}+07$ \\
\hline 15 & 288.000 & 288.000 & 1.0000 & $\odot .8044 \mathrm{E}+07$ \\
\hline 16 & $288.00 \odot$ & $288.00 \odot$ & $1.000 \odot$ & $\odot .8044 \mathrm{E}+07$ \\
\hline 17 & $288.00 \odot$ & 288.000 & 1.0000 & $\odot .8 \odot 44 \mathrm{E}+07$ \\
\hline 18 & 288.000 & 288.000 & 1.0000 & $\odot .8044 \mathrm{E}+07$ \\
\hline 19 & $288.0 \odot \odot$ & $288 . \odot \odot \odot$ & $1.000 \odot$ & $\odot .8044 \mathrm{E}+07$ \\
\hline 20 & $288.00 \odot$ & $288.0 \odot \odot$ & $1.000 \odot$ & $\odot .8 \odot 44 \mathrm{E}+07$ \\
\hline 21 & $288.00 \odot$ & 288.000 & $1.000 \odot$ & $\odot .8 \odot 44 \mathrm{E}+07$ \\
\hline 22 & $288 . \odot \odot \odot$ & $288.0 \odot \odot$ & 1.0000 & $\odot .8 \odot 44 \mathrm{E}+\odot 7$ \\
\hline 23 & 288.000 & 288.000 & 1.0000 & $\odot .8044 \mathrm{E}+07$ \\
\hline 24 & $288.00 \odot$ & 288.000 & $1.000 \odot$ & $\odot .8044 \mathrm{E}+07$ \\
\hline 25 & $288 . \odot \odot \odot$ & $288 . \odot \odot \odot$ & $1.000 \odot$ & $\odot .8 \odot 44 \mathrm{E}+07$ \\
\hline time & ( $\mathrm{sec})$ & 6.00 & $\mathrm{da}=$ & 9599307 \\
\hline node & $\mathrm{T}(\mathrm{K})$ & Twall(K) & $\mathrm{X}$ & $P(P a)$ \\
\hline 1 & 137.608 & 236.268 & - - - - - & $\odot .8829 E+06$ \\
\hline 2 & 159.625 & 250.674 & 0.9708 & $\odot .1628 \mathrm{E}+07$ \\
\hline 3 & 177.624 & 261.604 & 0.9966 & $\odot .3042 \mathrm{E}+07$ \\
\hline 4 & 185.364 & 266.807 & 0.9976 & $\odot .3918 \mathrm{E}+07$ \\
\hline 5 & 203.504 & 273.612 & $1.000 \odot$ & $\odot .4397 \mathrm{E}+07$ \\
\hline 6 & 211.388 & 278.020 & 1.0000 & $\odot .4986 \mathrm{E}+07$ \\
\hline 7 & 218.109 & 282.075 & 1.0000 & $\odot .5242 \mathrm{E}+07$ \\
\hline 8 & 222.111 & 284.943 & $1.000 \odot$ & $\odot .5443 \mathrm{E}+07$ \\
\hline 9 & 242.118 & 287.161 & 1.0000 & $0.6018 \mathrm{E}+07$ \\
\hline 10 & 274.367 & 287.938 & 1.0000 & $0.7121 \mathrm{E}+07$ \\
\hline 11 & 285.693 & $288.0 \odot \odot$ & $1.000 \odot$ & $\odot .7813 \mathrm{E}+\odot 7$ \\
\hline 12 & 287.807 & $288.00 \odot$ & $1.000 \odot$ & $\odot .8010 \mathrm{E}+07$ \\
\hline 13 & 288.000 & 288.000 & 1.0000 & $\odot .8 \odot 42 \mathrm{E}+07$ \\
\hline 14 & $288.00 \odot$ & $288 . \odot \odot \odot$ & 1.0000 & $\odot .8044 \mathrm{E}+07$ \\
\hline 15 & 288.000 & 288.000 & 1.0000 & $\odot .8044 \mathrm{E}+07$ \\
\hline 16 & 288.000 & $288.00 \odot$ & $1.000 \odot$ & $\odot .8 \odot 44 \mathrm{E}+07$ \\
\hline 17 & $288.0 \odot \odot$ & $288.00 \odot$ & 1.0000 & $\odot .8 \odot 44 \mathrm{E}+\odot 7$ \\
\hline 18 & 288.000 & 288.000 & 1.0000 & $\odot .8044 \mathrm{E}+07$ \\
\hline 19 & 288.000 & $288.00 \odot$ & $1.000 \odot$ & $\odot .8044 \mathrm{E}+07$ \\
\hline 20 & $288.0 \odot \odot$ & $288 . \odot \odot \odot$ & 1.0000 & $0.8044 \mathrm{E}+07$ \\
\hline 21 & $288.0 \odot \odot$ & $288 . \odot \odot \odot$ & $1.000 \odot$ & $\odot .8 \odot 44 \mathrm{E}+07$ \\
\hline 22 & $288.00 \odot$ & 288.000 & $1.000 \odot$ & $\odot .8044 \mathrm{E}+\odot 7$ \\
\hline 23 & 288.000 & 288.000 & $1.000 \odot$ & $\odot .8044 \mathrm{E}+\odot 7$ \\
\hline 24 & 288.000 & 288.000 & 1.0000 & $\odot .8044 \mathrm{E}+\odot 7$ \\
\hline 25 & $288 . \odot \odot \odot$ & $288 . \odot \odot \odot$ & $1.000 \odot$ & $0.8044 \mathrm{E}+07$ \\
\hline
\end{tabular}




\begin{tabular}{|c|c|c|c|c|}
\hline time & $(\sec )=$ & $7.00 \quad 1$ & \multicolumn{2}{|c|}{ lambda $=0.2592824697$} \\
\hline node & $\mathrm{T}(\mathrm{K})$ & Twall(K) & ) $\quad x$ & $\mathrm{P}(\mathrm{Pa})$ \\
\hline 1 & 136.416 & 230.222 & $-\ldots$ & $0.8381 \mathrm{E}+06$ \\
\hline 2 & 158.242 & 245.306 & $\odot .9702$ & $\odot .1546 \mathrm{E}+07$ \\
\hline 3 & 177.517 & 256.722 & $\odot .9946$ & $\odot .3030 \mathrm{E}+07$ \\
\hline 4 & 184.090 & 262.317 & $\odot .9983$ & $\odot .3774 \mathrm{E}+07$ \\
\hline 5 & 191.321 & 269.262 & 1.0000 & $\odot .4100 \mathrm{E}+07$ \\
\hline 6 & 210.593 & 274.652 & 1.0000 & $\odot .4822 \mathrm{E}+\odot 7$ \\
\hline 7 & 219.662 & 279.107 & 1.0000 & $0.5254 \mathrm{E}+07$ \\
\hline 8 & 225.191 & 282.028 & 1.0000 & $0.5513 \mathrm{E}+07$ \\
\hline 9 & 228.760 & 284.377 & 1.0000 & $\odot .5686 \mathrm{E}+07$ \\
\hline 10 & 233.187 & 286.431 & 1.0000 & $\odot .5856 \mathrm{E}+07$ \\
\hline 11 & 262.109 & 287.743 & 1.0000 & $\odot .6677 \mathrm{E}+07$ \\
\hline 12 & 282.009 & 288.000 & 1.0000 & $\odot .7565 \mathrm{E}+07$ \\
\hline 13 & 287.218 & $288.00 \odot$ & $1.000 \odot$ & $\odot .7948 \mathrm{E}+07$ \\
\hline 14 & 287.971 & 288.000 & 1.0000 & $\odot .8033 \mathrm{E}+07$ \\
\hline 15 & 288.000 & 288.000 & 1.0000 & $\odot .8044 \mathrm{E}+07$ \\
\hline 16 & 288.000 & 288.000 & 1.0000 & $\odot .8044 \mathrm{E}+07$ \\
\hline 17 & $288.00 \odot$ & 288.000 & 1.0000 & $\odot .8 \odot 44 \mathrm{E}+07$ \\
\hline 18 & 288.000 & 288.000 & 1.0000 & $\odot .8044 \mathrm{E}+07$ \\
\hline 19 & $288.0 \odot \odot$ & $288 . \odot \odot \odot$ & $1.000 \odot$ & $\odot .8 \odot 44 \mathrm{E}+07$ \\
\hline 20 & $288.00 \odot$ & $288.0 \odot \odot$ & $1.000 \odot$ & $\odot .8 \odot 44 \mathrm{E}+07$ \\
\hline 21 & $288.00 \odot$ & 288.000 & 1.0000 & $\odot .8 \odot 44 \mathrm{E}+07$ \\
\hline 22 & $288.00 \odot$ & 288.000 & 1.0000 & $\odot .8044 \mathrm{E}+07$ \\
\hline 23 & 288.000 & 288.000 & 1.0000 & $\odot .8044 \mathrm{E}+\odot 7$ \\
\hline 24 & 288.000 & 288.000 & $1.000 \odot$ & $\odot .8 \odot 44 \mathrm{E}+07$ \\
\hline 25 & $288 . \odot \odot \odot$ & $288 . \odot \odot \odot$ & $1.00 \odot \odot$ & $\odot .8044 \mathrm{E}+\odot 7$ \\
\hline time & $(\sec )=$ & 8.00 & $\mathrm{da}=$ & 33113360 \\
\hline node & $\mathrm{T}(\mathrm{K})$ & Twall(K) & $X$ & $P(P a)$ \\
\hline 1 & 135.858 & 224.703 & - - - - - & $\odot .8171 \mathrm{E}+06$ \\
\hline 2 & 157.595 & 240.240 & $\odot .9671$ & $\odot .1507 \mathrm{E}+07$ \\
\hline 3 & 176.778 & 252.234 & $\odot .9931$ & $\odot .2959 \mathrm{E}+07$ \\
\hline 4 & 182.163 & 258.349 & ๑. 9993 & $\odot .3556 \mathrm{E}+07$ \\
\hline 5 & 192.243 & 265.150 & 1.0000 & $\odot .4119 \mathrm{E}+07$ \\
\hline 6 & 201.806 & 270.949 & 1.0000 & $\odot .4577 \mathrm{E}+07$ \\
\hline 7 & 215.078 & 276.269 & 1.0000 & $0.5037 \mathrm{E}+07$ \\
\hline 8 & 225.160 & 279.564 & $1.000 \odot$ & $\odot .5448 \mathrm{E}+\odot 7$ \\
\hline 9 & 230.232 & 282.003 & $1.000 \odot$ & $\odot .5710 \mathrm{E}+07$ \\
\hline 10 & 233.789 & 284.101 & $1.000 \odot$ & $\odot .5870 \mathrm{E}+07$ \\
\hline 11 & 236.426 & 285.854 & $1.000 \odot$ & $\odot .5993 \mathrm{E}+\odot 7$ \\
\hline 12 & 247.721 & 287.302 & 1.0000 & $\odot .6328 \mathrm{E}+07$ \\
\hline 13 & 274.828 & 287.942 & 1.0000 & $\odot .7194 \mathrm{E}+\Theta 7$ \\
\hline 14 & 285.619 & $288 . \odot \odot \odot$ & $1.00 \odot \odot$ & $\odot .7816 \mathrm{E}+\odot 7$ \\
\hline 15 & 287.787 & 288.000 & 1.0000 & $\odot .8008 \mathrm{E}+07$ \\
\hline 16 & 288.000 & $288.00 \odot$ & $1.000 \odot$ & $\odot .8041 \mathrm{E}+07$ \\
\hline 17 & 288.000 & 288.000 & 1.0000 & $0.8044 \mathrm{E}+07$ \\
\hline 18 & $288.0 \odot \odot$ & $288.00 \odot$ & $1.000 \odot$ & $\odot .8044 \mathrm{E}+07$ \\
\hline 19 & 288.000 & $288.00 \odot$ & $1.000 \odot$ & $\odot .8044 \mathrm{E}+07$ \\
\hline 20 & $288.00 \odot$ & $288 . \odot \odot \odot$ & $1.000 \odot$ & $0.8044 \mathrm{E}+07$ \\
\hline 21 & $288.0 \odot \odot$ & $288.00 \odot$ & $1.000 \odot$ & $\odot .8 \odot 44 \mathrm{E}+07$ \\
\hline 22 & 288.000 & 288.000 & 1.0000 & $\odot .8044 \mathrm{E}+07$ \\
\hline 23 & 288.000 & $288.00 \odot$ & $1.000 \odot$ & $\odot .8 \odot 44 \mathrm{E}+07$ \\
\hline 24 & $288.00 \odot$ & $288.00 \odot$ & $1.000 \odot$ & $\odot .8 \odot 44 \mathrm{E}+\odot 7$ \\
\hline 25 & $288 . \odot \odot \odot$ & $288 . \odot \odot \odot$ & $1.000 \odot$ & $0.8044 \mathrm{E}+07$ \\
\hline
\end{tabular}




\begin{tabular}{|c|c|c|c|c|}
\hline time & $(\sec )=$ & $9.00 \quad l c$ & \multicolumn{2}{|c|}{ lambda $=0.2472027689$} \\
\hline node & $\mathrm{T}(\mathrm{K})$ & Twall(K) & ) $\quad x$ & $\mathrm{P}(\mathrm{Pa})$ \\
\hline 1 & 135.289 & 219.599 & 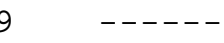 & $0.7957 \mathrm{E}+06$ \\
\hline 2 & 156.935 & 235.561 & 0.9649 & $0.1468 \mathrm{E}+07$ \\
\hline 3 & 175.194 & 248.210 & 0.9936 & $\odot .2820 \mathrm{E}+\odot 7$ \\
\hline 4 & 181.786 & 254.382 & 0.9992 & $\odot .3513 \mathrm{E}+\odot 7$ \\
\hline 5 & 193.952 & 261.318 & 1.0000 & $\odot .4124 \mathrm{E}+07$ \\
\hline 6 & 198.694 & 266.879 & 1.0000 & $\odot .4500 \mathrm{E}+07$ \\
\hline 7 & 205.650 & 272.825 & 1.0000 & $\odot .4753 \mathrm{E}+\odot 7$ \\
\hline 8 & 218.847 & 277.028 & 1.0000 & $0.5173 \mathrm{E}+07$ \\
\hline 9 & 228.792 & 279.867 & 1.0000 & $\odot .5593 \mathrm{E}+07$ \\
\hline 10 & 234.336 & 282.094 & 1.0000 & $\odot .5858 \mathrm{E}+\odot 7$ \\
\hline 11 & 237.861 & 283.894 & 1.0000 & $\odot .6025 \mathrm{E}+07$ \\
\hline 12 & 240.359 & 285.440 & 1.0000 & $0.6140 \mathrm{E}+07$ \\
\hline 13 & 242.441 & 286.753 & $1.000 \odot$ & $0.6233 \mathrm{E}+07$ \\
\hline 14 & 263.483 & 287.762 & 1.0000 & $\odot .6820 \mathrm{E}+07$ \\
\hline 15 & 281.981 & 288.000 & 1.0000 & $\odot .7578 \mathrm{E}+07$ \\
\hline 16 & 287.180 & $288.00 \odot$ & 1.0000 & $\odot .7946 \mathrm{E}+\odot 7$ \\
\hline 17 & 287.966 & $288.00 \odot$ & 1.0000 & $\odot .8033 \mathrm{E}+07$ \\
\hline 18 & 288.000 & $288.0 \odot \odot$ & 1.0000 & $\odot .8044 \mathrm{E}+07$ \\
\hline 19 & $288.0 \odot \odot$ & $288 . \odot \odot \odot$ & $1.000 \odot$ & $\odot .8044 \mathrm{E}+07$ \\
\hline 20 & $288.00 \odot$ & $288 . \odot \odot \odot$ & $1.000 \odot$ & $\odot .8 \odot 44 \mathrm{E}+07$ \\
\hline 21 & $288.00 \odot$ & $288.00 \odot$ & $1.000 \odot$ & $\odot .8 \odot 44 \mathrm{E}+07$ \\
\hline 22 & $288.00 \odot$ & 288.000 & 1.0000 & $\odot .8044 \mathrm{E}+07$ \\
\hline 23 & 288.000 & $288.0 \odot \odot$ & 1.0000 & $\odot .8044 \mathrm{E}+\odot 7$ \\
\hline 24 & 288.000 & $288.0 \odot \odot$ & $1.000 \odot$ & $\odot .8044 \mathrm{E}+07$ \\
\hline 25 & $288 . \odot \odot \odot$ & $288 . \odot \odot \odot$ & $1.000 \odot$ & $\odot .8044 \mathrm{E}+\odot 7$ \\
\hline time & ( $\mathrm{sec})$ & $10.0 \odot$ & $\mathrm{da}=$ & 2308563 \\
\hline node & $\mathrm{T}(\mathrm{K})$ & Twall(K) & $\mathrm{x}$ & $P(P a)$ \\
\hline 1 & 134.550 & 214.892 & - - - - - & $\odot .7680 \mathrm{E}+06$ \\
\hline 2 & 156.078 & 231.301 & $\odot .9651$ & $\odot .1417 \mathrm{E}+07$ \\
\hline 3 & 174.324 & 244.242 & $\odot .9939$ & $\odot .2744 \mathrm{E}+07$ \\
\hline 4 & 181.689 & 250.479 & 0.9984 & $\odot .3502 \mathrm{E}+\odot 7$ \\
\hline 5 & 188.045 & 257.715 & 1.0000 & $\odot .3910 \mathrm{E}+07$ \\
\hline 6 & 201.655 & 263.421 & 1.0000 & $\odot .4494 \mathrm{E}+07$ \\
\hline 7 & 205.918 & 269.226 & 1.0000 & $\odot .4759 \mathrm{E}+07$ \\
\hline 8 & 210.499 & 273.840 & $1.000 \odot$ & $\odot .4950 \mathrm{E}+\odot 7$ \\
\hline 9 & 221.811 & 277.543 & $1.000 \odot$ & $\odot .5309 \mathrm{E}+07$ \\
\hline 10 & 231.080 & 280.190 & 1.0000 & $\odot .5688 \mathrm{E}+07$ \\
\hline 11 & 237.325 & 282.063 & 1.0000 & $\odot .5965 \mathrm{E}+\odot 7$ \\
\hline 12 & 241.066 & 283.776 & $1.000 \odot$ & $\odot .6145 \mathrm{E}+07$ \\
\hline 13 & 243.453 & 285.115 & 1.0000 & $\odot .6258 \mathrm{E}+07$ \\
\hline 14 & 245.342 & 286.347 & 1.0000 & $\odot .6343 \mathrm{E}+07$ \\
\hline 15 & 251.934 & 287.393 & $1.000 \odot$ & $\odot .6545 \mathrm{E}+07$ \\
\hline 16 & 275.009 & 287.943 & 1.0000 & $0.7246 \mathrm{E}+07$ \\
\hline 17 & 285.561 & $288.00 \odot$ & 1.0000 & $\odot .7816 \mathrm{E}+07$ \\
\hline 18 & 287.775 & $288 . \odot \odot \odot$ & $1.000 \odot$ & $\odot .8007 \mathrm{E}+07$ \\
\hline 19 & 288.000 & $288.00 \odot$ & $1.000 \odot$ & $\odot .8041 \mathrm{E}+07$ \\
\hline 20 & $288.00 \odot$ & $288 . \odot \odot \odot$ & 1.0000 & $0.8044 \mathrm{E}+07$ \\
\hline 21 & $288.0 \odot \odot$ & $288 . \odot \odot \odot$ & $1.000 \odot$ & $0.8044 \mathrm{E}+07$ \\
\hline 22 & 288.000 & $288.00 \odot$ & $1.000 \odot$ & $\odot .8 \odot 44 \mathrm{E}+\odot 7$ \\
\hline 23 & 288.000 & $288.0 \odot \odot$ & $1.000 \odot$ & $\odot .8 \odot 44 \mathrm{E}+07$ \\
\hline 24 & $288.00 \odot$ & $288 . \odot \odot \odot$ & $1.000 \odot$ & $\odot .8044 \mathrm{E}+07$ \\
\hline 25 & $288 . \odot \odot \odot$ & $288 . \odot \odot \odot$ & 1.0000 & $0.8044 \mathrm{E}+07$ \\
\hline
\end{tabular}




\begin{tabular}{|c|c|c|c|c|}
\hline \multicolumn{5}{|c|}{$.00 \quad$ lambda $=0.2329435498$} \\
\hline node & $T^{\prime}(K)$ & Twall(K) & $x$ & $\mathrm{P}(\mathrm{Pa})$ \\
\hline 1 & 133.971 & 210.561 & - - _ - _ & $\odot .7462 \mathrm{E}+\odot 6$ \\
\hline 2 & 155.406 & 227.344 & $\odot .9654$ & $\odot .1376 \mathrm{E}+\odot 7$ \\
\hline 3 & 174.034 & 240.435 & $\odot .9929$ & $\odot .2718 E+\odot 7$ \\
\hline 4 & 180.602 & 247.014 & $\odot .9991$ & $\odot .3379 \mathrm{E}+\odot 7$ \\
\hline 5 & 184.657 & 253.820 & $\odot .9993$ & $\odot .3838 E+07$ \\
\hline 6 & 198.144 & 260.356 & 1.0000 & $\odot .4238 \mathrm{E}+\odot 7$ \\
\hline 7 & 208.166 & 266.279 & $1.000 \odot$ & $\odot .4775 \mathrm{E}+\odot 7$ \\
\hline 8 & 213.064 & $270.9 \odot 9$ & 1.0000 & $\odot .5010 \mathrm{E}+\odot 7$ \\
\hline 9 & 217.388 & 274.796 & $1.000 \odot$ & $\odot .520 \odot \mathrm{E}+\odot 7$ \\
\hline 10 & 223.556 & 277.920 & 1.0000 & $\odot .5435 \mathrm{E}+\odot 7$ \\
\hline 11 & 232.484 & 280.232 & $1.000 \odot$ & $\odot .5746 \mathrm{E}+\odot 7$ \\
\hline 12 & 239.364 & 282.250 & 1.0000 & $\odot .6038 \mathrm{E}+07$ \\
\hline 13 & 243.609 & 283.589 & 1.0000 & $\odot .6236 \mathrm{E}+\odot 7$ \\
\hline 14 & 246.095 & 284.880 & $1.000 \odot$ & $\odot .6358 \mathrm{E}+\odot 7$ \\
\hline 15 & 247.875 & 285.977 & $1.000 \odot$ & $\odot .6439 \mathrm{E}+\odot 7$ \\
\hline 16 & 249.186 & 287.007 & $1.000 \odot$ & $\odot .6501 \mathrm{E}+07$ \\
\hline 17 & 264.392 & 287.777 & 1.0000 & $\odot .6924 \mathrm{E}+\odot 7$ \\
\hline 18 & 281.937 & 288.000 & 1.0000 & $\odot .7586 \mathrm{E}+\odot 7$ \\
\hline 19 & 287.151 & $288.0 \odot \odot$ & $1.000 \odot$ & $\odot .7945 \mathrm{E}+\odot 7$ \\
\hline 20 & 287.963 & $288 . \odot$ & $1.000 \odot$ & $\odot .8032 \mathrm{E}+\odot 7$ \\
\hline 21 & 288.000 & 288.000 & 1.0000 & $\odot .8044 \mathrm{E}+07$ \\
\hline 22 & 288.000 & 288.000 & 1.0000 & $\odot .8044 \mathrm{E}+\odot 7$ \\
\hline 23 & 288.000 & 288.000 & 1.0000 & $\odot .8044 \mathrm{E}+07$ \\
\hline 24 & 288.000 & 288.000 & 1.0000 & $\odot .8 \odot 44 \mathrm{E}+\odot 7$ \\
\hline 25 & 288.000 & 288.000 & 1.0000 & $\odot .8044 \mathrm{E}+07$ \\
\hline
\end{tabular}

\begin{tabular}{|c|c|c|c|c|}
\hline \multicolumn{5}{|c|}{ lambda $=0.2287873030$} \\
\hline node & $\mathrm{T}(\mathrm{K})$ & Twall(K) & $\mathrm{x}$ & $\mathrm{P}(\mathrm{Pa})$ \\
\hline 1 & 133.589 & 206.525 & $\ldots$ & $\odot .7318 \mathrm{E}+06$ \\
\hline 2 & 154.963 & 223.615 & 0.9645 & $\odot .1350 \mathrm{E}+07$ \\
\hline 3 & 173.337 & 236.928 & $\odot .9928$ & $\odot .2657 \mathrm{E}+\odot 7$ \\
\hline 4 & 179.730 & 243.801 & 0.9990 & $\odot .3281 \mathrm{E}+\odot 7$ \\
\hline 5 & 184.385 & 250.364 & $\odot .9997$ & $\odot .3807 \mathrm{E}+07$ \\
\hline 6 & 188.747 & 256.880 & 1.0000 & $\odot .4013 \mathrm{E}+\odot 7$ \\
\hline 7 & 206.125 & 263.532 & 1.0000 & $\odot .4593 \mathrm{E}+07$ \\
\hline 8 & 214.377 & 268.396 & 1.0000 & $0.5014 \mathrm{E}+07$ \\
\hline 9 & 219.408 & 272.364 & 1.0000 & $\odot .5246 \mathrm{E}+\odot 7$ \\
\hline 10 & 222.294 & 275.486 & $1.000 \odot$ & $\odot .5404 \mathrm{E}+07$ \\
\hline 11 & 225.568 & 278.017 & 1.0000 & $\odot .5542 \mathrm{E}+07$ \\
\hline 12 & 233.091 & 280.507 & 1.0000 & $\odot .5787 \mathrm{E}+07$ \\
\hline 13 & 240.596 & 282.064 & 1.0000 & $\odot .6078 \mathrm{E}+07$ \\
\hline 14 & 245.467 & 283.659 & 1.0000 & $\odot .6301 \mathrm{E}+07$ \\
\hline 15 & 248.322 & 284.756 & 1.0000 & $\odot .6439 \mathrm{E}+07$ \\
\hline 16 & 249.954 & 285.787 & 1.0000 & $\odot .6521 \mathrm{E}+\odot 7$ \\
\hline 17 & 251.485 & 286.628 & 1.0000 & $0.6585 \mathrm{E}+07$ \\
\hline 18 & 255.435 & 287.476 & 1.0000 & $\odot .6711 \mathrm{E}+07$ \\
\hline 19 & 275.140 & 287.943 & $1.000 \odot$ & $\odot .7289 \mathrm{E}+07$ \\
\hline 20 & 285.516 & $288.0 \odot \odot$ & 1.0000 & $\odot .7816 \mathrm{E}+\odot 7$ \\
\hline 21 & 287.767 & $288.00 \odot$ & $1.00 \odot \odot$ & $\odot .80 \odot 6 \mathrm{E}+\odot 7$ \\
\hline 22 & 288.000 & 288.000 & 1.0000 & $\odot .8041 \mathrm{E}+07$ \\
\hline 23 & 288.000 & 288.000 & 1.0000 & $\odot .8044 \mathrm{E}+07$ \\
\hline 24 & 288.000 & 288.000 & 1.0000 & $\odot .8044 \mathrm{E}+07$ \\
\hline 25 & $288 . \odot \odot \odot$ & $288 . \odot \odot \odot$ & $1.00 \odot \odot$ & $\odot .8044 \mathrm{E}+\odot 7$ \\
\hline
\end{tabular}




\begin{tabular}{|c|c|c|c|c|}
\hline \multicolumn{5}{|c|}{ lambda $=0.2242530584$} \\
\hline node & $T^{\prime}(K)$ & Twall(K) & $\mathrm{x}$ & $\mathrm{P}(\mathrm{Pa})$ \\
\hline 1 & 133.173 & 202.767 & $-\ldots$ & $\odot .7162 \mathrm{E}+\odot 6$ \\
\hline 2 & 154.481 & 220.152 & $\odot .9642$ & $\odot .1321 \mathrm{E}+\odot 7$ \\
\hline 3 & 172.481 & 233.669 & $\odot .9930$ & $\odot .2582 E+\odot 7$ \\
\hline 4 & 179.392 & 240.632 & 0.9980 & $\odot .3242 \mathrm{E}+\odot 7$ \\
\hline 5 & 187.961 & 247.455 & $1.000 \odot$ & $\odot .3756 \mathrm{E}+\odot 7$ \\
\hline 6 & 194.731 & 253.811 & 1.0000 & $\odot .4140 \mathrm{E}+\odot 7$ \\
\hline 7 & 196.940 & 260.314 & 1.0000 & $\odot .4385 \mathrm{E}+\odot 7$ \\
\hline 8 & 210.172 & 265.914 & 1.0000 & $\odot .4806 \mathrm{E}+\odot 7$ \\
\hline 9 & 219.254 & 270.227 & $1.000 \odot$ & $\odot .5189 \mathrm{E}+\odot 7$ \\
\hline 10 & 223.603 & 273.350 & 1.0000 & $\odot .5426 \mathrm{E}+\odot 7$ \\
\hline 11 & 226.686 & 275.893 & $1.000 \odot$ & $\odot .5570 \mathrm{E}+\odot 7$ \\
\hline 12 & 229.482 & 278.379 & 1.0000 & $\odot .5693 \mathrm{E}+\odot 7$ \\
\hline 13 & 233.645 & 280.347 & 1.0000 & $\odot .5846 \mathrm{E}+\odot 7$ \\
\hline 14 & 240.598 & 282.267 & 1.0000 & $\odot .6087 \mathrm{E}+\odot 7$ \\
\hline 15 & 247.052 & 283.535 & $1.000 \odot$ & $\odot .6343 \mathrm{E}+\odot 7$ \\
\hline 16 & 249.901 & 284.566 & 1.0000 & $\odot .6501 \mathrm{E}+07$ \\
\hline 17 & 251.814 & 285.477 & 1.0000 & $\odot .6590 \mathrm{E}+\odot 7$ \\
\hline 18 & 253.249 & 286.373 & 1.0000 & $0.6653 E+07$ \\
\hline 19 & 254.327 & 287.179 & $1.000 \odot$ & $\odot .6701 \mathrm{E}+\odot 7$ \\
\hline 20 & 265.204 & 287.786 & $1.000 \odot$ & $\odot .7006 \mathrm{E}+\odot 7$ \\
\hline 21 & 281.907 & 288.000 & 1.0000 & $\odot .7594 \mathrm{E}+\odot 7$ \\
\hline 22 & 287.130 & 288.000 & 1.0000 & $\odot .7944 \mathrm{E}+\odot 7$ \\
\hline 23 & 287.960 & 288.000 & 1.0000 & $\odot .8032 \mathrm{E}+07$ \\
\hline 24 & 288.000 & 288.000 & 1.0000 & $\odot .8 \odot 44 \mathrm{E}+\odot 7$ \\
\hline 25 & 288.000 & 288.000 & 1.0000 & $\odot .8044 \mathrm{E}+07$ \\
\hline
\end{tabular}

\begin{tabular}{|c|c|c|c|c|}
\hline \multicolumn{5}{|c|}{ lambda $=0.2192834169$} \\
\hline node & $T(K)$ & Twall(K) & $\mathrm{x}$ & $\mathrm{P}(\mathrm{Pa})$ \\
\hline 1 & 132.719 & 199.251 & $\ldots$ & $\odot .6991 \mathrm{E}+06$ \\
\hline 2 & 153.954 & 216.913 & 0.9646 & $\odot .1290 \mathrm{E}+07$ \\
\hline 3 & 171.940 & 230.545 & $\odot .9929$ & $\odot .2535 \mathrm{E}+\odot 7$ \\
\hline 4 & 179.015 & 237.614 & 0.9976 & $\odot .3200 \mathrm{E}+\odot 7$ \\
\hline 5 & 186.070 & 244.678 & 1.0000 & $\odot .3634 \mathrm{E}+07$ \\
\hline 6 & 197.738 & 251.164 & 1.0000 & $\odot .4151 \mathrm{E}+\odot 7$ \\
\hline 7 & 199.542 & 257.249 & 1.0000 & $\odot .4443 \mathrm{E}+07$ \\
\hline 8 & 202.586 & 262.951 & 1.0000 & $\odot .4619 \mathrm{E}+07$ \\
\hline 9 & 212.878 & 267.956 & 1.0000 & $\odot .4945 \mathrm{E}+\odot 7$ \\
\hline 10 & 222.883 & 271.519 & $1.000 \odot$ & $\odot .5332 \mathrm{E}+\odot 7$ \\
\hline 11 & 227.721 & 274.062 & 1.0000 & $\odot .5580 \mathrm{E}+07$ \\
\hline 12 & 230.495 & 276.548 & 1.0000 & $\odot .5718 \mathrm{E}+07$ \\
\hline 13 & 233.458 & 278.516 & 1.0000 & $\odot .5840 \mathrm{E}+07$ \\
\hline 14 & 234.532 & 280.503 & 1.0000 & $\odot .5919 \mathrm{E}+07$ \\
\hline 15 & 240.874 & 282.184 & 1.0000 & $\odot .6100 \mathrm{E}+07$ \\
\hline 16 & 247.550 & 283.345 & 1.0000 & $\odot .6362 \mathrm{E}+\odot 7$ \\
\hline 17 & 251.066 & 284.562 & 1.0000 & $\odot .6541 \mathrm{E}+07$ \\
\hline 18 & 253.385 & 285.458 & 1.0000 & $\odot .6646 \mathrm{E}+07$ \\
\hline 19 & 254.837 & 286.264 & $1.000 \odot$ & $\odot .6713 \mathrm{E}+07$ \\
\hline 20 & 256.009 & 286.890 & $1.000 \odot$ & $\odot .6763 \mathrm{E}+07$ \\
\hline 21 & 258.414 & 287.520 & $1.00 \odot \odot$ & $\odot .6843 \mathrm{E}+07$ \\
\hline 22 & 275.298 & 287.944 & 1.0000 & $\odot .7329 \mathrm{E}+07$ \\
\hline 23 & 285.466 & 288.000 & 1.0000 & $\odot .7817 \mathrm{E}+07$ \\
\hline 24 & 287.770 & 288.000 & 1.0000 & $\odot .8006 \mathrm{E}+07$ \\
\hline 25 & 287.770 & 288.000 & 1.0000 & $\odot .8035 \mathrm{E}+07$ \\
\hline
\end{tabular}




\begin{tabular}{|c|c|c|c|c|}
\hline \multicolumn{5}{|c|}{ lambda $=0.2151512802$} \\
\hline node & $T^{\prime}(K)$ & Twall(K) & $\mathrm{x}$ & $\mathrm{P}(\mathrm{Pa})$ \\
\hline 1 & 132.343 & 195.968 & $-\ldots$ & $\odot .6850 \mathrm{E}+\odot 6$ \\
\hline 2 & 153.517 & 213.890 & 0.9647 & $0.1263 E+07$ \\
\hline 3 & 171.538 & 227.571 & $\odot .9924$ & $\odot .2499 \mathrm{E}+\odot 7$ \\
\hline 4 & 178.299 & 234.813 & $\odot .9981$ & $\odot .3119 \mathrm{E}+\odot 7$ \\
\hline 5 & 183.731 & 241.671 & $1.000 \odot$ & $\odot .3586 E+\odot 7$ \\
\hline 6 & 192.350 & 248.654 & 1.0000 & $\odot .3917 \mathrm{E}+\odot 7$ \\
\hline 7 & 201.617 & 254.629 & 1.0000 & $\odot .4441 E+07$ \\
\hline 8 & 204.771 & 260.145 & 1.0000 & $0.4669 \mathrm{E}+07$ \\
\hline 9 & 208.832 & 265.209 & $1.000 \odot$ & $\odot .485 \odot E+\odot 7$ \\
\hline 10 & 214.519 & 269.351 & 1.0000 & $\odot .5069 \mathrm{E}+\odot 7$ \\
\hline 11 & 227.668 & 272.231 & $1.000 \odot$ & $\odot .5476 \mathrm{E}+\odot 7$ \\
\hline 12 & 228.696 & 274.717 & 1.0000 & $\odot .5670 \mathrm{E}+\Theta 7$ \\
\hline 13 & 234.190 & 276.954 & 1.0000 & $\odot .5830 E+\odot 7$ \\
\hline 14 & 235.524 & 278.884 & 1.0000 & $\odot .5944 \mathrm{E}+\odot 7$ \\
\hline 15 & 238.456 & 280.658 & 1.0000 & $0.6039 \mathrm{E}+07$ \\
\hline 16 & 241.547 & 281.991 & 1.0000 & $\odot .6167 \mathrm{E}+07$ \\
\hline 17 & 247.326 & 283.442 & 1.0000 & $\odot .6367 \mathrm{E}+\odot 7$ \\
\hline 18 & 251.809 & 284.542 & 1.0000 & $0.6559 \mathrm{E}+07$ \\
\hline 19 & 254.619 & 285.348 & $1.000 \odot$ & $\odot .6688 \mathrm{E}+\odot 7$ \\
\hline 20 & 256.297 & 285.974 & $1.000 \odot$ & $\odot .6766 \mathrm{E}+\odot 7$ \\
\hline 21 & 257.378 & 286.604 & 1.0000 & $\odot .6816 E+\odot 7$ \\
\hline 22 & 258.444 & 287.242 & 1.0000 & $\odot .6859 \mathrm{E}+\odot 7$ \\
\hline 23 & 264.413 & 287.782 & 1.0000 & $0.7034 \mathrm{E}+07$ \\
\hline 24 & 282.506 & 288.000 & 1.0000 & $\odot .7600 \mathrm{E}+\odot 7$ \\
\hline 25 & 282.605 & 288.000 & 1.0000 & $\odot .7826 \mathrm{E}+07$ \\
\hline
\end{tabular}

\begin{tabular}{|c|c|c|c|c|}
\hline \multicolumn{5}{|c|}{ lambda $=0.2116734833$} \\
\hline node & $\mathrm{T}(\mathrm{K})$ & Twall(K) & $\mathrm{x}$ & $P(P a)$ \\
\hline 1 & 132.027 & 192.875 & - - - & $0.6731 \mathrm{E}+06$ \\
\hline 2 & 153.151 & 211.038 & 0.9643 & $\odot .1242 \mathrm{E}+07$ \\
\hline 3 & 170.981 & 224.789 & $\odot .9926$ & $\odot .2451 \mathrm{E}+07$ \\
\hline 4 & 177.889 & 232.067 & 0.9977 & $\odot .3072 \mathrm{E}+\odot 7$ \\
\hline 5 & 187.284 & 239.126 & 1.0000 & $\odot .3587 \mathrm{E}+07$ \\
\hline 6 & 187.608 & 245.788 & 1.0000 & $\odot .3813 E+07$ \\
\hline 7 & 199.030 & 252.181 & 1.0000 & $\odot .4271 \mathrm{E}+07$ \\
\hline 8 & 204.442 & 257.704 & 1.0000 & $0.4638 \mathrm{E}+07$ \\
\hline 9 & 210.205 & 262.832 & 1.0000 & $\odot .4868 \mathrm{E}+\odot 7$ \\
\hline 10 & 210.481 & 266.849 & $1.000 \odot$ & $\odot .4974 \mathrm{E}+\odot 7$ \\
\hline 11 & 225.629 & $270.40 \odot$ & 1.0000 & $0.5348 \mathrm{E}+07$ \\
\hline 12 & 225.658 & 272.886 & 1.0000 & $\odot .5578 \mathrm{E}+07$ \\
\hline 13 & 232.979 & 275.428 & 1.0000 & $\odot .5759 \mathrm{E}+07$ \\
\hline 14 & 236.370 & 277.358 & $1.000 \odot$ & $\odot .5949 \mathrm{E}+07$ \\
\hline 15 & 239.273 & 279.133 & 1.0000 & $\odot .6060 \mathrm{E}+07$ \\
\hline 16 & 241.334 & 280.501 & 1.0000 & $\odot .6161 \mathrm{E}+07$ \\
\hline 17 & 242.615 & 282.059 & 1.0000 & $0.6233 \mathrm{E}+07$ \\
\hline 18 & 246.872 & 283.428 & 1.0000 & $0.6367 \mathrm{E}+07$ \\
\hline 19 & 251.608 & 284.433 & $1.000 \odot$ & $\odot .6548 \mathrm{E}+07$ \\
\hline 20 & 255.879 & 285.059 & 1.0000 & $\odot .6718 \mathrm{E}+\odot 7$ \\
\hline 21 & 256.670 & 285.689 & 1.0000 & $\odot .6791 \mathrm{E}+07$ \\
\hline 22 & 258.531 & 286.455 & 1.0000 & $\odot .6851 \mathrm{E}+07$ \\
\hline 23 & 258.531 & 286.974 & 1.0000 & $\odot .6878 \mathrm{E}+\odot 7$ \\
\hline 24 & 258.531 & 287.702 & 1.0000 & $\odot .6878 \mathrm{E}+07$ \\
\hline 25 & 258.772 & 287.704 & 1.0000 & $\odot .6892 \mathrm{E}+07$ \\
\hline
\end{tabular}




\begin{tabular}{|c|c|c|c|c|}
\hline \multicolumn{5}{|c|}{ lambda $=0.2081392556$} \\
\hline node & $T^{\prime}(K)$ & Twall(K) & $\mathrm{x}$ & $\mathrm{P}(\mathrm{Pa})$ \\
\hline 1 & 131.706 & 189.974 & $-\ldots$ & $\odot .6610 \mathrm{E}+\odot 6$ \\
\hline 2 & 152.779 & 208.356 & $\odot .9645$ & $\odot .1219 \mathrm{E}+\odot 7$ \\
\hline 3 & 170.511 & 222.176 & $\odot .9926$ & $\odot .2409 \mathrm{E}+\odot 7$ \\
\hline 4 & 177.729 & 229.431 & $\odot .9967$ & $\odot .3054 \mathrm{E}+\odot 7$ \\
\hline 5 & 192.057 & 237.082 & $1.000 \odot$ & $\odot .3505 \mathrm{E}+\odot 7$ \\
\hline 6 & 192.057 & 243.315 & 1.0000 & $\odot .3903 \mathrm{E}+\odot 7$ \\
\hline 7 & 192.920 & 249.519 & 1.0000 & $\odot .4138 \mathrm{E}+07$ \\
\hline 8 & 196.147 & 255.122 & 1.0000 & $0.4385 E+07$ \\
\hline 9 & 211.928 & 260.779 & $1.000 \odot$ & $\odot .4809 \mathrm{E}+\odot 7$ \\
\hline 10 & 213.589 & 264.657 & 1.0000 & $\odot .5 \odot 47 \mathrm{E}+\odot 7$ \\
\hline 11 & 223.056 & 268.569 & $1.000 \odot$ & $\odot .5287 \mathrm{E}+\odot 7$ \\
\hline 12 & 225.211 & 271.055 & 1.0000 & $\odot .5544 \mathrm{E}+07$ \\
\hline 13 & 227.474 & 273.738 & 1.0000 & $\odot .5614 \mathrm{E}+\odot 7$ \\
\hline 14 & 236.529 & 275.964 & 1.0000 & $\odot .5881 \mathrm{E}+\odot 7$ \\
\hline 15 & 238.076 & 277.876 & $1.000 \odot$ & $\odot .6 \odot 27 \mathrm{E}+\odot 7$ \\
\hline 16 & 241.944 & 279.281 & 1.0000 & $\odot .6158 \mathrm{E}+07$ \\
\hline 17 & 242.964 & 280.836 & 1.0000 & $\odot .6242 \mathrm{E}+\odot 7$ \\
\hline 18 & 245.428 & 282.207 & 1.0000 & $0.6326 \mathrm{E}+07$ \\
\hline 19 & 245.428 & 283.255 & $1.000 \odot$ & $\odot .6365 \mathrm{E}+07$ \\
\hline 20 & 254.213 & 284.143 & $1.000 \odot$ & $\odot .6593 \mathrm{E}+\odot 7$ \\
\hline 21 & 254.213 & 284.773 & 1.0000 & $\odot .6708 \mathrm{E}+07$ \\
\hline 22 & 256.893 & 285.845 & 1.0000 & $\odot .6779 \mathrm{E}+\odot 7$ \\
\hline 23 & 256.893 & 286.364 & 1.0000 & $\odot .6816 E+\odot 7$ \\
\hline 24 & 256.893 & 287.092 & 1.0000 & $\odot .6816 \mathrm{E}+\odot 7$ \\
\hline 25 & 256.893 & 287.093 & 1.0000 & $\odot .6816 \mathrm{E}+07$ \\
\hline
\end{tabular}

\begin{tabular}{|c|c|c|c|c|}
\hline \multicolumn{5}{|c|}{ lambda $=0.2049267441$} \\
\hline node & $\mathrm{T}(\mathrm{K})$ & Twall(K) & $\mathrm{x}$ & $\mathrm{P}(\mathrm{Pa})$ \\
\hline 1 & 131.416 & 187.247 & $\ldots$ & $\odot .6501 \mathrm{E}+06$ \\
\hline 2 & 152.442 & 205.823 & 0.9645 & $\odot .1199 \mathrm{E}+07$ \\
\hline 3 & 170.221 & 219.658 & $\odot .9921$ & $\odot .2384 \mathrm{E}+\odot 7$ \\
\hline 4 & 177.112 & 227.013 & 0.9968 & $\odot .2988 \mathrm{E}+\odot 7$ \\
\hline 5 & 185.365 & 235.003 & 1.0000 & $\odot .3311 \mathrm{E}+07$ \\
\hline 6 & 190.695 & 241.117 & 1.0000 & $\odot .3833 \mathrm{E}+\odot 7$ \\
\hline 7 & 193.522 & 247.041 & 1.0000 & $\odot .4147 \mathrm{E}+07$ \\
\hline 8 & 194.212 & 252.305 & 1.0000 & $0.4341 \mathrm{E}+07$ \\
\hline 9 & 208.659 & 258.801 & 1.0000 & $\odot .4664 \mathrm{E}+\odot 7$ \\
\hline 10 & 214.976 & 262.651 & $1.000 \odot$ & $\odot .5044 \mathrm{E}+\odot 7$ \\
\hline 11 & 215.614 & 266.738 & 1.0000 & $0.5111 \mathrm{E}+07$ \\
\hline 12 & 226.066 & $269.50 \odot$ & 1.0000 & $\odot .5478 \mathrm{E}+07$ \\
\hline 13 & 227.495 & 272.125 & 1.0000 & $\odot .5614 \mathrm{E}+07$ \\
\hline 14 & 236.653 & 274.682 & $1.000 \odot$ & $\odot .5863 \mathrm{E}+\odot 7$ \\
\hline 15 & 236.653 & 276.350 & 1.0000 & $\odot .5988 \mathrm{E}+07$ \\
\hline 16 & 242.501 & 278.060 & 1.0000 & $\odot .6136 \mathrm{E}+\odot 7$ \\
\hline 17 & 242.501 & 279.615 & 1.0000 & $0.6231 \mathrm{E}+07$ \\
\hline 18 & 245.802 & 280.987 & 1.0000 & $\odot .6315 \mathrm{E}+07$ \\
\hline 19 & 245.802 & 282.034 & $1.000 \odot$ & $\odot .6375 \mathrm{E}+07$ \\
\hline 20 & 249.903 & 283.227 & $1.000 \odot$ & $\odot .6481 \mathrm{E}+07$ \\
\hline 21 & 249.903 & 283.857 & 1.0000 & $\odot .6547 \mathrm{E}+07$ \\
\hline 22 & 252.144 & 285.095 & 1.0000 & $\odot .6606 \mathrm{E}+07$ \\
\hline 23 & 252.144 & 285.753 & 1.0000 & $\odot .6636 \mathrm{E}+07$ \\
\hline 24 & 252.144 & 286.481 & 1.0000 & $\odot .6636 \mathrm{E}+07$ \\
\hline 25 & 252.144 & 286.483 & 1.0000 & $\odot .6636 \mathrm{E}+07$ \\
\hline
\end{tabular}




\begin{tabular}{|c|c|c|c|c|}
\hline \multicolumn{5}{|c|}{$.00 \quad$ lambda $=0.2022141218$} \\
\hline node & $T(K)$ & Twall(K) & $x$ & $P(P a)$ \\
\hline 1 & 131.171 & 184.668 & $\cdots$ & $\odot .6409 \mathrm{E}+06$ \\
\hline 2 & 152.158 & 203.418 & $\odot .9640$ & $\odot .1182 \mathrm{E}+\odot 7$ \\
\hline 3 & 169.704 & 217.309 & ๑.9915 & $\odot .2339 \mathrm{E}+\odot 7$ \\
\hline 4 & 175.582 & 224.840 & $\odot .9980$ & $\odot .2854 \mathrm{E}+\odot 7$ \\
\hline 5 & 179.587 & 232.568 & $1.000 \odot$ & $\odot .3207 \mathrm{E}+\odot 7$ \\
\hline 6 & 185.911 & 238.801 & 1.0000 & $\odot .3682 \mathrm{E}+\odot 7$ \\
\hline 7 & 191.962 & 244.778 & $1.000 \odot$ & $\odot .4 \odot 55 \mathrm{E}+\odot 7$ \\
\hline 8 & 197.296 & 249.898 & 1.0000 & $\odot .4391 \mathrm{E}+07$ \\
\hline 9 & 197.359 & 256.307 & 1.0000 & $\odot .4412 \mathrm{E}+07$ \\
\hline 10 & 216.248 & 260.979 & 1.0000 & $\odot .4942 \mathrm{E}+\odot 7$ \\
\hline 11 & 216.248 & 264.907 & $1.000 \odot$ & $\odot .5126 \mathrm{E}+\odot 7$ \\
\hline 12 & 227.407 & 267.974 & 1.0000 & $\odot .5479 \mathrm{E}+07$ \\
\hline 13 & 227.905 & 270.599 & $1.000 \odot$ & $\odot .5624 \mathrm{E}+\odot 7$ \\
\hline 14 & 237.666 & 273.461 & $1.000 \odot$ & $\odot .5871 \mathrm{E}+\odot 7$ \\
\hline 15 & 237.666 & 274.956 & 1.0000 & $\odot .6014 \mathrm{E}+07$ \\
\hline 16 & 242.441 & 276.839 & $1.000 \odot$ & $\odot .6135 \mathrm{E}+07$ \\
\hline 17 & 242.441 & 278.395 & 1.0000 & $\odot .6229 E+\odot 7$ \\
\hline 18 & 245.702 & 280.067 & 1.0000 & $\odot .6313 E+07$ \\
\hline 19 & 245.877 & 280.978 & 1.0000 & $0.6373 \mathrm{E}+07$ \\
\hline 20 & 245.877 & 282.312 & $1.000 \odot$ & $\odot .6375 \mathrm{E}+\odot 7$ \\
\hline 21 & 245.877 & 282.942 & 1.0000 & $\odot .6394 \mathrm{E}+\odot 7$ \\
\hline 22 & 245.877 & 284.179 & 1.0000 & $\odot .6394 \mathrm{E}+07$ \\
\hline 23 & 246.624 & 285.143 & 1.0000 & $\odot .6418 \mathrm{E}+07$ \\
\hline 24 & 246.624 & 285.871 & 1.0000 & $\odot .6430 \mathrm{E}+\odot 7$ \\
\hline 25 & 246.624 & 285.873 & 1.0000 & $\odot .6430 \mathrm{E}+07$ \\
\hline
\end{tabular}

\begin{tabular}{|c|c|c|c|c|}
\hline \multicolumn{5}{|c|}{ lambda $=0.1986758709$} \\
\hline node & $T(K)$ & Twall(K) & $\mathrm{x}$ & $P(P a)$ \\
\hline 1 & 130.852 & 182.232 & $-\ldots$ & $0.6289 E+06$ \\
\hline 2 & 151.789 & 201.163 & 0.9635 & $\odot .1160 \mathrm{E}+07$ \\
\hline 3 & 168.748 & 215.175 & $\odot .9918$ & $\odot .2255 \mathrm{E}+\odot 7$ \\
\hline 4 & 174.517 & 222.704 & 0.9986 & $\odot .2760 \mathrm{E}+\odot 7$ \\
\hline 5 & 178.990 & 230.127 & $\odot .9996$ & $\odot .3197 \mathrm{E}+07$ \\
\hline 6 & 184.651 & 236.410 & 1.0000 & $\odot .3619 \mathrm{E}+\odot 7$ \\
\hline 7 & 185.063 & 242.186 & 0.9994 & $\odot .3884 \mathrm{E}+07$ \\
\hline 8 & 198.884 & 247.979 & 1.0000 & $0.4235 \mathrm{E}+07$ \\
\hline 9 & 200.923 & 254.098 & 1.0000 & $\odot .4491 \mathrm{E}+07$ \\
\hline 10 & 218.259 & 259.453 & $1.000 \odot$ & $\odot .4979 \mathrm{E}+\odot 7$ \\
\hline 11 & 218.259 & 263.076 & 1.0000 & $\odot .5174 \mathrm{E}+07$ \\
\hline 12 & 228.868 & 266.508 & 1.0000 & $0.5474 \mathrm{E}+07$ \\
\hline 13 & 228.868 & 269.073 & 1.0000 & $0.5648 \mathrm{E}+07$ \\
\hline 14 & 236.733 & 272.240 & 1.0000 & $\odot .5842 \mathrm{E}+07$ \\
\hline 15 & 236.733 & 273.736 & 1.0000 & $\odot .5981 \mathrm{E}+07$ \\
\hline 16 & 240.750 & 275.618 & 1.0000 & $\odot .6083 \mathrm{E}+\odot 7$ \\
\hline 17 & 241.343 & 277.175 & 1.0000 & $0.6183 \mathrm{E}+07$ \\
\hline 18 & 241.343 & 279.056 & 1.0000 & $\odot .6189 \mathrm{E}+07$ \\
\hline 19 & 245.402 & 280.063 & $1.000 \odot$ & $\odot .6308 \mathrm{E}+07$ \\
\hline 20 & 245.402 & 281.396 & $1.000 \odot$ & $0.6358 \mathrm{E}+07$ \\
\hline 21 & 245.402 & 282.026 & 1.0000 & $\odot .6358 \mathrm{E}+07$ \\
\hline 22 & 245.402 & 283.264 & 1.0000 & $\odot .6358 \mathrm{E}+07$ \\
\hline 23 & 245.402 & 284.533 & 1.0000 & $\odot .6358 \mathrm{E}+07$ \\
\hline 24 & 245.402 & 285.261 & 1.0000 & $\odot .6358 \mathrm{E}+07$ \\
\hline 25 & 245.402 & 285.262 & 1.0000 & $\odot .6358 \mathrm{E}+07$ \\
\hline
\end{tabular}




\begin{tabular}{|c|c|c|c|c|}
\hline \multicolumn{5}{|c|}{$.00 \quad$ lambda $=0.1934487522$} \\
\hline node & $T^{\prime}(K)$ & Twall(K) & $x$ & $\mathrm{P}(\mathrm{Pa})$ \\
\hline 1 & 130.383 & 179.943 & - - _ - _ & $\odot .6113 \mathrm{E}+06$ \\
\hline 2 & 151.244 & 199.064 & $\odot .9640$ & $\odot .1128 \mathrm{E}+\odot 7$ \\
\hline 3 & 167.857 & 213.191 & $\odot .9924$ & $\odot .2177 \mathrm{E}+\odot 7$ \\
\hline 4 & 174.273 & 220.568 & $\odot .9989$ & $\odot .2739 \mathrm{E}+\odot 7$ \\
\hline 5 & 178.990 & 227.789 & $\odot .9990$ & $\odot .3197 \mathrm{E}+\odot 7$ \\
\hline 6 & 185.566 & 234.247 & 1.0000 & $\odot .3550 \mathrm{E}+\odot 7$ \\
\hline 7 & 185.156 & 239.589 & $\odot .9988$ & $\odot .3894 \mathrm{E}+07$ \\
\hline 8 & 202.457 & 246.148 & 1.0000 & $\odot .4249 E+07$ \\
\hline 9 & 202.457 & 251.859 & $1.000 \odot$ & $\odot .4526 \mathrm{E}+\odot 7$ \\
\hline 10 & 220.591 & 257.927 & 1.0000 & $\odot .4988 \mathrm{E}+\odot 7$ \\
\hline 11 & 220.591 & 261.385 & $1.000 \odot$ & $\odot .5229 \mathrm{E}+\odot 7$ \\
\hline 12 & 228.711 & 265.241 & 1.0000 & $\odot .5422 \mathrm{E}+\Theta 7$ \\
\hline 13 & 229.125 & 267.547 & 1.0000 & $\odot .5647 \mathrm{E}+\odot 7$ \\
\hline 14 & 232.060 & 270.980 & $1.000 \odot$ & $\odot .5722 \mathrm{E}+\odot 7$ \\
\hline 15 & 234.947 & 272.515 & $1.000 \odot$ & $\odot .5888 \mathrm{E}+\odot 7$ \\
\hline 16 & 234.947 & 274.398 & $1.000 \odot$ & $\odot .5917 \mathrm{E}+\odot 7$ \\
\hline 17 & 241.326 & 276.260 & 1.0000 & $\odot .6094 \mathrm{E}+\odot 7$ \\
\hline 18 & 241.326 & 278.037 & 1.0000 & $\odot .6187 E+07$ \\
\hline 19 & 241.326 & 279.147 & $1.000 \odot$ & $\odot .6187 \mathrm{E}+\odot 7$ \\
\hline 20 & 242.504 & 280.481 & $1.000 \odot$ & $\odot .6237 \mathrm{E}+\odot 7$ \\
\hline 21 & 242.504 & 281.111 & 1.0000 & $\odot .6250 \mathrm{E}+\odot 7$ \\
\hline 22 & 242.504 & 282.395 & 1.0000 & $\odot .6250 \mathrm{E}+\odot 7$ \\
\hline 23 & 242.504 & 283.922 & 1.0000 & $\odot .6250 \mathrm{E}+\odot 7$ \\
\hline 24 & 242.504 & 284.650 & 1.0000 & $\odot .6250 \mathrm{E}+\odot 7$ \\
\hline 25 & 242.504 & 284.652 & 1.0000 & $\odot .6250 \mathrm{E}+\odot 7$ \\
\hline
\end{tabular}

\begin{tabular}{|c|c|c|c|c|}
\hline \multicolumn{5}{|c|}{ lambda $=0.1880414188$} \\
\hline node & $\mathrm{T}(\mathrm{K})$ & Twall(K) & $\mathrm{x}$ & $P(P a)$ \\
\hline 1 & 129.899 & 177.806 & $-\ldots$ & $\odot .5931 \mathrm{E}+06$ \\
\hline 2 & 150.683 & 197.080 & 0.9652 & $\odot .1094 \mathrm{E}+07$ \\
\hline 3 & 167.457 & 211.207 & $\odot .9929$ & $\odot .2142 \mathrm{E}+07$ \\
\hline 4 & 174.188 & 218.458 & 0.9984 & $\odot .2732 \mathrm{E}+07$ \\
\hline 5 & 178.835 & 225.622 & $\odot .9989$ & $\odot .3179 \mathrm{E}+07$ \\
\hline 6 & 185.564 & 232.111 & 1.0000 & $\odot .3518 \mathrm{E}+07$ \\
\hline 7 & 185.156 & 236.995 & 0.9982 & $\odot .3894 \mathrm{E}+07$ \\
\hline 8 & 206.317 & 244.366 & 1.0000 & $\odot .4330 \mathrm{E}+07$ \\
\hline 9 & 206.317 & 249.673 & 1.0000 & $\odot .4612 \mathrm{E}+\odot 7$ \\
\hline 10 & 215.582 & 256.366 & $1.000 \odot$ & $\odot .4819 \mathrm{E}+\odot 7$ \\
\hline 11 & 221.315 & 259.859 & 1.0000 & $\odot .5198 \mathrm{E}+07$ \\
\hline 12 & 221.315 & 263.715 & 1.0000 & $\odot .5246 \mathrm{E}+07$ \\
\hline 13 & 229.569 & 266.277 & 1.0000 & $0.5565 \mathrm{E}+07$ \\
\hline 14 & 229.569 & 269.554 & $1.000 \odot$ & $\odot .5660 \mathrm{E}+07$ \\
\hline 15 & 235.168 & 271.294 & 1.0000 & $\odot .5812 \mathrm{E}+07$ \\
\hline 16 & 235.276 & 273.177 & 1.0000 & $\odot .5924 \mathrm{E}+\odot 7$ \\
\hline 17 & 235.276 & 275.208 & 1.0000 & $0.5925 \mathrm{E}+07$ \\
\hline 18 & 239.808 & 277.121 & 1.0000 & $\odot .6079 \mathrm{E}+07$ \\
\hline 19 & 239.808 & 278.231 & $1.000 \odot$ & $\odot .6132 \mathrm{E}+07$ \\
\hline 20 & 239.808 & 279.565 & 1.0000 & $\odot .6132 \mathrm{E}+07$ \\
\hline 21 & 239.808 & 280.195 & 1.0000 & $\odot .6140 \mathrm{E}+07$ \\
\hline 22 & 239.808 & 281.785 & 1.0000 & $\odot .6151 \mathrm{E}+07$ \\
\hline 23 & 239.808 & 283.312 & 1.0000 & $\odot .6151 \mathrm{E}+07$ \\
\hline 24 & 239.808 & 284.040 & 1.0000 & $\odot .6151 \mathrm{E}+07$ \\
\hline 25 & 239.808 & 284.042 & 1.0000 & $\odot .6151 \mathrm{E}+07$ \\
\hline
\end{tabular}




\begin{tabular}{|c|c|c|c|c|}
\hline time & $(\sec )=$ & & & \\
\hline node & $\mathrm{T}(\mathrm{K})$ & Twall(K) & $\mathrm{X}$ & $\mathrm{P}(\mathrm{Pa})$ \\
\hline 1 & 129.564 & 175.804 & - - - - - & $0.5805 \mathrm{E}+06$ \\
\hline 2 & 150.294 & 195.225 & 0.9660 & $\odot .1071 \mathrm{E}+07$ \\
\hline 3 & 167.356 & 209.226 & 0.9929 & $\odot .2133 E+07$ \\
\hline 4 & 173.904 & 216.474 & 0.9979 & $\odot .2707 \mathrm{E}+07$ \\
\hline 5 & 178.492 & 223.629 & 0.9994 & $\odot .3140 \mathrm{E}+\odot 7$ \\
\hline 6 & 185.762 & 230.027 & 1.0000 & $\odot .3522 \mathrm{E}+07$ \\
\hline 7 & 185.193 & 234.431 & 0.9978 & $\odot .3898 \mathrm{E}+07$ \\
\hline 8 & 198.658 & 242.581 & $1.000 \odot$ & $\odot .4169 \mathrm{E}+\odot 7$ \\
\hline 9 & 207.073 & 247.857 & 1.0000 & $0.4565 \mathrm{E}+07$ \\
\hline 10 & 207.073 & 254.391 & 1.0000 & $\odot .4629 \mathrm{E}+\odot 7$ \\
\hline 11 & 222.564 & 258.630 & $1.000 \odot$ & $\odot .5095 \mathrm{E}+07$ \\
\hline 12 & 222.564 & 262.189 & 1.0000 & $\odot .5276 \mathrm{E}+07$ \\
\hline 13 & 228.930 & 265.057 & $1.000 \odot$ & $\odot .5490 \mathrm{E}+07$ \\
\hline 14 & 229.975 & 268.333 & 1.0000 & $\odot .5660 \mathrm{E}+07$ \\
\hline 15 & 229.975 & 270.073 & 1.0000 & $\odot .5669 \mathrm{E}+\odot 7$ \\
\hline 16 & 235.282 & 272.152 & 1.0000 & $\odot .5854 \mathrm{E}+\odot 7$ \\
\hline 17 & 235.282 & 274.045 & $1.000 \odot$ & $\odot .5924 \mathrm{E}+07$ \\
\hline 18 & 235.282 & 276.206 & 1.0000 & $\odot .5924 \mathrm{E}+\Theta 7$ \\
\hline 19 & 236.758 & 277.316 & $1.000 \odot$ & $\odot .5997 \mathrm{E}+07$ \\
\hline 20 & 236.797 & 278.650 & 1.0000 & $\odot .6022 \mathrm{E}+\odot 7$ \\
\hline 21 & 236.797 & 279.280 & 1.0000 & $\odot .6 \odot 28 \mathrm{E}+\odot 7$ \\
\hline 22 & 236.916 & 281.174 & 1.0000 & $\odot .6 \odot 42 \mathrm{E}+07$ \\
\hline 23 & 236.916 & 282.702 & 1.0000 & $0.6044 \mathrm{E}+07$ \\
\hline 24 & 236.916 & 283.430 & $1.000 \odot$ & $0.6044 \mathrm{E}+07$ \\
\hline 25 & 236.916 & 283.431 & $1 . \odot \odot \odot \odot$ & $\odot .6 \odot 44 \mathrm{E}+\odot 7$ \\
\hline time & $(\sec )=$ & $24.00 \quad 10$ & & 22333485 \\
\hline node & $\mathrm{T}(\mathrm{K})$ & Twall(K) & $\mathrm{x}$ & $\mathrm{P}(\mathrm{Pa})$ \\
\hline 1 & 129.381 & 173.895 & - - - - - & $\odot .5736 \mathrm{E}+06$ \\
\hline 2 & 150.082 & 193.394 & 0.9660 & $\odot .1058 \mathrm{E}+07$ \\
\hline 3 & 167.222 & 207.395 & 0.9929 & $\odot .2121 \mathrm{E}+07$ \\
\hline 4 & 173.556 & 214.577 & 0.9979 & $\odot .2676 \mathrm{E}+\odot 7$ \\
\hline 5 & 178.238 & 221.673 & 0.9998 & $\odot .3112 \mathrm{E}+07$ \\
\hline 6 & 181.075 & 227.870 & 0.9996 & $\odot .3433 \mathrm{E}+07$ \\
\hline 7 & 184.480 & 232.229 & 0.9982 & $\odot .3818 \mathrm{E}+07$ \\
\hline 8 & 185.653 & 240.243 & 1.0000 & $\odot .3896 \mathrm{E}+\odot 7$ \\
\hline 9 & 208.982 & 246.412 & $1.000 \odot$ & $\odot .4416 \mathrm{E}+07$ \\
\hline 10 & 208.982 & 252.643 & 1.0000 & $\odot .4672 \mathrm{E}+07$ \\
\hline 11 & 220.977 & 257.409 & 1.0000 & $\odot .5 \odot 45 \mathrm{E}+\odot 7$ \\
\hline 12 & 223.357 & 260.815 & 1.0000 & $\odot .5274 \mathrm{E}+07$ \\
\hline 13 & 223.357 & 263.660 & 1.0000 & $\odot .5336 \mathrm{E}+07$ \\
\hline 14 & 230.211 & 267.113 & $1.00 \odot \odot$ & $\odot .5554 \mathrm{E}+\odot 7$ \\
\hline 15 & 230.287 & 268.853 & 1.0000 & $\odot .5677 \mathrm{E}+\odot 7$ \\
\hline 16 & 230.287 & 271.102 & 1.0000 & $\odot .5695 \mathrm{E}+07$ \\
\hline 17 & 234.828 & 273.130 & 1.0000 & $\odot .5857 \mathrm{E}+07$ \\
\hline 18 & 234.828 & 275.290 & 1.0000 & $\odot .5908 \mathrm{E}+07$ \\
\hline 19 & 234.828 & 276.400 & 1.0000 & $\odot .5908 \mathrm{E}+07$ \\
\hline 20 & 234.828 & 277.734 & 1.0000 & $\odot .5928 \mathrm{E}+\Theta 7$ \\
\hline 21 & 234.828 & 278.460 & $1.000 \odot$ & $\odot .5953 \mathrm{E}+07$ \\
\hline 22 & 234.828 & 280.564 & 1.0000 & $\odot .5953 \mathrm{E}+07$ \\
\hline 23 & 234.828 & 282.091 & $1.000 \odot$ & $\odot .5961 \mathrm{E}+07$ \\
\hline 24 & 234.828 & 282.819 & $1.000 \odot$ & $\odot .5963 \mathrm{E}+07$ \\
\hline 25 & 234.828 & 282.821 & $1.000 \odot$ & $\odot .5963 \mathrm{E}+07$ \\
\hline
\end{tabular}




\begin{tabular}{|c|c|c|c|c|}
\hline \multicolumn{5}{|c|}{ lambda $=0.1807483137$} \\
\hline node & $T^{\prime}(K)$ & Twall(K) & $\mathrm{x}$ & $\mathrm{P}(\mathrm{Pa})$ \\
\hline 1 & 129.249 & 172.064 & $-\ldots$ & $\odot .5687 \mathrm{E}+\odot 6$ \\
\hline 2 & 149.929 & 191.662 & $\odot .9657$ & $\odot .1049 \mathrm{E}+\odot 7$ \\
\hline 3 & 166.983 & 205.629 & $\odot .9929$ & $\odot .210 \odot E+\odot 7$ \\
\hline 4 & 173.268 & 212.746 & $\odot .9979$ & $\odot .2651 \mathrm{E}+\odot 7$ \\
\hline 5 & 177.842 & 219.842 & $\odot .9999$ & $\odot .3067 \mathrm{E}+\odot 7$ \\
\hline 6 & 181.075 & 225.788 & $\odot .9986$ & $\odot .3433 \mathrm{E}+\odot 7$ \\
\hline 7 & 182.911 & 230.398 & 0.9988 & $\odot .3640 \mathrm{E}+\odot 7$ \\
\hline 8 & 190.625 & 238.214 & 1.0000 & $0.4001 \mathrm{E}+07$ \\
\hline 9 & 209.678 & 245.023 & $1.000 \odot$ & $\odot .440 \odot E+\odot 7$ \\
\hline 10 & 211.233 & 250.972 & 1.0000 & $\odot .4715 \mathrm{E}+\odot 7$ \\
\hline 11 & 211.233 & 255.751 & $1.000 \odot$ & $\odot .4813 E+\odot 7$ \\
\hline 12 & 223.517 & 259.595 & 1.0000 & $\odot .5146 \mathrm{E}+07$ \\
\hline 13 & 223.973 & 262.440 & 1.0000 & $\odot .5347 \mathrm{E}+\odot 7$ \\
\hline 14 & 223.973 & 265.892 & 1.0000 & $\odot .5357 \mathrm{E}+\odot 7$ \\
\hline 15 & 230.397 & 267.851 & 1.0000 & $\odot .5608 \mathrm{E}+\odot 7$ \\
\hline 16 & 230.397 & 269.913 & 1.0000 & $\odot .5697 \mathrm{E}+\odot 7$ \\
\hline 17 & 230.397 & 272.202 & 1.0000 & $\odot .5697 \mathrm{E}+\odot 7$ \\
\hline 18 & 232.336 & 274.374 & 1.0000 & $\odot .5798 \mathrm{E}+07$ \\
\hline 19 & 232.349 & 275.485 & $1.000 \odot$ & $\odot .5819 \mathrm{E}+\odot 7$ \\
\hline 20 & 232.349 & 276.8 & $1.000 \odot$ & $\odot .5828 \mathrm{E}+\odot 7$ \\
\hline 21 & 232.563 & 277.850 & 1.0000 & $\odot .5853 E+\odot 7$ \\
\hline 22 & 232.563 & 279.954 & 1.0000 & $\odot .5870 \mathrm{E}+\odot 7$ \\
\hline 23 & 232.563 & 281.481 & 1.0000 & $\odot .5872 \mathrm{E}+\odot 7$ \\
\hline 24 & 232.563 & 282.209 & 1.0000 & $\odot .5878 \mathrm{E}+\odot 7$ \\
\hline 25 & 232.563 & 282.211 & 1.0000 & $\odot .5878 \mathrm{E}+\odot 7$ \\
\hline
\end{tabular}

\begin{tabular}{|c|c|c|c|c|}
\hline \multicolumn{5}{|c|}{ lambda $=0.1792172343$} \\
\hline node & $T(K)$ & Twall(K) & $\mathrm{x}$ & $\mathrm{P}(\mathrm{Pa})$ \\
\hline 1 & 129.113 & 170.349 & $-\ldots$ & $\odot .5636 \mathrm{E}+06$ \\
\hline 2 & 149.771 & 189.984 & 0.9657 & $\odot .1040 \mathrm{E}+07$ \\
\hline 3 & 166.717 & 203.951 & $\odot .9929$ & $\odot .2077 \mathrm{E}+\odot 7$ \\
\hline 4 & 172.940 & 211.044 & $\odot .9979$ & $\odot .2622 \mathrm{E}+\odot 7$ \\
\hline 5 & 177.633 & 218.018 & $\odot .9993$ & $0.3043 \mathrm{E}+07$ \\
\hline 6 & 180.601 & 223.968 & 0.9989 & $\odot .3379 \mathrm{E}+\odot 7$ \\
\hline 7 & 182.733 & 228.432 & 0.9991 & $\odot .3620 \mathrm{E}+07$ \\
\hline 8 & 193.058 & 236.257 & 1.0000 & $\odot .4048 \mathrm{E}+07$ \\
\hline 9 & 197.045 & 243.216 & 1.0000 & $\odot .4135 \mathrm{E}+07$ \\
\hline 10 & 211.995 & 249.516 & $1.000 \odot$ & $\odot .4615 \mathrm{E}+\odot 7$ \\
\hline 11 & 212.362 & 254.062 & 1.0000 & $\odot .4837 \mathrm{E}+07$ \\
\hline 12 & 212.362 & 258.139 & 1.0000 & $\odot .4851 \mathrm{E}+07$ \\
\hline 13 & 224.379 & 261.219 & 1.0000 & $\odot .5237 \mathrm{E}+07$ \\
\hline 14 & 224.388 & 264.671 & $1.000 \odot$ & $\odot .5367 \mathrm{E}+\odot 7$ \\
\hline 15 & 224.388 & 266.755 & 1.0000 & $\odot .5421 \mathrm{E}+07$ \\
\hline 16 & 230.101 & 268.997 & 1.0000 & $\odot .5623 \mathrm{E}+\odot 7$ \\
\hline 17 & 230.101 & 271.199 & 1.0000 & $0.5686 \mathrm{E}+07$ \\
\hline 18 & 230.101 & 273.459 & 1.0000 & $\odot .5686 \mathrm{E}+07$ \\
\hline 19 & 230.470 & 274.569 & $1.000 \odot$ & $\odot .5734 \mathrm{E}+07$ \\
\hline 20 & 230.475 & 275.903 & 1.0000 & $\odot .5762 \mathrm{E}+\odot 7$ \\
\hline 21 & 230.475 & 277.191 & 1.0000 & $\odot .5762 \mathrm{E}+\odot 7$ \\
\hline 22 & 230.475 & 279.343 & 1.0000 & $\odot .5783 \mathrm{E}+\odot 7$ \\
\hline 23 & 230.475 & 280.871 & 1.0000 & $\odot .5795 \mathrm{E}+07$ \\
\hline 24 & 230.475 & 281.599 & 1.0000 & $\odot .5795 \mathrm{E}+07$ \\
\hline 25 & 230.475 & 281.600 & 1.0000 & $\odot .5795 \mathrm{E}+\odot 7$ \\
\hline
\end{tabular}




\begin{tabular}{|c|c|c|c|c|}
\hline \multicolumn{5}{|c|}{ lambda $=0.1775712520$} \\
\hline node & $T(K)$ & Twall(K) & $\mathrm{x}$ & $P(P a)$ \\
\hline 1 & 128.967 & 168.671 & - - - & $\odot .5581 E+\odot 6$ \\
\hline 2 & 149.602 & 188.428 & $\odot .9657$ & $\odot .1029 \mathrm{E}+\odot 7$ \\
\hline 3 & 166.471 & 202.334 & $\odot .9929$ & $\odot .2055 \mathrm{E}+\odot 7$ \\
\hline 4 & 172.645 & 209.366 & $\odot .9975$ & $\odot .2596 \mathrm{E}+\odot 7$ \\
\hline 5 & 177.326 & 216.339 & $\odot .9988$ & $\odot .3009 \mathrm{E}+\odot 7$ \\
\hline 6 & 179.993 & 222.290 & $\odot .9992$ & $\odot .3310 \mathrm{E}+\odot 7$ \\
\hline 7 & 182.732 & 226.566 & $\odot .9988$ & $\odot .3620 \mathrm{E}+\odot 7$ \\
\hline 8 & 194.353 & 234.627 & 1.0000 & $\odot .3979 \mathrm{E}+\odot 7$ \\
\hline 9 & 198.128 & 241.394 & 1.0000 & $\odot .4158 \mathrm{E}+\odot 7$ \\
\hline 10 & 200.837 & 247.849 & 1.0000 & $\odot .4351 \mathrm{E}+\odot 7$ \\
\hline 11 & 212.879 & 252.645 & 1.0000 & $\odot .4746 \mathrm{E}+\odot 7$ \\
\hline 12 & 213.173 & 256.591 & $1.000 \odot$ & $\odot .4869 \mathrm{E}+\odot 7$ \\
\hline 13 & 219.724 & 259.998 & 1.0000 & $\odot .5083 \mathrm{E}+\odot 7$ \\
\hline 14 & 224.669 & 263.451 & 1.0000 & $\odot .5327 \mathrm{E}+07$ \\
\hline 15 & 224.669 & 265.534 & 1.0000 & $\odot .5427 \mathrm{E}+\odot 7$ \\
\hline 16 & 224.669 & 267.989 & 1.0000 & $\odot .5427 \mathrm{E}+\odot 7$ \\
\hline 17 & 228.889 & 270.283 & 1.0000 & $\odot .5598 \mathrm{E}+\odot 7$ \\
\hline 18 & 228.889 & 272.543 & $1.000 \odot$ & $\odot .5644 \mathrm{E}+\odot 7$ \\
\hline 19 & 228.889 & 273.654 & 1.0000 & $\odot .5650 \mathrm{E}+\odot 7$ \\
\hline 20 & 228.908 & 274.988 & 1.0000 & $\odot .5682 \mathrm{E}+\odot 7$ \\
\hline 21 & 228.9 & 276.337 & 1.0000 & $\odot .5706 \mathrm{E}+07$ \\
\hline 22 & 228.908 & 278.733 & 1.0000 & $\odot .5708 \mathrm{E}+07$ \\
\hline 23 & 228.908 & 280.260 & 1.0000 & $\odot .5724 \mathrm{E}+\odot 7$ \\
\hline 24 & 228.908 & 280.988 & 1.0000 & $\odot .5728 \mathrm{E}+\odot 7$ \\
\hline 25 & 228.908 & 280.990 & 1.0000 & $\odot .5728 \mathrm{E}+07$ \\
\hline
\end{tabular}

\begin{tabular}{|c|c|c|c|c|}
\hline time & $(\sec )=$ & 3.00 & $a=0$ & 8563221 \\
\hline node & $\mathrm{T}(\mathrm{K})$ & Twall(K) & $x$ & $\mathrm{P}(\mathrm{Pa})$ \\
\hline 1 & 128.815 & 167.120 & $-\ldots$ & $\odot .5524 \mathrm{E}+06$ \\
\hline 2 & 149.425 & 186.902 & 0.9657 & $\odot .1019 \mathrm{E}+07$ \\
\hline 3 & 166.234 & 200.808 & $\odot .9929$ & $\odot .2034 \mathrm{E}+07$ \\
\hline 4 & 172.307 & 207.811 & ๑. 9971 & $\odot .2567 \mathrm{E}+\odot 7$ \\
\hline 5 & 176.752 & 214.765 & $\odot .9988$ & $\odot .2956 \mathrm{E}+07$ \\
\hline 6 & 179.891 & 220.611 & 0.9992 & $\odot .3299 \mathrm{E}+\Theta 7$ \\
\hline 7 & 182.733 & 224.734 & 0.9984 & $0.3620 \mathrm{E}+07$ \\
\hline 8 & 192.868 & 233.101 & 1.0000 & $0.3821 \mathrm{E}+07$ \\
\hline 9 & 199.810 & 239.796 & 1.0000 & $0.4173 \mathrm{E}+07$ \\
\hline 10 & 199.810 & 245.940 & $1.000 \odot$ & $\odot .4329 E+\odot 7$ \\
\hline 11 & 212.382 & 251.368 & 1.0000 & $\odot .4638 \mathrm{E}+07$ \\
\hline 12 & 214.211 & 255.159 & 1.0000 & $\odot .4884 \mathrm{E}+07$ \\
\hline 13 & 214.552 & 258.531 & 1.0000 & $\odot .4963 \mathrm{E}+07$ \\
\hline 14 & 217.600 & 262.190 & $1.00 \odot \odot$ & $\odot .5102 \mathrm{E}+07$ \\
\hline 15 & 224.690 & 264.475 & 1.0000 & $\odot .5355 \mathrm{E}+07$ \\
\hline 16 & 224.690 & 266.769 & 1.0000 & $\odot .5427 \mathrm{E}+\odot 7$ \\
\hline 17 & 224.690 & 269.337 & 1.0000 & $\odot .5427 \mathrm{E}+\Theta 7$ \\
\hline 18 & 227.429 & 271.628 & 1.0000 & $\odot .5561 \mathrm{E}+07$ \\
\hline 19 & 227.429 & 272.738 & $1.000 \odot$ & $\odot .5598 \mathrm{E}+\odot 7$ \\
\hline 20 & 227.429 & 274.072 & 1.0000 & $\odot .5605 \mathrm{E}+\odot 7$ \\
\hline 21 & 227.429 & 275.727 & 1.0000 & $0.5638 \mathrm{E}+07$ \\
\hline 22 & 227.429 & 278.123 & 1.0000 & $\odot .5650 \mathrm{E}+\odot 7$ \\
\hline 23 & 227.429 & 279.650 & 1.0000 & $\odot .5650 \mathrm{E}+07$ \\
\hline 24 & 227.429 & 280.378 & 1.0000 & $\odot .5662 \mathrm{E}+\Theta 7$ \\
\hline 25 & 227.429 & 280.380 & 1.0000 & $\odot .5662 \mathrm{E}+\odot 7$ \\
\hline
\end{tabular}




\begin{tabular}{|c|c|c|c|c|}
\hline \multicolumn{5}{|c|}{$.00 \quad$ lambda $=0.1741027832$} \\
\hline node & $T^{\prime}(K)$ & Twall(K) & $x$ & $P(P a)$ \\
\hline 1 & 128.659 & 165.595 & $----_{-}$ & $\odot .5465 \mathrm{E}+06$ \\
\hline 2 & 149.245 & 185.479 & $\odot .9657$ & $\odot .1008 \mathrm{E}+\odot 7$ \\
\hline 3 & 165.978 & 199.308 & $\odot .9929$ & $\odot .2012 \mathrm{E}+\odot 7$ \\
\hline 4 & 171.890 & 206.285 & $\odot .9971$ & $\odot .2530 \mathrm{E}+\odot 7$ \\
\hline 5 & 176.487 & 213.239 & $\odot .9988$ & $\odot .2933 \mathrm{E}+07$ \\
\hline 6 & 179.870 & 218.933 & ๑. 9988 & $\odot .3296 \mathrm{E}+\odot 7$ \\
\hline 7 & 182.211 & 223.079 & $\odot .9988$ & $\odot .3561 E+\odot 7$ \\
\hline 8 & 187.765 & 231.366 & 1.0000 & $\odot .3717 \mathrm{E}+07$ \\
\hline 9 & 200.684 & 238.326 & 1.0000 & $\odot .4109 \mathrm{E}+07$ \\
\hline 10 & 201.451 & 244.171 & 1.0000 & $\odot .4362 \mathrm{E}+\odot 7$ \\
\hline 11 & 202.737 & 249.710 & $1.000 \odot$ & $\odot .4418 \mathrm{E}+\odot 7$ \\
\hline 12 & 213.374 & 253.786 & 1.0000 & $\odot .4772 \mathrm{E}+\Theta 7$ \\
\hline 13 & 215.217 & 257.124 & $1.000 \odot$ & $\odot .4969 \mathrm{E}+\odot 7$ \\
\hline 14 & 215.217 & 260.664 & $1.000 \odot$ & $\odot .5 \odot 46 \mathrm{E}+\odot 7$ \\
\hline 15 & 218.658 & 263.385 & 1.0000 & $\odot .5127 \mathrm{E}+\odot 7$ \\
\hline 16 & 224.631 & 265.852 & $1.000 \odot$ & $\odot .5364 \mathrm{E}+07$ \\
\hline 17 & 224.631 & 268.296 & 1.0000 & $\odot .5424 \mathrm{E}+\odot 7$ \\
\hline 18 & 224.631 & 270.712 & 1.0000 & $\odot .5430 \mathrm{E}+07$ \\
\hline 19 & 226.046 & 271.823 & 1.0000 & $\odot .5522 \mathrm{E}+\odot 7$ \\
\hline 20 & 226.046 & 273.157 & $1.000 \odot$ & $\odot .5556 \mathrm{E}+\odot 7$ \\
\hline 21 & 226.046 & 275.117 & 1.0000 & $\odot .5560 \mathrm{E}+\odot 7$ \\
\hline 22 & 226.046 & 277.512 & 1.0000 & $\odot .5588 \mathrm{E}+\odot 7$ \\
\hline 23 & 226.046 & 279.039 & 1.0000 & $\odot .5593 E+\odot 7$ \\
\hline 24 & 226.046 & 279.768 & 1.0000 & $\odot .5593 \mathrm{E}+\odot 7$ \\
\hline 25 & 226.046 & 279.769 & 1.0000 & $\odot .5593 \mathrm{E}+07$ \\
\hline
\end{tabular}

\begin{tabular}{|c|c|c|c|c|}
\hline \multicolumn{5}{|c|}{ lambda $=0.1722757071$} \\
\hline node & $T^{\prime}(K)$ & Twall(K) & $x$ & $\mathrm{P}(\mathrm{Pa})$ \\
\hline 1 & 128.466 & 164.178 & - - - - & $0.5404 \mathrm{E}+0$ \\
\hline 2 & 149.020 & 184.105 & 0.9657 & $\odot .9967 \mathrm{E}+\odot 6$ \\
\hline 3 & 165.601 & 197.934 & $\odot .9929$ & $\odot .1986 \mathrm{E}+\odot 7$ \\
\hline 4 & 171.610 & 204.866 & 0.9971 & $\odot .2506 \mathrm{E}+\odot 7$ \\
\hline 5 & 176.406 & 211.713 & $\odot .9983$ & $\odot .2926 \mathrm{E}+\odot 7$ \\
\hline 6 & 179.458 & 217.370 & 0.9985 & $\odot .3250 \mathrm{E}+\odot 7$ \\
\hline 7 & 181.475 & 221.553 & $\odot .9992$ & $\odot .3478 \mathrm{E}+\odot$ \\
\hline 8 & 191.102 & 229.803 & 1.0000 & $\odot .3779 E+\odot$ \\
\hline 9 & 191.102 & 236.647 & $1.000 \odot$ & $\odot .3909 \mathrm{E}+\odot$ \\
\hline 10 & 202.316 & 242.683 & 1.0000 & $0.4277 \mathrm{E}+\mathrm{C}$ \\
\hline 11 & 203.334 & 247.996 & 1.0000 & $\odot .4431 \mathrm{E}+07$ \\
\hline 12 & 206.774 & 252.260 & $1.000 \odot$ & $\odot .4614 \mathrm{E}+\odot 7$ \\
\hline 13 & 211.981 & 255.865 & 1.0000 & $\odot .4831 \mathrm{E}+\odot$ \\
\hline 14 & 215.367 & 259.338 & $1.0 \odot \odot \odot$ & $\odot .5018 \mathrm{E}+\mathrm{C}$ \\
\hline 15 & 215.391 & 261.970 & $1.000 \odot$ & $\odot .5050 E+\odot$ \\
\hline 16 & 219.378 & 264.822 & $1.00 \odot \odot$ & $0.5155 \mathrm{E}+\mathrm{C}$ \\
\hline 17 & 224.441 & 267.380 & 1.0000 & $0.5366 \mathrm{E}+07$ \\
\hline 18 & 224.441 & 269.797 & $1.000 \odot$ & $0.5423 E+07$ \\
\hline 19 & 224.441 & 270.9 & 1.0000 & $0.5425 \mathrm{E}+\mathrm{C}$ \\
\hline 20 & 224.441 & 272.241 & $1.00 \odot \odot$ & $\odot .5479 \mathrm{E}+\odot$ \\
\hline 21 & 224.441 & 274.248 & $1.000 \odot$ & $\odot .5500 \mathrm{E}+\odot$ \\
\hline 22 & 224.441 & $276.9 \odot 2$ & $1.00 \odot \odot$ & $\odot .5507 \mathrm{E}+\odot$ \\
\hline 23 & 224.441 & 278.429 & 1.0000 & $\odot .5526 \mathrm{E}+07$ \\
\hline 24 & 224.441 & 279.157 & 1.0000 & $\odot .5526 \mathrm{E}+\odot 7$ \\
\hline & 224.441 & 279.159 & 1.0000 & $\odot .5526 \mathrm{E}+\odot$ \\
\hline
\end{tabular}

lambda_avg $=0.248135760$ 
Compressor station distance $=5$ miles, Pressure $=1167$ psi, 50 axial node elements

\begin{tabular}{|c|c|c|c|c|}
\hline \multicolumn{5}{|c|}{$.0 \odot \quad$ lambda $=0.4904261529$} \\
\hline node & $\mathrm{T}(\mathrm{K})$ & Twall(K) & $x$ & $P(P a)$ \\
\hline 1 & 153.583 & 277.616 & - _ - _ n & $\odot .1682 \mathrm{E}+07$ \\
\hline 2 & 178.156 & 280.928 & $\odot .9580$ & $\odot .3102 \mathrm{E}+07$ \\
\hline 3 & 179.025 & 286.150 & $\odot .9976$ & $0.3201 \mathrm{E}+07$ \\
\hline 4 & 266.427 & 287.932 & 1.0000 & $\odot .6230 \mathrm{E}+07$ \\
\hline 5 & 286.017 & $288 . \odot \odot \odot$ & $1.0 \odot \odot \odot$ & $\odot .7741 \mathrm{E}+\odot 7$ \\
\hline 6 & 287.899 & $288.0 \odot \odot$ & $1.00 \odot \odot$ & $\odot .8017 \mathrm{E}+\odot 7$ \\
\hline 7 & $288.00 \odot$ & 288.000 & $1.00 \odot \odot$ & $\odot .8 \odot 43 \mathrm{E}+\odot 7$ \\
\hline 8 & 288.000 & 288.000 & 1.0000 & $\odot .8044 \mathrm{E}+07$ \\
\hline 9 & $288.00 \odot$ & $288.0 \odot \odot$ & $1.0 \odot \odot \odot$ & $\odot .8 \odot 44 \mathrm{E}+07$ \\
\hline 10 & 288.000 & $288.0 \odot \odot$ & $1.00 \odot \odot$ & $\odot .8 \odot 44 \mathrm{E}+\odot 7$ \\
\hline 11 & $288.0 \odot \odot$ & $288.00 \odot$ & $1.00 \odot \odot$ & $\odot .8 \odot 44 \mathrm{E}+\odot 7$ \\
\hline 12 & 288.000 & $288.00 \odot$ & 1.0000 & $\odot .8044 \mathrm{E}+\odot 7$ \\
\hline 13 & $288.00 \odot$ & $288.0 \odot \odot$ & $1.00 \odot \odot$ & $\odot .8 \odot 44 \mathrm{E}+\odot 7$ \\
\hline 14 & $288.00 \odot$ & 288.000 & 1.0000 & $\odot .8 \odot 44 \mathrm{E}+\odot 7$ \\
\hline 15 & $288 . \odot \odot \odot$ & $288.0 \odot \odot$ & $1.000 \odot$ & $\odot .8 \odot 44 \mathrm{E}+\odot 7$ \\
\hline 16 & $288.00 \odot$ & 288.000 & 1.0000 & $\odot .8044 \mathrm{E}+\odot 7$ \\
\hline 17 & $288.00 \odot$ & $288.0 \odot \odot$ & $1.00 \odot \odot$ & $\odot .8 \odot 44 \mathrm{E}+\odot 7$ \\
\hline 18 & 288.000 & 288.000 & $1.000 \odot$ & $\odot .8044 \mathrm{E}+07$ \\
\hline 19 & 288.000 & $288.00 \odot$ & 1.0000 & $\odot .8044 \mathrm{E}+\odot 7$ \\
\hline 20 & $288.00 \odot$ & $288.0 \odot \odot$ & $1.00 \odot \odot$ & $\odot .8 \odot 44 \mathrm{E}+07$ \\
\hline 21 & $288.00 \odot$ & $288.0 \odot \odot$ & $1.0 \odot \odot \odot$ & $\odot .8 \odot 44 \mathrm{E}+\odot 7$ \\
\hline 22 & $288.0 \odot \odot$ & $288.0 \odot \odot$ & $1.0 \odot \odot \odot$ & $\odot .8 \odot 44 \mathrm{E}+\odot 7$ \\
\hline 23 & 288.000 & 288.000 & $1.000 \odot$ & $\odot .8044 \mathrm{E}+\odot 7$ \\
\hline 24 & 288.000 & 288.000 & 1.0000 & $\odot .8044 \mathrm{E}+07$ \\
\hline 25 & 288.000 & 288.000 & $1.000 \odot$ & $\odot .8044 \mathrm{E}+\odot 7$ \\
\hline 26 & 288.000 & $288.0 \odot \odot$ & $1.000 \odot$ & $\odot .8044 \mathrm{E}+\odot 7$ \\
\hline 27 & $288.00 \odot$ & $288.00 \odot$ & $1.0 \odot \odot \odot$ & $\odot .8 \odot 44 \mathrm{E}+\odot 7$ \\
\hline 28 & $288.00 \odot$ & $288.0 \odot \odot$ & $1.00 \odot \odot$ & $\odot .8 \odot 44 \mathrm{E}+\odot 7$ \\
\hline 29 & 288.000 & $288.0 \odot \odot$ & 1.0000 & $\odot .8044 \mathrm{E}+\odot 7$ \\
\hline 30 & 288.000 & 288.000 & 1.0000 & $\odot .8044 \mathrm{E}+07$ \\
\hline 31 & 288.000 & $288.0 \odot \odot$ & $1.000 \odot$ & $\odot .8044 \mathrm{E}+07$ \\
\hline 32 & 288.000 & 288.000 & 1.0000 & $\odot .8044 \mathrm{E}+07$ \\
\hline 33 & $288.00 \odot$ & $288.0 \odot \odot$ & $1.00 \odot \odot$ & $\odot .8 \odot 44 \mathrm{E}+07$ \\
\hline 34 & $288.00 \odot$ & $288.00 \odot$ & $1.0 \odot \odot \odot$ & $\odot .8 \odot 44 \mathrm{E}+\odot 7$ \\
\hline 35 & 288.000 & 288.000 & 1.0000 & $\odot .8044 \mathrm{E}+07$ \\
\hline 36 & 288.000 & 288.000 & 1.0000 & $\odot .8044 \mathrm{E}+07$ \\
\hline 37 & 288.000 & 288.000 & $1.00 \odot \odot$ & $\odot .8 \odot 44 \mathrm{E}+\odot 7$ \\
\hline 38 & $288.00 \odot$ & $288.00 \odot$ & $1.0 \odot \odot \odot$ & $\odot .8 \odot 44 \mathrm{E}+\odot 7$ \\
\hline 39 & $288.00 \odot$ & 288.000 & $1.000 \odot$ & $\odot .8044 \mathrm{E}+\odot 7$ \\
\hline 40 & $288.00 \odot$ & $288.0 \odot \odot$ & $1.000 \odot$ & $\odot .8 \odot 44 \mathrm{E}+\odot 7$ \\
\hline 41 & 288.000 & 288.000 & 1.0000 & $\odot .8 \odot 44 \mathrm{E}+\odot 7$ \\
\hline 42 & 288.000 & $288.0 \odot \odot$ & $1.000 \odot$ & $\odot .8044 \mathrm{E}+\odot 7$ \\
\hline 43 & 288.000 & $288.0 \odot \odot$ & $1.00 \odot \odot$ & $\odot .8044 \mathrm{E}+07$ \\
\hline 44 & $288.00 \odot$ & $288.00 \odot$ & $1.0 \odot \odot \odot$ & $\odot .8 \odot 44 \mathrm{E}+\odot 7$ \\
\hline 45 & 288.000 & $288.00 \odot$ & 1.0000 & $\odot .8044 \mathrm{E}+07$ \\
\hline 46 & 288.000 & 288.000 & $1.000 \odot$ & $\odot .8044 \mathrm{E}+\odot 7$ \\
\hline 47 & 288.000 & 288.000 & 1.0000 & $\odot .8044 \mathrm{E}+07$ \\
\hline 48 & 288.000 & 288.000 & 1.0000 & $\odot .8044 \mathrm{E}+07$ \\
\hline 49 & 288.000 & $288.0 \odot \odot$ & 1.0000 & $\odot .8 \odot 44 \mathrm{E}+07$ \\
\hline 50 & $288.00 \odot$ & $288.0 \odot \odot$ & $1.0 \odot \odot \odot$ & $\odot .8044 \mathrm{E}+07$ \\
\hline
\end{tabular}




\begin{tabular}{|c|c|c|c|c|}
\hline \multicolumn{5}{|c|}{ lambda $=0.3496202528$} \\
\hline node & $\mathrm{T}(\mathrm{K})$ & Twall(K) & ) $\quad x$ & $\mathrm{P}(\mathrm{Pa})$ \\
\hline 1 & 144.387 & 266.586 & - . - . & $\odot .1163 \mathrm{E}+\odot 7$ \\
\hline 2 & 167.488 & 273.275 & $\odot .9798$ & $\odot .2144 \mathrm{E}+\odot 7$ \\
\hline 3 & 179.005 & 277.687 & $\odot .9908$ & $\odot .3199 \mathrm{E}+\odot 7$ \\
\hline 4 & 184.892 & 280.944 & $\odot .9929$ & $\odot .3865 \mathrm{E}+\odot 7$ \\
\hline 5 & 185.680 & 284.347 & $\odot .9961$ & $\odot .3954 \mathrm{E}+\odot 7$ \\
\hline 6 & 219.462 & 287.221 & 1.0000 & $\odot .4905 \mathrm{E}+\odot 7$ \\
\hline 7 & 271.307 & 287.956 & 1.0000 & $\odot .6807 \mathrm{E}+07$ \\
\hline 8 & 285.583 & 288.000 & 1.0000 & $\odot .7776 \mathrm{E}+\odot 7$ \\
\hline 9 & 287.793 & 288.000 & 1.0000 & $\odot .8008 \mathrm{E}+07$ \\
\hline 10 & 288.000 & 288.000 & 1.0000 & $\odot .8041 \mathrm{E}+\odot 7$ \\
\hline 11 & 288.000 & $288.00 \odot$ & $1.000 \odot$ & $\odot .8044 \mathrm{E}+\odot 7$ \\
\hline 12 & 288.000 & 288.000 & 1.0000 & $\odot .8044 \mathrm{E}+07$ \\
\hline 13 & 288.000 & 288.000 & 1.0000 & $\odot .8044 \mathrm{E}+07$ \\
\hline 14 & 288.000 & 288.000 & 1.0000 & $\odot .8044 \mathrm{E}+07$ \\
\hline 15 & 288.000 & $288.00 \odot$ & $1.000 \odot$ & $\odot .8044 \mathrm{E}+\odot 7$ \\
\hline 16 & 288.000 & 288.000 & 1.0000 & $\odot .8044 \mathrm{E}+07$ \\
\hline 17 & 288.000 & 288.000 & 1.0000 & $\odot .8044 \mathrm{E}+\odot 7$ \\
\hline 18 & 288.000 & $288.00 \odot$ & 1.0000 & $\odot .8044 \mathrm{E}+07$ \\
\hline 19 & 288.000 & 288.000 & 1.0000 & $\odot .8044 \mathrm{E}+\odot 7$ \\
\hline 20 & $288.00 \odot$ & $288.00 \odot$ & 1.0000 & $\odot .8044 \mathrm{E}+07$ \\
\hline 21 & 288.000 & 288.000 & 1.0000 & $\odot .8044 \mathrm{E}+07$ \\
\hline 22 & 288.000 & 288.000 & 1.0000 & $\odot .8044 \mathrm{E}+\odot 7$ \\
\hline 23 & 288.000 & $288.00 \odot$ & 1.0000 & $\odot .8044 \mathrm{E}+07$ \\
\hline 24 & 288.000 & $288.00 \odot$ & 1.0000 & $\odot .8044 \mathrm{E}+\odot 7$ \\
\hline 25 & 288.000 & $288.00 \odot$ & 1.0000 & $\odot .8044 \mathrm{E}+07$ \\
\hline 26 & $288.00 \odot$ & 288.000 & 1.0000 & $\odot .8044 \mathrm{E}+\odot 7$ \\
\hline 27 & 288.000 & $288.00 \odot$ & 1.0000 & $\odot .8044 \mathrm{E}+\odot 7$ \\
\hline 28 & $288.00 \odot$ & $288.00 \odot$ & $1.000 \odot$ & $\odot .8044 \mathrm{E}+\odot 7$ \\
\hline 29 & 288.000 & 288.000 & 1.0000 & $\odot .8044 \mathrm{E}+\odot 7$ \\
\hline 30 & 288.000 & $288.00 \odot$ & 1.0000 & $\odot .8044 \mathrm{E}+07$ \\
\hline 31 & 288.000 & 288.000 & 1.0000 & $\odot .8044 \mathrm{E}+07$ \\
\hline 32 & 288.000 & $288.00 \odot$ & 1.0000 & $\odot .8044 \mathrm{E}+07$ \\
\hline 33 & 288.000 & 288.000 & 1.0000 & $\odot .8044 \mathrm{E}+\odot 7$ \\
\hline 34 & $288 . \odot \odot \odot$ & $288.00 \odot$ & $1.000 \odot$ & $\odot .8044 \mathrm{E}+\odot 7$ \\
\hline 35 & 288.000 & 288.000 & 1.0000 & $\odot .8044 \mathrm{E}+07$ \\
\hline 36 & 288.000 & 288.000 & 1.0000 & $\odot .8044 \mathrm{E}+\odot 7$ \\
\hline 37 & $288.00 \odot$ & 288.000 & 1.0000 & $\odot .8044 \mathrm{E}+07$ \\
\hline 38 & $288 . \odot \odot \odot$ & $288.0 \odot \odot$ & $1.000 \odot$ & $\odot .8044 \mathrm{E}+\odot 7$ \\
\hline 39 & $288.00 \odot$ & $288.00 \odot$ & $1.000 \odot$ & $\odot .8 \odot 44 \mathrm{E}+\odot 7$ \\
\hline 40 & 288.000 & $288.00 \odot$ & 1.0000 & $\odot .8044 \mathrm{E}+\odot 7$ \\
\hline 41 & 288.000 & $288.00 \odot$ & 1.0000 & $\odot .8044 \mathrm{E}+07$ \\
\hline 42 & 288.000 & 288.000 & 1.0000 & $\odot .8044 \mathrm{E}+07$ \\
\hline 43 & 288.000 & $288.00 \odot$ & 1.0000 & $\odot .8044 \mathrm{E}+\odot 7$ \\
\hline 44 & $288.00 \odot$ & $288.00 \odot$ & $1.000 \odot$ & $\odot .8 \odot 44 \mathrm{E}+\odot 7$ \\
\hline 45 & $288 . \odot \odot \odot$ & $288.00 \odot$ & $1.000 \odot$ & $\odot .8044 \mathrm{E}+\odot 7$ \\
\hline 46 & 288.000 & $288.00 \odot$ & 1.0000 & $\odot .8044 \mathrm{E}+07$ \\
\hline 47 & 288.000 & $288.00 \odot$ & $1.000 \odot$ & $\odot .8 \odot 44 \mathrm{E}+\odot 7$ \\
\hline 48 & 288.000 & $288.00 \odot$ & 1.0000 & $\odot .8044 \mathrm{E}+07$ \\
\hline 49 & 288.000 & 288.000 & 1.0000 & $\odot .8044 \mathrm{E}+\odot 7$ \\
\hline 50 & 288.000 & 288.000 & 1.0000 & $\odot .8044 \mathrm{E}+\odot 7$ \\
\hline time & $(\sec )=$ & $3.00 \quad 1$ & lambda $=0$. & 17281306 \\
\hline node & $\mathrm{T}(\mathrm{K})$ & Twall(K) & $\mathrm{X}$ & $\mathrm{P}(\mathrm{Pa})$ \\
\hline 1 & 143.227 & 257.656 & $-\ldots$ & $\odot .1099 \mathrm{E}+\odot 7$ \\
\hline 2 & 166.143 & 265.765 & 0.9645 & $\odot .2027 \mathrm{E}+07$ \\
\hline
\end{tabular}




\begin{tabular}{|c|c|c|c|c|}
\hline 3 & 176.618 & 270.833 & $\odot .9868$ & $\odot .2945 E+\odot 7$ \\
\hline 4 & 181.208 & 274.798 & $\odot .9969$ & $0.3448 \mathrm{E}+07$ \\
\hline 5 & 185.680 & $277.7 \odot 2$ & $\odot .9981$ & $\odot .3954 \mathrm{E}+07$ \\
\hline 6 & 199.724 & 280.834 & $1.00 \odot \odot$ & $\odot .44 \odot 3 E+\odot 7$ \\
\hline 7 & 200.816 & 283.210 & 1. $0 \odot \odot \odot$ & $\odot .4645 E+\odot 7$ \\
\hline 8 & 201.975 & 285.578 & $1.000 \odot$ & $\odot .4742 \mathrm{E}+\odot 7$ \\
\hline 9 & 232.995 & 287.435 & 1.0000 & $\odot .5561 \mathrm{E}+07$ \\
\hline 10 & 272.530 & 287.961 & 1.0000 & $\odot .6973 \mathrm{E}+\odot 7$ \\
\hline 11 & 285.323 & $288.00 \odot$ & $1.000 \odot$ & $\odot .7782 \mathrm{E}+07$ \\
\hline 12 & 287.722 & $288 . \odot \odot \odot$ & $1.000 \odot$ & $\odot .8003 \mathrm{E}+\odot 7$ \\
\hline 13 & $288.0 \odot \odot$ & $288 . \odot \odot \odot$ & $1.00 \odot \odot$ & $\odot .8041 \mathrm{E}+07$ \\
\hline 14 & 288.000 & $288.00 \odot$ & 1.0000 & $\odot .8044 \mathrm{E}+\odot 7$ \\
\hline 15 & $288.00 \odot$ & $288.0 \odot \odot$ & 1.0000 & $0.8044 \mathrm{E}+07$ \\
\hline 16 & 288.000 & $288.00 \odot$ & 1.0000 & $\odot .8044 \mathrm{E}+07$ \\
\hline 17 & $288.00 \odot$ & $288.00 \odot$ & $1.000 \odot$ & $\odot .8044 \mathrm{E}+\odot 7$ \\
\hline 18 & $288.00 \odot$ & $288.00 \odot$ & 1.0000 & $\odot .8044 \mathrm{E}+07$ \\
\hline 19 & $288.00 \odot$ & $288 . \odot \odot \odot$ & $1.000 \odot$ & $\odot .8044 \mathrm{E}+07$ \\
\hline 20 & $288.0 \odot \odot$ & $288 . \odot \odot \odot$ & $1 . \odot \odot \odot \odot$ & $\odot .8 \odot 44 \mathrm{E}+\odot 7$ \\
\hline 21 & 288.000 & $288.00 \odot$ & 1.0000 & $\odot .8044 \mathrm{E}+\odot 7$ \\
\hline 22 & $288.00 \odot$ & $288 . \odot \odot \odot$ & $1.000 \odot$ & $\odot .8044 \mathrm{E}+07$ \\
\hline 23 & 288.000 & $288.00 \odot$ & 1.0000 & $0.8044 \mathrm{E}+07$ \\
\hline 24 & 288.000 & $288.00 \odot$ & 1.0000 & $\odot .8044 \mathrm{E}+\odot 7$ \\
\hline 25 & 288.000 & $288.00 \odot$ & $1.000 \odot$ & $\odot .8044 \mathrm{E}+07$ \\
\hline 26 & $288.0 \odot \odot$ & $288 . \odot \odot \odot$ & $1.0 \odot \odot \odot$ & $0.8044 \mathrm{E}+07$ \\
\hline 27 & $288.00 \odot$ & 288.000 & 1.0000 & $\odot .8 \odot 44 \mathrm{E}+\odot 7$ \\
\hline 28 & $288.00 \odot$ & $288.00 \odot$ & 1.0000 & $\odot .8044 \mathrm{E}+\odot 7$ \\
\hline 29 & 288.000 & 288.000 & 1.0000 & $0.8044 \mathrm{E}+07$ \\
\hline 30 & $288 . \odot \odot \odot$ & $288 . \odot \odot \odot$ & $1.00 \odot \odot$ & $\odot .8044 \mathrm{E}+\odot 7$ \\
\hline 31 & 288.000 & $288.00 \odot$ & 1.0000 & $\odot .8044 \mathrm{E}+\odot 7$ \\
\hline 32 & 288.000 & $288.00 \odot$ & $1.00 \odot \odot$ & $\odot .8044 \mathrm{E}+07$ \\
\hline 33 & $288.0 \odot \odot$ & $288 . \odot \odot \odot$ & $1 . \odot \odot \odot \odot$ & $\odot .8044 \mathrm{E}+\odot 7$ \\
\hline 34 & 288.000 & 288.000 & 1.0000 & $0.8044 \mathrm{E}+07$ \\
\hline 35 & 288.000 & 288.000 & 1.0000 & $\odot .8044 \mathrm{E}+07$ \\
\hline 36 & $288.00 \odot$ & $288 . \odot \odot \odot$ & $1 . \odot \odot \odot \odot$ & $0.8044 \mathrm{E}+07$ \\
\hline 37 & $288 . \odot \odot \odot$ & $288 . \odot \odot \odot$ & $1.0 \odot \odot \odot$ & $\odot .8 \odot 44 \mathrm{E}+\odot 7$ \\
\hline 38 & $288.00 \odot$ & $288.00 \odot$ & $1.000 \odot$ & $0.8044 \mathrm{E}+07$ \\
\hline 39 & $288 . \odot \odot \odot$ & $288 . \odot \odot \odot$ & $1.0 \odot \odot \odot$ & $\odot .8044 \mathrm{E}+\odot 7$ \\
\hline 40 & 288.000 & 288.000 & 1.0000 & $0.8044 \mathrm{E}+07$ \\
\hline 41 & 288.000 & $288.00 \odot$ & $1.000 \odot$ & $\odot .8044 \mathrm{E}+07$ \\
\hline 42 & $288.00 \odot$ & $288.0 \odot \odot$ & $1.000 \odot$ & $0.8044 \mathrm{E}+07$ \\
\hline 43 & $288.00 \odot$ & $288 . \odot \odot \odot$ & $1.0 \odot \odot \odot$ & $\odot .8044 \mathrm{E}+\odot 7$ \\
\hline 44 & 288.000 & 288.000 & 1.0000 & $\odot .8044 \mathrm{E}+07$ \\
\hline 45 & 288.000 & 288.000 & 1.0000 & $0.8044 \mathrm{E}+07$ \\
\hline 46 & $288 . c$ & 288.000 & 1.0000 & $0.8044 \mathrm{E}+07$ \\
\hline 47 & 288. & 288.000 & 1.0000 & $\odot .8044 \mathrm{E}+07$ \\
\hline 48 & $288.00 \odot$ & $288.00 \odot$ & $1.000 \odot$ & $0.8044 \mathrm{E}+07$ \\
\hline 49 & $288.00 \odot$ & $288.00 \odot$ & $1.000 \odot$ & $0.8044 \mathrm{E}+07$ \\
\hline 50 & 288.000 & 288.000 & 1.0000 & $0.8044 \mathrm{E}+07$ \\
\hline time & & \multicolumn{3}{|c|}{ lambda $=0.3022502661$} \\
\hline node & $\mathrm{T}(\mathrm{K})$ & Twall(K) & $\mathrm{x}$ & $P(P a)$ \\
\hline 1 & 140.496 & 249.425 & - . - - - & $\odot .9915 \mathrm{E}+06$ \\
\hline 2 & 162.975 & 258.994 & $\odot .9665$ & $\odot .1829 \mathrm{E}+07$ \\
\hline 3 & 173.874 & 264.583 & 0.9874 & $\odot .27 \odot 4 \mathrm{E}+07$ \\
\hline 4 & 180.556 & 268.781 & 0.9948 & $\odot .3374 \mathrm{E}+\odot 7$ \\
\hline 5 & 184.132 & 271.856 & 0.9983 & $\odot .3779 \mathrm{E}+\odot 7$ \\
\hline 6 & 189.048 & 275.461 & 1.0000 & $0.4023 \mathrm{E}+07$ \\
\hline
\end{tabular}




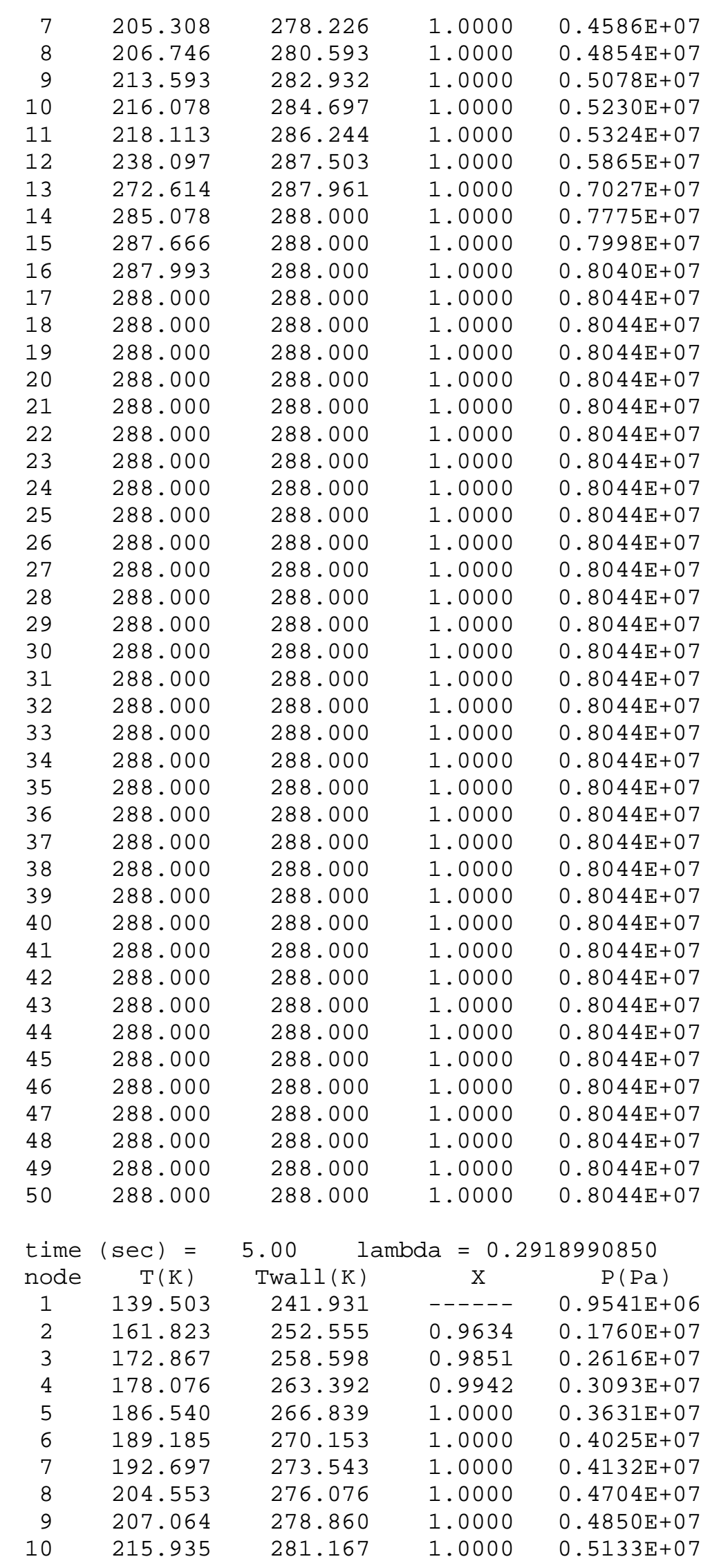




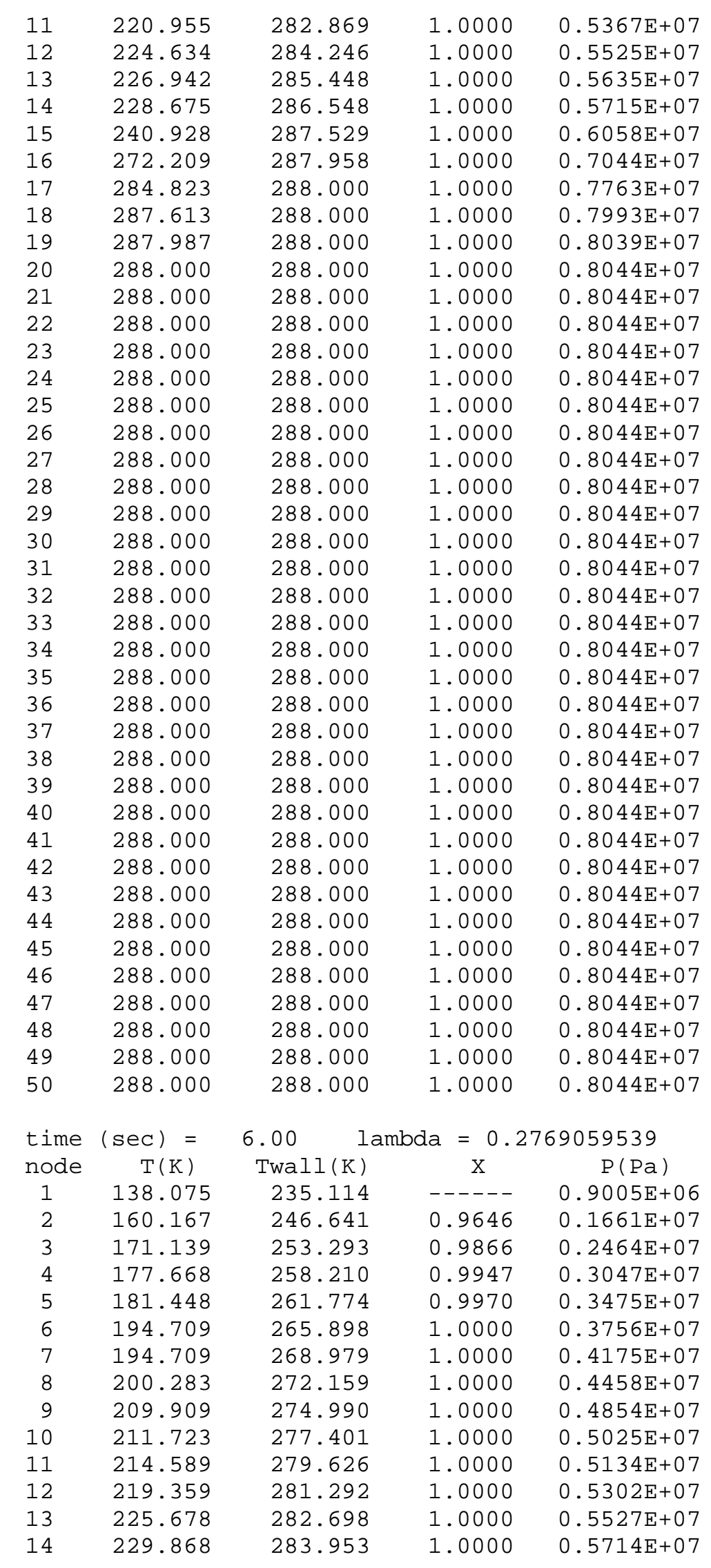




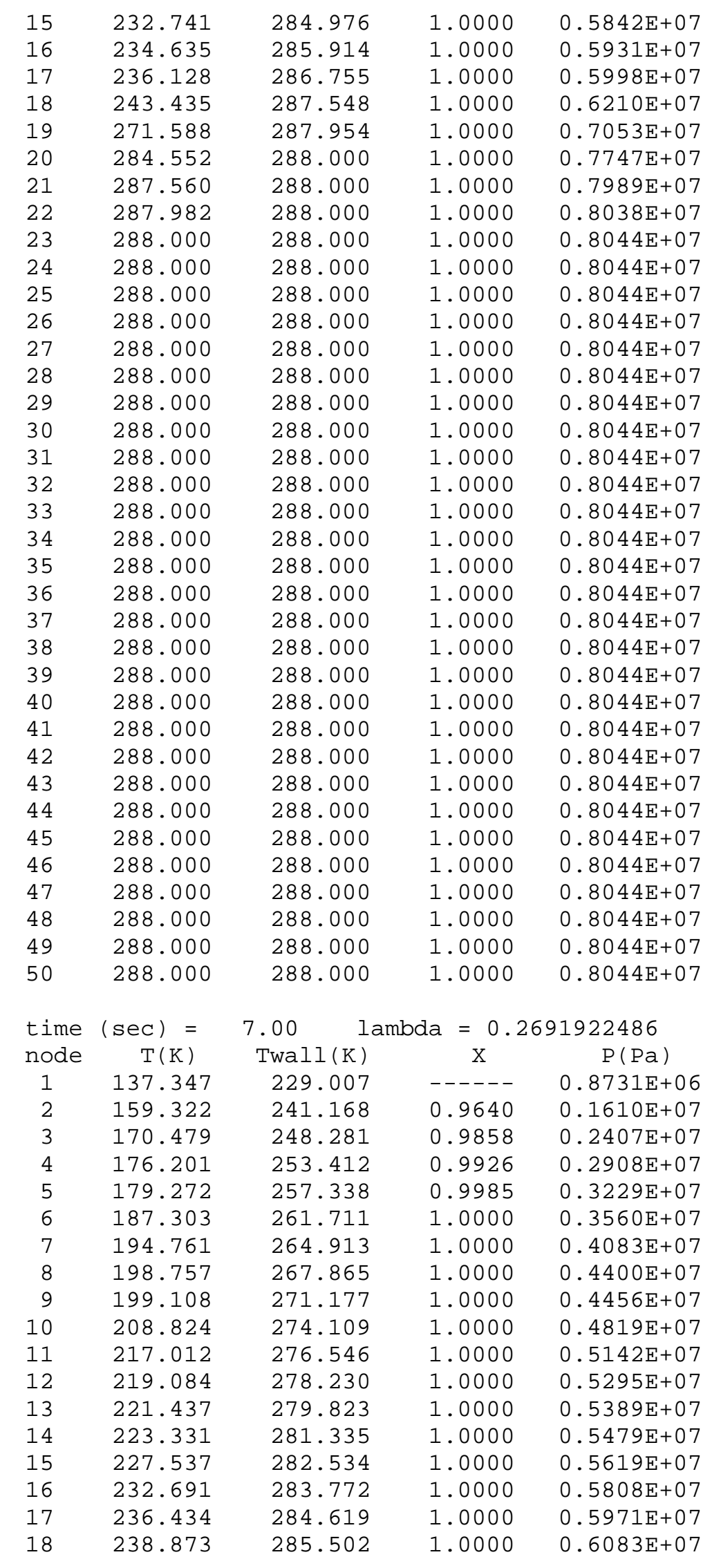




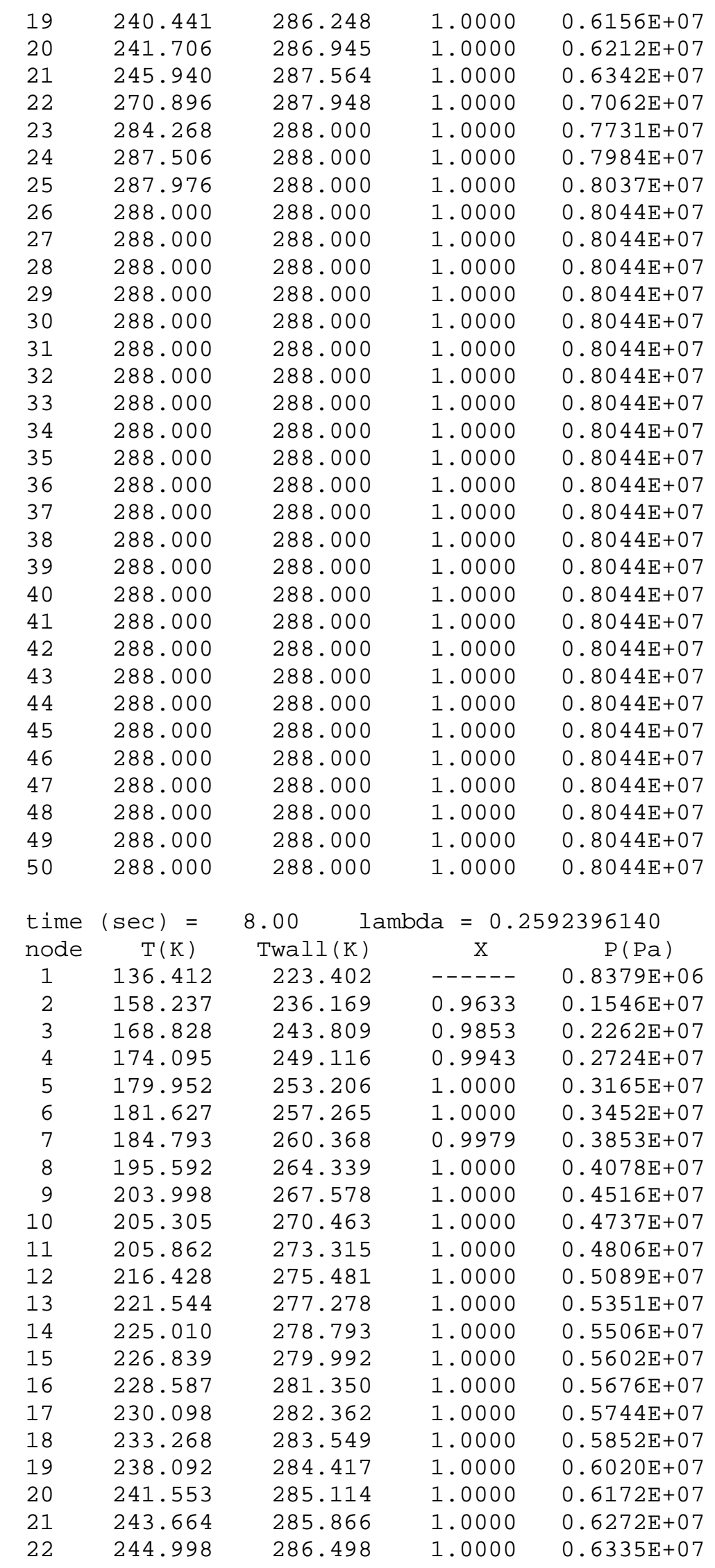




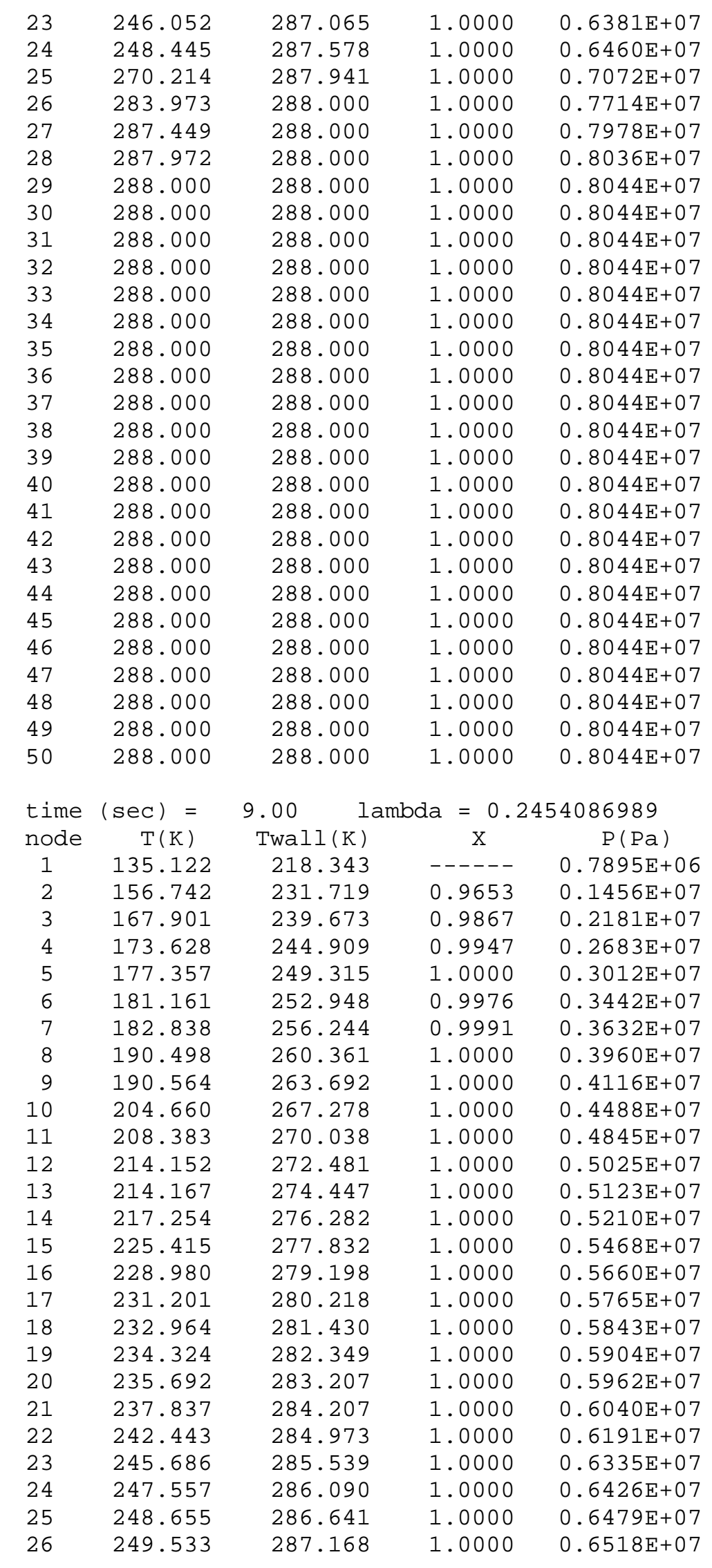




\begin{tabular}{|c|c|c|c|c|}
\hline 27 & 250.885 & 287.602 & 1.0000 & $0.6567 \mathrm{E}+07$ \\
\hline 28 & 269.577 & 287.935 & $1.000 \odot$ & $\odot .7083 \mathrm{E}+07$ \\
\hline 29 & 283.669 & $288.00 \odot$ & 1.0000 & $\odot .7697 \mathrm{E}+\odot 7$ \\
\hline 30 & 287.388 & 288.000 & 1.0000 & $\odot .7973 \mathrm{E}+\Theta 7$ \\
\hline 31 & 287.967 & 288.000 & 1.0000 & $\odot .8035 \mathrm{E}+07$ \\
\hline 32 & 288.000 & 288.000 & 1.0000 & $0.8044 \mathrm{E}+07$ \\
\hline 33 & 288.000 & $288.00 \odot$ & 1.0000 & $\odot .8044 \mathrm{E}+\odot 7$ \\
\hline 34 & 288.000 & $288.0 \odot \odot$ & $1.000 \odot$ & $\odot .8044 \mathrm{E}+07$ \\
\hline 35 & 288.000 & 288.000 & 1.0000 & $0.8044 \mathrm{E}+07$ \\
\hline 36 & $288.00 \odot$ & $288.0 \odot \odot$ & $1.000 \odot$ & $0.8044 \mathrm{E}+\odot 7$ \\
\hline 37 & 288.000 & 288.000 & 1.0000 & $0.8044 \mathrm{E}+07$ \\
\hline 38 & 288.000 & 288.000 & 1.0000 & $0.8044 \mathrm{E}+07$ \\
\hline 39 & 288.000 & 288.000 & 1.0000 & $0.8044 \mathrm{E}+07$ \\
\hline 40 & 288.000 & $288.0 \odot \odot$ & 1.0000 & $\odot .8044 \mathrm{E}+\odot 7$ \\
\hline 41 & 288.000 & 288.000 & 1.0000 & $0.8044 \mathrm{E}+07$ \\
\hline 42 & $288.00 \odot$ & $288.00 \odot$ & 1.0000 & $\odot .8044 \mathrm{E}+07$ \\
\hline 43 & $288.00 \odot$ & $288.0 \odot \odot$ & $1.000 \odot$ & $\odot .8044 \mathrm{E}+\odot 7$ \\
\hline 44 & 288.000 & 288.000 & 1.0000 & $0.8044 \mathrm{E}+07$ \\
\hline 45 & 288.000 & 288.000 & 1.0000 & $0.8044 \mathrm{E}+07$ \\
\hline 46 & 288.000 & 288.000 & 1.0000 & $0.8044 \mathrm{E}+07$ \\
\hline 47 & 288.000 & 288.000 & 1.0000 & $0.8044 \mathrm{E}+07$ \\
\hline 48 & $288.00 \odot$ & $288.0 \odot \odot$ & $1.000 \odot$ & $0.8044 \mathrm{E}+\odot 7$ \\
\hline 49 & 288.000 & 288.000 & 1.0000 & $\odot .8044 \mathrm{E}+07$ \\
\hline 50 & 288.000 & 288.000 & 1.0000 & $0.8044 \mathrm{E}+07$ \\
\hline tim & $(\sec )=$ & \multicolumn{3}{|c|}{.$\odot \odot \quad l$ lambda $=0.2398249656$} \\
\hline node & $\mathrm{T}(\mathrm{K})$ & Twall(K) & $\mathrm{X}$ & $\mathrm{P}(\mathrm{Pa})$ \\
\hline 1 & 134.605 & 213.732 & - - - - - & $\odot .7700 \mathrm{E}+06$ \\
\hline 2 & 156.142 & 227.569 & $\odot .9651$ & $\odot .1420 \mathrm{E}+\odot 7$ \\
\hline 3 & 167.124 & 235.754 & $\odot .9876$ & $0.2113 \mathrm{E}+07$ \\
\hline 4 & 172.547 & 241.042 & $\odot .9957$ & $\odot .2588 \mathrm{E}+\Theta 7$ \\
\hline 5 & 177.283 & 245.312 & 0.9970 & $0.3004 \mathrm{E}+07$ \\
\hline 6 & 179.446 & 249.194 & $\odot .9983$ & $\odot .3248 \mathrm{E}+\odot 7$ \\
\hline 7 & 182.565 & 252.339 & $\odot .9990$ & $0.3601 \mathrm{E}+07$ \\
\hline 8 & 185.314 & 256.699 & 1.0000 & $0.3742 \mathrm{E}+07$ \\
\hline 9 & 198.295 & 260.160 & 1.0000 & $0.4177 \mathrm{E}+07$ \\
\hline 10 & 199.779 & 263.627 & 1.0000 & $0.4377 \mathrm{E}+07$ \\
\hline 11 & 199.822 & 266.431 & 1.0000 & $\odot .4569 \mathrm{E}+07$ \\
\hline 12 & 201.724 & 269.503 & $1.000 \odot$ & $\odot .4613 \mathrm{E}+07$ \\
\hline 13 & 215.937 & 271.814 & $1.000 \odot$ & $\odot .5031 \mathrm{E}+\odot 7$ \\
\hline 14 & 218.339 & 273.690 & 1.0000 & $\odot .5236 \mathrm{E}+\Theta 7$ \\
\hline 15 & 221.246 & 275.394 & 1.0000 & $0.5346 \mathrm{E}+07$ \\
\hline 16 & 222.896 & 276.942 & $1.000 \odot$ & $\odot .5440 \mathrm{E}+07$ \\
\hline 17 & 226.523 & 278.082 & 1.0000 & $\odot .5569 \mathrm{E}+07$ \\
\hline 18 & 231.592 & 279.599 & 1.0000 & $\odot .5744 \mathrm{E}+07$ \\
\hline 19 & 234.422 & 280.518 & 1.00 & $\odot .5883 \mathrm{E}+\odot 7$ \\
\hline 20 & 236.426 & 281.376 & 1.0000 & $\odot .5972 \mathrm{E}+07$ \\
\hline 21 & 237.867 & 282.376 & 1.0000 & $0.6039 \mathrm{E}+07$ \\
\hline 22 & 239.095 & 283.160 & 1.0000 & $0.6093 \mathrm{E}+07$ \\
\hline 23 & 240.184 & 283.875 & $1.000 \odot$ & $\odot .6140 \mathrm{E}+\odot 7$ \\
\hline 24 & 241.538 & 284.654 & 1.0000 & $0.6193 \mathrm{E}+07$ \\
\hline 25 & 246.358 & 285.392 & $1.000 \odot$ & $\odot .6340 \mathrm{E}+\odot 7$ \\
\hline 26 & 249.152 & 285.948 & $1.000 \odot$ & $0.6476 \mathrm{E}+07$ \\
\hline 27 & 250.637 & 286.381 & 1.0000 & $\odot .6552 \mathrm{E}+07$ \\
\hline 28 & 251.672 & 286.790 & 1.0000 & $\odot .6598 \mathrm{E}+07$ \\
\hline 29 & 252.457 & 287.200 & 1.0000 & $\odot .6632 \mathrm{E}+07$ \\
\hline 30 & 253.164 & 287.612 & $1.000 \odot$ & $0.6661 \mathrm{E}+07$ \\
\hline
\end{tabular}




\begin{tabular}{|c|c|c|c|c|}
\hline 31 & 268.993 & 287.928 & $1.000 \odot$ & $\odot .7094 \mathrm{E}+07$ \\
\hline 32 & 283.359 & $288.00 \odot$ & 1.0000 & $\odot .7681 \mathrm{E}+07$ \\
\hline 33 & 287.325 & $288.0 \odot \odot$ & 1.0000 & $\odot .7967 \mathrm{E}+\odot 7$ \\
\hline 34 & 287.961 & $288.0 \odot \odot$ & 1.0000 & $\odot .8034 \mathrm{E}+07$ \\
\hline 35 & $288.0 \odot \odot$ & $288.00 \odot$ & 1.0000 & $\odot .8044 \mathrm{E}+07$ \\
\hline 36 & 288.000 & $288.0 \odot \odot$ & 1.0000 & $\odot .8044 \mathrm{E}+07$ \\
\hline 37 & $288.00 \odot$ & $288.0 \odot \odot$ & 1.0000 & $\odot .8044 \mathrm{E}+\odot 7$ \\
\hline 38 & $288.00 \odot$ & $288.0 \odot \odot$ & 1.0000 & $\odot .8044 \mathrm{E}+07$ \\
\hline 39 & $288.0 \odot \odot$ & $288.0 \odot \odot$ & 1.0000 & $\odot .8044 \mathrm{E}+07$ \\
\hline 40 & $288 . \odot \odot \odot$ & $288 . \odot \odot \odot$ & $1.00 \odot \odot$ & $\odot .8 \odot 44 \mathrm{E}+07$ \\
\hline 41 & $288.0 \odot \odot$ & $288.00 \odot$ & 1.0000 & $0.8044 \mathrm{E}+07$ \\
\hline 42 & $288.0 \odot \odot$ & $288.00 \odot$ & 1.0000 & $\odot .8044 \mathrm{E}+07$ \\
\hline 43 & $288.0 \odot \odot$ & 288.000 & 1.0000 & $\odot .8044 \mathrm{E}+07$ \\
\hline 44 & $288.00 \odot$ & $288.0 \odot \odot$ & 1.0000 & $\odot .8044 \mathrm{E}+\odot 7$ \\
\hline 45 & $288.00 \odot$ & $288.00 \odot$ & 1.0000 & $\odot .8044 \mathrm{E}+07$ \\
\hline 46 & $288 . \odot \odot \odot$ & $288 . \odot \odot \odot$ & $1.000 \odot$ & $\odot .8 \odot 44 \mathrm{E}+\odot 7$ \\
\hline 47 & $288 . \odot \odot \odot$ & $288 . \odot \odot \odot$ & $1.00 \odot \odot$ & $\odot .8044 \mathrm{E}+07$ \\
\hline 48 & $288.00 \odot$ & $288.00 \odot$ & 1.0000 & $\odot .8044 \mathrm{E}+07$ \\
\hline 49 & $288.0 \odot \odot$ & $288.00 \odot$ & 1.0000 & $\odot .8044 \mathrm{E}+07$ \\
\hline 50 & $288.0 \odot \odot$ & $288.0 \odot \odot$ & 1.0000 & $0.8044 \mathrm{E}+07$ \\
\hline time & $(\sec )=$ & 11.00 & $\mathrm{a} a=0$. & 9272777 \\
\hline node & $\mathrm{T}(\mathrm{K})$ & Twall(K) & X & $P(P a)$ \\
\hline 1 & 133.969 & 209.456 & - - - - - & $0.7461 \mathrm{E}+06$ \\
\hline 2 & 155.404 & 223.745 & $\odot .9668$ & $0.1376 \mathrm{E}+07$ \\
\hline 3 & 166.689 & 232.072 & $\odot .9872$ & $\odot .2074 \mathrm{E}+07$ \\
\hline 4 & 171.897 & 237.335 & $\odot .9943$ & $0.2531 \mathrm{E}+07$ \\
\hline 5 & 175.949 & 241.759 & $\odot .9970$ & $\odot .2886 \mathrm{E}+07$ \\
\hline 6 & 179.286 & 245.522 & $\odot .9978$ & $\odot .3230 \mathrm{E}+07$ \\
\hline 7 & 183.173 & 248.812 & 1.0000 & $\odot .3491 \mathrm{E}+07$ \\
\hline 8 & 188.539 & 253.056 & 1.0000 & $\odot .3803 \mathrm{E}+07$ \\
\hline 9 & 192.045 & 257.030 & 1.0000 & $\odot .3896 \mathrm{E}+07$ \\
\hline 10 & 193.833 & 260.270 & 1.00 & $\odot .42 \odot 4 \mathrm{E}+07$ \\
\hline 11 & 202.498 & 263.202 & $1 .($ & $1 \mathrm{E}+07$ \\
\hline 12 & 204.293 & 266.175 & $1.000 \odot$ & $\odot .4672 \mathrm{E}+07$ \\
\hline 13 & 211.136 & 269.117 & $1.000 \odot$ & $\odot .4873 \mathrm{E}+07$ \\
\hline 14 & 214.750 & 271.190 & $1.000 \odot$ & $\odot .5089 \mathrm{E}+07$ \\
\hline 15 & 218.254 & 273.103 & 1.0000 & $\odot .5221 \mathrm{E}+07$ \\
\hline 16 & 223.700 & 274.708 & 1.0 & $0.5415 \mathrm{E}+07$ \\
\hline 17 & 224.904 & 275.843 & & $6 \mathrm{E}+\odot 7$ \\
\hline 18 & 226.783 & 277.487 & 1.0000 & $\odot .5588 \mathrm{E}+07$ \\
\hline 19 & 229.256 & 278.579 & $1.00 \odot \odot$ & $\odot .5681 \mathrm{E}+07$ \\
\hline 20 & 231.702 & 279.544 & $1.00 \odot \odot$ & $\odot .5785 \mathrm{E}+07$ \\
\hline 21 & 237.011 & 280.821 & 1.0000 & $\odot .5954 \mathrm{E}+07$ \\
\hline 22 & 237.962 & 281.633 & $1.00 \odot \odot$ & $\odot .6 \odot 49 \mathrm{E}+07$ \\
\hline 23 & 240.883 & 282.349 & 1.0 & $0.6137 \mathrm{E}+07$ \\
\hline 24 & 241.872 & 283.128 & 1.0000 & $\odot .6202 \mathrm{E}+07$ \\
\hline 25 & 243.164 & 283.866 & 1.0000 & $\odot .6249 \mathrm{E}+07$ \\
\hline 26 & 244.248 & 284.541 & 1.0000 & $\odot .6298 \mathrm{E}+07$ \\
\hline 27 & 245.539 & 285.106 & 1.0000 & $\odot .6352 \mathrm{E}+07$ \\
\hline 28 & 249.269 & 285.569 & $1.000 \odot$ & $\odot .6470 \mathrm{E}+07$ \\
\hline 29 & 251. & 285.979 & $1.00 \odot \odot$ & $\odot .6586 \mathrm{E}+07$ \\
\hline 30 & 253.318 & 286.520 & $1.00 \odot \odot$ & $\odot .6656 \mathrm{E}+07$ \\
\hline 31 & 254.268 & 286.958 & $1.000 \odot$ & $\odot .6699 \mathrm{E}+07$ \\
\hline 32 & 254.954 & 287.337 & 1.0000 & $\odot .6729 \mathrm{E}+07$ \\
\hline 33 & 255.359 & 287.642 & $1.000 \odot$ & $\odot .6749 \mathrm{E}+\odot 7$ \\
\hline & 268.472 & 287.923 & $1.000 \odot$ & $\odot .7106 \mathrm{E}+07$ \\
\hline
\end{tabular}




\begin{tabular}{|c|c|c|c|c|}
\hline 35 & 283.046 & 288.000 & 1.0000 & $\odot .7666 \mathrm{E}+07$ \\
\hline 36 & 287.258 & $288.0 \odot \odot$ & 1.0000 & $\odot .7962 \mathrm{E}+\odot 7$ \\
\hline 37 & 287.952 & 288.000 & 1.0000 & $\odot .8033 \mathrm{E}+07$ \\
\hline 38 & 288.000 & 288.000 & 1.0000 & $\odot .8043 \mathrm{E}+07$ \\
\hline 39 & $288.00 \odot$ & 288.000 & 1.0000 & $\odot .8044 \mathrm{E}+07$ \\
\hline 40 & $288.00 \odot$ & $288 . \odot \odot \odot$ & 1.0000 & $\odot .8 \odot 44 \mathrm{E}+\odot 7$ \\
\hline 41 & 288.000 & 288.000 & 1.0000 & $\odot .8044 \mathrm{E}+07$ \\
\hline 42 & 288.000 & 288.000 & 1.0000 & $\odot .8044 \mathrm{E}+07$ \\
\hline 43 & 288.000 & $288 . \odot \odot \odot$ & 1.0000 & $\odot .8044 \mathrm{E}+07$ \\
\hline 44 & 288.000 & 288.000 & 1.0000 & $\odot .8044 \mathrm{E}+07$ \\
\hline 45 & 288.000 & 288.000 & 1.0000 & $\odot .8044 \mathrm{E}+07$ \\
\hline 46 & $288.00 \odot$ & 288.000 & 1.0000 & $\odot .8044 \mathrm{E}+07$ \\
\hline 47 & $288.00 \odot$ & $288.00 \odot$ & 1.0000 & $\odot .8044 \mathrm{E}+07$ \\
\hline 48 & 288.000 & $288.00 \odot$ & 1.0000 & $\odot .8044 \mathrm{E}+07$ \\
\hline 49 & $288.00 \odot$ & 288.000 & 1.0000 & $\odot .8044 \mathrm{E}+07$ \\
\hline 50 & 288.000 & $288 . \odot \odot \odot$ & 1.0000 & $\odot .8044 \mathrm{E}+\odot 7$ \\
\hline
\end{tabular}

\begin{tabular}{|c|c|c|c|c|}
\hline \multicolumn{5}{|c|}{$.0 \odot \quad$ lambda $=0.2288985699$} \\
\hline node & $T(K)$ & Twall(K) & $x$ & $\mathrm{P}(\mathrm{Pa})$ \\
\hline 1 & 133.599 & 205.478 & - - - - - & $\odot .7322 \mathrm{E}+06$ \\
\hline 2 & 154.974 & 220.156 & $\odot .9659$ & $\odot .1351 \mathrm{E}+07$ \\
\hline 3 & 165.895 & 228.622 & $\odot .9876$ & $\odot .2 \odot \odot 5 E+\odot 7$ \\
\hline 4 & 171.300 & 233.839 & $\odot .9938$ & $\odot .2479 \mathrm{E}+\odot 7$ \\
\hline 5 & 175.462 & 238.311 & $\odot .9966$ & $\odot .2843 \mathrm{E}+\odot 7$ \\
\hline 6 & 178.584 & 242.155 & $\odot .9990$ & $\odot .3151 \mathrm{E}+07$ \\
\hline 7 & 181.272 & 245.250 & $\odot .9984$ & $\odot .3455 \mathrm{E}+07$ \\
\hline 8 & 195.573 & 250.163 & 1.0000 & $\odot .3728 \mathrm{E}+07$ \\
\hline 9 & 195.573 & 253.861 & 1.0000 & $\odot .3968 \mathrm{E}+\odot 7$ \\
\hline 10 & 195.573 & 256.879 & 1.0000 & $\odot .4204 \mathrm{E}+07$ \\
\hline 11 & 195.573 & 260.015 & 1.0000 & $\odot .4228 \mathrm{E}+07$ \\
\hline 12 & 207.472 & 263.274 & 1.0000 & $\odot .4632 \mathrm{E}+\odot 7$ \\
\hline 13 & 207.472 & 266.104 & 1.0000 & $\odot .4789 \mathrm{E}+07$ \\
\hline 14 & 212.890 & 268.678 & $1.000 \odot$ & $\odot .4943 \mathrm{E}+\odot 7$ \\
\hline 15 & 215.653 & 270.557 & 1.0000 & $\odot .5129 \mathrm{E}+07$ \\
\hline 16 & 215.793 & 272.393 & 1.0000 & $\odot .5160 \mathrm{E}+07$ \\
\hline 17 & 226.520 & 273.908 & 1.0000 & $\odot .5450 \mathrm{E}+\odot 7$ \\
\hline 18 & 226.520 & 275.351 & 1.0000 & $\odot .5581 \mathrm{E}+07$ \\
\hline 19 & 230.508 & 276.703 & 1.0000 & $\odot .5680 \mathrm{E}+07$ \\
\hline 20 & 230.801 & 277.627 & 1.0000 & $\odot .5763 \mathrm{E}+\odot 7$ \\
\hline 21 & 233.598 & 279.082 & 1.0000 & $\odot .5832 \mathrm{E}+\odot 7$ \\
\hline 22 & 235.968 & 279.938 & 1.0000 & $\odot .5962 \mathrm{E}+07$ \\
\hline 23 & 235.968 & 280.775 & 1.0000 & $\odot .5985 \mathrm{E}+\odot 7$ \\
\hline 24 & 242.505 & 281.603 & 1.0000 & $\odot .6151 \mathrm{E}+07$ \\
\hline 25 & 242.506 & 282.340 & 1.0000 & $\odot .6231 \mathrm{E}+07$ \\
\hline 26 & 244.157 & 283.177 & 1.0000 & $\odot .6277 \mathrm{E}+07$ \\
\hline 27 & 245.498 & 283.738 & 1.0000 & $\odot .6345 \mathrm{E}+07$ \\
\hline 28 & 246.364 & 284.318 & $1.000 \odot$ & $\odot .6385 \mathrm{E}+07$ \\
\hline 29 & 247.368 & 284.759 & 1.0000 & $0.6425 \mathrm{E}+07$ \\
\hline 30 & 248.448 & $285.4 \odot 4$ & 1.0000 & $\odot .6469 \mathrm{E}+07$ \\
\hline 31 & 251.606 & 285.991 & 1.0000 & $\odot .6568 \mathrm{E}+\odot 7$ \\
\hline 32 & 254.215 & 286.421 & 1.0000 & $\odot .6676 \mathrm{E}+07$ \\
\hline 33 & 255.650 & 286.726 & 1.0000 & $\odot .6746 \mathrm{E}+07$ \\
\hline 34 & 256.526 & 287.032 & 1.0000 & $\odot .6787 \mathrm{E}+\odot 7$ \\
\hline 35 & 257.116 & 287.339 & 1.0000 & $\odot .6813 \mathrm{E}+07$ \\
\hline 36 & 257.462 & 287.646 & 1.0000 & $\odot .6830 \mathrm{E}+07$ \\
\hline 37 & 268.020 & 287.917 & 1.0000 & $\odot .7119 \mathrm{E}+\odot 7$ \\
\hline 38 & 282.730 & $288.0 \odot \odot$ & 1.0000 & $0.7651 \mathrm{E}+07$ \\
\hline
\end{tabular}




\begin{tabular}{|c|c|c|c|c|}
\hline 39 & 287.188 & $288.00 \odot$ & 1.0000 & $\odot .7956 \mathrm{E}+07$ \\
\hline 40 & 287.943 & $288 . \odot \odot \odot$ & $1.00 \odot \odot$ & $\odot .8032 \mathrm{E}+07$ \\
\hline 41 & $288.00 \odot$ & $288.0 \odot \odot$ & $1.000 \odot$ & $\odot .8043 \mathrm{E}+07$ \\
\hline 42 & $288.0 \odot \odot$ & $288.0 \odot \odot$ & 1. $\odot \odot \odot \odot$ & $\odot .8 \odot 44 \mathrm{E}+\odot 7$ \\
\hline 43 & $288.00 \odot$ & $288.00 \odot$ & 1.0000 & $\odot .8 \odot 44 \mathrm{E}+\odot 7$ \\
\hline 44 & $288.0 \odot \odot$ & $288.0 \odot \odot$ & $1.0 \odot \odot \odot$ & $\odot .8044 \mathrm{E}+\odot 7$ \\
\hline 45 & 288.000 & 288.000 & 1.0000 & $0.8044 \mathrm{E}+07$ \\
\hline 46 & 288.000 & $288.00 \odot$ & 1.0000 & $\odot .8044 \mathrm{E}+07$ \\
\hline 47 & $288.00 \odot$ & $288.0 \odot \odot$ & 1.0000 & $0.8044 \mathrm{E}+07$ \\
\hline 48 & $288.0 \odot \odot$ & $288.0 \odot \odot$ & 1. $\odot \odot \odot \odot$ & $\odot .8 \odot 44 \mathrm{E}+07$ \\
\hline 49 & $288.00 \odot$ & $288.00 \odot$ & 1.0000 & $\odot .8044 \mathrm{E}+07$ \\
\hline 50 & 288.000 & $288.00 \odot$ & $1.000 \odot$ & $\odot .8044 \mathrm{E}+07$ \\
\hline time & $(\sec )=$ & & $a=0$ & 29794115 \\
\hline node & $\mathrm{T}(\mathrm{K})$ & Twall(K) & X & $\mathrm{P}(\mathrm{Pa})$ \\
\hline 1 & 133.057 & 201.772 & - . - _ & $\odot .7118 \mathrm{E}+06$ \\
\hline 2 & 154.346 & 216.855 & $\odot .9669$ & $0.1313 \mathrm{E}+07$ \\
\hline 3 & 165.557 & 225.304 & $\odot .9868$ & $\odot .1983 E+07$ \\
\hline 4 & 170.611 & 230.572 & $\odot .9939$ & $\odot .2418 \mathrm{E}+\odot 7$ \\
\hline 5 & 175.010 & 234.965 & $\odot .9970$ & $\odot .2804 \mathrm{E}+07$ \\
\hline 6 & 178.565 & 238.824 & 0.9985 & $\odot .3149 \mathrm{E}+07$ \\
\hline 7 & 181.261 & 241.800 & $\odot .9974$ & $\odot .3454 \mathrm{E}+07$ \\
\hline 8 & 191.126 & 247.511 & 1.0000 & $\odot .3642 \mathrm{E}+07$ \\
\hline 9 & 192.333 & 250.996 & 1.0000 & $\odot .3863 \mathrm{E}+07$ \\
\hline 10 & 199.277 & 254.222 & 1.0000 & $0.4063 \mathrm{E}+07$ \\
\hline 11 & 202.474 & 257.215 & 1.0000 & $0.4370 \mathrm{E}+07$ \\
\hline 12 & 202.474 & 260.402 & 1.0000 & $\odot .4517 \mathrm{E}+07$ \\
\hline 13 & 209.413 & 263.529 & $1.000 \odot$ & $\odot .4700 \mathrm{E}+\odot 7$ \\
\hline 14 & 209.956 & 266.030 & 1.0000 & $\odot .4868 \mathrm{E}+07$ \\
\hline 15 & 218.342 & 268.421 & 1.0000 & $\odot .5086 \mathrm{E}+07$ \\
\hline 16 & 218.811 & 270.227 & 1.0000 & $\odot .5232 \mathrm{E}+\odot 7$ \\
\hline 17 & 219.261 & 271.972 & 1.0000 & $\odot .5267 \mathrm{E}+07$ \\
\hline 18 & 226.209 & 273.475 & 1.0000 & $0.5495 \mathrm{E}+07$ \\
\hline 19 & 226.209 & 274.872 & 1.0000 & $\odot .5566 \mathrm{E}+\odot 7$ \\
\hline 20 & 231.643 & 275.971 & $1.000 \odot$ & $\odot .5700 \mathrm{E}+\odot 7$ \\
\hline 21 & 232.045 & 277.365 & 1.0000 & $\odot .5794 \mathrm{E}+07$ \\
\hline 22 & 235.310 & 278.412 & 1.0000 & $\odot .5899 \mathrm{E}+\odot 7$ \\
\hline 23 & 236.983 & 279.250 & 1.0000 & $\odot .5997 \mathrm{E}+07$ \\
\hline 24 & 237.039 & 280.077 & 1.0000 & $0.6012 \mathrm{E}+07$ \\
\hline 25 & 242.965 & 281.060 & 1.0000 & $\odot .6170 \mathrm{E}+07$ \\
\hline 26 & 243.158 & 281.956 & 1.0000 & $\odot .6249 \mathrm{E}+\odot 7$ \\
\hline 27 & 244.242 & 282.518 & 1.0000 & $\odot .6291 \mathrm{E}+07$ \\
\hline 28 & 246.206 & 283.098 & 1.0000 & $0.6361 \mathrm{E}+07$ \\
\hline 29 & 246.994 & 283.538 & $1.000 \odot$ & $\odot .6408 \mathrm{E}+07$ \\
\hline 30 & 248.630 & 284.183 & 1.0000 & $0.6464 \mathrm{E}+07$ \\
\hline 31 & 249.205 & 284.770 & 1.0000 & $0.6501 \mathrm{E}+07$ \\
\hline 32 & 250.108 & 285.284 & 1.0000 & $0.6533 \mathrm{E}+07$ \\
\hline 33 & 250.876 & 285.715 & 1.0000 & $\odot .6567 \mathrm{E}+07$ \\
\hline 34 & 253.615 & 286.117 & 1.0000 & $0.6650 \mathrm{E}+07$ \\
\hline 35 & 256.270 & 286.423 & 1.0000 & $\odot .6754 \mathrm{E}+07$ \\
\hline 36 & 257.694 & 286.730 & $1.000 \odot$ & $0.6824 \mathrm{E}+07$ \\
\hline 37 & 258.497 & 287.039 & 1.0000 & $0.6863 \mathrm{E}+07$ \\
\hline 38 & 259.018 & 287.348 & 1.0000 & $\odot .6887 \mathrm{E}+07$ \\
\hline 39 & 259.369 & 287.660 & $1.000 \odot$ & $\odot .6904 \mathrm{E}+07$ \\
\hline 40 & 267.741 & 287.912 & 1.0000 & $0.7134 \mathrm{E}+07$ \\
\hline 41 & 282.416 & 288.000 & 1.0000 & $0.7638 \mathrm{E}+07$ \\
\hline 42 & 287.116 & 288.000 & 1.0000 & $\odot .7950 \mathrm{E}+07$ \\
\hline
\end{tabular}




\begin{tabular}{|c|c|c|c|c|}
\hline 43 & 287.935 & 288.000 & $1.00 \odot \odot$ & $\odot .8031 \mathrm{E}+07$ \\
\hline 44 & $288.00 \odot$ & 288.000 & 1.0000 & $\odot .8043 \mathrm{E}+07$ \\
\hline 45 & $288.00 \odot$ & 288.000 & 1.0000 & $\odot .8044 \mathrm{E}+07$ \\
\hline 46 & $288.00 \odot$ & 288.000 & 1.0000 & $\odot .8044 \mathrm{E}+07$ \\
\hline 47 & $288 . \odot \odot \odot$ & $288.00 \odot$ & $1.000 \odot$ & $\odot .8044 \mathrm{E}+07$ \\
\hline 48 & $288 . \odot \odot \odot$ & $288.0 \odot \odot$ & $1.000 \odot$ & $\odot .8044 \mathrm{E}+07$ \\
\hline 49 & 288.000 & 288.000 & 1.0000 & $\odot .8044 \mathrm{E}+07$ \\
\hline 50 & $288.00 \odot$ & $288.00 \odot$ & 1.0000 & $\odot .8044 \mathrm{E}+07$ \\
\hline
\end{tabular}

\begin{tabular}{|c|c|c|c|c|}
\hline $\begin{array}{l}\text { time } \\
\text { node }\end{array}$ & $\begin{array}{l}(\mathrm{sec})= \\
T(\mathrm{~K})\end{array}$ & $\begin{array}{c}14.00 \text { lo } \\
\text { Twall(K) }\end{array}$ & $a=0$ & $\begin{array}{l}46148 \\
P(P a)\end{array}$ \\
\hline 1 & 132.767 & 198.317 & $\ldots$ & $0.7009 \mathrm{E}+06$ \\
\hline 2 & 154.009 & 213.728 & $\odot .9666$ & $\odot .1293 E+\odot 7$ \\
\hline 3 & 164.766 & 222.184 & 0.9877 & $\odot .1936 \mathrm{E}+07$ \\
\hline 4 & 170.482 & 227.424 & $\odot .9937$ & $\odot .24 \odot 7 \mathrm{E}+\odot 7$ \\
\hline 5 & 174.865 & 231.790 & $\odot .9959$ & $\odot .2791 \mathrm{E}+07$ \\
\hline 6 & 177.964 & 235.714 & $\odot .9968$ & $\odot .3081 \mathrm{E}+07$ \\
\hline 7 & 179.655 & 238.853 & 0.9987 & $\odot .3272 \mathrm{E}+07$ \\
\hline 8 & 185.184 & 244.568 & 1.0000 & $\odot .3509 \mathrm{E}+07$ \\
\hline 9 & 197.851 & 248.384 & 1.0000 & $\odot .3820 \mathrm{E}+07$ \\
\hline 10 & 197.851 & 251.425 & 1.0000 & $\odot .4031 \mathrm{E}+07$ \\
\hline 11 & 198.246 & 254.583 & 1.0000 & $\odot .4221 \mathrm{E}+07$ \\
\hline 12 & 203.963 & 257.653 & 1.0000 & $0.4505 \mathrm{E}+07$ \\
\hline 13 & 203.963 & 260.990 & 1.0000 & $\odot .4547 \mathrm{E}+07$ \\
\hline 14 & 211.708 & 263.656 & 1.0000 & $\odot .4829 \mathrm{E}+07$ \\
\hline 15 & 211.708 & 266.057 & 1.0000 & $\odot .4926 \mathrm{E}+07$ \\
\hline 16 & 220.537 & 268.259 & 1.0000 & $\odot .5159 \mathrm{E}+07$ \\
\hline 17 & 220.569 & 269.836 & 1.0000 & $\odot .5298 \mathrm{E}+\odot 7$ \\
\hline 18 & 223.157 & 271.643 & 1.0000 & $\odot .5360 \mathrm{E}+07$ \\
\hline 19 & 226.981 & 273.041 & 1.0000 & $0.5544 \mathrm{E}+07$ \\
\hline 20 & 226.981 & $274.30 \odot$ & 1.0000 & $\odot .5582 \mathrm{E}+07$ \\
\hline 21 & 233.080 & 275.840 & 1.0000 & $\odot .5741 \mathrm{E}+07$ \\
\hline 22 & 233.080 & 276.886 & 1.0000 & $\odot .5843 \mathrm{E}+07$ \\
\hline 23 & 236.434 & 277.724 & $1.000 \odot$ & $\odot .5927 \mathrm{E}+\odot 7$ \\
\hline 24 & 238.433 & 278.586 & 1.0000 & $0.6034 \mathrm{E}+07$ \\
\hline 25 & 238.433 & 279.715 & 1.0000 & $\odot .6055 \mathrm{E}+07$ \\
\hline 26 & 243.811 & 280.735 & 1.0000 & $\odot .6205 \mathrm{E}+07$ \\
\hline 27 & 243.811 & 281.297 & 1.0000 & $\odot .6280 \mathrm{E}+07$ \\
\hline 28 & 246.088 & 281.877 & 1.0000 & $\odot .6339 \mathrm{E}+07$ \\
\hline 29 & 246.640 & 282.317 & 1.0000 & $\odot .6394 \mathrm{E}+07$ \\
\hline 30 & 247.092 & 282.962 & $1.000 \odot$ & $\odot .6410 \mathrm{E}+07$ \\
\hline 31 & 249.184 & 283.549 & 1.0000 & $0.6478 \mathrm{E}+07$ \\
\hline 32 & 249.772 & 284.063 & $1.000 \odot$ & $\odot .6521 \mathrm{E}+07$ \\
\hline 33 & 251.129 & 284.637 & 1.0000 & $\odot .6567 \mathrm{E}+07$ \\
\hline 34 & 251.738 & 285.074 & 1.0000 & $\odot .6600 \mathrm{E}+07$ \\
\hline 35 & 252.504 & 285.508 & 1.0000 & $0.6628 \mathrm{E}+07$ \\
\hline 36 & 253.140 & 285.815 & 1.0000 & $\odot .6655 \mathrm{E}+07$ \\
\hline 37 & 255.384 & 286.123 & 1.0000 & $\odot .6724 \mathrm{E}+07$ \\
\hline 38 & 258.080 & 286.433 & 1.0000 & $\odot .6824 \mathrm{E}+07$ \\
\hline 39 & 259.502 & 286.944 & 1.0000 & $\odot .6894 \mathrm{E}+07$ \\
\hline 40 & 260.222 & 287.265 & 1.0000 & $\odot .6931 E+07$ \\
\hline 41 & 260.675 & 287.503 & 1.0000 & $\odot .6952 \mathrm{E}+07$ \\
\hline 42 & 261.037 & 287.705 & 1.0000 & $\odot .6969 \mathrm{E}+07$ \\
\hline 43 & 267.659 & $287.9 \odot 7$ & 1.0000 & $\odot .7152 \mathrm{E}+07$ \\
\hline 44 & 282.103 & 288.000 & 1.0000 & $\odot .7628 \mathrm{E}+07$ \\
\hline 45 & 287.041 & 288.000 & 1.0000 & $\odot .7944 \mathrm{E}+07$ \\
\hline 46 & 287.927 & $288.00 \odot$ & $1.000 \odot$ & $\odot .8030 \mathrm{E}+07$ \\
\hline
\end{tabular}




$\begin{array}{lllll}47 & 288.00 \odot & 288.00 \odot & 1.000 \odot & \odot .8043 \mathrm{E}+\odot 7 \\ 48 & 288.00 \odot & 288.00 \odot & 1.00 \odot \odot & 0.8044 \mathrm{E}+07 \\ 49 & 288.00 \odot & 288.00 \odot & 1.000 \odot & \odot .8044 \mathrm{E}+07 \\ 5 \odot & 288.00 \odot & 288.00 \odot & 1.000 \odot & 0.8044 \mathrm{E}+07\end{array}$

\begin{tabular}{|c|c|c|c|c|}
\hline time & & & & \\
\hline node & $\mathrm{T}(\mathrm{K})$ & Twall(K) & $\mathrm{x}$ & $\mathrm{P}(\mathrm{Pa})$ \\
\hline 1 & 132.512 & 195.062 & & $\odot .6913 \mathrm{E}+\odot 6$ \\
\hline 2 & 153.714 & 210.798 & $\odot .9670$ & $\odot .1275 E+\odot 7$ \\
\hline 3 & 164.721 & 219.159 & $\odot .9863$ & $\odot .1933 E+07$ \\
\hline 4 & 169.971 & 224.480 & $\odot .9921$ & $\odot .2362 \mathrm{E}+07$ \\
\hline 5 & 173.569 & 228.897 & $\odot .9952$ & $\odot .2677 \mathrm{E}+\odot 7$ \\
\hline 6 & 176.733 & 232.941 & $\odot .9979$ & $\odot .2955 \mathrm{E}+\odot 7$ \\
\hline 7 & 179.396 & 235.998 & $\odot .9991$ & $\odot .3243 E+07$ \\
\hline 8 & 190.518 & 241.771 & 1.0000 & $0.3571 \mathrm{E}+07$ \\
\hline 9 & 194.355 & 245.945 & 1.0000 & $\odot .3683 \mathrm{E}+07$ \\
\hline 10 & 197.941 & 248.867 & $1.0 \odot \odot \odot$ & $0.3993 \mathrm{E}+07$ \\
\hline 11 & 198.571 & 251.775 & 1.0000 & $\odot .4214 \mathrm{E}+\odot 7$ \\
\hline 12 & 205.713 & 255.333 & 1.0000 & $0.4365 E+07$ \\
\hline 13 & 206.831 & 258.489 & 1.0000 & $\odot .4604 \mathrm{E}+\odot 7$ \\
\hline 14 & 206.831 & 261.451 & 1.0000 & $\odot .4629 \mathrm{E}+07$ \\
\hline 15 & 213.765 & 263.825 & 1.0000 & $\odot .4913 \mathrm{E}+07$ \\
\hline 16 & 213.765 & 266.149 & 1.0000 & $\odot .4999 \mathrm{E}+\odot 7$ \\
\hline 17 & 221.841 & 267.980 & 1.0000 & $\odot .5205 \mathrm{E}+\odot 7$ \\
\hline 18 & 221.841 & 269.743 & 1.0000 & $\odot .5329 \mathrm{E}+07$ \\
\hline 19 & 224.308 & 271.414 & 1.0000 & $\odot .5409 \mathrm{E}+\odot 7$ \\
\hline 20 & 227.951 & 272.584 & 1.0000 & $\odot .5570 \mathrm{E}+\Theta 7$ \\
\hline 21 & 227.951 & 274.238 & 1.0000 & $0.5606 \mathrm{E}+07$ \\
\hline 22 & 233.893 & 275.360 & 1.0000 & $\odot .5783 \mathrm{E}+07$ \\
\hline 23 & 234.170 & 276.198 & 1.0000 & $\odot .5870 \mathrm{E}+\odot 7$ \\
\hline 24 & 237.968 & 277.366 & 1. $0 \odot \odot \odot$ & $\odot .5967 \mathrm{E}+\odot 7$ \\
\hline 25 & 239.574 & 278.470 & 1.0000 & $0.6071 \mathrm{E}+07$ \\
\hline 26 & 239.574 & 279.514 & 1.0000 & $0.6094 \mathrm{E}+07$ \\
\hline 27 & 244.085 & 280.076 & 1.0000 & $\odot .6220 \mathrm{E}+07$ \\
\hline 28 & 244.085 & 280.656 & 1.0000 & $0.6286 \mathrm{E}+07$ \\
\hline 29 & 247.205 & 281.196 & 1.0000 & $0.6366 \mathrm{E}+07$ \\
\hline 30 & 247.205 & 281.742 & 1.0000 & $0.6413 \mathrm{E}+07$ \\
\hline 31 & 249.115 & 282.585 & 1.0000 & $0.6463 \mathrm{E}+07$ \\
\hline 32 & 249.449 & 283.040 & 1.0000 & $\odot .6509 \mathrm{E}+\odot 7$ \\
\hline 33 & 250.152 & 283.721 & 1.0000 & $\odot .6530 \mathrm{E}+\odot 7$ \\
\hline 34 & 251.541 & 284.159 & 1.0000 & $\odot .6581 \mathrm{E}+07$ \\
\hline 35 & 252.172 & 284.592 & 1.0000 & $\odot .6615 \mathrm{E}+\odot 7$ \\
\hline 36 & 253.356 & 284.899 & $1.000 \odot$ & $\odot .6655 \mathrm{E}+07$ \\
\hline 37 & 254.006 & 285.208 & 1.0000 & $0.6687 \mathrm{E}+07$ \\
\hline 38 & 254.592 & 285.517 & 1.0000 & $0.6711 \mathrm{E}+07$ \\
\hline 39 & 255.164 & 286.101 & 1.0000 & $0.6734 \mathrm{E}+07$ \\
\hline 40 & 257.030 & 286.560 & 1.0000 & $\odot .6792 \mathrm{E}+\odot 7$ \\
\hline 41 & 259.774 & 286.893 & 1.0000 & $\odot .6889 \mathrm{E}+07$ \\
\hline 42 & 261.080 & 287.095 & 1.0000 & $\odot .6957 \mathrm{E}+\odot 7$ \\
\hline 43 & 261.715 & 287.297 & $1.000 \odot$ & $\odot .6990 \mathrm{E}+\Theta 7$ \\
\hline 44 & 262.136 & 287.499 & 1.0000 & $0.7010 \mathrm{E}+07$ \\
\hline 45 & 262.488 & 287.703 & 1.0000 & $\odot .7025 \mathrm{E}+07$ \\
\hline 46 & 267.714 & 287.906 & 1.0000 & $\odot .7171 \mathrm{E}+\odot 7$ \\
\hline 47 & 281.797 & $288 . \odot \odot \odot$ & 1.0000 & $\odot .7620 \mathrm{E}+\odot 7$ \\
\hline 48 & 286.959 & 288.000 & 1.0000 & $\odot .7938 \mathrm{E}+\odot 7$ \\
\hline 49 & 287.921 & $288.0 \odot \odot$ & $1.000 \odot$ & $\odot .8029 \mathrm{E}+\odot 7$ \\
\hline 50 & 287.921 & 288.000 & $1.0 \odot \odot \odot$ & $0.8041 E+07$ \\
\hline
\end{tabular}




\begin{tabular}{|c|c|c|c|c|}
\hline \multicolumn{5}{|c|}{ lambda $=0.2146503627$} \\
\hline node & $\mathrm{T}(\mathrm{K})$ & Twall(K) & $\mathrm{x}$ & $\mathrm{P}(\mathrm{Pa})$ \\
\hline 1 & 132.297 & 191.984 & - - _ - _ & $\odot .6833 \mathrm{E}+06$ \\
\hline 2 & 153.465 & 208.013 & 0.9652 & $\odot .1260 \mathrm{E}+07$ \\
\hline 3 & 163.540 & 216.352 & 0.9858 & $\odot .1862 \mathrm{E}+07$ \\
\hline 4 & 169.056 & 221.820 & 0.9924 & $\odot .2282 \mathrm{E}+\odot 7$ \\
\hline 5 & 173.132 & 226.150 & 0.9963 & $\odot .2639 \mathrm{E}+07$ \\
\hline 6 & 176.725 & 230.195 & 0.9989 & $\odot .2954 \mathrm{E}+07$ \\
\hline 7 & 179.396 & 233.057 & 0.9970 & $0.3243 \mathrm{E}+07$ \\
\hline 8 & 199.975 & 239.634 & 1.0000 & $\odot .3615 \mathrm{E}+\odot 7$ \\
\hline 9 & 199.975 & 243.619 & 1.0000 & $\odot .3789 \mathrm{E}+\odot 7$ \\
\hline 10 & 199.975 & 246.333 & 1.0000 & $\odot .4012 \mathrm{E}+\odot 7$ \\
\hline 11 & 201.889 & 249.398 & 1.0000 & $0.4081 \mathrm{E}+07$ \\
\hline 12 & 201.889 & 252.785 & 1.0000 & $\odot .4280 \mathrm{E}+07$ \\
\hline 13 & 209.039 & 256.303 & 1.0000 & $\odot .4504 \mathrm{E}+07$ \\
\hline 14 & 209.214 & 259.105 & 1.0000 & $\odot .4682 \mathrm{E}+07$ \\
\hline 15 & 209.214 & 261.772 & 1.0000 & $\odot .4733 \mathrm{E}+07$ \\
\hline 16 & 215.366 & 264.099 & 1.0000 & $\odot .4973 \mathrm{E}+\odot 7$ \\
\hline 17 & 215.366 & 266.013 & 1.0000 & $0.5036 \mathrm{E}+07$ \\
\hline 18 & 223.218 & 268.040 & 1.0000 & $\odot .5269 \mathrm{E}+07$ \\
\hline 19 & 223.218 & 269.583 & 1.0000 & $\odot .5383 \mathrm{E}+07$ \\
\hline 20 & 227.323 & 271.059 & 1.0000 & $\odot .5482 \mathrm{E}+07$ \\
\hline 21 & 229.427 & 272.712 & 1.0000 & $\odot .5625 \mathrm{E}+\Theta 7$ \\
\hline 22 & 229.427 & 273.834 & 1.0000 & $\odot .5669 \mathrm{E}+07$ \\
\hline 23 & 234.978 & 274.822 & 1.0000 & $\odot .5808 \mathrm{E}+07$ \\
\hline 24 & 235.345 & 275.917 & 1.0000 & $\odot .5901 \mathrm{E}+07$ \\
\hline 25 & 240.526 & 277.250 & 1.0000 & $0.6042 \mathrm{E}+07$ \\
\hline 26 & 240.633 & 278.294 & 1.0000 & $\odot .6121 \mathrm{E}+07$ \\
\hline 27 & 242.602 & 278.856 & 1.0000 & $\odot .6171 \mathrm{E}+07$ \\
\hline 28 & 244.047 & 279.435 & 1.0000 & $\odot .6264 \mathrm{E}+07$ \\
\hline 29 & 244.047 & 280.137 & 1.0000 & $\odot .6279 \mathrm{E}+07$ \\
\hline 30 & 247.462 & 280.762 & 1.0000 & $\odot .6367 \mathrm{E}+07$ \\
\hline 31 & 247.462 & 281.669 & 1.0000 & $\odot .6419 \mathrm{E}+07$ \\
\hline 32 & 249.876 & 282.124 & 1.0000 & $0.6488 \mathrm{E}+07$ \\
\hline 33 & 249.876 & 282.806 & 1.0000 & $\odot .6523 \mathrm{E}+07$ \\
\hline 34 & 251.501 & 283.243 & 1.0000 & $\odot .6565 \mathrm{E}+07$ \\
\hline 35 & 251.879 & 283.677 & 1.0000 & $0.6605 \mathrm{E}+07$ \\
\hline 36 & 252.570 & 283.984 & $1.000 \odot$ & $0.6628 \mathrm{E}+07$ \\
\hline 37 & 253.567 & 284.292 & 1.0000 & $\odot .6663 \mathrm{E}+\odot 7$ \\
\hline 38 & 254.421 & 284.602 & 1.0000 & $\odot .6699 \mathrm{E}+07$ \\
\hline 39 & 255.357 & 285.185 & 1.0000 & $\odot .6735 \mathrm{E}+\odot 7$ \\
\hline 40 & 255.925 & 285.645 & $1.000 \odot$ & $0.6762 \mathrm{E}+07$ \\
\hline 41 & 256.475 & 286.014 & $1.000 \odot$ & $\odot .6785 \mathrm{E}+07$ \\
\hline 42 & 256.950 & 286.342 & 1.0000 & $\odot .6805 \mathrm{E}+07$ \\
\hline 43 & 258.863 & 286.671 & 1.0000 & $\odot .6863 \mathrm{E}+07$ \\
\hline 44 & 261.265 & 286.889 & $1.000 \odot$ & $\odot .6951 \mathrm{E}+07$ \\
\hline 45 & 262.565 & 287.092 & 1.0000 & $\odot .7015 \mathrm{E}+07$ \\
\hline 46 & 262.880 & 287.296 & $1.000 \odot$ & $\odot .7 \odot 41 \mathrm{E}+\odot 7$ \\
\hline 47 & 263.530 & 287.500 & $1.000 \odot$ & $0.7062 \mathrm{E}+07$ \\
\hline 48 & 263.530 & 287.692 & 1.0000 & $0.7071 \mathrm{E}+07$ \\
\hline 49 & 268.711 & 287.940 & 1.0000 & $\odot .7210 \mathrm{E}+07$ \\
\hline 50 & 269.076 & 287.940 & 1.0000 & $\odot .7290 \mathrm{E}+07$ \\
\hline & & & $\mathrm{la}=0$ & 75638473 \\
\hline node & $T(K)$ & Twall(K) & $\mathrm{X}$ & $P(P a)$ \\
\hline 1 & 131.654 & 189.129 & 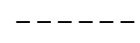 & $\odot .6591 \mathrm{E}+06$ \\
\hline
\end{tabular}




\begin{tabular}{|c|c|c|c|c|}
\hline 2 & 152.719 & 205.467 & 0.9665 & $\odot .1216 \mathrm{E}+07$ \\
\hline 3 & 163.098 & 213.629 & $\odot .9864$ & $0.1836 \mathrm{E}+07$ \\
\hline 4 & 168.930 & 219.226 & $\odot .993 \odot$ & $\odot .2271 \mathrm{E}+\odot 7$ \\
\hline 5 & 173.074 & 223.471 & $\odot .9968$ & $\odot .2634 \mathrm{E}+07$ \\
\hline 6 & 176.725 & 227.472 & $\odot .9986$ & $\odot .2954 \mathrm{E}+07$ \\
\hline 7 & 179.396 & 230.158 & $\odot .9950$ & $\odot .3243 \mathrm{E}+07$ \\
\hline 8 & 202.828 & 237.976 & 1.0000 & $0.3666 \mathrm{E}+07$ \\
\hline 9 & 202.836 & 241.689 & 1.0000 & $\odot .3833 \mathrm{E}+07$ \\
\hline 10 & 207.329 & 244.480 & $1.00 \odot \odot$ & $\odot .3980 \mathrm{E}+\odot 7$ \\
\hline 11 & 207.329 & 247.396 & 1.0000 & $0.4180 \mathrm{E}+07$ \\
\hline 12 & 207.329 & 250.610 & $1.000 \odot$ & $\odot .4349 \mathrm{E}+07$ \\
\hline 13 & 207.329 & 254.074 & $1.000 \odot$ & $0.4434 \mathrm{E}+07$ \\
\hline 14 & 211.185 & 257.094 & 1.0000 & $0.4625 \mathrm{E}+07$ \\
\hline 15 & 211.455 & 259.600 & 1.0000 & $0.4783 \mathrm{E}+07$ \\
\hline 16 & 213.900 & 262.232 & $1.000 \odot$ & $\odot .4839 \mathrm{E}+\odot 7$ \\
\hline 17 & 217.737 & 264.182 & 1.0000 & $\odot .5064 \mathrm{E}+07$ \\
\hline 18 & 217.737 & 266.250 & $1.00 \odot \odot$ & $\odot .5139 \mathrm{E}+\odot 7$ \\
\hline 19 & 224.384 & 267.955 & $1.000 \odot$ & $\odot .5299 \mathrm{E}+\odot 7$ \\
\hline 20 & 225.012 & 269.394 & $1.000 \odot$ & $\odot .5426 \mathrm{E}+\Theta 7$ \\
\hline 21 & 230.795 & 271.187 & 1.0000 & $\odot .5608 \mathrm{E}+07$ \\
\hline 22 & 230.857 & 272.308 & 1.0000 & $\odot .5704 \mathrm{E}+\odot 7$ \\
\hline 23 & 232.191 & 273.498 & $1.000 \odot$ & $\odot .5737 \mathrm{E}+07$ \\
\hline 24 & 235.984 & 274.523 & $1.000 \odot$ & $0.5878 \mathrm{E}+07$ \\
\hline 25 & 235.984 & 275.929 & 1.0000 & $\odot .5923 \mathrm{E}+07$ \\
\hline 26 & 241.296 & 277.073 & 1.0000 & $\odot .6056 \mathrm{E}+07$ \\
\hline 27 & 241.296 & 277.635 & $1.00 \odot \odot$ & $0.6138 \mathrm{E}+07$ \\
\hline 28 & 244.795 & 278.215 & 1.0000 & $0.6227 \mathrm{E}+07$ \\
\hline 29 & 244.795 & 278.916 & $1.000 \odot$ & $\odot .6298 \mathrm{E}+\odot 7$ \\
\hline 30 & 246.800 & 279.846 & 1.0000 & $\odot .6350 \mathrm{E}+07$ \\
\hline 31 & 247.797 & 280.753 & 1.0000 & $0.6416 \mathrm{E}+07$ \\
\hline 32 & 247.797 & 281.209 & $1.000 \odot$ & $0.6427 \mathrm{E}+\odot 7$ \\
\hline 33 & 250.126 & 281.890 & 1.0000 & $\odot .6491 \mathrm{E}+07$ \\
\hline 34 & 250.178 & 282.328 & 1.0000 & $0.6530 \mathrm{E}+07$ \\
\hline 35 & 252.191 & 282.761 & 00 & $\odot .6594 \mathrm{E}+07$ \\
\hline 36 & 252.191 & 283.068 & $1.000 \odot$ & $0.6618 \mathrm{E}+07$ \\
\hline 37 & 253.828 & 283.376 & 1.0000 & $0.6661 \mathrm{E}+07$ \\
\hline 38 & 253.994 & 283.686 & 1.0000 & $0.6688 \mathrm{E}+07$ \\
\hline 39 & 254.867 & 284.270 & 1.0000 & $\odot .6715 \mathrm{E}+07$ \\
\hline 40 & 254.867 & 284.729 & 1.0000 & $\odot .6727 E+\odot 7$ \\
\hline 41 & 256.780 & 114 & $\odot \odot$ & $E+07$ \\
\hline 42 & 256.780 & 285.427 & $\odot \odot \odot$ & $\odot .6800 \mathrm{E}+07$ \\
\hline 43 & 257.943 & 285.866 & 1.0000 & $0.6831 \mathrm{E}+07$ \\
\hline 44 & 257.943 & 286.143 & $1.000 \odot$ & $\odot .6849 \mathrm{E}+07$ \\
\hline 45 & 259.962 & 286.482 & 1.0000 & $\odot .6903 \mathrm{E}+07$ \\
\hline 46 & 259.962 & 286.686 & 1.0000 & $\odot .6932 \mathrm{E}+07$ \\
\hline 47 & L. 274 & 286.890 & 1.00 & $0.6967 \mathrm{E}+07$ \\
\hline 48 & 261.274 & 287.082 & 1.0 & $0.6984 \mathrm{E}+07$ \\
\hline 49 & 261.274 & 287.635 & 1.0000 & $0.6984 \mathrm{E}+\Theta 7$ \\
\hline 50 & 261.274 & 287.635 & 1.0000 & $\odot .6984 \mathrm{E}+\Theta 7$ \\
\hline time & $(\sec )=$ & \multicolumn{3}{|c|}{ lambda $=0.2063198984$} \\
\hline node & $\mathrm{T}(\mathrm{K})$ & Twall(K) & $\mathrm{X}$ & $\mathrm{P}(\mathrm{Pa})$ \\
\hline 1 & 131.542 & 186.439 & - - - - - & $\odot .6549 \mathrm{E}+06$ \\
\hline 2 & 152.588 & 203.026 & 0.9664 & $0.1208 \mathrm{E}+07$ \\
\hline 3 & 162.969 & 211.035 & $\odot .9869$ & $\odot .1828 \mathrm{E}+\odot 7$ \\
\hline 4 & 168.876 & 216.737 & 0.9932 & $\odot .2266 \mathrm{E}+07$ \\
\hline 5 & 173.028 & 220.881 & 966 & $\odot .2630 \mathrm{E}+\odot 7$ \\
\hline
\end{tabular}




\begin{tabular}{|c|c|c|c|c|}
\hline 6 & 176.690 & 224.878 & $\odot .9977$ & $\odot .2951 E+\odot 7$ \\
\hline 7 & 179.396 & 227.419 & $\odot .9937$ & $0.3243 E+07$ \\
\hline 8 & 191.237 & 236.180 & $1.000 \odot$ & $0.3457 \mathrm{E}+07$ \\
\hline 9 & 204.896 & 239.959 & $1.00 \odot \odot$ & $\odot .3776 \mathrm{E}+\odot 7$ \\
\hline 10 & 204.896 & 242.749 & 1.0000 & $\odot .3920 \mathrm{E}+\odot 7$ \\
\hline 11 & 204.896 & 245.536 & $1.00 \odot \odot$ & $0.4103 \mathrm{E}+07$ \\
\hline 12 & 204.896 & 248.817 & $1.000 \odot$ & $\odot .4179 \mathrm{E}+07$ \\
\hline 13 & 210.339 & 252.189 & 1.0000 & $\odot .4456 \mathrm{E}+\odot 7$ \\
\hline 14 & 210.339 & 255.053 & $1.000 \odot$ & $\odot .4607 \mathrm{E}+\odot 7$ \\
\hline 15 & 213.064 & 257.711 & 1.0000 & $0.4688 \mathrm{E}+07$ \\
\hline 16 & 214.098 & 260.276 & $1.00 \odot \odot$ & $\odot .4844 \mathrm{E}+07$ \\
\hline 17 & 219.704 & 262.425 & 1.0000 & $\odot .5080 \mathrm{E}+\odot 7$ \\
\hline 18 & 219.795 & 264.419 & $1.000 \odot$ & $0.5187 \mathrm{E}+07$ \\
\hline 19 & 221.486 & 266.357 & 1.0000 & $\odot .5227 \mathrm{E}+07$ \\
\hline 20 & 225.969 & 267.868 & 1.0000 & $\odot .5408 \mathrm{E}+\odot 7$ \\
\hline 21 & 225.969 & 269.585 & 1.0000 & $\odot .5481 E+07$ \\
\hline 22 & 231.860 & 270.885 & $1.000 \odot$ & $\odot .5624 \mathrm{E}+\odot 7$ \\
\hline 23 & 231.860 & 271.972 & 1.0000 & $\odot .5729 \mathrm{E}+07$ \\
\hline 24 & 236.758 & 273.302 & 1.0000 & $\odot .5850 \mathrm{E}+\odot 7$ \\
\hline 25 & 237.124 & 274.708 & $1.000 \odot$ & $\odot .5949 \mathrm{E}+\odot 7$ \\
\hline 26 & 237.278 & 275.852 & 1.0000 & $\odot .5955 \mathrm{E}+07$ \\
\hline 27 & 241.792 & 276.414 & 1.0000 & $0.6100 \mathrm{E}+07$ \\
\hline 28 & 241.792 & 276.994 & $1.000 \odot$ & $\odot .6150 \mathrm{E}+07$ \\
\hline 29 & 244.975 & 277.897 & $1.0 \odot \odot \odot$ & $\odot .6232 \mathrm{E}+\odot 7$ \\
\hline 30 & 244.975 & 278.803 & 1.0000 & $0.6301 \mathrm{E}+07$ \\
\hline 31 & 248.422 & 279.838 & 1.0000 & $\odot .6389 \mathrm{E}+07$ \\
\hline 32 & 248.422 & 280.293 & 1.0000 & $43 E+07$ \\
\hline 33 & 250.584 & 280.975 & 1.0000 & $\odot .6499 \mathrm{E}+07$ \\
\hline 34 & 250.584 & 281.412 & 1.0000 & $\odot .6541 \mathrm{E}+\odot 7$ \\
\hline 35 & 252.592 & 281.846 & $1.00 \odot \odot$ & $\odot .6593 \mathrm{E}+07$ \\
\hline 36 & 252.592 & 282.153 & 1.0000 & $\odot .6629 \mathrm{E}+07$ \\
\hline 37 & 254.113 & 282.485 & 1.0000 & $\odot .6669 \mathrm{E}+07$ \\
\hline 38 & 254.113 & 282.770 & 1.0000 & $0.6691 \mathrm{E}+07$ \\
\hline 39 & 255.020 & 283.491 & $1.0 \odot \odot \odot$ & $0.6715 \mathrm{E}+07$ \\
\hline 40 & 255.143 & 283.892 & 1.0000 & $0.6734 \mathrm{E}+07$ \\
\hline 41 & 257.009 & 284.504 & 1.0000 & $0.6783 \mathrm{E}+07$ \\
\hline 42 & 257.014 & 284.769 & $1.00 \odot \odot$ & $0.6806 \mathrm{E}+07$ \\
\hline 43 & 257.014 & 285.256 & 1.0000 & $0.6806 \mathrm{E}+07$ \\
\hline 44 & 257.989 & 285.533 & 1.0000 & $0.6837 \mathrm{E}+07$ \\
\hline 45 & 257.989 & 285.872 & $1.000 \odot$ & $\odot .6850 \mathrm{E}+07$ \\
\hline 46 & 257.989 & 286.075 & $1.00 \odot \odot$ & $\odot .6850 \mathrm{E}+\odot 7$ \\
\hline 47 & 257.989 & 286.279 & 1.0000 & $\odot .6850 \mathrm{E}+\odot 7$ \\
\hline 48 & 257.989 & 286.471 & $1.000 \odot$ & $\odot .6850 \mathrm{E}+07$ \\
\hline 49 & 257.989 & 287.045 & 1.00 & $0.6850 \mathrm{E}+07$ \\
\hline 50 & 257.989 & 287.045 & 1.0000 & $\odot .6850 \mathrm{E}+\odot 7$ \\
\hline time & $(\sec )=$ & \multicolumn{3}{|c|}{$.00 \quad$ lambda $=0.2059085816$} \\
\hline node & $\mathrm{T}(\mathrm{K})$ & Twall(K) & $\mathrm{X}$ & $\mathrm{P}(\mathrm{Pa})$ \\
\hline 1 & 131.505 & 183.869 & - - - - - & $0.6535 \mathrm{E}+06$ \\
\hline 2 & 152.545 & 200.705 & 0.9666 & $\odot .1205 \mathrm{E}+07$ \\
\hline 3 & 162.907 & 208.581 & $\odot .9869$ & $0.1825 \mathrm{E}+07$ \\
\hline 4 & 168.830 & 214.351 & $\odot .9930$ & $\odot .2262 \mathrm{E}+\odot 7$ \\
\hline 5 & 172.966 & 218.439 & $\odot .9954$ & $\odot .2625 \mathrm{E}+\odot 7$ \\
\hline 6 & 176.259 & 222.455 & $\odot .9954$ & $\odot .2913 \mathrm{E}+07$ \\
\hline 7 & 178.044 & 225.119 & $\odot .9961$ & $\odot .3090 \mathrm{E}+\odot 7$ \\
\hline 8 & 187.055 & 233.738 & 1.0000 & $\odot .3380 \mathrm{E}+07$ \\
\hline 9 & 203.603 & 238.501 & $1.0 \odot \odot \odot$ & $\odot .3679 \mathrm{E}+07$ \\
\hline
\end{tabular}




\begin{tabular}{|c|c|c|c|c|}
\hline 10 & 203.603 & 241.141 & 1.0000 & $\odot .3874 \mathrm{E}+07$ \\
\hline 11 & 210.063 & 243.943 & 1.0000 & $\odot .4092 \mathrm{E}+\odot 7$ \\
\hline 12 & 210.063 & 247.046 & 1.0000 & $\odot .4284 \mathrm{E}+07$ \\
\hline 13 & 210.063 & 250.299 & 1.0000 & $\odot .4433 \mathrm{E}+07$ \\
\hline 14 & 210.845 & 253.118 & $1.00 \odot \odot$ & $\odot .4548 E+07$ \\
\hline 15 & 212.450 & 255.880 & 1.0000 & $\odot .4652 \mathrm{E}+\Theta 7$ \\
\hline 16 & 215.851 & 258.445 & 1.0000 & $\odot .4863 \mathrm{E}+07$ \\
\hline 17 & 215.851 & 260.559 & 1.0000 & $\odot .4971 \mathrm{E}+07$ \\
\hline 18 & 221.414 & 262.842 & 1.0000 & $\odot .5099 \mathrm{E}+07$ \\
\hline 19 & 221.459 & 264.722 & $1.000 \odot$ & $\odot .5227 \mathrm{E}+\odot 7$ \\
\hline 20 & 225.658 & 266.342 & $1.000 \odot$ & $\odot .5372 \mathrm{E}+\odot 7$ \\
\hline 21 & 227.263 & 268.059 & 1.0000 & $\odot .5498 \mathrm{E}+07$ \\
\hline 22 & 227.263 & 269.359 & 1.0000 & $\odot .5513 E+07$ \\
\hline 23 & 232.734 & 270.600 & 1.0000 & $\odot .5681 \mathrm{E}+07$ \\
\hline 24 & 232.734 & 271.920 & $1.000 \odot$ & $\odot .5751 \mathrm{E}+07$ \\
\hline 25 & 238.026 & 273.487 & $1.000 \odot$ & $\odot .5885 E+07$ \\
\hline 26 & 238.026 & 274.632 & $1.000 \odot$ & $\odot .5974 \mathrm{E}+\odot 7$ \\
\hline 27 & 242.901 & 275.193 & $1.000 \odot$ & $\odot .6 \odot 96 \mathrm{E}+\odot 7$ \\
\hline 28 & 242.901 & 275.773 & 1.0000 & $0.6178 \mathrm{E}+07$ \\
\hline 29 & 246.041 & 276.981 & 1.0000 & $\odot .6258 \mathrm{E}+\odot 7$ \\
\hline 30 & 246.041 & 277.634 & $1.000 \odot$ & $\odot .6328 \mathrm{E}+07$ \\
\hline 31 & 249.048 & 278.922 & 1.0000 & $\odot .6406 \mathrm{E}+07$ \\
\hline 32 & 249.048 & 279.378 & $1.000 \odot$ & $\odot .6459 \mathrm{E}+07$ \\
\hline 33 & 251.134 & 280.059 & $1.000 \odot$ & $\odot .6513 \mathrm{E}+07$ \\
\hline 34 & 251.134 & 280.497 & 1.0000 & $\odot .6555 \mathrm{E}+07$ \\
\hline 35 & 253.029 & 280.930 & $1.000 \odot$ & $\odot .6605 \mathrm{E}+07$ \\
\hline 36 & 253.029 & 281.237 & $1.000 \odot$ & $\odot .6640 \mathrm{E}+07$ \\
\hline 37 & 254.165 & 281.874 & 1.0000 & $\odot .6670 \mathrm{E}+07$ \\
\hline 38 & 254.170 & 282.123 & 1.0000 & $\odot .6691 \mathrm{E}+07$ \\
\hline 39 & 254.264 & 282.881 & $1.000 \odot$ & $\odot .6694 \mathrm{E}+07$ \\
\hline 40 & 255.295 & 283.282 & 1.0000 & $\odot .6728 \mathrm{E}+07$ \\
\hline 41 & 255.295 & 283.893 & $1.000 \odot$ & $\odot .6738 \mathrm{E}+07$ \\
\hline 42 & 255.935 & 284.159 & 1.0000 & $\odot .6755 \mathrm{E}+\odot 7$ \\
\hline 43 & 256.248 & 284.645 & 1.0000 & $0.6773 \mathrm{E}+07$ \\
\hline 44 & 256.248 & 284.922 & $1.000 \odot$ & $\odot .6778 \mathrm{E}+\odot 7$ \\
\hline 45 & 256.248 & 285.261 & 1.0000 & $\odot .6778 \mathrm{E}+\odot 7$ \\
\hline 46 & 256.248 & 285.465 & 1.0000 & $\odot .6778 \mathrm{E}+07$ \\
\hline 47 & 256.248 & 285.669 & 1.0000 & $0.6778 \mathrm{E}+07$ \\
\hline 48 & 256.248 & 285.861 & 1.0000 & $\odot .6778 \mathrm{E}+\odot 7$ \\
\hline 49 & 256.248 & 286.569 & 1.0000 & $0.6778 \mathrm{E}+07$ \\
\hline 50 & 256.248 & 286.568 & $1.000 \odot$ & $\odot .6778 \mathrm{E}+07$ \\
\hline tim & $(\sec )=$ & \multicolumn{3}{|l|}{20.00} \\
\hline node & $\mathrm{T}(\mathrm{K})$ & Twall(K) & $x$ & $P(P a)$ \\
\hline 1 & 131.483 & 181.427 & - - - - - & $\odot .6527 E+06$ \\
\hline 2 & 152.520 & 198.479 & 0.9663 & $\odot .12 \odot 4 E+07$ \\
\hline 3 & 162.819 & 206.238 & 0.9860 & $\odot .1819 E+\odot 7$ \\
\hline 4 & 168.500 & 212.112 & 0.9915 & $\odot .2233 \mathrm{E}+\odot 7$ \\
\hline 5 & 171.927 & 216.217 & 0.9943 & $\odot .2534 \mathrm{E}+\odot 7$ \\
\hline 6 & 174.974 & 220.292 & ๑.9964 & $\odot .2801 E+\odot 7$ \\
\hline 7 & 177.909 & 222.859 & 0.9980 & $0.3075 \mathrm{E}+07$ \\
\hline 8 & 182.475 & 231.346 & 1.0000 & $\odot .3297 \mathrm{E}+07$ \\
\hline 9 & 196.103 & 236.787 & $1.000 \odot$ & $\odot .3544 \mathrm{E}+\odot 7$ \\
\hline 10 & 203.605 & 239.373 & 1.0000 & $\odot .3825 \mathrm{E}+\odot 7$ \\
\hline 11 & 203.610 & 242.277 & $1.000 \odot$ & $\odot .3914 \mathrm{E}+\odot 7$ \\
\hline 12 & 206.331 & 245.332 & $1.000 \odot$ & $0.4165 \mathrm{E}+07$ \\
\hline 13 & & 248.438 & $1.0 \odot \odot \odot$ & $\odot .4334 \mathrm{E}+07$ \\
\hline
\end{tabular}




\begin{tabular}{|c|c|c|c|c|}
\hline 14 & 213.969 & 251.424 & $1.000 \odot$ & $\odot .4517 \mathrm{E}+07$ \\
\hline 15 & 213.969 & 254.027 & $1.00 \odot \odot$ & $\odot .4685 \mathrm{E}+\odot 7$ \\
\hline 16 & 213.969 & 256.614 & 1.0000 & $\odot .4802 \mathrm{E}+\odot 7$ \\
\hline 17 & 217.177 & 258.782 & 1.0000 & $\odot .4958 \mathrm{E}+\odot 7$ \\
\hline 18 & 217.177 & 261.166 & 1.0000 & $\odot .5001 \mathrm{E}+07$ \\
\hline 19 & 223.075 & 263.158 & 1.0000 & $\odot .5199 \mathrm{E}+07$ \\
\hline 20 & 223.075 & 264.741 & 1.0000 & $\odot .5305 \mathrm{E}+07$ \\
\hline 21 & 228.847 & 266.586 & 1.0000 & $0.5442 \mathrm{E}+07$ \\
\hline 22 & 228.847 & 267.833 & 1.0000 & $\odot .5551 \mathrm{E}+07$ \\
\hline 23 & 234.377 & 269.379 & 1.0000 & $\odot .5692 \mathrm{E}+\odot 7$ \\
\hline 24 & 234.521 & 270.699 & 1.0000 & $0.5795 \mathrm{E}+07$ \\
\hline 25 & 239.630 & 272.267 & 1.0000 & $\odot .5921 \mathrm{E}+07$ \\
\hline 26 & 239.630 & 273.411 & 1.0000 & $\odot .6014 \mathrm{E}+07$ \\
\hline 27 & 243.795 & 273.973 & 1.0000 & $\odot .6119 \mathrm{E}+07$ \\
\hline 28 & 243.795 & 274.553 & 1.0000 & $\odot .6201 \mathrm{E}+07$ \\
\hline 29 & 246.959 & 276.066 & $1.000 \odot$ & $\odot .6281 \mathrm{E}+\odot 7$ \\
\hline 30 & 246.959 & 276.697 & $1.00 \odot \odot$ & $\odot .6352 \mathrm{E}+07$ \\
\hline 31 & 249.157 & $278.0 \odot 7$ & 1.0000 & $\odot .6408 \mathrm{E}+07$ \\
\hline 32 & 249.157 & 278.462 & 1.0000 & $\odot .6460 \mathrm{E}+07$ \\
\hline 33 & 249.824 & 279.143 & 1.0000 & $\odot .6477 \mathrm{E}+07$ \\
\hline 34 & 250.811 & 279.581 & 1.0000 & $\odot .6530 \mathrm{E}+07$ \\
\hline 35 & 250.811 & 280.015 & $1.00 \odot \odot$ & $\odot .6541 \mathrm{E}+07$ \\
\hline 36 & 252.238 & 280.428 & $1.000 \odot$ & $\odot .6578 \mathrm{E}+\odot 7$ \\
\hline 37 & 252.238 & 281.115 & 1.0000 & $0.6610 \mathrm{E}+07$ \\
\hline 38 & 253.050 & 281.512 & 1.0000 & $\odot .6631 \mathrm{E}+07$ \\
\hline 39 & 254.158 & 282.270 & 1.0000 & $\odot .6677 \mathrm{E}+07$ \\
\hline 40 & 254.158 & 282.672 & 1.0000 & $\odot .6690 \mathrm{E}+07$ \\
\hline 41 & 254.636 & 283.283 & 1.0 & ๑3E+๑7 \\
\hline 42 & 254.636 & 283.548 & $1.00 \odot \odot$ & $\odot .6713 \mathrm{E}+07$ \\
\hline 43 & 254.643 & 284.035 & 1.0000 & $\odot .6713 \mathrm{E}+07$ \\
\hline 44 & 254.648 & 284.312 & 1.0000 & $\odot .6713 \mathrm{E}+07$ \\
\hline 45 & 254.670 & 284.651 & 1.0000 & $\odot .6714 \mathrm{E}+07$ \\
\hline 46 & 254.670 & 284.855 & 1.0 & $\odot .6714 \mathrm{E}+07$ \\
\hline 47 & 254.670 & 285. & & $4 \mathrm{E}+07$ \\
\hline 48 & 254.670 & 285.251 & $1.000 \odot$ & $\odot .6714 \mathrm{E}+07$ \\
\hline 49 & 254.670 & 286.263 & $1.000 \odot$ & $\odot .6714 \mathrm{E}+07$ \\
\hline 50 & 254.670 & 286.263 & 1.0000 & $\odot .6714 \mathrm{E}+07$ \\
\hline time & $(\sec )=$ & 21.00 & $\mathrm{da}=0.2$ & 35088986 \\
\hline & $\mathrm{T}(\mathrm{K})$ & Twall(K) & $\mathrm{X}$ & $P(P a)$ \\
\hline 1 & 131.288 & 179.120 & -- & $0.6453 \mathrm{E}+06$ \\
\hline 2 & 152.294 & 196.376 & $\odot .9650$ & $\odot .1190 \mathrm{E}+07$ \\
\hline 3 & 161.962 & 204.053 & $\odot .9852$ & $\odot .1768 \mathrm{E}+07$ \\
\hline 4 & 167.640 & 210.074 & ๑.9916 & $\odot .2158 \mathrm{E}+07$ \\
\hline 5 & 171.537 & 214.081 & ๑. 9949 & $\odot .2499 \mathrm{E}+07$ \\
\hline 6 & 174.974 & 218.156 & & $\odot .2801 \mathrm{E}+07$ \\
\hline 7 & 177.771 & 220.725 & $\odot .9977$ & $\odot .3059 \mathrm{E}+07$ \\
\hline 8 & 188.493 & 229.269 & $1.00 \odot \odot$ & $\odot .3256 \mathrm{E}+07$ \\
\hline 9 & 191.896 & 234.621 & 1.0000 & $\odot .3468 \mathrm{E}+07$ \\
\hline 10 & 210.599 & 237.998 & 1.0000 & $0.3805 \mathrm{E}+07$ \\
\hline 11 & 210.599 & 240.715 & $1.000 \odot$ & $\odot .4 \odot 48 \mathrm{E}+07$ \\
\hline 12 & 210.599 & 243.595 & $1.00 \odot \odot$ & $\odot .4244 \mathrm{E}+07$ \\
\hline 13 & 210.599 & 246.596 & $1.000 \odot$ & $\odot .4362 \mathrm{E}+\odot 7$ \\
\hline 14 & 210.645 & 249.701 & 1.0000 & $\odot .4423 E+07$ \\
\hline 15 & 212.228 & 252.316 & 1.0000 & $\odot .4610 \mathrm{E}+07$ \\
\hline 16 & 212.228 & 254.783 & 1.0000 & $\odot .4746 \mathrm{E}+07$ \\
\hline 17 & 219.968 & 257.256 & $1.000 \odot$ & $\odot .4919 E+\odot 7$ \\
\hline
\end{tabular}




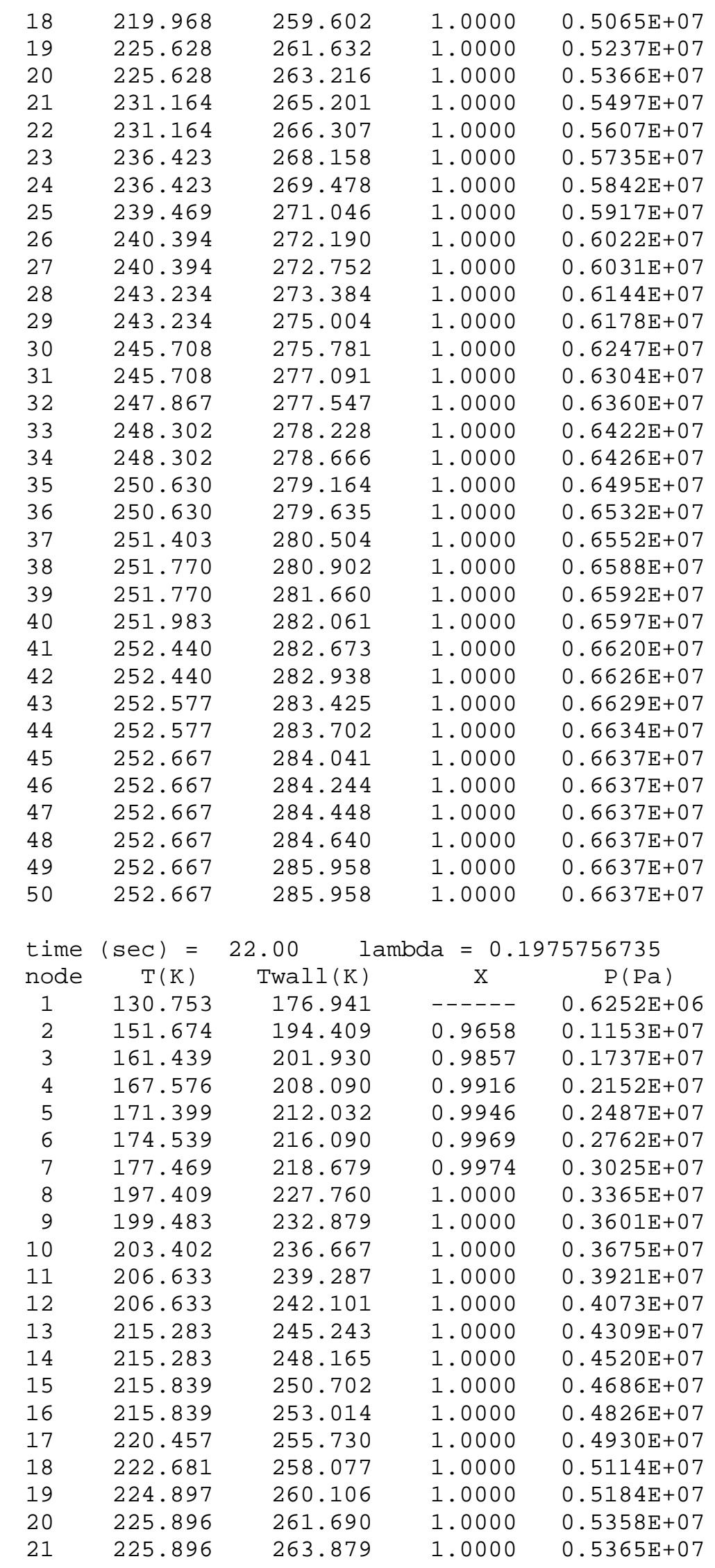




\begin{tabular}{|c|c|c|c|c|}
\hline 22 & 232.393 & 264.967 & 1.0000 & $\odot .5568 \mathrm{E}+\Theta 7$ \\
\hline 23 & 232.393 & 266.883 & $1.000 \odot$ & $\odot .5637 \mathrm{E}+07$ \\
\hline 24 & 237.107 & 268.258 & 1.0000 & $\odot .5776 \mathrm{E}+\odot 7$ \\
\hline 25 & 237.107 & 269.825 & 1.0000 & $\odot .5858 \mathrm{E}+\Theta 7$ \\
\hline 26 & 239.350 & 270.970 & 1.0000 & $\odot .5914 \mathrm{E}+07$ \\
\hline 27 & 240.786 & 271.562 & $1.000 \odot$ & $\odot .6024 \mathrm{E}+\odot 7$ \\
\hline 28 & 240.786 & 272.391 & 1.0000 & $0.6039 \mathrm{E}+07$ \\
\hline 29 & 243.420 & 273.994 & 1.0000 & $0.6137 \mathrm{E}+07$ \\
\hline 30 & 243.420 & 274.865 & 1.0000 & $\odot .6180 \mathrm{E}+07$ \\
\hline 31 & 245.484 & 276.176 & $1.000 \odot$ & $0.6232 \mathrm{E}+07$ \\
\hline 32 & 245.675 & 276.631 & 1.0000 & $\odot .6299 \mathrm{E}+\Theta 7$ \\
\hline 33 & 245.675 & 277.312 & $1.000 \odot$ & $0.6301 \mathrm{E}+07$ \\
\hline 34 & 248.319 & 277.750 & 1.0000 & $0.6386 \mathrm{E}+07$ \\
\hline 35 & 248.319 & 278.304 & 1.0000 & $0.6425 \mathrm{E}+07$ \\
\hline 36 & 248.851 & 278.980 & 1.0000 & $0.6439 \mathrm{E}+07$ \\
\hline 37 & 249.563 & 279.834 & $1.000 \odot$ & $\odot .6484 \mathrm{E}+07$ \\
\hline 38 & 249.563 & 280.292 & $1.000 \odot$ & $\odot .6492 \mathrm{E}+\odot 7$ \\
\hline 39 & 249.795 & 281.050 & 1.0000 & $\odot .6498 \mathrm{E}+07$ \\
\hline 40 & 250.011 & 281.451 & 1.0000 & $0.6524 \mathrm{E}+07$ \\
\hline 41 & 250.011 & 282.062 & 1.0000 & $\odot .6526 \mathrm{E}+07$ \\
\hline 42 & 250.120 & 282.328 & 1.0000 & $\odot .6529 \mathrm{E}+07$ \\
\hline 43 & 250.533 & 282.814 & $1.0 \odot \odot \odot$ & $\odot .6550 \mathrm{E}+\odot 7$ \\
\hline 44 & 250.533 & 283.091 & 1.0000 & $\odot .6556 \mathrm{E}+07$ \\
\hline 45 & 250.683 & 283.430 & 1.0000 & $0.6560 \mathrm{E}+\Theta 7$ \\
\hline 46 & 250.683 & 283.634 & 1.0000 & $0.6561 \mathrm{E}+07$ \\
\hline 47 & 250.683 & 283.838 & 1.0000 & $0.6561 \mathrm{E}+07$ \\
\hline 48 & 250.683 & 284.030 & 1.0000 & $0.6561 \mathrm{E}+07$ \\
\hline 49 & 250.683 & 285.653 & 1.0000 & $0.6561 \mathrm{E}+07$ \\
\hline 50 & 250.683 & 285.653 & $1.000 \odot$ & $\odot .6561 \mathrm{E}+07$ \\
\hline time & $=$ & 23.00 & \multicolumn{2}{|c|}{ lambda $=0.1959706545$} \\
\hline node & $\mathrm{T}(\mathrm{K})$ & Twall(K) & $\mathrm{x}$ & $P(P a)$ \\
\hline 1 & 130.609 & 174.894 & $--\cdots$ & $0.6198 \mathrm{E}+06$ \\
\hline 2 & 151.507 & 192.578 & 0.9655 & $3 \mathrm{E}+07$ \\
\hline 3 & 161.318 & 199.946 & 0.9854 & $\odot .1730 \mathrm{E}+\odot 7$ \\
\hline 4 & 167.263 & 206.182 & $\odot .9916$ & $0.2125 \mathrm{E}+\odot 7$ \\
\hline 5 & 171.040 & 210.065 & 0.9950 & $0.2456 \mathrm{E}+\Theta 7$ \\
\hline 6 & 174.419 & 214.106 & 0.9967 & $\odot .2752 \mathrm{E}+\Theta 7$ \\
\hline 7 & 176.761 & 216.708 & $\odot .9981$ & $\odot .2957 \mathrm{E}+\odot 7$ \\
\hline 8 & 181. & 225.862 & $\odot \odot$ & $3101 \mathrm{E}+07$ \\
\hline 9 & 206.454 & 231.577 & 1.0000 & $\odot .3521 \mathrm{E}+07$ \\
\hline 10 & 206.454 & 235.257 & $1.000 \odot$ & $\odot .3731 \mathrm{E}+07$ \\
\hline 11 & 206.454 & 237.889 & 1.0000 & $\odot .3911 \mathrm{E}+07$ \\
\hline 12 & 210.925 & 240.624 & 1.0000 & $0.4127 \mathrm{E}+07$ \\
\hline 13 & 214.071 & 243.875 & 1.0000 & $\odot .4248 \mathrm{E}+07$ \\
\hline 14 & 214 & 246.675 & $1.0 \odot$ & $5 E+07$ \\
\hline 15 & 214.071 & 249.099 & 1.0000 & $\odot .4631 \mathrm{E}+07$ \\
\hline 16 & 217.556 & 251.531 & $1.000 \odot$ & $0.4722 \mathrm{E}+\odot 7$ \\
\hline 17 & 217.556 & 254.204 & 1.0000 & $\odot .4865 \mathrm{E}+07$ \\
\hline 18 & 224.225 & 256.753 & 1.0000 & $\odot .5079 \mathrm{E}+\odot 7$ \\
\hline 19 & 224.225 & 258.656 & $1.000 \odot$ & $0.5168 \mathrm{E}+07$ \\
\hline 20 & 226.939 & 260.392 & 1.0000 & $0.5296 \mathrm{E}+07$ \\
\hline 21 & 227.387 & 262.474 & $1.000 \odot$ & $\odot .5400 \mathrm{E}+\odot 7$ \\
\hline 22 & 229.076 & 263.746 & 1.0000 & $\odot .5444 \mathrm{E}+07$ \\
\hline 23 & 233.352 & 265.662 & 1.0000 & $\odot .5618 \mathrm{E}+07$ \\
\hline 24 & 233.352 & 267.037 & 1.0000 & $\odot .5667 \mathrm{E}+07$ \\
\hline 25 & 237.639 & 268.711 & 1.0000 & $\odot .5785 \mathrm{E}+07$ \\
\hline
\end{tabular}




\begin{tabular}{|c|c|c|c|c|}
\hline 26 & 237.737 & 269.749 & 1.0000 & $\odot .5873 \mathrm{E}+\Theta 7$ \\
\hline 27 & 237.862 & 270.605 & $1.000 \odot$ & $\odot .5889 E+07$ \\
\hline 28 & 241.165 & 271.461 & 1.0000 & $0.6013 \mathrm{E}+07$ \\
\hline 29 & 241.165 & 273.078 & 1.0000 & $0.6048 \mathrm{E}+07$ \\
\hline 30 & 243.389 & 273.950 & 1.0000 & $\odot .6120 \mathrm{E}+07$ \\
\hline 31 & 243.623 & 275.260 & $1.000 \odot$ & $\odot .6182 \mathrm{E}+07$ \\
\hline 32 & 243.623 & 275.715 & 1.0000 & $0.6186 \mathrm{E}+07$ \\
\hline 33 & 245.823 & 276.397 & 1.0000 & $0.6271 \mathrm{E}+07$ \\
\hline 34 & 245.823 & 276.835 & 1.0000 & $0.6304 \mathrm{E}+07$ \\
\hline 35 & 245.913 & 277.389 & $1.000 \odot$ & $0.6307 \mathrm{E}+07$ \\
\hline 36 & 247.326 & 278.129 & 1.0000 & $0.6372 \mathrm{E}+07$ \\
\hline 37 & 247.326 & 279.094 & $1.000 \odot$ & $0.6388 \mathrm{E}+07$ \\
\hline 38 & 247.475 & 279.681 & $1.000 \odot$ & $\odot .6392 \mathrm{E}+\odot 7$ \\
\hline 39 & 248.304 & 280.439 & 1.0000 & $0.6436 \mathrm{E}+07$ \\
\hline 40 & 248.304 & 280.841 & 1.0000 & $0.6446 \mathrm{E}+07$ \\
\hline 41 & 248.371 & 281.452 & 1.0000 & $\odot .6447 \mathrm{E}+07$ \\
\hline 42 & 248.547 & 281.717 & $1.000 \odot$ & $\odot .6469 \mathrm{E}+\odot 7$ \\
\hline 43 & 248.547 & 282.204 & 1.0000 & $0.6471 \mathrm{E}+07$ \\
\hline 44 & 248.547 & 282.481 & 1.0000 & $0.6474 \mathrm{E}+07$ \\
\hline 45 & 248.547 & 282.820 & 1.0000 & $0.6474 \mathrm{E}+07$ \\
\hline 46 & 248.547 & 283.024 & 1.0000 & $0.6480 \mathrm{E}+07$ \\
\hline 47 & 248.547 & 283.228 & $1.000 \odot$ & $0.6480 \mathrm{E}+07$ \\
\hline 48 & 248.547 & 283.420 & 1.0000 & $\odot .6481 \mathrm{E}+07$ \\
\hline 49 & 248.547 & 285.348 & 1.0000 & $0.6481 \mathrm{E}+07$ \\
\hline 50 & 248.547 & 285.348 & 1.0000 & $0.6481 \mathrm{E}+07$ \\
\hline time & sec) $=$ & \multicolumn{3}{|l|}{$24.00 \quad 1$} \\
\hline node & $\mathrm{T}(\mathrm{K})$ & Twall(K) & $\mathrm{X}$ & $\mathrm{P}(\mathrm{Pa})$ \\
\hline 1 & 130.479 & 172.912 & - - - - - & $0.6149 \mathrm{E}+06$ \\
\hline 2 & 151.355 & 190.782 & 0.9654 & $0.1134 \mathrm{E}+07$ \\
\hline 3 & 160.913 & 198.054 & 0.9854 & $0.1705 \mathrm{E}+07$ \\
\hline 4 & 167.080 & 204.351 & 0.9911 & $0.2109 \mathrm{E}+07$ \\
\hline 5 & 170.548 & 208.254 & $\odot .9943$ & $\odot .2413 \mathrm{E}+07$ \\
\hline 6 & 173.099 & 212.261 & 0.9974 & $0.2636 \mathrm{E}+\Theta 7$ \\
\hline 7 & 175.922 & 214.919 & $\odot .9993$ & $\odot .2884 \mathrm{E}+\odot 7$ \\
\hline 8 & 186.264 & 224.080 & $1.000 \odot$ & $\odot .3175 \mathrm{E}+\odot 7$ \\
\hline 9 & 205.964 & 230.509 & 1.0000 & $0.3510 \mathrm{E}+07$ \\
\hline 10 & 207.276 & 234.026 & 1.0000 & $\odot .3737 \mathrm{E}+07$ \\
\hline 11 & 207.276 & 236.507 & $1.000 \odot$ & $\odot .3927 \mathrm{E}+\odot 7$ \\
\hline 12 & 217.198 & 239.492 & $1.000 \odot$ & $\odot .4119 \mathrm{E}+\odot 7$ \\
\hline 13 & 217.198 & 242.654 & 1.0000 & $\odot .4310 \mathrm{E}+07$ \\
\hline 14 & 217.198 & 245.316 & 1.0000 & $\odot .4507 \mathrm{E}+07$ \\
\hline 15 & 218.180 & 247.767 & 1.0000 & $0.4603 \mathrm{E}+07$ \\
\hline 16 & 218.307 & 250.237 & 1.0000 & $0.4700 \mathrm{E}+07$ \\
\hline 17 & 220.341 & 252.787 & $1.000 \odot$ & $\odot .4911 \mathrm{E}+07$ \\
\hline 18 & 220 & 255.473 & 1.00 & $\odot .4927 \mathrm{E}+\odot 7$ \\
\hline 19 & 225.626 & 257.436 & 1.0000 & $\odot .5138 \mathrm{E}+07$ \\
\hline 20 & 225.626 & 259.171 & 1.0000 & $\odot .5250 \mathrm{E}+\Theta 7$ \\
\hline 21 & 228.328 & 261.254 & 1.0000 & $0.5313 \mathrm{E}+07$ \\
\hline 22 & 228.705 & 262.525 & $1.000 \odot$ & $\odot .5435 \mathrm{E}+\odot 7$ \\
\hline 23 & 228.895 & 264.441 & $1.000 \odot$ & $0.5477 \mathrm{E}+07$ \\
\hline 24 & 234.108 & 265.886 & $1.000 \odot$ & $\odot .5631 \mathrm{E}+\odot 7$ \\
\hline 25 & 234.157 & 267.501 & $1.000 \odot$ & $\odot .5695 \mathrm{E}+\odot 7$ \\
\hline 26 & 236.402 & 268.793 & 1.0000 & $\odot .5779 \mathrm{E}+07$ \\
\hline 27 & 238.160 & $269.6 \odot 4$ & 1.0000 & $\odot .5879 \mathrm{E}+07$ \\
\hline 28 & 238.160 & 270.545 & 1.0000 & $\odot .5897 \mathrm{E}+07$ \\
\hline 29 & 241.276 & 272.163 & 1.0000 & $\odot .5997 \mathrm{E}+07$ \\
\hline
\end{tabular}




\begin{tabular}{|c|c|c|c|c|}
\hline 30 & 241.495 & 273.034 & 1.0000 & $0.6062 \mathrm{E}+07$ \\
\hline 31 & 241.495 & 274.345 & 1.0000 & $0.6067 \mathrm{E}+07$ \\
\hline 32 & 243.793 & 274.800 & 1.0000 & $0.6158 \mathrm{E}+07$ \\
\hline 33 & 243.812 & 275.481 & $1.00 \odot \odot$ & $\odot .6197 \mathrm{E}+\odot 7$ \\
\hline 34 & 243.812 & 275.919 & $1.000 \odot$ & $\odot .6198 \mathrm{E}+\odot 7$ \\
\hline 35 & 245.733 & 276.502 & 1.0000 & $\odot .6276 \mathrm{E}+07$ \\
\hline 36 & 245.733 & 277.298 & 1.0000 & $\odot .6301 \mathrm{E}+07$ \\
\hline 37 & 245.733 & 278.435 & 1.0000 & $0.6301 \mathrm{E}+07$ \\
\hline 38 & 246.153 & 279.071 & 1.0000 & $0.6337 \mathrm{E}+07$ \\
\hline 39 & 246.153 & 279.829 & $1.00 \odot \odot$ & $0.6345 \mathrm{E}+07$ \\
\hline 40 & 246.153 & 280.230 & $1.000 \odot$ & $0.6345 \mathrm{E}+07$ \\
\hline 41 & 246.442 & 280.842 & 1.0000 & $0.6371 \mathrm{E}+07$ \\
\hline 42 & 246.442 & 281.107 & $1.000 \odot$ & $0.6376 \mathrm{E}+07$ \\
\hline 43 & 246.442 & 281.594 & 1.0000 & $0.6381 \mathrm{E}+07$ \\
\hline 44 & 246.442 & 281.871 & $1.000 \odot$ & $0.6387 E+07$ \\
\hline 45 & 246.452 & 282.210 & 1.0000 & $\odot .6393 \mathrm{E}+07$ \\
\hline 46 & 246.452 & 282.413 & $1.000 \odot$ & $0.6394 \mathrm{E}+07$ \\
\hline 47 & 246.452 & 282.617 & $1.000 \odot$ & $\odot .6397 \mathrm{E}+07$ \\
\hline 48 & 246.452 & 282.809 & 1.0000 & $\odot .6399 \mathrm{E}+\odot 7$ \\
\hline 49 & 246.452 & 285.043 & 1.0000 & $\odot .6399 \mathrm{E}+07$ \\
\hline 50 & 246.452 & 285.043 & $1.000 \odot$ & $\odot .6399 \mathrm{E}+07$ \\
\hline time & & \multicolumn{3}{|l|}{25.00} \\
\hline node & $\mathrm{T}(\mathrm{K})$ & Twall(K) & $\mathrm{X}$ & $P(P a)$ \\
\hline 1 & 130.296 & 171.081 & $-\ldots$ & $\odot .6080 \mathrm{E}+06$ \\
\hline 2 & 151.143 & 189.103 & 0.9649 & $0.1122 \mathrm{E}+07$ \\
\hline 3 & 160.510 & 196.243 & $\odot .9846$ & $0.1681 \mathrm{E}+07$ \\
\hline 4 & 166.279 & 202.656 & 0.9912 & $0.2039 \mathrm{E}+07$ \\
\hline 5 & 169.764 & 206.575 & 0.9955 & $\odot .2344 \mathrm{E}+07$ \\
\hline 6 & 172.992 & 210.430 & $\odot .9991$ & $\odot .2627 \mathrm{E}+\odot 7$ \\
\hline 7 & 175.921 & 213.088 & $\odot .9998$ & $\odot .2884 \mathrm{E}+07$ \\
\hline 8 & $188.9 \odot 2$ & 222.229 & $1.000 \odot$ & $\odot .3211 \mathrm{E}+07$ \\
\hline 9 & 195.669 & 229.094 & $1.000 \odot$ & $0.3335 \mathrm{E}+07$ \\
\hline 10 & .055 & 232.816 & 1.0000 & $0.3625 \mathrm{E}+07$ \\
\hline 11 & 209.011 & 235.188 & $1.000 \odot$ & $\odot .3943 \mathrm{E}+\odot 7$ \\
\hline 12 & 212.288 & 238.520 & 1.0000 & $\odot .4019 \mathrm{E}+07$ \\
\hline 13 & 216.346 & 241.580 & 1.0000 & $0.4246 \mathrm{E}+07$ \\
\hline 14 & 216.346 & 244.195 & 1.0000 & $0.4361 \mathrm{E}+07$ \\
\hline 15 & 218.277 & 246.654 & ८०९ & $0.4536 \mathrm{E}+07$ \\
\hline 16 & .268 & 248.998 & 1.0000 & $0.4707 \mathrm{E}+07$ \\
\hline 17 & 219.717 & 251.605 & $1.000 \odot$ & $\odot .4758 \mathrm{E}+\odot 7$ \\
\hline 18 & 222.815 & 254.226 & 1.0000 & $0.4968 \mathrm{E}+07$ \\
\hline 19 & 222.815 & 256.215 & 1.0000 & $\odot .5071 \mathrm{E}+07$ \\
\hline 20 & 225.990 & 257.950 & $1.000 \odot$ & $\odot .5154 \mathrm{E}+\odot 7$ \\
\hline 21 & 225.990 & 260.033 & $0 \odot \odot$ & $0.5254 \mathrm{E}+07$ \\
\hline 22 & 94 & 261.3 & $1.000 \odot$ & $0.5382 \mathrm{E}+07$ \\
\hline 23 & 229.512 & 263.221 & 1.0000 & $\odot .5479 \mathrm{E}+07$ \\
\hline 24 & 229.512 & 264.771 & 1.0000 & $\odot .5492 \mathrm{E}+07$ \\
\hline 25 & 234.285 & 266.478 & 1.0000 & $\odot .5638 \mathrm{E}+\Theta 7$ \\
\hline 26 & 234.808 & 267.608 & $1.000 \odot$ & $0.5736 \mathrm{E}+07$ \\
\hline 27 & 234.808 & 268.639 & 1.0000 & $0.5740 \mathrm{E}+07$ \\
\hline 28 & 238.582 & 269.6 & $1.0 \odot \odot \odot$ & $\odot .5863 \mathrm{E}+\odot 7$ \\
\hline 29 & 238.621 & 271.247 & $1.000 \odot$ & $\odot .5926 \mathrm{E}+\odot 7$ \\
\hline 30 & 239.020 & 272.119 & 1.0000 & $\odot .5938 \mathrm{E}+07$ \\
\hline 31 & 241.663 & 273.429 & $1.000 \odot$ & $0.6043 \mathrm{E}+07$ \\
\hline 32 & 241.711 & 273.884 & 1.0000 & $\odot .6086 \mathrm{E}+07$ \\
\hline 33 & 241.711 & 274.566 & $1.00 \odot \odot$ & $\odot .6087 \mathrm{E}+07$ \\
\hline
\end{tabular}




\begin{tabular}{|c|c|c|c|c|}
\hline 34 & 243.839 & 275.004 & 1.0000 & $\odot .6169 \mathrm{E}+07$ \\
\hline 35 & 243.839 & 275.625 & 1.0000 & $\odot .6198 \mathrm{E}+07$ \\
\hline 36 & 243.839 & 276.585 & 1.0000 & $\odot .6198 \mathrm{E}+07$ \\
\hline 37 & 244.248 & 277.699 & $1.00 \odot \odot$ & $0.6236 \mathrm{E}+\odot 7$ \\
\hline 38 & 244.248 & 278.461 & 1.0000 & $\odot .6246 \mathrm{E}+\odot 7$ \\
\hline 39 & 244.248 & 279.219 & 1.0000 & $\odot .6251 \mathrm{E}+07$ \\
\hline 40 & 244.392 & 279.620 & 1.0000 & $\odot .6276 \mathrm{E}+07$ \\
\hline 41 & 244.392 & 280.231 & 1.0000 & $\odot .6280 \mathrm{E}+07$ \\
\hline 42 & 244.392 & 280.496 & 1.0000 & $0.6283 \mathrm{E}+07$ \\
\hline 43 & 244.392 & 280.983 & $1.000 \odot$ & $0.6299 \mathrm{E}+07$ \\
\hline 44 & 244.392 & 281.260 & $1.000 \odot$ & $0.6303 \mathrm{E}+07$ \\
\hline 45 & 244.392 & 281.599 & 1.0000 & $0.6307 \mathrm{E}+07$ \\
\hline 46 & 244.399 & 281.803 & $1.000 \odot$ & $0.6313 \mathrm{E}+07$ \\
\hline 47 & 244.399 & 282.007 & 1.0000 & $0.6317 \mathrm{E}+07$ \\
\hline 48 & 244.399 & 282.199 & $1.00 \odot \odot$ & $\odot .6317 \mathrm{E}+07$ \\
\hline 49 & 244.399 & 284.737 & 1.0000 & $\odot .6317 \mathrm{E}+07$ \\
\hline 50 & 244.399 & 284.737 & $1.000 \odot$ & $0.6317 \mathrm{E}+07$ \\
\hline time & ec) $=$ & \multicolumn{3}{|l|}{$26.0 \odot$} \\
\hline node & $\mathrm{T}(\mathrm{K})$ & Twall(K) & $\mathrm{X}$ & $P(P a)$ \\
\hline 1 & 129.886 & 169.317 & $-\ldots$ & $\odot .5926 \mathrm{E}+06$ \\
\hline 2 & 150.668 & 187.512 & 0.9651 & $0.1093 \mathrm{E}+07$ \\
\hline 3 & 159.654 & 194.565 & ๑. 9856 & $\odot .1630 \mathrm{E}+07$ \\
\hline 4 & 166.073 & $20 \odot .978$ & $\odot .9923$ & $0.2020 \mathrm{E}+\odot 7$ \\
\hline 5 & 169.764 & 204.897 & 0.9966 & $\odot .2344 \mathrm{E}+\odot 7$ \\
\hline 6 & 172.992 & 208.717 & $\odot .9996$ & $0.2627 \mathrm{E}+\Theta 7$ \\
\hline 7 & 175.921 & 211.268 & 0.9986 & $\odot .2884 \mathrm{E}+07$ \\
\hline 8 & .250 & 220.773 & 000 & $020 E+07$ \\
\hline 9 & 203.848 & 227.801 & 1.0000 & $0.3393 \mathrm{E}+07$ \\
\hline 10 & 203.848 & 231.362 & $1.000 \odot$ & $\odot .3587 \mathrm{E}+\odot 7$ \\
\hline 11 & 214.601 & 234.188 & 1.0000 & $\odot .3848 \mathrm{E}+07$ \\
\hline 12 & 214.601 & 237.366 & $1.000 \odot$ & $0.4062 \mathrm{E}+07$ \\
\hline 13 & 217.462 & 240.618 & $1.000 \odot$ & $0.4231 \mathrm{E}+07$ \\
\hline 14 & .462 & 243.089 & 1.0000 & $\odot .4380 E+07$ \\
\hline 15 & 217.651 & 245.434 & $1.000 \odot$ & $\odot .4501 \mathrm{E}+\odot 7$ \\
\hline 16 & 220.321 & 247.917 & 1.0000 & $0.4571 \mathrm{E}+07$ \\
\hline 17 & 220.674 & 250.385 & 1.0000 & $0.4774 \mathrm{E}+07$ \\
\hline 18 & 220.674 & 253.005 & $1.00 \odot \odot$ & $\odot .4892 \mathrm{E}+07$ \\
\hline 19 & 222.177 & 254.994 & $1.000 \odot$ & $0.5010 \mathrm{E}+07$ \\
\hline 20 & .327 & 256.729 & 1.0000 & $0.5071 \mathrm{E}+07$ \\
\hline 21 & 225.558 & 258.812 & $1.000 \odot$ & $\odot .5213 \mathrm{E}+\odot 7$ \\
\hline 22 & 226.211 & 260.084 & 1.0000 & $\odot .5325 E+07$ \\
\hline 23 & 226.407 & 262.000 & 1.0000 & $\odot .5335 \mathrm{E}+07$ \\
\hline 24 & 230.598 & 263.603 & $1.000 \odot$ & $\odot .5481 \mathrm{E}+07$ \\
\hline 25 & 230.598 & 265.258 & & $0.5549 \mathrm{E}+07$ \\
\hline 26 & & 266.670 & 1.0000 & $0.56 \odot 4 \mathrm{E}+\odot 7$ \\
\hline 27 & 235.245 & 267.708 & 1.0000 & $\odot .5724 \mathrm{E}+07$ \\
\hline 28 & 235.245 & 268.714 & 1.0000 & $0.5769 \mathrm{E}+07$ \\
\hline 29 & 237.197 & 270.331 & 1.0000 & $0.5826 \mathrm{E}+\Theta 7$ \\
\hline 30 & 238.893 & 271.203 & $1.000 \odot$ & $\odot .5918 \mathrm{E}+07$ \\
\hline 31 & 238.904 & 272.514 & 1.0000 & $0.5957 \mathrm{E}+07$ \\
\hline 32 & 238.948 & 272.9 & 1.0000 & $\odot .5958 \mathrm{E}+07$ \\
\hline 33 & 241.756 & 273.650 & $1.000 \odot$ & $0.6057 \mathrm{E}+07$ \\
\hline 34 & 241.762 & 274.088 & 1.0000 & $\odot .6090 \mathrm{E}+\odot 7$ \\
\hline 35 & 241.762 & 274.869 & $1.000 \odot$ & $\odot .6090 \mathrm{E}+\odot 7$ \\
\hline 36 & 242.690 & 275.846 & 1.0000 & $\odot .6140 \mathrm{E}+07$ \\
\hline 37 & 242.690 & 277.089 & $1.00 \odot \odot$ & $\odot .6156 \mathrm{E}+07$ \\
\hline
\end{tabular}









$\begin{array}{lllll}42 & 241.377 & 279.276 & 1.00 \odot \odot & \odot .6144 \mathrm{E}+\odot 7 \\ 43 & 241.377 & 279.762 & 1.00 \odot \odot & \odot .6153 \mathrm{E}+\odot 7 \\ 44 & 241.377 & 280.04 \odot & 1.00 \odot \odot & \odot .6163 \mathrm{E}+\odot 7 \\ 45 & 241.377 & 280.378 & 1.00 \odot \odot & \odot .6164 \mathrm{E}+\odot 7 \\ 46 & 241.377 & 280.582 & 1.00 \odot \odot & 0.6169 \mathrm{E}+\odot 7 \\ 47 & 241.377 & 28 \odot .786 & 1.00 \odot \odot & \odot .6175 \mathrm{E}+\odot 7 \\ 48 & 241.377 & 281.372 & 1.00 \odot \odot & \odot .6175 \mathrm{E}+\odot 7 \\ 49 & 241.377 & 284.127 & 1.00 \odot \odot & \odot .6175 \mathrm{E}+\odot 7 \\ 5 \odot & 241.377 & 284.127 & 1.00 \odot \odot & 0.6175 \mathrm{E}+\odot 7\end{array}$

\begin{tabular}{|c|c|c|c|c|}
\hline \multicolumn{5}{|c|}{$.00 \quad$ lambda $=0.1847556233$} \\
\hline node & $T^{\prime}(\mathrm{K})$ & Twall(K) & $x$ & $\mathrm{P}(\mathrm{Pa})$ \\
\hline 1 & 129.606 & 166.085 & - - - - - & $0.5821 \mathrm{E}+06$ \\
\hline 2 & 150.343 & 184.490 & $\odot .9659$ & $\odot .1074 \mathrm{E}+\odot 7$ \\
\hline 3 & 159.493 & 191.341 & $\odot .9861$ & $\odot .1621 \mathrm{E}+\odot 7$ \\
\hline 4 & 165.674 & 197.869 & $\odot .9923$ & $\odot .1990 \mathrm{E}+07$ \\
\hline 5 & 168.989 & 201.763 & $\odot .9964$ & $\odot .2276 \mathrm{E}+\odot 7$ \\
\hline 6 & 172.022 & 205.552 & $\odot .999 \odot$ & $\odot .2542 \mathrm{E}+\odot 7$ \\
\hline 7 & 174.875 & 208.013 & $\odot .9973$ & $\odot .2792 \mathrm{E}+07$ \\
\hline 8 & 195.665 & 218.146 & 1.0000 & $\odot .3124 \mathrm{E}+07$ \\
\hline 9 & 200.579 & 225.557 & $1.00 \odot \odot$ & $0.3288 \mathrm{E}+07$ \\
\hline 10 & 218.698 & 229.728 & 1.0000 & $\odot .3703 \mathrm{E}+\odot 7$ \\
\hline 11 & 218.698 & 232.496 & 1.0000 & $\odot .3886 \mathrm{E}+07$ \\
\hline 12 & 218.698 & 235.684 & 1.0000 & $\odot .4 \odot 87 \mathrm{E}+07$ \\
\hline 13 & 218.698 & 238.645 & 1.0000 & $\odot .4237 \mathrm{E}+\odot 7$ \\
\hline 14 & 219.376 & 240.967 & $1.000 \odot$ & $\odot .4352 \mathrm{E}+07$ \\
\hline 15 & 222.522 & 243.539 & 1.0000 & $\odot .4454 \mathrm{E}+07$ \\
\hline 16 & 222.522 & 245.868 & 1.0000 & $\odot .4610 \mathrm{E}+\odot 7$ \\
\hline 17 & 222.744 & 248.226 & 1.0000 & $\odot .4668 \mathrm{E}+\odot 7$ \\
\hline 18 & 223.749 & 250.688 & 1.0000 & $\odot .4827 \mathrm{E}+\odot 7$ \\
\hline 19 & 223.749 & 252.654 & 1.0000 & $\odot .4952 \mathrm{E}+\odot 7$ \\
\hline 20 & 223.973 & 254.349 & 1.0000 & $\odot .5 \odot 22 \mathrm{E}+\odot 7$ \\
\hline 21 & 223.973 & 256.371 & 1.0000 & $\odot .5061 \mathrm{E}+07$ \\
\hline 22 & 224.166 & 257.643 & 1.0000 & $\odot .5159 \mathrm{E}+07$ \\
\hline 23 & 224.166 & 259.558 & 1.0000 & $\odot .5200 \mathrm{E}+\odot 7$ \\
\hline 24 & 227.607 & 261.315 & 1.0000 & $0.5317 \mathrm{E}+07$ \\
\hline 25 & 227.651 & 263.017 & 1.0000 & $\odot .5399 \mathrm{E}+07$ \\
\hline 26 & 227.901 & 264.506 & 1.0000 & $\odot .5428 \mathrm{E}+07$ \\
\hline 27 & 231.567 & 265.735 & 1.0000 & $\odot .5561 \mathrm{E}+07$ \\
\hline 28 & 231.803 & 266.840 & 1.0000 & $\odot .5621 \mathrm{E}+07$ \\
\hline 29 & 231.803 & 268.373 & 1.0000 & $\odot .5651 \mathrm{E}+07$ \\
\hline 30 & 234.083 & 269.372 & 1.0000 & $\odot .5706 \mathrm{E}+07$ \\
\hline 31 & 235.751 & 270.683 & 1.0000 & $\odot .5799 \mathrm{E}+07$ \\
\hline 32 & 235.769 & 271.138 & 1.0000 & $\odot .5817 \mathrm{E}+07$ \\
\hline 33 & 236.693 & 271.819 & 1.0000 & $\odot .5850 \mathrm{E}+07$ \\
\hline 34 & 239.069 & 272.257 & 1.0000 & $\odot .5940 \mathrm{E}+\odot 7$ \\
\hline 35 & 239.069 & 273.226 & 1.0000 & $\odot .5966 \mathrm{E}+07$ \\
\hline 36 & 239.069 & 274.398 & 1.0000 & $\odot .5966 \mathrm{E}+07$ \\
\hline 37 & 240.294 & 275.797 & 1.0000 & $\odot .6 \odot 24 \mathrm{E}+\odot 7$ \\
\hline 38 & 240.294 & 276.630 & 1.0000 & $0.6043 \mathrm{E}+07$ \\
\hline 39 & 240.294 & 277.388 & $1.000 \odot$ & $0.6046 \mathrm{E}+\odot 7$ \\
\hline 40 & 240.306 & 277.789 & 1.0000 & $\odot .6070 \mathrm{E}+\odot 7$ \\
\hline 41 & 240.306 & 278.400 & 1.0000 & $0.6076 \mathrm{E}+07$ \\
\hline 42 & 240.306 & 278.665 & 1.0000 & $\odot .6084 \mathrm{E}+07$ \\
\hline 43 & 240.306 & 279.152 & 1.0000 & $\odot .6097 \mathrm{E}+07$ \\
\hline 44 & 240.306 & 279.429 & 1.0000 & $\odot .6097 \mathrm{E}+07$ \\
\hline 45 & 240.306 & 279.768 & 1.0000 & $0.6106 \mathrm{E}+07$ \\
\hline
\end{tabular}




\begin{tabular}{|c|c|c|c|c|}
\hline 46 & 240.306 & 279.972 & 1.0000 & $\odot .6110 E+07$ \\
\hline 47 & 240.306 & 280.176 & 1.0000 & $0.6110 \mathrm{E}+07$ \\
\hline 48 & 240.306 & 281.067 & $1.000 \odot$ & $0.6111 \mathrm{E}+07$ \\
\hline 49 & 240.306 & 283.822 & $1.000 \odot$ & $0.6112 \mathrm{E}+07$ \\
\hline 50 & 240.306 & 283.822 & 1.0000 & $0.6112 \mathrm{E}+07$ \\
\hline time & & & \multicolumn{2}{|c|}{ lambda $=0.1829307526$} \\
\hline node & $\mathrm{T}(\mathrm{K})$ & Twall(K) & $\mathrm{x}$ & $P(P a)$ \\
\hline 1 & 129.443 & 164.559 & $-\ldots$ & $\odot .5760 \mathrm{E}+\odot 6$ \\
\hline 2 & 150.154 & 183.117 & $\odot .9659$ & $0.1062 \mathrm{E}+07$ \\
\hline 3 & 158.945 & 189.855 & $\odot .9864$ & $\odot .1588 \mathrm{E}+07$ \\
\hline 4 & 165.036 & 196.452 & $\odot .9930$ & $\odot .1952 \mathrm{E}+\odot 7$ \\
\hline 5 & 168.881 & 200.390 & $\odot .9968$ & $\odot .2266 \mathrm{E}+\odot 7$ \\
\hline 6 & 172.022 & 204.026 & 0.9986 & $0.2542 \mathrm{E}+\Theta 7$ \\
\hline 7 & 174.876 & 206.410 & $\odot .9956$ & $\odot .2792 \mathrm{E}+\odot 7$ \\
\hline 8 & 205.022 & 217.447 & 1.0000 & $\odot .3273 \mathrm{E}+07$ \\
\hline 9 & 211.384 & 224.818 & $1.000 \odot$ & $\odot .3465 \mathrm{E}+07$ \\
\hline 10 & 225.664 & 229.466 & 1.0000 & $\odot .3816 \mathrm{E}+07$ \\
\hline 11 & 225.664 & 232.101 & 1.0000 & $\odot .4009 \mathrm{E}+\odot 7$ \\
\hline 12 & 225.664 & 235.159 & 1.0000 & $\odot .4150 \mathrm{E}+07$ \\
\hline 13 & 225.664 & 238.045 & 1.0000 & $\odot .4286 \mathrm{E}+07$ \\
\hline 14 & 226.075 & 240.282 & 1.0000 & $0.4411 \mathrm{E}+07$ \\
\hline 15 & 226.075 & 242.773 & $1.000 \odot$ & $\odot .4525 E+\odot 7$ \\
\hline 16 & 226.075 & 245.031 & 1.0000 & $\odot .4639 \mathrm{E}+\odot 7$ \\
\hline 17 & 226.075 & 247.295 & 1.0000 & $0.4731 \mathrm{E}+07$ \\
\hline 18 & 226.075 & 249.676 & 1.0000 & $\odot .4861 \mathrm{E}+\odot 7$ \\
\hline 19 & 226.075 & 251.645 & 1.0000 & $\odot .4896 \mathrm{E}+07$ \\
\hline 20 & 226.075 & 253.333 & 1.0000 & $\odot .4980 \mathrm{E}+\odot 7$ \\
\hline 21 & 226.075 & 255.264 & 1.0000 & $\odot .5083 \mathrm{E}+\odot 7$ \\
\hline 22 & 226.075 & 256.612 & 1.0000 & $\odot .5152 \mathrm{E}+07$ \\
\hline 23 & 226.075 & 258.420 & 1.0000 & $\odot .5216 \mathrm{E}+07$ \\
\hline 24 & 226.075 & 260.111 & 1.0000 & $\odot .5267 \mathrm{E}+07$ \\
\hline 25 & 226.583 & 261.926 & 1.0000 & $\odot .5319 \mathrm{E}+07$ \\
\hline 26 & 228.233 & 263.317 & $1.0 \odot \odot \odot$ & $0.5421 E+07$ \\
\hline 27 & 228.233 & 264.610 & 1.0000 & $0.5463 \mathrm{E}+07$ \\
\hline 28 & 228.233 & 265.806 & $1.000 \odot$ & $0.5495 \mathrm{E}+07$ \\
\hline 29 & 231.430 & 267.429 & 1.0000 & $\odot .5574 \mathrm{E}+\odot 7$ \\
\hline 30 & 231.960 & 268.420 & 1.0000 & $0.5649 \mathrm{E}+07$ \\
\hline 31 & 231.960 & 269.767 & 1.0 & $\odot .5654 \mathrm{E}+07$ \\
\hline 32 & 234.342 & 270.222 & $1.000 \odot$ & $\odot .5729 \mathrm{E}+\odot 7$ \\
\hline 33 & 235.916 & $27 \odot .9 \odot 4$ & $1.0 \odot \odot \odot$ & $\odot .5815 \mathrm{E}+\odot 7$ \\
\hline 34 & 235.921 & 271.341 & 1.0000 & $\odot .5832 \mathrm{E}+\odot 7$ \\
\hline 35 & 236.768 & 272.441 & 1.0000 & $\odot .5854 \mathrm{E}+\odot 7$ \\
\hline 36 & 238.950 & 273.614 & 1.0000 & $\odot .5937 \mathrm{E}+07$ \\
\hline 37 & 238. & 274.9 & 1.0 & $\odot .5962 \mathrm{E}+\odot 7$ \\
\hline 38 & 238.950 & 276.019 & $1.000 \odot$ & $\odot .5962 \mathrm{E}+\odot 7$ \\
\hline 39 & 239.101 & 276.777 & $1.000 \odot$ & $\odot .5991 \mathrm{E}+\odot 7$ \\
\hline 40 & 239.101 & 277.178 & 1.0000 & $0.6001 \mathrm{E}+07$ \\
\hline 41 & 239.101 & 277.790 & 1.0000 & $\odot .6008 \mathrm{E}+\odot 7$ \\
\hline 42 & 239.101 & 278.055 & 1.0000 & $\odot .6026 \mathrm{E}+07$ \\
\hline 43 & 239.101 & 278.542 & 1.0000 & $0.6027 \mathrm{E}+07$ \\
\hline 44 & 239.101 & 278.819 & 1.0000 & $0.6037 \mathrm{E}+07$ \\
\hline 45 & 239.101 & 279.158 & $1.000 \odot$ & $0.6041 \mathrm{E}+07$ \\
\hline 46 & 239.101 & 279.361 & 1.0000 & $0.6041 \mathrm{E}+07$ \\
\hline 47 & 239.101 & 279.604 & 1.0000 & $0.6046 \mathrm{E}+\odot 7$ \\
\hline 48 & 239.101 & 280.762 & 1.0000 & $\odot .6046 \mathrm{E}+07$ \\
\hline 49 & 239.101 & 283.517 & 1.0000 & $0.6046 \mathrm{E}+07$ \\
\hline
\end{tabular}




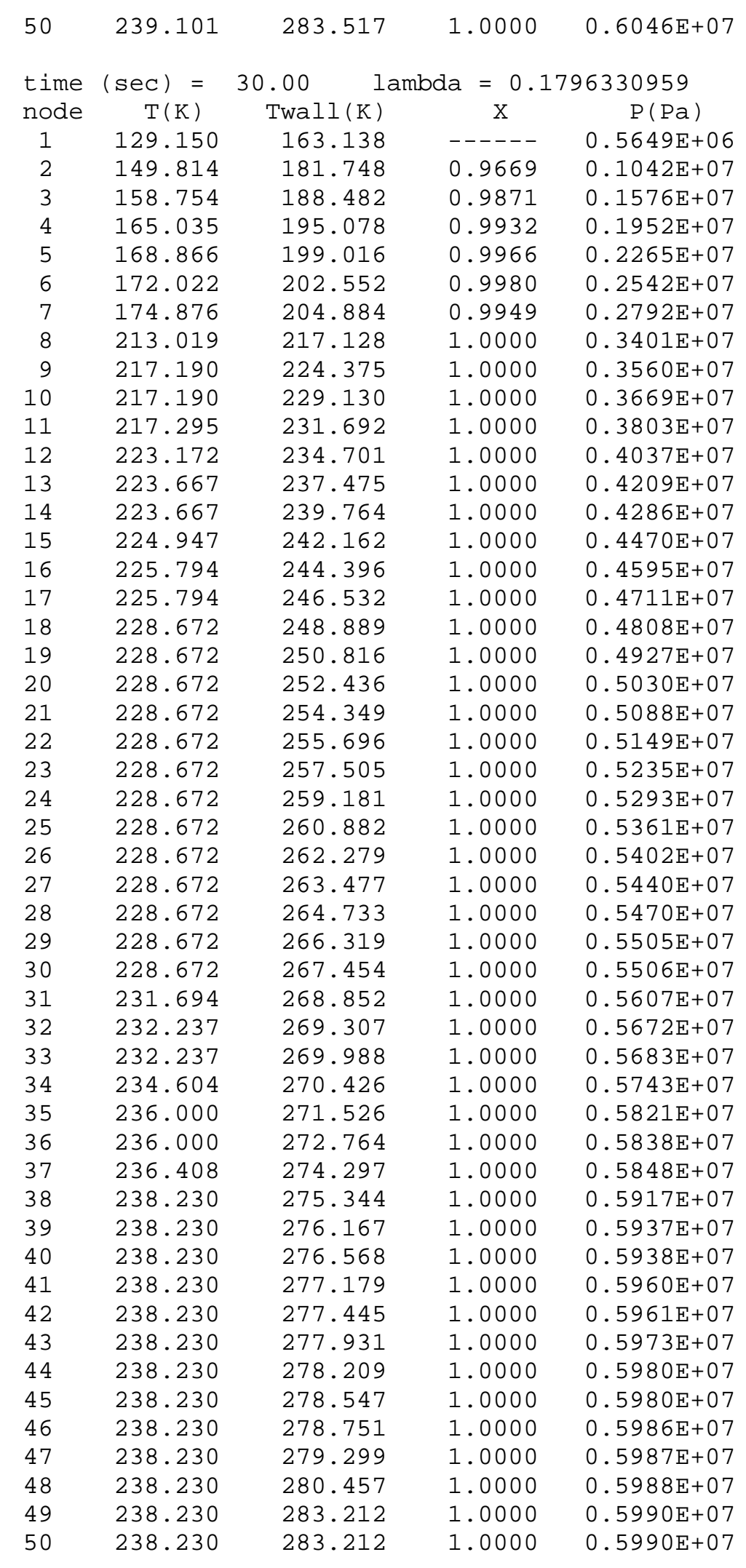

lambda_avg $=0.245157793$ 
Compressor station distance $=5$ miles, Pressure $=2100$ psi, 5 axial node elements

\begin{tabular}{|c|c|c|c|c|}
\hline time & $(\sec )=$ & $1.00 \quad 1$ & \multicolumn{2}{|c|}{ lambda $=0.8513534665$} \\
\hline node & $\mathrm{T}(\mathrm{K})$ & Twall(K) & ) $\quad x$ & $\mathrm{P}(\mathrm{Pa})$ \\
\hline 1 & 247.451 & 277.435 & $-\ldots$ & $\odot .6669 \mathrm{E}+07$ \\
\hline 2 & 287.043 & 288.000 & $1.0 \odot \odot \odot$ & $\odot .1230 E+08$ \\
\hline 3 & 288.000 & $288.00 \odot$ & 1.0000 & $\odot .1445 E+\odot 8$ \\
\hline 4 & 288.000 & $288.00 \odot$ & 1.0000 & $\odot .1448 \mathrm{E}+08$ \\
\hline 5 & 288.000 & 288.000 & 1.0000 & $\odot .1448 \mathrm{E}+08$ \\
\hline time & $(\sec )=$ & \multirow{2}{*}{$\begin{array}{c}2.00 \quad \text { lambda }=0.7250236869 \\
\text { Twall }(\mathrm{K})\end{array}$} & \multicolumn{2}{|c|}{ lambda $=0.7250236869$} \\
\hline node & $\mathrm{T}(\mathrm{K})$ & & ) $\quad x$ & $\mathrm{P}(\mathrm{Pa})$ \\
\hline 1 & 241.665 & 269.991 & 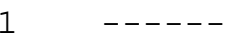 & $\odot .5613 \mathrm{E}+07$ \\
\hline 2 & 280.330 & 287.886 & 1.0000 & $\odot .1035 \mathrm{E}+08$ \\
\hline 3 & 287.973 & $288 . \odot \odot \odot$ & 1.0000 & $\odot .1433 E+08$ \\
\hline 4 & 288.000 & \multirow{2}{*}{$\begin{array}{l}288.00 \odot \\
288.000\end{array}$} & 1.0000 & $\odot .1448 \mathrm{E}+\odot 8$ \\
\hline 5 & 288.000 & & $1.0 \odot \odot \odot$ & $\odot .1448 E+08$ \\
\hline time & $(\sec )=$ & \multicolumn{3}{|l|}{$3.00 \quad 1$} \\
\hline node & $\mathrm{T}(\mathrm{K})$ & Twall(K) & $\mathrm{x}$ & $P(P a)$ \\
\hline 1 & 227.833 & \multirow{2}{*}{263.088} & 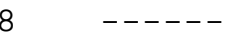 & $\odot .4652 \mathrm{E}+07$ \\
\hline 2 & 264.284 & & $1.000 \odot$ & $\odot .8581 \mathrm{E}+07$ \\
\hline 3 & 287.511 & \multirow{2}{*}{$\begin{array}{l}288.000 \\
288.00 \odot\end{array}$} & 1.0000 & $\odot .1404 \mathrm{E}+\odot 8$ \\
\hline 4 & 288.000 & & 1.0000 & $0.1446 \mathrm{E}+08$ \\
\hline 5 & 288.000 & 288.000 & 1.0000 & $\odot .1448 \mathrm{E}+08$ \\
\hline time & $(\sec )=$ & \multirow{2}{*}{$\begin{array}{ll}4.00 \quad 1 \\
\text { Twall (K) }\end{array}$} & \multicolumn{2}{|c|}{ lambda $=0.5314630866$} \\
\hline node & $\mathrm{T}(\mathrm{K})$ & & ) $\quad x$ & $\mathrm{P}(\mathrm{Pa})$ \\
\hline 1 & 207.465 & 255.063 & $-\ldots-$ & $0.3812 \mathrm{E}+07$ \\
\hline 2 & 240.657 & 284.300 & 1.0000 & $\odot .7032 \mathrm{E}+07$ \\
\hline 3 & 286.046 & 288.000 & 1.0000 & $\odot .1360 E+\odot 8$ \\
\hline 4 & 288.000 & 288.000 & 1.0000 & $\odot .1443 \mathrm{E}+08$ \\
\hline 5 & $288.0 \odot \odot$ & $288.00 \odot$ & $1 . \odot \odot \odot \odot$ & $\odot .1448 E+\odot 8$ \\
\hline time & $(\sec )=$ & $5.00 \quad 1$ & nbda & 20520473 \\
\hline node & $\mathrm{T}(\mathrm{K})$ & Twall(K) & $\mathrm{X}$ & $P(P a)$ \\
\hline 1 & 185.777 & 245.729 & - - - - - & $\odot .3136 \mathrm{E}+07$ \\
\hline 2 & 215.499 & 279.384 & $1.000 \odot$ & $0.5785 \mathrm{E}+07$ \\
\hline 3 & 282.752 & 287.997 & $1.00 \odot \odot$ & $0.1306 \mathrm{E}+08$ \\
\hline 4 & 287.865 & 288.000 & 1.0000 & $\odot .1435 \mathrm{E}+08$ \\
\hline 5 & 287.866 & 288.000 & 1.0000 & $\odot .1447 \mathrm{E}+08$ \\
\hline time & $(\sec )=$ & $6.00 \quad 1$ & lambda $=0.4$ & 94182253 \\
\hline node & $\mathrm{T}(\mathrm{K})$ & Twall(K) & $\mathrm{x}$ & $\mathrm{P}(\mathrm{Pa})$ \\
\hline 1 & 167.340 & 235.695 & $-\ldots$ & $\odot .2637 \mathrm{E}+07$ \\
\hline 2 & 194.113 & 272.270 & 1.0000 & $\odot .4865 \mathrm{E}+07$ \\
\hline 3 & 276.996 & 287.689 & $1.000 \odot$ & $\odot .1245 E+08$ \\
\hline 4 & 287.430 & 288.000 & $1.000 \odot$ & $\odot .1420 \mathrm{E}+08$ \\
\hline 5 & 287.435 & 288.000 & 1.0000 & $\odot .1443 \mathrm{E}+08$ \\
\hline
\end{tabular}




\begin{tabular}{|c|c|c|c|c|}
\hline time & $(\sec )=$ & $7.00 \quad 1$ & \multicolumn{2}{|c|}{ lambda $=0.3815264106$} \\
\hline node & $\mathrm{T}(\mathrm{K})$ & Twall(K) & ) $\quad x$ & $\mathrm{P}(\mathrm{Pa})$ \\
\hline 1 & 163.687 & 225.921 & 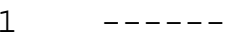 & $0.2431 \mathrm{E}+07$ \\
\hline 2 & 189.877 & 264.893 & $\odot .8566$ & $0.4484 \mathrm{E}+07$ \\
\hline 3 & 268.727 & 286.953 & 1.0000 & $\odot .1180 \mathrm{E}+08$ \\
\hline 4 & 286.433 & 288.000 & 1.0000 & $\odot .1397 \mathrm{E}+\odot 8$ \\
\hline 5 & 286.465 & $288.00 \odot$ & $1.000 \odot$ & $0.1436 \mathrm{E}+08$ \\
\hline time & $(\sec )=$ & $8.00 \quad l$ & \multicolumn{2}{|c|}{ lambda $=0.3581233919$} \\
\hline node & $\mathrm{T}(\mathrm{K})$ & Twall(K) & ) $\quad x$ & $P(P a)$ \\
\hline 1 & 161.654 & 217.607 & $-\ldots$ & $0.2268 \mathrm{E}+07$ \\
\hline 2 & 187.519 & 258.289 & 0.8608 & $\odot .4182 \mathrm{E}+\odot 7$ \\
\hline 3 & 258.588 & 285.533 & 1.0000 & $0.1117 \mathrm{E}+08$ \\
\hline 4 & 284.500 & 288.000 & 1.0000 & $0.1365 \mathrm{E}+08$ \\
\hline 5 & 284.593 & 288.000 & 1.0000 & $\odot .1422 \mathrm{E}+08$ \\
\hline time & $(\mathrm{sec})=$ & $9.00 \quad 1$ & \multicolumn{2}{|c|}{ lambda $=0.3367220461$} \\
\hline node & $\mathrm{T}(\mathrm{K})$ & Twall(K) & ) $\quad x$ & $P(P a)$ \\
\hline 1 & 159.730 & 210.369 & $--_{-}^{-}$ & $\odot .2119 \mathrm{E}+07$ \\
\hline 2 & 185.286 & 252.389 & $\odot .8677$ & $\odot .3909 \mathrm{E}+07$ \\
\hline 3 & 247.714 & 283.258 & $1.000 \odot$ & $0.1058 \mathrm{E}+08$ \\
\hline 4 & 281.292 & 287.955 & 1.0000 & $0.1325 E+08$ \\
\hline 5 & 281.540 & 287.951 & 1.0000 & $0.1400 E+08$ \\
\hline time & $(\sec )=$ & $10.00 \quad l$ & \multicolumn{2}{|c|}{ lambda $=0.3169826567$} \\
\hline node & $\mathrm{T}(\mathrm{K})$ & Twall(K) & ) $\quad x$ & $\mathrm{P}(\mathrm{Pa})$ \\
\hline 1 & 157.815 & 204.071 & 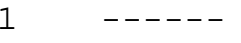 & $\odot .1983 \mathrm{E}+\odot 7$ \\
\hline 2 & 183.065 & 247.081 & $\odot .8749$ & $\odot .3658 \mathrm{E}+07$ \\
\hline 3 & 237.477 & 280.102 & 1.0000 & $\odot .1008 \mathrm{E}+08$ \\
\hline 4 & 276.570 & 287.650 & 1.0000 & $\odot .1278 \mathrm{E}+\odot 8$ \\
\hline 5 & 277.093 & 287.646 & 1.0000 & $0.1367 \mathrm{E}+08$ \\
\hline time & $(\sec )=$ & $11.00 \quad 1$ & \multicolumn{2}{|c|}{ lambda $=0.2989589870$} \\
\hline node & $\mathrm{T}(\mathrm{K})$ & Twall(K) & ) $\quad x$ & $P(P a)$ \\
\hline 1 & 156.085 & 198.653 & 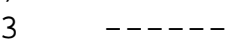 & $\odot .186 \odot \mathrm{E}+\odot 7$ \\
\hline 2 & 181.059 & 242.253 & $\odot .8821$ & $0.3431 \mathrm{E}+07$ \\
\hline 3 & 228.760 & 276.258 & 1.0000 & $0.9687 \mathrm{E}+07$ \\
\hline 4 & 270.306 & 287.133 & 1.0000 & $0.1226 \mathrm{E}+08$ \\
\hline 5 & 271.255 & 287.123 & 1.0000 & $\odot .1325 E+08$ \\
\hline time & $(\sec )=$ & $12.00 \quad 1$ & \multicolumn{2}{|c|}{ lambda $=0.2825615108$} \\
\hline node & $\mathrm{T}(\mathrm{K})$ & Twall(K) & ) $\quad x$ & $\mathrm{P}(\mathrm{Pa})$ \\
\hline 1 & 154.528 & 193.974 & 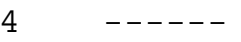 & $\odot .1749 \mathrm{E}+07$ \\
\hline 2 & 179.252 & 237.825 & 0.8888 & $0.3226 \mathrm{E}+07$ \\
\hline 3 & 221.786 & 272.006 & 1.0000 & $\odot .9381 \mathrm{E}+07$ \\
\hline 4 & 262.695 & 286.265 & 1.0000 & $0.1172 \mathrm{E}+08$ \\
\hline 5 & 264.199 & 286.246 & $1 . \odot \odot \odot \odot$ & $\odot .1274 \mathrm{E}+\odot 8$ \\
\hline time & $(\sec )=$ & $13.00 \quad 1$ & \multicolumn{2}{|c|}{ lambda $=0.2677095830$} \\
\hline node & $\mathrm{T}(\mathrm{K})$ & Twall(K) & $\mathrm{X}$ & $P(P a)$ \\
\hline 1 & 153.130 & 189.907 & $-\ldots$ & $\odot .1650 \mathrm{E}+\odot 7$ \\
\hline 2 & 177.630 & 233.723 & $\odot .8954$ & $\odot .3043 \mathrm{E}+07$ \\
\hline 3 & 216.471 & 267.733 & 1.0000 & $\odot .9148 \mathrm{E}+07$ \\
\hline 4 & 254.089 & 284.927 & 1.0000 & $0.1118 \mathrm{E}+08$ \\
\hline 5 & 256.240 & 284.910 & $1.00 \odot \odot$ & $0.1218 \mathrm{E}+08$ \\
\hline
\end{tabular}




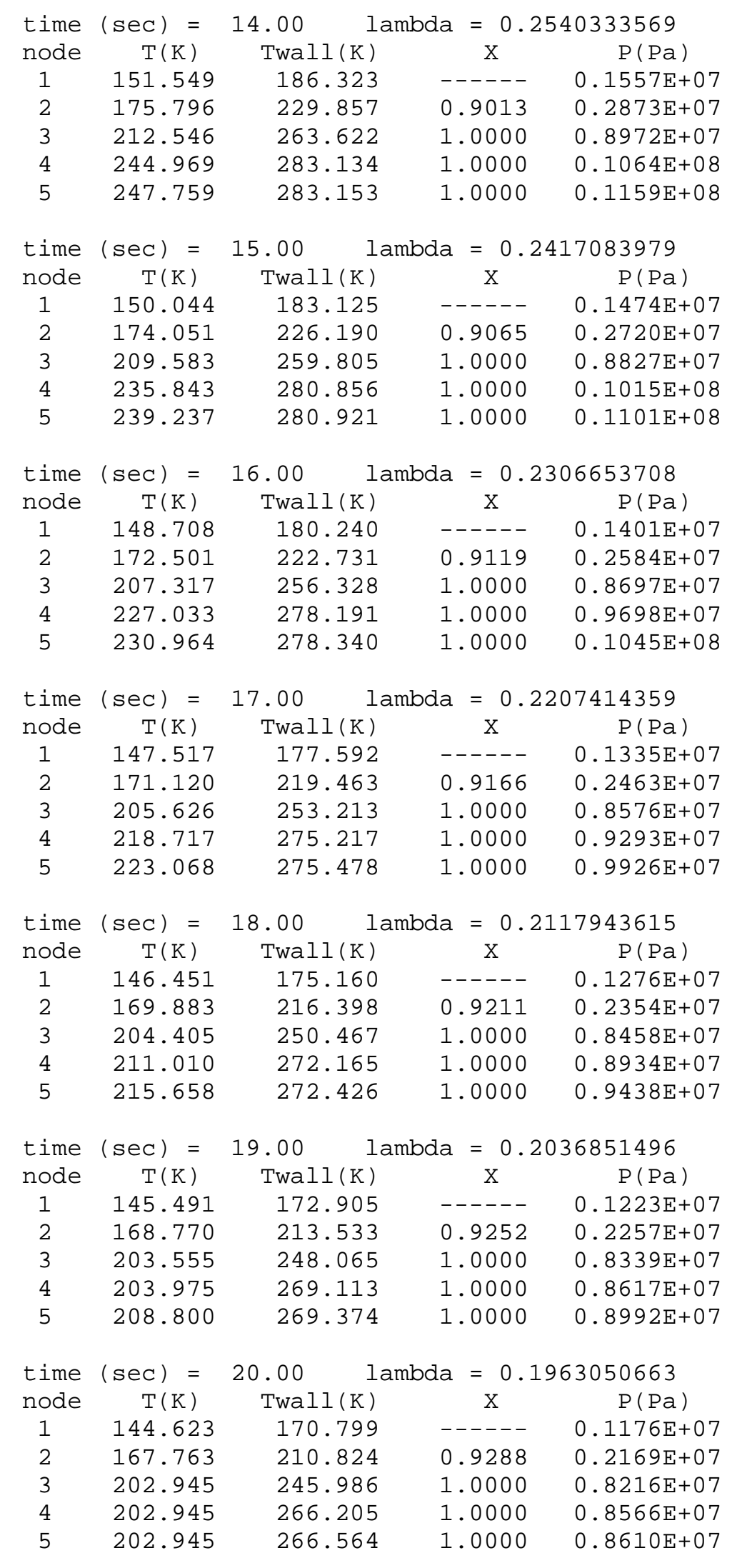




\begin{tabular}{|c|c|c|c|c|}
\hline time & ec) $=$ & & \multicolumn{2}{|c|}{ lambda $=0.1895456612$} \\
\hline node & $\mathrm{T}(\mathrm{K})$ & Twall(K) & $\mathrm{X}$ & $\mathrm{P}(\mathrm{Pa})$ \\
\hline 1 & 143.832 & 168.821 & 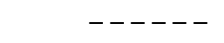 & $\odot .1132 \mathrm{E}+07$ \\
\hline 2 & 166.845 & 208.253 & $\odot .9323$ & $0.2088 \mathrm{E}+07$ \\
\hline 3 & 202.093 & 244.109 & 1.0000 & $\odot .8075 \mathrm{E}+07$ \\
\hline 4 & 202.093 & 263.676 & 1.0000 & $\odot .8516 \mathrm{E}+07$ \\
\hline 5 & 202.093 & 264.022 & $1.000 \odot$ & $\odot .8516 \mathrm{E}+07$ \\
\hline time & $(\sec )=$ & $22.00 \quad 1$ & \multicolumn{2}{|c|}{ lambda $=0.1832952052$} \\
\hline node & $\mathrm{T}(\mathrm{K})$ & Twall(K) & $\mathrm{x}$ & $P(P a)$ \\
\hline 1 & 143.105 & 166.990 & $-\ldots$ & $0.1092 \mathrm{E}+07$ \\
\hline 2 & 166.001 & 205.812 & $\odot .9353$ & $\odot .2014 \mathrm{E}+07$ \\
\hline 3 & 200.716 & 242.278 & $1.000 \odot$ & $\odot .7912 \mathrm{E}+\odot 7$ \\
\hline 4 & 200.716 & 261.491 & 1.0000 & $0.8434 \mathrm{E}+07$ \\
\hline 5 & 200.716 & 261.825 & 1.0000 & $\odot .8434 \mathrm{E}+07$ \\
\hline time & $(\sec )=$ & 23.00 & \multicolumn{2}{|c|}{ lambda $=0.1772957444$} \\
\hline node & $\mathrm{T}(\mathrm{K})$ & Twall(K) & $\mathrm{X}$ & $\mathrm{P}(\mathrm{Pa})$ \\
\hline 1 & 142.122 & 165.265 & $-\ldots$ & $0.1053 \mathrm{E}+07$ \\
\hline 2 & 164.861 & 203.514 & 0.9383 & $\odot .1942 \mathrm{E}+07$ \\
\hline 3 & 198.782 & 240.496 & 1.0000 & $\odot .7736 \mathrm{E}+07$ \\
\hline 4 & 198.782 & 259.539 & 1.0000 & $\odot .8320 \mathrm{E}+\odot 7$ \\
\hline 5 & 198.782 & 259.857 & 1.0000 & $\odot .8320 \mathrm{E}+07$ \\
\hline time & $(\sec )=$ & 24.00 & \multicolumn{2}{|c|}{ lambda $=0.1716509759$} \\
\hline node & $\mathrm{T}(\mathrm{K})$ & Twall(K) & $\mathrm{X}$ & $\mathrm{P}(\mathrm{Pa})$ \\
\hline 1 & 141.136 & 163.587 & - - - - - & $0.1016 \mathrm{E}+07$ \\
\hline 2 & 163.718 & 201.243 & $\odot .9408$ & $\odot .1873 \mathrm{E}+07$ \\
\hline 3 & 196.348 & 238.665 & 1.0000 & $\odot .7547 \mathrm{E}+\odot 7$ \\
\hline 4 & 196.348 & 257.708 & 1.0000 & $\odot .8175 \mathrm{E}+07$ \\
\hline 5 & 196.348 & 258.026 & 1.0000 & $\odot .8175 \mathrm{E}+07$ \\
\hline time & $(\sec )=$ & $25.0 \odot$ & \multicolumn{2}{|c|}{ lambda $=0.1663479954$} \\
\hline node & $\mathrm{T}(\mathrm{K})$ & Twall(K) & $\mathrm{X}$ & $P(P a)$ \\
\hline 1 & 140.216 & 161.986 & - - - - - & $0.9809 \mathrm{E}+06$ \\
\hline 2 & 162.651 & 199.107 & 0.9432 & $\odot .1809 \mathrm{E}+07$ \\
\hline 3 & 193.516 & 236.834 & 1.0000 & $0.7353 \mathrm{E}+07$ \\
\hline 4 & 193.516 & 256.103 & 1.0000 & $\odot .8006 \mathrm{E}+\odot 7$ \\
\hline 5 & 193.516 & 256.400 & $1.000 \odot$ & $0.8006 \mathrm{E}+07$ \\
\hline time & $(\sec )=$ & 26.00 & \multicolumn{2}{|c|}{ lambda $=0.1613388956$} \\
\hline node & $\mathrm{T}(\mathrm{K})$ & Twall(K) & $\mathrm{X}$ & $\mathrm{P}(\mathrm{Pa})$ \\
\hline 1 & 139.352 & 160.461 & 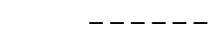 & $0.9485 \mathrm{E}+06$ \\
\hline 2 & 161.649 & 197.042 & 0.9456 & $\odot .1749 \mathrm{E}+07$ \\
\hline 3 & 190.404 & 235.003 & 1.0000 & $\odot .7157 \mathrm{E}+07$ \\
\hline 4 & 190.723 & 254.577 & 1.0000 & $0.7832 \mathrm{E}+\Theta 7$ \\
\hline 5 & 190.723 & 254.874 & $1.000 \odot$ & $\odot .7832 \mathrm{E}+07$ \\
\hline time & $(\sec )=$ & $27.00 \quad 1$ & \multicolumn{2}{|c|}{ lambda $=0.1565725803$} \\
\hline node & $\mathrm{T}(\mathrm{K})$ & Twall(K) & $\mathrm{X}$ & $\mathrm{P}(\mathrm{Pa})$ \\
\hline 1 & 138.535 & 159.054 & - - - - - & $0.9177 \mathrm{E}+06$ \\
\hline 2 & 160.701 & 195.059 & 0.9476 & $\odot .1693 \mathrm{E}+07$ \\
\hline 3 & 187.110 & 233.039 & 1.0000 & $\odot .6964 \mathrm{E}+\Theta 7$ \\
\hline 4 & 188.235 & 253.111 & 1.0000 & $\odot .7665 \mathrm{E}+\odot 7$ \\
\hline 5 & 188.236 & 253.394 & $1.000 \odot$ & $\odot .7677 \mathrm{E}+\odot 7$ \\
\hline
\end{tabular}




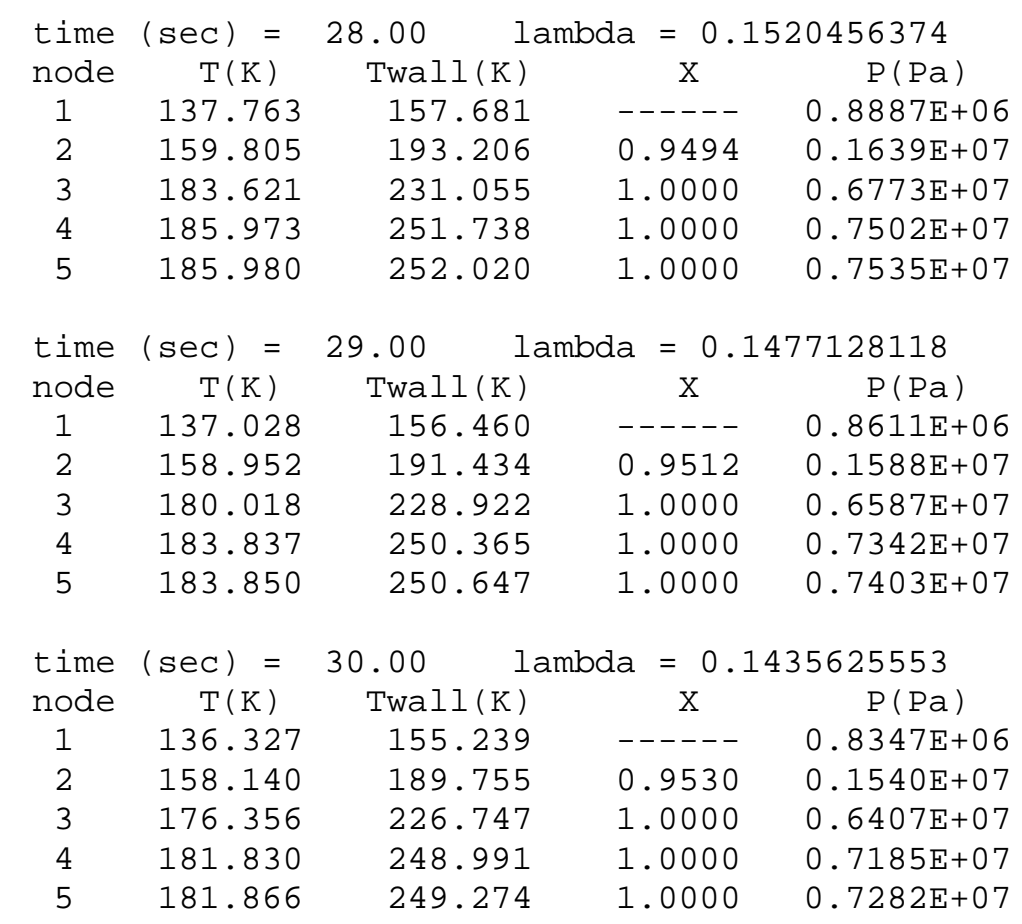

lambda_avg $=0.315512955$ 
Compressor station distance $=5$ miles, Pressure $=2100$ psi, 25 axial node elements

\begin{tabular}{|c|c|c|c|c|}
\hline time & $(\sec )=$ & $1.00 \quad 1$ & lambda $=0$. & 6956453 \\
\hline node & $\mathrm{T}(\mathrm{K})$ & Twall(K) & $\mathrm{x}$ & $P(P a)$ \\
\hline 1 & 166.460 & 275.450 & $\ldots$ & $\odot .2979 \mathrm{E}+07$ \\
\hline 2 & 193.077 & 284.864 & 1.0000 & $\odot .5495 \mathrm{E}+\odot 7$ \\
\hline 3 & 281.770 & 287.986 & $1.000 \odot$ & $\odot .1258 \mathrm{E}+\odot 8$ \\
\hline 4 & 287.788 & $288 . \odot \odot \odot$ & $1.00 \odot \odot$ & $\odot .1432 E+\odot 8$ \\
\hline 5 & 288.000 & $288.00 \odot$ & $1.00 \odot \odot$ & $\odot .1447 \mathrm{E}+\odot 8$ \\
\hline 6 & $288.0 \odot \odot$ & $288.00 \odot$ & $1.000 \odot$ & $\odot .1448 \mathrm{E}+\odot 8$ \\
\hline 7 & $288 . \odot \odot \odot$ & $288.00 \odot$ & $1.000 \odot$ & $\odot .1448 \mathrm{E}+\odot 8$ \\
\hline 8 & $288 . \odot \odot \odot$ & $288.00 \odot$ & $1.0 \odot \odot \odot$ & $\odot .1448 \mathrm{E}+\odot 8$ \\
\hline 9 & $288.0 \odot \odot$ & $288.00 \odot$ & 1.0000 & $\odot .1448 \mathrm{E}+\odot 8$ \\
\hline 10 & $288 . \odot \odot \odot$ & $288 . \odot \odot \odot$ & $1.00 \odot \odot$ & $\odot .1448 \mathrm{E}+\odot 8$ \\
\hline 11 & 288.000 & 288.000 & 1.0000 & $\odot .1448 \mathrm{E}+\odot 8$ \\
\hline 12 & 288.000 & 288.000 & 1.0000 & $\odot .1448 \mathrm{E}+08$ \\
\hline 13 & $288 . \odot \odot \odot$ & 288.000 & $1.00 \odot \odot$ & $\odot .1448 \mathrm{E}+08$ \\
\hline 14 & $288.0 \odot \odot$ & $288.0 \odot \odot$ & $1.0 \odot \odot \odot$ & $\odot .1448 \mathrm{E}+\odot 8$ \\
\hline 15 & $288.00 \odot$ & 288.000 & 1.0000 & $\odot .1448 \mathrm{E}+\odot 8$ \\
\hline 16 & $288 . \odot \odot \odot$ & $288 . \odot \odot \odot$ & $1.000 \odot$ & $\odot .1448 \mathrm{E}+\odot 8$ \\
\hline 17 & $288 . \odot \odot \odot$ & $288.00 \odot$ & $1.00 \odot \odot$ & $\odot .1448 \mathrm{E}+\odot 8$ \\
\hline 18 & $288.00 \odot$ & 288.000 & 1.0000 & $0.1448 \mathrm{E}+08$ \\
\hline 19 & $288.0 \odot \odot$ & $288 . \odot \odot \odot$ & $1.000 \odot$ & $\odot .1448 \mathrm{E}+\odot 8$ \\
\hline 20 & $288 . \odot \odot \odot$ & $288 . \odot \odot \odot$ & $1.00 \odot \odot$ & $\odot .1448 \mathrm{E}+\odot 8$ \\
\hline 21 & $288 . \odot \odot \odot$ & $288 . \odot \odot \odot$ & $1.00 \odot \odot$ & $\odot .1448 \mathrm{E}+\odot 8$ \\
\hline 22 & $288.00 \odot$ & $288.00 \odot$ & $1.000 \odot$ & $\odot .1448 \mathrm{E}+\odot 8$ \\
\hline 23 & 288.000 & 288.000 & 1.0000 & $\odot .1448 \mathrm{E}+08$ \\
\hline 24 & $288.00 \odot$ & 288.000 & 1.0000 & $\odot .1448 \mathrm{E}+08$ \\
\hline 25 & $288.0 \odot \odot$ & 288.000 & 1.0000 & $\odot .1448 \mathrm{E}+\odot 8$ \\
\hline time & $(\sec )=$ & $2.00 \quad$ lambda $=0.4411576390$ & \multicolumn{2}{|c|}{ lambda $=0.4411576390$} \\
\hline node & $\mathrm{T}(\mathrm{K})$ & Twall(K) & $\mathrm{X}$ & $\mathrm{P}(\mathrm{Pa})$ \\
\hline 1 & 161.769 & 257.302 & - . - . - & $\odot .2794 \mathrm{E}+\odot 7$ \\
\hline 2 & 187.655 & 269.135 & 0.0306 & $\odot .5154 \mathrm{E}+\odot 7$ \\
\hline 3 & 197.292 & 283.436 & $1.000 \odot$ & $\odot .7071 \mathrm{E}+\odot 7$ \\
\hline 4 & 269.845 & 287.811 & 1.0000 & $\odot .1168 \mathrm{E}+\odot 8$ \\
\hline 5 & 286.273 & $288 . \odot \odot \odot$ & $1.000 \odot$ & $\odot .1398 \mathrm{E}+\odot 8$ \\
\hline 6 & 287.928 & $288.00 \odot$ & $1.000 \odot$ & $0.1443 \mathrm{E}+08$ \\
\hline 7 & $288.00 \odot$ & 288.000 & 1.0000 & $\odot .1447 \mathrm{E}+\odot 8$ \\
\hline 8 & $288.00 \odot$ & 288.000 & 1.0000 & $\odot .1448 \mathrm{E}+08$ \\
\hline 9 & $288 . \odot \odot \odot$ & $288.00 \odot$ & $1.00 \odot \odot$ & $\odot .1448 \mathrm{E}+\odot 8$ \\
\hline 10 & $288 . \odot \odot \odot$ & $288 . \odot \odot \odot$ & $1.0 \odot \odot \odot$ & $\odot .1448 \mathrm{E}+\odot 8$ \\
\hline 11 & $288.00 \odot$ & 288.000 & 1.0000 & $\odot .1448 \mathrm{E}+\odot 8$ \\
\hline 12 & $288.00 \odot$ & $288.00 \odot$ & $1.000 \odot$ & $\odot .1448 \mathrm{E}+\odot 8$ \\
\hline 13 & $288.0 \odot \odot$ & 288.000 & 1.0000 & $\odot .1448 \mathrm{E}+08$ \\
\hline 14 & $288 . \odot \odot \odot$ & $288 . \odot \odot \odot$ & $1.0 \odot \odot \odot$ & $\odot .1448 \mathrm{E}+\odot 8$ \\
\hline 15 & $288 . \odot \odot \odot$ & $288.00 \odot$ & $1.000 \odot$ & $\odot .1448 \mathrm{E}+\odot 8$ \\
\hline 16 & $288 . \odot \odot \odot$ & $288.00 \odot$ & $1.000 \odot$ & $\odot .1448 \mathrm{E}+\odot 8$ \\
\hline 17 & 288.000 & 288.000 & 1.0000 & $\odot .1448 \mathrm{E}+08$ \\
\hline 18 & $288.0 \odot \odot$ & $288 . \odot \odot \odot$ & $1.000 \odot$ & $\odot .1448 \mathrm{E}+\odot 8$ \\
\hline 19 & $288.0 \odot \odot$ & $288.00 \odot$ & 1.0000 & $\odot .1448 \mathrm{E}+\odot 8$ \\
\hline 20 & $288 . \odot \odot \odot$ & $288.00 \odot$ & $1.0 \odot \odot \odot$ & $\odot .1448 E+\odot 8$ \\
\hline 21 & $288 . \odot \odot \odot$ & $288 . \odot \odot \odot$ & $1.000 \odot$ & $\odot .1448 \mathrm{E}+\odot 8$ \\
\hline 22 & $288 . \odot \odot \odot$ & $288.00 \odot$ & $1.0 \odot \odot \odot$ & $\odot .1448 \mathrm{E}+\odot 8$ \\
\hline 23 & $288.00 \odot$ & 288.000 & 1.0000 & $\odot .1448 \mathrm{E}+\odot 8$ \\
\hline 24 & $288.0 \odot \odot$ & $288.00 \odot$ & $1.000 \odot$ & $\odot .1448 \mathrm{E}+\odot 8$ \\
\hline 25 & $288 . \odot \odot \odot$ & $288 . \odot \odot \odot$ & $1.000 \odot$ & $\odot .1448 \mathrm{E}+\odot 8$ \\
\hline
\end{tabular}




\begin{tabular}{|c|c|c|c|c|}
\hline time & $(\sec )=$ & & \multicolumn{2}{|c|}{ lambda $=0.3848655522$} \\
\hline node & $\mathrm{T}(\mathrm{K})$ & Twall(K) & $x$ & $\mathrm{P}(\mathrm{Pa})$ \\
\hline 1 & 188.431 & 246.123 & $-\ldots$ & $\odot .2631 \mathrm{E}+07$ \\
\hline 2 & 218.579 & 261.800 & 0.0306 & $\odot .4853 \mathrm{E}+07$ \\
\hline 3 & 220.068 & 272.812 & 1.0000 & $\odot .7887 \mathrm{E}+07$ \\
\hline 4 & 220.068 & 282.002 & 1.0000 & $\odot .8458 \mathrm{E}+07$ \\
\hline 5 & 254.207 & 287.299 & 1.0000 & $\odot .1074 \mathrm{E}+08$ \\
\hline 6 & 282.337 & 288.000 & 1.0000 & $\odot .1344 \mathrm{E}+08$ \\
\hline 7 & 287.476 & 288.000 & 1.0000 & $\odot .1432 \mathrm{E}+08$ \\
\hline 8 & 288.000 & 288.000 & 1.0000 & $\odot .1446 \mathrm{E}+08$ \\
\hline 9 & 288.000 & 288.000 & 1.0000 & $0.1448 \mathrm{E}+08$ \\
\hline 10 & 288.000 & 288.000 & 1.0000 & $0.1448 \mathrm{E}+08$ \\
\hline 11 & 288.000 & 288.000 & 1.0000 & $0.1448 \mathrm{E}+08$ \\
\hline 12 & 288.000 & 288.000 & 1.0000 & $\odot .1448 \mathrm{E}+08$ \\
\hline 13 & $288.00 \odot$ & $288.00 \odot$ & $1.000 \odot$ & $0.1448 \mathrm{E}+08$ \\
\hline 14 & 288.000 & 288.000 & 1.0000 & $\odot .1448 \mathrm{E}+08$ \\
\hline 15 & 288.000 & 288.000 & 1.0000 & $\odot .1448 \mathrm{E}+08$ \\
\hline 16 & 288.000 & 288.000 & 1.0000 & $\odot .1448 \mathrm{E}+08$ \\
\hline 17 & 288.000 & 288.000 & 1.0000 & $\odot .1448 \mathrm{E}+08$ \\
\hline 18 & 288.000 & 288.000 & 1.0000 & $\odot .1448 \mathrm{E}+08$ \\
\hline 19 & $288.0 \odot \odot$ & $288 . \odot \odot \odot$ & $1.000 \odot$ & $0.1448 \mathrm{E}+08$ \\
\hline 20 & $288.00 \odot$ & $288.0 \odot \odot$ & $1.000 \odot$ & $\odot .1448 \mathrm{E}+08$ \\
\hline 21 & $288.00 \odot$ & 288.000 & 1.0000 & $0.1448 \mathrm{E}+08$ \\
\hline 22 & 288.000 & 288.000 & 1.0000 & $0.1448 \mathrm{E}+08$ \\
\hline 23 & 288.000 & 288.000 & 1.0000 & $\odot .1448 \mathrm{E}+\odot 8$ \\
\hline 24 & $288.00 \odot$ & 288.000 & $1.000 \odot$ & $0.1448 \mathrm{E}+08$ \\
\hline 25 & $288 . \odot \odot \odot$ & $288 . \odot \odot \odot$ & $1.00 \odot \odot$ & $0.1448 \mathrm{E}+08$ \\
\hline time & $(\sec )=$ & 4.00 & $\mathrm{da}=$ & 79782832 \\
\hline node & $\mathrm{T}(\mathrm{K})$ & Twall(K) & $X$ & $P(P a)$ \\
\hline 1 & 158.879 & 235.862 & - - - - - & $\odot .2059 \mathrm{E}+07$ \\
\hline 2 & 184.299 & 253.676 & ๑. 3694 & $\odot .3797 \mathrm{E}+07$ \\
\hline 3 & 226.999 & 267.750 & 1.0000 & $\odot .7548 \mathrm{E}+07$ \\
\hline 4 & 227.359 & 275.508 & $1.0 \odot \odot \odot$ & $\odot .8738 \mathrm{E}+07$ \\
\hline 5 & 227.359 & 281.115 & 1.0000 & $\odot .9386 \mathrm{E}+\odot 7$ \\
\hline 6 & 237.460 & 286.225 & 1.0000 & $\odot .1014 \mathrm{E}+08$ \\
\hline 7 & 274.785 & 287.886 & 1.0000 & $0.1267 \mathrm{E}+08$ \\
\hline 8 & 286.079 & $288.00 \odot$ & $1.000 \odot$ & $\odot .1407 \mathrm{E}+\odot 8$ \\
\hline 9 & 287.868 & 288.000 & $1.000 \odot$ & $0.1442 \mathrm{E}+08$ \\
\hline 10 & $288.00 \odot$ & $288.00 \odot$ & $1.000 \odot$ & $0.1447 \mathrm{E}+08$ \\
\hline 11 & 288.000 & 288.000 & 1.0000 & $\odot .1448 \mathrm{E}+08$ \\
\hline 12 & 288.000 & $288.00 \odot$ & 1.0000 & $\odot .1448 \mathrm{E}+08$ \\
\hline 13 & 288.000 & 288.000 & 1.0000 & $\odot .1448 \mathrm{E}+08$ \\
\hline 14 & $288.0 \odot \odot$ & $288 . \odot \odot \odot$ & $1.00 \odot \odot$ & $\odot .1448 \mathrm{E}+08$ \\
\hline 15 & 288.000 & 288.000 & 1.0000 & $\odot .1448 \mathrm{E}+08$ \\
\hline 16 & 288.000 & 288.000 & 1.0000 & $0.1448 E+08$ \\
\hline 17 & 288.000 & 288.000 & 1.0000 & $0.1448 \mathrm{E}+08$ \\
\hline 18 & $288.0 \odot \odot$ & $288.00 \odot$ & $1.000 \odot$ & $0.1448 \mathrm{E}+08$ \\
\hline 19 & 288.000 & $288.00 \odot$ & $1.000 \odot$ & $0.1448 \mathrm{E}+08$ \\
\hline 20 & $288.00 \odot$ & $288 . \odot \odot \odot$ & $1.000 \odot$ & $0.1448 \mathrm{E}+08$ \\
\hline 21 & $288.0 \odot \odot$ & $288 . \odot \odot \odot$ & $1.000 \odot$ & $0.1448 \mathrm{E}+08$ \\
\hline 22 & 288.000 & 288.000 & 1.0000 & $\odot .1448 \mathrm{E}+08$ \\
\hline 23 & 288.000 & $288.00 \odot$ & $1.000 \odot$ & $\odot .1448 \mathrm{E}+08$ \\
\hline 24 & 288.000 & 288.000 & 1.0000 & $\odot .1448 \mathrm{E}+08$ \\
\hline 25 & $288.0 \odot \odot$ & $288 . \odot \odot \odot$ & $1.000 \odot$ & $0.1448 \mathrm{E}+08$ \\
\hline
\end{tabular}




\begin{tabular}{|c|c|c|c|c|}
\hline time & $(\sec )=$ & $5.00 \quad 1$ & \multicolumn{2}{|c|}{ lambda $=0.3159569800$} \\
\hline node & $\mathrm{T}^{\prime}(\mathrm{K})$ & Twall(K) & $x$ & $\mathrm{P}(\mathrm{Pa})$ \\
\hline 1 & 157.716 & 226.493 & $-\ldots$ & $\odot .1976 \mathrm{E}+07$ \\
\hline 2 & 182.950 & 245.823 & 0.6212 & $\odot .3645 \mathrm{E}+07$ \\
\hline 3 & 212.493 & 263.359 & 1.0000 & $0.6373 \mathrm{E}+07$ \\
\hline 4 & 225.503 & 270.934 & $1.000 \odot$ & $\odot .8508 \mathrm{E}+\odot 7$ \\
\hline 5 & 230.329 & 276.040 & 1.0000 & $\odot .9465 \mathrm{E}+07$ \\
\hline 6 & 230.961 & 280.590 & 1.0000 & $\odot .9856 \mathrm{E}+07$ \\
\hline 7 & 230.961 & 284.808 & 1.0000 & $0.1011 \mathrm{E}+08$ \\
\hline 8 & 263.027 & 287.565 & 1.0000 & $\odot .1186 \mathrm{E}+08$ \\
\hline 9 & 282.800 & 288.000 & 1.0000 & $0.1364 \mathrm{E}+08$ \\
\hline 10 & 287.394 & 288.000 & 1.0000 & $\odot .1432 E+08$ \\
\hline 11 & 287.993 & 288.000 & 1.0000 & $\odot .1446 \mathrm{E}+08$ \\
\hline 12 & 288.000 & 288.000 & 1.0000 & $0.1448 \mathrm{E}+08$ \\
\hline 13 & $288.00 \odot$ & $288.00 \odot$ & $1.000 \odot$ & $\odot .1448 \mathrm{E}+08$ \\
\hline 14 & 288.000 & 288.000 & 1.0000 & $\odot .1448 \mathrm{E}+08$ \\
\hline 15 & 288.000 & 288.000 & 1.0000 & $\odot .1448 \mathrm{E}+08$ \\
\hline 16 & 288.000 & 288.000 & 1.0000 & $\odot .1448 \mathrm{E}+\odot 8$ \\
\hline 17 & 288.000 & 288.000 & 1.0000 & $\odot .1448 \mathrm{E}+08$ \\
\hline 18 & 288.000 & 288.000 & 1.0000 & $\odot .1448 \mathrm{E}+08$ \\
\hline 19 & $288.0 \odot \odot$ & $288.00 \odot$ & $1.000 \odot$ & $\odot .1448 \mathrm{E}+08$ \\
\hline 20 & $288.00 \odot$ & $288.00 \odot$ & $1.000 \odot$ & $0.1448 \mathrm{E}+08$ \\
\hline 21 & $288.00 \odot$ & $288.00 \odot$ & 1.0000 & $\odot .1448 \mathrm{E}+08$ \\
\hline 22 & $288.00 \odot$ & 288.000 & 1.0000 & $\odot .1448 \mathrm{E}+08$ \\
\hline 23 & 288.000 & 288.000 & 1.0000 & $\odot .1448 \mathrm{E}+\odot 8$ \\
\hline 24 & 288.000 & $288.00 \odot$ & $1.000 \odot$ & $\odot .1448 \mathrm{E}+08$ \\
\hline 25 & $288 . \odot \odot \odot$ & $288.0 \odot \odot$ & $1.00 \odot \odot$ & $\odot .1448 \mathrm{E}+08$ \\
\hline time & $(\sec )=$ & $6.00 \quad l$ & $\mathrm{da}=$ & 5358422 \\
\hline node & $\mathrm{T}(\mathrm{K})$ & Twall(K) & $X$ & $P(P a)$ \\
\hline 1 & 154.809 & 218.610 & - - - - - & $\odot .1769 \mathrm{E}+07$ \\
\hline 2 & 179.578 & 239.545 & $\odot .7667$ & $\odot .3263 \mathrm{E}+07$ \\
\hline 3 & 191.680 & 256.068 & 1.0000 & $\odot .5710 \mathrm{E}+07$ \\
\hline 4 & 207.965 & 265.778 & $1.0 \odot \odot \odot$ & $\odot .7383 \mathrm{E}+07$ \\
\hline 5 & 227.822 & 272.039 & 1.0000 & $\odot .9006 \mathrm{E}+07$ \\
\hline 6 & 232.676 & 276.470 & 1.0000 & $\odot .9880 \mathrm{E}+07$ \\
\hline 7 & 233.972 & 280.311 & 1.0000 & $0.1024 \mathrm{E}+08$ \\
\hline 8 & 234.480 & 283.828 & $1.000 \odot$ & $\odot .1047 \mathrm{E}+08$ \\
\hline 9 & 249.103 & 286.841 & $1.000 \odot$ & $\odot .1128 \mathrm{E}+08$ \\
\hline 10 & 276.459 & 287.917 & $1.000 \odot$ & $\odot .1300 \mathrm{E}+08$ \\
\hline 11 & 286.073 & 288.000 & 1.0000 & $\odot .1411 \mathrm{E}+08$ \\
\hline 12 & 287.851 & $288.00 \odot$ & 1.0000 & $\odot .1442 \mathrm{E}+08$ \\
\hline 13 & 288.000 & 288.000 & 1.0000 & $\odot .1447 \mathrm{E}+08$ \\
\hline 14 & $288.0 \odot \odot$ & $288.0 \odot \odot$ & $1.00 \odot \odot$ & $\odot .1448 \mathrm{E}+08$ \\
\hline 15 & 288.000 & $288.00 \odot$ & 1.0000 & $\odot .1448 \mathrm{E}+08$ \\
\hline 16 & 288.000 & $288.00 \odot$ & 1.0000 & $\odot .1448 \mathrm{E}+08$ \\
\hline 17 & 288.000 & 288.000 & 1.0000 & $\odot .1448 \mathrm{E}+08$ \\
\hline 18 & $288.0 \odot \odot$ & $288.00 \odot$ & $1.000 \odot$ & $0.1448 \mathrm{E}+08$ \\
\hline 19 & 288.000 & $288.00 \odot$ & $1.000 \odot$ & $\odot .1448 \mathrm{E}+08$ \\
\hline 20 & $288.00 \odot$ & $288.00 \odot$ & $1.000 \odot$ & $0.1448 \mathrm{E}+08$ \\
\hline 21 & $288.0 \odot \odot$ & $288 . \odot \odot \odot$ & $1.000 \odot$ & $0.1448 \mathrm{E}+08$ \\
\hline 22 & 288.000 & $288.00 \odot$ & 1.0000 & $\odot .1448 \mathrm{E}+08$ \\
\hline 23 & 288.000 & $288.00 \odot$ & $1.000 \odot$ & $\odot .1448 \mathrm{E}+08$ \\
\hline 24 & 288.000 & 288.000 & 1.0000 & $\odot .1448 \mathrm{E}+\odot 8$ \\
\hline 25 & $288 . \odot \odot \odot$ & $288 . \odot \odot \odot$ & $1.000 \odot$ & $0.1448 \mathrm{E}+08$ \\
\hline
\end{tabular}




\begin{tabular}{|c|c|c|c|c|}
\hline time & $(\sec )=$ & $7.00 \quad 1$ & \multicolumn{2}{|c|}{ lambda $=0.2632044852$} \\
\hline node & $\mathrm{T}(\mathrm{K})$ & Twall(K) & $x$ & $\mathrm{P}(\mathrm{Pa})$ \\
\hline 1 & 152.678 & 212.080 & $-\ldots$ & $\odot .1620 \mathrm{E}+07$ \\
\hline 2 & 177.106 & 233.958 & 0.8494 & $\odot .2987 \mathrm{E}+\Theta 7$ \\
\hline 3 & 197.086 & 249.578 & 1.0000 & $\odot .5848 \mathrm{E}+07$ \\
\hline 4 & 197.086 & 258.719 & 1.0000 & $\odot .6920 \mathrm{E}+07$ \\
\hline 5 & 211.433 & 267.320 & 1.0000 & $\odot .7912 \mathrm{E}+07$ \\
\hline 6 & 229.357 & 272.864 & 1.0000 & $\odot .9390 \mathrm{E}+07$ \\
\hline 7 & 235.210 & 276.806 & 1.0000 & $\odot .1022 \mathrm{E}+08$ \\
\hline 8 & 237.035 & 280.159 & 1.0000 & $0.1059 \mathrm{E}+08$ \\
\hline 9 & 239.412 & 283.225 & 1.0000 & $0.1084 \mathrm{E}+08$ \\
\hline 10 & 242.333 & 285.893 & 1.0000 & $\odot .1109 \mathrm{E}+08$ \\
\hline 11 & 266.429 & 287.655 & 1.0000 & $0.1233 \mathrm{E}+08$ \\
\hline 12 & 283.065 & 288.000 & 1.0000 & $\odot .1372 \mathrm{E}+08$ \\
\hline 13 & 287.378 & $288.00 \odot$ & $1.000 \odot$ & $0.1433 \mathrm{E}+08$ \\
\hline 14 & 287.990 & 288.000 & 1.0000 & $0.1446 \mathrm{E}+08$ \\
\hline 15 & 288.000 & 288.000 & 1.0000 & $\odot .1448 \mathrm{E}+08$ \\
\hline 16 & 288.000 & 288.000 & 1.0000 & $\odot .1448 \mathrm{E}+08$ \\
\hline 17 & 288.000 & 288.000 & 1.0000 & $\odot .1448 \mathrm{E}+08$ \\
\hline 18 & 288.000 & 288.000 & 1.0000 & $0.1448 \mathrm{E}+08$ \\
\hline 19 & $288.0 \odot \odot$ & $288 . \odot \odot \odot$ & $1.000 \odot$ & $0.1448 \mathrm{E}+08$ \\
\hline 20 & $288.00 \odot$ & $288.0 \odot \odot$ & $1.000 \odot$ & $\odot .1448 \mathrm{E}+08$ \\
\hline 21 & $288.00 \odot$ & 288.000 & 1.0000 & $0.1448 \mathrm{E}+08$ \\
\hline 22 & 288.000 & 288.000 & 1.0000 & $0.1448 \mathrm{E}+08$ \\
\hline 23 & 288.000 & 288.000 & 1.0000 & $\odot .1448 \mathrm{E}+\odot 8$ \\
\hline 24 & 288.000 & 288.000 & $1.000 \odot$ & $0.1448 \mathrm{E}+08$ \\
\hline 25 & $288 . \odot \odot \odot$ & $288 . \odot \odot \odot$ & $1.00 \odot \odot$ & $0.1448 \mathrm{E}+08$ \\
\hline time & $(\sec )=$ & 8.00 & $\mathrm{da}=$ & 4961443 \\
\hline node & $\mathrm{T}(\mathrm{K})$ & Twall(K) & $\mathrm{X}$ & $P(P a)$ \\
\hline 1 & 151.728 & 206.409 & - - - - - & $\odot .1567 \mathrm{E}+07$ \\
\hline 2 & 176.005 & 228.583 & $\odot .8936$ & $\odot .2891 \mathrm{E}+07$ \\
\hline 3 & 194.940 & 244.582 & 1.0000 & $\odot .5559 \mathrm{E}+07$ \\
\hline 4 & 202.120 & 253.019 & $1.0 \odot \odot \odot$ & $\odot .7 \odot 43 \mathrm{E}+\odot 7$ \\
\hline 5 & 202.417 & 261.021 & 1.0000 & $\odot .7544 \mathrm{E}+\odot 7$ \\
\hline 6 & 213.800 & 268.342 & 1.0000 & $\odot .8434 \mathrm{E}+07$ \\
\hline 7 & 231.735 & 273.600 & 1.0000 & $\odot .9702 \mathrm{E}+07$ \\
\hline 8 & 237.115 & 277.060 & $1.000 \odot$ & $\odot .1050 \mathrm{E}+\odot 8$ \\
\hline 9 & 240.558 & 280.169 & $1.000 \odot$ & $0.1087 \mathrm{E}+08$ \\
\hline 10 & 242.805 & 282.783 & 1.0000 & $0.1111 \mathrm{E}+08$ \\
\hline 11 & 244.569 & 285.085 & 1.0000 & $\odot .1127 \mathrm{E}+08$ \\
\hline 12 & 254.664 & 287.089 & 1.0000 & $\odot .1181 \mathrm{E}+08$ \\
\hline 13 & 277.287 & 287.925 & 1.0000 & $\odot .1316 \mathrm{E}+08$ \\
\hline 14 & 286.110 & $288 . \odot \odot \odot$ & $1.00 \odot \odot$ & $\odot .1413 \mathrm{E}+08$ \\
\hline 15 & 287.848 & 288.000 & 1.0000 & $\odot .1442 \mathrm{E}+08$ \\
\hline 16 & 288.000 & 288.000 & 1.0000 & $\odot .1447 \mathrm{E}+08$ \\
\hline 17 & 288.000 & 288.000 & 1.0000 & $0.1448 \mathrm{E}+08$ \\
\hline 18 & $288.0 \odot \odot$ & $288.00 \odot$ & $1.000 \odot$ & $0.1448 \mathrm{E}+08$ \\
\hline 19 & 288.000 & $288.00 \odot$ & $1.000 \odot$ & $0.1448 \mathrm{E}+08$ \\
\hline 20 & $288.00 \odot$ & $288 . \odot \odot \odot$ & $1.000 \odot$ & $0.1448 \mathrm{E}+08$ \\
\hline 21 & $288.0 \odot \odot$ & $288.00 \odot$ & $1.000 \odot$ & $0.1448 \mathrm{E}+08$ \\
\hline 22 & 288.000 & 288.000 & 1.0000 & $\odot .1448 \mathrm{E}+08$ \\
\hline 23 & 288.000 & $288.00 \odot$ & $1.000 \odot$ & $\odot .1448 \mathrm{E}+08$ \\
\hline 24 & 288.000 & 288.000 & 1.0000 & $\odot .1448 \mathrm{E}+08$ \\
\hline 25 & $288 . \odot \odot \odot$ & $288 . \odot \odot \odot$ & $1.000 \odot$ & $0.1448 \mathrm{E}+08$ \\
\hline
\end{tabular}




\begin{tabular}{|c|c|c|c|c|}
\hline time & $(\sec )=$ & $9.00 \quad 1$ & \multicolumn{2}{|c|}{ lambda $=0.2458254844$} \\
\hline node & $\mathrm{T}(\mathrm{K})$ & Twall(K) & ) $\quad x$ & $\mathrm{P}(\mathrm{Pa})$ \\
\hline 1 & 150.545 & 201.363 & 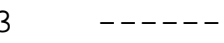 & $\odot .1502 \mathrm{E}+07$ \\
\hline 2 & 174.632 & 223.770 & 0.9214 & $\odot .2771 \mathrm{E}+07$ \\
\hline 3 & 187.520 & 239.349 & 1.0000 & $\odot .5285 \mathrm{E}+07$ \\
\hline 4 & 205.563 & 248.963 & $1.000 \odot$ & $\odot .6755 \mathrm{E}+07$ \\
\hline 5 & 207.708 & 256.170 & 1.0000 & $\odot .7741 \mathrm{E}+07$ \\
\hline 6 & 209.453 & 262.967 & 1.0000 & $\odot .8262 \mathrm{E}+07$ \\
\hline 7 & 217.602 & 269.365 & 1.0000 & $\odot .8907 \mathrm{E}+07$ \\
\hline 8 & 232.602 & 273.949 & 1.0000 & $\odot .9957 \mathrm{E}+07$ \\
\hline 9 & 239.090 & 277.423 & 1.0000 & $\odot .1068 \mathrm{E}+08$ \\
\hline 10 & 243.402 & 280.204 & 1.0000 & $0.1108 \mathrm{E}+08$ \\
\hline 11 & 245.816 & 282.494 & 1.0000 & $0.1132 \mathrm{E}+08$ \\
\hline 12 & 247.740 & 284.543 & 1.0000 & $0.1149 \mathrm{E}+08$ \\
\hline 13 & 249.697 & 286.375 & $1.000 \odot$ & $0.1164 \mathrm{E}+08$ \\
\hline 14 & 268.199 & 287.704 & 1.0000 & $\odot .1259 \mathrm{E}+08$ \\
\hline 15 & 283.262 & 288.000 & 1.0000 & $\odot .1377 \mathrm{E}+08$ \\
\hline 16 & 287.386 & 288.000 & 1.0000 & $\odot .1433 E+\odot 8$ \\
\hline 17 & 287.990 & 288.000 & 1.0000 & $\odot .1446 \mathrm{E}+08$ \\
\hline 18 & 288.000 & 288.000 & 1.0000 & $\odot .1448 \mathrm{E}+08$ \\
\hline 19 & $288.0 \odot \odot$ & $288.00 \odot$ & $1.000 \odot$ & $0.1448 \mathrm{E}+08$ \\
\hline 20 & $288.00 \odot$ & $288.00 \odot$ & $1.000 \odot$ & $0.1448 \mathrm{E}+08$ \\
\hline 21 & $288.00 \odot$ & $288.00 \odot$ & $1.000 \odot$ & $0.1448 \mathrm{E}+08$ \\
\hline 22 & $288.00 \odot$ & 288.000 & 1.0000 & $0.1448 \mathrm{E}+08$ \\
\hline 23 & 288.000 & 288.000 & 1.0000 & $\odot .1448 \mathrm{E}+\odot 8$ \\
\hline 24 & 288.000 & $288.00 \odot$ & $1.000 \odot$ & $0.1448 \mathrm{E}+08$ \\
\hline 25 & $288 . \odot \odot \odot$ & $288.0 \odot \odot$ & $1.000 \odot$ & $\odot .1448 \mathrm{E}+08$ \\
\hline time & $(\mathrm{sec})$ & $10.0 \odot$ & $\mathrm{da}=$ & 1351143 \\
\hline node & $\mathrm{T}(\mathrm{K})$ & Twall(K) & $\mathrm{x}$ & $P(P a)$ \\
\hline 1 & 149.611 & 196.876 & - - - - - & $\odot .1451 \mathrm{E}+07$ \\
\hline 2 & 173.548 & 219.369 & 0.9363 & $\odot .2676 \mathrm{E}+07$ \\
\hline 3 & 193.847 & 234.625 & 1.0000 & $\odot .5406 \mathrm{E}+07$ \\
\hline 4 & 193.971 & 244.657 & $1.000 \odot$ & $\odot .6150 \mathrm{E}+\odot 7$ \\
\hline 5 & 209.207 & 252.330 & 1.0000 & $\odot .7608 \mathrm{E}+\odot 7$ \\
\hline 6 & 212.893 & 258.616 & 1.0000 & $0.8394 \mathrm{E}+07$ \\
\hline 7 & 215.182 & 264.540 & 1.0000 & $\odot .8808 \mathrm{E}+07$ \\
\hline 8 & 222.035 & 270.041 & $1.000 \odot$ & $\odot .9355 \mathrm{E}+\odot 7$ \\
\hline 9 & 233.065 & 274.456 & $1.000 \odot$ & $0.1013 \mathrm{E}+08$ \\
\hline 10 & 240.980 & 277.763 & 1.0000 & $0.1081 \mathrm{E}+08$ \\
\hline 11 & 245.673 & 280.269 & 1.0000 & $\odot .1124 \mathrm{E}+08$ \\
\hline 12 & 248.389 & 282.322 & $1.000 \odot$ & $\odot .1150 \mathrm{E}+08$ \\
\hline 13 & 250.015 & 284.135 & 1.0000 & $\odot .1166 \mathrm{E}+08$ \\
\hline 14 & 251.583 & 285.781 & $1.000 \odot$ & $\odot .1179 \mathrm{E}+\odot 8$ \\
\hline 15 & 258.279 & 287.230 & $1.000 \odot$ & $\odot .1215 \mathrm{E}+08$ \\
\hline 16 & 277.830 & 287.931 & 1.0000 & $0.1326 \mathrm{E}+08$ \\
\hline 17 & 286.164 & $288.00 \odot$ & 1.0000 & $0.1414 \mathrm{E}+08$ \\
\hline 18 & 287.851 & $288 . \odot \odot \odot$ & $1.000 \odot$ & $0.1442 \mathrm{E}+08$ \\
\hline 19 & 288.000 & $288.00 \odot$ & $1.000 \odot$ & $0.1447 \mathrm{E}+08$ \\
\hline 20 & $288.00 \odot$ & $288 . \odot \odot \odot$ & 1.0000 & $0.1448 \mathrm{E}+08$ \\
\hline 21 & $288.0 \odot \odot$ & $288 . \odot \odot \odot$ & $1.000 \odot$ & $0.1448 \mathrm{E}+08$ \\
\hline 22 & 288.000 & $288.00 \odot$ & $1.000 \odot$ & $\odot .1448 \mathrm{E}+08$ \\
\hline 23 & 288.000 & $288.0 \odot \odot$ & $1.000 \odot$ & $\odot .1448 \mathrm{E}+08$ \\
\hline 24 & 288.000 & $288.00 \odot$ & 1.0000 & $\odot .1448 \mathrm{E}+08$ \\
\hline 25 & $288 . \odot \odot \odot$ & $288 . \odot \odot \odot$ & $1.000 \odot$ & $0.1448 \mathrm{E}+08$ \\
\hline
\end{tabular}




\begin{tabular}{|c|c|c|c|c|}
\hline time & $(\cos )_{-}^{-}$ & $1.00 \quad l$ & - & \\
\hline node & $T^{\prime}(K)$ & Twall(K) & $x$ & $\mathrm{P}(\mathrm{Pa})$ \\
\hline 1 & 149.493 & 192.801 & - - _ - _ & $\odot .1444 \mathrm{E}+\odot 7$ \\
\hline 2 & 173.412 & 215.234 & $\odot .9393$ & $\odot .2664 \mathrm{E}+\odot 7$ \\
\hline 3 & 206.519 & 231.690 & $1.000 \odot$ & $\odot .5365 E+\odot 7$ \\
\hline 4 & 206.519 & 240.493 & $1.000 \odot$ & $\odot .6548 \mathrm{E}+\odot 7$ \\
\hline 5 & 206.519 & 248.388 & $1.000 \odot$ & $\odot .7281 E+\odot 7$ \\
\hline 6 & 214.809 & 255.123 & 1.0000 & $\odot .8266 \mathrm{E}+\odot 7$ \\
\hline 7 & 218.268 & 260.635 & $1.000 \odot$ & $\odot .8934 \mathrm{E}+07$ \\
\hline 8 & 221.366 & 265.872 & 1.0000 & $\odot .9327 \mathrm{E}+\odot 7$ \\
\hline 9 & 225.064 & 270.694 & $1.000 \odot$ & $\odot .9706 \mathrm{E}+\odot 7$ \\
\hline 10 & 233.957 & 274.880 & 1.0000 & $\odot .1025 \mathrm{E}+08$ \\
\hline 11 & 241.784 & $278.0 \odot 3$ & $1.000 \odot$ & $\odot .1088 \mathrm{E}+\odot 8$ \\
\hline 12 & 247.614 & 280.311 & 1.0000 & $\odot .1136 \mathrm{E}+08$ \\
\hline 13 & 250.111 & 282.204 & $1.000 \odot$ & $0.1164 \mathrm{E}+08$ \\
\hline 14 & 252.126 & 283.847 & 1.0000 & $\odot .1181 E+08$ \\
\hline 15 & 253.603 & 285.354 & 1.0000 & $0.1193 E+08$ \\
\hline 16 & 254.841 & 286.672 & $1.000 \odot$ & $0.1203 E+08$ \\
\hline 17 & 269.428 & 287.731 & 1.0000 & $\odot .1277 \mathrm{E}+\odot 8$ \\
\hline 18 & 283.439 & 288.000 & 1.0000 & $\odot .1381 E+08$ \\
\hline 19 & 287.403 & $288.0 \odot \odot$ & $1.000 \odot$ & $\odot .1434 \mathrm{E}+08$ \\
\hline 20 & 287.992 & $288.0 \odot \odot$ & $1.000 \odot$ & $\odot .1446 \mathrm{E}+\odot 8$ \\
\hline 21 & $288.00 \odot$ & 288.000 & 1.0000 & $0.1448 \mathrm{E}+08$ \\
\hline 22 & 288.000 & 288.000 & 1.0000 & $\odot .1448 \mathrm{E}+08$ \\
\hline 23 & 288.000 & 288.000 & 1.0000 & $0.1448 \mathrm{E}+08$ \\
\hline 24 & 288.000 & 288.000 & 1.0000 & $\odot .1448 \mathrm{E}+\odot 8$ \\
\hline 25 & 288.000 & 288.000 & 1.0000 & $\odot .1448 \mathrm{E}+08$ \\
\hline
\end{tabular}

\begin{tabular}{|c|c|c|c|c|}
\hline \multicolumn{5}{|c|}{ lambda $=0.2330709547$} \\
\hline node & $\mathrm{T}(\mathrm{K})$ & Twall(K) & $\mathrm{x}$ & $\mathrm{P}(\mathrm{Pa})$ \\
\hline 1 & 148.998 & 189.094 & . . - & $\odot .1417 \mathrm{E}+07$ \\
\hline 2 & 172.838 & 211.524 & 0.9445 & $\odot .2613 \mathrm{E}+07$ \\
\hline 3 & 197.015 & 229.208 & $1.000 \odot$ & $\odot .4842 \mathrm{E}+\odot 7$ \\
\hline 4 & 207.985 & 237.647 & $1.000 \odot$ & $\odot .6531 \mathrm{E}+\odot 7$ \\
\hline 5 & 208.096 & 244.727 & 1.0000 & $0.7337 \mathrm{E}+07$ \\
\hline 6 & 208.096 & 251.563 & 1.0000 & $\odot .7811 \mathrm{E}+07$ \\
\hline 7 & 218.831 & 257.402 & 1.0000 & $\odot .8804 \mathrm{E}+07$ \\
\hline 8 & 223.321 & 262.434 & 1.0000 & $\odot .9397 \mathrm{E}+07$ \\
\hline 9 & 225.850 & 266.992 & 1.0000 & $\odot .9740 \mathrm{E}+\odot 7$ \\
\hline 10 & 229.087 & 271.327 & $1.000 \odot$ & $\odot .1000 \mathrm{E}+\odot 8$ \\
\hline 11 & 233.373 & 275.082 & 1.0000 & $\odot .1034 \mathrm{E}+08$ \\
\hline 12 & 242.952 & 278.120 & 1.0000 & $\odot .1091 \mathrm{E}+08$ \\
\hline 13 & 248.157 & 280.373 & 1.0000 & $0.1143 E+08$ \\
\hline 14 & 251.703 & 282.016 & $1.000 \odot$ & $\odot .1174 \mathrm{E}+\odot 8$ \\
\hline 15 & 253.824 & 283.636 & 1.0000 & $\odot .1193 \mathrm{E}+08$ \\
\hline 16 & 255.303 & 284.955 & 1.0000 & $\odot .1205 \mathrm{E}+\odot 8$ \\
\hline 17 & 256.515 & 286.242 & 1.0000 & $0.1215 \mathrm{E}+08$ \\
\hline 18 & 261.091 & 287.338 & 1.0000 & $0.1240 \mathrm{E}+08$ \\
\hline 19 & 278.286 & 287.937 & $1.000 \odot$ & $0.1335 \mathrm{E}+08$ \\
\hline 20 & 286.229 & $288.0 \odot \odot$ & 1.0000 & $\odot .1416 \mathrm{E}+\odot 8$ \\
\hline 21 & 287.856 & 288.000 & 1.0000 & $\odot .1443 \mathrm{E}+\odot 8$ \\
\hline 22 & 288.000 & 288.000 & 1.0000 & $\odot .1447 \mathrm{E}+08$ \\
\hline 23 & 288.000 & 288.000 & 1.0000 & $\odot .1448 \mathrm{E}+08$ \\
\hline 24 & 288.000 & 288.000 & 1.0000 & $\odot .1448 \mathrm{E}+08$ \\
\hline 25 & 288.000 & 288.000 & 1.0000 & $\odot .1448 \mathrm{E}+08$ \\
\hline
\end{tabular}




\begin{tabular}{|c|c|c|c|c|}
\hline \multicolumn{5}{|c|}{ lambda $=0.2206054032$} \\
\hline node & $\mathrm{T}^{\prime}(\mathrm{K})$ & Twall(K) & $x$ & $\mathrm{P}(\mathrm{Pa})$ \\
\hline 1 & 147.501 & 185.724 & $-\ldots$ & $\odot .1334 \mathrm{E}+\odot 7$ \\
\hline 2 & 171.101 & 208.311 & $\odot .9552$ & $\odot .2461 \mathrm{E}+\odot 7$ \\
\hline 3 & 189.328 & 225.686 & $1.000 \odot$ & $\odot .465 \odot E+\odot 7$ \\
\hline 4 & 197.424 & 234.736 & $1.000 \odot$ & $\odot .5947 \mathrm{E}+\odot 7$ \\
\hline 5 & 212.101 & 241.918 & $1.000 \odot$ & $\odot .7349 \mathrm{E}+\odot 7$ \\
\hline 6 & 212.101 & 248.011 & 1.0000 & $\odot .7962 \mathrm{E}+\odot 7$ \\
\hline 7 & 212.101 & 254.055 & 1.0000 & $\odot .8381 E+\odot 7$ \\
\hline 8 & 221.920 & 259.382 & 1.0000 & $\odot .9178 \mathrm{E}+07$ \\
\hline 9 & 226.796 & 263.971 & $1.000 \odot$ & $\odot .9740 \mathrm{E}+\odot 7$ \\
\hline 10 & 230.700 & 268.242 & 1.0000 & $\odot .1006 \mathrm{E}+08$ \\
\hline 11 & 232.255 & 271.841 & $1.000 \odot$ & $\odot .1029 \mathrm{E}+\odot 8$ \\
\hline 12 & 235.379 & 275.296 & 1.0000 & $\odot .1049 \mathrm{E}+08$ \\
\hline 13 & 242.617 & 278.206 & $1.000 \odot$ & $\odot .1097 \mathrm{E}+08$ \\
\hline 14 & 248.693 & 280.185 & 1.0000 & $\odot .1145 \mathrm{E}+\odot 8$ \\
\hline 15 & 252.698 & 282.110 & $1.000 \odot$ & $\odot .1180 \mathrm{E}+\odot 8$ \\
\hline 16 & 255.252 & 283.430 & 1.0000 & $\odot .1202 \mathrm{E}+0 \varepsilon$ \\
\hline 17 & 256.865 & 284.716 & 1.0000 & $\odot .1216 \mathrm{E}+\odot 8$ \\
\hline 18 & 257.990 & 285.812 & 1.0000 & $\odot .1225 \mathrm{E}+08$ \\
\hline 19 & 258.817 & 286.880 & $1.000 \odot$ & $\odot .1232 \mathrm{E}+08$ \\
\hline 20 & 270.469 & 287.758 & $1.000 \odot$ & $\odot .1291 \mathrm{E}+08$ \\
\hline 21 & 283.618 & 288.000 & 1.0000 & $0.1384 \mathrm{E}+08$ \\
\hline 22 & 287.427 & 288.000 & 1.0000 & $\odot .1434 \mathrm{E}+08$ \\
\hline 23 & 287.995 & 288.000 & 1.0000 & $0.1446 \mathrm{E}+08$ \\
\hline 24 & 288.000 & 288.000 & 1.0000 & $\odot .1448 \mathrm{E}+\odot 8$ \\
\hline 25 & 288.000 & 288.000 & 1.0000 & $\odot .1448 \mathrm{E}+08$ \\
\hline
\end{tabular}

\begin{tabular}{|c|c|c|c|c|}
\hline \multicolumn{5}{|c|}{ lambda $=0.2117832005$} \\
\hline node & $\mathrm{T}(\mathrm{K})$ & Twall(K) & $\mathrm{x}$ & $P(P a)$ \\
\hline 1 & 146.450 & 182.640 & $\ldots$ & $\odot .1276 \mathrm{E}+\odot 7$ \\
\hline 2 & 169.882 & 205.333 & 0.9590 & $\odot .2354 \mathrm{E}+07$ \\
\hline 3 & 190.532 & 222.506 & $1.000 \odot$ & $\odot .4670 \mathrm{E}+\odot 7$ \\
\hline 4 & 190.824 & 230.770 & $1.000 \odot$ & $\odot .5731 \mathrm{E}+07$ \\
\hline 5 & 207.318 & 239.476 & 1.0000 & $\odot .6792 \mathrm{E}+07$ \\
\hline 6 & 214.690 & 245.281 & 1.0000 & $\odot .8000 \mathrm{E}+\odot 7$ \\
\hline 7 & 214.737 & 250.703 & 1.0000 & $\odot .8485 \mathrm{E}+07$ \\
\hline 8 & 214.737 & 256.119 & 1.0000 & $\odot .8765 \mathrm{E}+07$ \\
\hline 9 & 224.168 & 261.225 & 1.0000 & $\odot .9445 \mathrm{E}+\odot 7$ \\
\hline 10 & 230.745 & 265.671 & $1.000 \odot$ & $\odot .9983 \mathrm{E}+\odot 7$ \\
\hline 11 & 233.639 & 269.180 & 1.0000 & $0.1034 \mathrm{E}+08$ \\
\hline 12 & 236.573 & 272.585 & 1.0000 & $\odot .1054 \mathrm{E}+08$ \\
\hline 13 & 238.616 & 275.544 & 1.0000 & $\odot .1077 \mathrm{E}+08$ \\
\hline 14 & 242.508 & 278.021 & $1.000 \odot$ & $\odot .1104 \mathrm{E}+08$ \\
\hline 15 & 248.630 & 280.378 & 1.0000 & $\odot .1145 \mathrm{E}+08$ \\
\hline 16 & 253.205 & 281.904 & 1.0000 & $\odot .1182 \mathrm{E}+\odot 8$ \\
\hline 17 & 256.323 & 283.342 & 1.0000 & $0.1208 \mathrm{E}+08$ \\
\hline 18 & 258.176 & 284.495 & 1.0000 & $0.1224 \mathrm{E}+08$ \\
\hline 19 & 259.322 & 285.552 & $1.000 \odot$ & $0.1234 \mathrm{E}+08$ \\
\hline 20 & 260.243 & 286.580 & 1.0000 & $\odot .1242 \mathrm{E}+\odot 8$ \\
\hline 21 & 263.492 & 287.421 & 1.0000 & $\odot .1260 \mathrm{E}+08$ \\
\hline 22 & 278.728 & 287.943 & 1.0000 & $\odot .1343 \mathrm{E}+08$ \\
\hline 23 & 286.294 & 288.000 & 1.0000 & $\odot .1417 \mathrm{E}+\odot 8$ \\
\hline 24 & 287.867 & 288.000 & 1.0000 & $\odot .1443 \mathrm{E}+08$ \\
\hline 25 & 287.867 & 288.000 & 1.0000 & $\odot .1447 \mathrm{E}+08$ \\
\hline
\end{tabular}




\begin{tabular}{|c|c|c|c|c|}
\hline \multicolumn{5}{|c|}{$.00 \quad$ lambda $=0.2085444629$} \\
\hline node & $\mathrm{T}^{\prime}(\mathrm{K})$ & Twall(K) & $\mathrm{x}$ & $\mathrm{P}(\mathrm{Pa})$ \\
\hline 1 & 146.066 & 179.809 & - - _ - _ & $\odot .1255 \mathrm{E}+\odot 7$ \\
\hline 2 & 169.436 & 202.515 & $\odot .9580$ & $\odot .2315 \mathrm{E}+\odot 7$ \\
\hline 3 & 190.743 & 219.939 & $1.000 \odot$ & $\odot .4586 E+\odot 7$ \\
\hline 4 & 198.459 & 227.577 & $1.000 \odot$ & $\odot .5931 E+\odot 7$ \\
\hline 5 & 198.459 & 235.916 & $1.000 \odot$ & $\odot .6436 \mathrm{E}+07$ \\
\hline 6 & 208.570 & 242.755 & 1.0000 & $\odot .7463 \mathrm{E}+\odot 7$ \\
\hline 7 & 216.459 & 248.064 & $1.000 \odot$ & $\odot .8463 E+\odot 7$ \\
\hline 8 & 216.683 & 252.980 & 1.0000 & $\odot .8845 \mathrm{E}+\odot 7$ \\
\hline 9 & 219.285 & 258.158 & $1.000 \odot$ & $\odot .9185 \mathrm{E}+\odot 7$ \\
\hline 10 & 225.783 & 263.054 & 1.0000 & $\odot .9627 \mathrm{E}+\odot 7$ \\
\hline 11 & 234.949 & 266.917 & $1.000 \odot$ & $\odot .1025 \mathrm{E}+\odot 8$ \\
\hline 12 & 236.973 & 270.268 & 1.0000 & $\odot .1056 \mathrm{E}+08$ \\
\hline 13 & 239.696 & 273.202 & $1.000 \odot$ & $\odot .1081 \mathrm{E}+08$ \\
\hline 14 & 241.265 & 275.620 & $1.000 \odot$ & $\odot .1099 \mathrm{E}+\odot 8$ \\
\hline 15 & 243.347 & 278.191 & $1.000 \odot$ & $\odot .1115 \mathrm{E}+\odot 8$ \\
\hline 16 & 248.119 & 280.169 & $1.000 \odot$ & $0.1144 \mathrm{E}+08$ \\
\hline 17 & 253.192 & 281.816 & 1.0000 & $\odot .1181 E+08$ \\
\hline 18 & 256.953 & 283.274 & 1.0000 & $0.1211 \mathrm{E}+08$ \\
\hline 19 & 259.240 & 284.331 & $1.000 \odot$ & $\odot .1231 \mathrm{E}+08$ \\
\hline 20 & 260.548 & 285.359 & $1.000 \odot$ & $\odot .1243 E+08$ \\
\hline 21 & 261.404 & 286.201 & 1.0000 & $\odot .1250 \mathrm{E}+08$ \\
\hline 22 & 262.168 & 287.049 & 1.0000 & $\odot .1256 \mathrm{E}+\odot 8$ \\
\hline 23 & 270.319 & 287.770 & 1.0000 & $\odot .1298 \mathrm{E}+08$ \\
\hline 24 & 284.181 & 288.000 & 1.0000 & $\odot .1386 \mathrm{E}+08$ \\
\hline 25 & 284.235 & 288.000 & 1.0000 & $\odot .1420 \mathrm{E}+08$ \\
\hline
\end{tabular}

\begin{tabular}{|c|c|c|c|c|}
\hline \multicolumn{5}{|c|}{ lambda $=0.2057363540$} \\
\hline node & $T(K)$ & Twall(K) & $\mathrm{x}$ & $\mathrm{P}(\mathrm{Pa})$ \\
\hline 1 & 145.733 & 177.179 & $\ldots$ & $\odot .1237 \mathrm{E}+07$ \\
\hline 2 & 169.051 & 199.918 & 0.9579 & $\odot .2281 \mathrm{E}+07$ \\
\hline 3 & 190.526 & 217.409 & $\odot .9440$ & $\odot .4567 \mathrm{E}+\odot 7$ \\
\hline 4 & 205.801 & 225.587 & $1.000 \odot$ & $\odot .5845 \mathrm{E}+\odot 7$ \\
\hline 5 & 205.871 & 233.091 & 1.0000 & $0.6676 \mathrm{E}+07$ \\
\hline 6 & 205.871 & 239.404 & 1.0000 & $\odot .7352 \mathrm{E}+07$ \\
\hline 7 & 214.594 & 245.754 & 1.0000 & $\odot .8017 \mathrm{E}+07$ \\
\hline 8 & 217.182 & 250.370 & 1.0000 & $\odot .8837 \mathrm{E}+07$ \\
\hline 9 & 220.379 & 255.299 & 1.0000 & $\odot .9219 \mathrm{E}+\odot 7$ \\
\hline 10 & 220.444 & 259.953 & $1.000 \odot$ & $\odot .9395 \mathrm{E}+\odot 7$ \\
\hline 11 & 233.580 & 264.780 & 1.0000 & $\odot .9992 \mathrm{E}+07$ \\
\hline 12 & 235.000 & 268.131 & 1.0000 & $0.1044 \mathrm{E}+08$ \\
\hline 13 & 239.609 & 271.066 & 1.0000 & $\odot .1074 \mathrm{E}+08$ \\
\hline 14 & 242.035 & 273.542 & 1.0000 & $\odot .1101 \mathrm{E}+08$ \\
\hline 15 & 243.933 & 276.068 & 1.0000 & $\odot .1117 \mathrm{E}+08$ \\
\hline 16 & 245.524 & 278.033 & 1.0000 & $\odot .1132 \mathrm{E}+08$ \\
\hline 17 & 247.602 & 280.000 & 1.0000 & $0.1147 \mathrm{E}+08$ \\
\hline 18 & 252.676 & 281.821 & 1.0000 & $\odot .1177 \mathrm{E}+08$ \\
\hline 19 & 256.987 & 283.110 & $1.000 \odot$ & $0.1210 \mathrm{E}+08$ \\
\hline 20 & 260.160 & 284.138 & $1.000 \odot$ & $\odot .1235 \mathrm{E}+08$ \\
\hline 21 & 261.101 & 284.980 & 1.0000 & $\odot .1248 \mathrm{E}+\odot 8$ \\
\hline 22 & 262.343 & 285.930 & 1.0000 & $\odot .1256 \mathrm{E}+08$ \\
\hline 23 & 262.343 & 286.635 & 1.0000 & $\odot .1260 \mathrm{E}+08$ \\
\hline 24 & 264.511 & 287.657 & 1.0000 & $\odot .1270 \mathrm{E}+08$ \\
\hline 25 & 264.966 & 287.660 & 1.0000 & $\odot .1280 E+08$ \\
\hline
\end{tabular}




\begin{tabular}{|c|c|c|c|c|}
\hline \multicolumn{5}{|c|}{ lambda $=0.2040060908$} \\
\hline node & $\mathrm{T}^{\prime}(\mathrm{K})$ & Twall(K) & $x$ & $\mathrm{P}(\mathrm{Pa})$ \\
\hline 1 & 145.529 & 174.770 & $-\ldots$ & $\odot .1226 \mathrm{E}+\odot 7$ \\
\hline 2 & 168.814 & 197.519 & $\odot .9559$ & $\odot .2261 \mathrm{E}+\odot 7$ \\
\hline 3 & 190.299 & 215.119 & $\odot .9189$ & $\odot .4538 \mathrm{E}+\odot 7$ \\
\hline 4 & 204.463 & 224.062 & $1.000 \odot$ & $\odot .5562 \mathrm{E}+\odot 7$ \\
\hline 5 & 204.463 & 230.955 & $1.000 \odot$ & $\odot .6626 \mathrm{E}+\odot 7$ \\
\hline 6 & 208.571 & 236.798 & 1.0000 & $\odot .7414 \mathrm{E}+\odot 7$ \\
\hline 7 & 208.571 & 242.898 & 1.0000 & $\odot .7710 \mathrm{E}+\odot 7$ \\
\hline 8 & 210.964 & 247.762 & 1.0000 & $\odot .8398 \mathrm{E}+\odot 7$ \\
\hline 9 & 221.731 & 252.941 & $1.000 \odot$ & $\odot .9128 \mathrm{E}+\odot 7$ \\
\hline 10 & 223.618 & 257.315 & 1.0000 & $\odot .9530 E+\odot 7$ \\
\hline 11 & 230.268 & 262.367 & $1.000 \odot$ & $\odot .9847 \mathrm{E}+\odot 7$ \\
\hline 12 & 233.752 & 265.995 & 1.0000 & $\odot .1031 \mathrm{E}+08$ \\
\hline 13 & 236.290 & 268.930 & $1.000 \odot$ & $0.1054 \mathrm{E}+08$ \\
\hline 14 & 241.491 & 271.711 & 1.0000 & $\odot .1089 \mathrm{E}+\odot 8$ \\
\hline 15 & 244.110 & 274.237 & $1.000 \odot$ & $\odot .1116 \mathrm{E}+\odot 8$ \\
\hline 16 & 246.151 & 276.185 & 1.0000 & $0.1134 \mathrm{E}+08$ \\
\hline 17 & 247.526 & 278.120 & 1.0000 & $\odot .1146 \mathrm{E}+\odot 8$ \\
\hline 18 & 249.482 & 280.004 & 1.0000 & $0.1160 E+08$ \\
\hline 19 & 249.772 & 281.524 & $1.000 \odot$ & $\odot .1168 \mathrm{E}+08$ \\
\hline 20 & 258.057 & 282.918 & $1.000 \odot$ & $\odot .1208 \mathrm{E}+08$ \\
\hline 21 & 258.660 & 283.759 & 1.0000 & $\odot .1231 \mathrm{E}+08$ \\
\hline 22 & 261.266 & 285.015 & 1.0000 & $\odot .1245 \mathrm{E}+\odot 8$ \\
\hline 23 & 261.266 & 285.719 & 1.0000 & $\odot .1252 \mathrm{E}+08$ \\
\hline 24 & 261.266 & 286.741 & 1.0000 & $\odot .1252 \mathrm{E}+\odot 8$ \\
\hline 25 & 261.266 & 286.745 & 1.0000 & $\odot .1252 \mathrm{E}+08$ \\
\hline
\end{tabular}

\begin{tabular}{|c|c|c|c|c|}
\hline \multicolumn{5}{|c|}{ lambda $=0.2018307447$} \\
\hline node & $T(K)$ & Twall(K) & $\mathrm{x}$ & $\mathrm{P}(\mathrm{Pa})$ \\
\hline 1 & 145.273 & 172.536 & - _ _ _ _ & $0.1211 \mathrm{E}+07$ \\
\hline 2 & 168.516 & 195.305 & 0.9511 & $\odot .2235 E+\odot 7$ \\
\hline 3 & 189.007 & 213.106 & $\odot .9349$ & $\odot .4373 E+\odot 7$ \\
\hline 4 & 196.093 & 221.975 & $1.0 \odot \odot \odot$ & $\odot .5318 \mathrm{E}+\odot 7$ \\
\hline 5 & 196.093 & 228.349 & $1.000 \odot$ & $\odot .6324 \mathrm{E}+07$ \\
\hline 6 & 211.750 & 234.885 & $1.000 \odot$ & $\odot .7239 \mathrm{E}+\odot 7$ \\
\hline 7 & 211.750 & 240.439 & 1.0000 & $\odot .7827 E+\odot 7$ \\
\hline 8 & 211.750 & 244.828 & 1.0000 & $\odot .8427 \mathrm{E}+07$ \\
\hline 9 & 217.571 & 250.660 & $1.000 \odot$ & $\odot .8735 \mathrm{E}+\odot 7$ \\
\hline 10 & 225.224 & 255.064 & $1.000 \odot$ & $\odot .9508 \mathrm{E}+\odot 7$ \\
\hline 11 & 227.428 & 260.010 & 1.0000 & $\odot .9725 \mathrm{E}+\odot 7$ \\
\hline 12 & 234.735 & 263.961 & 1.0000 & $0.1026 \mathrm{E}+08$ \\
\hline 13 & 234.806 & 266.794 & 1.0000 & $0.1047 \mathrm{E}+08$ \\
\hline 14 & 238.343 & 269.759 & 1.0000 & $0.1068 \mathrm{E}+08$ \\
\hline 15 & 241.772 & 272.406 & $1.000 \odot$ & $\odot .1097 \mathrm{E}+\odot 8$ \\
\hline 16 & 246.676 & 274.583 & $1.00 \odot \odot$ & $\odot .1129 E+08$ \\
\hline 17 & 246.676 & 276.403 & 1.0000 & $0.1142 \mathrm{E}+08$ \\
\hline 18 & 249.743 & 278.389 & $1.00 \odot \odot$ & $0.1158 \mathrm{E}+\odot 8$ \\
\hline 19 & 249.743 & 279.803 & 1.0000 & $0.1168 \mathrm{E}+08$ \\
\hline 20 & 253.242 & 281.542 & 1.0000 & $0.1184 \mathrm{E}+08$ \\
\hline 21 & 253.950 & 282.537 & $1.000 \odot$ & $\odot .1198 \mathrm{E}+\odot 8$ \\
\hline 22 & 256.856 & 283.985 & $1.00 \odot \odot$ & $\odot .1213 E+\odot 8$ \\
\hline 23 & 256.917 & 284.804 & 1.0000 & $\odot .1222 \mathrm{E}+08$ \\
\hline 24 & 256.917 & 285.825 & 1.0000 & $\odot .1222 \mathrm{E}+\odot 8$ \\
\hline 25 & 256.917 & 285.829 & $1.00 \odot \odot$ & $\odot .1222 \mathrm{E}+\odot 8$ \\
\hline
\end{tabular}




\begin{tabular}{|c|c|c|c|c|}
\hline \multicolumn{5}{|c|}{$.00 \quad$ lambda $=0.1967642754$} \\
\hline node & $\mathrm{T}^{\prime}(\mathrm{K})$ & Twall(K) & $x$ & $\mathrm{P}(\mathrm{Pa})$ \\
\hline 1 & 144.677 & 170.460 & - - - - - & $\odot .1179 \mathrm{E}+07$ \\
\hline 2 & 167.825 & 193.283 & $\odot .9473$ & $\odot .2174 \mathrm{E}+\odot 7$ \\
\hline 3 & 187.533 & 211.275 & $\odot .9519$ & $\odot .4184 \mathrm{E}+07$ \\
\hline 4 & 193.376 & 219.686 & $1.00 \odot \odot$ & $\odot .5245 \mathrm{E}+\odot 7$ \\
\hline 5 & 194.440 & 225.476 & $1.00 \odot \odot$ & $\odot .6270 \mathrm{E}+\odot 7$ \\
\hline 6 & 212.765 & 233.301 & 1.0000 & $\odot .6966 \mathrm{E}+\odot 7$ \\
\hline 7 & 212.765 & 238.359 & 1.0000 & $\odot .7862 \mathrm{E}+\odot 7$ \\
\hline 8 & 214.104 & 242.576 & $1.00 \odot \odot$ & $\odot .8375 \mathrm{E}+\odot 7$ \\
\hline 9 & 214.104 & 248.025 & 1.0000 & $\odot .8592 \mathrm{E}+07$ \\
\hline 10 & 226.559 & 253.170 & 1.0000 & $\odot .9352 \mathrm{E}+\odot 7$ \\
\hline 11 & 226.559 & 257.874 & $1.00 \odot \odot$ & $\odot .9688 \mathrm{E}+\odot 7$ \\
\hline 12 & 234.117 & 262.130 & 1.0000 & $0.1015 \mathrm{E}+08$ \\
\hline 13 & 235.547 & 264.845 & $1.000 \odot$ & $0.1048 \mathrm{E}+08$ \\
\hline 14 & 238.166 & 267.785 & 1.0000 & $\odot .1067 \mathrm{E}+08$ \\
\hline 15 & 238.166 & 270.334 & $1.00 \odot \odot$ & $\odot .1079 \mathrm{E}+\odot 8$ \\
\hline 16 & 246.157 & 273.057 & 1.0000 & $\odot .1115 \mathrm{E}+08$ \\
\hline 17 & 246.171 & 274.877 & $1.000 \odot$ & $0.1139 \mathrm{E}+08$ \\
\hline 18 & 249.241 & 276.863 & 1.0000 & $\odot .1153 \mathrm{E}+08$ \\
\hline 19 & 249.935 & 278.278 & 1.0000 & $0.1167 \mathrm{E}+08$ \\
\hline 20 & 249.935 & 280.016 & $1.000 \odot$ & $0.1169 \mathrm{E}+08$ \\
\hline 21 & 249.935 & 281.012 & 1.0000 & $\odot .1171 \mathrm{E}+\odot 8$ \\
\hline 22 & 249.935 & 282.698 & 1.0000 & $0.1171 \mathrm{E}+08$ \\
\hline 23 & 251.615 & 283.849 & 1.0000 & $\odot .1179 \mathrm{E}+\odot 8$ \\
\hline 24 & 251.615 & 284.910 & $1.000 \odot$ & $0.1185 \mathrm{E}+08$ \\
\hline 25 & 251.615 & 284.913 & $1.000 \odot$ & $\odot .1185 \mathrm{E}+\odot 8$ \\
\hline time & $(\sec )=$ & & & 5764788 \\
\hline node & $\mathrm{T}(\mathrm{K})$ & Twall(K) & $\mathrm{x}$ & $\mathrm{P}(\mathrm{Pa})$ \\
\hline 1 & 143.953 & 168.515 & - - - - - & $\odot .1139 \mathrm{E}+07$ \\
\hline 2 & 166.985 & 191.429 & $\odot .9466$ & $\odot .2100 \mathrm{E}+\odot 7$ \\
\hline 3 & 186.800 & 209.444 & $\odot .9645$ & $\odot .4091 \mathrm{E}+07$ \\
\hline 4 & 190.593 & 217.370 & 1.0000 & $\odot .5169 \mathrm{E}+07$ \\
\hline 5 & 200.539 & 223.249 & 1.0000 & $\odot .6297 \mathrm{E}+\odot 7$ \\
\hline 6 & 205.188 & 231.302 & 1.0000 & $\odot .6680 \mathrm{E}+\odot 7$ \\
\hline 7 & 205.188 & 236.008 & 1.0000 & $\odot .7518 \mathrm{E}+07$ \\
\hline 8 & 215.964 & 240.792 & 1.0000 & $\odot .8135 \mathrm{E}+\odot 7$ \\
\hline 9 & 215.964 & 245.807 & 1.0000 & $\odot .8667 \mathrm{E}+\odot 7$ \\
\hline 10 & 227.090 & 251.449 & 1.0000 & $\odot .9322 \mathrm{E}+\odot 7$ \\
\hline 11 & 227.687 & 255.756 & 1.0000 & $\odot .9733 \mathrm{E}+\odot 7$ \\
\hline 12 & 229.179 & 260.119 & 1.0000 & $\odot .9895 \mathrm{E}+\odot 7$ \\
\hline 13 & 233.537 & 263.014 & 1.0000 & $\odot .1028 \mathrm{E}+08$ \\
\hline 14 & 238.716 & 266.092 & $1.00 \odot \odot$ & $\odot .1063 \mathrm{E}+\odot 8$ \\
\hline 15 & 238.982 & 268.496 & 1.0000 & $\odot .1082 \mathrm{E}+08$ \\
\hline 16 & 241.481 & 271.409 & 1.0000 & $\odot .1094 \mathrm{E}+\odot 8$ \\
\hline 17 & 245.419 & 273.351 & 1.0000 & $0.1125 \mathrm{E}+08$ \\
\hline 18 & 245.419 & 275.337 & 1.0000 & $0.1133 \mathrm{E}+08$ \\
\hline 19 & 249.180 & 277.037 & $1.000 \odot$ & $0.1152 \mathrm{E}+08$ \\
\hline 20 & 249.203 & 278.663 & 1.0000 & $\odot .1164 \mathrm{E}+08$ \\
\hline 21 & 249.219 & 279.700 & $1.000 \odot$ & $\odot .1164 \mathrm{E}+08$ \\
\hline 22 & 249.219 & 281.393 & 1.0000 & $0.1164 \mathrm{E}+08$ \\
\hline 23 & 249.219 & 282.628 & 1.0000 & $\odot .1164 \mathrm{E}+08$ \\
\hline 24 & 249.219 & 283.994 & 1.0000 & $0.1164 \mathrm{E}+08$ \\
\hline 25 & 249.219 & 283.998 & $1.000 \odot$ & $0.1164 \mathrm{E}+08$ \\
\hline
\end{tabular}




\begin{tabular}{|c|c|c|c|c|}
\hline \multicolumn{5}{|c|}{$.00 \quad$ lambda $=0.1858711243$} \\
\hline node & $T^{\prime}(K)$ & Twall(K) & $x$ & $\mathrm{P}(\mathrm{Pa})$ \\
\hline 1 & 143.404 & 166.685 & - - _ - _ & $\odot .1108 \mathrm{E}+07$ \\
\hline 2 & 166.349 & 189.660 & $\odot .9476$ & $\odot .2 \odot 45 \mathrm{E}+\odot 7$ \\
\hline 3 & 186.327 & 207.750 & $\odot .9742$ & $\odot .4 \odot 3 \odot E+\odot 7$ \\
\hline 4 & 186.328 & 214.820 & $1.0 \odot \odot \odot$ & $\odot .5 \odot 53 E+\odot 7$ \\
\hline 5 & 206.498 & 221.824 & $1.000 \odot$ & $\odot .6052 \mathrm{E}+07$ \\
\hline 6 & 206.498 & 229.427 & 1.0000 & $\odot .6722 \mathrm{E}+\odot 7$ \\
\hline 7 & 206.985 & 233.709 & $1.000 \odot$ & $\odot .7583 E+\odot 7$ \\
\hline 8 & 215.580 & 239.090 & 1.0000 & $\odot .7934 \mathrm{E}+\odot 7$ \\
\hline 9 & 217.557 & 243.708 & $1.000 \odot$ & $\odot .8712 \mathrm{E}+\odot 7$ \\
\hline 10 & 218.753 & 249.464 & 1.0000 & $\odot .8918 \mathrm{E}+\odot 7$ \\
\hline 11 & 228.439 & 253.978 & $1.000 \odot$ & $\odot .9587 \mathrm{E}+\odot 7$ \\
\hline 12 & 229.266 & 258.086 & 1.0000 & $\odot .9899 \mathrm{E}+\odot 7$ \\
\hline 13 & 229.510 & 260.942 & $1.000 \odot$ & $\odot .1008 \mathrm{E}+08$ \\
\hline 14 & 234.454 & 264.451 & $1.000 \odot$ & $\odot .1031 \mathrm{E}+\odot 8$ \\
\hline 15 & 239.244 & 266.908 & $1.000 \odot$ & $\odot .1074 \mathrm{E}+\odot 8$ \\
\hline 16 & 239.247 & 269.672 & $1.000 \odot$ & $\odot .1084 \mathrm{E}+08$ \\
\hline 17 & 244.837 & 271.881 & 1.0000 & $\odot .1113 E+08$ \\
\hline 18 & 245.134 & 273.811 & 1.0000 & $0.1130 \mathrm{E}+08$ \\
\hline 19 & 245.134 & 275.664 & $1.000 \odot$ & $\odot .1131 \mathrm{E}+08$ \\
\hline 20 & 247.062 & 277.442 & $1.000 \odot$ & $0.1142 \mathrm{E}+08$ \\
\hline 21 & 247.433 & 278.479 & 1.0000 & $\odot .1150 \mathrm{E}+08$ \\
\hline 22 & 247.433 & 280.172 & 1.0000 & $\odot .1152 \mathrm{E}+08$ \\
\hline 23 & 247.433 & 281.683 & 1.0000 & $\odot .1152 \mathrm{E}+08$ \\
\hline 24 & 247.433 & 283.079 & 1.0000 & $\odot .1152 \mathrm{E}+\odot 8$ \\
\hline 25 & 247.433 & 283.082 & 1.0000 & $\odot .1152 \mathrm{E}+08$ \\
\hline
\end{tabular}

\begin{tabular}{|c|c|c|c|c|}
\hline \multicolumn{5}{|c|}{ lambda $=0.1824505627$} \\
\hline node & $\mathrm{T}(\mathrm{K})$ & Twall(K) & $\mathrm{x}$ & $P(P a)$ \\
\hline 1 & 143.007 & $165.0 \odot 7$ & . . - & $\odot .1087 \mathrm{E}+07$ \\
\hline 2 & 165.888 & 187.982 & 0.9492 & $\odot .2004 \mathrm{E}+\odot 7$ \\
\hline 3 & 185.941 & 206.169 & $\odot .9801$ & $\odot .3983 E+\odot 7$ \\
\hline 4 & 194.113 & 212.665 & $1.000 \odot$ & $\odot .5265 \mathrm{E}+\odot 7$ \\
\hline 5 & 213.692 & 221.025 & 1.0000 & $\odot .5954 \mathrm{E}+07$ \\
\hline 6 & 213.692 & 227.953 & 1.0000 & $\odot .6956 \mathrm{E}+\odot 7$ \\
\hline 7 & 213.737 & 232.107 & 1.0000 & $\odot .7590 \mathrm{E}+07$ \\
\hline 8 & 213.737 & 237.233 & $1.000 \odot$ & $\odot .7866 \mathrm{E}+07$ \\
\hline 9 & 218.644 & 241.956 & 1.0000 & $\odot .8656 \mathrm{E}+\odot 7$ \\
\hline 10 & 218.715 & 247.289 & $1.000 \odot$ & $\odot .8917 \mathrm{E}+\odot 7$ \\
\hline 11 & 224.315 & 252.208 & 1.0000 & $\odot .9301 \mathrm{E}+07$ \\
\hline 12 & 227.686 & 256.255 & 1.0000 & $\odot .9748 \mathrm{E}+\odot 7$ \\
\hline 13 & 229.840 & 259.013 & 1.0000 & $0.1005 \mathrm{E}+08$ \\
\hline 14 & 229.855 & 262.399 & $1.000 \odot$ & $\odot .1011 \mathrm{E}+\odot 8$ \\
\hline 15 & 238.892 & 265.382 & 1.0000 & $0.1054 \mathrm{E}+08$ \\
\hline 16 & 239.835 & 268.146 & 1.0000 & $\odot .1085 \mathrm{E}+08$ \\
\hline 17 & 239.835 & 270.355 & 1.0000 & $\odot .1090 \mathrm{E}+08$ \\
\hline 18 & 244.571 & 272.577 & 1.0000 & $0.1114 \mathrm{E}+08$ \\
\hline 19 & 244.598 & 274.304 & $1.000 \odot$ & $\odot .1127 \mathrm{E}+08$ \\
\hline 20 & 244.598 & 276.221 & 1.0000 & $\odot .1127 \mathrm{E}+\odot 8$ \\
\hline 21 & 245.270 & 277.258 & 1.0000 & $\odot .1131 \mathrm{E}+\odot 8$ \\
\hline 22 & 245.270 & 278.951 & 1.0000 & $\odot .1137 \mathrm{E}+08$ \\
\hline 23 & 245.270 & 280.768 & 1.0000 & $\odot .1137 \mathrm{E}+08$ \\
\hline 24 & 245.270 & 282.163 & 1.0000 & $\odot .1137 \mathrm{E}+08$ \\
\hline 25 & 245.270 & 282.167 & 1.0000 & $\odot .1137 \mathrm{E}+08$ \\
\hline
\end{tabular}




\begin{tabular}{|c|c|c|c|c|}
\hline \multicolumn{5}{|c|}{ lambda $=0.1802584529$} \\
\hline node & $T^{\prime}(K)$ & Twall(K) & $\mathrm{x}$ & $\mathrm{P}(\mathrm{Pa})$ \\
\hline 1 & 142.642 & 163.424 & $-\ldots$ & $\odot .1072 \mathrm{E}+07$ \\
\hline 2 & 165.465 & 186.428 & $\odot .9521$ & $\odot .1978 \mathrm{E}+\odot 7$ \\
\hline 3 & 185.940 & 204.643 & $\odot .9794$ & $\odot .3983 E+\odot 7$ \\
\hline 4 & 203.967 & 211.645 & $1.000 \odot$ & $\odot .5328 \mathrm{E}+\odot 7$ \\
\hline 5 & 209.944 & 220.308 & $1.000 \odot$ & $\odot .5836 E+\odot 7$ \\
\hline 6 & 209.944 & 226.643 & 1.0000 & $\odot .6826 \mathrm{E}+\odot 7$ \\
\hline 7 & 216.889 & 231.014 & 1.0000 & $\odot .7350 \mathrm{E}+\odot 7$ \\
\hline 8 & 216.889 & 235.740 & 1.0000 & $\odot .7982 E+\odot 7$ \\
\hline 9 & 218.974 & 240.451 & $1.000 \odot$ & $\odot .8579 \mathrm{E}+\odot 7$ \\
\hline 10 & 220.116 & 245.439 & 1.0000 & $\odot .8970 \mathrm{E}+\odot 7$ \\
\hline 11 & 221.168 & 250.137 & $1.000 \odot$ & $\odot .9170 \mathrm{E}+\odot 7$ \\
\hline 12 & 221.168 & 254.129 & 1.0000 & $\odot .9399 \mathrm{E}+\odot 7$ \\
\hline 13 & 230.402 & 257.466 & 1.0000 & $\odot .9853 E+\odot 7$ \\
\hline 14 & 231.448 & 260.586 & 1.0000 & $\odot .1018 \mathrm{E}+\odot 8$ \\
\hline 15 & 233.927 & 263.806 & $1.000 \odot$ & $\odot .1032 \mathrm{E}+\odot 8$ \\
\hline 16 & 239.759 & 266.754 & 1.0000 & $\odot .1072 \mathrm{E}+08$ \\
\hline 17 & 239.924 & 268.830 & 1.0000 & $\odot .1090 \mathrm{E}+08$ \\
\hline 18 & 239.924 & 271.192 & 1.0000 & $0.1091 \mathrm{E}+08$ \\
\hline 19 & 242.908 & 273.084 & $1.000 \odot$ & $\odot .1107 \mathrm{E}+08$ \\
\hline 20 & 242.950 & 275.001 & $1.000 \odot$ & $0.1116 \mathrm{E}+08$ \\
\hline 21 & 242.950 & 276.038 & 1.0000 & $0.1116 \mathrm{E}+08$ \\
\hline 22 & 242.950 & 277.830 & 1.0000 & $\odot .1118 \mathrm{E}+08$ \\
\hline 23 & 242.950 & 279.852 & 1.0000 & $0.1118 \mathrm{E}+08$ \\
\hline 24 & 242.950 & 281.248 & 1.0000 & $\odot .1118 \mathrm{E}+\odot 8$ \\
\hline 25 & 242.950 & 281.251 & 1.0000 & $\odot .1118 \mathrm{E}+08$ \\
\hline
\end{tabular}

\begin{tabular}{|c|c|c|c|c|}
\hline \multicolumn{5}{|c|}{ lambda $=0.1793928295$} \\
\hline node & $\mathrm{T}(\mathrm{K})$ & Twall(K) & $\mathrm{x}$ & $\mathrm{P}(\mathrm{Pa})$ \\
\hline 1 & 142.490 & 161.914 & $\ldots$ & $\odot .1066 \mathrm{E}+07$ \\
\hline 2 & 165.288 & 184.903 & 0.9531 & $\odot .1967 \mathrm{E}+07$ \\
\hline 3 & 185.940 & 203.152 & $\odot .9770$ & $\odot .3983 E+\odot 7$ \\
\hline 4 & 214.544 & 211.444 & $1.000 \odot$ & $\odot .5153 \mathrm{E}+\odot 7$ \\
\hline 5 & 214.544 & 219.610 & 1.0000 & $\odot .5964 \mathrm{E}+07$ \\
\hline 6 & 214.544 & 225.469 & 1.0000 & $\odot .6959 \mathrm{E}+\odot 7$ \\
\hline 7 & 215.269 & 230.049 & 1.0000 & $\odot .7204 \mathrm{E}+07$ \\
\hline 8 & 215.721 & 234.367 & 1.0000 & $\odot .7917 \mathrm{E}+07$ \\
\hline 9 & 215.721 & 238.885 & 1.0000 & $\odot .8326 \mathrm{E}+\odot 7$ \\
\hline 10 & 220.999 & 243.861 & $1.000 \odot$ & $\odot .8877 \mathrm{E}+\odot 7$ \\
\hline 11 & 221.941 & 248.292 & 1.0000 & $\odot .9202 \mathrm{E}+07$ \\
\hline 12 & 222.050 & 251.981 & 1.0000 & $\odot .9436 \mathrm{E}+07$ \\
\hline 13 & 223.983 & 255.686 & 1.0000 & $\odot .9519 \mathrm{E}+07$ \\
\hline 14 & 232.051 & 259.060 & $1.000 \odot$ & $\odot .1006 \mathrm{E}+\odot 8$ \\
\hline 15 & 232.895 & 262.114 & 1.0000 & $\odot .1027 \mathrm{E}+08$ \\
\hline 16 & 233.751 & 265.190 & 1.0000 & $0.1041 \mathrm{E}+08$ \\
\hline 17 & 239.690 & 267.571 & 1.0000 & $0.1076 \mathrm{E}+08$ \\
\hline 18 & 239.699 & 269.766 & 1.0000 & $\odot .1089 \mathrm{E}+08$ \\
\hline 19 & 239.699 & 271.863 & $1.000 \odot$ & $\odot .1089 \mathrm{E}+08$ \\
\hline 20 & 241.044 & 273.780 & 1.0000 & $\odot .1097 \mathrm{E}+\odot 8$ \\
\hline 21 & 241.044 & 274.817 & 1.0000 & $\odot .1103 \mathrm{E}+\odot 8$ \\
\hline 22 & 241.044 & 276.915 & 1.0000 & $\odot .1103 \mathrm{E}+08$ \\
\hline 23 & 241.044 & 278.937 & 1.0000 & $\odot .1103 \mathrm{E}+08$ \\
\hline 24 & 241.044 & 280.332 & 1.0000 & $\odot .1103 \mathrm{E}+08$ \\
\hline 25 & 241.044 & 280.336 & 1.0000 & $\odot .1103 \mathrm{E}+08$ \\
\hline
\end{tabular}




\begin{tabular}{|c|c|c|c|c|}
\hline \multicolumn{5}{|c|}{$.00 \quad$ lambda $=0.1791149378$} \\
\hline node & $T^{\prime}(K)$ & Twall(K) & $x$ & $P(P a)$ \\
\hline 1 & 142.441 & 160.541 & $----_{-}$ & $\odot .1065 \mathrm{E}+07$ \\
\hline 2 & 165.232 & 183.527 & $\odot .9525$ & $\odot .1964 \mathrm{E}+\odot 7$ \\
\hline 3 & 185.941 & 201.803 & $\odot .9781$ & $\odot .3983 E+\odot 7$ \\
\hline 4 & 228.189 & 212.194 & $1.000 \odot$ & $\odot .5160 \mathrm{E}+\odot 7$ \\
\hline 5 & 228.189 & 219.837 & $1.000 \odot$ & $\odot .6343 E+07$ \\
\hline 6 & 228.306 & 225.267 & 1.0000 & $\odot .7080 \mathrm{E}+\odot 7$ \\
\hline 7 & 228.306 & 229.524 & $1.000 \odot$ & $\odot .7641 E+\odot 7$ \\
\hline 8 & 228.306 & 233.518 & 1.0000 & $\odot .8375 \mathrm{E}+\odot 7$ \\
\hline 9 & 228.306 & 237.728 & 1.0000 & $\odot .8769 \mathrm{E}+07$ \\
\hline 10 & 228.306 & 242.549 & 1.0000 & $\odot .9009 \mathrm{E}+\odot 7$ \\
\hline 11 & 228.306 & 246.780 & $1.000 \odot$ & $\odot .9417 \mathrm{E}+\odot 7$ \\
\hline 12 & 228.306 & 250.337 & 1.0000 & $\odot .9608 \mathrm{E}+07$ \\
\hline 13 & 228.306 & 253.822 & $1.000 \odot$ & $\odot .9702 \mathrm{E}+\odot 7$ \\
\hline 14 & 229.551 & 257.534 & $1.000 \odot$ & $9 \mathrm{E}+07$ \\
\hline 15 & 233.256 & 260.588 & 1.0000 & $\odot .1023 \mathrm{E}+08$ \\
\hline 16 & 233.257 & 263.489 & $1.000 \odot$ & $\odot .1039 \mathrm{E}+08$ \\
\hline 17 & 233.947 & 266.175 & 1.0000 & $\odot .1042 \mathrm{E}+08$ \\
\hline 18 & 238.829 & 268.545 & 1.0000 & $\odot .1072 \mathrm{E}+08$ \\
\hline 19 & 238.829 & 270.642 & 1.0000 & $\odot .1083 \mathrm{E}+08$ \\
\hline 20 & 238.829 & 272.559 & $1.000 \odot$ & $\odot .1083 \mathrm{E}+08$ \\
\hline 21 & 238.829 & 273.661 & 1.0000 & $0.1085 \mathrm{E}+08$ \\
\hline 22 & 238.829 & 275.999 & 1.0000 & $\odot .1086 \mathrm{E}+08$ \\
\hline 23 & 238.829 & 278.021 & 1.0000 & $\odot .1087 \mathrm{E}+08$ \\
\hline 24 & 238.829 & 279.417 & 1.0000 & $\odot .1087 \mathrm{E}+\odot 8$ \\
\hline 25 & 238.829 & 279.420 & 1.0000 & $\odot .1087 \mathrm{E}+08$ \\
\hline
\end{tabular}

\begin{tabular}{|c|c|c|c|c|}
\hline \multicolumn{5}{|c|}{ lambda $=0.1790664345$} \\
\hline node & $\mathrm{T}(\mathrm{K})$ & Twall(K) & $\mathrm{x}$ & $\mathrm{P}(\mathrm{Pa})$ \\
\hline 1 & 142.433 & 159.254 & $\ldots$ & $\odot .1064 \mathrm{E}+07$ \\
\hline 2 & 165.222 & 182.217 & 0.9521 & $\odot .1963 \mathrm{E}+07$ \\
\hline 3 & 185.858 & 200.601 & $\odot .9832$ & $\odot .3974 \mathrm{E}+\odot 7$ \\
\hline 4 & 217.253 & 213.073 & $1.000 \odot$ & $\odot .4858 \mathrm{E}+\odot 7$ \\
\hline 5 & 218.356 & 220.160 & 1.0000 & $\odot .6019 \mathrm{E}+07$ \\
\hline 6 & 234.526 & 225.701 & 1.0000 & $\odot .7000 \mathrm{E}+\odot 7$ \\
\hline 7 & 234.526 & 229.679 & 1.0000 & $\odot .7849 \mathrm{E}+07$ \\
\hline 8 & 234.526 & 233.383 & 1.0000 & $\odot .8480 \mathrm{E}+07$ \\
\hline 9 & 234.526 & 237.328 & 1.0000 & $\odot .8892 \mathrm{E}+\odot 7$ \\
\hline 10 & 234.526 & 241.819 & $1.000 \odot$ & $\odot .9251 \mathrm{E}+\odot 7$ \\
\hline 11 & 234.526 & 245.863 & 1.0000 & $\odot .9491 \mathrm{E}+07$ \\
\hline 12 & 234.526 & 249.329 & 1.0000 & $\odot .9724 \mathrm{E}+\odot 7$ \\
\hline 13 & 234.526 & 252.628 & 1.0000 & $\odot .9959 \mathrm{E}+07$ \\
\hline 14 & 234.526 & 256.145 & $1.000 \odot$ & $\odot .1008 \mathrm{E}+\odot 8$ \\
\hline 15 & 234.526 & 259.101 & 1.0000 & $\odot .1025 \mathrm{E}+08$ \\
\hline 16 & 234.526 & 262.024 & 1.0000 & $\odot .1036 \mathrm{E}+08$ \\
\hline 17 & 234.526 & 264.590 & 1.0000 & $0.1044 \mathrm{E}+08$ \\
\hline 18 & 234.526 & 267.208 & 1.0000 & $0.1044 \mathrm{E}+08$ \\
\hline 19 & 236.007 & 269.422 & $1.000 \odot$ & $\odot .1059 \mathrm{E}+08$ \\
\hline 20 & 236.066 & 271.339 & 1.0000 & $\odot .1065 \mathrm{E}+\odot 8$ \\
\hline 21 & 236.066 & 272.599 & 1.0000 & $\odot .1065 \mathrm{E}+\odot 8$ \\
\hline 22 & 236.066 & 275.084 & 1.0000 & $\odot .1067 \mathrm{E}+08$ \\
\hline 23 & 236.323 & 277.106 & 1.0000 & $\odot .1069 \mathrm{E}+08$ \\
\hline 24 & 236.323 & 278.501 & 1.0000 & $\odot .1070 \mathrm{E}+08$ \\
\hline 25 & 236.323 & 278.505 & 1.0000 & $\odot .1070 \mathrm{E}+08$ \\
\hline
\end{tabular}




\begin{tabular}{|c|c|c|c|c|}
\hline \multicolumn{5}{|c|}{ lambda $=0.1781143695$} \\
\hline node & $T^{\prime}(K)$ & Twall(K) & $\mathrm{x}$ & $\mathrm{P}(\mathrm{Pa})$ \\
\hline 1 & 142.266 & 158.033 & $-\ldots$ & $\odot .1058 \mathrm{E}+07$ \\
\hline 2 & 165.028 & 180.997 & $\odot .9521$ & $\odot .1951 \mathrm{E}+\odot 7$ \\
\hline 3 & 184.824 & 199.533 & $\odot .9884$ & $\odot .3857 E+\odot 7$ \\
\hline 4 & 215.902 & 213.246 & $1.000 \odot$ & $\odot .4828 \mathrm{E}+\odot 7$ \\
\hline 5 & 216.141 & 219.846 & $1.000 \odot$ & $\odot .5954 \mathrm{E}+\odot 7$ \\
\hline 6 & 227.313 & 226.195 & 1.0000 & $\odot .6662 \mathrm{E}+\odot 7$ \\
\hline 7 & 228.302 & 229.916 & 1.0000 & $\odot .7575 \mathrm{E}+\odot 7$ \\
\hline 8 & 230.221 & 233.382 & 1.0000 & $\odot .8133 E+\odot 7$ \\
\hline 9 & 230.261 & 237.072 & $1.000 \odot$ & $\odot .8638 \mathrm{E}+\odot 7$ \\
\hline 10 & 231.633 & 241.297 & 1.0000 & $\odot .9067 \mathrm{E}+\odot 7$ \\
\hline 11 & 232.223 & 245.156 & $1.000 \odot$ & $\odot .9280 E+\odot 7$ \\
\hline 12 & 232.223 & 248.472 & 1.0000 & $\odot .9553 \mathrm{E}+\odot 7$ \\
\hline 13 & 235.082 & 251.712 & 1.0000 & $\odot .9888 \mathrm{E}+\odot 7$ \\
\hline 14 & 235.082 & 255.046 & 1.0000 & $\odot .1011 \mathrm{E}+\odot 8$ \\
\hline 15 & 235.082 & 257.881 & $1.000 \odot$ & $\odot .1023 \mathrm{E}+\odot 8$ \\
\hline 16 & 235.082 & 260.803 & 1.0000 & $\odot .1029 E+0 \varepsilon$ \\
\hline 17 & 235.082 & 263.330 & 1.0000 & $\odot .1041 \mathrm{E}+08$ \\
\hline 18 & 235.082 & 265.809 & 1.0000 & $0.1046 \mathrm{E}+08$ \\
\hline 19 & 235.082 & 268.201 & $1.000 \odot$ & $\odot .1046 \mathrm{E}+08$ \\
\hline 20 & 235.082 & 270.1 & $1.000 \odot$ & $\odot .1051 \mathrm{E}+08$ \\
\hline 21 & 235.082 & 271.520 & 1.0000 & $0.1056 \mathrm{E}+08$ \\
\hline 22 & 235.082 & 274.168 & 1.0000 & $\odot .1057 \mathrm{E}+08$ \\
\hline 23 & 235.082 & 276.190 & 1.0000 & $0.1057 \mathrm{E}+08$ \\
\hline 24 & 235.082 & 277.586 & 1.0000 & $\odot .1058 \mathrm{E}+\odot 8$ \\
\hline 25 & 235.082 & 277.589 & 1.0000 & $\odot .1058 \mathrm{E}+08$ \\
\hline
\end{tabular}

\begin{tabular}{|c|c|c|c|c|}
\hline time & $(\sec )=$ & 3.00 & $\mathrm{da}=0$ & 19094308 \\
\hline node & $\mathrm{T}(\mathrm{K})$ & Twall(K) & $\mathrm{x}$ & $P(P a)$ \\
\hline 1 & 141.720 & 156.914 & - - - - - & $0.1037 \mathrm{E}+07$ \\
\hline 2 & 164.395 & 179.889 & 0.9540 & $\odot .1914 \mathrm{E}+07$ \\
\hline 3 & 183.868 & 198.465 & $\odot .9923$ & $\odot .3749 E+\odot 7$ \\
\hline 4 & 203.141 & 213.071 & $1.00 \odot \odot$ & $\odot .4543 E+\odot 7$ \\
\hline 5 & 222.567 & 219.787 & 1.0000 & $0.6027 \mathrm{E}+07$ \\
\hline 6 & 222.567 & 225.865 & 1.0000 & $\odot .6523 \mathrm{E}+07$ \\
\hline 7 & 222.567 & 229.497 & 1.0000 & $\odot .7259 \mathrm{E}+\odot 7$ \\
\hline 8 & 224.259 & 232.922 & 1.0000 & $\odot .7815 \mathrm{E}+07$ \\
\hline 9 & 224.259 & 236.372 & 1.0000 & $\odot .8340 E+\odot 7$ \\
\hline 10 & 230.213 & 240.687 & $1.000 \odot$ & $\odot .8812 \mathrm{E}+\odot 7$ \\
\hline 11 & 230.826 & 244.373 & 1.0000 & $\odot .9203 \mathrm{E}+07$ \\
\hline 12 & 230.830 & 247.407 & 1.0000 & $\odot .9479 E+\odot 7$ \\
\hline 13 & 231.496 & 250.738 & 1.0000 & $\odot .9608 \mathrm{E}+\odot 7$ \\
\hline 14 & 231.907 & 253.957 & $1.00 \odot \odot$ & $\odot .9905 E+\odot 7$ \\
\hline 15 & 234.127 & 256.660 & 1.0000 & $\odot .1010 \mathrm{E}+08$ \\
\hline 16 & 234.127 & 259.583 & 1.0000 & $\odot .1023 E+08$ \\
\hline 17 & 234.127 & 262.109 & 1.0000 & $\odot .1027 \mathrm{E}+08$ \\
\hline 18 & 234.127 & 264.588 & 1.0000 & $\odot .1037 \mathrm{E}+08$ \\
\hline 19 & 234.127 & 266.980 & $1.000 \odot$ & $\odot .1038 \mathrm{E}+08$ \\
\hline 20 & 234.127 & 268.897 & 1.0000 & $\odot .1039 E+08$ \\
\hline 21 & 234.127 & 270.604 & 1.0000 & $0.1044 \mathrm{E}+08$ \\
\hline 22 & 234.127 & 273.253 & 1.0000 & $\odot .1047 \mathrm{E}+08$ \\
\hline 23 & 234.127 & 275.275 & 1.0000 & $0.1048 \mathrm{E}+08$ \\
\hline 24 & 234.127 & 276.921 & 1.0000 & $\odot .1048 \mathrm{E}+08$ \\
\hline 25 & 234.127 & 276.924 & 1.0000 & $\odot .1048 \mathrm{E}+08$ \\
\hline
\end{tabular}




\begin{tabular}{|c|c|c|c|c|}
\hline \multicolumn{5}{|c|}{$.00 \quad$ lambda $=0.1706423014$} \\
\hline node & $T^{\prime}(K)$ & Twall(K) & $x$ & $P(P a)$ \\
\hline 1 & 140.961 & 155.845 & $\cdots$ & $\odot .1009 \mathrm{E}+07$ \\
\hline 2 & 163.515 & 178.821 & $\odot .9557$ & $\odot .1861 E+\odot 7$ \\
\hline 3 & 182.366 & 197.514 & ๑. 9951 & $\odot .3579 \mathrm{E}+\odot 7$ \\
\hline 4 & 195.850 & 211.916 & $1.000 \odot$ & $\odot .4380 E+\odot 7$ \\
\hline 5 & 231.950 & 220.263 & $1.000 \odot$ & $\odot .6089 \mathrm{E}+07$ \\
\hline 6 & 231.950 & 225.950 & 1.0000 & $\odot .6798 \mathrm{E}+\odot 7$ \\
\hline 7 & 231.950 & 229.313 & $1.000 \odot$ & $\odot .7527 \mathrm{E}+\odot 7$ \\
\hline 8 & 231.950 & 232.507 & 1.0000 & $\odot .8044 \mathrm{E}+07$ \\
\hline 9 & 231.950 & 235.723 & 1.0000 & $\odot .8514 \mathrm{E}+07$ \\
\hline 10 & 231.950 & 239.964 & 1.0000 & $\odot .8754 \mathrm{E}+\odot 7$ \\
\hline 11 & 231.950 & 243.448 & $1.000 \odot$ & $\odot .9179 \mathrm{E}+\odot 7$ \\
\hline 12 & 231.950 & 246.449 & 1.0000 & $\odot .9401 \mathrm{E}+07$ \\
\hline 13 & 231.950 & 249.581 & $1.000 \odot$ & $\odot .9620 \mathrm{E}+\odot 7$ \\
\hline 14 & 231.950 & 252.742 & $1.000 \odot$ & $\odot .9797 \mathrm{E}+\odot 7$ \\
\hline 15 & 231.950 & 255.439 & 1.0000 & $\odot .9901 \mathrm{E}+\odot 7$ \\
\hline 16 & 232.323 & 258.362 & $1.000 \odot$ & $0.1006 \mathrm{E}+08$ \\
\hline 17 & 232.323 & 260.888 & 1.0000 & $\odot .1015 \mathrm{E}+08$ \\
\hline 18 & 232.323 & 263.367 & 1.0000 & $\odot .1018 \mathrm{E}+08$ \\
\hline 19 & 232.921 & 265.760 & 1.0000 & $0.1027 \mathrm{E}+08$ \\
\hline 20 & 232.921 & 267.677 & $1.000 \odot$ & $0.1031 \mathrm{E}+08$ \\
\hline 21 & 232.921 & 269.689 & 1.0000 & $\odot .1031 \mathrm{E}+\odot 8$ \\
\hline 22 & 232.921 & 272.337 & 1.0000 & $\odot .1035 \mathrm{E}+08$ \\
\hline 23 & 232.921 & 274.359 & 1.0000 & $0.1037 \mathrm{E}+08$ \\
\hline 24 & 232.921 & 276.311 & 1.0000 & $\odot .1037 \mathrm{E}+\odot 8$ \\
\hline 25 & 232.921 & 276.313 & 1.0000 & $\odot .1037 \mathrm{E}+08$ \\
\hline
\end{tabular}

\begin{tabular}{|c|c|c|c|c|}
\hline \multicolumn{5}{|c|}{ lambda $=0.1648330837$} \\
\hline node & $T(K)$ & Twall(K) & $\mathrm{x}$ & $\mathrm{P}(\mathrm{Pa})$ \\
\hline 1 & 139.954 & 154.777 & $-\ldots$ & $\odot .9711 E+06$ \\
\hline 2 & 162.347 & 177.752 & 0.9585 & $\odot .1791 \mathrm{E}+07$ \\
\hline 3 & 181.234 & 196.524 & $\odot .9975$ & $\odot .3451 E+\odot 7$ \\
\hline 4 & 198.909 & 210.801 & $1.000 \odot$ & $0.4448 \mathrm{E}+07$ \\
\hline 5 & 239.101 & 221.357 & 1.0000 & $0.6119 \mathrm{E}+07$ \\
\hline 6 & 239.101 & 226.725 & 1.0000 & $\odot .7007 \mathrm{E}+07$ \\
\hline 7 & 239.101 & 229.824 & 1.0000 & $0.7648 \mathrm{E}+07$ \\
\hline 8 & 239.101 & 232.791 & 1.0000 & $0.8246 \mathrm{E}+\Theta 7$ \\
\hline 9 & 239.101 & 235.757 & 1.0000 & $\odot .8582 \mathrm{E}+07$ \\
\hline 10 & 239.101 & 239.737 & $1.000 \odot$ & $\odot .9024 \mathrm{E}+\odot 7$ \\
\hline 11 & 239.101 & 243.010 & 1.0000 & $\odot .9316 \mathrm{E}+07$ \\
\hline 12 & 239.101 & 245.878 & 1.0000 & $\odot .9589 \mathrm{E}+\odot 7$ \\
\hline 13 & 239.101 & 248.838 & 1.0000 & $\odot .9875 \mathrm{E}+\Theta 7$ \\
\hline 14 & 239.101 & 251.906 & 1.0000 & $\odot .9998 \mathrm{E}+07$ \\
\hline 15 & 239.101 & 254.513 & 1.0000 & $0.1015 \mathrm{E}+08$ \\
\hline 16 & 239.101 & 257.399 & 1.0000 & $0.1023 E+08$ \\
\hline 17 & 239.101 & 259.902 & 1.0000 & $0.1037 \mathrm{E}+08$ \\
\hline 18 & 239.101 & 262.386 & 1.0000 & $0.1042 \mathrm{E}+08$ \\
\hline 19 & 239.101 & 264.806 & $1.000 \odot$ & $0.1047 \mathrm{E}+08$ \\
\hline 20 & 239.101 & 266.724 & 1.0000 & $0.1053 \mathrm{E}+08$ \\
\hline 21 & 239.101 & 268.773 & 1.0000 & $0.1054 \mathrm{E}+08$ \\
\hline 22 & 239.101 & 271.422 & 1.0000 & $0.1055 \mathrm{E}+08$ \\
\hline 23 & 239.101 & 273.638 & 1.0000 & $0.1058 \mathrm{E}+08$ \\
\hline 24 & 239.101 & 275.700 & 1.0000 & $0.1059 \mathrm{E}+08$ \\
\hline 25 & 239.101 & 275.703 & 1.0000 & $0.1059 \mathrm{E}+08$ \\
\hline
\end{tabular}

lambda_avg $=0.248590544$ 
Compressor station distance $=20$ miles, Pressure $=1167 \mathrm{psi}, 20$ axial node elements

\begin{tabular}{|c|c|c|c|c|}
\hline time & $(\sec )=$ & $1.00 \quad 1$ & $\mathrm{a} a=c$ & \\
\hline node & $\mathrm{T}(\mathrm{K})$ & Twall(K) & $x$ & $P(P a)$ \\
\hline 1 & 247.398 & 281.591 & - - - - - & $0.3705 \mathrm{E}+07$ \\
\hline 2 & 286.982 & 288.000 & 1.0000 & $\odot .6835 \mathrm{E}+\odot 7$ \\
\hline 3 & 288.000 & $288.0 \odot \odot$ & $1.000 \odot$ & $\odot .8033 \mathrm{E}+\odot 7$ \\
\hline 4 & 288.000 & 288.000 & 1.0000 & $\odot .8044 \mathrm{E}+\odot 7$ \\
\hline 5 & 288.000 & $288.00 \odot$ & 1.0000 & $\odot .8044 \mathrm{E}+\odot 7$ \\
\hline 6 & $288.00 \odot$ & $288 . \odot \odot \odot$ & $1.000 \odot$ & $\odot .8 \odot 44 \mathrm{E}+\odot 7$ \\
\hline 7 & 288.000 & 288.000 & 1.0000 & $\odot .8044 \mathrm{E}+\odot 7$ \\
\hline 8 & 288.000 & $288.00 \odot$ & 1.0000 & $\odot .8044 \mathrm{E}+07$ \\
\hline 9 & 288.000 & $288 . \odot \odot \odot$ & $1.000 \odot$ & $\odot .8044 \mathrm{E}+\odot 7$ \\
\hline 10 & 288.000 & 288.000 & 1.0000 & $0.8044 \mathrm{E}+07$ \\
\hline 11 & 288.000 & 288.000 & 1.0000 & $\odot .8044 \mathrm{E}+\odot 7$ \\
\hline 12 & 288.000 & 288.000 & 1.0000 & $0.8044 \mathrm{E}+07$ \\
\hline 13 & 288.000 & $288.00 \odot$ & 1.0000 & $0.8044 \mathrm{E}+07$ \\
\hline 14 & 288.000 & 288.000 & 1.0000 & $\odot .8044 \mathrm{E}+07$ \\
\hline 15 & $288.00 \odot$ & $288 . \odot \odot \odot$ & $1.000 \odot$ & $\odot .8044 \mathrm{E}+07$ \\
\hline 16 & 288.000 & 288.000 & 1.0000 & $\odot .8044 \mathrm{E}+07$ \\
\hline 17 & 288.000 & 288.000 & 1.0000 & $\odot .8044 \mathrm{E}+\odot 7$ \\
\hline 18 & 288.000 & $288.00 \odot$ & $1.000 \odot$ & $\odot .8044 \mathrm{E}+07$ \\
\hline 19 & 288.000 & 288.000 & 1.00 & $0.8044 \mathrm{E}+07$ \\
\hline 20 & 288.000 & $288.0 \odot \odot$ & 1.0000 & $\odot .8044 \mathrm{E}+07$ \\
\hline
\end{tabular}

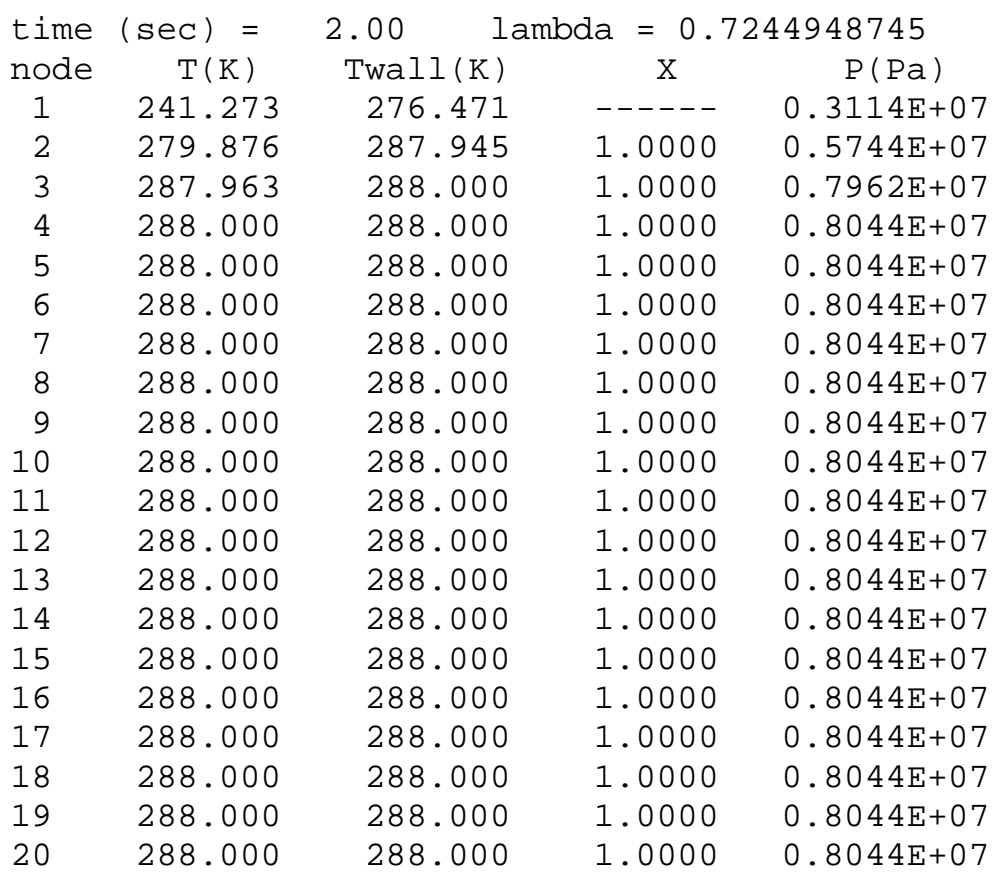

$\begin{array}{ccccc}\text { time } & (\mathrm{sec})= & 3.0 \odot \quad \text { lambda }=\odot .6175189018 \\ \text { node } & \mathrm{T}(\mathrm{K}) & \text { Twall }(\mathrm{K}) & \mathrm{X} & \mathrm{P}(\mathrm{Pa}) \\ 1 & 226.629 & 271.401 & --.- & \odot .2573 \mathrm{E}+07 \\ 2 & 262.888 & 287.379 & 1.000 \odot & 0.4745 \mathrm{E}+07 \\ 3 & 287.470 & 288.00 \odot & 1.000 \odot & 0.7800 \mathrm{E}+07 \\ 4 & 288.00 \odot & 288.00 \odot & 1.000 \odot & 0.8038 \mathrm{E}+07 \\ 5 & 288.00 \odot & 288.00 \odot & 1.000 \odot & 0.8044 \mathrm{E}+\odot 7 \\ 6 & 288.00 \odot & 288.00 \odot & 1.000 \odot & 0.8044 \mathrm{E}+07\end{array}$




\begin{tabular}{|c|c|c|c|c|}
\hline 7 & 288.000 & $288 . \odot \odot \odot$ & $1.00 \odot \odot$ & $\odot .8044 \mathrm{E}+07$ \\
\hline 8 & $288.00 \odot$ & 288.000 & 1.0000 & $\odot .8044 \mathrm{E}+\odot 7$ \\
\hline 9 & $288.00 \odot$ & $288.00 \odot$ & 1.0000 & $\odot .8044 \mathrm{E}+07$ \\
\hline 10 & 288.000 & 288.000 & 1.0000 & $\odot .8044 \mathrm{E}+07$ \\
\hline 11 & 288.000 & $288.00 \odot$ & 1.0000 & $0.8044 \mathrm{E}+07$ \\
\hline 12 & 288.000 & 288.000 & 1.0000 & $\odot .8044 \mathrm{E}+07$ \\
\hline 13 & $288.00 \odot$ & $288 . \odot \odot \odot$ & $1.00 \odot \odot$ & $\odot .8 \odot 44 \mathrm{E}+\odot 7$ \\
\hline 14 & 288.000 & $288.00 \odot$ & 1.0000 & $\odot .8044 \mathrm{E}+07$ \\
\hline 15 & $288.00 \odot$ & $288 . \odot \odot \odot$ & 1.0000 & $\odot .8 \odot 44 \mathrm{E}+\odot 7$ \\
\hline 16 & 288.000 & 288.000 & 1.0000 & $0.8044 \mathrm{E}+07$ \\
\hline 17 & 288.000 & 288.000 & 1.0000 & $\odot .8044 \mathrm{E}+07$ \\
\hline 18 & 288.000 & $288 . \odot \odot \odot$ & 1.0000 & $\odot .8 \odot 44 \mathrm{E}+07$ \\
\hline 19 & $288.00 \odot$ & $288 . \odot \odot \odot$ & 1.0000 & $\odot .8 \odot 44 \mathrm{E}+\odot 7$ \\
\hline 20 & 288.000 & 288.000 & 1.0000 & $\odot .8044 \mathrm{E}+07$ \\
\hline time & $(\sec )=$ & lambda $=0.5290004611$ & \multicolumn{2}{|c|}{ lambda $=0.5290004611$} \\
\hline node & $\mathrm{T}(\mathrm{K})$ & Twall(K) & $\mathrm{x}$ & $\mathrm{P}(\mathrm{Pa})$ \\
\hline 1 & 205.044 & 265.588 & $-\ldots$ & $\odot .2096 \mathrm{E}+\odot 7$ \\
\hline 2 & 237.849 & 285.711 & 1.0000 & $\odot .3867 \mathrm{E}+\odot 7$ \\
\hline 3 & 285.901 & 288.000 & $1.000 \odot$ & $\odot .7555 \mathrm{E}+\odot 7$ \\
\hline 4 & 288.000 & $288.00 \odot$ & 1.0000 & $\odot .8019 \mathrm{E}+\odot 7$ \\
\hline 5 & 288.000 & $288.00 \odot$ & 1.0000 & $\odot .8044 \mathrm{E}+07$ \\
\hline 6 & 288.000 & 288.000 & 1.0000 & $\odot .8044 \mathrm{E}+07$ \\
\hline 7 & 288.000 & 288.000 & 1.0000 & $\odot .8044 \mathrm{E}+07$ \\
\hline 8 & 288.000 & $288.00 \odot$ & 1.0000 & $\odot .8044 \mathrm{E}+\odot 7$ \\
\hline 9 & $288.00 \odot$ & 288.000 & 1.0000 & $\odot .8044 \mathrm{E}+07$ \\
\hline 10 & 288.000 & 288.000 & 1.0000 & $\odot .8044 \mathrm{E}+07$ \\
\hline 11 & 288.000 & $288.00 \odot$ & 1.0000 & $\odot .8044 \mathrm{E}+\odot 7$ \\
\hline 12 & 288.000 & $288.00 \odot$ & 1.0000 & $0.8044 \mathrm{E}+07$ \\
\hline 13 & 288. & $288.00 \odot$ & 1.0000 & $\odot .8044 \mathrm{E}+07$ \\
\hline 14 & 288.000 & $288.00 \odot$ & 1.0000 & $\odot .8044 \mathrm{E}+07$ \\
\hline 15 & 288.000 & $288.00 \odot$ & 1.0000 & $\odot .8044 \mathrm{E}+07$ \\
\hline 16 & 288.000 & 288.000 & 1.0000 & $\odot .8044 \mathrm{E}+07$ \\
\hline 17 & 288.000 & 288.000 & 1.0000 & $\odot .8044 \mathrm{E}+07$ \\
\hline 18 & 288. & $288 . \odot \odot \odot$ & $1.000 \odot$ & $\odot .8044 \mathrm{E}+07$ \\
\hline 19 & 288.000 & 288.000 & 1.0000 & $0.8044 \mathrm{E}+07$ \\
\hline 20 & 288.000 & $288.00 \odot$ & 1.0000 & $\odot .8044 \mathrm{E}+07$ \\
\hline time & $(\sec )=$ & $5.00 \quad 10$ & \multicolumn{2}{|c|}{ lambda $=0.4580084682$} \\
\hline node & $\mathrm{T}(\mathrm{K})$ & Twall(K) & $\mathrm{X}$ & $P(P a)$ \\
\hline 1 & 181.553 & 258.902 & - - - - - & $\odot .1708 \mathrm{E}+07$ \\
\hline 2 & 210.599 & 282.639 & $1.000 \odot$ & $\odot .3150 \mathrm{E}+\odot 7$ \\
\hline 3 & 282.363 & $288.00 \odot$ & 1.0000 & $0.7246 \mathrm{E}+07$ \\
\hline 4 & 287.846 & $288.00 \odot$ & 1.0000 & $\odot .7973 \mathrm{E}+\odot 7$ \\
\hline 5 & 288.000 & $288 . \odot \odot \odot$ & $1.000 \odot$ & $\odot .8 \odot 43 \mathrm{E}+\odot 7$ \\
\hline 6 & 288.6 & 288.6 & 1.00 & $\odot .8044 \mathrm{E}+07$ \\
\hline 7 & 288. & $288 . \odot$ & 1.00 & $0.8044 \mathrm{E}+07$ \\
\hline 8 & 288.000 & $288.00 \odot$ & 1.0000 & $\odot .8044 \mathrm{E}+\odot 7$ \\
\hline 9 & $288.00 \odot$ & 288.000 & 1.0000 & $\odot .8044 \mathrm{E}+07$ \\
\hline 10 & 288.000 & $288.00 \odot$ & 1.0000 & $\odot .8044 \mathrm{E}+07$ \\
\hline 11 & $288.00 \odot$ & $288 . \odot \odot \odot$ & 1.0000 & $\odot .8044 \mathrm{E}+\odot 7$ \\
\hline 12 & $288.00 \odot$ & $288.00 \odot$ & 1.0000 & $\odot .8044 \mathrm{E}+07$ \\
\hline 13 & $288.00 \odot$ & $288 . \odot \odot \odot$ & $1.000 \odot$ & $\odot .8 \odot 44 \mathrm{E}+\odot 7$ \\
\hline 14 & 288.000 & $288.00 \odot$ & $1.000 \odot$ & $\odot .8044 \mathrm{E}+07$ \\
\hline 15 & 288.000 & $288 . \odot \odot \odot$ & 1.0000 & $\odot .8044 \mathrm{E}+\odot 7$ \\
\hline 16 & 288.000 & $288.00 \odot$ & 1.0000 & $\odot .8044 \mathrm{E}+07$ \\
\hline 17 & 288.000 & $288.00 \odot$ & 1.0000 & $\odot .8044 \mathrm{E}+07$ \\
\hline
\end{tabular}




$\begin{array}{lllll}18 & 288.000 & 288.00 \odot & 1.000 \odot & \odot .8044 \mathrm{E}+07 \\ 19 & 288.000 & 288.00 \odot & 1.000 \odot & 0.8044 \mathrm{E}+07 \\ 20 & 288.000 & 288.00 \odot & 1.000 \odot & 0.8044 \mathrm{E}+07\end{array}$

\begin{tabular}{|c|c|c|c|c|}
\hline \multicolumn{5}{|c|}{$6.00 \quad$ lambda $=0.4031239450$} \\
\hline node & $T(K)$ & Twall(K) & $x$ & $\mathrm{P}(\mathrm{Pa})$ \\
\hline 1 & 160.575 & 251.608 & - - - - _ n & $\odot .1414 \mathrm{E}+\odot 7$ \\
\hline 2 & 186.265 & 278.269 & 1.0000 & $\odot .26 \odot 8 \mathrm{E}+\odot 7$ \\
\hline 3 & 276.172 & 287.806 & 1.0000 & $\odot .6894 \mathrm{E}+\odot 7$ \\
\hline 4 & 287.379 & $288 . \odot \odot \odot$ & 1.0000 & $\odot .7888 \mathrm{E}+\odot 7$ \\
\hline 5 & $288.00 \odot$ & $288.00 \odot$ & 1.0000 & $\odot .8037 \mathrm{E}+\odot 7$ \\
\hline 6 & $288.00 \odot$ & 288.000 & 1.0000 & $\odot .8044 \mathrm{E}+\odot 7$ \\
\hline 7 & 288.000 & $288.00 \odot$ & $1.000 \odot$ & $\odot .8044 \mathrm{E}+\odot 7$ \\
\hline 8 & $288.00 \odot$ & $288 . \odot \odot \odot$ & 1.0000 & $0.8044 \mathrm{E}+07$ \\
\hline 9 & 288.000 & 288.000 & 1.0000 & $\odot .8044 \mathrm{E}+07$ \\
\hline 10 & $288.00 \odot$ & $288.00 \odot$ & 1.0000 & $\odot .8044 \mathrm{E}+07$ \\
\hline 11 & $288.00 \odot$ & $288 . \odot \odot \odot$ & $1.000 \odot$ & $\odot .8 \odot 44 \mathrm{E}+\odot 7$ \\
\hline 12 & 288.000 & $288.00 \odot$ & 1.0000 & $\odot .8044 \mathrm{E}+07$ \\
\hline 13 & $288.00 \odot$ & $288 . \odot \odot \odot$ & $1.000 \odot$ & $\odot .8 \odot 44 \mathrm{E}+07$ \\
\hline 14 & $288.00 \odot$ & $288.00 \odot$ & $1.000 \odot$ & $\odot .8044 \mathrm{E}+07$ \\
\hline 15 & 288.000 & $288.00 \odot$ & 1.0000 & $\odot .8044 \mathrm{E}+07$ \\
\hline 16 & 288.000 & 288.000 & 1.0000 & $0.8044 \mathrm{E}+07$ \\
\hline 17 & $288.00 \odot$ & 288.000 & 1.0000 & $\odot .8044 \mathrm{E}+\odot 7$ \\
\hline 18 & 288.000 & 288.000 & 1.0000 & $\odot .8044 \mathrm{E}+07$ \\
\hline 19 & $288.00 \odot$ & $288 . \odot \odot \odot$ & $1.000 \odot$ & $\odot .8 \odot 44 \mathrm{E}+07$ \\
\hline 20 & $288.00 \odot$ & $288.0 \odot \odot$ & 1.0000 & $\odot .8044 \mathrm{E}+\odot 7$ \\
\hline
\end{tabular}

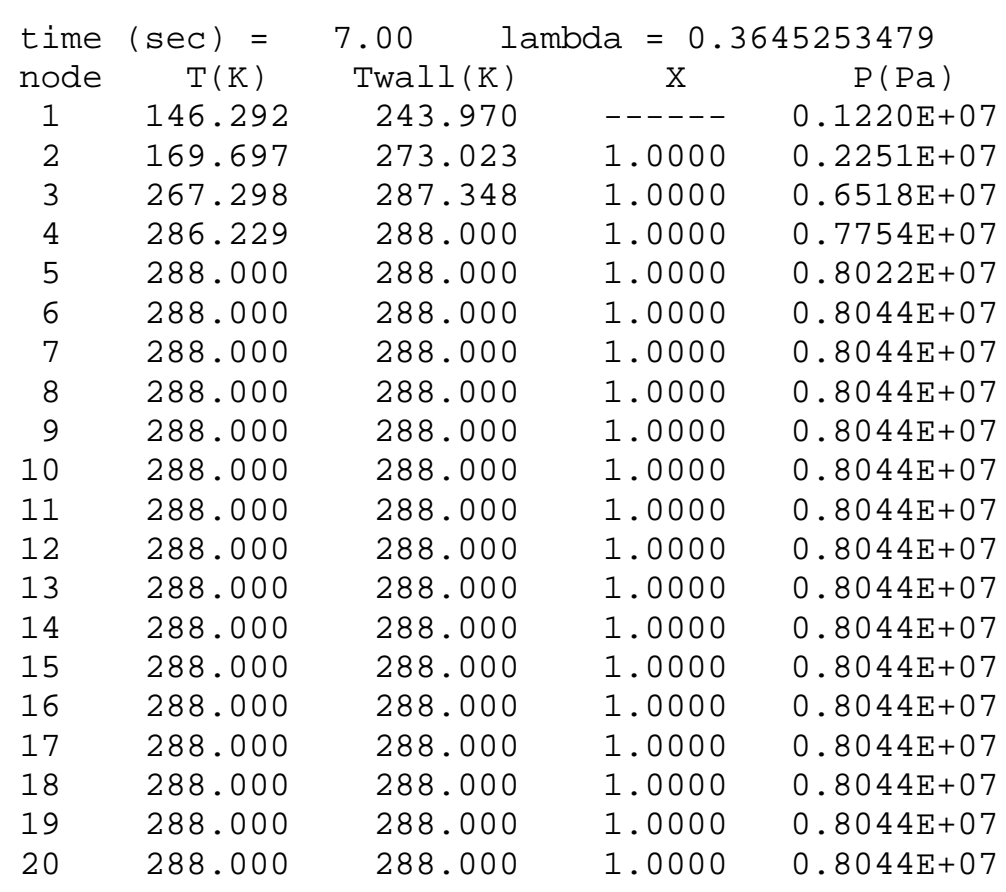

\begin{tabular}{ccccc} 
time & $(\mathrm{sec})=$ & 8.00 & \multicolumn{2}{c}{ lambda $=0.3445852697$} \\
node & $\mathrm{T}(\mathrm{K})$ & Twall $(\mathrm{K})$ & $\mathrm{X}$ & $\mathrm{P}(\mathrm{Pa})$ \\
1 & 144.059 & 236.554 &.--- & $0.1145 \mathrm{E}+07$ \\
2 & 167.109 & 267.633 & 0.9988 & $0.2111 \mathrm{E}+07$ \\
3 & 256.532 & 286.444 & $1.000 \odot$ & $0.6147 \mathrm{E}+07$ \\
4 & 283.992 & $288.00 \odot$ & $1.000 \odot$ & $0.7570 \mathrm{E}+07$ \\
5 & 287.867 & $288.00 \odot$ & $1.00 \odot \odot$ & $0.7991 \mathrm{E}+07$
\end{tabular}




\begin{tabular}{|c|c|c|c|c|}
\hline 6 & 288.000 & 288.000 & 1.0000 & $\odot .8043 \mathrm{E}+07$ \\
\hline 7 & 288.000 & $288 . \odot \odot \odot$ & $1.000 \odot$ & $\odot .8 \odot 44 \mathrm{E}+\odot 7$ \\
\hline 8 & $288.00 \odot$ & $288.0 \odot \odot$ & 1.0000 & $\odot .8 \odot 44 \mathrm{E}+\odot 7$ \\
\hline 9 & 288.000 & 288.000 & 1.0000 & $\odot .8044 \mathrm{E}+07$ \\
\hline 10 & 288.000 & 288.000 & 1.0000 & $\odot .8044 \mathrm{E}+\odot 7$ \\
\hline 11 & $288.00 \odot$ & $288.00 \odot$ & 1.0000 & $\odot .8044 \mathrm{E}+07$ \\
\hline 12 & 288.000 & 288.000 & 1.0000 & $\odot .8044 \mathrm{E}+07$ \\
\hline 13 & 288.000 & 288.000 & 1.0000 & $\odot .8044 \mathrm{E}+07$ \\
\hline 14 & 288.000 & $288.00 \odot$ & 1.0000 & $\odot .8044 \mathrm{E}+\odot 7$ \\
\hline 15 & $288.00 \odot$ & 288.000 & $1.000 \odot$ & $\odot .8044 \mathrm{E}+07$ \\
\hline 16 & $288.0 \odot \odot$ & $288.0 \odot \odot$ & $1.000 \odot$ & $\odot .8 \odot 44 \mathrm{E}+\odot 7$ \\
\hline 17 & $288.0 \odot \odot$ & $288.00 \odot$ & 1.0000 & $\odot .8 \odot 44 \mathrm{E}+07$ \\
\hline 18 & $288.00 \odot$ & $288.00 \odot$ & 1.0000 & $\odot .8044 \mathrm{E}+07$ \\
\hline 19 & 288.000 & 288.000 & 1.0000 & $\odot .8044 \mathrm{E}+07$ \\
\hline 20 & 288.000 & $288 . \odot \odot \odot$ & $1.00 \odot \odot$ & $\odot .8044 \mathrm{E}+07$ \\
\hline
\end{tabular}

\begin{tabular}{|c|c|c|c|c|}
\hline \multicolumn{5}{|c|}{$.00 \quad$ lambda $=0.3272083998$} \\
\hline node & $\mathrm{T}(\mathrm{K})$ & Twall(K) & $x$ & $P(P a)$ \\
\hline 1 & 142.919 & 229.786 & - - - - - & $0.1083 \mathrm{E}+07$ \\
\hline 2 & 165.786 & 262.603 & $\odot .9977$ & $\odot .1997 E+\odot 7$ \\
\hline 3 & 245.305 & 284.999 & 1.0000 & $\odot .5810 E+\odot 7$ \\
\hline 4 & 280.220 & $288.0 \odot \odot$ & 1.0000 & $\odot .7338 \mathrm{E}+07$ \\
\hline 5 & 287.480 & $288.00 \odot$ & $1.000 \odot$ & $\odot .7935 E+\odot 7$ \\
\hline 6 & $288.00 \odot$ & $288.00 \odot$ & 1.0000 & $\odot .8038 \mathrm{E}+07$ \\
\hline 7 & $288.00 \odot$ & $288.00 \odot$ & $1.000 \odot$ & $\odot .8044 \mathrm{E}+\odot 7$ \\
\hline 8 & 288.000 & 288.000 & 1.0000 & $\odot .8044 \mathrm{E}+07$ \\
\hline 9 & $288.00 \odot$ & 288.000 & $1.000 \odot$ & $\odot .8044 \mathrm{E}+\odot 7$ \\
\hline 10 & $288.00 \odot$ & 288.000 & 1.0000 & $\odot .8044 \mathrm{E}+\odot 7$ \\
\hline 11 & $288.00 \odot$ & $288 . \odot \odot \odot$ & 1.0000 & $\odot .8044 \mathrm{E}+07$ \\
\hline 12 & 288.000 & $288.00 \odot$ & 1.0000 & $\odot .8044 \mathrm{E}+07$ \\
\hline 13 & $288.00 \odot$ & 288.000 & 1.0000 & $\odot .8044 \mathrm{E}+\odot 7$ \\
\hline 14 & 288.000 & 288.000 & 1.0000 & $\odot .8044 \mathrm{E}+07$ \\
\hline 15 & 288.000 & $288.00 \odot$ & 1.0000 & $\odot .8044 \mathrm{E}+07$ \\
\hline 16 & 288.000 & $288.00 \odot$ & $1.000 \odot$ & $\odot .8044 \mathrm{E}+\odot 7$ \\
\hline 17 & 288.000 & 288.000 & 1.0000 & $0.8044 \mathrm{E}+07$ \\
\hline 18 & 288.000 & 288.000 & 1.0000 & $0.8044 \mathrm{E}+07$ \\
\hline 19 & 288.000 & 288.000 & 1.0000 & $\odot .8044 \mathrm{E}+\odot 7$ \\
\hline 20 & $288.00 \odot$ & $288 . \odot \odot \odot$ & $1.000 \odot$ & $\odot .8044 \mathrm{E}+\odot 7$ \\
\hline
\end{tabular}

\begin{tabular}{|c|c|c|c|c|}
\hline $\begin{array}{l}\text { time } \\
\text { node }\end{array}$ & $\begin{array}{c}(\mathrm{sec})= \\
T(\mathrm{~K})=\end{array}$ & . $00 \quad 1$ & $a=0$ & $\begin{array}{r}2678955 \\
P(P a)\end{array}$ \\
\hline 1 & 141.270 & 223.549 & $\ldots$ & $\odot .1021 \mathrm{E}+07$ \\
\hline 2 & 163.873 & 257.853 & $\odot .9965$ & $\odot .1882 \mathrm{E}+\odot 7$ \\
\hline 3 & 235.196 & 283.017 & 1.0000 & $\odot .5533 \mathrm{E}+07$ \\
\hline 4 & 274.683 & 287.722 & 1.0000 & $\odot .7 \odot 71 \mathrm{E}+\odot 7$ \\
\hline 5 & 286.652 & 288.000 & 1.0000 & $\odot .7846 \mathrm{E}+\odot 7$ \\
\hline 6 & $288.0 \odot \odot$ & $288.00 \odot$ & $1.000 \odot$ & $\odot .8 \odot 28 \mathrm{E}+07$ \\
\hline 7 & $288.00 \odot$ & $288 . \odot \odot \odot$ & 1.0000 & $\odot .8 \odot 44 \mathrm{E}+\odot 7$ \\
\hline 8 & 288.000 & 288.000 & 1.0000 & $\odot .8044 \mathrm{E}+07$ \\
\hline 9 & 288.000 & 288.000 & 1.0000 & $\odot .8044 \mathrm{E}+07$ \\
\hline 10 & $288.00 \odot$ & 288.000 & 1.0000 & $\odot .8 \odot 44 \mathrm{E}+\odot 7$ \\
\hline 11 & 288.000 & 288.000 & 1.0000 & $\odot .8044 \mathrm{E}+07$ \\
\hline 12 & $288.00 \odot$ & $288.00 \odot$ & 1.0000 & $\odot .8 \odot 44 \mathrm{E}+\odot 7$ \\
\hline 13 & 288.000 & $288.00 \odot$ & $1.000 \odot$ & $\odot .8044 \mathrm{E}+07$ \\
\hline 14 & 288.000 & 288.000 & 1.0000 & $\odot .8044 \mathrm{E}+07$ \\
\hline 15 & 288.000 & 288.000 & 1.0000 & $\odot .8044 \mathrm{E}+07$ \\
\hline 16 & 288.000 & $288.00 \odot$ & 1.0000 & $\odot .8044 \mathrm{E}+\odot 7$ \\
\hline
\end{tabular}




\begin{tabular}{|c|c|c|c|c|}
\hline 17 & 288.000 & 288.000 & $1.000 \odot$ & $\odot .8044 \mathrm{E}+\odot 7$ \\
\hline 18 & 288.000 & $288.00 \odot$ & 1.0000 & $\odot .8 \odot 44 \mathrm{E}+\odot 7$ \\
\hline 19 & $288.00 \odot$ & 288.000 & 1.0000 & $\odot .8044 \mathrm{E}+\odot 7$ \\
\hline 20 & 288.000 & $288 . \odot \odot \odot$ & 1.0000 & $\odot .8044 \mathrm{E}+\odot 7$ \\
\hline
\end{tabular}

\begin{tabular}{|c|c|c|c|c|}
\hline \multicolumn{5}{|c|}{ lambda $=0.2945762873$} \\
\hline node & $\mathrm{T}(\mathrm{K})$ & Twall(K) & $\mathrm{x}$ & $\mathrm{P}(\mathrm{Pa})$ \\
\hline 1 & 139.759 & 217.766 & $-\ldots$ & $0.9637 \mathrm{E}+06$ \\
\hline 2 & 162.120 & 253.347 & 0.9957 & $\odot .1778 \mathrm{E}+07$ \\
\hline 3 & 227.287 & 280.640 & 1.0000 & $\odot .5327 E+\odot 7$ \\
\hline 4 & 267.547 & 287.277 & 1.0000 & $\odot .6785 E+07$ \\
\hline 5 & 285.079 & 288.000 & 1.0000 & $0.7717 \mathrm{E}+07$ \\
\hline 6 & 287.951 & $288.0 \odot \odot$ & 1.0000 & $\odot .80 \odot 7 E+\odot 7$ \\
\hline 7 & 288.000 & 288.000 & 1.0000 & $0.8044 \mathrm{E}+07$ \\
\hline 8 & 288.000 & $288.00 \odot$ & 1.0000 & $\odot .8044 \mathrm{E}+07$ \\
\hline 9 & 288.000 & 288.000 & 1.0000 & $\odot .8044 \mathrm{E}+\odot 7$ \\
\hline 10 & $288.00 \odot$ & $288.0 \odot \odot$ & 1.0000 & $\odot .8044 \mathrm{E}+\odot 7$ \\
\hline 11 & 288.000 & 288.000 & 1.0000 & $\odot .8044 \mathrm{E}+07$ \\
\hline 12 & 288.000 & $288.00 \odot$ & 1.0000 & $\odot .8044 \mathrm{E}+07$ \\
\hline 13 & 288.000 & 288.000 & 1.0000 & $\odot .8044 \mathrm{E}+\odot 7$ \\
\hline 14 & 288.000 & 288.000 & 1.0000 & $\odot .8044 \mathrm{E}+07$ \\
\hline 15 & 288.000 & $288.00 \odot$ & 1.0000 & $\odot .8044 \mathrm{E}+07$ \\
\hline 16 & 288.000 & 288.000 & 1.0000 & $\odot .8044 \mathrm{E}+\odot 7$ \\
\hline 17 & 288.000 & 288.000 & 1.0000 & $0.8044 \mathrm{E}+07$ \\
\hline 18 & 288.000 & 288.000 & 1.0000 & $0.8044 \mathrm{E}+07$ \\
\hline 19 & $288.00 \odot$ & 288.000 & 1.0000 & $\odot .8044 \mathrm{E}+\odot 7$ \\
\hline 20 & $288.0 \odot \odot$ & $288.00 \odot$ & 1.0000 & $\odot .8 \odot 44 \mathrm{E}+\odot 7$ \\
\hline
\end{tabular}

\begin{tabular}{|c|c|c|c|c|}
\hline \multicolumn{5}{|c|}{ lambda $=0.2801606953$} \\
\hline node & $T(K)$ & Twall(K) & $x$ & $\mathrm{P}(\mathrm{Pa})$ \\
\hline 1 & 138.384 & 212.526 & - - - - - & $0.9121 \mathrm{E}+06$ \\
\hline 2 & 160.525 & 249.066 & 0.9951 & $\odot .1682 \mathrm{E}+07$ \\
\hline 3 & 221.984 & 278.041 & 1.0000 & $5193 E+\odot 7$ \\
\hline 4 & 259.419 & 286.509 & 1.0000 & $\odot .6504 \mathrm{E}+\odot$ \\
\hline 5 & 282.437 & 288.000 & 1.0000 & $\odot .7548 \mathrm{E}+\odot 7$ \\
\hline 6 & 287.646 & 288.000 & 1.0000 & $0.7966 \mathrm{E}+\Theta 7$ \\
\hline 7 & 288.000 & 288.000 & 1.0000 & $\odot .8040 \mathrm{E}+\Theta$ \\
\hline 8 & 288.000 & 288.000 & 1.0000 & $\odot .8044 \mathrm{E}+\odot$ \\
\hline 9 & $288.00 \odot$ & $288.00 \odot$ & $1.000 \odot$ & $\odot .8044 \mathrm{E}+\odot$ \\
\hline 10 & 288.000 & 288.000 & 1.0000 & $0.8044 \mathrm{E}+\mathrm{C}$ \\
\hline$\perp \perp$ & 288.000 & 288.000 & 1.0000 & $0.8044 \mathrm{E}+07$ \\
\hline 12 & $288.00 \odot$ & 288.000 & $1.000 \odot$ & $\odot .8044 \mathrm{E}+\odot 7$ \\
\hline & 288.000 & $288 . \odot$ & 1.0000 & $\odot .8044 \mathrm{E}+\odot$ \\
\hline-4 & $288.00 \odot$ & $288.00 \odot$ & $1.000 \odot$ & $\odot .8044 \mathrm{E}+\odot$ \\
\hline-5 & 288.000 & 288.000 & 1.0000 & $\odot .8044 \mathrm{E}+\odot$ \\
\hline 16 & 288.000 & $288.00 \odot$ & $1.000 \odot$ & $\odot .8044 \mathrm{E}+\odot$ \\
\hline 17 & 288.000 & 288.000 & 1.0000 & $\odot .8044 \mathrm{E}+\odot$ \\
\hline 18 & 288.000 & 288.000 & 1.0000 & $0.8044 \mathrm{E}+\odot$ \\
\hline 19 & 288.000 & $288.00 \odot$ & $1.00 \odot \odot$ & $\odot .8044 \mathrm{E}+\odot$ \\
\hline & 288.000 & $288.0 \odot \odot$ & 1.0000 & $\odot .8044 \mathrm{E}+\odot$ \\
\hline
\end{tabular}

\begin{tabular}{ccccc} 
time & $(\mathrm{sec})=$ & 13.00 & \multicolumn{2}{c}{ lambda $=0.2670564651$} \\
node & $\mathrm{T}(\mathrm{K})$ & Twall $(\mathrm{K})$ & $\mathrm{X}$ & $\mathrm{P}(\mathrm{Pa})$ \\
1 & 137.145 & 207.815 &.--- & $0.8655 \mathrm{E}+06$ \\
2 & 159.089 & 245.017 & 0.9945 & $0.1596 \mathrm{E}+07$ \\
3 & 219.157 & 275.416 & $1.000 \odot$ & $0.5119 \mathrm{E}+07$ \\
4 & 251.252 & 285.381 & $1.000 \odot$ & $0.6251 \mathrm{E}+07$
\end{tabular}




\begin{tabular}{|c|c|c|c|c|}
\hline 5 & 278.477 & 287.931 & 1.0000 & $\odot .7342 \mathrm{E}+07$ \\
\hline 6 & 287.025 & $288 . \odot \odot \odot$ & $1.000 \odot$ & $\odot .790 \odot \mathrm{E}+\odot 7$ \\
\hline 7 & $288.00 \odot$ & $288 . \odot \odot \odot$ & 1.0000 & $\odot .8032 \mathrm{E}+\odot 7$ \\
\hline 8 & 288.000 & 288.000 & 1.0000 & $\odot .8044 \mathrm{E}+07$ \\
\hline 9 & 288.000 & 288.000 & 1.0000 & $\odot .8044 \mathrm{E}+\odot 7$ \\
\hline 10 & 288.000 & $288.00 \odot$ & 1.0000 & $\odot .8044 \mathrm{E}+07$ \\
\hline 11 & 288.000 & 288.000 & 1.0000 & $\odot .8044 \mathrm{E}+07$ \\
\hline 12 & 288.000 & 288.000 & 1.0000 & $\odot .8044 \mathrm{E}+07$ \\
\hline 13 & 288.000 & 288.000 & 1.0000 & $\odot .8044 \mathrm{E}+\odot 7$ \\
\hline 14 & $288.00 \odot$ & $288.00 \odot$ & 1.0000 & $\odot .8044 \mathrm{E}+07$ \\
\hline 15 & $288.0 \odot \odot$ & $288.00 \odot$ & $1.000 \odot$ & $\odot .8 \odot 44 \mathrm{E}+\odot 7$ \\
\hline 16 & $288.0 \odot \odot$ & $288.00 \odot$ & 1.0000 & $\odot .8 \odot 44 \mathrm{E}+07$ \\
\hline 17 & $288.00 \odot$ & $288.00 \odot$ & 1.0000 & $\odot .8044 \mathrm{E}+07$ \\
\hline 18 & $288.00 \odot$ & $288.00 \odot$ & 1.0000 & $\odot .8 \odot 44 \mathrm{E}+\odot 7$ \\
\hline 19 & 288.000 & 288.000 & 1.0000 & $\odot .8044 \mathrm{E}+07$ \\
\hline 20 & 288.000 & $288.00 \odot$ & 1.0000 & $\odot .8044 \mathrm{E}+07$ \\
\hline
\end{tabular}

\begin{tabular}{|c|c|c|c|c|}
\hline \multicolumn{5}{|c|}{$.00 \quad$ lambda $=0.2552609444$} \\
\hline node & $\mathrm{T}(\mathrm{K})$ & Twall(K) & $x$ & $\mathrm{P}(\mathrm{Pa})$ \\
\hline 1 & 136.040 & 203.548 & $-\ldots$ & $\odot .8239 E+06$ \\
\hline 2 & 157.806 & 241.221 & $\odot .9936$ & $\odot .152 \odot E+\odot 7$ \\
\hline 3 & 218.303 & 272.974 & $1.0 \odot \odot \odot$ & $\odot .5085 E+\odot 7$ \\
\hline 4 & 243.997 & 283.896 & 1.0000 & $\odot .6 \odot 46 \mathrm{E}+\odot 7$ \\
\hline 5 & 273.164 & 287.626 & 1.0000 & $\odot .7112 \mathrm{E}+\odot 7$ \\
\hline 6 & 285.866 & $288.00 \odot$ & 1.0000 & $\odot .7804 \mathrm{E}+07$ \\
\hline 7 & 288.000 & $288.00 \odot$ & 1.0000 & $\odot .8018 \mathrm{E}+07$ \\
\hline 8 & $288.00 \odot$ & $288 . \odot \odot \odot$ & $1.000 \odot$ & $\odot .8 \odot 44 \mathrm{E}+\odot 7$ \\
\hline 9 & $288 . \odot \odot \odot$ & $288 . \odot \odot \odot$ & $1.00 \odot \odot$ & $\odot .8 \odot 44 \mathrm{E}+\odot 7$ \\
\hline 10 & $288.00 \odot$ & $288 . \odot \odot \odot$ & $1.0 \odot \odot \odot$ & $\odot .8 \odot 44 \mathrm{E}+\odot 7$ \\
\hline 11 & $288.00 \odot$ & $288 . \odot \odot \odot$ & $1.00 \odot \odot$ & $\odot .8 \odot 44 \mathrm{E}+\odot 7$ \\
\hline 12 & 288.000 & $288.00 \odot$ & 1.0000 & $\odot .8044 \mathrm{E}+07$ \\
\hline 13 & 288.000 & 288.000 & 1.0000 & $\odot .8044 \mathrm{E}+07$ \\
\hline 14 & 288.000 & $288.00 \odot$ & 1.0000 & $\odot .8 \odot 44 \mathrm{E}+\odot 7$ \\
\hline 15 & $288.00 \odot$ & $288 . \odot \odot \odot$ & $1.000 \odot$ & $\odot .8 \odot 44 \mathrm{E}+\odot 7$ \\
\hline 16 & $288.00 \odot$ & $288 . \odot \odot \odot$ & 1.0000 & $\odot .8 \odot 44 \mathrm{E}+\odot 7$ \\
\hline 17 & $288.00 \odot$ & $288.0 \odot \odot$ & 1.0000 & $\odot .8044 \mathrm{E}+\odot 7$ \\
\hline 18 & 288.000 & $288.00 \odot$ & 1.0000 & $\odot .8044 \mathrm{E}+07$ \\
\hline 19 & 288.000 & $288.00 \odot$ & 1.0000 & $\odot .8044 \mathrm{E}+07$ \\
\hline 20 & 288.000 & $288.0 \odot \odot$ & $1.000 \odot$ & $\odot .8044 \mathrm{E}+\odot 7$ \\
\hline
\end{tabular}

\begin{tabular}{|c|c|c|c|c|}
\hline \multicolumn{5}{|c|}{ lambda $=0.2447123379$} \\
\hline node & $T(K)$ & Twall(K) & $\mathrm{x}$ & $\mathrm{P}(\mathrm{Pa})$ \\
\hline 1 & 135.058 & 199.661 & - - - _ & $\odot .7870 \mathrm{E}+06$ \\
\hline 2 & 156.667 & 237.637 & 0.9924 & $\odot .1452 \mathrm{E}+\odot 7$ \\
\hline 3 & 218.757 & 270.700 & 1.0000 & $\odot .5076 \mathrm{E}+07$ \\
\hline 4 & 238.470 & 282.117 & 1.0000 & $\odot .5903 \mathrm{E}+07$ \\
\hline 5 & 266.764 & 287.151 & $1.000 \odot$ & $\odot .6873 \mathrm{E}+07$ \\
\hline 6 & 283.900 & 288.000 & 1.0000 & $\odot .7672 \mathrm{E}+\odot 7$ \\
\hline 7 & 287.762 & 288.000 & 1.0000 & $\odot .7987 \mathrm{E}+\odot 7$ \\
\hline 8 & 288.000 & 288.000 & 1.0000 & $\odot .8 \odot 42 \mathrm{E}+07$ \\
\hline 9 & 288.000 & 288.000 & 1.0000 & $\odot .8044 \mathrm{E}+07$ \\
\hline 10 & 288.000 & 288.000 & 1.0000 & $\odot .8044 \mathrm{E}+07$ \\
\hline 11 & 288.000 & 288.000 & 1.0000 & $\odot .8044 \mathrm{E}+07$ \\
\hline 12 & 288.000 & 288.000 & 1.0000 & $\odot .8044 \mathrm{E}+07$ \\
\hline 13 & 288.000 & 288.000 & 1.0000 & $\odot .8044 \mathrm{E}+07$ \\
\hline 14 & 288.000 & $288 . \odot \odot \odot$ & 1.0000 & $\odot .8044 \mathrm{E}+07$ \\
\hline 15 & 288.000 & $288.00 \odot$ & 1.0000 & $\odot .8044 \mathrm{E}+\odot 7$ \\
\hline
\end{tabular}




\begin{tabular}{|c|c|c|c|c|}
\hline 16 & 288.000 & 288.000 & 1.0000 & $\odot .8044 \mathrm{E}+07$ \\
\hline 17 & 288.000 & $288 . \odot \odot \odot$ & $1.000 \odot$ & $0.8044 \mathrm{E}+07$ \\
\hline 18 & 288.000 & $288 . \odot \odot \odot$ & 1.0000 & $\odot .8044 \mathrm{E}+07$ \\
\hline 19 & 288.000 & 288.000 & 1.0000 & $\odot .8044 \mathrm{E}+\odot 7$ \\
\hline 20 & 288.000 & $288.00 \odot$ & 1.0000 & $0.8044 \mathrm{E}+07$ \\
\hline time & $(\mathrm{sec})=$ & lambda $=0.2353087068$ & \multicolumn{2}{|c|}{ lambda $=0.2353087068$} \\
\hline node & $\mathrm{T}(\mathrm{K})$ & Twall(K) & \multirow[t]{2}{*}{$\mathrm{X}$} & $P(P a)$ \\
\hline 1 & 134.188 & 196.102 & & $\odot .7543 \mathrm{E}+06$ \\
\hline 2 & 155.658 & 234.237 & $\odot .9912$ & $\odot .1391 \mathrm{E}+07$ \\
\hline 3 & 219.924 & 268.669 & 1.0000 & $0.5075 \mathrm{E}+07$ \\
\hline 4 & 234.958 & 280.286 & 1.0000 & $\odot .5816 \mathrm{E}+07$ \\
\hline 5 & 259.876 & 286.408 & $1.00 \odot \odot$ & $0.6645 \mathrm{E}+07$ \\
\hline 6 & 280.886 & 288.000 & 1.0000 & $\odot .7506 \mathrm{E}+07$ \\
\hline 7 & 287.322 & $288 . \odot \odot \odot$ & $1.000 \odot$ & $\odot .7938 \mathrm{E}+07$ \\
\hline 8 & 88.000 & $288.0 \odot \odot$ & $1.000 \odot$ & $\odot .8036 \mathrm{E}+\odot 7$ \\
\hline 9 & $288 . \odot \odot \odot$ & $288.00 \odot$ & $1.00 \odot \odot$ & $\odot .8044 \mathrm{E}+07$ \\
\hline 10 & 288.000 & 288.000 & $1.000 \odot$ & $\odot .8044 \mathrm{E}+07$ \\
\hline 11 & 288.000 & 288.000 & $1.00 \odot \odot$ & $\odot .8044 \mathrm{E}+07$ \\
\hline 12 & 288.000 & 288.000 & 1.0000 & $0.8044 \mathrm{E}+07$ \\
\hline 13 & 288.000 & $288.00 \odot$ & 1.0000 & $\odot .8044 \mathrm{E}+07$ \\
\hline 14 & 288.000 & $288.00 \odot$ & $1.000 \odot$ & $0.8044 \mathrm{E}+07$ \\
\hline 15 & $288.00 \odot$ & $288 . \odot \odot \odot$ & $1.00 \odot \odot$ & $\odot .8044 \mathrm{E}+07$ \\
\hline 16 & 88.000 & 288.000 & 1.0000 & $0.8044 \mathrm{E}+07$ \\
\hline 17 & 288.000 & 288.000 & 1.0000 & $0.8044 \mathrm{E}+07$ \\
\hline 18 & $288.00 \odot$ & $288.00 \odot$ & $1.00 \odot \odot$ & $\odot .8044 \mathrm{E}+\odot 7$ \\
\hline 19 & 38.000 & $288.00 \odot$ & 1.0000 & $0.8044 \mathrm{E}+07$ \\
\hline 20 & 38.000 & 288.000 & 1.0000 & $\odot .8044 \mathrm{E}+07$ \\
\hline time & sec) $=$ & $17.00 \quad 1$ & $1 a=0.2$ & 69305140 \\
\hline node & $\mathrm{T}(\mathrm{K})$ & Twall(K) & X & $\mathrm{P}(\mathrm{Pa})$ \\
\hline 1 & 133.418 & 192.826 & - - - - - & $\odot .7254 \mathrm{E}+06$ \\
\hline 2 & 154.765 & 231.011 & $\odot .9901$ & $0.1338 \mathrm{E}+07$ \\
\hline 3 & 221.263 & 266.838 & 1.0000 & $0.5071 \mathrm{E}+07$ \\
\hline 4 & 233.384 & 278.455 & $1.000 \odot$ & $0.5777 \mathrm{E}+\odot 7$ \\
\hline 5 & 253.332 & 285.378 & 1.0000 & $0.6450 \mathrm{E}+07$ \\
\hline 6 & 76.699 & 287.820 & 1.0000 & $0.7313 \mathrm{E}+07$ \\
\hline 7 & 36.473 & 288. & 1.0000 & $0.7863 \mathrm{E}+07$ \\
\hline 8 & 88.000 & $288.00 \odot$ & $1.00 \odot \odot$ & $\odot .8026 \mathrm{E}+07$ \\
\hline 9 & 88.000 & 288.000 & 1.0000 & $\odot .8044 \mathrm{E}+07$ \\
\hline 10 & 38.000 & $288.00 \odot$ & 1.0000 & $\odot .8044 \mathrm{E}+07$ \\
\hline 11 & 288.000 & $288.00 \odot$ & 1.0000 & $0.8044 \mathrm{E}+07$ \\
\hline 12 & 88.000 & 288.000 & 1.0000 & $\odot .8044 \mathrm{E}+07$ \\
\hline 13 & 0 & 288. & 1.0000 & $\odot .8044 \mathrm{E}+07$ \\
\hline 14 & $\odot \odot$ & 288.000 & 1.0000 & $0.8044 \mathrm{E}+07$ \\
\hline 15 & 38.000 & 288.000 & 1.0000 & $0.8044 \mathrm{E}+07$ \\
\hline 16 & 38.000 & 288.000 & 1.0000 & $0.8044 \mathrm{E}+07$ \\
\hline 17 & 288.000 & 288.000 & 1.0000 & $0.8044 \mathrm{E}+07$ \\
\hline 18 & 288.000 & $288.00 \odot$ & 1.0000 & $0.8044 \mathrm{E}+07$ \\
\hline 19 & $288 . c$ & 288.000 & 1.0000 & $0.8044 \mathrm{E}+07$ \\
\hline 20 & 288.000 & 288.000 & 1.0000 & $\odot .8044 \mathrm{E}+07$ \\
\hline time & $(\mathrm{sec})=$ & $18.00 \quad 1$ & $a=0.2$ & 94225043 \\
\hline node & $\mathrm{T}(\mathrm{K})$ & Twall(K) & $\mathrm{X}$ & $\mathrm{P}(\mathrm{Pa})$ \\
\hline 1 & 132.732 & 189.805 & - - - - - & $0.6996 \mathrm{E}+06$ \\
\hline 2 & 153.969 & 227.949 & $\odot .9889$ & $\odot .1290 \mathrm{E}+07$ \\
\hline 3 & 222.267 & 265.278 & 1.0000 & $\odot .5055 \mathrm{E}+07$ \\
\hline
\end{tabular}




\begin{tabular}{|c|c|c|c|c|}
\hline 4 & 233.412 & 276.706 & $1.00 \odot \odot$ & $\odot .5778 \mathrm{E}+\odot 7$ \\
\hline 5 & 247.823 & 284.149 & 1.0000 & $0.6301 \mathrm{E}+07$ \\
\hline 6 & 271.441 & 287.515 & $1.000 \odot$ & $\odot .7104 \mathrm{E}+07$ \\
\hline 7 & 284.976 & 288.000 & 1.0000 & $0.7757 \mathrm{E}+07$ \\
\hline 8 & 287.895 & $288.0 \odot \odot$ & $1.000 \odot$ & $\odot .80 \odot 4 \mathrm{E}+\odot 7$ \\
\hline 9 & $288.00 \odot$ & $288 . \odot \odot \odot$ & $1.00 \odot \odot$ & $\odot .8 \odot 44 \mathrm{E}+\odot 7$ \\
\hline 10 & $288.00 \odot$ & $288.00 \odot$ & $1.000 \odot$ & $\odot .8044 \mathrm{E}+07$ \\
\hline 11 & $288.00 \odot$ & $288 . \odot \odot \odot$ & $1.00 \odot \odot$ & $\odot .8044 \mathrm{E}+07$ \\
\hline 12 & 288.000 & $288 . \odot \odot \odot$ & $1.00 \odot \odot$ & $\odot .8 \odot 44 \mathrm{E}+\odot 7$ \\
\hline 13 & 288.000 & 288.000 & $1.000 \odot$ & $0.8044 \mathrm{E}+07$ \\
\hline 14 & $288.00 \odot$ & $288 . \odot \odot \odot$ & $1.00 \odot \odot$ & $\odot .8044 \mathrm{E}+07$ \\
\hline 15 & 288.000 & $288 . \odot \odot \odot$ & 1.0000 & $\odot .8044 \mathrm{E}+\odot 7$ \\
\hline 16 & 288.000 & $288 . \odot \odot \odot$ & $1.000 \odot$ & $0.8044 \mathrm{E}+07$ \\
\hline 17 & $288.00 \odot$ & $288 . \odot \odot \odot$ & $1.000 \odot$ & $\odot .8044 \mathrm{E}+07$ \\
\hline 18 & $288.0 \odot \odot$ & $288 . \odot \odot \odot$ & $1.00 \odot \odot$ & $\odot .8044 \mathrm{E}+07$ \\
\hline 19 & $288.00 \odot$ & $288 . \odot \odot \odot$ & $1.000 \odot$ & $\odot .8044 \mathrm{E}+\odot 7$ \\
\hline 20 & 88.000 & $288.00 \odot$ & 1.0000 & $0.8044 \mathrm{E}+07$ \\
\hline time & $(\sec )=$ & \multicolumn{3}{|c|}{$\mathrm{mbda}=0.2$} \\
\hline node & $\mathrm{T}(\mathrm{K})$ & Twall(K) & $x$ & $P(P a)$ \\
\hline 1 & 132.116 & 186.993 & - - - - - & $\odot .6764 \mathrm{E}+06$ \\
\hline 2 & 153.254 & 225.043 & $\odot .9877$ & $0.1248 \mathrm{E}+07$ \\
\hline 3 & 222.527 & 263.752 & 1.0000 & $\odot .5018 \mathrm{E}+07$ \\
\hline 4 & 234.229 & 275.180 & $1.00 \odot \odot$ & $\odot .5796 \mathrm{E}+\odot 7$ \\
\hline 5 & 244.211 & 282.623 & $1.00 \odot \odot$ & $\odot .6210 \mathrm{E}+07$ \\
\hline 6 & 265.479 & 286.976 & 1.0000 & $\odot .6899 \mathrm{E}+07$ \\
\hline 7 & 282.618 & $288.0 \odot \odot$ & 1.0000 & $\odot .7620 \mathrm{E}+\odot 7$ \\
\hline 8 & 287.590 & $288 . \odot \odot \odot$ & $1.00 \odot \odot$ & $\odot .7966 \mathrm{E}+\odot 7$ \\
\hline 9 & $288.0 \odot \odot$ & $288 . \odot \odot \odot$ & $1.00 \odot \odot$ & $\odot .8039 \mathrm{E}+\odot 7$ \\
\hline 10 & $288.00 \odot$ & $288 . \odot \odot \odot$ & 1.0000 & $\odot .8044 \mathrm{E}+07$ \\
\hline 11 & $288.00 \odot$ & $288 . \odot \odot \odot$ & $1.0 \odot \odot \odot$ & $\odot .8044 \mathrm{E}+\odot 7$ \\
\hline 12 & $288.0 \odot \odot$ & $288 . \odot \odot \odot$ & $1.0 \odot \odot \odot$ & $\odot .8 \odot 44 \mathrm{E}+\odot 7$ \\
\hline 13 & 288.000 & $288 . \odot \odot \odot$ & $1.000 \odot$ & $\odot .8044 \mathrm{E}+07$ \\
\hline 14 & $288.00 \odot$ & $288 . \odot \odot \odot$ & $1.0 \odot \odot \odot$ & $0.8044 \mathrm{E}+07$ \\
\hline 15 & $288 . \odot \odot \odot$ & $288 . \odot \odot \odot$ & $1.00 \odot \odot$ & $\odot .8 \odot 44 \mathrm{E}+07$ \\
\hline 16 & $288.00 \odot$ & $288 . \odot \odot \odot$ & $1.000 \odot$ & $0.8044 \mathrm{E}+07$ \\
\hline 17 & $288.00 \odot$ & $288 . \odot \odot \odot$ & $1.00 \odot \odot$ & $\odot .8044 \mathrm{E}+\odot 7$ \\
\hline 18 & $288.00 \odot$ & $288 . \odot \odot \odot$ & $1.00 \odot \odot$ & $\odot .8044 \mathrm{E}+\odot 7$ \\
\hline 19 & $288.00 \odot$ & $288 . \odot \odot \odot$ & 1.0000 & $\odot .8044 \mathrm{E}+07$ \\
\hline 20 & 288.000 & 288.000 & 1.0000 & $\odot .8044 \mathrm{E}+07$ \\
\hline time & $(\sec )=$ & \multicolumn{3}{|l|}{$20.00 \quad 1 a$} \\
\hline node & $\mathrm{T}(\mathrm{K})$ & Twall(K) & $\mathrm{x}$ & $\mathrm{P}(\mathrm{Pa})$ \\
\hline 1 & 131.555 & 184.368 & - - - - - & $\odot .6554 \mathrm{E}+06$ \\
\hline 2 & 152.604 & 222.287 & $\odot .9865$ & $\odot .1209 \mathrm{E}+07$ \\
\hline 3 & 221.778 & 262.226 & $1.000 \odot$ & $0.4963 \mathrm{E}+07$ \\
\hline 4 & 234.886 & 273.832 & $1.000 \odot$ & $\odot .5802 \mathrm{E}+\odot 7$ \\
\hline 5 & 243.094 & 281.321 & 1.0000 & $0.6181 \mathrm{E}+07$ \\
\hline 6 & 259.470 & 286.219 & 1.0000 & $0.6724 \mathrm{E}+07$ \\
\hline 7 & 279.219 & $288 . \odot \odot \odot$ & $1.000 \odot$ & $0.7453 \mathrm{E}+07$ \\
\hline 8 & 286.933 & $288.00 \odot$ & 1.0000 & $0.7906 \mathrm{E}+07$ \\
\hline 9 & $288.00 \odot$ & 288.000 & $1.000 \odot$ & $\odot .8031 \mathrm{E}+07$ \\
\hline 10 & 288.000 & $288 . \odot \odot \odot$ & $1.000 \odot$ & $\odot .8044 \mathrm{E}+\odot 7$ \\
\hline 11 & $288.00 \odot$ & $288 . \odot \odot \odot$ & $1.000 \odot$ & $\odot .8044 \mathrm{E}+07$ \\
\hline 12 & $288.0 \odot \odot$ & $288 . \odot \odot \odot$ & $1.00 \odot \odot$ & $\odot .8 \odot 44 \mathrm{E}+07$ \\
\hline 13 & 288.000 & 288.000 & 1.0000 & $\odot .8044 \mathrm{E}+07$ \\
\hline 14 & $288.00 \odot$ & $288 . \odot \odot \odot$ & $1.00 \odot \odot$ & $\odot .8044 \mathrm{E}+07$ \\
\hline
\end{tabular}




\begin{tabular}{|c|c|c|c|c|}
\hline 15 & 288.000 & 288.000 & 1.0000 & $0.8044 \mathrm{E}+07$ \\
\hline 16 & 288.000 & 288.000 & 1.0000 & $0.8044 \mathrm{E}+07$ \\
\hline 17 & 288.000 & $288 . \odot \odot \odot$ & $1.000 \odot$ & $\odot .8 \odot 44 \mathrm{E}+\odot 7$ \\
\hline 8 & $288 . \odot \odot \odot$ & $288.00 \odot$ & $1.000 \odot$ & $\odot .8 \odot 44 \mathrm{E}+\odot 7$ \\
\hline 19 & 288.000 & 288.000 & 1.0000 & $0.8044 \mathrm{E}+07$ \\
\hline 20 & 288.000 & 288.000 & 1.0000 & $\odot .8044 \mathrm{E}+07$ \\
\hline time & $(\sec )=$ & $\mathrm{nbda}=0.2007577866$ & \multicolumn{2}{|c|}{$\mathrm{nbda}=0.2007577866$} \\
\hline node & $\mathrm{T}(\mathrm{K})$ & \multirow{2}{*}{$\begin{array}{r}\text { Twall(K) } \\
181.920\end{array}$} & $\mathrm{x}$ & $P(P a)$ \\
\hline 1 & 131.040 & & ----- & $\odot .6360 \mathrm{E}+06$ \\
\hline 2 & 152.006 & 219.673 & $\odot .9853$ & $0.1173 \mathrm{E}+07$ \\
\hline 3 & 220.072 & 260.700 & $1.000 \odot$ & $\odot .4891 \mathrm{E}+\odot 7$ \\
\hline 4 & 235.496 & 272.612 & $1.000 \odot$ & $\odot .5796 \mathrm{E}+\odot 7$ \\
\hline 5 & 243.541 & 280.100 & 1.0000 & $\odot .6192 \mathrm{E}+07$ \\
\hline 6 & 254.276 & 285.303 & 1.0000 & $0.6588 \mathrm{E}+07$ \\
\hline 7 & 74.785 & 287.696 & 1.0000 & $\odot .7269 \mathrm{E}+\odot 7$ \\
\hline 8 & 85.797 & $288.0 \odot \odot$ & $1.000 \odot$ & $\odot .7820 \mathrm{E}+\odot 7$ \\
\hline 9 & $288 . \odot \odot \odot$ & $288.00 \odot$ & $1.00 \odot \odot$ & $\odot .8017 \mathrm{E}+\odot 7$ \\
\hline 10 & 288.000 & 288.000 & 1.0000 & $0.8044 \mathrm{E}+07$ \\
\hline 11 & 288.000 & 288.000 & $1.000 \odot$ & $\odot .8044 \mathrm{E}+07$ \\
\hline 12 & $288.00 \odot$ & $288.0 \odot \odot$ & $1.000 \odot$ & $\odot .8 \odot 44 \mathrm{E}+\odot 7$ \\
\hline 13 & 88.000 & $288 . \odot \odot \odot$ & $1.00 \odot \odot$ & $44 \mathrm{E}+07$ \\
\hline 14 & 88.000 & 288.000 & 1.0000 & $\odot .8044 \mathrm{E}+07$ \\
\hline 15 & 88.000 & $288.0 \odot \odot$ & $1.000 \odot$ & $\odot .8044 \mathrm{E}+07$ \\
\hline 16 & 88.000 & 288.000 & $1.00 \odot \odot$ & $\odot .8 \odot 44 \mathrm{E}+\odot 7$ \\
\hline 17 & 88.000 & 288.000 & 1.0000 & $0.8044 \mathrm{E}+07$ \\
\hline 18 & $38.00 \odot$ & \multirow{2}{*}{$\begin{array}{l}288.00 \odot \\
288.00 \odot\end{array}$} & $1.00 \odot \odot$ & $4 \mathrm{E}+07$ \\
\hline 19 & 88.000 & & 1.0000 & $\odot .8044 \mathrm{E}+07$ \\
\hline 20 & $38.0 \odot \odot$ & $288 . \odot \odot \odot$ & $1.00 \odot \odot$ & $\odot .8 \odot 44 \mathrm{E}+\odot 7$ \\
\hline time & $(\mathrm{sec})=$ & $22.00 \quad 1$ & $a=0$. & 00566 \\
\hline node & $\mathrm{T}(\mathrm{K})$ & Twall(K) & $x$ & $\mathrm{P}(\mathrm{Pa})$ \\
\hline 1 & 130.560 & 179.631 & - - - - - & $0.6179 \mathrm{E}+\odot 6$ \\
\hline 2 & 151.449 & 217.193 & $\odot .9845$ & $0.1140 \mathrm{E}+\odot 7$ \\
\hline 3 & 17.618 & 259.174 & $1.00 \odot \odot$ & $\odot .4808 \mathrm{E}+07$ \\
\hline 4 & 236.009 & 271.391 & $1.000 \odot$ & $\odot .5780 \mathrm{E}+07$ \\
\hline 5 & 44.030 & 278.879 & $1.00 \odot \odot$ & $0.6205 \mathrm{E}+07$ \\
\hline 6 & 51.213 & 284.388 & $1.00 \odot \odot$ & $\odot .6509 \mathrm{E}+\odot 7$ \\
\hline 7 & 99.554 & 287.375 & 1.0000 & $\odot .7090 \mathrm{E}+\odot 7$ \\
\hline 8 & 83.947 & $288 . \odot \odot \odot$ & $1.000 \odot$ & $\odot .7706 \mathrm{E}+\odot 7$ \\
\hline 9 & 37.732 & $288 . \odot \odot \odot$ & $1.0 \odot \odot \odot$ & $\odot .7986 \mathrm{E}+\odot 7$ \\
\hline 10 & 288.000 & $288.00 \odot$ & 1.0000 & $\odot .8041 \mathrm{E}+07$ \\
\hline 11 & 288.000 & $288 . \odot \odot \odot$ & $1.00 \odot \odot$ & $\odot .8044 \mathrm{E}+07$ \\
\hline 12 & 0 & 288. & $1.00 \odot \odot$ & $\odot .8044 \mathrm{E}+07$ \\
\hline 13 & $\odot \odot$ & 288. & $1.000 \odot$ & $4 E+\odot 7$ \\
\hline 14 & 38.000 & $288 . \odot \odot \odot$ & $1.000 \odot$ & $0.8044 \mathrm{E}+07$ \\
\hline 15 & 88.000 & $288 . \odot \odot \odot$ & $1.000 \odot$ & $0.8044 \mathrm{E}+07$ \\
\hline 16 & 288.000 & 288.000 & 1.0000 & $0.8044 \mathrm{E}+07$ \\
\hline 17 & 288.000 & $288.00 \odot$ & 1.0000 & $0.8044 \mathrm{E}+07$ \\
\hline 18 & $\odot \odot$ & $288 . \odot \odot \odot$ & $1.000 \odot$ & $0.8044 \mathrm{E}+07$ \\
\hline 19 & 88.000 & 288.000 & 1.0000 & $0.8044 \mathrm{E}+07$ \\
\hline 20 & $288.00 \odot$ & 288.000 & $1.000 \odot$ & $\odot .8044 \mathrm{E}+07$ \\
\hline time & $(\sec )=$ & $23.00 \quad 1$ & $\mathrm{a} a=0$. & 03892905 \\
\hline node & $\mathrm{T}(\mathrm{K})$ & Twall(K) & $x$ & $\mathrm{P}(\mathrm{Pa})$ \\
\hline 1 & 130.109 & 177.492 & - - - - - & $\odot .6010 \mathrm{E}+06$ \\
\hline 2 & 150.926 & 214.834 & $\odot .9839$ & $\odot .1109 \mathrm{E}+07$ \\
\hline
\end{tabular}




\begin{tabular}{|c|c|c|c|c|}
\hline 3 & 214.628 & 257.602 & 1.0000 & $\odot .4721 \mathrm{E}+07$ \\
\hline 4 & 236.081 & 270.359 & $1.000 \odot$ & $\odot .5747 \mathrm{E}+\odot 7$ \\
\hline 5 & 244.462 & 277.901 & 1.0000 & $\odot .6214 \mathrm{E}+07$ \\
\hline 6 & 250.297 & 283.472 & 1.0000 & $\odot .6485 \mathrm{E}+07$ \\
\hline 7 & 264.061 & 286.764 & 1.0000 & $\odot .6931 \mathrm{E}+\odot 7$ \\
\hline 8 & 281.169 & $288.00 \odot$ & 1.0000 & $\odot .7560 \mathrm{E}+07$ \\
\hline 9 & 287.279 & 288.000 & 1.0000 & $\odot .7940 \mathrm{E}+07$ \\
\hline 10 & 288.000 & 288.000 & 1.0000 & $\odot .8036 \mathrm{E}+07$ \\
\hline 11 & $288.00 \odot$ & 288.000 & 1.0000 & $\odot .8044 \mathrm{E}+\odot 7$ \\
\hline 12 & $288.00 \odot$ & $288.00 \odot$ & 1.0000 & $\odot .8044 \mathrm{E}+07$ \\
\hline 13 & $288 . \odot \odot \odot$ & $288.00 \odot$ & $1.000 \odot$ & $\odot .8 \odot 44 \mathrm{E}+\odot 7$ \\
\hline 14 & $288 . \odot \odot \odot$ & $288.00 \odot$ & 1.0000 & $\odot .8 \odot 44 \mathrm{E}+07$ \\
\hline 15 & $288.00 \odot$ & $288.00 \odot$ & 1.0000 & $\odot .8044 \mathrm{E}+07$ \\
\hline 16 & $288.00 \odot$ & 288.000 & 1.0000 & $\odot .8044 \mathrm{E}+07$ \\
\hline 17 & 288.000 & 288.000 & 1.0000 & $\odot .8044 \mathrm{E}+07$ \\
\hline 18 & 288.000 & 288.000 & 1.0000 & $\odot .8044 \mathrm{E}+07$ \\
\hline 19 & $288 . \odot 0 \odot$ & $288.00 \odot$ & 1.0000 & $\odot .8044 \mathrm{E}+07$ \\
\hline 20 & $288 . \odot \odot \odot$ & $288 . \odot \odot \odot$ & $1.00 \odot \odot$ & $\odot .8044 \mathrm{E}+\odot 7$ \\
\hline
\end{tabular}

\begin{tabular}{|c|c|c|c|c|}
\hline $\begin{array}{l}\text { time } \\
\text { node }\end{array}$ & $\begin{array}{r}(\mathrm{sec})= \\
T(\mathrm{~K})\end{array}$ & $\begin{array}{c}4.00 \\
\text { Twall(K) }\end{array}$ & $a=0$ & $\begin{array}{r}6184751 \\
P(\mathrm{~Pa})\end{array}$ \\
\hline 1 & 129.683 & 175.484 & $\ldots$ & $0.5850 E+06$ \\
\hline 2 & 150.432 & 212.574 & $\odot .9834$ & $\odot .1079 \mathrm{E}+\odot 7$ \\
\hline 3 & 211.335 & 255.771 & $1.000 \odot$ & $\odot .4634 \mathrm{E}+07$ \\
\hline 4 & 235.585 & 269.444 & 1.0000 & $\odot .5695 \mathrm{E}+07$ \\
\hline 5 & 244.767 & 276.986 & 1.0000 & $\odot .6214 \mathrm{E}+07$ \\
\hline 6 & 250.600 & 282.556 & 1.0000 & $\odot .6493 \mathrm{E}+\odot 7$ \\
\hline 7 & 259.320 & 286.040 & 1.0000 & $\odot .6806 \mathrm{E}+\odot 7$ \\
\hline 8 & 277.402 & 287.869 & $1.000 \odot$ & $\odot .7398 \mathrm{E}+\odot 7$ \\
\hline 9 & 286.435 & $288.00 \odot$ & 1.0000 & $\odot .7870 \mathrm{E}+\odot 7$ \\
\hline 10 & $288.00 \odot$ & $288.0 \odot \odot$ & 1.0000 & $\odot .8 \odot 25 \mathrm{E}+\odot 7$ \\
\hline 11 & 288.000 & 288.000 & 1.0000 & $\odot .8044 \mathrm{E}+07$ \\
\hline 12 & 288.000 & 288.000 & 1.0000 & $\odot .8044 \mathrm{E}+07$ \\
\hline 13 & $288.00 \odot$ & 288.000 & 1.0000 & $\odot .8044 \mathrm{E}+\odot 7$ \\
\hline 14 & 288.000 & $288.00 \odot$ & 1.0000 & $\odot .8 \odot 44 \mathrm{E}+\odot 7$ \\
\hline 15 & 288.000 & 288.000 & 1.0000 & $\odot .8044 \mathrm{E}+07$ \\
\hline 16 & 288.000 & $288.00 \odot$ & 1.0000 & $\odot .8044 \mathrm{E}+\odot 7$ \\
\hline 17 & 288.000 & 288.000 & 1.0000 & $\odot .8044 \mathrm{E}+07$ \\
\hline 18 & 288.000 & 288.000 & 1.0000 & $\odot .8044 \mathrm{E}+07$ \\
\hline 19 & 288.000 & 288.000 & 1.0000 & $\odot .8044 \mathrm{E}+07$ \\
\hline 20 & $288 . \odot \odot \odot$ & $288.00 \odot$ & 1.0000 & $\odot .8044 \mathrm{E}+\odot 7$ \\
\hline
\end{tabular}

\begin{tabular}{|c|c|c|c|c|}
\hline \multicolumn{5}{|c|}{ lambda $=0.1810748130$} \\
\hline node & $T(K)$ & Twall(K) & $\mathrm{x}$ & $P(P a)$ \\
\hline 1 & 129.278 & 173.591 & - - - - - & $\odot .5698 \mathrm{E}+06$ \\
\hline 2 & 149.962 & 210.437 & 0.9828 & $\odot .1051 \mathrm{E}+07$ \\
\hline 3 & 207.994 & 253.891 & 1.0000 & $\odot .4552 \mathrm{E}+07$ \\
\hline 4 & 234.557 & 268.528 & 1.0000 & $\odot .5630 \mathrm{E}+\odot 7$ \\
\hline 5 & 245.073 & 276.070 & 1.0000 & $\odot .6208 \mathrm{E}+07$ \\
\hline 6 & 250.902 & 281.641 & 1.0000 & $\odot .6501 \mathrm{E}+07$ \\
\hline 7 & 256.569 & 285.124 & 1.0000 & $0.6734 \mathrm{E}+07$ \\
\hline 8 & 272.783 & 287.564 & 1.0000 & $\odot .7239 \mathrm{E}+07$ \\
\hline 9 & 284.973 & 288.000 & 1.0000 & $\odot .7772 \mathrm{E}+07$ \\
\hline 10 & 287.879 & 288.000 & 1.0000 & $\odot .8003 \mathrm{E}+\odot 7$ \\
\hline 11 & $288.00 \odot$ & $288.00 \odot$ & 1.0000 & $\odot .8044 \mathrm{E}+07$ \\
\hline 12 & 288.000 & 288.000 & $1.000 \odot$ & $\odot .8044 \mathrm{E}+07$ \\
\hline & 288.000 & 288.000 & 1.0000 & $\odot .8044 \mathrm{E}+07$ \\
\hline
\end{tabular}




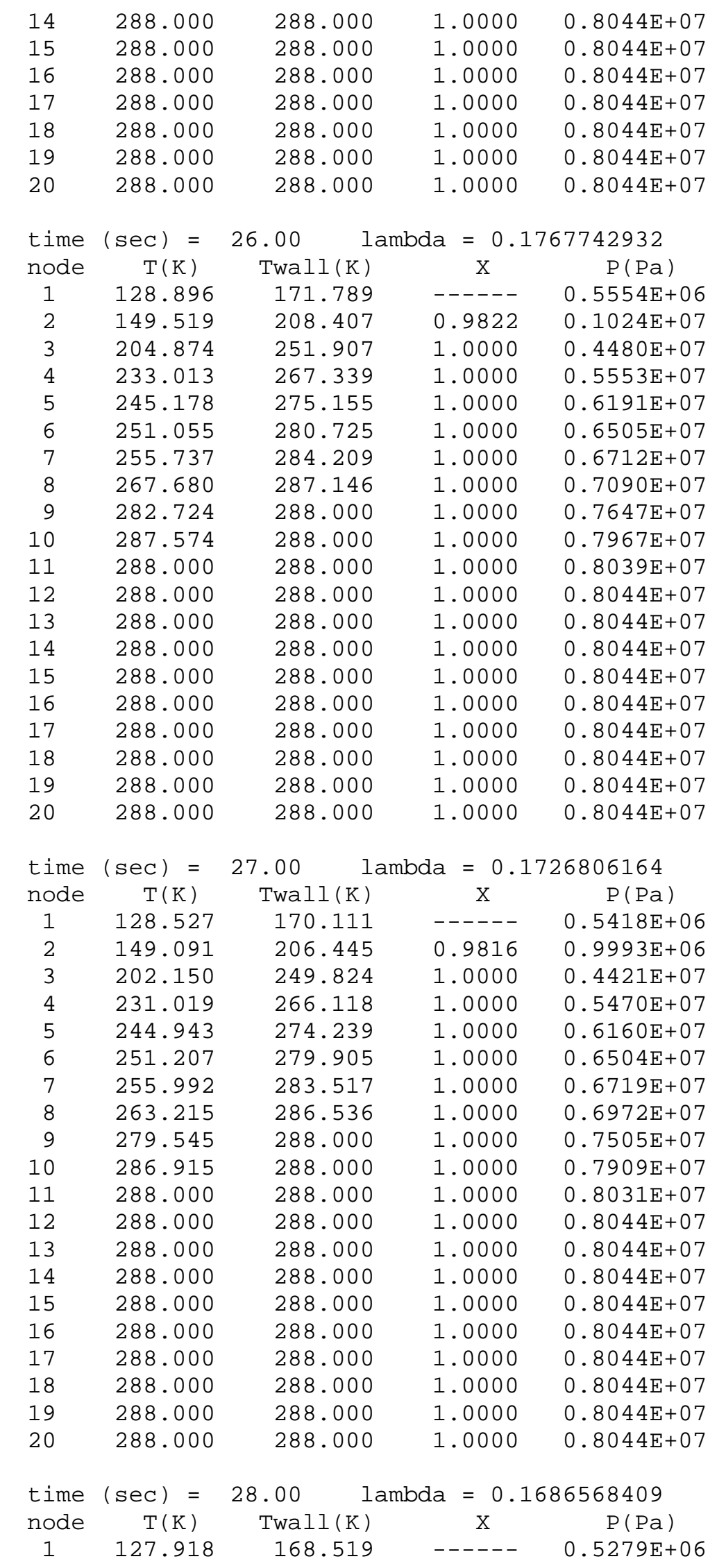




\begin{tabular}{|c|c|c|c|c|}
\hline 2 & 148.385 & 204.614 & $\odot .9810$ & $\odot .9737 \mathrm{E}+06$ \\
\hline 3 & 199.940 & 247.688 & 1.0000 & $\odot .4372 \mathrm{E}+07$ \\
\hline 4 & 228.681 & 264.897 & 1.0000 & $\odot .5384 \mathrm{E}+07$ \\
\hline 5 & 244.373 & 273.324 & 1.0000 & $\odot .6117 \mathrm{E}+07$ \\
\hline 6 & 251.360 & 279.295 & 1.0000 & $\odot .6500 \mathrm{E}+07$ \\
\hline 7 & 256.263 & 282.906 & $1.00 \odot \odot$ & $\odot .6726 \mathrm{E}+07$ \\
\hline 8 & 260.554 & 285.925 & 1.0000 & $\odot .6901 \mathrm{E}+\odot 7$ \\
\hline 9 & 275.504 & 287.733 & 1.0000 & $0.7361 \mathrm{E}+07$ \\
\hline 10 & 285.799 & $288.00 \odot$ & 1.0000 & $\odot .7828 \mathrm{E}+07$ \\
\hline 11 & $288.00 \odot$ & $288.0 \odot \odot$ & $1.00 \odot \odot$ & $\odot .8017 \mathrm{E}+07$ \\
\hline 12 & $288.00 \odot$ & $288.00 \odot$ & 1.0000 & $\odot .8044 \mathrm{E}+07$ \\
\hline 13 & 288.000 & 288.000 & 1.0000 & $\odot .8044 \mathrm{E}+07$ \\
\hline 14 & 288.000 & 288.000 & 1.0000 & $\odot .8044 \mathrm{E}+07$ \\
\hline 15 & $288.00 \odot$ & $288.00 \odot$ & 1.0000 & $\odot .8044 \mathrm{E}+\odot 7$ \\
\hline 16 & 288.000 & $288.00 \odot$ & 1.0000 & $\odot .8044 \mathrm{E}+07$ \\
\hline 17 & $288.0 \odot \odot$ & $288.00 \odot$ & $1.000 \odot$ & $\odot .8 \odot 44 \mathrm{E}+\odot 7$ \\
\hline 18 & $288.00 \odot$ & $288 . \odot \odot \odot$ & $1.00 \odot \odot$ & $\odot .8 \odot 44 \mathrm{E}+07$ \\
\hline 19 & 288.000 & $288.0 \odot \odot$ & 1.0000 & $\odot .8044 \mathrm{E}+07$ \\
\hline 20 & 288.000 & 288.000 & 1.0000 & $\odot .8044 \mathrm{E}+07$ \\
\hline time & ec) $=$ & \multicolumn{3}{|l|}{$29.0 \odot$} \\
\hline node & $\mathrm{T}(\mathrm{K})$ & Twall(K) & $\mathrm{X}$ & $P(P a)$ \\
\hline 1 & 127.354 & 166.993 & $---{ }_{-1}$ & $\odot .5150 \mathrm{E}+06$ \\
\hline 2 & 147.731 & 202.783 & $\odot .9804$ & $0.9500 \mathrm{E}+06$ \\
\hline 3 & 198.404 & 245.640 & 1.0000 & $\odot .4339 \mathrm{E}+07$ \\
\hline 4 & 226.165 & 263.677 & 1.0000 & $\odot .5300 \mathrm{E}+07$ \\
\hline 5 & 243.471 & 272.408 & 1.0000 & $0.6062 \mathrm{E}+07$ \\
\hline 6 & 251.347 & 278.685 & 1.0000 & $488 \mathrm{E}+07$ \\
\hline 7 & 256.263 & 282.296 & $1.00 \odot \odot$ & $\odot .6726 \mathrm{E}+07$ \\
\hline 8 & 259.689 & 285.315 & 1.0000 & $\odot .6878 \mathrm{E}+07$ \\
\hline 9 & 270.870 & 287.428 & 1.0000 & $\odot .7224 \mathrm{E}+07$ \\
\hline 10 & 283.989 & $288.0 \odot \odot$ & 1.0000 & $\odot .7721 \mathrm{E}+07$ \\
\hline 11 & 287.725 & $288.0 \odot \odot$ & $\odot \odot \odot$ & $\odot .7987 \mathrm{E}+07$ \\
\hline 12 & $288.00 \odot$ & $288.00 \odot$ & 1.0 & $041 \mathrm{E}+07$ \\
\hline 13 & $288.00 \odot$ & $288 . \odot \odot \odot$ & 1.0000 & $\odot .8044 \mathrm{E}+07$ \\
\hline 14 & $288.0 \odot \odot$ & $288.00 \odot$ & $1.000 \odot$ & $\odot .8 \odot 44 \mathrm{E}+07$ \\
\hline 15 & $288.00 \odot$ & $288.00 \odot$ & 1.0000 & $\odot .8044 \mathrm{E}+07$ \\
\hline 16 & 288.000 & 288.000 & 1.0000 & $\odot .8 \odot 44 \mathrm{E}+07$ \\
\hline 17 & $288.00 \odot$ & 288.0 & $\bullet \odot$ & $\odot .8 \odot 44 \mathrm{E}+07$ \\
\hline 18 & $288.00 \odot$ & 288 & 1.0 & $044 \mathrm{E}+07$ \\
\hline 19 & $288.00 \odot$ & $288.00 \odot$ & 1.0000 & $\odot .8044 \mathrm{E}+07$ \\
\hline 20 & $288.0 \odot \odot$ & $288 . \odot \odot \odot$ & $1.00 \odot \odot$ & $\odot .8 \odot 44 \mathrm{E}+\odot 7$ \\
\hline time & $(\sec )=$ & \multicolumn{3}{|l|}{30.00} \\
\hline node & $\mathrm{T}(\mathrm{K})$ & Twall(K) & $\mathrm{X}$ & $P(P a)$ \\
\hline 1 & 126.836 & 165.555 & - - - - - & $0.5032 \mathrm{E}+06$ \\
\hline 2 & 147.130 & 200.952 & $\odot .9798$ & $\odot .9282 \mathrm{E}+\odot 6$ \\
\hline 3 & 197.549 & 243.657 & 1.0000 & $\odot .4320 \mathrm{E}+07$ \\
\hline 4 & 223.643 & 262.401 & 1.0000 & $\odot .5222 \mathrm{E}+\odot 7$ \\
\hline 5 & 242.233 & 271.493 & $1.000 \odot$ & $\odot .5998 \mathrm{E}+07$ \\
\hline 6 & 251.062 & 278.074 & 1.0000 & $\odot .6465 \mathrm{E}+07$ \\
\hline 7 & 256.263 & 281.685 & $1.00 \odot \odot$ & $\odot .6721 \mathrm{E}+\odot 7$ \\
\hline 8 & 259.957 & 284.705 & $1.00 \odot \odot$ & $\odot .6886 \mathrm{E}+07$ \\
\hline 9 & 266.636 & 286.954 & 1.0000 & $\odot .7111 \mathrm{E}+07$ \\
\hline 10 & 281.337 & $288.0 \odot \odot$ & 1.0000 & $\odot .7595 \mathrm{E}+07$ \\
\hline 11 & 287.273 & $288.00 \odot$ & $1.000 \odot$ & $\odot .7941 \mathrm{E}+\odot 7$ \\
\hline 12 & $288.00 \odot$ & $288.00 \odot$ & 1.0000 & $\odot .8036 \mathrm{E}+07$ \\
\hline
\end{tabular}




\begin{tabular}{|c|c|c|c|c|}
\hline 13 & 288.000 & $288.0 \odot \odot$ & 1.0000 & $\odot .8044 \mathrm{E}+\odot 7$ \\
\hline 14 & $288.00 \odot$ & $288 . \odot \odot \odot$ & 1.0000 & $\odot .8 \odot 44 \mathrm{E}+\odot 7$ \\
\hline 15 & $288 . \odot \odot \odot$ & $288 . \odot \odot \odot$ & $1.000 \odot$ & $\odot .8 \odot 44 \mathrm{E}+\odot 7$ \\
\hline 16 & 288.000 & 288.000 & 1.0000 & $\odot .8044 \mathrm{E}+07$ \\
\hline 17 & $288.0 \odot \odot$ & $288 . \odot \odot \odot$ & $1.00 \odot \odot$ & $\odot .8 \odot 44 \mathrm{E}+07$ \\
\hline 18 & $288 . \odot \odot \odot$ & $288 . \odot \odot \odot$ & 1.0000 & $\odot .8 \odot 44 \mathrm{E}+\odot 7$ \\
\hline 19 & $288.0 \odot \odot$ & $288 . \odot \odot \odot$ & $1.000 \odot$ & $\odot .8 \odot 44 \mathrm{E}+\odot 7$ \\
\hline & $288.0 \odot \odot$ & $288 . \odot \odot \odot$ & $1.00 \odot \odot$ & $\odot .8 \odot 44 \mathrm{E}+07$ \\
\hline
\end{tabular}

lambda_avg $=0.319210589$ 
Compressor station distance $=20$ miles, Pressure $=2100$ psi, 20 axial node elements

\begin{tabular}{|c|c|c|c|c|}
\hline \multicolumn{5}{|c|}{$.00 \quad$ lambda $=0.8513534665$} \\
\hline node & $\mathrm{T}(\mathrm{K})$ & Twall(K) & $\mathrm{X}$ & $P(P a)$ \\
\hline 1 & 247.451 & 277.435 & $\ldots$ & $\odot .6669 \mathrm{E}+\odot$ \\
\hline 2 & 287.043 & 288.000 & 1.0000 & $\odot .1230 E+\odot 8$ \\
\hline 3 & 288.000 & 288.000 & 1.0000 & $0.1445 \mathrm{E}+\mathrm{C}$ \\
\hline 4 & 288.000 & $288.00 \odot$ & 1.0000 & $0.1448 \mathrm{E}+\odot$ \\
\hline 5 & 288.000 & 288.000 & 1.0000 & $\odot .1448 \mathrm{E}+08$ \\
\hline 6 & 288.000 & 288.000 & 1.0000 & $\odot .1448 \mathrm{E}+08$ \\
\hline 7 & 288.000 & 288.000 & 1.0000 & 0.144 \\
\hline 8 & 288.000 & 288.000 & 1.0000 & $\odot .1448 \mathrm{E}+08$ \\
\hline 9 & 288.000 & 288.000 & 1.0000 & $\odot .1448 \mathrm{E}+\odot 8$ \\
\hline 10 & 288.000 & 288.000 & 1.0000 & $\odot .1448 \mathrm{E}+08$ \\
\hline 11 & 288.000 & 288.000 & 1.0000 & $\odot .1448 \mathrm{E}+08$ \\
\hline 12 & 288.000 & 288.000 & 1.0000 & $\odot .1448 \mathrm{E}+08$ \\
\hline 13 & 288.0 & $288.00 \odot$ & $1.000 \odot$ & 0.14 \\
\hline 14 & 288.000 & 288.000 & 1.0000 & 0.14 \\
\hline 15 & 288.000 & 288.000 & 1.0000 & $0.1448 \mathrm{E}+08$ \\
\hline 16 & 288.000 & 288.000 & 1.0000 & $0.1448 \mathrm{E}+08$ \\
\hline 17 & 288.000 & $288.00 \odot$ & $1.000 \odot$ & $\odot .1448 \mathrm{E}+\odot 8$ \\
\hline 18 & $288.00 \odot$ & $288.00 \odot$ & 1.0000 & $0.1448 \mathrm{E}+08$ \\
\hline 19 & $288 . \odot \odot \odot$ & $288.00 \odot$ & 1.0000 & $\odot .1448 \mathrm{E}+08$ \\
\hline 20 & $288.00 \odot$ & 288.000 & $1.000 \odot$ & $\odot .1448 \mathrm{E}+\odot 8$ \\
\hline
\end{tabular}

\begin{tabular}{|c|c|c|c|c|}
\hline \multicolumn{5}{|c|}{ lambda $=0.7250236869$} \\
\hline node & $\mathrm{T}(\mathrm{K})$ & Twall(K) & $\mathrm{x}$ & $P(P a)$ \\
\hline 1 & 241.665 & 269.991 & - . - _ & $0.5613 \mathrm{E}+\odot$ \\
\hline 2 & 280.330 & 287.886 & $1.000 \odot$ & $\odot .1035 \mathrm{E}+\odot 8$ \\
\hline 3 & 287.973 & 288.000 & 1.0000 & $0.1433 \mathrm{E}+\odot$ \\
\hline 4 & 288.000 & 288.000 & 1.0000 & $0.1448 \mathrm{E}+08$ \\
\hline 5 & 288.000 & 288.000 & 1.0000 & $0.1448 \mathrm{E}+\mathrm{C}$ \\
\hline 6 & 288.000 & 288.000 & 1.0000 & $\odot .1448 \mathrm{E}+\odot$ \\
\hline 7 & 288.000 & 288.000 & 1.0000 & $\odot .1448 \mathrm{E}+\odot 8$ \\
\hline 8 & $288.00 \odot$ & $288.00 \odot$ & $1.000 \odot$ & $\odot .1448 \mathrm{E}+\odot 8$ \\
\hline 9 & 288.000 & 288.000 & 1.0000 & $\odot .1448 \mathrm{E}+\odot$ \\
\hline 10 & 288.000 & 288.000 & 1.0000 & $\odot .1448 \mathrm{E}+\odot$ \\
\hline 11 & 288.000 & 288.000 & 1.0000 & $0.1448 \mathrm{E}+0$ \\
\hline 12 & 288.000 & 288.000 & 1.0000 & $\odot .1448 \mathrm{E}+\odot$ \\
\hline 13 & 288.000 & 288.000 & 1.0000 & $\odot .1448 \mathrm{E}+\odot$ \\
\hline 14 & $288.00 \odot$ & $288.0 \odot \odot$ & $1.000 \odot$ & $\odot .1448 \mathrm{E}+\odot$ \\
\hline 15 & 288.000 & 288.000 & 1.0000 & $\odot .1448 \mathrm{E}+\odot$ \\
\hline 16 & 288.000 & 288.000 & 1.0000 & $\odot .1448 \mathrm{E}+\odot$ \\
\hline 17 & $288.00 \odot$ & 288.000 & 1.0000 & $0.1448 \mathrm{E}+\odot$ \\
\hline 18 & 288.000 & $288.0 \odot \odot$ & 1.0000 & $\odot .1448 \mathrm{E}+\Theta$ \\
\hline 19 & 288.000 & 288.000 & 1.0000 & $\odot .1448 \mathrm{E}+\Theta$ \\
\hline 20 & 288.000 & $288 . \odot \odot \odot$ & $1.000 \odot$ & $\odot .1448 \mathrm{E}+\odot$ \\
\hline
\end{tabular}

\begin{tabular}{ccccc} 
time & $(\mathrm{sec})=$ & 3.00 & \multicolumn{2}{c}{ lambda $=0.6188996434$} \\
node & $\mathrm{T}(\mathrm{K})$ & Twall $(\mathrm{K})$ & $\mathrm{X}$ & $\mathrm{P}(\mathrm{Pa})$ \\
1 & 227.833 & 263.088 &.--- & $0.4652 \mathrm{E}+07$ \\
2 & 264.284 & 286.981 & $1.000 \odot$ & $0.8581 \mathrm{E}+07$ \\
3 & 287.511 & $288.00 \odot$ & $1.000 \odot$ & $0.1404 \mathrm{E}+08$ \\
4 & $288.00 \odot$ & $288.00 \odot$ & $1.000 \odot$ & $0.1446 \mathrm{E}+08$ \\
5 & $288.00 \odot$ & $288.00 \odot$ & $1.000 \odot$ & $0.1448 \mathrm{E}+08$ \\
6 & $288.00 \odot$ & $288.00 \odot$ & $1.000 \odot$ & $0.1448 \mathrm{E}+08$
\end{tabular}




\begin{tabular}{|c|c|c|c|c|}
\hline 7 & 288.000 & $288 . \odot \odot \odot$ & $1.00 \odot \odot$ & $\odot .1448 E+\odot 8$ \\
\hline 8 & $288.00 \odot$ & 288.000 & 1.0000 & $\odot .1448 \mathrm{E}+\odot 8$ \\
\hline 9 & $288.00 \odot$ & $288 . \odot \odot \odot$ & $1.00 \odot \odot$ & $\odot .1448 \mathrm{E}+08$ \\
\hline 10 & 288.000 & 288.000 & 1.0000 & $\odot .1448 \mathrm{E}+08$ \\
\hline 11 & 288.000 & $288.00 \odot$ & 1.0000 & $\odot .1448 \mathrm{E}+08$ \\
\hline 12 & 288.000 & 288.000 & 1.0000 & $\odot .1448 \mathrm{E}+08$ \\
\hline 13 & $288.00 \odot$ & $288 . \odot \odot \odot$ & $1.00 \odot \odot$ & $\odot .1448 \mathrm{E}+08$ \\
\hline 14 & 288.000 & $288.00 \odot$ & 1.0000 & $\odot .1448 \mathrm{E}+08$ \\
\hline 15 & $288.00 \odot$ & $288 . \odot \odot \odot$ & 1.0000 & $\odot .1448 \mathrm{E}+\odot 8$ \\
\hline 16 & 288.000 & 288.000 & 1.0000 & $0.1448 \mathrm{E}+08$ \\
\hline 17 & 288.000 & 288.000 & 1.0000 & $0.1448 \mathrm{E}+08$ \\
\hline 18 & 288.000 & 288.000 & 1.0000 & $\odot .1448 \mathrm{E}+08$ \\
\hline 19 & $288.00 \odot$ & $288 . \odot \odot \odot$ & 1.0000 & $\odot .1448 \mathrm{E}+\odot 8$ \\
\hline 20 & $288.00 \odot$ & $288 . \odot \odot \odot$ & $1.00 \odot \odot$ & $\odot .1448 \mathrm{E}+08$ \\
\hline time & $(\sec )=$ & \multirow{2}{*}{\multicolumn{3}{|c|}{$\begin{array}{l}4.00 \quad \text { lambda }=0.5314630866 \\
\text { Twall }(K)\end{array}$}} \\
\hline node & $\mathrm{T}(\mathrm{K})$ & & & \\
\hline 1 & 207.465 & 255.063 & $-\ldots$ & $\odot .3812 \mathrm{E}+07$ \\
\hline 2 & 240.657 & 284.300 & 1.0000 & $\odot .7 \odot 32 \mathrm{E}+\odot 7$ \\
\hline 3 & 286.046 & 288.000 & 1.0000 & $\odot .1360 E+08$ \\
\hline 4 & 288.000 & $288.00 \odot$ & 1.0000 & $\odot .1443 E+\odot 8$ \\
\hline 5 & 288.000 & $288.00 \odot$ & 1.0000 & $\odot .1448 \mathrm{E}+\odot 8$ \\
\hline 6 & 288.000 & 288.000 & 1.0000 & $\odot .1448 \mathrm{E}+\odot 8$ \\
\hline 7 & 288. & 288.000 & 1.0000 & $0.1448 \mathrm{E}+08$ \\
\hline 8 & 288.000 & $288.00 \odot$ & 1.0000 & $\odot .1448 \mathrm{E}+08$ \\
\hline 9 & $288.00 \odot$ & $288 . \odot \odot \odot$ & 1.0000 & $\odot .1448 \mathrm{E}+08$ \\
\hline 10 & 288.000 & 288.000 & 1.0000 & $0.1448 \mathrm{E}+08$ \\
\hline 11 & 288.000 & $288.00 \odot$ & 1.0000 & $\odot .1448 \mathrm{E}+\odot 8$ \\
\hline 12 & 288. & $288.00 \odot$ & 1.0000 & $0.1448 \mathrm{E}+08$ \\
\hline 13 & 288. & $288 . \odot \odot \odot$ & $1.00 \odot \odot$ & $\odot .1448 \mathrm{E}+08$ \\
\hline 14 & 288.000 & $288.00 \odot$ & 1.0000 & $0.1448 \mathrm{E}+08$ \\
\hline 15 & 288.000 & $288.00 \odot$ & 1.0000 & $\odot .1448 \mathrm{E}+\odot 8$ \\
\hline 16 & 288.000 & 288.000 & 1.0000 & $\odot .1448 \mathrm{E}+08$ \\
\hline 17 & 288.000 & 288.000 & 1.0000 & $48 \mathrm{E}+08$ \\
\hline 18 & 288. & $288.00 \odot$ & 1.0000 & $\odot .1448 \mathrm{E}+\odot 8$ \\
\hline 19 & 288. & 288.000 & 1.0000 & $0.1448 \mathrm{E}+08$ \\
\hline 20 & 288.000 & $288.00 \odot$ & 1.0000 & $\odot .1448 \mathrm{E}+08$ \\
\hline time & $(\sec )=$ & $5.00 \quad 10$ & \multicolumn{2}{|c|}{ lambda $=0.4620520473$} \\
\hline node & $\mathrm{T}(\mathrm{K})$ & Twall(K) & $\mathrm{x}$ & $\mathrm{P}(\mathrm{Pa})$ \\
\hline 1 & 185.777 & 245.729 & - - - - - & $\odot .3136 \mathrm{E}+07$ \\
\hline 2 & 215.499 & 279.384 & $1.000 \odot$ & $\odot .5785 \mathrm{E}+07$ \\
\hline 3 & 282.753 & 287.998 & $1.00 \odot \odot$ & $0.1306 \mathrm{E}+08$ \\
\hline 4 & 287.865 & $288.00 \odot$ & 1.0000 & $\odot .1435 \mathrm{E}+08$ \\
\hline 5 & 288.000 & $288 . \odot \odot \odot$ & $1.000 \odot$ & $\odot .1447 \mathrm{E}+\odot 8$ \\
\hline 6 & 288.6 & 288.6 & 1.00 & $\odot .1448 \mathrm{E}+08$ \\
\hline 7 & 288. & $288 . \odot$ & 1.00 & $0.1448 \mathrm{E}+08$ \\
\hline 8 & 288.000 & $288.00 \odot$ & 1.0000 & $\odot .1448 \mathrm{E}+\odot 8$ \\
\hline 9 & $288.00 \odot$ & $288 . \odot \odot \odot$ & $1.000 \odot$ & $\odot .1448 \mathrm{E}+\odot 8$ \\
\hline 10 & 288.000 & $288.00 \odot$ & 1.0000 & $\odot .1448 \mathrm{E}+08$ \\
\hline 11 & $288.00 \odot$ & $288 . \odot \odot \odot$ & 1.0000 & $\odot .1448 \mathrm{E}+\odot 8$ \\
\hline 12 & $288.00 \odot$ & 288.000 & 1.0000 & $\odot .1448 \mathrm{E}+08$ \\
\hline 13 & $288.00 \odot$ & $288 . \odot \odot \odot$ & $1.000 \odot$ & $\odot .1448 \mathrm{E}+\odot 8$ \\
\hline 14 & 288.000 & $288.00 \odot$ & $1.000 \odot$ & $\odot .1448 \mathrm{E}+08$ \\
\hline 15 & 288.000 & $288 . \odot \odot \odot$ & 1.0000 & $\odot .1448 \mathrm{E}+\odot 8$ \\
\hline 16 & 288.000 & $288.00 \odot$ & $1.000 \odot$ & $\odot .1448 \mathrm{E}+\odot 8$ \\
\hline 17 & 288.000 & $288.00 \odot$ & 1.0000 & $0.1448 \mathrm{E}+08$ \\
\hline
\end{tabular}




$\begin{array}{lllll}18 & 288.000 & 288.000 & 1.000 \odot & \odot .1448 \mathrm{E}+08 \\ 19 & 288.000 & 288.000 & 1.000 \odot & 0.1448 \mathrm{E}+08 \\ 20 & 288.000 & 288.000 & 1.000 \odot & 0.1448 \mathrm{E}+08\end{array}$

\begin{tabular}{|c|c|c|c|c|}
\hline \multicolumn{5}{|c|}{ lambda $=0.4094181657$} \\
\hline node & $\mathrm{T}^{\prime}(\mathrm{K})$ & Twall(K) & $\mathrm{x}$ & $\mathrm{P}(\mathrm{Pa})$ \\
\hline 1 & 167.340 & 235.695 & $-\ldots$ & $\odot .2637 \mathrm{E}+\odot 7$ \\
\hline 2 & 194.113 & 272.270 & 1.0000 & $\odot .4865 \mathrm{E}+07$ \\
\hline 3 & 277.016 & 287.690 & 1.0000 & $\odot .1245 E+\odot 8$ \\
\hline 4 & 287.421 & $288 . \odot \odot \odot$ & 1.0000 & $\odot .1420 E+\odot 8$ \\
\hline 5 & 288.000 & 288.000 & 1.0000 & $\odot .1446 \mathrm{E}+\odot 8$ \\
\hline 6 & 288.000 & 288.000 & 1.0000 & $\odot .1448 \mathrm{E}+\odot 8$ \\
\hline 7 & 288.000 & $288 . \odot \odot \odot$ & 1.0000 & $\odot .1448 \mathrm{E}+\odot 8$ \\
\hline 8 & 288.000 & 288.000 & 1.0000 & $0.1448 \mathrm{E}+08$ \\
\hline 9 & 288.000 & $288.00 \odot$ & 1.0000 & $\odot .1448 \mathrm{E}+08$ \\
\hline 10 & 288.000 & 288.000 & 1.0000 & $\odot .1448 \mathrm{E}+\odot 8$ \\
\hline 11 & 288.000 & 288.000 & 1.0000 & $\odot .1448 \mathrm{E}+\odot 8$ \\
\hline 12 & 288.000 & 288.000 & 1.0000 & $\odot .1448 \mathrm{E}+08$ \\
\hline 13 & 288.000 & 288.000 & 1.0000 & $\odot .1448 \mathrm{E}+\odot 8$ \\
\hline 14 & 288.000 & 288.000 & 1.0000 & $0.1448 \mathrm{E}+08$ \\
\hline 15 & 288.000 & 288.000 & 1.0000 & $\odot .1448 \mathrm{E}+08$ \\
\hline 16 & 288.000 & 288.000 & 1.0000 & $\odot .1448 \mathrm{E}+08$ \\
\hline 17 & 288.000 & 288.000 & 1.0000 & $\odot .1448 \mathrm{E}+\odot 8$ \\
\hline 18 & 288.000 & 288.000 & 1.0000 & $\odot .1448 \mathrm{E}+\odot 8$ \\
\hline 19 & 288.000 & 288.000 & 1.0000 & $\odot .1448 \mathrm{E}+08$ \\
\hline 20 & 288.000 & $288.00 \odot$ & 1.0000 & $\odot .1448 \mathrm{E}+\odot 8$ \\
\hline
\end{tabular}

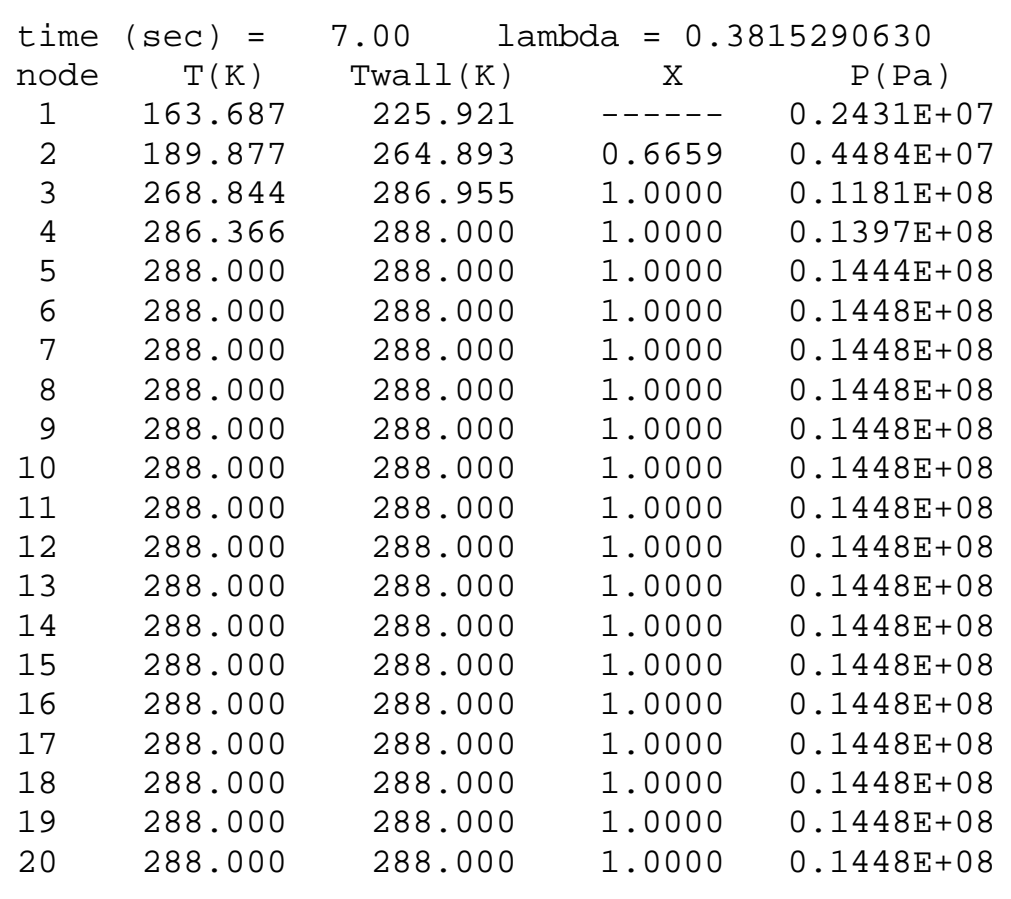

\begin{tabular}{ccccc} 
time & $(\mathrm{sec})=$ & 8.00 & \multicolumn{2}{c}{ lambda $=0.3581381738$} \\
node & $\mathrm{T}(\mathrm{K})$ & Twall $(\mathrm{K})$ & $\mathrm{X}$ & $\mathrm{P}(\mathrm{Pa})$ \\
1 & 161.655 & 217.607 &.--- & $0.2268 \mathrm{E}+07$ \\
2 & 187.520 & 258.286 & 0.6835 & $0.4183 \mathrm{E}+07$ \\
3 & 259.029 & 285.554 & $1.000 \odot$ & $0.1119 \mathrm{E}+08$ \\
4 & 284.308 & $288.00 \odot$ & $1.000 \odot$ & $0.1365 \mathrm{E}+08$ \\
5 & 287.895 & $288.00 \odot$ & $1.000 \odot$ & $0.1438 \mathrm{E}+08$
\end{tabular}




\begin{tabular}{|c|c|c|c|c|}
\hline 6 & 288.000 & 288.000 & 1.0000 & $\odot .1448 \mathrm{E}+08$ \\
\hline 7 & 288.000 & $288 . \odot \odot \odot$ & $1.000 \odot$ & $\odot .1448 \mathrm{E}+\odot 8$ \\
\hline 8 & $288.00 \odot$ & $288.0 \odot \odot$ & 1.0000 & $\odot .1448 \mathrm{E}+08$ \\
\hline 9 & 288.000 & 288.000 & 1.0000 & $\odot .1448 \mathrm{E}+08$ \\
\hline 10 & 288.000 & 288.000 & 1.0000 & $\odot .1448 \mathrm{E}+\odot 8$ \\
\hline 11 & $288.00 \odot$ & $288.00 \odot$ & 1.0000 & $\odot .1448 \mathrm{E}+08$ \\
\hline 12 & $288.00 \odot$ & $288.0 \odot \odot$ & 1.0000 & $\odot .1448 \mathrm{E}+\odot 8$ \\
\hline 13 & 288.000 & 288.000 & 1.0000 & $\odot .1448 \mathrm{E}+08$ \\
\hline 14 & 288.000 & $288.00 \odot$ & 1.0000 & $\odot .1448 \mathrm{E}+\odot 8$ \\
\hline 15 & $288.00 \odot$ & 288.000 & 1.0000 & $0.1448 \mathrm{E}+08$ \\
\hline 16 & 288.000 & 288.000 & 1.0000 & $\odot .1448 \mathrm{E}+08$ \\
\hline 17 & $288.0 \odot \odot$ & $288.00 \odot$ & 1.0000 & $\odot .1448 \mathrm{E}+08$ \\
\hline 18 & $288.00 \odot$ & $288.0 \odot \odot$ & 1.0000 & $\odot .1448 \mathrm{E}+\odot 8$ \\
\hline 19 & 288.000 & 288.000 & 1.0000 & $\odot .1448 \mathrm{E}+08$ \\
\hline 20 & 288.000 & $288 . \odot \odot \odot$ & $1.00 \odot \odot$ & $\odot .1448 E+\odot 8$ \\
\hline
\end{tabular}

\begin{tabular}{|c|c|c|c|c|}
\hline \multicolumn{5}{|c|}{$9.00 \quad$ lambda $=0.3367696702$} \\
\hline node & $\mathrm{T}(\mathrm{K})$ & Twall(K) & $x$ & $P(P a)$ \\
\hline 1 & 159.734 & 210.369 & - - - - - - & $\odot .2120 \mathrm{E}+\odot 7$ \\
\hline 2 & 185.292 & 252.383 & $\odot .7 \odot 27$ & $\odot .3910 \mathrm{E}+\odot 7$ \\
\hline 3 & 248.826 & 283.343 & 1.0000 & $\odot .1063 \mathrm{E}+\odot 8$ \\
\hline 4 & 280.856 & 287.888 & 1.0000 & $\odot .1325 E+\odot 8$ \\
\hline 5 & 287.542 & $288.00 \odot$ & $1.000 \odot$ & $\odot .1429 E+\odot 8$ \\
\hline 6 & $288.00 \odot$ & $288 . \odot \odot \odot$ & 1.0000 & $\odot .1446 \mathrm{E}+\odot 8$ \\
\hline 7 & $288.00 \odot$ & $288.00 \odot$ & $1.000 \odot$ & $\odot .1448 \mathrm{E}+\odot 8$ \\
\hline 8 & 288.000 & $288.00 \odot$ & 1.0000 & $\odot .1448 \mathrm{E}+08$ \\
\hline 9 & 288.000 & $288.00 \odot$ & 1.0000 & $\odot .1448 \mathrm{E}+08$ \\
\hline 10 & $288.00 \odot$ & $288 . \odot \odot \odot$ & 1.0000 & $\odot .1448 \mathrm{E}+\odot 8$ \\
\hline 11 & 288.000 & $288.0 \odot \odot$ & 1.0000 & $\odot .1448 \mathrm{E}+08$ \\
\hline 12 & 288.000 & $288.00 \odot$ & 1.0000 & $\odot .1448 \mathrm{E}+\odot 8$ \\
\hline 13 & 288.000 & $288.0 \odot \odot$ & 1.0000 & $\odot .1448 \mathrm{E}+08$ \\
\hline 14 & 288.000 & $288.0 \odot \odot$ & 1.0000 & $\odot .1448 \mathrm{E}+08$ \\
\hline 15 & $288.00 \odot$ & $288.0 \odot \odot$ & 1.0000 & $\odot .1448 \mathrm{E}+08$ \\
\hline 16 & 288.000 & 288.000 & $1.000 \odot$ & $\odot .1448 \mathrm{E}+\odot 8$ \\
\hline 17 & 288.000 & 288.000 & 1.0000 & $\odot .1448 \mathrm{E}+08$ \\
\hline 18 & 288.000 & 288.000 & 1.0000 & $\odot .1448 \mathrm{E}+\odot 8$ \\
\hline 19 & $288.00 \odot$ & $288.00 \odot$ & $1.000 \odot$ & $0.1448 \mathrm{E}+08$ \\
\hline 20 & $288.00 \odot$ & $288.0 \odot \odot$ & 1.0000 & $\odot .1448 \mathrm{E}+08$ \\
\hline
\end{tabular}

\begin{tabular}{|c|c|c|c|c|}
\hline time & $(\sec )=$ & 0.00 & $=0$. & 1127141 \\
\hline node & $\mathrm{T}(\mathrm{K})$ & Twall(K) & $\mathrm{X}$ & $P(P a)$ \\
\hline 1 & 157.827 & 204.071 & - - - & $\odot .1984 \mathrm{E}+\odot 7$ \\
\hline 2 & 183.079 & 247.059 & $\odot .7219$ & $\odot .3659 \mathrm{E}+07$ \\
\hline 3 & 239.833 & 280.323 & 1.0000 & $\odot .1018 \mathrm{E}+\odot 8$ \\
\hline 4 & 275.810 & 287.560 & 1.0000 & $\odot .1279 \mathrm{E}+\odot 8$ \\
\hline 5 & 286.787 & $288.0 \odot \odot$ & 1.0000 & $\odot .1413 E+08$ \\
\hline 6 & $288.0 \odot \odot$ & $288 . \odot \odot \odot$ & $1.000 \odot$ & $\odot .1445 E+08$ \\
\hline 7 & 288.000 & 288.000 & 1.0000 & $\odot .1448 \mathrm{E}+\odot 8$ \\
\hline 8 & 288.000 & $288.00 \odot$ & 1.0000 & $\odot .1448 \mathrm{E}+08$ \\
\hline 9 & 288.000 & $288.00 \odot$ & 1.0000 & $\odot .1448 \mathrm{E}+\odot 8$ \\
\hline 10 & 288.000 & $288.0 \odot \odot$ & 1.0000 & $\odot .1448 \mathrm{E}+\odot 8$ \\
\hline 11 & 288.000 & $288.00 \odot$ & 1.0000 & $\odot .1448 \mathrm{E}+08$ \\
\hline 12 & 288.000 & $288.0 \odot \odot$ & 1.0000 & $\odot .1448 \mathrm{E}+08$ \\
\hline 13 & $288.00 \odot$ & $288.00 \odot$ & $1.000 \odot$ & $\odot .1448 \mathrm{E}+\odot 8$ \\
\hline 14 & 288.000 & 288.000 & 1.0000 & $\odot .1448 \mathrm{E}+\odot 8$ \\
\hline 15 & 288.000 & $288.00 \odot$ & 1.0000 & $\odot .1448 \mathrm{E}+08$ \\
\hline 16 & 288.000 & $288.0 \odot \odot$ & 1.0000 & $\odot .1448 \mathrm{E}+\odot 8$ \\
\hline
\end{tabular}




$\begin{array}{lllll}17 & 288.00 \odot & 288.00 \odot & 1.000 \odot & \odot .1448 \mathrm{E}+\odot 8 \\ 18 & 288.00 \odot & 288.00 \odot & 1.000 \odot & \odot .1448 \mathrm{E}+08 \\ 19 & 288.00 \odot & 288.00 \odot & 1.000 \odot & 0.1448 \mathrm{E}+08 \\ 20 & 288.00 \odot & 288.00 \odot & 1.000 \odot & \odot .1448 \mathrm{E}+08\end{array}$

\begin{tabular}{|c|c|c|c|c|}
\hline \multicolumn{5}{|c|}{ lambda $=0.2992591262$} \\
\hline node & $T^{\prime}(K)$ & Twall(K) & $\mathrm{x}$ & $P(P a)$ \\
\hline 1 & 156.114 & 198.651 & -- & $\odot .1862 \mathrm{E}+07$ \\
\hline 2 & 181.092 & 242.201 & 0.7401 & $\odot .3435 E+07$ \\
\hline 3 & 233.062 & 276.763 & 1.0000 & $\odot .9857 \mathrm{E}+07$ \\
\hline 4 & 269.330 & 286.861 & 1.0000 & $\odot .1231 \mathrm{E}+08$ \\
\hline 5 & 285.347 & 288.000 & 1.0000 & $\odot .1391 \mathrm{E}+08$ \\
\hline 6 & 287.989 & $288.0 \odot \odot$ & 1.0000 & $\odot .1441 \mathrm{E}+08$ \\
\hline 7 & 288.000 & $288.00 \odot$ & 1.0000 & $\odot .1448 \mathrm{E}+\odot 8$ \\
\hline 8 & 288.000 & 288.000 & 1.0000 & $\odot .1448 \mathrm{E}+\odot 8$ \\
\hline 9 & $288.00 \odot$ & 288.000 & 1.0000 & $\odot .1448 \mathrm{E}+\odot 8$ \\
\hline 10 & 288.000 & $288.0 \odot \odot$ & 1.0000 & $\odot .1448 \mathrm{E}+\odot 8$ \\
\hline 11 & 288.000 & 288.000 & 1.0000 & $\odot .1448 \mathrm{E}+08$ \\
\hline 12 & 288.000 & $288.00 \odot$ & 1.0000 & $\odot .1448 \mathrm{E}+\odot 8$ \\
\hline 13 & 288.000 & 288.000 & 1.0000 & $0.1448 \mathrm{E}+08$ \\
\hline 14 & 288.000 & 288.000 & 1.0000 & $\odot .1448 \mathrm{E}+08$ \\
\hline 15 & 288.000 & $288.00 \odot$ & 1.0000 & $0.1448 \mathrm{E}+08$ \\
\hline 16 & 288.000 & 288.000 & 1.0000 & $\odot .1448 \mathrm{E}+\odot 8$ \\
\hline 17 & 288.000 & 288.000 & 1.0000 & $0.1448 \mathrm{E}+08$ \\
\hline 18 & 288.000 & 288.000 & 1.0000 & $\odot .1448 \mathrm{E}+08$ \\
\hline 19 & 288.000 & 288.000 & 1.0000 & $0.1448 \mathrm{E}+08$ \\
\hline 20 & 288.000 & 288.000 & 1.0000 & $\odot .1448 \mathrm{E}+08$ \\
\hline
\end{tabular}

\begin{tabular}{|c|c|c|c|c|}
\hline \multicolumn{5}{|c|}{$.00 \quad$ lambda $=0.2831389308$} \\
\hline node & $\mathrm{T}(\mathrm{K})$ & Twall(K) & $x$ & $P(P a)$ \\
\hline 1 & 154.582 & 193.969 & - - - - - - & $\odot .1753 \mathrm{E}+\odot 7$ \\
\hline 2 & 179.315 & 237.735 & $\odot .7574$ & $\odot .3234 \mathrm{E}+07$ \\
\hline 3 & 228.743 & 273.101 & 1.0000 & $\odot .9654 \mathrm{E}+\odot 7$ \\
\hline 4 & 262.007 & 285.718 & 1.0000 & $\odot .1185 E+08$ \\
\hline 5 & 282.957 & $288.00 \odot$ & $1.000 \odot$ & $\odot .1362 \mathrm{E}+\odot 8$ \\
\hline 6 & 287.683 & $288 . \odot \odot \odot$ & 1.0000 & $\odot .1434 \mathrm{E}+08$ \\
\hline 7 & $288.0 \odot \odot$ & $288.00 \odot$ & 1.0000 & $\odot .1447 E+08$ \\
\hline 8 & 288.000 & $288.00 \odot$ & 1.0000 & $\odot .1448 \mathrm{E}+08$ \\
\hline 9 & $288.00 \odot$ & $288.00 \odot$ & $1.000 \odot$ & $\odot .1448 \mathrm{E}+\odot 8$ \\
\hline 10 & 288.000 & $288 . \odot \odot \odot$ & 1.0000 & $\odot .1448 \mathrm{E}+\odot 8$ \\
\hline 11 & 288.000 & $288.0 \odot \odot$ & 1.0000 & $\odot .1448 \mathrm{E}+08$ \\
\hline 12 & 288.000 & $288.00 \odot$ & 1.0000 & $\odot .1448 \mathrm{E}+\odot 8$ \\
\hline 13 & 288.000 & $288.0 \odot \odot$ & 1.0000 & $\odot .1448 \mathrm{E}+08$ \\
\hline 14 & 288.000 & $288.0 \odot \odot$ & 1.0000 & $\odot .1448 \mathrm{E}+08$ \\
\hline 15 & 288.000 & 288.000 & 1.0000 & $\odot .1448 \mathrm{E}+\odot 8$ \\
\hline 16 & 288.000 & 288.000 & $1.000 \odot$ & $\odot .1448 \mathrm{E}+\odot 8$ \\
\hline 17 & 288.000 & 288.000 & 1.0000 & $\odot .1448 \mathrm{E}+08$ \\
\hline 18 & 288.000 & 288.000 & 1.0000 & $\odot .1448 \mathrm{E}+\odot 8$ \\
\hline 19 & $288.00 \odot$ & $288.00 \odot$ & $1.000 \odot$ & $0.1448 \mathrm{E}+08$ \\
\hline 20 & $288.00 \odot$ & $288.0 \odot \odot$ & 1.0000 & $\odot .1448 \mathrm{E}+08$ \\
\hline
\end{tabular}

\begin{tabular}{ccccc} 
time & $(\mathrm{sec})=$ & 13.00 & \multicolumn{2}{c}{ lambda $=0.2687073946$} \\
node & $\mathrm{T}(\mathrm{K})$ & Twall $(\mathrm{K})$ & $\mathrm{X}$ & $\mathrm{P}(\mathrm{Pa})$ \\
1 & 153.223 & 189.897 & ---- & $0.1656 \mathrm{E}+07$ \\
2 & 177.739 & 233.562 & 0.7738 & $0.3055 \mathrm{E}+07$ \\
3 & 226.663 & 269.451 & $1.000 \odot$ & $0.9546 \mathrm{E}+07$ \\
4 & 254.659 & 284.032 & $1.000 \odot$ & $0.1143 \mathrm{E}+08$
\end{tabular}




\begin{tabular}{|c|c|c|c|c|}
\hline 5 & 279.388 & 287.782 & 1.0000 & $0.1328 \mathrm{E}+08$ \\
\hline 6 & 287.153 & $288 . \odot \odot \odot$ & $1.000 \odot$ & $\odot .1423 E+\odot 8$ \\
\hline 7 & $288.00 \odot$ & $288 . \odot \odot \odot$ & 1.0000 & $\odot .1446 \mathrm{E}+\odot 8$ \\
\hline 8 & 288.000 & $288.00 \odot$ & 1.0000 & $\odot .1448 \mathrm{E}+08$ \\
\hline 9 & 288.000 & 288.000 & 1.0000 & $\odot .1448 \mathrm{E}+\odot 8$ \\
\hline 10 & 288.000 & $288.00 \odot$ & 1.0000 & $\odot .1448 \mathrm{E}+08$ \\
\hline 11 & $288.00 \odot$ & $288.0 \odot \odot$ & 1.0000 & $\odot .1448 \mathrm{E}+\odot 8$ \\
\hline 12 & 288.000 & 288.000 & 1.0000 & $\odot .1448 \mathrm{E}+08$ \\
\hline 13 & 288.000 & 288.000 & 1.0000 & $\odot .1448 \mathrm{E}+\odot 8$ \\
\hline 14 & $288.00 \odot$ & $288.00 \odot$ & 1.0000 & $0.1448 \mathrm{E}+08$ \\
\hline 15 & $288.0 \odot \odot$ & $288.00 \odot$ & $1.000 \odot$ & $\odot .1448 \mathrm{E}+\odot 8$ \\
\hline 16 & $288.0 \odot \odot$ & $288.00 \odot$ & 1.0000 & $\odot .1448 \mathrm{E}+08$ \\
\hline 17 & $288.00 \odot$ & $288.00 \odot$ & 1.0000 & $0.1448 \mathrm{E}+08$ \\
\hline 18 & 288.000 & 288.000 & 1.0000 & $\odot .1448 \mathrm{E}+08$ \\
\hline 19 & 288.000 & 288.000 & 1.0000 & $\odot .1448 \mathrm{E}+08$ \\
\hline 20 & 288.000 & 288.000 & 1.0000 & $\odot .1448 \mathrm{E}+\odot 8$ \\
\hline
\end{tabular}

\begin{tabular}{|c|c|c|c|c|}
\hline \multicolumn{5}{|c|}{$.00 \quad$ lambda $=0.2556441426$} \\
\hline node & $\mathrm{T}(\mathrm{K})$ & Twall(K) & X & $\mathrm{P}(\mathrm{Pa})$ \\
\hline 1 & 151.747 & 186.308 & $\ldots$ & $\odot .1568 \mathrm{E}+07$ \\
\hline 2 & 176.026 & 229.633 & $\odot .7893$ & $\odot .2893 \mathrm{E}+\odot 7$ \\
\hline 3 & 226.345 & 266.167 & 1.0000 & $\odot .9503 \mathrm{E}+07$ \\
\hline 4 & 248.141 & 281.831 & 1.0000 & $\odot .1109 \mathrm{E}+\odot 8$ \\
\hline 5 & 274.624 & 287.408 & 1.0000 & $\odot .1289 \mathrm{E}+\odot 8$ \\
\hline 6 & 286.111 & $288.00 \odot$ & 1.0000 & $\odot .1407 \mathrm{E}+08$ \\
\hline 7 & $288.0 \odot \odot$ & $288 . \odot \odot \odot$ & 1.0000 & $\odot .1443 E+\odot 8$ \\
\hline 8 & $288.00 \odot$ & $288 . \odot \odot \odot$ & 1.0000 & $\odot .1448 \mathrm{E}+08$ \\
\hline 9 & $288 . \odot \odot \odot$ & $288 . \odot \odot \odot$ & 1.0000 & $\odot .1448 \mathrm{E}+\odot 8$ \\
\hline 10 & $288.00 \odot$ & $288 . \odot \odot \odot$ & 1.0000 & $\odot .1448 \mathrm{E}+\odot 8$ \\
\hline 11 & $288.00 \odot$ & $288 . \odot \odot \odot$ & 1.0000 & $\odot .1448 \mathrm{E}+\odot 8$ \\
\hline 12 & 288.000 & $288.00 \odot$ & 1.0000 & $\odot .1448 \mathrm{E}+08$ \\
\hline 13 & 288.000 & 288.000 & 1.0000 & $\odot .1448 \mathrm{E}+08$ \\
\hline 14 & 288.000 & $288.00 \odot$ & 1.0000 & $\odot .1448 \mathrm{E}+\odot 8$ \\
\hline 15 & $288.00 \odot$ & $288 . \odot \odot \odot$ & 1.0000 & $\odot .1448 E+\odot 8$ \\
\hline 16 & $288.00 \odot$ & $288 . \odot \odot \odot$ & 1.0000 & $\odot .1448 \mathrm{E}+\odot 8$ \\
\hline 17 & $288.00 \odot$ & $288.0 \odot \odot$ & 1.0000 & $\odot .1448 \mathrm{E}+\odot 8$ \\
\hline 18 & 288.000 & $288.00 \odot$ & 1.0000 & $\odot .1448 \mathrm{E}+\odot 8$ \\
\hline 19 & 288.000 & $288.00 \odot$ & 1.0000 & $0.1448 E+08$ \\
\hline 20 & 288.000 & $288.0 \odot \odot$ & 1.0000 & $\odot .1448 \mathrm{E}+\odot 8$ \\
\hline
\end{tabular}

\begin{tabular}{|c|c|c|c|c|}
\hline \multicolumn{5}{|c|}{ lambda $=0.2440384477$} \\
\hline node & $\mathrm{T}(\mathrm{K})$ & Twall(K) & $\mathrm{x}$ & $\mathrm{P}(\mathrm{Pa})$ \\
\hline 1 & 150.328 & 183.105 & - - - _ & $\odot .1490 \mathrm{E}+\odot 7$ \\
\hline 2 & 174.380 & 225.877 & 0.8023 & $\odot .2748 \mathrm{E}+\odot 7$ \\
\hline 3 & 227.110 & 263.277 & 1.0000 & $\odot .9492 \mathrm{E}+07$ \\
\hline 4 & 243.187 & 279.311 & 1.0000 & $\odot .1086 \mathrm{E}+08$ \\
\hline 5 & 268.916 & 286.704 & $1.000 \odot$ & $\odot .1250 E+\odot 8$ \\
\hline 6 & 284.342 & 288.000 & 1.0000 & $0.1384 \mathrm{E}+08$ \\
\hline 7 & 287.810 & 288.000 & 1.0000 & $\odot .1438 \mathrm{E}+\odot 8$ \\
\hline 8 & 288.000 & 288.000 & 1.0000 & $\odot .1447 \mathrm{E}+08$ \\
\hline 9 & 288.000 & 288.000 & 1.0000 & $\odot .1448 \mathrm{E}+\odot 8$ \\
\hline 10 & 288.000 & 288.000 & 1.0000 & $\odot .1448 \mathrm{E}+08$ \\
\hline 11 & 288.000 & $288.00 \odot$ & 1.0000 & $\odot .1448 \mathrm{E}+08$ \\
\hline 12 & 288.000 & 288.000 & 1.0000 & $\odot .1448 \mathrm{E}+08$ \\
\hline 13 & 288.000 & 288.000 & 1.0000 & $\odot .1448 \mathrm{E}+08$ \\
\hline 14 & 288.000 & $288.00 \odot$ & 1.0000 & $0.1448 \mathrm{E}+08$ \\
\hline 15 & 288.000 & 288.000 & $1.0 \odot \odot \odot$ & $\odot .1448 E+\odot 8$ \\
\hline
\end{tabular}




\begin{tabular}{|c|c|c|c|c|}
\hline 16 & 288.000 & $288.0 \odot \odot$ & 1.0000 & $\odot .1448 E+\odot 8$ \\
\hline 17 & 288.000 & 288.000 & 1.0000 & $0.1448 \mathrm{E}+08$ \\
\hline 18 & 288.000 & $288 . \odot \odot \odot$ & 1.0000 & $0.1448 \mathrm{E}+08$ \\
\hline 19 & $288.00 \odot$ & $288.00 \odot$ & $1.000 \odot$ & $0.1448 \mathrm{E}+08$ \\
\hline 20 & 288.000 & 288.000 & 1.0000 & $0.1448 \mathrm{E}+08$ \\
\hline time & $(\sec )=$ & $16.00 \quad$ lambda $=0.2338320762$ & \multicolumn{2}{|c|}{ lambda $=0.2338320762$} \\
\hline node & $\mathrm{T}(\mathrm{K})$ & Twall(K) & $\mathrm{x}$ & $P(P a)$ \\
\hline 1 & 149.090 & \multirow{2}{*}{180.218} & $-\ldots$ & $\odot .1422 \mathrm{E}+07$ \\
\hline 2 & 172.944 & & 0.8162 & $0.2623 \mathrm{E}+07$ \\
\hline 3 & 228.393 & 260.799 & $1.000 \odot$ & $0.9488 \mathrm{E}+07$ \\
\hline 4 & 240.073 & 276.565 & 1.0000 & $0.1072 \mathrm{E}+08$ \\
\hline 5 & 262.812 & 285.625 & $1.000 \odot$ & $0.1212 \mathrm{E}+08$ \\
\hline 6 & 281.663 & 287.974 & 1.0000 & $0.1357 \mathrm{E}+08$ \\
\hline 7 & 287.425 & 288.000 & 1.0000 & $0.1430 E+08$ \\
\hline 8 & 288 & 288.000 & 1.0000 & $6 E+08$ \\
\hline 9 & $288.00 \odot$ & $288.0 \odot \odot$ & $1.00 \odot \odot$ & $\odot .1448 E+\odot 8$ \\
\hline 10 & 288.000 & 288.000 & 1.0000 & $0.1448 E+08$ \\
\hline 11 & $288.00 \odot$ & $288.00 \odot$ & $1.000 \odot$ & $0.1448 \mathrm{E}+08$ \\
\hline 12 & 288.000 & 288.000 & $1.00 \odot \odot$ & $\odot .1448 \mathrm{E}+08$ \\
\hline 13 & 288.000 & 288.000 & 1.0000 & $0.1448 \mathrm{E}+08$ \\
\hline 14 & 288 & \multirow{2}{*}{$\begin{array}{l}288.000 \\
288.000\end{array}$} & $\bullet \odot \odot$ & $8 \mathrm{E}+08$ \\
\hline 15 & 288.000 & & $1.000 \odot$ & $\odot .1448 E+08$ \\
\hline 16 & 288.000 & 288.000 & 1.0000 & $0.1448 \mathrm{E}+08$ \\
\hline 17 & 288.000 & 288.000 & 1.0000 & $0.1448 E+08$ \\
\hline 18 & $288.00 \odot$ & 288.000 & $1.000 \odot$ & $0.1448 \mathrm{E}+08$ \\
\hline 19 & 288 & $288 . \odot \odot \odot$ & 1.0000 & $8 \mathrm{E}+08$ \\
\hline 20 & $288.0 \odot \odot$ & $288.00 \odot$ & $1.000 \odot$ & $\odot .1448 \mathrm{E}+08$ \\
\hline time & ec) $=$ & $17.0 \odot$ & $a=0$. & 48311490 \\
\hline node & $\mathrm{T}(\mathrm{K})$ & Twall(K) & $x$ & $P(P a)$ \\
\hline 1 & 148.007 & 177.570 & - - - - & $0.1362 \mathrm{E}+07$ \\
\hline 2 & 171 & .905 & $\odot .8291$ & $3 E+\odot 7$ \\
\hline 3 & 229 & 258.711 & 1.0000 & $\odot .9474 \mathrm{E}+07$ \\
\hline 4 & 238.689 & 274.010 & 1.0000 & $0.1066 \mathrm{E}+08$ \\
\hline 5 & 256.978 & 284.162 & $1.00 \odot \odot$ & $0.1180 \mathrm{E}+\odot 8$ \\
\hline 6 & 277.924 & 287.669 & 1.0000 & $0.1325 \mathrm{E}+08$ \\
\hline 7 & 286.672 & $288.00 \odot$ & $1.000 \odot$ & $0.1417 \mathrm{E}+08$ \\
\hline 8 & $28 \varepsilon$ & 288 & & $E+\odot 8$ \\
\hline 9 & 288 & 288.000 & 1.0000 & $8 \mathrm{E}+\odot 8$ \\
\hline 10 & 288.000 & 288.000 & 1.0000 & $0.1448 E+08$ \\
\hline 11 & $288.00 \odot$ & $288.00 \odot$ & $1.000 \odot$ & $0.1448 \mathrm{E}+08$ \\
\hline 12 & 288.000 & 288.000 & 1.0000 & $\odot .1448 \mathrm{E}+08$ \\
\hline 13 & $288.0 \odot \odot$ & $288.0 \odot \odot$ & $1.000 \odot$ & $0.1448 \mathrm{E}+08$ \\
\hline 14 & & 288 & & $8 \mathrm{E}+\odot 8$ \\
\hline 15 & 288 & $288 . \odot \odot \odot$ & $1.000 \odot$ & $3 E+\odot 8$ \\
\hline 16 & 288.000 & 288.000 & 1.0000 & $0.1448 \mathrm{E}+08$ \\
\hline 17 & 288.000 & 288.000 & 1.0000 & $0.1448 E+08$ \\
\hline 18 & 288.000 & 288.000 & 1.0000 & $\odot .1448 \mathrm{E}+\odot 8$ \\
\hline 19 & 288.000 & 288.000 & 1.0000 & $\odot .1448 \mathrm{E}+08$ \\
\hline 20 & 288.000 & $288.0 \odot \odot$ & 1.0000 & $\odot .1448 E+\odot 8$ \\
\hline time & $(\sec )=$ & $18.00 \quad 1$ & $a=0$. & 68445587 \\
\hline node & $\mathrm{T}(\mathrm{K})$ & Twall(K) & $\mathrm{X}$ & $\mathrm{P}(\mathrm{Pa})$ \\
\hline 1 & 147.052 & 175.151 & - . - - & $0.1310 \mathrm{E}+07$ \\
\hline 2 & 170.580 & 215.729 & 0.8411 & $\odot .2415 \mathrm{E}+07$ \\
\hline 3 & 230.718 & 256.880 & 1.0000 & $\odot .9436 \mathrm{E}+\odot 7$ \\
\hline
\end{tabular}




\begin{tabular}{|c|c|c|c|c|}
\hline 4 & 238.701 & 271.669 & 1.0000 & $0.1066 \mathrm{E}+08$ \\
\hline 5 & 252.070 & 282.336 & $1.000 \odot$ & $\odot .1156 \mathrm{E}+\odot 8$ \\
\hline 6 & 273.267 & 287.222 & 1.0000 & $\odot .1290 \mathrm{E}+\odot 8$ \\
\hline 7 & 285.363 & 288.000 & 1.0000 & $\odot .1400 \mathrm{E}+08$ \\
\hline 8 & 287.953 & 288.000 & 1.0000 & $\odot .1441 \mathrm{E}+08$ \\
\hline 9 & $288.00 \odot$ & $288.00 \odot$ & 1.0000 & $\odot .1448 \mathrm{E}+08$ \\
\hline 10 & 288.000 & 288.000 & 1.0000 & $\odot .1448 \mathrm{E}+08$ \\
\hline 11 & $288.00 \odot$ & 288.000 & 1.0000 & $\odot .1448 \mathrm{E}+08$ \\
\hline 12 & $288.00 \odot$ & 288.000 & 1.0000 & $\odot .1448 \mathrm{E}+\odot 8$ \\
\hline 13 & $288.00 \odot$ & $288.00 \odot$ & 1.0000 & $0.1448 \mathrm{E}+08$ \\
\hline 14 & $288.00 \odot$ & 288.000 & 1.0000 & $\odot .1448 \mathrm{E}+08$ \\
\hline 15 & $288 . \odot \odot \odot$ & $288.00 \odot$ & 1.0000 & $\odot .1448 \mathrm{E}+08$ \\
\hline 16 & $288.0 \odot \odot$ & $288.00 \odot$ & 1.0000 & $\odot .1448 \mathrm{E}+\odot 8$ \\
\hline 17 & $288.00 \odot$ & 288.000 & 1.0000 & $\odot .1448 \mathrm{E}+08$ \\
\hline 18 & 288.000 & 288.000 & 1.0000 & $\odot .1448 \mathrm{E}+08$ \\
\hline 19 & $288.00 \odot$ & 288.000 & 1.0000 & $\odot .1448 \mathrm{E}+\odot 8$ \\
\hline 20 & $288 . \odot 0 \odot$ & $288 . \odot \odot \odot$ & $1.000 \odot$ & $\odot .1448 \mathrm{E}+\odot 8$ \\
\hline
\end{tabular}

\begin{tabular}{|c|c|c|c|c|}
\hline $\begin{array}{l}\text { time } \\
\text { node }\end{array}$ & $\begin{array}{r}(\mathrm{sec})= \\
T(K)\end{array}$ & $\begin{array}{l}.0 \odot \quad l \\
\text { Twall(K) }\end{array}$ & $a=0$ & $\begin{array}{l}3489 \\
P(P a)\end{array}$ \\
\hline 1 & 146.201 & 172.909 & $\ldots$ & $\odot .1263 \mathrm{E}+07$ \\
\hline 2 & 169.593 & 212.745 & 0.8522 & $\odot .2329 \mathrm{E}+07$ \\
\hline 3 & 231.051 & 255.314 & $1.000 \odot$ & $\odot .9370 \mathrm{E}+\odot 7$ \\
\hline 4 & 239.527 & 269.642 & 1.0000 & $\odot .1070 \mathrm{E}+\odot 8$ \\
\hline 5 & 248.677 & 280.249 & 1.0000 & $\odot .1140 \mathrm{E}+08$ \\
\hline 6 & 267.971 & 286.477 & 1.0000 & $\odot .1256 \mathrm{E}+08$ \\
\hline 7 & 283.272 & 288.000 & 1.0000 & $\odot .1377 \mathrm{E}+\odot 8$ \\
\hline 8 & 287.648 & $288.0 \odot \odot$ & $1.00 \odot \odot$ & $\odot .1435 \mathrm{E}+\odot 8$ \\
\hline 9 & 288.000 & 288.000 & 1.0000 & $\odot .1447 \mathrm{E}+08$ \\
\hline 10 & 288.000 & 288.000 & 1.0000 & $\odot .1448 \mathrm{E}+08$ \\
\hline 11 & $288.00 \odot$ & 288.000 & 1.0000 & $\odot .1448 \mathrm{E}+08$ \\
\hline 12 & 288.000 & 288.000 & 1.0000 & $\odot .1448 \mathrm{E}+08$ \\
\hline 13 & 288.000 & 288.000 & 1.0000 & $\odot .1448 \mathrm{E}+08$ \\
\hline 14 & 288.000 & $288.00 \odot$ & 1.0000 & $\odot .1448 \mathrm{E}+\odot 8$ \\
\hline 15 & $288.00 \odot$ & $288.0 \odot \odot$ & $1.00 \odot \odot$ & $\odot .1448 \mathrm{E}+\odot 8$ \\
\hline 16 & 288.000 & 288.000 & 1.0000 & $0.1448 \mathrm{E}+08$ \\
\hline 17 & $288.00 \odot$ & $288.0 \odot \odot$ & $1.000 \odot$ & $0.1448 \mathrm{E}+08$ \\
\hline 18 & $288.00 \odot$ & 288.000 & $1.000 \odot$ & $0.1448 \mathrm{E}+08$ \\
\hline 19 & 288.000 & 288.000 & 1.0000 & $0.1448 \mathrm{E}+08$ \\
\hline 20 & 288.000 & $288 . \odot \odot \odot$ & 1.0000 & $\odot .1448 E+\odot 8$ \\
\hline
\end{tabular}

\begin{tabular}{|c|c|c|c|c|}
\hline \multicolumn{5}{|c|}{ lambda $=0.2032227069$} \\
\hline node & $T(K)$ & Twall(K) & $\mathrm{x}$ & $\mathrm{P}(\mathrm{Pa})$ \\
\hline 1 & 145.437 & 170.822 & - . - & $\odot .1220 \mathrm{E}+\odot 7$ \\
\hline 2 & 168.707 & 209.938 & 0.8624 & $\odot .2251 \mathrm{E}+07$ \\
\hline 3 & 230.485 & 253.788 & 1.0000 & $\odot .9271 \mathrm{E}+\odot 7$ \\
\hline 4 & 240.224 & 267.892 & 1.0000 & $\odot .1071 \mathrm{E}+08$ \\
\hline 5 & 247.452 & 278.418 & 1.0000 & $\odot .1135 \mathrm{E}+\odot 8$ \\
\hline 6 & 262.618 & 285.393 & 1.0000 & $\odot .1227 \mathrm{E}+08$ \\
\hline 7 & 280.286 & 287.853 & 1.0000 & $\odot .1349 \mathrm{E}+08$ \\
\hline 8 & 287.113 & 288.000 & 1.0000 & $0.1425 \mathrm{E}+08$ \\
\hline 9 & 288.000 & 288.000 & 1.0000 & $\odot .1446 \mathrm{E}+08$ \\
\hline 10 & $288.00 \odot$ & $288.00 \odot$ & 1.0000 & $0.1448 \mathrm{E}+08$ \\
\hline 11 & 288.000 & 288.000 & 1.0000 & $\odot .1448 \mathrm{E}+08$ \\
\hline 12 & $288.00 \odot$ & $288 . \odot \odot \odot$ & $1.000 \odot$ & $\odot .1448 \mathrm{E}+08$ \\
\hline 13 & $288.00 \odot$ & $288.00 \odot$ & 1.0000 & $0.1448 \mathrm{E}+08$ \\
\hline 14 & 288.000 & 288.000 & 1.0000 & $\odot .1448 E+08$ \\
\hline
\end{tabular}




\begin{tabular}{|c|c|c|c|c|}
\hline 15 & 288.000 & $288 . \odot \odot \odot$ & 1.0000 & $\odot .1448 E+\odot 8$ \\
\hline 16 & $288.00 \odot$ & 288.000 & 1.0000 & $0.1448 \mathrm{E}+08$ \\
\hline 17 & $288.00 \odot$ & $288 . \odot \odot \odot$ & $1.000 \odot$ & $\odot .1448 \mathrm{E}+\odot 8$ \\
\hline 8 & $288.00 \odot$ & $288.0 \odot \odot$ & $1.000 \odot$ & $\odot .1448 \mathrm{E}+08$ \\
\hline 19 & 288.000 & 288.000 & 1.0000 & $\odot .1448 \mathrm{E}+08$ \\
\hline 20 & $288.0 \odot \odot$ & 288.000 & 1.0000 & $\odot .1448 E+08$ \\
\hline time & $(\sec )=$ & lambda $=0.1973002851$ & \multicolumn{2}{|c|}{ lambda $=0.1973002851$} \\
\hline node & $T(K)$ & \multirow{2}{*}{$\begin{array}{r}\text { Twall (K) } \\
168.871\end{array}$} & $\mathrm{x}$ & $P(P a)$ \\
\hline 1 & 144.740 & & - - - - - & $\odot .1182 \mathrm{E}+07$ \\
\hline 2 & 167.898 & 207.298 & $\odot .8718$ & $\odot .2180 \mathrm{E}+07$ \\
\hline 3 & 229.044 & 252.262 & 1.0000 & $\odot .9147 \mathrm{E}+07$ \\
\hline 4 & 240.834 & 266.366 & $1.000 \odot$ & $\odot .1070 \mathrm{E}+\odot 8$ \\
\hline 5 & 247.740 & $\begin{array}{l}276.657 \\
283.987\end{array}$ & $1.000 \odot$ & $\odot .1136 \mathrm{E}+\odot 8$ \\
\hline 6 & 257.910 & \multirow{2}{*}{$\begin{array}{l}283.987 \\
287.548\end{array}$} & 1.0000 & $\odot .1205 E+\odot 8$ \\
\hline 7 & 276.376 & & 1.0000 & $0.1319 \mathrm{E}+08$ \\
\hline 8 & 286.114 & $288.0 \odot \odot$ & $1.000 \odot$ & $\odot .1411 \mathrm{E}+08$ \\
\hline 9 & $288 . \odot \odot \odot$ & 288.000 & $1.0 \odot \odot \odot$ & $\odot .1443 E+\odot 8$ \\
\hline 10 & 288.000 & 288.000 & 1.0000 & $\odot .1448 \mathrm{E}+\odot 8$ \\
\hline 11 & $288.00 \odot$ & 288.000 & $1.000 \odot$ & $\odot .1448 \mathrm{E}+\odot 8$ \\
\hline 12 & $288 . \odot \odot \odot$ & $288.0 \odot \odot$ & 1. $0 \odot \odot \odot$ & $\odot .1448 E+\odot 8$ \\
\hline 13 & $288 .(-1)(x)$ & $288 . \odot \odot \odot$ & 1. $\odot \odot \odot \odot$ & $\odot .1448 \mathrm{E}+\odot 8$ \\
\hline 14 & $288 . \odot \odot \odot$ & $288.00 \odot$ & $1.000 \odot$ & $\odot .1448 \mathrm{E}+08$ \\
\hline 15 & $288 . \odot \odot \odot$ & $288 . \odot \odot \odot$ & $1.00 \odot \odot$ & $\odot .1448 \mathrm{E}+\odot 8$ \\
\hline 16 & $288 . \odot \odot \odot$ & 288.000 & 1.0000 & $\odot .1448 \mathrm{E}+08$ \\
\hline 17 & $288.00 \odot$ & $288 . \odot \odot \odot$ & 1.0000 & $8 \mathrm{E}+08$ \\
\hline 18 & 288. & \multirow{2}{*}{$\begin{array}{l}288.00 \odot \\
288.00 \odot\end{array}$} & $1.00 \odot \odot$ & $8 \mathrm{E}+08$ \\
\hline 19 & $288.0 \odot \odot$ & & 1.0000 & $\odot .1448 \mathrm{E}+08$ \\
\hline 20 & $288 . \odot \odot \odot$ & 288.000 & 1. $\odot \odot \odot \odot$ & $\odot .1448 \mathrm{E}+\odot 8$ \\
\hline time & ec) $=$ & 22.00 & $a=0$. & 44822 \\
\hline node & $\mathrm{T}(\mathrm{K})$ & Twall(K) & $\mathrm{x}$ & $P(P a)$ \\
\hline 1 & 144.097 & 167.040 & - - - - - & $\odot .1147 \mathrm{E}+07$ \\
\hline 2 & 167.153 & 204.813 & $\odot .8804$ & $\odot .2115 \mathrm{E}+07$ \\
\hline 3 & 226.929 & 250.734 & $1.00 \odot \odot$ & $\odot .9 \odot \odot 7 \mathrm{E}+\odot 7$ \\
\hline 4 & 241.387 & 265.051 & $1.000 \odot$ & $\odot .1067 \mathrm{E}+\odot 8$ \\
\hline 5 & 248.194 & 275.131 & $1.00 \odot \odot$ & $\odot .1138 \mathrm{E}+\odot 8$ \\
\hline 6 & 254. & 282.2 & $1.00 \odot \odot$ & $1 E+08$ \\
\hline 7 & 271 & $287 .(1-x)$ & $1.00 \odot \odot$ & $9 E+\odot 8$ \\
\hline 8 & 284.511 & $288 . \odot \odot \odot$ & $1.000 \odot$ & $\odot .1392 \mathrm{E}+\odot 8$ \\
\hline 9 & 287.8 & $288 . \odot \odot \odot$ & $1.0 \odot \odot \odot$ & $\odot .1438 \mathrm{E}+\odot 8$ \\
\hline 10 & $288.00 \odot$ & $288.00 \odot$ & 1.0000 & $\odot .1447 \mathrm{E}+\odot 8$ \\
\hline 11 & $288.0 \odot \odot$ & $288 . \odot \odot \odot$ & $1.00 \odot \odot$ & $\odot .1448 \mathrm{E}+\odot 8$ \\
\hline 12 & 288 & 288. & 1.06 & $8 \mathrm{E}+08$ \\
\hline 13 & 288 & 288. & 1. $00 \odot \odot$ & $8 \mathrm{E}+08$ \\
\hline 14 & 288. & $288 . \odot \odot \odot$ & $1.00 \odot \odot$ & $\odot .1448 \mathrm{E}+\odot 8$ \\
\hline 15 & $288.0 \odot \odot$ & $288 . \odot \odot \odot$ & $1.000 \odot$ & $\odot .1448 \mathrm{E}+\odot 8$ \\
\hline 16 & $288.0 \odot \odot$ & 288.000 & 1.0000 & $\odot .1448 \mathrm{E}+08$ \\
\hline 17 & $288.0 \odot \odot$ & $288.00 \odot$ & 1.0000 & $\odot .1448 \mathrm{E}+\odot 8$ \\
\hline 18 & $288 . C$ & $288 . \odot$ & $1.000 \odot$ & $\odot .1448 \mathrm{E}+\odot 8$ \\
\hline 19 & 288.000 & 288.000 & 1.0000 & $\odot .1448 \mathrm{E}+08$ \\
\hline 20 & $288.0 \odot \odot$ & 288.000 & $1.000 \odot$ & $\odot .1448 E+\odot 8$ \\
\hline time & $(\sec )=$ & 23.00 & $a=0$. & 67003143 \\
\hline node & $T(K)$ & Twall(K) & $x$ & $P(P a)$ \\
\hline 1 & 143.501 & 165.357 & & $\odot .1114 \mathrm{E}+07$ \\
\hline 2 & 166.461 & 202.470 & $\odot .8882$ & $\odot .2054 \mathrm{E}+\odot 7$ \\
\hline
\end{tabular}




\begin{tabular}{|c|c|c|c|c|}
\hline 3 & 224.343 & 249.055 & 1.0000 & $\odot .8862 \mathrm{E}+07$ \\
\hline 4 & 241.588 & 263.830 & $1.000 \odot$ & $\odot .1062 \mathrm{E}+\odot 8$ \\
\hline 5 & 248.499 & 273.750 & 1.0000 & $\odot .1139 \mathrm{E}+08$ \\
\hline 6 & 253.894 & 280.935 & 1.0000 & $\odot .1186 \mathrm{E}+08$ \\
\hline 7 & 266.873 & 286.198 & 1.0000 & $\odot .1262 \mathrm{E}+\odot 8$ \\
\hline 8 & 282.093 & $288.00 \odot$ & 1.0000 & $0.1368 \mathrm{E}+08$ \\
\hline 9 & 287.425 & 288.000 & 1.0000 & $\odot .1431 \mathrm{E}+08$ \\
\hline 10 & 288.000 & 288.000 & 1.0000 & $\odot .1446 \mathrm{E}+08$ \\
\hline 11 & $288.00 \odot$ & 288.000 & 1.0000 & $\odot .1448 \mathrm{E}+\odot 8$ \\
\hline 12 & $288.00 \odot$ & $288.00 \odot$ & 1.0000 & $0.1448 \mathrm{E}+08$ \\
\hline 13 & $288 . \odot \odot \odot$ & $288.00 \odot$ & $1.000 \odot$ & $\odot .1448 \mathrm{E}+\odot 8$ \\
\hline 14 & $288 . \odot \odot \odot$ & $288.00 \odot$ & 1.0000 & $\odot .1448 \mathrm{E}+08$ \\
\hline 15 & $288.00 \odot$ & $288.00 \odot$ & 1.0000 & $0.1448 \mathrm{E}+08$ \\
\hline 16 & $288.00 \odot$ & 288.000 & 1.0000 & $\odot .1448 \mathrm{E}+08$ \\
\hline 17 & 288.000 & 288.000 & 1.0000 & $\odot .1448 \mathrm{E}+08$ \\
\hline 18 & 288.000 & 288.000 & 1.0000 & $\odot .1448 \mathrm{E}+\odot 8$ \\
\hline 19 & $288 . \odot 0 \odot$ & $288.00 \odot$ & 1.0000 & $\odot .1448 \mathrm{E}+08$ \\
\hline 20 & 288.000 & $288.0 \odot \odot$ & 1.0000 & $\odot .1448 \mathrm{E}+08$ \\
\hline
\end{tabular}

\begin{tabular}{|c|c|c|c|c|}
\hline \multicolumn{5}{|c|}{ lambda $=0.1819036007$} \\
\hline node & $T(K)$ & Twall(K) & $\mathrm{x}$ & $\mathrm{P}(\mathrm{Pa})$ \\
\hline 1 & 142.931 & 163.762 & 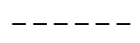 & $0.1083 \mathrm{E}+07$ \\
\hline 2 & 165.800 & 200.249 & $\odot .8950$ & $\odot .1998 \mathrm{E}+07$ \\
\hline 3 & 221.511 & 247.289 & 1.0000 & $\odot .8720 \mathrm{E}+\odot 7$ \\
\hline 4 & 241.320 & 262.609 & 1.0000 & $\odot .1054 \mathrm{E}+08$ \\
\hline 5 & 248.804 & 272.529 & 1.0000 & $\odot .1139 \mathrm{E}+\odot 8$ \\
\hline 6 & 254.106 & 279.629 & 1.0000 & $\odot .1187 \mathrm{E}+\odot 8$ \\
\hline 7 & 262.460 & 285.099 & 1.0000 & $\odot .1241 \mathrm{E}+08$ \\
\hline 8 & 278.806 & 287.727 & 1.0000 & $0.1341 \mathrm{E}+08$ \\
\hline 9 & 286.693 & $288.00 \odot$ & 1.0000 & $\odot .1419 \mathrm{E}+\odot 8$ \\
\hline 10 & $288.00 \odot$ & 288.000 & 1.0000 & $\odot .1444 \mathrm{E}+\odot 8$ \\
\hline 11 & 288.000 & 288.000 & 1.0000 & $\odot .1448 \mathrm{E}+08$ \\
\hline 12 & 288.000 & 288.000 & 1.0000 & $\odot .1448 \mathrm{E}+\odot 8$ \\
\hline 13 & 288.000 & 288.000 & 1.0000 & $0.1448 \mathrm{E}+08$ \\
\hline 14 & 288.000 & 288.000 & 1.0000 & $\odot .1448 \mathrm{E}+\odot 8$ \\
\hline 15 & 288.000 & 288.000 & 1.0000 & $0.1448 \mathrm{E}+08$ \\
\hline 16 & 288.000 & 288.000 & 1.0000 & $\odot .1448 \mathrm{E}+08$ \\
\hline 17 & 288.000 & 288.000 & 1.0000 & $0.1448 \mathrm{E}+08$ \\
\hline 18 & 288.000 & 288.000 & 1.0000 & $\odot .1448 \mathrm{E}+08$ \\
\hline 19 & 288.000 & 288.000 & 1.0000 & $\odot .1448 \mathrm{E}+\odot 8$ \\
\hline 20 & $288 . \odot \odot \odot$ & $288 . \odot \odot \odot$ & 1. $0 \odot \odot \odot$ & $\odot .1448 \mathrm{E}+\odot 8$ \\
\hline
\end{tabular}

\begin{tabular}{|c|c|c|c|c|}
\hline \multicolumn{5}{|c|}{ lambda $=0.1772223115$} \\
\hline node & $\mathrm{T}(\mathrm{K})$ & Twall(K) & $\mathrm{x}$ & $\mathrm{P}(\mathrm{Pa})$ \\
\hline 1 & 142.109 & 162.236 & $-\ldots$ & $\odot .1052 \mathrm{E}+07$ \\
\hline 2 & 164.847 & 198.112 & 0.9017 & $\odot .1941 \mathrm{E}+07$ \\
\hline 3 & 218.676 & 245.455 & 1.0000 & $\odot .8588 \mathrm{E}+\odot 7$ \\
\hline 4 & 240.589 & 261.389 & $1.00 \odot \odot$ & $\odot .1043 \mathrm{E}+08$ \\
\hline 5 & 249.098 & 271.308 & $1.000 \odot$ & $\odot .1139 \mathrm{E}+\odot 8$ \\
\hline 6 & 254.399 & 278.409 & $1.000 \odot$ & $\odot .1188 \mathrm{E}+08$ \\
\hline 7 & 259.720 & 283.878 & 1.0000 & $\odot .1228 \mathrm{E}+08$ \\
\hline 8 & 274.757 & 287.388 & $1.000 \odot$ & $\odot .1314 \mathrm{E}+08$ \\
\hline 9 & 285.454 & $288.0 \odot \odot$ & $1.000 \odot$ & $\odot .1403 \mathrm{E}+\odot 8$ \\
\hline 10 & 287.960 & $288.0 \odot \odot$ & $1.000 \odot$ & $\odot .1441 \mathrm{E}+\odot 8$ \\
\hline 11 & 288.000 & $288.00 \odot$ & 1.0000 & $\odot .1448 \mathrm{E}+\odot 8$ \\
\hline 12 & $288.00 \odot$ & 288.000 & $1.000 \odot$ & $\odot .1448 \mathrm{E}+\odot 8$ \\
\hline 13 & 288.000 & 288.000 & 1.0000 & $\odot .1448 E+08$ \\
\hline
\end{tabular}




\begin{tabular}{|c|c|c|c|c|}
\hline 14 & $288.00 \odot$ & 288.000 & 1.0000 & $0.1448 \mathrm{E}+08$ \\
\hline 15 & $288 . \odot \odot \odot$ & 288.000 & 1.0000 & $\odot .1448 \mathrm{E}+\odot 8$ \\
\hline 16 & $288.00 \odot$ & $288.00 \odot$ & 1.0000 & $\odot .1448 \mathrm{E}+\odot 8$ \\
\hline 17 & $288.00 \odot$ & 288.000 & 1.0000 & $\odot .1448 \mathrm{E}+08$ \\
\hline 18 & 288.000 & 288.000 & 1.0000 & $\odot .1448 \mathrm{E}+\odot 8$ \\
\hline 19 & $288 . \odot \odot \odot$ & 288.000 & $1.000 \odot$ & $\odot .1448 \mathrm{E}+08$ \\
\hline 20 & $288 . \odot \odot \odot$ & 288.000 & 1.0000 & $\odot .1448 E+08$ \\
\hline time & & lambda $=0.1728569865$ & \multicolumn{2}{|c|}{ lambda $=0.1728569865$} \\
\hline node & $\mathrm{T}(\mathrm{K})$ & \multirow{2}{*}{ Twall(K) } & & $\mathrm{P}(\mathrm{Pa})$ \\
\hline 1 & 141.346 & & & $0.1023 \mathrm{E}+07$ \\
\hline 2 & 163.962 & 196.089 & 0.9078 & $\odot .1888 \mathrm{E}+07$ \\
\hline 3 & 216.056 & 243.471 & 1.0000 & $\odot .8474 \mathrm{E}+07$ \\
\hline 4 & 239.426 & 260.168 & 1.0000 & $\odot .1031 \mathrm{E}+08$ \\
\hline 5 & 249.179 & 270.377 & 1.0000 & $0.1136 \mathrm{E}+08$ \\
\hline 6 & 254.552 & 277.232 & 1.0000 & $0.1189 \mathrm{E}+08$ \\
\hline 7 & 258.703 & 282.657 & 1.0000 & $\odot .1224 \mathrm{E}+\odot 8$ \\
\hline 8 & 270 & 286.778 & 1.0000 & $\odot .1290 \mathrm{E}+08$ \\
\hline 9 & 283.531 & $288 . \odot \odot \odot$ & 1.0000 & $0.1383 \mathrm{E}+08$ \\
\hline 10 & 287.655 & 288.000 & 1.0000 & $\odot .1435 E+08$ \\
\hline 11 & 288.000 & 288.000 & 1.0000 & $\odot .1447 \mathrm{E}+08$ \\
\hline 12 & 288.000 & 288.000 & 1.0000 & $0.1448 \mathrm{E}+08$ \\
\hline 13 & 288. & $288.00 \odot$ & $1 .($ & $0.1448 \mathrm{E}+08$ \\
\hline 14 & 288. & $288.00 \odot$ & $1.000 \odot$ & $\odot .1448 \mathrm{E}+\odot 8$ \\
\hline 15 & $288.00 \odot$ & $288 . \odot \odot \odot$ & 1.0000 & $0.1448 \mathrm{E}+08$ \\
\hline 16 & 288.000 & 288.000 & 1.0000 & $\odot .1448 \mathrm{E}+08$ \\
\hline 17 & $288.00 \odot$ & 288.000 & $1.000 \odot$ & $0.1448 \mathrm{E}+08$ \\
\hline 18 & $288.0 \odot \odot$ & 288.000 & 1.6 & $\odot .1448 \mathrm{E}+08$ \\
\hline 19 & 288. & 288.000 & 1.6 & $18 \mathrm{E}+08$ \\
\hline 20 & 288.000 & 288.000 & 1.0000 & $\odot .1448 E+08$ \\
\hline time & $(\sec )=$ & 27.00 & $\mathrm{da}=0.1$ & 87866598 \\
\hline node & $\mathrm{T}(\mathrm{K})$ & Twall(K) & $\mathrm{x}$ & $\mathrm{P}(\mathrm{Pa})$ \\
\hline 1 & 140.639 & 159.410 & $-\ldots$ & $\odot .9968 \mathrm{E}+06$ \\
\hline 2 & 163.141 & 194.106 & 0.9133 & $\odot .1839 \mathrm{E}+07$ \\
\hline 3 & 213.794 & 241.487 & 1.0000 & $\odot .8377 \mathrm{E}+07$ \\
\hline 4 & 237.892 & 258.947 & 1.0000 & $\odot .1017 \mathrm{E}+\odot 8$ \\
\hline 5 & 248.950 & 269.462 & 1.0000 & $\odot .1131 \mathrm{E}+08$ \\
\hline 6 & $254.7 \odot 4$ & 276.316 & $1.000 \odot$ & $\odot .1189 \mathrm{E}+08$ \\
\hline 7 & 258. & 281. & & $5 E+\odot 8$ \\
\hline 8 & 266. & $285 . \varepsilon$ & 1.0 & $\odot .1270 \mathrm{E}+\odot 8$ \\
\hline 9 & 280.790 & $287.9 \odot 4$ & 1.0000 & $0.1360 \mathrm{E}+08$ \\
\hline 10 & 287.137 & 288.000 & 1.0000 & $\odot .1426 \mathrm{E}+08$ \\
\hline 11 & $288.00 \odot$ & 288.000 & 1.0000 & $0.1446 \mathrm{E}+08$ \\
\hline 12 & 288.000 & 288.000 & & $0.1448 \mathrm{E}+08$ \\
\hline 13 & 288. & 288.6 & & $18 E+08$ \\
\hline 14 & 288. & 288.0 & 1.00 & $\odot .1448 \mathrm{E}+\odot 8$ \\
\hline 15 & 288.000 & 288.000 & 1.0000 & $0.1448 \mathrm{E}+08$ \\
\hline 16 & 288.000 & 288.000 & 1.0000 & $\odot .1448 \mathrm{E}+08$ \\
\hline 17 & $288.00 \odot$ & $288 . \odot \odot \odot$ & $1.000 \odot$ & $\odot .1448 \mathrm{E}+08$ \\
\hline 18 & 288.000 & 288.000 & 1.0000 & $\odot .1448 \mathrm{E}+08$ \\
\hline 19 & $288.0 \odot \odot$ & $288.00 \odot$ & & $\odot .1448 E+08$ \\
\hline 20 & $288.00 \odot$ & $288.0 \odot \odot$ & $1.000 \odot$ & $\odot .1448 \mathrm{E}+08$ \\
\hline time & $(\sec )=$ & $8.00 \quad 1$ & -6 & 50236696 \\
\hline node & $\mathrm{T}(\mathrm{K})$ & Twall(K) & $\mathrm{X}$ & $P(P a)$ \\
\hline 1 & 139.987 & 158.048 & - & $0.9723 \mathrm{E}+\Theta$ \\
\hline
\end{tabular}




\begin{tabular}{|c|c|c|c|c|}
\hline 2 & 162.385 & 192.256 & $\odot .9184$ & $\odot .1794 \mathrm{E}+07$ \\
\hline 3 & 211.979 & 239.504 & $1.00 \odot \odot$ & $\odot .8304 \mathrm{E}+07$ \\
\hline 4 & 236.104 & 257.727 & 1.0000 & $\odot .10 \odot 4 \mathrm{E}+\odot 8$ \\
\hline 5 & 248.417 & 268.546 & 1.0000 & $\odot .1124 \mathrm{E}+08$ \\
\hline 6 & 254.857 & 275.401 & 1.0000 & $\odot .1188 \mathrm{E}+08$ \\
\hline 7 & 259.198 & 280.665 & $1.00 \odot \odot$ & $\odot .1226 \mathrm{E}+08$ \\
\hline 8 & 263.389 & 284.979 & 1.0000 & $\odot .1257 \mathrm{E}+\odot 8$ \\
\hline 9 & 277.272 & 287.598 & 1.0000 & $0.1336 \mathrm{E}+08$ \\
\hline 10 & 286.194 & $288.00 \odot$ & 1.0000 & $\odot .1413 \mathrm{E}+08$ \\
\hline 11 & $288.00 \odot$ & $288.0 \odot \odot$ & $1.00 \odot \odot$ & $0.1443 \mathrm{E}+08$ \\
\hline 12 & $288.00 \odot$ & $288.00 \odot$ & 1.0000 & $0.1448 \mathrm{E}+08$ \\
\hline 13 & 288.000 & 288.000 & 1.0000 & $0.1448 \mathrm{E}+08$ \\
\hline 14 & 288.000 & 288.000 & 1.0000 & $\odot .1448 \mathrm{E}+08$ \\
\hline 15 & $288.00 \odot$ & $288.00 \odot$ & 1.0000 & $\odot .1448 \mathrm{E}+\odot 8$ \\
\hline 16 & 288.000 & $288.00 \odot$ & 1.0000 & $\odot .1448 \mathrm{E}+08$ \\
\hline 17 & $288.0 \odot \odot$ & $288.00 \odot$ & $1.000 \odot$ & $\odot .1448 \mathrm{E}+\odot 8$ \\
\hline 18 & $288.00 \odot$ & $288 . \odot \odot \odot$ & $1.00 \odot \odot$ & $\odot .1448 \mathrm{E}+08$ \\
\hline 19 & 288.000 & $288.0 \odot \odot$ & 1.0000 & $\odot .1448 \mathrm{E}+08$ \\
\hline 20 & 288.000 & 288.000 & 1.0000 & $\odot .1448 \mathrm{E}+08$ \\
\hline time & $(\sec )=$ & \multicolumn{3}{|l|}{29.00} \\
\hline node & $\mathrm{T}(\mathrm{K})$ & Twall(K) & $\mathrm{X}$ & $P(P a)$ \\
\hline 1 & 139.388 & 156.828 & - - - - - & $\odot .9498 \mathrm{E}+06$ \\
\hline 2 & 161.690 & 190.425 & $\odot .9230$ & $\odot .1752 \mathrm{E}+07$ \\
\hline 3 & 210.633 & 237.597 & 1.0000 & $\odot .8252 \mathrm{E}+07$ \\
\hline 4 & 234.171 & 256.457 & 1.0000 & $\odot .9906 \mathrm{E}+07$ \\
\hline 5 & 247.588 & 267.631 & 1.0000 & $0.1115 \mathrm{E}+08$ \\
\hline 6 & 254.909 & 274.485 & 1.0 & $\odot .1186 \mathrm{E}+08$ \\
\hline 7 & 259.198 & 279.749 & 1.0 & $\odot .1226 \mathrm{E}+08$ \\
\hline 8 & 262.316 & 284.063 & 1.0000 & $\odot .1252 \mathrm{E}+\odot 8$ \\
\hline 9 & 273.189 & 287.156 & 1.0000 & $0.1313 \mathrm{E}+08$ \\
\hline 10 & 284.673 & $288.0 \odot \odot$ & 1.0000 & $\odot .1396 \mathrm{E}+08$ \\
\hline 11 & 287.817 & $288.0 \odot \odot$ & $1.00 \odot \odot$ & $\odot .1439 \mathrm{E}+08$ \\
\hline 12 & $288.00 \odot$ & $288.00 \odot$ & 1.0 & $7 \mathrm{E}+08$ \\
\hline 13 & 288.000 & 288.000 & 1.0 & $0.1448 \mathrm{E}+08$ \\
\hline 14 & 288.000 & 288.000 & 1.0000 & $\odot .1448 \mathrm{E}+08$ \\
\hline 15 & 288.000 & $288.00 \odot$ & 1.0000 & $\odot .1448 \mathrm{E}+\odot 8$ \\
\hline 16 & 288.000 & 288.000 & 1.0000 & $\odot .1448 \mathrm{E}+08$ \\
\hline 17 & 288.000 & 288.000 & 1.0 & $\odot .1448 \mathrm{E}+08$ \\
\hline 18 & 288.0 & 288. & 1.0 & $8 \mathrm{E}+08$ \\
\hline 19 & $288.00 \odot$ & 288.0 & 1.0000 & $\odot .1448 \mathrm{E}+08$ \\
\hline 20 & $288 . \odot \odot \odot$ & $288 . \odot \odot \odot$ & $1.0 \odot \odot \odot$ & $\odot .1448 E+\odot 8$ \\
\hline time & $(\sec )=$ & \multicolumn{3}{|l|}{$30.00 \quad 1 a$} \\
\hline node & $\mathrm{T}(\mathrm{K})$ & Twall(K) & $\mathrm{x}$ & $P(P a)$ \\
\hline 1 & 138.837 & 155.608 & - - - - - & $\odot .9291 \mathrm{E}+06$ \\
\hline 2 & 161.051 & 188.740 & $\odot .9272$ & $\odot .1714 \mathrm{E}+07$ \\
\hline 3 & 209.740 & 235.766 & 1.0000 & $0.8213 \mathrm{E}+07$ \\
\hline 4 & 232.266 & 255.038 & 1.0000 & $\odot .9786 \mathrm{E}+\odot 7$ \\
\hline 5 & 246.468 & 266.715 & 1.0000 & $\odot .1104 \mathrm{E}+08$ \\
\hline 6 & 254.695 & 273.569 & 1.0000 & $0.1182 \mathrm{E}+08$ \\
\hline 7 & 259.198 & 278.834 & $1.00 \odot \odot$ & $\odot .1225 E+08$ \\
\hline 8 & 262.478 & 283.148 & 1.0000 & $\odot .1253 \mathrm{E}+08$ \\
\hline 9 & 269.199 & 286.545 & 1.0000 & $\odot .1294 \mathrm{E}+08$ \\
\hline 10 & 282.431 & $288.0 \odot \odot$ & 1.0000 & $0.1375 \mathrm{E}+08$ \\
\hline 11 & 287.464 & $288.00 \odot$ & $1.000 \odot$ & $\odot .1432 \mathrm{E}+08$ \\
\hline 12 & $288.00 \odot$ & $288.00 \odot$ & 1.0000 & $0.1446 \mathrm{E}+08$ \\
\hline
\end{tabular}




\begin{tabular}{|c|c|c|c|c|}
\hline 13 & 288.000 & $288.00 \odot$ & 1.0000 & $\odot .1448 \mathrm{E}+\odot 8$ \\
\hline 14 & $288.00 \odot$ & $288.00 \odot$ & 1.0000 & $\odot .1448 \mathrm{E}+08$ \\
\hline 15 & $288 . \odot \odot \odot$ & 288.000 & 1.0000 & $0.1448 E+08$ \\
\hline 16 & $288.0 \odot \odot$ & $288.0 \odot \odot$ & 1.0000 & $\odot .1448 \mathrm{E}+\odot 8$ \\
\hline 17 & $288.0 \odot \odot$ & $288 . \odot \odot \odot$ & $1.00 \odot \odot$ & $\odot .1448 \mathrm{E}+08$ \\
\hline 18 & $288 . \odot \odot \odot$ & $288 . \odot \odot \odot$ & 1.0000 & $\odot .1448 \mathrm{E}+\odot 8$ \\
\hline 19 & $288 . \odot \odot \odot$ & $288.00 \odot$ & $1.000 \odot$ & $\odot .1448 \mathrm{E}+\odot 8$ \\
\hline & $288.0 \odot \odot$ & $288.00 \odot$ & $1.00 \odot \odot$ & $\odot .1448 \mathrm{E}+08$ \\
\hline
\end{tabular}

lambda_avg $=0.320041120$ 


\section{Compressor station distance $=60$ miles, Pressure $=1167$ psi, 60 axial node elements}

\begin{tabular}{|c|c|c|c|c|}
\hline \multicolumn{5}{|c|}{$.00 \quad$ lambda $=0.8512680531$} \\
\hline node & $\mathrm{T}(\mathrm{K})$ & Twall(K) & $\mathrm{X}$ & $\mathrm{P}(\mathrm{Pa})$ \\
\hline 1 & 247.398 & 281.591 & $-\ldots$ & $\odot .3705 \mathrm{E}+07$ \\
\hline 2 & 286.982 & 288.000 & 1.0000 & $\odot .6835 \mathrm{E}+\odot 7$ \\
\hline 3 & 288.000 & 288.000 & 1.0000 & $0.8033 E+07$ \\
\hline 4 & $288.00 \odot$ & $288.0 \odot \odot$ & 1.0000 & $\odot .8044 \mathrm{E}+\odot 7$ \\
\hline 5 & 288.000 & 288.000 & 1.0000 & $\odot .8044 \mathrm{E}+07$ \\
\hline 6 & 288.000 & 288.000 & 1.0000 & $\odot .8044 \mathrm{E}+\odot 7$ \\
\hline 7 & 288.000 & $288 . \odot \odot \odot$ & $1.000 \odot$ & $\odot .8044 \mathrm{E}+07$ \\
\hline 8 & $288.0 \odot \odot$ & $288 . \odot \odot \odot$ & $1.000 \odot$ & $\odot .8 \odot 44 \mathrm{E}+\odot 7$ \\
\hline 9 & 288.000 & 288.000 & 1.0000 & $\odot .8044 \mathrm{E}+\odot 7$ \\
\hline 10 & 288.000 & 288.000 & $1.000 \odot$ & $\odot .8044 \mathrm{E}+\odot 7$ \\
\hline 11 & 288.000 & 288.000 & 1.0000 & $\odot .8044 \mathrm{E}+\odot 7$ \\
\hline 12 & 288.000 & 288.000 & 1.0000 & $\odot .8044 \mathrm{E}+07$ \\
\hline 13 & 288.000 & 288.000 & 1.0000 & $\odot .8044 \mathrm{E}+\odot 7$ \\
\hline 14 & $288.00 \odot$ & $288 . \odot \odot \odot$ & $1.000 \odot$ & $\odot .8 \odot 44 \mathrm{E}+\odot 7$ \\
\hline 15 & 288.000 & 288.000 & 1.0000 & $\odot .8044 \mathrm{E}+07$ \\
\hline 16 & $288.00 \odot$ & $288.00 \odot$ & 1.0000 & $\odot .8044 \mathrm{E}+\odot 7$ \\
\hline 17 & 288.000 & 288.000 & $1.000 \odot$ & $\odot .8 \odot 44 \mathrm{E}+\odot 7$ \\
\hline 18 & 288.000 & $288.00 \odot$ & 1.0000 & $\odot .8044 \mathrm{E}+07$ \\
\hline 19 & 288.000 & 288.000 & 1.0000 & $\odot .8044 \mathrm{E}+07$ \\
\hline 20 & $288.00 \odot$ & $288.00 \odot$ & 1.0000 & $\odot .8044 \mathrm{E}+\odot 7$ \\
\hline 21 & $288.00 \odot$ & $288.0 \odot \odot$ & $1.000 \odot$ & $\odot .8044 \mathrm{E}+\odot 7$ \\
\hline 22 & 288.000 & 288.000 & 1.0000 & $\odot .8044 \mathrm{E}+\odot 7$ \\
\hline 23 & 288.000 & 288.000 & 1.0000 & $0.8044 \mathrm{E}+07$ \\
\hline 24 & 288.000 & $288.00 \odot$ & 1.0000 & $\odot .8 \odot 44 \mathrm{E}+\odot 7$ \\
\hline 25 & 288.000 & 288.000 & 1.0000 & $\odot .8044 \mathrm{E}+\odot 7$ \\
\hline 26 & 288.000 & $288.00 \odot$ & 1.0000 & $\odot .8044 \mathrm{E}+\odot 7$ \\
\hline 27 & $288.00 \odot$ & 288.000 & 1.0000 & $\odot .8044 \mathrm{E}+\odot 7$ \\
\hline 28 & 288.000 & 288.000 & 1.0000 & $\odot .8044 \mathrm{E}+07$ \\
\hline 29 & 288.000 & 288.000 & 1.0000 & $\odot .8044 \mathrm{E}+\odot 7$ \\
\hline 30 & $288.00 \odot$ & $288.00 \odot$ & 1.0000 & $\odot .8044 \mathrm{E}+\odot 7$ \\
\hline 31 & $288.0 \odot \odot$ & $288.00 \odot$ & $1.000 \odot$ & $\odot .8 \odot 44 \mathrm{E}+\odot 7$ \\
\hline 32 & 288.000 & 288.000 & 1.0000 & $\odot .8044 \mathrm{E}+\odot 7$ \\
\hline 33 & $288.00 \odot$ & $288.00 \odot$ & 1.0000 & $\odot .8044 \mathrm{E}+\odot 7$ \\
\hline 34 & 288.000 & $288.0 \odot \odot$ & $1.000 \odot$ & $\odot .8044 \mathrm{E}+\odot 7$ \\
\hline 35 & 288.000 & 288.000 & 1.0000 & $\odot .8044 \mathrm{E}+\odot 7$ \\
\hline 36 & 288.000 & 288.000 & 1.0000 & $\odot .8044 \mathrm{E}+\odot 7$ \\
\hline 37 & $288.00 \odot$ & $288.0 \odot \odot$ & $1.000 \odot$ & $\odot .8044 \mathrm{E}+\odot 7$ \\
\hline 38 & 288.000 & 288.000 & 1.0000 & $\odot .8044 \mathrm{E}+07$ \\
\hline 39 & 288.000 & 288.000 & 1.0000 & $\odot .8044 \mathrm{E}+\odot 7$ \\
\hline 40 & 288.000 & $288.00 \odot$ & 1.0000 & $\odot .8044 \mathrm{E}+\odot 7$ \\
\hline 41 & 288.000 & 288.000 & 1.0000 & $\odot .8044 \mathrm{E}+07$ \\
\hline 42 & 288.0 & 288.000 & 1.0000 & $\odot .8044 \mathrm{E}+\odot 7$ \\
\hline 43 & 288.000 & 288.000 & 1.0000 & $\odot .8044 \mathrm{E}+07$ \\
\hline 44 & 288.000 & 288.000 & 1.0000 & $\odot .8044 \mathrm{E}+07$ \\
\hline 45 & 288.000 & $288.0 \odot \odot$ & 1.0000 & $\odot .8044 \mathrm{E}+07$ \\
\hline 46 & 288.000 & 288.000 & 1.0000 & $\odot .8044 \mathrm{E}+07$ \\
\hline 47 & 288.000 & 288.000 & $1.000 \odot$ & $\odot .8044 \mathrm{E}+\odot 7$ \\
\hline 48 & 288.000 & 288.000 & 1.0000 & $\odot .8044 \mathrm{E}+07$ \\
\hline 49 & 288.000 & 288.000 & 1.0000 & $\odot .8044 \mathrm{E}+\odot 7$ \\
\hline 50 & 288.000 & 288.000 & 1.0000 & $\odot .8044 \mathrm{E}+07$ \\
\hline 51 & 288.000 & 288.000 & 1.0000 & $\odot .8044 \mathrm{E}+07$ \\
\hline 52 & 288.000 & 288.000 & 1.0000 & $\odot .8044 \mathrm{E}+07$ \\
\hline
\end{tabular}









\begin{tabular}{|c|c|c|c|c|}
\hline 47 & 288.000 & $288.00 \odot$ & 1.0000 & $\odot .8 \odot 44 \mathrm{E}+\odot 7$ \\
\hline 48 & 288.000 & 288.000 & 1.0000 & $0.8044 \mathrm{E}+07$ \\
\hline 49 & 288.000 & $288.0 \odot \odot$ & $1.000 \odot$ & $\odot .8 \odot 44 \mathrm{E}+\odot 7$ \\
\hline 50 & $288.00 \odot$ & $288.00 \odot$ & $1.00 \odot \odot$ & $\odot .8 \odot 44 \mathrm{E}+\odot 7$ \\
\hline 51 & 288.000 & 288.000 & 1.0000 & $\odot .8044 \mathrm{E}+07$ \\
\hline 52 & 288.000 & $288.0 \odot \odot$ & $1.000 \odot$ & $\odot .8044 \mathrm{E}+\odot 7$ \\
\hline 53 & $288.00 \odot$ & $288.00 \odot$ & $1.000 \odot$ & $\odot .8044 \mathrm{E}+07$ \\
\hline 54 & 288.000 & 288.000 & 1.0000 & $0.8044 \mathrm{E}+07$ \\
\hline 55 & $288.00 \odot$ & $288.00 \odot$ & $1.000 \odot$ & $\odot .8044 \mathrm{E}+07$ \\
\hline 56 & 288.000 & $288.0 \odot \odot$ & $1.000 \odot$ & $0.8044 \mathrm{E}+07$ \\
\hline 57 & $288.00 \odot$ & $288.00 \odot$ & $1.00 \odot \odot$ & $\odot .8044 \mathrm{E}+07$ \\
\hline 58 & 288.000 & $288.00 \odot$ & 1.0000 & $\odot .8044 \mathrm{E}+\odot 7$ \\
\hline 59 & 288.000 & $288.0 \odot \odot$ & $1.000 \odot$ & $0.8044 \mathrm{E}+07$ \\
\hline 60 & 288.000 & 288.000 & 1.0000 & $\odot .8044 \mathrm{E}+07$ \\
\hline & & & \multirow{2}{*}{\multicolumn{2}{|c|}{$\begin{array}{c}\text { lambda }=0.6175189018 \\
\mathrm{~K})\end{array}$}} \\
\hline nod & $\mathrm{T}^{\prime}(\mathrm{K})$ & Twall(K) & & \\
\hline 1 & 226.629 & 271.401 & $-\ldots$ & $0.2573 \mathrm{E}+07$ \\
\hline 2 & 262.888 & 287.379 & 1.0000 & $\odot .4745 \mathrm{E}+07$ \\
\hline 3 & 287.470 & $288.0 \odot \odot$ & $1.000 \odot$ & $\odot .7800 \mathrm{E}+\odot 7$ \\
\hline 4 & $288.00 \odot$ & $288.0 \odot \odot$ & $1.000 \odot$ & $\odot .8038 \mathrm{E}+07$ \\
\hline 5 & 288.000 & $288.00 \odot$ & 1.0000 & $0.8044 \mathrm{E}+07$ \\
\hline 6 & 288.000 & 288.000 & $1.000 \odot$ & $\odot .8044 \mathrm{E}+07$ \\
\hline 7 & $288.00 \odot$ & $288.00 \odot$ & $1.00 \odot \odot$ & $0.8044 \mathrm{E}+07$ \\
\hline 8 & 288.000 & 288.000 & 1.0000 & $0.8044 \mathrm{E}+07$ \\
\hline 9 & 288.000 & $288.0 \odot \odot$ & 1.0000 & $0.8044 \mathrm{E}+07$ \\
\hline 10 & $288.00 \odot$ & $288.00 \odot$ & $1.00 \odot \odot$ & $\odot .8044 \mathrm{E}+\odot 7$ \\
\hline 11 & 288.000 & 288.000 & 1.0000 & $\odot .8044 \mathrm{E}+07$ \\
\hline 12 & 288.000 & $288.0 \odot \odot$ & 1.0000 & $\odot .8044 \mathrm{E}+07$ \\
\hline 13 & $88.00 \odot$ & $288.00 \odot$ & 1.0000 & $\odot .8044 \mathrm{E}+07$ \\
\hline 14 & 288.000 & 288.000 & 1.0000 & $\odot .8044 \mathrm{E}+07$ \\
\hline 15 & 288.000 & 288.000 & 1.0000 & $0.8044 \mathrm{E}+07$ \\
\hline 16 & 288.000 & $288.0 \odot \odot$ & $1.000 \odot$ & $\odot .8044 \mathrm{E}+07$ \\
\hline 17 & 88.000 & $288.00 \odot$ & $1.0 \odot \odot \odot$ & $0.8044 \mathrm{E}+07$ \\
\hline 18 & $88.00 \odot$ & $288.00 \odot$ & $1.00 \odot \odot$ & $\odot .8 \odot 44 \mathrm{E}+07$ \\
\hline 19 & $288.00 \odot$ & $288.0 \odot \odot$ & $1.000 \odot$ & $0.8044 \mathrm{E}+07$ \\
\hline 20 & $288.00 \odot$ & $288.00 \odot$ & $1.00 \odot \odot$ & $\odot .8044 \mathrm{E}+\odot 7$ \\
\hline 21 & $288.00 \odot$ & $288.00 \odot$ & $1.00 \odot \odot$ & $\odot .8044 \mathrm{E}+\odot 7$ \\
\hline 22 & $288.00 \odot$ & $288.00 \odot$ & 1.0000 & $\odot .8044 \mathrm{E}+07$ \\
\hline 23 & $288.00 \odot$ & $288 . \odot \odot \odot$ & $1.000 \odot$ & $\odot .8044 \mathrm{E}+\odot 7$ \\
\hline 24 & $88.00 \odot$ & $288.0 \odot \odot$ & $1.0 \odot \odot \odot$ & $\odot .8044 \mathrm{E}+\odot 7$ \\
\hline 25 & 288.000 & $288.0 \odot \odot$ & 1.0000 & $\odot .8044 \mathrm{E}+07$ \\
\hline 26 & 288.000 & $288.0 \odot \odot$ & $1.00 \odot \odot$ & $\odot .8044 \mathrm{E}+07$ \\
\hline 27 & $288.00 \odot$ & $288.00 \odot$ & $1.00 \odot \odot$ & $\odot .8044 \mathrm{E}+07$ \\
\hline 28 & 88. & 288.000 & 1.0000 & $\odot .8044 \mathrm{E}+07$ \\
\hline 29 & 288.000 & $288 . \odot \odot \odot$ & $1.000 \odot$ & $0.8044 \mathrm{E}+07$ \\
\hline 30 & 88.000 & $288.0 \odot \odot$ & $1.000 \odot$ & $0.8044 \mathrm{E}+07$ \\
\hline 31 & 288.000 & 288.000 & 1.0000 & $0.8044 \mathrm{E}+07$ \\
\hline 32 & 288.000 & $288.0 \odot \odot$ & 1.0000 & $0.8044 \mathrm{E}+07$ \\
\hline 33 & 288.000 & $288 . \odot \odot \odot$ & $1.000 \odot$ & $0.8044 \mathrm{E}+07$ \\
\hline 34 & 288.000 & $288.00 \odot$ & 1.0000 & $0.8044 \mathrm{E}+07$ \\
\hline 35 & 288.000 & 288.000 & 1.0000 & $\odot .8044 \mathrm{E}+07$ \\
\hline 36 & 288.000 & $288 . \odot \odot \odot$ & $1.000 \odot$ & $\odot .8044 \mathrm{E}+\odot 7$ \\
\hline 37 & 288.000 & 288.000 & 1.0000 & $\odot .8044 \mathrm{E}+07$ \\
\hline 38 & 288.000 & $288.00 \odot$ & 1.0000 & $0.8044 \mathrm{E}+07$ \\
\hline 39 & 288.000 & $288.00 \odot$ & 1.0000 & $\odot .8044 \mathrm{E}+07$ \\
\hline $4 \odot$ & $288.00 \odot$ & $288.00 \odot$ & $1.0 \odot \odot \odot$ & $\odot .8044 \mathrm{E}+07$ \\
\hline
\end{tabular}




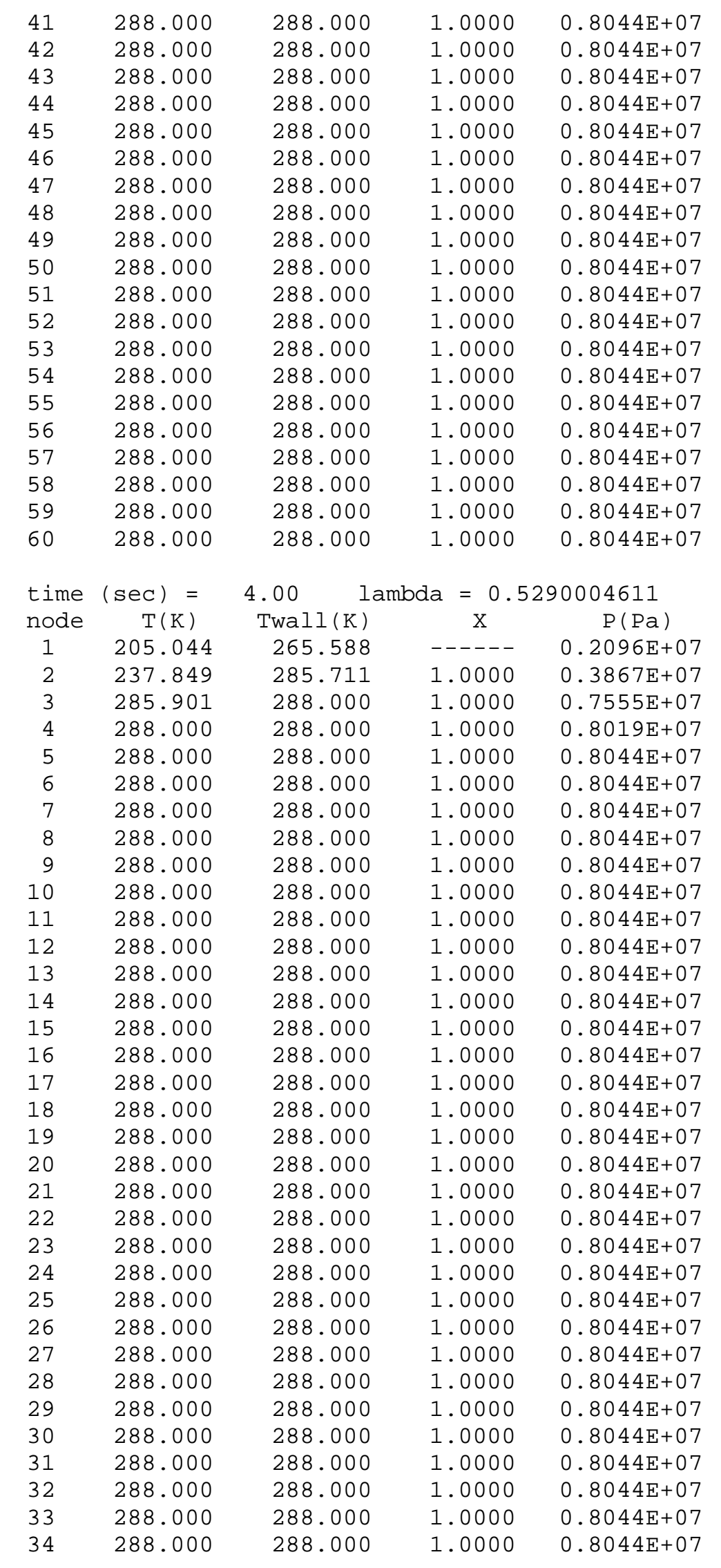




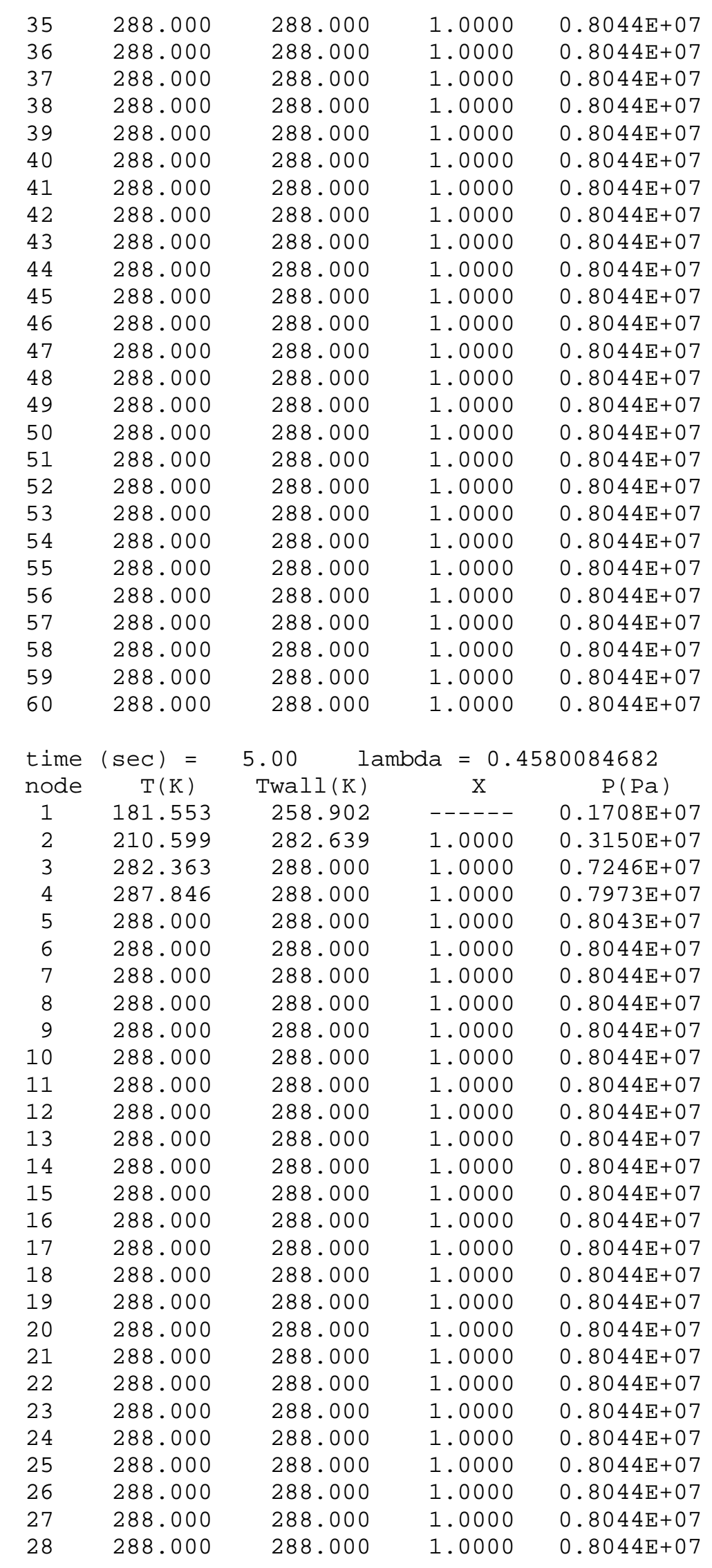




\begin{tabular}{|c|c|c|c|c|}
\hline 29 & 288.000 & 288.000 & 1.0000 & $\odot .8044 \mathrm{E}+07$ \\
\hline 30 & 288.000 & 288.000 & 1.0000 & $0.8044 \mathrm{E}+07$ \\
\hline 31 & 288.000 & 288.000 & 1.0000 & $0.8044 \mathrm{E}+07$ \\
\hline 32 & $288 . \odot \odot \odot$ & $288.0 \odot \odot$ & $1.000 \odot$ & $\odot .8044 \mathrm{E}+\odot 7$ \\
\hline 33 & $288.00 \odot$ & $288.00 \odot$ & 1.0000 & $0.8044 \mathrm{E}+07$ \\
\hline 34 & 288.000 & 288.000 & 1.0000 & $\odot .8044 \mathrm{E}+07$ \\
\hline 35 & 288.000 & 288.000 & 1.0000 & $0.8044 \mathrm{E}+07$ \\
\hline 36 & 288.000 & 288.000 & 1.0000 & $0.8044 \mathrm{E}+07$ \\
\hline 37 & 288.000 & 288.000 & 1.0000 & $0.8044 \mathrm{E}+07$ \\
\hline 38 & 88.000 & $288.00 \odot$ & $1.000 \odot$ & $0.8044 \mathrm{E}+07$ \\
\hline 39 & $288.0 \odot \odot$ & $288.0 \odot \odot$ & $1.000 \odot$ & $0.8044 \mathrm{E}+\odot 7$ \\
\hline 40 & 88.000 & 288.000 & 1.0000 & $0.8044 \mathrm{E}+07$ \\
\hline 41 & 288.000 & $288.0 \odot \odot$ & $1.000 \odot$ & $0.8044 \mathrm{E}+07$ \\
\hline 42 & 288.000 & 288.000 & 1.0000 & $0.8044 \mathrm{E}+07$ \\
\hline 43 & $288.00 \odot$ & $288 . \odot \odot \odot$ & $1.000 \odot$ & $\odot .8044 \mathrm{E}+\odot 7$ \\
\hline 44 & 88.000 & 288.000 & 1.0000 & $\odot .8044 \mathrm{E}+07$ \\
\hline 45 & $288.00 \odot$ & $288.00 \odot$ & $1.000 \odot$ & $\odot .8044 \mathrm{E}+07$ \\
\hline 46 & $288.00 \odot$ & $288.0 \odot \odot$ & $1.000 \odot$ & $\odot .8044 \mathrm{E}+\odot 7$ \\
\hline 47 & 288.000 & $288.00 \odot$ & 1.0000 & $0.8044 \mathrm{E}+07$ \\
\hline 48 & 288.000 & 288.000 & 1.0000 & $0.8044 \mathrm{E}+07$ \\
\hline 49 & $288.00 \odot$ & $288.0 \odot \odot$ & $1.000 \odot$ & $\odot .8044 \mathrm{E}+\odot 7$ \\
\hline 50 & 288.000 & 288.000 & 1.0000 & $0.8044 \mathrm{E}+07$ \\
\hline 51 & 288.000 & 288.000 & 1.0000 & $0.8044 \mathrm{E}+07$ \\
\hline 52 & $288.0 \odot \odot$ & $288.0 \odot \odot$ & $1.000 \odot$ & $0.8044 \mathrm{E}+\odot 7$ \\
\hline 53 & 288.000 & 288.000 & 1.0000 & $0.8044 \mathrm{E}+07$ \\
\hline 54 & 88.000 & 288.000 & 1.0000 & $0.8044 \mathrm{E}+07$ \\
\hline 55 & $288.0 \odot \odot$ & $288.0 \odot \odot$ & $1.000 \odot$ & $\odot .8044 \mathrm{E}+\odot 7$ \\
\hline 56 & 288.000 & 288.000 & 1.0000 & $0.8044 \mathrm{E}+07$ \\
\hline 57 & 288.000 & 288.000 & 1.0000 & $\odot .8044 \mathrm{E}+07$ \\
\hline 58 & 288.000 & $288.00 \odot$ & 1.0000 & $\odot .8044 \mathrm{E}+07$ \\
\hline 59 & 288.000 & 288.000 & 1.0000 & $\odot .8044 \mathrm{E}+07$ \\
\hline 60 & $288.00 \odot$ & $288.0 \odot \odot$ & $1.000 \odot$ & $\odot .8044 \mathrm{E}+\odot 7$ \\
\hline tim & $=$ & \multicolumn{3}{|c|}{ lambda $=0.4031239450$} \\
\hline node & $\mathrm{T}(\mathrm{K})$ & Twall(K) & $\mathrm{x}$ & $\mathrm{P}(\mathrm{Pa})$ \\
\hline 1 & 160.575 & 251.608 & $--\ldots$ & $0.1414 \mathrm{E}+07$ \\
\hline 2 & 186.265 & 278.269 & 1.0000 & $0.2608 \mathrm{E}+07$ \\
\hline 3 & 276.172 & 287.806 & 1.0000 & $0.6894 \mathrm{E}+07$ \\
\hline 4 & 79 & 288. & 1.0000 & $0.7888 \mathrm{E}+07$ \\
\hline 5 & $288.00 \odot$ & 288.000 & 1.0000 & $0.8037 \mathrm{E}+07$ \\
\hline 6 & $288.00 \odot$ & $288.00 \odot$ & 1.0000 & $\odot .8044 \mathrm{E}+\odot 7$ \\
\hline 7 & 288.000 & $288.00 \odot$ & 1.0000 & $\odot .8044 \mathrm{E}+07$ \\
\hline 8 & 288.000 & 288.000 & 1.0000 & $0.8044 \mathrm{E}+07$ \\
\hline 9 & $288.00 \odot$ & $288.00 \odot$ & 1.0000 & $\odot .8044 \mathrm{E}+\odot 7$ \\
\hline 10 & & & 1.0000 & $14 \mathrm{E}+07$ \\
\hline 11 & 88.6 & 288.000 & 1.0000 & $0.8044 \mathrm{E}+07$ \\
\hline 12 & 288.000 & 288.000 & 1.0000 & $\odot .8044 \mathrm{E}+07$ \\
\hline 13 & 288.000 & 288.000 & 1.0000 & $0.8044 \mathrm{E}+07$ \\
\hline 14 & 288.000 & $288.0 \odot \odot$ & $1.00 \odot \odot$ & $0.8044 \mathrm{E}+07$ \\
\hline 15 & $288.00 \odot$ & 288.000 & 1.0000 & $0.8044 \mathrm{E}+07$ \\
\hline 16 & & 288 . & 1.0000 & $0.8044 \mathrm{E}+07$ \\
\hline 17 & 288.000 & 288.000 & 1.0000 & $0.8044 \mathrm{E}+07$ \\
\hline 18 & 288.000 & $288 . \odot \odot \odot$ & $1.000 \odot$ & $\odot .8044 \mathrm{E}+07$ \\
\hline 19 & 288.000 & 288.000 & 1.0000 & $\odot .8044 \mathrm{E}+07$ \\
\hline 20 & $288.00 \odot$ & $288.0 \odot \odot$ & $1.000 \odot$ & $\odot .8044 \mathrm{E}+\odot 7$ \\
\hline 21 & 88.000 & $288.00 \odot$ & 1.0000 & $\odot .8044 \mathrm{E}+07$ \\
\hline 22 & 288.000 & 288.000 & 1.0000 & $\odot .8044 \mathrm{E}+07$ \\
\hline
\end{tabular}




\begin{tabular}{|c|c|c|c|c|}
\hline 23 & $288 . \odot \odot \odot$ & $288.00 \odot$ & 1.0000 & $0.8044 \mathrm{E}+\odot 7$ \\
\hline 24 & 288.000 & 288.000 & 1.0000 & $0.8044 \mathrm{E}+07$ \\
\hline 25 & $288.00 \odot$ & $288 . \odot \odot \odot$ & $1.00 \odot \odot$ & $0.8044 \mathrm{E}+07$ \\
\hline 26 & $288.0 \odot \odot$ & $288.00 \odot$ & $1.00 \odot \odot$ & $\odot .8044 \mathrm{E}+\odot 7$ \\
\hline 27 & $288.00 \odot$ & $288.0 \odot \odot$ & $1.000 \odot$ & $\odot .8044 \mathrm{E}+07$ \\
\hline 28 & $288.00 \odot$ & $288.00 \odot$ & $1.000 \odot$ & $\odot .8 \odot 44 \mathrm{E}+\odot 7$ \\
\hline 29 & 288.000 & $288.00 \odot$ & 1.0000 & $\odot .8044 \mathrm{E}+07$ \\
\hline 30 & 288.000 & 288.000 & 1.0000 & $0.8044 \mathrm{E}+07$ \\
\hline 31 & 288.000 & $288.00 \odot$ & $1.000 \odot$ & $\odot .8044 \mathrm{E}+07$ \\
\hline 32 & $288.00 \odot$ & $288.00 \odot$ & $1.000 \odot$ & $0.8044 \mathrm{E}+07$ \\
\hline 33 & $288.0 \odot \odot$ & $288.00 \odot$ & $1.00 \odot \odot$ & $\odot .8044 \mathrm{E}+07$ \\
\hline 34 & $288.00 \odot$ & $288.00 \odot$ & 1.0000 & $\odot .8044 \mathrm{E}+\odot 7$ \\
\hline 35 & $288.00 \odot$ & $288.0 \odot \odot$ & $1.00 \odot \odot$ & $0.8044 \mathrm{E}+07$ \\
\hline 36 & 288.000 & $288.00 \odot$ & 1.0000 & $\odot .8044 \mathrm{E}+07$ \\
\hline 37 & $288 . \odot \odot \odot$ & $288.0 \odot \odot$ & 1. $\odot \odot \odot \odot$ & $\odot .8044 \mathrm{E}+07$ \\
\hline 38 & $288.00 \odot$ & $288.0 \odot \odot$ & $1.000 \odot$ & $\odot .8044 \mathrm{E}+07$ \\
\hline 39 & $288.00 \odot$ & $288 . \odot \odot \odot$ & $1.000 \odot$ & $\odot .8044 \mathrm{E}+\odot 7$ \\
\hline 40 & $288.0 \odot \odot$ & $288.0 \odot \odot$ & $1.00 \odot \odot$ & $\odot .8 \odot 44 \mathrm{E}+\odot 7$ \\
\hline 41 & 288.000 & $288.00 \odot$ & 1.0000 & $0.8044 \mathrm{E}+07$ \\
\hline 42 & $288.00 \odot$ & $288.0 \odot \odot$ & $1.000 \odot$ & $\odot .8044 \mathrm{E}+07$ \\
\hline 43 & $288.00 \odot$ & $288.0 \odot \odot$ & $1.000 \odot$ & $\odot .8 \odot 44 \mathrm{E}+07$ \\
\hline 44 & $288.00 \odot$ & $288.0 \odot \odot$ & $1.00 \odot \odot$ & $\odot .8044 \mathrm{E}+07$ \\
\hline 45 & $288.00 \odot$ & $288.00 \odot$ & 1.0000 & $\odot .8 \odot 44 \mathrm{E}+\odot 7$ \\
\hline 46 & $288.0 \odot \odot$ & $288.00 \odot$ & $1.00 \odot \odot$ & $0.8044 \mathrm{E}+07$ \\
\hline 47 & $288.00 \odot$ & 288.000 & 1.0000 & $0.8044 \mathrm{E}+07$ \\
\hline 48 & $288.00 \odot$ & $288.0 \odot \odot$ & 1.0000 & $0.8044 \mathrm{E}+07$ \\
\hline 49 & $288.0 \odot \odot$ & $288.00 \odot$ & $1.00 \odot \odot$ & $\odot .8044 \mathrm{E}+\odot 7$ \\
\hline 50 & $288 . \odot \odot \odot$ & $288.0 \odot \odot$ & $1.00 \odot \odot$ & $\odot .8 \odot 44 \mathrm{E}+\odot 7$ \\
\hline 51 & $288.0 \odot \odot$ & $288.0 \odot \odot$ & $1.00 \odot \odot$ & $\odot .8 \odot 44 \mathrm{E}+\odot 7$ \\
\hline 52 & 288.000 & $288.00 \odot$ & $1.00 \odot \odot$ & $\odot .8044 \mathrm{E}+07$ \\
\hline 53 & $288.0 \odot \odot$ & $288.0 \odot \odot$ & $1 . \odot \odot \odot \odot$ & $\odot .8044 \mathrm{E}+\odot 7$ \\
\hline 54 & $288.0 \odot \odot$ & $288.0 \odot \odot$ & $1.0 \odot \odot \odot$ & $\odot .8 \odot 44 \mathrm{E}+\odot 7$ \\
\hline 55 & $288.00 \odot$ & 288.000 & 1.0000 & $\odot .8044 \mathrm{E}+07$ \\
\hline 56 & $288.00 \odot$ & $288.00 \odot$ & $1.00 \odot \odot$ & $0.8044 \mathrm{E}+07$ \\
\hline 57 & $288 . \odot \odot \odot$ & $288.00 \odot$ & $1.000 \odot$ & $\odot .8044 \mathrm{E}+07$ \\
\hline 58 & $288.00 \odot$ & $288.00 \odot$ & 1.0000 & $0.8044 \mathrm{E}+07$ \\
\hline 59 & $288 . \odot \odot \odot$ & $288.00 \odot$ & $1.00 \odot \odot$ & $0.8044 \mathrm{E}+\odot 7$ \\
\hline 60 & 288.000 & 288.000 & 1.0000 & $0.8044 \mathrm{E}+07$ \\
\hline time & $(\sec )=$ & \multicolumn{3}{|c|}{$7.00 \quad$ lambda $=0.3645253479$} \\
\hline node & $\mathrm{T}(\mathrm{K})$ & Twall(K) & $x$ & $\mathrm{P}(\mathrm{Pa})$ \\
\hline 1 & 146.292 & 243.970 & $-\ldots$ & $\odot .1220 \mathrm{E}+\odot 7$ \\
\hline 2 & 169.697 & 273.023 & $1.000 \odot$ & $\odot .2251 \mathrm{E}+07$ \\
\hline 3 & 267.298 & 287.348 & $1.00 \odot \odot$ & $\odot .6518 \mathrm{E}+07$ \\
\hline 4 & 286.229 & 288.000 & 1.0000 & $\odot .7754 \mathrm{E}+07$ \\
\hline 5 & $288.00 \odot$ & $288.00 \odot$ & $1.00 \odot \odot$ & $\odot .8022 \mathrm{E}+\odot 7$ \\
\hline 6 & $288.00 \odot$ & $288.00 \odot$ & $1.000 \odot$ & $0.8044 \mathrm{E}+07$ \\
\hline 7 & 288.000 & 288.000 & 1.0000 & $0.8044 \mathrm{E}+07$ \\
\hline 8 & 288.000 & $288.00 \odot$ & 1.0000 & $0.8044 \mathrm{E}+07$ \\
\hline 9 & 288.000 & 288.000 & 1.0000 & $0.8044 \mathrm{E}+07$ \\
\hline 10 & $288 . \odot \odot \odot$ & $288.00 \odot$ & $1.0 \odot \odot \odot$ & $\odot .8044 \mathrm{E}+07$ \\
\hline 11 & $288.00 \odot$ & 288.000 & 1.0000 & $\odot .8044 \mathrm{E}+\odot 7$ \\
\hline 12 & $288.00 \odot$ & $288 . \odot \odot \odot$ & $1.00 \odot \odot$ & $\odot .8044 \mathrm{E}+\odot 7$ \\
\hline 13 & 288.000 & $288.00 \odot$ & 1.0000 & $\odot .8044 \mathrm{E}+07$ \\
\hline 14 & 288.000 & $288.00 \odot$ & 1.0000 & $0.8044 \mathrm{E}+07$ \\
\hline 15 & 288.000 & 288.000 & 1.0000 & $\odot .8044 \mathrm{E}+07$ \\
\hline 16 & $288.00 \odot$ & $288.00 \odot$ & 1.0000 & $\odot .8044 \mathrm{E}+07$ \\
\hline
\end{tabular}




\begin{tabular}{|c|c|c|c|c|}
\hline 17 & $288 . \odot \odot \odot$ & $288.00 \odot$ & $1.0 \odot \odot \odot$ & $0.8044 \mathrm{E}+\odot 7$ \\
\hline 18 & 288.000 & 288.000 & 1.0000 & $0.8044 \mathrm{E}+07$ \\
\hline 19 & $288.00 \odot$ & $288.0 \odot \odot$ & $1.000 \odot$ & $0.8044 \mathrm{E}+07$ \\
\hline 20 & $288.0 \odot \odot$ & $288 . \odot \odot \odot$ & $1.00 \odot \odot$ & $\odot .8044 \mathrm{E}+\odot 7$ \\
\hline 21 & $288.00 \odot$ & $288.0 \odot \odot$ & $1.000 \odot$ & $\odot .8044 \mathrm{E}+07$ \\
\hline 22 & $288.00 \odot$ & $288.00 \odot$ & 1.0000 & $\odot .8 \odot 44 \mathrm{E}+\odot 7$ \\
\hline 23 & 288.000 & 288.000 & $1.000 \odot$ & $\odot .8044 \mathrm{E}+07$ \\
\hline 24 & 288.000 & $288.00 \odot$ & 1.0000 & $0.8044 \mathrm{E}+07$ \\
\hline 25 & 288.000 & $288.00 \odot$ & $1.000 \odot$ & $\odot .8044 \mathrm{E}+07$ \\
\hline 26 & 288.000 & 288.000 & 1.0000 & $0.8044 \mathrm{E}+07$ \\
\hline 27 & $288.0 \odot \odot$ & $288 . \odot \odot \odot$ & $1.00 \odot \odot$ & $\odot .8044 \mathrm{E}+07$ \\
\hline 28 & $288.00 \odot$ & $288.00 \odot$ & 1.0000 & $0.8044 \mathrm{E}+07$ \\
\hline 29 & $288.00 \odot$ & $288.0 \odot \odot$ & $1.000 \odot$ & $0.8044 \mathrm{E}+07$ \\
\hline 30 & 288.000 & $288.00 \odot$ & $1.000 \odot$ & $\odot .8044 \mathrm{E}+07$ \\
\hline 31 & $288 . \odot \odot \odot$ & $288 . \odot \odot \odot$ & $1.00 \odot \odot$ & $\odot .8044 \mathrm{E}+07$ \\
\hline 32 & $288.00 \odot$ & $288.0 \odot \odot$ & $1.000 \odot$ & $\odot .8044 \mathrm{E}+07$ \\
\hline 33 & $288.00 \odot$ & $288 . \odot \odot \odot$ & $1.000 \odot$ & $\odot .8044 \mathrm{E}+\odot 7$ \\
\hline 34 & $288.0 \odot \odot$ & $288.0 \odot \odot$ & $1.00 \odot \odot$ & $\odot .8 \odot 44 \mathrm{E}+\odot 7$ \\
\hline 35 & 288.000 & $288.00 \odot$ & 1.0000 & $0.8044 \mathrm{E}+07$ \\
\hline 36 & $288.00 \odot$ & $288.0 \odot \odot$ & $1.000 \odot$ & $\odot .8044 \mathrm{E}+07$ \\
\hline 37 & $288.00 \odot$ & $288.0 \odot \odot$ & $1.000 \odot$ & $\odot .8 \odot 44 \mathrm{E}+07$ \\
\hline 38 & $288.00 \odot$ & $288.0 \odot \odot$ & $1.00 \odot \odot$ & $\odot .8044 \mathrm{E}+07$ \\
\hline 39 & $288.00 \odot$ & $288.00 \odot$ & 1.0000 & $\odot .8 \odot 44 \mathrm{E}+\odot 7$ \\
\hline 40 & $288.0 \odot \odot$ & $288.00 \odot$ & $1.00 \odot \odot$ & $0.8044 \mathrm{E}+07$ \\
\hline 41 & 288.000 & 288.000 & 1.0000 & $0.8044 \mathrm{E}+07$ \\
\hline 42 & $288.00 \odot$ & $288.0 \odot \odot$ & 1.0000 & $0.8044 \mathrm{E}+07$ \\
\hline 43 & $288.0 \odot \odot$ & $288.00 \odot$ & $1.00 \odot \odot$ & $\odot .8044 \mathrm{E}+\odot 7$ \\
\hline 44 & $288 . \odot \odot \odot$ & $288.0 \odot \odot$ & $1.00 \odot \odot$ & $\odot .8 \odot 44 \mathrm{E}+\odot 7$ \\
\hline 45 & $288.0 \odot \odot$ & $288.0 \odot \odot$ & $1.00 \odot \odot$ & $\odot .8 \odot 44 \mathrm{E}+\odot 7$ \\
\hline 46 & 288.000 & $288.00 \odot$ & $1.00 \odot \odot$ & $\odot .8044 \mathrm{E}+07$ \\
\hline 47 & $288.0 \odot \odot$ & $288.0 \odot \odot$ & $1 . \odot \odot \odot \odot$ & $\odot .8044 \mathrm{E}+\odot 7$ \\
\hline 48 & $288.0 \odot \odot$ & $288.0 \odot \odot$ & $1 . \odot \odot \odot \odot$ & $\odot .8 \odot 44 \mathrm{E}+\odot 7$ \\
\hline 49 & 288.000 & $288.00 \odot$ & 1.0000 & $\odot .8044 \mathrm{E}+07$ \\
\hline 50 & $288.00 \odot$ & $288.00 \odot$ & $1.0 \odot \odot \odot$ & $0.8044 \mathrm{E}+07$ \\
\hline 51 & $288 . \odot \odot \odot$ & $288.00 \odot$ & $1 . \odot \odot \odot \odot$ & $\odot .8 \odot 44 \mathrm{E}+07$ \\
\hline 52 & $288.00 \odot$ & $288.0 \odot \odot$ & $1.000 \odot$ & $0.8044 \mathrm{E}+07$ \\
\hline 53 & $288 . \odot \odot \odot$ & $288.00 \odot$ & $1.0 \odot \odot \odot$ & $\odot .8044 \mathrm{E}+\odot 7$ \\
\hline 54 & $288.0 \odot \odot$ & $288.00 \odot$ & $1.0 \odot \odot \odot$ & $\odot .8044 \mathrm{E}+\odot 7$ \\
\hline 55 & $288.0 \odot \odot$ & $288 . \odot \odot \odot$ & $1.00 \odot \odot$ & $\odot .8 \odot 44 \mathrm{E}+\odot 7$ \\
\hline 56 & $288.00 \odot$ & $288.0 \odot \odot$ & $1.000 \odot$ & $\odot .8044 \mathrm{E}+\odot 7$ \\
\hline 57 & $288.0 \odot \odot$ & $288.0 \odot \odot$ & $1.0 \odot \odot \odot$ & $\odot .8044 \mathrm{E}+\odot 7$ \\
\hline 58 & 288.000 & $288.0 \odot \odot$ & 1.0000 & $\odot .8044 \mathrm{E}+07$ \\
\hline 59 & $288.00 \odot$ & $288.0 \odot \odot$ & $1.000 \odot$ & $\odot .8044 \mathrm{E}+07$ \\
\hline 60 & 288.000 & 288.000 & 1.0000 & $\odot .8044 \mathrm{E}+07$ \\
\hline time & $(\sec )=$ & \multicolumn{3}{|c|}{$.00 \quad$ lambda $=0.3445852697$} \\
\hline node & $\mathrm{T}(\mathrm{K})$ & Twall(K) & $x$ & $\mathrm{P}(\mathrm{Pa})$ \\
\hline 1 & 144.059 & 236.554 & - - - - - & $0.1145 \mathrm{E}+07$ \\
\hline 2 & 167.109 & 267.633 & $\odot .9988$ & $0.2111 \mathrm{E}+07$ \\
\hline 3 & 256.532 & 286.444 & $1.000 \odot$ & $0.6147 \mathrm{E}+07$ \\
\hline 4 & 283.992 & $288 . \odot \odot \odot$ & $1.0 \odot \odot \odot$ & $\odot .7570 \mathrm{E}+\odot 7$ \\
\hline 5 & 287.867 & 288.000 & 1.0000 & $\odot .7991 \mathrm{E}+07$ \\
\hline 6 & $288.00 \odot$ & $288 . \odot \odot \odot$ & $1.00 \odot \odot$ & $\odot .8043 \mathrm{E}+\odot 7$ \\
\hline 7 & $288.00 \odot$ & $288.0 \odot \odot$ & $1.00 \odot \odot$ & $\odot .8044 \mathrm{E}+07$ \\
\hline 8 & $288 . \odot \odot \odot$ & $288 . \odot \odot \odot$ & $1 . \odot \odot \odot \odot$ & $\odot .8 \odot 44 \mathrm{E}+07$ \\
\hline 9 & 288.000 & 288.000 & 1.0000 & $\odot .8044 \mathrm{E}+07$ \\
\hline 10 & $288.00 \odot$ & $288 . \odot \odot \odot$ & $1.0 \odot \odot \odot$ & $\odot .8044 \mathrm{E}+07$ \\
\hline
\end{tabular}




\begin{tabular}{|c|c|c|c|c|}
\hline 11 & $288 . \odot \odot \odot$ & $288.00 \odot$ & 1.0000 & $0.8044 \mathrm{E}+\odot 7$ \\
\hline 12 & 288.000 & 288.000 & 1.0000 & $0.8044 \mathrm{E}+07$ \\
\hline 13 & $288.00 \odot$ & $288.0 \odot \odot$ & $1.00 \odot \odot$ & $0.8044 \mathrm{E}+07$ \\
\hline 14 & $288.0 \odot \odot$ & $288 . \odot \odot \odot$ & $1.00 \odot \odot$ & $\odot .8044 \mathrm{E}+\odot 7$ \\
\hline 15 & $288.00 \odot$ & $288 . \odot \odot \odot$ & 1. $0 \odot \odot \odot$ & $\odot .8044 \mathrm{E}+07$ \\
\hline 16 & $288.00 \odot$ & $288.00 \odot$ & $1.000 \odot$ & $\odot .8 \odot 44 \mathrm{E}+\odot 7$ \\
\hline 17 & 288.000 & 288.000 & 1.0000 & $\odot .8044 \mathrm{E}+07$ \\
\hline 18 & 288.000 & $288.00 \odot$ & 1.0000 & $0.8044 \mathrm{E}+07$ \\
\hline 19 & 288.000 & $288.00 \odot$ & $1.000 \odot$ & $\odot .8044 \mathrm{E}+07$ \\
\hline 20 & 288.000 & 288.000 & $1.000 \odot$ & $0.8044 \mathrm{E}+07$ \\
\hline 21 & $288.0 \odot \odot$ & $288 . \odot \odot \odot$ & $1.00 \odot \odot$ & $\odot .8044 \mathrm{E}+07$ \\
\hline 22 & $288.00 \odot$ & $288.00 \odot$ & 1.0000 & $0.8044 \mathrm{E}+07$ \\
\hline 23 & $288.00 \odot$ & $288.0 \odot \odot$ & $1.00 \odot \odot$ & $0.8044 \mathrm{E}+07$ \\
\hline 24 & 288.000 & $288.00 \odot$ & 1.0000 & $\odot .8044 \mathrm{E}+07$ \\
\hline 25 & $288 . \odot \odot \odot$ & $288 . \odot \odot \odot$ & 1. $\odot \odot \odot \odot$ & $\odot .8044 \mathrm{E}+07$ \\
\hline 26 & $288.00 \odot$ & $288.0 \odot \odot$ & $1.000 \odot$ & $\odot .8044 \mathrm{E}+07$ \\
\hline 27 & $288.00 \odot$ & $288 . \odot \odot \odot$ & $1.000 \odot$ & $\odot .8044 \mathrm{E}+\odot 7$ \\
\hline 28 & $288.0 \odot \odot$ & $288.0 \odot \odot$ & $1.00 \odot \odot$ & $\odot .8 \odot 44 \mathrm{E}+\odot 7$ \\
\hline 29 & 288.000 & $288.00 \odot$ & 1.0000 & $0.8044 \mathrm{E}+07$ \\
\hline 30 & $288.00 \odot$ & $288.0 \odot \odot$ & $1.000 \odot$ & $\odot .8044 \mathrm{E}+07$ \\
\hline 31 & $288.00 \odot$ & $288.0 \odot \odot$ & $1.000 \odot$ & $\odot .8 \odot 44 \mathrm{E}+07$ \\
\hline 32 & $288.00 \odot$ & $288.0 \odot \odot$ & $1.00 \odot \odot$ & $\odot .8044 \mathrm{E}+07$ \\
\hline 33 & 288.000 & $288.00 \odot$ & 1.0000 & $\odot .8 \odot 44 \mathrm{E}+\odot 7$ \\
\hline 34 & $288.0 \odot \odot$ & $288.00 \odot$ & $1.00 \odot \odot$ & $0.8044 \mathrm{E}+07$ \\
\hline 35 & $288.00 \odot$ & 288.000 & 1.0000 & $0.8044 \mathrm{E}+07$ \\
\hline 36 & $288.00 \odot$ & $288.0 \odot \odot$ & 1.0000 & $0.8044 \mathrm{E}+07$ \\
\hline 37 & $288.0 \odot \odot$ & $288.00 \odot$ & $1.00 \odot \odot$ & $\odot .8044 \mathrm{E}+\odot 7$ \\
\hline 38 & $288 . \odot \odot \odot$ & $288.0 \odot \odot$ & $1.00 \odot \odot$ & $\odot .8 \odot 44 \mathrm{E}+\odot 7$ \\
\hline 39 & $288.0 \odot \odot$ & $288.0 \odot \odot$ & $1.00 \odot \odot$ & $\odot .8 \odot 44 \mathrm{E}+\odot 7$ \\
\hline 40 & 288.000 & $288.00 \odot$ & $1.00 \odot \odot$ & $\odot .8044 \mathrm{E}+07$ \\
\hline 41 & $288.0 \odot \odot$ & $288.0 \odot \odot$ & $1 . \odot \odot \odot \odot$ & $\odot .8044 \mathrm{E}+\odot 7$ \\
\hline 42 & $288.0 \odot \odot$ & $288.0 \odot \odot$ & $1 . \odot \odot \odot \odot$ & $\odot .8 \odot 44 \mathrm{E}+\odot 7$ \\
\hline 43 & 288.000 & $288.00 \odot$ & $1.000 \odot$ & $\odot .8044 \mathrm{E}+07$ \\
\hline 44 & $288.00 \odot$ & $288.00 \odot$ & $1.0 \odot \odot \odot$ & $0.8044 \mathrm{E}+07$ \\
\hline 45 & $288 . \odot \odot \odot$ & $288.00 \odot$ & $1 . \odot \odot \odot \odot$ & $\odot .8 \odot 44 \mathrm{E}+07$ \\
\hline 46 & $288.00 \odot$ & $288.0 \odot \odot$ & $1.000 \odot$ & $0.8044 \mathrm{E}+07$ \\
\hline 47 & $288 . \odot \odot \odot$ & $288.00 \odot$ & $1.0 \odot \odot \odot$ & $\odot .8044 \mathrm{E}+\odot 7$ \\
\hline 48 & $288.0 \odot \odot$ & $288.00 \odot$ & $1.0 \odot \odot \odot$ & $\odot .8044 \mathrm{E}+\odot 7$ \\
\hline 49 & $288.00 \odot$ & $288.00 \odot$ & 1.0000 & $\odot .8044 \mathrm{E}+07$ \\
\hline 50 & $288.00 \odot$ & $288.0 \odot \odot$ & $1.000 \odot$ & $\odot .8044 \mathrm{E}+\odot 7$ \\
\hline 51 & $288.0 \odot \odot$ & $288.0 \odot \odot$ & $1.0 \odot \odot \odot$ & $\odot .8044 \mathrm{E}+\odot 7$ \\
\hline 52 & 288.000 & $288.0 \odot \odot$ & 1.0000 & $\odot .8044 \mathrm{E}+07$ \\
\hline 53 & $288.00 \odot$ & $288.0 \odot \odot$ & $1.000 \odot$ & $\odot .8044 \mathrm{E}+07$ \\
\hline 54 & $288 . \odot$ & $288.00 \odot$ & $1.0 \odot \odot \odot$ & $\odot .8044 \mathrm{E}+07$ \\
\hline 55 & 288.000 & 288.000 & 1.0000 & $\odot .8044 \mathrm{E}+07$ \\
\hline 56 & $288.00 \odot$ & $288 . \odot \odot \odot$ & $1.00 \odot \odot$ & $0.8044 \mathrm{E}+07$ \\
\hline 57 & $288.00 \odot$ & $288 . \odot \odot \odot$ & $1.00 \odot \odot$ & $0.8044 \mathrm{E}+07$ \\
\hline 58 & 288.000 & 288.000 & 1.0000 & $\odot .8044 \mathrm{E}+\odot 7$ \\
\hline 59 & $288.00 \odot$ & $288.00 \odot$ & 1.0000 & $0.8044 \mathrm{E}+07$ \\
\hline 60 & $288.00 \odot$ & $288 . \odot \odot \odot$ & $1.00 \odot \odot$ & $0.8044 \mathrm{E}+07$ \\
\hline time & ec) $=$ & \multicolumn{3}{|c|}{$9 . \odot \odot \quad$ lambda $=0.3272083998$} \\
\hline node & $\mathrm{T}(\mathrm{K})$ & Twall(K) & $\mathrm{x}$ & $\mathrm{P}(\mathrm{Pa})$ \\
\hline 1 & 142.919 & 229.786 & - - - - - & $0.1083 \mathrm{E}+07$ \\
\hline 2 & 165.786 & 262.603 & 0.9977 & $\odot .1997 \mathrm{E}+07$ \\
\hline 3 & 245.305 & 284.999 & 1.0000 & $\odot .5810 \mathrm{E}+07$ \\
\hline 4 & 280.220 & $288 . \odot \odot \odot$ & $1.000 \odot$ & $\odot .7338 \mathrm{E}+07$ \\
\hline
\end{tabular}




\begin{tabular}{|c|c|c|c|c|}
\hline 5 & 287.480 & $288.00 \odot$ & 1.0000 & $\odot .7935 \mathrm{E}+07$ \\
\hline 6 & $288 . \odot \odot \odot$ & $288 . \odot \odot \odot$ & $1.000 \odot$ & $\odot .8 \odot 38 \mathrm{E}+\odot 7$ \\
\hline 7 & $288 . \odot \odot \odot$ & $288 . \odot \odot \odot$ & 1.0000 & $\odot .8 \odot 44 \mathrm{E}+\odot 7$ \\
\hline 8 & 288.000 & $288.00 \odot$ & 1.0000 & $\odot .8 \odot 44 \mathrm{E}+\odot 7$ \\
\hline 9 & $288.0 \odot \odot$ & $288.00 \odot$ & 1.0000 & $\odot .8044 \mathrm{E}+\odot 7$ \\
\hline 10 & $288.00 \odot$ & $288 . \odot \odot \odot$ & 1.0000 & $\odot .8044 \mathrm{E}+07$ \\
\hline 11 & $288 . \odot \odot \odot$ & $288 . \odot \odot \odot$ & 1.0000 & $\odot .8 \odot 44 \mathrm{E}+\odot 7$ \\
\hline 12 & $288.00 \odot$ & $288.0 \odot \odot$ & 1.0000 & $\odot .8044 \mathrm{E}+07$ \\
\hline 13 & $288.0 \odot \odot$ & $288.0 \odot \odot$ & 1.0000 & $\odot .8044 \mathrm{E}+\odot 7$ \\
\hline 14 & 288.000 & $288.0 \odot \odot$ & 1.0000 & $\odot .8044 \mathrm{E}+07$ \\
\hline 15 & $288.00 \odot$ & 288.000 & 1.0000 & $\odot .8044 \mathrm{E}+07$ \\
\hline 16 & $288.0 \odot \odot$ & $288.00 \odot$ & 1.0000 & $\odot .8044 \mathrm{E}+\odot 7$ \\
\hline 17 & $288.0 \odot \odot$ & $288.0 \odot \odot$ & 1.0000 & $\odot .8044 \mathrm{E}+\odot 7$ \\
\hline 18 & $288 . \odot \odot \odot$ & $288.00 \odot$ & 1.0000 & $\odot .8 \odot 44 \mathrm{E}+\odot 7$ \\
\hline 19 & $288.0 \odot \odot$ & $288.00 \odot$ & 1.0000 & $\odot .8044 \mathrm{E}+07$ \\
\hline 20 & 288.000 & $288.00 \odot$ & 1.0000 & $\odot .8 \odot 44 \mathrm{E}+\odot 7$ \\
\hline 21 & $288.00 \odot$ & $288 . \odot \odot \odot$ & 1.0000 & $\odot .8044 \mathrm{E}+07$ \\
\hline 22 & $288.00 \odot$ & 288.000 & 1.0000 & $\odot .8044 \mathrm{E}+07$ \\
\hline 23 & $288.0 \odot \odot$ & $288.00 \odot$ & 1.0000 & $\odot .8044 \mathrm{E}+\odot 7$ \\
\hline 24 & $288.0 \odot \odot$ & $288 . \odot \odot \odot$ & $1.000 \odot$ & $\odot .8 \odot 44 \mathrm{E}+\odot 7$ \\
\hline 25 & $288.00 \odot$ & $288.00 \odot$ & $1.000 \odot$ & $\odot .8044 \mathrm{E}+\odot 7$ \\
\hline 26 & $288.0 \odot \odot$ & $288 . \odot \odot \odot$ & 1.0000 & $\odot .8 \odot 44 \mathrm{E}+07$ \\
\hline 27 & 288.000 & 288.000 & 1.0000 & $\odot .8 \odot 44 \mathrm{E}+07$ \\
\hline 28 & $288.00 \odot$ & 288.000 & 1.0000 & $\odot .8044 \mathrm{E}+07$ \\
\hline 29 & $288 . \odot \odot \odot$ & $288.00 \odot$ & $1.000 \odot$ & $\odot .8 \odot 44 \mathrm{E}+\odot 7$ \\
\hline 30 & $288.0 \odot \odot$ & $288.0 \odot \odot$ & 1.0000 & $\odot .8044 \mathrm{E}+\odot 7$ \\
\hline 31 & $288.00 \odot$ & $288.00 \odot$ & 1.0000 & $\odot .8044 \mathrm{E}+07$ \\
\hline 32 & $288 . \odot \odot \odot$ & $288 . \odot \odot \odot$ & 1.0000 & $\odot .8 \odot 44 \mathrm{E}+\odot 7$ \\
\hline 33 & 288.000 & $288.0 \odot \odot$ & 1.0000 & $\odot .8044 \mathrm{E}+07$ \\
\hline 34 & $288.00 \odot$ & $288.00 \odot$ & 1.0000 & $\odot .8044 \mathrm{E}+07$ \\
\hline 35 & $288 . \odot \odot \odot$ & $288 . \odot \odot \odot$ & 1.0000 & $\odot .8 \odot 44 \mathrm{E}+\odot 7$ \\
\hline 36 & $288.00 \odot$ & $288.0 \odot \odot$ & 1.0000 & $\odot .8044 \mathrm{E}+07$ \\
\hline 37 & $288.00 \odot$ & $288.0 \odot \odot$ & 1.0000 & $\odot .8044 \mathrm{E}+07$ \\
\hline 38 & $288.00 \odot$ & $288 . \odot \odot \odot$ & $1.000 \odot$ & $\odot .8 \odot 44 \mathrm{E}+\odot 7$ \\
\hline 39 & $288.00 \odot$ & $288.0 \odot \odot$ & 1.0000 & $\odot .8044 \mathrm{E}+07$ \\
\hline 40 & $288 . \odot \odot \odot$ & $288 . \odot \odot \odot$ & 1..$\odot \odot \odot$ & $\odot .8044 \mathrm{E}+\odot 7$ \\
\hline 41 & $288 . \odot \odot \odot$ & $288 . \odot \odot \odot$ & 1.0000 & $\odot .8 \odot 44 \mathrm{E}+\odot 7$ \\
\hline 42 & $288.0 \odot \odot$ & $288.0 \odot \odot$ & 1.0000 & $\odot .8044 \mathrm{E}+\odot 7$ \\
\hline 43 & $288.00 \odot$ & $288 . \odot \odot \odot$ & 1.0000 & $\odot .8044 \mathrm{E}+07$ \\
\hline 44 & $288.00 \odot$ & $288.0 \odot \odot$ & 1.0000 & $\odot .8044 \mathrm{E}+07$ \\
\hline 45 & $288.00 \odot$ & $288.00 \odot$ & 1.0000 & $\odot .8044 \mathrm{E}+07$ \\
\hline 46 & $288.0 \odot \odot$ & $288.0 \odot \odot$ & 1.0000 & $\odot .8044 \mathrm{E}+\odot 7$ \\
\hline 47 & $288.0 \odot \odot$ & $288 . \odot \odot \odot$ & $1.000 \odot$ & $\odot .8044 \mathrm{E}+\odot 7$ \\
\hline 48 & $288.00 \odot$ & $288.0 \odot \odot$ & 1.0000 & $\odot .8044 \mathrm{E}+07$ \\
\hline 49 & $288 . \odot \odot \odot$ & $288 . \odot \odot \odot$ & 1.0000 & $\odot .8044 \mathrm{E}+07$ \\
\hline 50 & $288.0 \odot \odot$ & 288.000 & 1.0000 & $\odot .8044 \mathrm{E}+\odot 7$ \\
\hline 51 & 288.000 & $288.0 \odot \odot$ & 1.0000 & $\odot .8044 \mathrm{E}+07$ \\
\hline 52 & $288.0 \odot \odot$ & $288 . \odot \odot \odot$ & $1.000 \odot$ & $\odot .8 \odot 44 \mathrm{E}+\odot 7$ \\
\hline 53 & $288.00 \odot$ & 288.000 & 1.0000 & $\odot .8044 \mathrm{E}+07$ \\
\hline 54 & $288.00 \odot$ & $288 . \odot \odot \odot$ & 1.0000 & $\odot .8044 \mathrm{E}+07$ \\
\hline 55 & $288.00 \odot$ & $288.0 \odot \odot$ & 1.0000 & $\odot .8044 \mathrm{E}+07$ \\
\hline 56 & 288.000 & $288.0 \odot \odot$ & 1.0000 & $\odot .8044 \mathrm{E}+07$ \\
\hline 57 & $288.0 \odot \odot$ & $288 . \odot \odot \odot$ & $1.00 \odot \odot$ & $\odot .8 \odot 44 \mathrm{E}+07$ \\
\hline 58 & $288 . \odot \odot \odot$ & $288 . \odot \odot \odot$ & 1.0000 & $\odot .8 \odot 44 \mathrm{E}+\odot 7$ \\
\hline 59 & $288.00 \odot$ & $288.0 \odot \odot$ & $1.0 \odot \odot \odot$ & $\odot .8044 \mathrm{E}+\odot 7$ \\
\hline 60 & 288.000 & 288.000 & 1.0000 & $\odot .8044 \mathrm{E}+\odot 7$ \\
\hline
\end{tabular}




\begin{tabular}{|c|c|c|c|c|}
\hline $\begin{array}{l}\text { time } \\
\text { node }\end{array}$ & $\begin{array}{l}(\sec )= \\
T(K)\end{array}$ & $\begin{array}{cc}10.00 \quad 1 \\
\text { Twall(K) }\end{array}$ & $a=0$ & $\begin{array}{l}7855 \\
P(P a)\end{array}$ \\
\hline $\begin{array}{c}\text { node } \\
1\end{array}$ & 141.270 & $\begin{array}{r}\text { IWaLI(K) } \\
223.549\end{array}$ & $\begin{array}{c}\lambda \\
-\ldots\end{array}$ & $0.1021 \mathrm{E}+07$ \\
\hline 2 & 163.873 & 257.853 & $\odot .9965$ & $\odot .1882 \mathrm{E}+\odot 7$ \\
\hline 3 & 235.196 & 283.017 & 1.0000 & $\odot .5533 \mathrm{E}+\odot 7$ \\
\hline 4 & 274.683 & 287.722 & $1.00 \odot \odot$ & $\odot .7071 \mathrm{E}+\odot 7$ \\
\hline 5 & 286.652 & $288 . \odot \odot \odot$ & $1.00 \odot \odot$ & $\odot .7846 \mathrm{E}+07$ \\
\hline 6 & $288.00 \odot$ & 288.000 & 1.0000 & $\odot .8028 \mathrm{E}+\odot 7$ \\
\hline 7 & $288.00 \odot$ & $288.00 \odot$ & 1.0000 & $\odot .8044 \mathrm{E}+\odot 7$ \\
\hline 8 & 288.000 & 288.000 & 1.0000 & $\odot .8044 \mathrm{E}+\odot 7$ \\
\hline 9 & 288.000 & 288.000 & 1.0000 & $0.8044 \mathrm{E}+07$ \\
\hline 10 & 288.000 & 288.000 & 1..$\odot \odot \odot$ & $\odot .8044 \mathrm{E}+\odot 7$ \\
\hline 11 & $288.00 \odot$ & $288 . \odot \odot \odot$ & $1.00 \odot \odot$ & $\odot .8044 \mathrm{E}+\odot 7$ \\
\hline 12 & 288.000 & $288.00 \odot$ & 1.0000 & $\odot .8044 \mathrm{E}+07$ \\
\hline 13 & $288.00 \odot$ & $288.00 \odot$ & $1.000 \odot$ & $\odot .8044 \mathrm{E}+\odot 7$ \\
\hline 14 & $288.00 \odot$ & $288.00 \odot$ & $1.000 \odot$ & $\odot .8044 \mathrm{E}+\odot 7$ \\
\hline 15 & $288 . \odot \odot \odot$ & $288.0 \odot \odot$ & $1.0 \odot \odot \odot$ & $\odot .8044 \mathrm{E}+\odot 7$ \\
\hline 16 & 288.000 & 288.000 & 1.0000 & $\odot .8 \odot 44 \mathrm{E}+\odot 7$ \\
\hline 17 & 288.000 & $288.00 \odot$ & $1.000 \odot$ & $\odot .8044 \mathrm{E}+\odot 7$ \\
\hline 18 & 288.000 & 288.000 & 1.0000 & $0.8044 \mathrm{E}+07$ \\
\hline 19 & 288.000 & 288.000 & 1.0000 & $\odot .8044 \mathrm{E}+\odot 7$ \\
\hline 20 & 288.000 & $288.00 \odot$ & 1.0000 & $\odot .8044 \mathrm{E}+07$ \\
\hline 21 & $288.00 \odot$ & 288.000 & 1.0000 & $\odot .8044 \mathrm{E}+\odot 7$ \\
\hline 22 & $288.0 \odot \odot$ & $288.00 \odot$ & $1.00 \odot \odot$ & $\odot .8044 \mathrm{E}+\odot 7$ \\
\hline 23 & 288.000 & 288.000 & 1.0000 & $0.8044 \mathrm{E}+07$ \\
\hline 24 & $288.00 \odot$ & $288.00 \odot$ & $1.000 \odot$ & $\odot .8044 \mathrm{E}+\odot 7$ \\
\hline 25 & $288.0 \odot \odot$ & $288.00 \odot$ & $1.00 \odot \odot$ & $\odot .8044 \mathrm{E}+\odot 7$ \\
\hline 26 & $288 . \odot \odot \odot$ & 288.000 & 1.0000 & $\odot .8044 \mathrm{E}+\odot 7$ \\
\hline 27 & 288.000 & $288.00 \odot$ & 1. $0 \odot \odot \odot$ & $\odot .8044 \mathrm{E}+\odot 7$ \\
\hline 28 & 288.000 & $288.00 \odot$ & $1.00 \odot \odot$ & $\odot .8 \odot 44 \mathrm{E}+\odot 7$ \\
\hline 29 & 288.000 & 288.000 & 1.0000 & $\odot .8044 \mathrm{E}+07$ \\
\hline 30 & $288.00 \odot$ & $288.00 \odot$ & $1.000 \odot$ & $\odot .8044 \mathrm{E}+07$ \\
\hline 31 & $288.00 \odot$ & $288 . \odot \odot \odot$ & $1.000 \odot$ & $\odot .8 \odot 44 \mathrm{E}+\odot 7$ \\
\hline 32 & 288.000 & 288.000 & 1.0000 & $\odot .8044 \mathrm{E}+07$ \\
\hline 33 & $288 . \odot \odot \odot$ & $288.00 \odot$ & 1. $\odot \odot \odot \odot$ & $\odot .8 \odot 44 \mathrm{E}+\odot 7$ \\
\hline 34 & $288.00 \odot$ & $288 . \odot \odot \odot$ & $1.000 \odot$ & $\odot .8 \odot 44 \mathrm{E}+\odot 7$ \\
\hline 35 & 288.000 & $288.00 \odot$ & 1.0000 & $\odot .8044 \mathrm{E}+07$ \\
\hline 36 & 288.000 & 288.000 & 1.0000 & $\odot .8044 \mathrm{E}+07$ \\
\hline 37 & $288.00 \odot$ & $288 . \odot \odot \odot$ & $1.000 \odot$ & $\odot .8 \odot 44 \mathrm{E}+\odot 7$ \\
\hline 38 & $288 . \odot \odot \odot$ & $288.0 \odot \odot$ & $1.000 \odot$ & $\odot .8044 \mathrm{E}+\odot 7$ \\
\hline 39 & $288.00 \odot$ & $288 . \odot \odot \odot$ & $1.0 \odot \odot \odot$ & $\odot .8 \odot 44 \mathrm{E}+\odot 7$ \\
\hline 40 & 288.000 & $288.00 \odot$ & $1.000 \odot$ & $\odot .8044 \mathrm{E}+\odot 7$ \\
\hline 41 & 288.000 & 288.000 & 1.0000 & $\odot .8 \odot 44 \mathrm{E}+07$ \\
\hline 42 & $288.00 \odot$ & $288.00 \odot$ & $1.0 \odot \odot \odot$ & $\odot .8 \odot 44 \mathrm{E}+\odot 7$ \\
\hline 43 & 288.000 & 288.000 & 1.0000 & $\odot .8044 \mathrm{E}+\odot 7$ \\
\hline 44 & 288.000 & $288.0 \odot \odot$ & 1.0000 & $\odot .8044 \mathrm{E}+07$ \\
\hline 45 & $288.00 \odot$ & $288 . \odot \odot \odot$ & $1.000 \odot$ & $\odot .8 \odot 44 \mathrm{E}+\odot 7$ \\
\hline 46 & 288.000 & 288.000 & 1.0000 & $\odot .8044 \mathrm{E}+07$ \\
\hline 47 & $288.00 \odot$ & $288.00 \odot$ & $1.000 \odot$ & $\odot .8044 \mathrm{E}+07$ \\
\hline 48 & 288.000 & $288 . \odot \odot \odot$ & $1.000 \odot$ & $\odot .8 \odot 44 \mathrm{E}+\odot 7$ \\
\hline 49 & 288.000 & 288.000 & 1.0000 & $\odot .8044 \mathrm{E}+07$ \\
\hline 50 & 288.000 & 288.000 & 1. $\odot \odot \odot \odot$ & $\odot .8044 \mathrm{E}+\odot 7$ \\
\hline 51 & $288.00 \odot$ & $288 . \odot \odot \odot$ & $1.00 \odot \odot$ & $\odot .8 \odot 44 \mathrm{E}+\odot 7$ \\
\hline 52 & 288.000 & 288.000 & $1.0 \odot \odot \odot$ & $\odot .8044 \mathrm{E}+\odot 7$ \\
\hline 53 & $288.00 \odot$ & $288.00 \odot$ & $1.000 \odot$ & $\odot .8 \odot 44 \mathrm{E}+07$ \\
\hline 54 & 288.000 & 288.000 & 1.0000 & $\odot .8044 \mathrm{E}+\odot 7$ \\
\hline 55 & 288.000 & 288.000 & 1.0000 & $0.8044 \mathrm{E}+07$ \\
\hline
\end{tabular}




$\begin{array}{lllll}56 & 288.00 \odot & 288.00 \odot & 1.00 \odot \odot & \odot .8 \odot 44 \mathrm{E}+\odot 7 \\ 57 & 288.00 \odot & 288.00 \odot & 1.00 \odot \odot & \odot .8044 \mathrm{E}+\odot 7 \\ 58 & 288.00 \odot & 288.00 \odot & 1.000 \odot & \odot .8044 \mathrm{E}+\odot 7 \\ 59 & 288.00 \odot & 288.00 \odot & 1.00 \odot \odot & \odot .8044 \mathrm{E}+\odot 7 \\ 6 \odot & 288.00 \odot & 288.00 \odot & 1.000 \odot & \odot .8044 \mathrm{E}+\odot 7\end{array}$

\begin{tabular}{|c|c|c|c|c|}
\hline \multicolumn{5}{|c|}{ lambda $=0.2945762873$} \\
\hline node & $\mathrm{T}^{\prime}(\mathrm{K})$ & Twall(K) & $x$ & $\mathrm{P}(\mathrm{Pa})$ \\
\hline 1 & 139.759 & 217.766 & 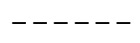 & $\odot .9637 \mathrm{E}+06$ \\
\hline 2 & 162.120 & 253.347 & $\odot .9957$ & $\odot .1778 \mathrm{E}+07$ \\
\hline 3 & 227.287 & 280.640 & $1.000 \odot$ & $\odot .5327 \mathrm{E}+\odot 7$ \\
\hline 4 & 267.547 & 287.277 & 1.0000 & $\odot .6785 \mathrm{E}+07$ \\
\hline 5 & 285.079 & 288.000 & 1.0000 & $\odot .7717 \mathrm{E}+\odot 7$ \\
\hline 6 & 287.951 & 288.000 & 1.0000 & $\odot .8007 \mathrm{E}+07$ \\
\hline 7 & 288.000 & 288.000 & 1.0000 & $0.8044 \mathrm{E}+07$ \\
\hline 8 & $288.00 \odot$ & $288.0 \odot \odot$ & 1.0000 & $\odot .8 \odot 44 \mathrm{E}+\odot 7$ \\
\hline 9 & 288.000 & $288.00 \odot$ & 1.0000 & $\odot .8 \odot 44 \mathrm{E}+\odot 7$ \\
\hline 10 & 288.000 & $288.00 \odot$ & 1.0000 & $\odot .8044 \mathrm{E}+\odot 7$ \\
\hline 11 & 288.000 & $288.0 \odot \odot$ & 1.0000 & $\odot .8 \odot 44 \mathrm{E}+\odot 7$ \\
\hline 12 & 288.000 & 288.000 & 1.0000 & $\odot .8044 \mathrm{E}+07$ \\
\hline 13 & 288.000 & $288.00 \odot$ & 1.0000 & $\odot .8044 \mathrm{E}+07$ \\
\hline 14 & 288.000 & 288.000 & 1.0000 & $\odot .8044 \mathrm{E}+\odot 7$ \\
\hline 15 & 288.000 & $288.00 \odot$ & 1.0000 & $\odot .8 \odot 44 \mathrm{E}+07$ \\
\hline 16 & 288.000 & 288.000 & 1.0000 & $\odot .8044 \mathrm{E}+07$ \\
\hline 17 & 288.000 & $288.00 \odot$ & 1.0000 & $\odot .8044 \mathrm{E}+\odot 7$ \\
\hline 18 & 288.000 & 288.000 & 1.0000 & $\odot .8044 \mathrm{E}+07$ \\
\hline 19 & 288.000 & 288.000 & 1.0000 & $\odot .8044 \mathrm{E}+07$ \\
\hline 20 & 288.000 & $288.00 \odot$ & 1.0000 & $\odot .8044 \mathrm{E}+\odot 7$ \\
\hline 21 & $288.00 \odot$ & 288.000 & $1.000 \odot$ & $\odot .8 \odot 44 \mathrm{E}+\odot 7$ \\
\hline 22 & 288.000 & 288.000 & 1.0000 & $\odot .8044 \mathrm{E}+07$ \\
\hline 23 & 288.000 & 288.000 & 1.0000 & $\odot .8044 \mathrm{E}+07$ \\
\hline 24 & 288.000 & 288.000 & 1.0000 & $\odot .8044 \mathrm{E}+07$ \\
\hline 25 & 288.000 & 288.000 & 1.0000 & $\odot .8044 \mathrm{E}+\odot 7$ \\
\hline 26 & 288.000 & 288.000 & 1.0000 & $\odot .8044 \mathrm{E}+07$ \\
\hline 27 & 288.000 & $288.00 \odot$ & 1.0000 & $\odot .8044 \mathrm{E}+\odot 7$ \\
\hline 28 & 288.000 & 288.000 & 1.0000 & $\odot .8044 \mathrm{E}+07$ \\
\hline 29 & 288.000 & 288.000 & 1.0000 & $\odot .8044 \mathrm{E}+07$ \\
\hline 30 & $288.00 \odot$ & $288 . \odot \odot \odot$ & $1.000 \odot$ & $\odot .8 \odot 44 \mathrm{E}+07$ \\
\hline 31 & 288.000 & 288.000 & 1.0000 & $\odot .8044 \mathrm{E}+07$ \\
\hline 32 & 288.000 & 288.000 & 1.0000 & $\odot .8044 \mathrm{E}+\odot 7$ \\
\hline 33 & 288.000 & 288.000 & 1.0000 & $\odot .8044 \mathrm{E}+07$ \\
\hline 34 & 288.000 & 288.000 & 1.0000 & $\odot .8044 \mathrm{E}+07$ \\
\hline 35 & 288.000 & 288.000 & 1.0000 & $\odot .8044 \mathrm{E}+07$ \\
\hline 36 & $288.00 \odot$ & $288.00 \odot$ & $1.000 \odot$ & $\odot .8 \odot 44 \mathrm{E}+\odot 7$ \\
\hline 37 & 288.000 & 288.000 & 1.0000 & $\odot .8044 \mathrm{E}+07$ \\
\hline 38 & 288.000 & 288.000 & 1.0000 & $\odot .8044 \mathrm{E}+07$ \\
\hline 39 & 288.000 & 288.000 & 1.0000 & $\odot .8044 \mathrm{E}+\odot 7$ \\
\hline 40 & 288.000 & $288.00 \odot$ & 1.0000 & $\odot .8044 \mathrm{E}+\odot 7$ \\
\hline 41 & 288.000 & 288.000 & 1.0000 & $\odot .8044 \mathrm{E}+07$ \\
\hline 42 & $288.0 \odot \odot$ & $288 . \odot \odot \odot$ & 1.0000 & $\odot .8 \odot 44 \mathrm{E}+\odot 7$ \\
\hline 43 & 288.000 & $288.0 \odot \odot$ & 1.0000 & $\odot .8044 \mathrm{E}+\odot 7$ \\
\hline 44 & 288.000 & 288.000 & 1.0000 & $\odot .8044 \mathrm{E}+07$ \\
\hline 45 & 288.000 & 288.000 & 1.0000 & $\odot .8044 \mathrm{E}+07$ \\
\hline 46 & 288.000 & 288.000 & 1.0000 & $\odot .8044 \mathrm{E}+07$ \\
\hline 47 & 288.000 & 288.000 & 1.0000 & $\odot .8 \odot 44 \mathrm{E}+07$ \\
\hline 48 & $288.00 \odot$ & $288.00 \odot$ & 1.0000 & $\odot .8044 \mathrm{E}+07$ \\
\hline 49 & 288.000 & 288.000 & 1.0000 & $\odot .8044 \mathrm{E}+07$ \\
\hline
\end{tabular}




\begin{tabular}{|c|c|c|c|c|}
\hline 50 & 288.000 & $288.00 \odot$ & 1.0000 & $\odot .8044 \mathrm{E}+\odot 7$ \\
\hline 51 & $288.00 \odot$ & $288 . \odot \odot \odot$ & 1.0000 & $\odot .8 \odot 44 \mathrm{E}+\odot 7$ \\
\hline 52 & $288 . \odot \odot \odot$ & 288.000 & $1.000 \odot$ & $0.8044 \mathrm{E}+07$ \\
\hline 53 & $288.0 \odot \odot$ & $288.00 \odot$ & $1.000 \odot$ & $\odot .8 \odot 44 \mathrm{E}+\odot 7$ \\
\hline 54 & $288.00 \odot$ & $288.00 \odot$ & $1.00 \odot \odot$ & $\odot .8 \odot 44 \mathrm{E}+07$ \\
\hline 55 & $288.0 \odot \odot$ & $288 . \odot \odot \odot$ & 1.0000 & $\odot .8044 \mathrm{E}+$ \\
\hline 56 & $288.0 \odot \odot$ & $288.00 \odot$ & $1.000 \odot$ & $\odot .8044 \mathrm{E}+\mathrm{C}$ \\
\hline 57 & $288.00 \odot$ & $288.00 \odot$ & $1.00 \odot \odot$ & $0.8044 \mathrm{E}+\mathrm{C}$ \\
\hline 58 & $288.00 \odot$ & $288 . \odot \odot \odot$ & 1.0000 & $\odot .8044 \mathrm{E}+\mathrm{C}$ \\
\hline 59 & 288.000 & 288.000 & 1.0000 & $0.8044 \mathrm{E}+\Theta$ \\
\hline 60 & 288.000 & $288 . \odot \odot \odot$ & $1.000 \odot$ & $0.8044 \mathrm{E}+\mathrm{C}$ \\
\hline
\end{tabular}

\begin{tabular}{|c|c|c|c|c|}
\hline $\begin{array}{l}\text { time } \\
\text { node }\end{array}$ & $\begin{array}{r}(\mathrm{sec})= \\
\mathrm{T}(\mathrm{K})\end{array}$ & $\begin{array}{l}2.00 \text { lo } \\
\text { Twall(K) }\end{array}$ & $l a=0$. & $\begin{array}{r}1606953 \\
P(P a)\end{array}$ \\
\hline 1 & 138.384 & 212.526 & $\ldots$ & $0.9121 \mathrm{E}+06$ \\
\hline 2 & 160.525 & 249.066 & $\odot .9951$ & $\odot .1682 \mathrm{E}+\odot 7$ \\
\hline 3 & 221.984 & 278.041 & $1.000 \odot$ & $\odot .5193 \mathrm{E}+\odot 7$ \\
\hline 4 & 259.419 & 286.509 & 1.0000 & $\odot .65 \odot 4 \mathrm{E}+\odot 7$ \\
\hline 5 & 282.437 & 288.000 & 1.0000 & $\odot .7548 \mathrm{E}+\odot 7$ \\
\hline 6 & 287.646 & 288.000 & 1.0000 & $\odot .7966 \mathrm{E}+\odot 7$ \\
\hline 7 & 288.000 & $288.0 \odot \odot$ & 1.0000 & $\odot .8040 \mathrm{E}+\odot 7$ \\
\hline 8 & 288.000 & $288.0 \odot \odot$ & 1.0000 & $\odot .8044 \mathrm{E}+07$ \\
\hline 9 & 288.000 & 288.000 & 1.0000 & $\odot .8044 \mathrm{E}+\odot 7$ \\
\hline 10 & $288.00 \odot$ & 288.000 & $1.000 \odot$ & $\odot .8 \odot 44 \mathrm{E}+\odot 7$ \\
\hline 11 & 288.000 & 288.000 & 1.0000 & $\odot .8 \odot 44 \mathrm{E}+\odot 7$ \\
\hline 12 & $288.00 \odot$ & $288 . \odot \odot \odot$ & $1.000 \odot$ & $\odot .8 \odot 44 \mathrm{E}+07$ \\
\hline 13 & $288.00 \odot$ & $288 . \odot \odot \odot$ & $1.000 \odot$ & $\odot .8044 \mathrm{E}+07$ \\
\hline 14 & 288.000 & $288.0 \odot \odot$ & 1.0000 & $\odot .8044 \mathrm{E}+\odot 7$ \\
\hline 15 & 288.000 & $288.00 \odot$ & 1.0000 & $\odot .8 \odot 44 \mathrm{E}+\odot 7$ \\
\hline 16 & $288.00 \odot$ & $288 . \odot \odot \odot$ & 1.0000 & $\odot .8 \odot 44 \mathrm{E}+\odot 7$ \\
\hline 17 & 288.000 & $288.00 \odot$ & 1.0000 & $\odot .8044 \mathrm{E}+07$ \\
\hline 18 & $288.00 \odot$ & $288.0 \odot \odot$ & $1.000 \odot$ & $\odot .8 \odot 44 \mathrm{E}+\odot 7$ \\
\hline 19 & 288.000 & $288.0 \odot \odot$ & $1.000 \odot$ & $\odot .8 \odot 44 \mathrm{E}+07$ \\
\hline 20 & 288.000 & $288 . \odot \odot \odot$ & $1.000 \odot$ & $\odot .8044 \mathrm{E}+\odot 7$ \\
\hline 21 & $288.0 \odot \odot$ & $288 . \odot \odot \odot$ & 1.0000 & $\odot .8 \odot 44 \mathrm{E}+\odot 7$ \\
\hline 22 & 288.000 & 288.000 & 1.0000 & $0.8044 \mathrm{E}+07$ \\
\hline 23 & 288.000 & 288.000 & 1.0000 & $\odot .8044 \mathrm{E}+07$ \\
\hline 24 & 288.000 & 288.000 & 1.0000 & $\odot .8044 \mathrm{E}+07$ \\
\hline 25 & 288.000 & $288.0 \odot \odot$ & 1.0000 & $\odot .8044 \mathrm{E}+\odot 7$ \\
\hline 26 & $288.00 \odot$ & $288 . \odot \odot \odot$ & 1.0000 & $\odot .8044 \mathrm{E}+\odot 7$ \\
\hline 27 & $288.00 \odot$ & $288 . \odot \odot \odot$ & 1.0000 & $\odot .8 \odot 44 \mathrm{E}+\odot 7$ \\
\hline 28 & 288.000 & $288.0 \odot \odot$ & 1.0000 & $\odot .8044 \mathrm{E}+07$ \\
\hline 29 & $288.00 \odot$ & $288.0 \odot \odot$ & $1.000 \odot$ & $\odot .8 \odot 44 \mathrm{E}+07$ \\
\hline 30 & 288.000 & $288 . \odot \odot \odot$ & 1.0000 & $\odot .8044 \mathrm{E}+07$ \\
\hline 31 & $288.0 \odot \odot$ & $288 . \odot \odot \odot$ & 1.0000 & $\odot .8044 \mathrm{E}+\odot 7$ \\
\hline 32 & 288.000 & 288.000 & 1.0000 & $\odot .8 \odot 44 \mathrm{E}+07$ \\
\hline 33 & $288.00 \odot$ & $288 . \odot \odot \odot$ & 1.0000 & $\odot .8 \odot 44 \mathrm{E}+\odot 7$ \\
\hline 34 & 288.000 & 288.000 & 1.0000 & $\odot .8044 \mathrm{E}+07$ \\
\hline 35 & $288 . \odot \odot \odot$ & $288 . \odot \odot \odot$ & 1.0000 & $\odot .8 \odot 44 \mathrm{E}+07$ \\
\hline 36 & 288.000 & $288.00 \odot$ & 1.0000 & $\odot .8044 \mathrm{E}+\odot 7$ \\
\hline 37 & 288.000 & 288.000 & 1.0000 & $\odot .8044 \mathrm{E}+\odot 7$ \\
\hline 38 & 288.000 & $288 . \odot \odot \odot$ & 1.0000 & $\odot .8044 \mathrm{E}+\odot 7$ \\
\hline 39 & 288.000 & $288.00 \odot$ & 1.0000 & $\odot .8044 \mathrm{E}+\odot 7$ \\
\hline 40 & 288.000 & $288.0 \odot \odot$ & 1.0000 & $\odot .8044 \mathrm{E}+07$ \\
\hline 41 & $288.00 \odot$ & $288 . \odot \odot \odot$ & $1.000 \odot$ & $\odot .8044 \mathrm{E}+07$ \\
\hline 42 & 288.000 & 288.000 & 1.0000 & $\odot .8044 \mathrm{E}+\odot 7$ \\
\hline 43 & 288.000 & 288.000 & 1.0000 & $\odot .8044 \mathrm{E}+07$ \\
\hline
\end{tabular}




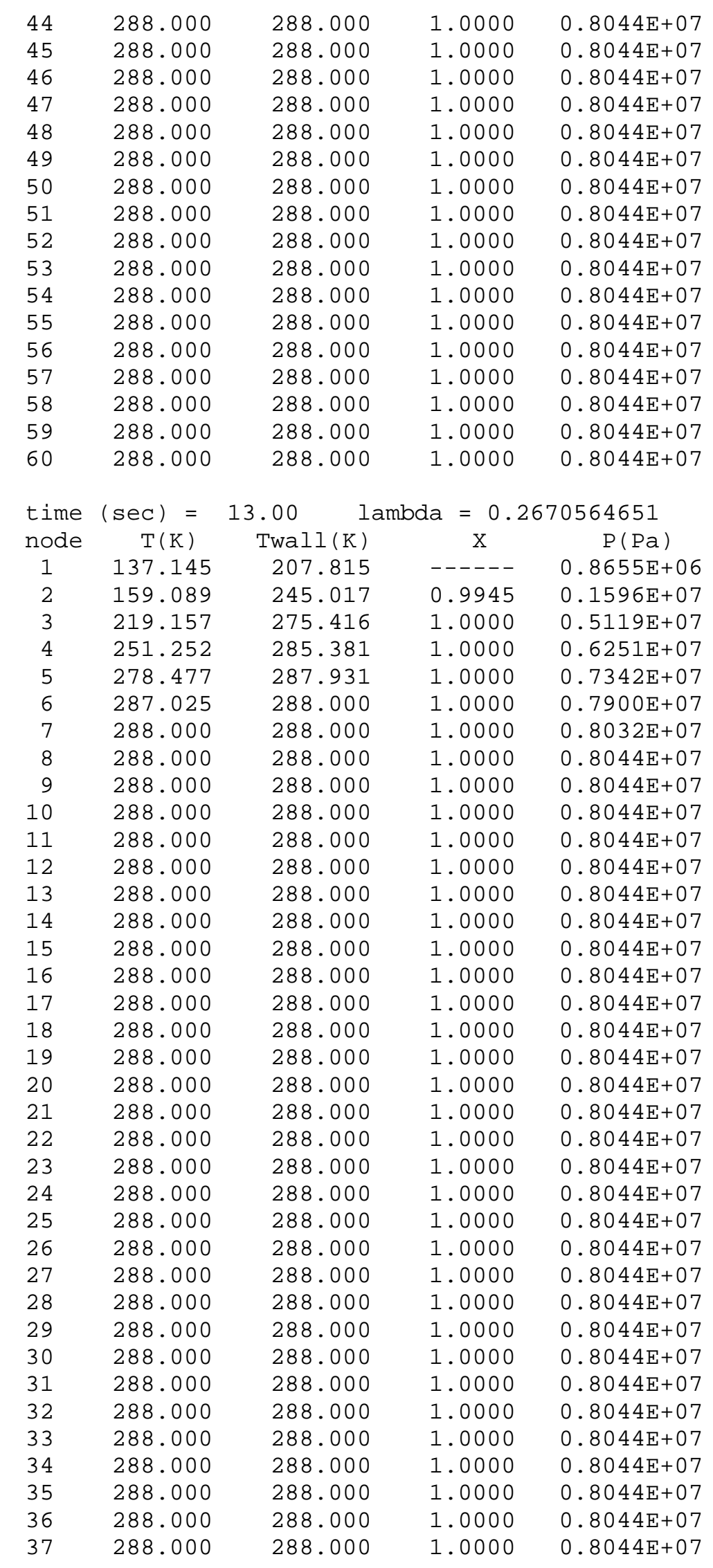




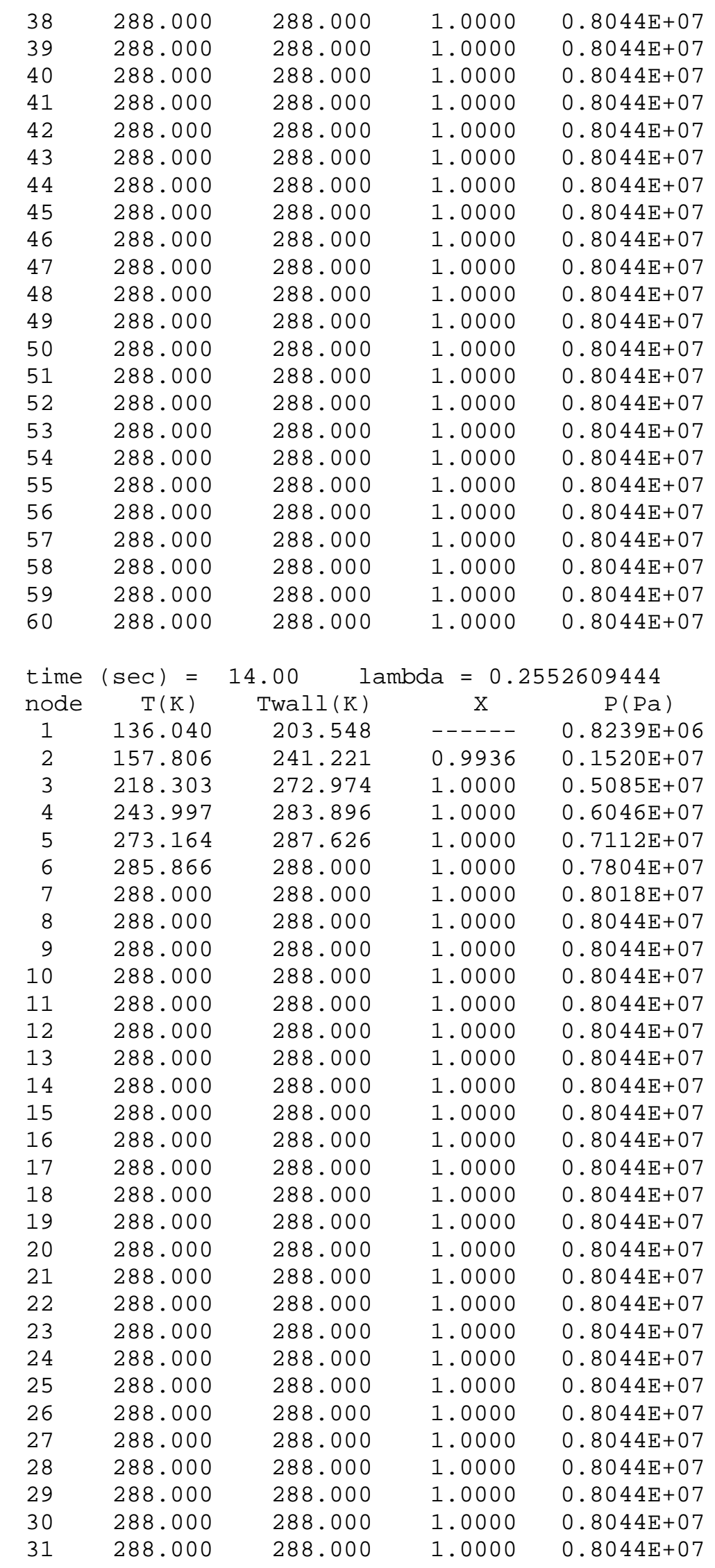




\begin{tabular}{|c|c|c|c|c|}
\hline 32 & 288.000 & $288.00 \odot$ & 1.0000 & $0.8044 \mathrm{E}+07$ \\
\hline 33 & $288.00 \odot$ & 288.000 & $1.000 \odot$ & $\odot .8044 \mathrm{E}+07$ \\
\hline 34 & 288.000 & $288.00 \odot$ & 1.0000 & $0.8044 \mathrm{E}+07$ \\
\hline 35 & 288.000 & 288.000 & 1.0000 & $\odot .8044 \mathrm{E}+07$ \\
\hline 36 & 288.000 & 288.000 & 1.0000 & $\odot .8044 \mathrm{E}+07$ \\
\hline 37 & $288.00 \odot$ & $288.0 \odot \odot$ & $1.000 \odot$ & $\odot .8044 \mathrm{E}+\odot 7$ \\
\hline 38 & 288.000 & $288.00 \odot$ & 1.0000 & $0.8044 \mathrm{E}+07$ \\
\hline 39 & 288.000 & $288.0 \odot \odot$ & $1.000 \odot$ & $\odot .8044 \mathrm{E}+07$ \\
\hline 40 & 288.000 & 288.000 & 1.0000 & $0.8044 \mathrm{E}+07$ \\
\hline 41 & $288.00 \odot$ & $288.0 \odot \odot$ & $1.000 \odot$ & $\odot .8044 \mathrm{E}+\odot 7$ \\
\hline 42 & 288.000 & 288.000 & 1.0000 & $0.8044 \mathrm{E}+07$ \\
\hline 43 & 288.000 & 288.000 & 1.0000 & $0.8044 \mathrm{E}+07$ \\
\hline 44 & 288.000 & 288.000 & 1.0000 & $0.8044 \mathrm{E}+07$ \\
\hline 45 & 288.000 & $288.0 \odot \odot$ & 1.0000 & $0.8044 \mathrm{E}+07$ \\
\hline 46 & 288.000 & 288.000 & 1.0000 & $0.8044 \mathrm{E}+07$ \\
\hline 47 & $288.00 \odot$ & $288.00 \odot$ & $1.000 \odot$ & $\odot .8044 \mathrm{E}+07$ \\
\hline 48 & $288.00 \odot$ & $288.0 \odot \odot$ & $1.000 \odot$ & $\odot .8044 \mathrm{E}+\odot 7$ \\
\hline 49 & 288.000 & 288.000 & 1.0000 & $0.8044 \mathrm{E}+07$ \\
\hline 50 & 288.000 & 288.000 & 1.0000 & $\odot .8044 \mathrm{E}+07$ \\
\hline 51 & 288.000 & 288.000 & 1.0000 & $0.8044 \mathrm{E}+07$ \\
\hline 52 & 288.000 & 288.000 & 1.0000 & $0.8044 \mathrm{E}+07$ \\
\hline 53 & $288.00 \odot$ & $288.0 \odot \odot$ & $1.0 \odot \odot \odot$ & $\odot .8044 \mathrm{E}+\odot 7$ \\
\hline 54 & 288.000 & 288.000 & 1.0000 & $0.8044 \mathrm{E}+07$ \\
\hline 55 & 288.000 & 288.000 & 1.0000 & $0.8044 \mathrm{E}+07$ \\
\hline 56 & 288.000 & 288.000 & 1.0000 & $0.8044 \mathrm{E}+07$ \\
\hline 57 & 288.000 & 288.000 & 1.0000 & $0.8044 \mathrm{E}+07$ \\
\hline 58 & 88.000 & 288.000 & 1.0000 & $0.8044 \mathrm{E}+07$ \\
\hline 59 & $288.00 \odot$ & 288.000 & 1.0000 & $0.8044 \mathrm{E}+07$ \\
\hline 60 & $288.00 \odot$ & $288.0 \odot \odot$ & $1.000 \odot$ & $\odot .8044 \mathrm{E}+\odot 7$ \\
\hline time & & 15.00 & \multicolumn{2}{|c|}{ lambda $=0.2447123379$} \\
\hline node & $\mathrm{T}(\mathrm{K})$ & Twall(K) & $\mathrm{x}$ & $P(P a)$ \\
\hline 1 & 135.058 & 199.661 & $--\cdots$ & $\odot .7870 \mathrm{E}+06$ \\
\hline 2 & 156.667 & 237.637 & 0.9924 & $0.1452 \mathrm{E}+\Theta 7$ \\
\hline 3 & 218.757 & $27 \odot .700$ & 1.0000 & $0.5076 \mathrm{E}+07$ \\
\hline 4 & 238.470 & 282.117 & 1.0000 & $\odot .5903 \mathrm{E}+\odot 7$ \\
\hline 5 & 266.764 & 287.151 & 1.0000 & $0.6873 \mathrm{E}+07$ \\
\hline 6 & 283.900 & 288.000 & 1.0000 & $\odot .7672 \mathrm{E}+07$ \\
\hline 7 & 287.762 & $288.00 \odot$ & $1.000 \odot$ & $\odot .7987 \mathrm{E}+\odot 7$ \\
\hline 8 & $\odot \odot$ & $288.00 \odot$ & 1.00 & $0.8042 \mathrm{E}+\odot 7$ \\
\hline 9 & 288.000 & 288.000 & 1.0000 & $\odot .8044 \mathrm{E}+07$ \\
\hline 10 & $288.00 \odot$ & $288.00 \odot$ & $1.000 \odot$ & $\odot .8044 \mathrm{E}+\odot 7$ \\
\hline 11 & $288.00 \odot$ & $288.00 \odot$ & $1.000 \odot$ & $0.8044 \mathrm{E}+\odot 7$ \\
\hline 12 & 288.000 & 288.000 & 1.0000 & $0.8044 \mathrm{E}+07$ \\
\hline 13 & $288.00 \odot$ & $288.00 \odot$ & $1.00 \odot \odot$ & $0.8044 \mathrm{E}+\odot 7$ \\
\hline 14 & 288.0 & $288.00 \odot$ & 1.00 & $0.8044 \mathrm{E}+\odot 7$ \\
\hline 15 & 288.000 & 288.000 & 1.0000 & $\odot .8044 \mathrm{E}+07$ \\
\hline 16 & 288.000 & 288.000 & 1.0000 & $0.8044 \mathrm{E}+07$ \\
\hline 17 & 288.000 & 288.000 & 1.0000 & $0.8044 \mathrm{E}+07$ \\
\hline 18 & 288.000 & 288.000 & $1.000 \odot$ & $\odot .8044 \mathrm{E}+\odot 7$ \\
\hline 19 & $288.00 \odot$ & $288.00 \odot$ & $1.000 \odot$ & $0.8044 \mathrm{E}+07$ \\
\hline 20 & $288 . \odot \odot \odot$ & $288.00 \odot$ & $1.0 \odot \odot \odot$ & $\odot .8044 \mathrm{E}+\odot 7$ \\
\hline 21 & $288.00 \odot$ & $288.00 \odot$ & $1.000 \odot$ & $\odot .8044 \mathrm{E}+\odot 7$ \\
\hline 22 & 288.000 & 288.000 & 1.0000 & $\odot .8044 \mathrm{E}+07$ \\
\hline 23 & 288.000 & 288.000 & 1.0000 & $0.8044 \mathrm{E}+07$ \\
\hline 24 & 288.000 & $288.00 \odot$ & 1.0000 & $0.8044 \mathrm{E}+07$ \\
\hline 25 & & 88.000 & $1.000 \odot$ & $0.8044 \mathrm{E}+07$ \\
\hline
\end{tabular}




\begin{tabular}{|c|c|c|c|c|}
\hline 26 & 288.000 & $288.00 \odot$ & 1.0000 & $0.8044 \mathrm{E}+07$ \\
\hline 27 & $288.00 \odot$ & 288.000 & $1.000 \odot$ & $\odot .8044 \mathrm{E}+07$ \\
\hline 28 & 288.000 & $288.00 \odot$ & 1.0000 & $0.8044 \mathrm{E}+07$ \\
\hline 29 & 288.000 & 288.000 & 1.0000 & $\odot .8044 \mathrm{E}+07$ \\
\hline 30 & 288.000 & 288.000 & 1.0000 & $\odot .8044 \mathrm{E}+07$ \\
\hline 31 & 288.000 & 288.000 & $1.000 \odot$ & $\odot .8044 \mathrm{E}+\odot 7$ \\
\hline 32 & 288.000 & $288.00 \odot$ & 1.0000 & $0.8044 \mathrm{E}+07$ \\
\hline 33 & 288.000 & $288.0 \odot \odot$ & $1.000 \odot$ & $\odot .8044 \mathrm{E}+07$ \\
\hline 34 & 288.000 & 288.000 & 1.0000 & $0.8044 \mathrm{E}+07$ \\
\hline 35 & $288.00 \odot$ & $288.0 \odot \odot$ & $1.000 \odot$ & $\odot .8044 \mathrm{E}+\odot 7$ \\
\hline 36 & 288.000 & 288.000 & 1.0000 & $0.8044 \mathrm{E}+07$ \\
\hline 37 & 288.000 & 288.000 & 1.0000 & $0.8044 \mathrm{E}+07$ \\
\hline 38 & 288.000 & 288.000 & 1.0000 & $0.8044 \mathrm{E}+07$ \\
\hline 39 & 288.000 & $288.0 \odot \odot$ & 1.0000 & $0.8044 \mathrm{E}+07$ \\
\hline 40 & 288.000 & 288.000 & 1.0000 & $0.8044 \mathrm{E}+07$ \\
\hline 41 & $288.00 \odot$ & $288.00 \odot$ & $1.000 \odot$ & $\odot .8044 \mathrm{E}+07$ \\
\hline 42 & $288.00 \odot$ & $288.0 \odot \odot$ & $1.000 \odot$ & $\odot .8044 \mathrm{E}+\odot 7$ \\
\hline 43 & 288.000 & 288.000 & 1.0000 & $0.8044 \mathrm{E}+07$ \\
\hline 44 & 288.000 & 288.000 & 1.0000 & $\odot .8044 \mathrm{E}+07$ \\
\hline 45 & 288.000 & 288.000 & 1.0000 & $0.8044 \mathrm{E}+07$ \\
\hline 46 & 288.000 & 288.000 & 1.0000 & $0.8044 \mathrm{E}+07$ \\
\hline 47 & $288.00 \odot$ & $288.0 \odot \odot$ & $1.0 \odot \odot \odot$ & $\odot .8044 \mathrm{E}+\odot 7$ \\
\hline 48 & 288.000 & 288.000 & 1.0000 & $0.8044 \mathrm{E}+07$ \\
\hline 49 & 288.000 & 288.000 & 1.0000 & $0.8044 \mathrm{E}+07$ \\
\hline 50 & 288.000 & 288.000 & 1.0000 & $0.8044 \mathrm{E}+07$ \\
\hline 51 & 288.000 & 288.000 & 1.0000 & $0.8044 \mathrm{E}+07$ \\
\hline 52 & 288.000 & 288.000 & 1.0000 & $0.8044 \mathrm{E}+07$ \\
\hline 53 & $288.00 \odot$ & 288.000 & 1.0000 & $0.8044 \mathrm{E}+07$ \\
\hline 54 & $288.00 \odot$ & $288.0 \odot \odot$ & $1.000 \odot$ & $\odot .8044 \mathrm{E}+\odot 7$ \\
\hline 55 & 288.000 & 288.000 & 1.0000 & $\odot .8044 \mathrm{E}+07$ \\
\hline 56 & 288.000 & 288.000 & 1.0000 & $0.8044 \mathrm{E}+07$ \\
\hline 57 & 288.000 & 288.000 & 1.0000 & $0.8044 \mathrm{E}+07$ \\
\hline 58 & 88.000 & $288.00 \odot$ & 1.0000 & $\odot .8044 \mathrm{E}+\odot 7$ \\
\hline 59 & 38.000 & 288.000 & 1.0000 & $0.8044 \mathrm{E}+07$ \\
\hline 60 & $288.00 \odot$ & $288.00 \odot$ & 1.0000 & $0.8044 \mathrm{E}+07$ \\
\hline time & ec) $=$ & 16.00 & \multicolumn{2}{|c|}{ lambda $=0.2353087068$} \\
\hline node & $\mathrm{T}(\mathrm{K})$ & Twall(K) & $\mathrm{x}$ & $P(P a)$ \\
\hline 1 & 134.188 & 196.102 & $-\ldots$ & $0.7543 \mathrm{E}+06$ \\
\hline 2 & 155.658 & 234.237 & 0.9912 & $\odot .1391 \mathrm{E}+07$ \\
\hline 3 & 219.924 & 268.669 & 1.0000 & $\odot .5075 \mathrm{E}+\Theta 7$ \\
\hline 4 & 234.958 & 280.286 & 1.0000 & $\odot .5816 \mathrm{E}+07$ \\
\hline 5 & 259.876 & 286.408 & 1.0000 & $0.6645 \mathrm{E}+07$ \\
\hline 6 & 280.886 & 288.000 & 1.0000 & $0.7506 \mathrm{E}+07$ \\
\hline 7 & 287.322 & $288.00 \odot$ & $1.00 \odot \odot$ & $\odot .7938 \mathrm{E}+\odot 7$ \\
\hline 8 & 288.0 & $288.00 \odot$ & 1.0000 & $0.8036 \mathrm{E}+\odot 7$ \\
\hline 9 & 288.000 & 288.000 & 1.0000 & $\odot .8044 \mathrm{E}+07$ \\
\hline 10 & 288.000 & 288.000 & 1.0000 & $0.8044 \mathrm{E}+07$ \\
\hline 11 & 288.000 & 288.000 & 1.0000 & $0.8044 \mathrm{E}+07$ \\
\hline 12 & $288.00 \odot$ & $288.00 \odot$ & $1.000 \odot$ & $0.8044 \mathrm{E}+\odot 7$ \\
\hline 13 & $288.00 \odot$ & 288.000 & 1.0000 & $0.8044 \mathrm{E}+07$ \\
\hline 14 & $288.00 \odot$ & $288.00 \odot$ & $1.000 \odot$ & $\odot .8044 \mathrm{E}+\odot 7$ \\
\hline 15 & $288.00 \odot$ & $288.00 \odot$ & $1.000 \odot$ & $\odot .8044 \mathrm{E}+\odot 7$ \\
\hline 16 & 288.000 & 288.000 & 1.0000 & $\odot .8044 \mathrm{E}+07$ \\
\hline 17 & 288.000 & 288.000 & 1.0000 & $0.8044 \mathrm{E}+07$ \\
\hline 18 & 288.000 & $288.00 \odot$ & 1.0000 & $0.8044 \mathrm{E}+07$ \\
\hline 19 & & 288.000 & 1.0000 & $\odot .8044 \mathrm{E}+07$ \\
\hline
\end{tabular}




\begin{tabular}{|c|c|c|c|c|}
\hline 20 & $288.00 \odot$ & $288.00 \odot$ & $1.0 \odot \odot \odot$ & $0.8044 \mathrm{E}+\odot 7$ \\
\hline 21 & 288.000 & 288.000 & 1.0000 & $0.8044 \mathrm{E}+07$ \\
\hline 22 & 288.000 & $288 . \odot \odot \odot$ & $1.00 \odot \odot$ & $0.8044 \mathrm{E}+07$ \\
\hline 23 & $288.00 \odot$ & $288.00 \odot$ & $1.00 \odot \odot$ & $\odot .8044 \mathrm{E}+\odot 7$ \\
\hline 24 & $288.00 \odot$ & $288.0 \odot \odot$ & 1. $0 \odot \odot \odot$ & $\odot .8044 \mathrm{E}+07$ \\
\hline 25 & 288.000 & $288.00 \odot$ & $1.000 \odot$ & $\odot .8 \odot 44 \mathrm{E}+\odot 7$ \\
\hline 26 & $288.00 \odot$ & $288.00 \odot$ & $1.00 \odot \odot$ & $\odot .8044 \mathrm{E}+07$ \\
\hline 27 & 288.000 & 288.000 & 1.0000 & $0.8044 \mathrm{E}+07$ \\
\hline 28 & $288.00 \odot$ & $288.00 \odot$ & $1.000 \odot$ & $\odot .8044 \mathrm{E}+07$ \\
\hline 29 & $288.00 \odot$ & $288.00 \odot$ & $1.000 \odot$ & $0.8044 \mathrm{E}+07$ \\
\hline 30 & $288.00 \odot$ & $288.00 \odot$ & $1.00 \odot \odot$ & $\odot .8044 \mathrm{E}+07$ \\
\hline 31 & 288.000 & 288.000 & $1.000 \odot$ & $0.8044 \mathrm{E}+07$ \\
\hline 32 & 288.000 & $288.0 \odot \odot$ & $1.00 \odot \odot$ & $0.8044 \mathrm{E}+07$ \\
\hline 33 & $288.00 \odot$ & $288.00 \odot$ & 1.0000 & $\odot .8044 \mathrm{E}+07$ \\
\hline 34 & 288.000 & $288.0 \odot \odot$ & $1.000 \odot$ & $0.8044 \mathrm{E}+07$ \\
\hline 35 & $288.00 \odot$ & $288.0 \odot \odot$ & $1.000 \odot$ & $\odot .8044 \mathrm{E}+07$ \\
\hline 36 & 288.000 & $288 . \odot \odot \odot$ & $1.000 \odot$ & $\odot .8044 \mathrm{E}+\odot 7$ \\
\hline 37 & $288.0 \odot \odot$ & $288.0 \odot \odot$ & $1.00 \odot \odot$ & $\odot .8 \odot 44 \mathrm{E}+\odot 7$ \\
\hline 38 & 288.000 & $288.00 \odot$ & 1.0000 & $0.8044 \mathrm{E}+07$ \\
\hline 39 & 288.000 & $288.0 \odot \odot$ & $1.000 \odot$ & $\odot .8044 \mathrm{E}+07$ \\
\hline 40 & $288.00 \odot$ & $288.0 \odot \odot$ & $1.000 \odot$ & $\odot .8 \odot 44 \mathrm{E}+07$ \\
\hline 41 & $288.0 \odot \odot$ & $288.0 \odot \odot$ & $1.00 \odot \odot$ & $\odot .8044 \mathrm{E}+07$ \\
\hline 42 & 288.000 & $288.00 \odot$ & 1.0000 & $\odot .8 \odot 44 \mathrm{E}+\odot 7$ \\
\hline 43 & $288.00 \odot$ & $288.00 \odot$ & $1.00 \odot \odot$ & $0.8044 \mathrm{E}+07$ \\
\hline 44 & 288.000 & 288.000 & 1.0000 & $0.8044 \mathrm{E}+07$ \\
\hline 45 & 288.000 & $288.0 \odot \odot$ & 1.0000 & $0.8044 \mathrm{E}+07$ \\
\hline 46 & 288.000 & $288.0 \odot \odot$ & 1.0000 & $\odot .8044 \mathrm{E}+\odot 7$ \\
\hline 47 & $288 . \odot \odot \odot$ & $288.0 \odot \odot$ & $1.00 \odot \odot$ & $\odot .8 \odot 44 \mathrm{E}+\odot 7$ \\
\hline 48 & $288.0 \odot \odot$ & $288.0 \odot \odot$ & $1.00 \odot \odot$ & $\odot .8 \odot 44 \mathrm{E}+\odot 7$ \\
\hline 49 & $288.00 \odot$ & $288.00 \odot$ & $1.00 \odot \odot$ & $\odot .8044 \mathrm{E}+07$ \\
\hline 50 & $288.00 \odot$ & $288.0 \odot \odot$ & $1 . \odot \odot \odot \odot$ & $\odot .8044 \mathrm{E}+\odot 7$ \\
\hline 51 & $288.0 \odot \odot$ & $288.0 \odot \odot$ & $1 . \odot \odot \odot \odot$ & $\odot .8 \odot 44 \mathrm{E}+\odot 7$ \\
\hline 52 & 288.000 & 288.000 & 1.0000 & $\odot .8044 \mathrm{E}+07$ \\
\hline 53 & $288.00 \odot$ & $288.00 \odot$ & $1.0 \odot \odot \odot$ & $0.8044 \mathrm{E}+07$ \\
\hline 54 & $288 . \odot \odot \odot$ & $288.00 \odot$ & $1.00 \odot \odot$ & $\odot .8 \odot 44 \mathrm{E}+07$ \\
\hline 55 & $288.00 \odot$ & $288.0 \odot \odot$ & $1.000 \odot$ & $0.8044 \mathrm{E}+07$ \\
\hline 56 & $288.00 \odot$ & $288.00 \odot$ & $1.0 \odot \odot \odot$ & $\odot .8044 \mathrm{E}+\odot 7$ \\
\hline 57 & $288.00 \odot$ & $288.00 \odot$ & $1.00 \odot \odot$ & $\odot .8044 \mathrm{E}+\odot 7$ \\
\hline 58 & $288.0 \odot \odot$ & $288 . \odot \odot \odot$ & $1.00 \odot \odot$ & $\odot .8 \odot 44 \mathrm{E}+\odot 7$ \\
\hline 59 & $288.00 \odot$ & $288.0 \odot \odot$ & $1.000 \odot$ & $\odot .8044 \mathrm{E}+\odot 7$ \\
\hline 60 & $288.00 \odot$ & $288.0 \odot \odot$ & $1.0 \odot \odot \odot$ & $\odot .8044 \mathrm{E}+\odot 7$ \\
\hline time & $(\sec )=$ & $17.00 \quad 1$ & $\mathrm{da}=0$. & 69305140 \\
\hline node & $\mathrm{T}(\mathrm{K})$ & Twall(K) & $x$ & $\mathrm{P}(\mathrm{Pa})$ \\
\hline 1 & 133.418 & 192.826 & $\ldots$ & $0.7254 \mathrm{E}+06$ \\
\hline 2 & 154.765 & 231.011 & 0.9901 & $0.1338 \mathrm{E}+07$ \\
\hline 3 & 221.263 & 266.838 & $1.00 \odot \odot$ & $0.5071 \mathrm{E}+07$ \\
\hline 4 & 233.384 & 278.455 & 1.0000 & $\odot .5777 \mathrm{E}+\odot 7$ \\
\hline 5 & 253.332 & 285.378 & 1.0000 & $\odot .6450 \mathrm{E}+07$ \\
\hline 6 & 276.699 & 287.820 & $1.00 \odot \odot$ & $0.7313 \mathrm{E}+07$ \\
\hline 7 & 286.473 & $288.0 \odot \odot$ & $1.0 \odot \odot \odot$ & $\odot .7863 \mathrm{E}+07$ \\
\hline 8 & $288 . \odot \odot \odot$ & 288.000 & 1.0000 & $\odot .8 \odot 26 \mathrm{E}+\odot 7$ \\
\hline 9 & 288.000 & $288 . \odot \odot \odot$ & $1.00 \odot \odot$ & $\odot .8044 \mathrm{E}+\odot 7$ \\
\hline 10 & 288.000 & 288.000 & 1.0000 & $\odot .8044 \mathrm{E}+07$ \\
\hline 11 & 288.000 & $288.00 \odot$ & $1.000 \odot$ & $0.8044 \mathrm{E}+07$ \\
\hline 12 & 288.000 & $288.00 \odot$ & 1.0000 & $\odot .8044 \mathrm{E}+07$ \\
\hline 13 & $288.00 \odot$ & $288.00 \odot$ & $1.0 \odot \odot \odot$ & $\odot .8044 \mathrm{E}+07$ \\
\hline
\end{tabular}




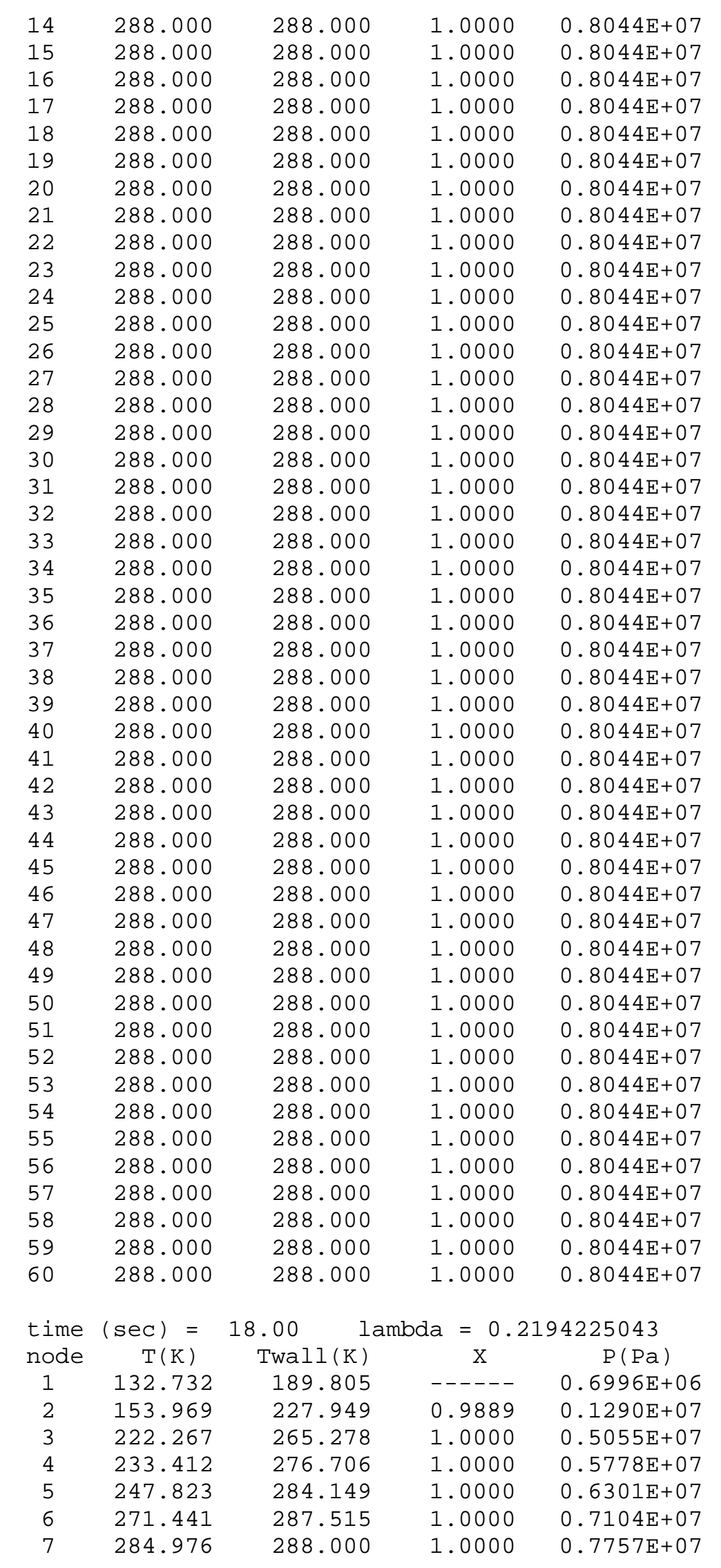




\begin{tabular}{|c|c|c|c|c|}
\hline 8 & 287.895 & 288.000 & $1.0 \odot \odot \odot$ & $\odot .80 \odot 4 \mathrm{E}+\odot 7$ \\
\hline 9 & $288.0 \odot \odot$ & 288.000 & $1.000 \odot$ & $\odot .8044 \mathrm{E}+07$ \\
\hline 10 & $288 . \odot \odot \odot$ & $288.00 \odot$ & $1.000 \odot$ & $\odot .8 \odot 44 \mathrm{E}+\odot 7$ \\
\hline 11 & $288.00 \odot$ & $288.00 \odot$ & $1.00 \odot \odot$ & $\odot .8 \odot 44 \mathrm{E}+\odot 7$ \\
\hline 12 & 288.000 & $288.00 \odot$ & 1.0000 & $\odot .8044 \mathrm{E}+07$ \\
\hline 13 & $288.0 \odot \odot$ & $288.00 \odot$ & $1.00 \odot \odot$ & $\odot .8 \odot 44 \mathrm{E}+\odot 7$ \\
\hline 14 & $288 . \odot \odot \odot$ & $288.00 \odot$ & $1.00 \odot \odot$ & $\odot .8 \odot 44 \mathrm{E}+\odot 7$ \\
\hline 15 & $288.0 \odot \odot$ & 288.000 & 1.0000 & $\odot .8044 \mathrm{E}+07$ \\
\hline 16 & $288.0 \odot \odot$ & $288.00 \odot$ & $1.000 \odot$ & $\odot .8 \odot 44 \mathrm{E}+\odot 7$ \\
\hline 17 & $288.00 \odot$ & 288.000 & 1.0000 & $\odot .8044 \mathrm{E}+07$ \\
\hline 18 & $288 . \odot \odot \odot$ & $288.00 \odot$ & $1.00 \odot \odot$ & $\odot .8 \odot 44 \mathrm{E}+\odot 7$ \\
\hline 19 & $288.0 \odot \odot$ & 288.000 & $1.000 \odot$ & $\odot .8044 \mathrm{E}+\odot 7$ \\
\hline 20 & $288.0 \odot \odot$ & $288.00 \odot$ & $1.00 \odot \odot$ & $\odot .8 \odot 44 \mathrm{E}+\odot 7$ \\
\hline 21 & 288.000 & 288.000 & $1.00 \odot \odot$ & $\odot .8 \odot 44 \mathrm{E}+\odot 7$ \\
\hline 22 & $288.00 \odot$ & $288.0 \odot \odot$ & $1.000 \odot$ & $\odot .8044 \mathrm{E}+\odot 7$ \\
\hline 23 & $288.0 \odot \odot$ & 288.000 & $1.000 \odot$ & $\odot .8044 \mathrm{E}+\odot 7$ \\
\hline 24 & $288.0 \odot \odot$ & $288 . \odot \odot \odot$ & $1.00 \odot \odot$ & $\odot .8 \odot 44 \mathrm{E}+\odot 7$ \\
\hline 25 & $288.0 \odot \odot$ & $288.00 \odot$ & 1.0000 & $\odot .8044 \mathrm{E}+07$ \\
\hline 26 & $288.00 \odot$ & $288.00 \odot$ & $1.000 \odot$ & $\odot .8044 \mathrm{E}+\odot 7$ \\
\hline 27 & $288.0 \odot \odot$ & $288 . \odot \odot \odot$ & $1.00 \odot \odot$ & $\odot .8 \odot 44 \mathrm{E}+\odot 7$ \\
\hline 28 & $288.0 \odot \odot$ & $288.00 \odot$ & 1.0000 & $\odot .8044 \mathrm{E}+07$ \\
\hline 29 & $288 . \odot \odot \odot$ & $288 . \odot \odot \odot$ & $1.000 \odot$ & $\odot .8044 \mathrm{E}+07$ \\
\hline 30 & $288.0 \odot \odot$ & $288 . \odot \odot \odot$ & $1.000 \odot$ & $\odot .8044 \mathrm{E}+\odot 7$ \\
\hline 31 & $288.00 \odot$ & $288.0 \odot \odot$ & $1.000 \odot$ & $\odot .8 \odot 44 \mathrm{E}+07$ \\
\hline 32 & $288 . \odot \odot \odot$ & $288.0 \odot \odot$ & 1.0000 & $\odot .8044 \mathrm{E}+07$ \\
\hline 33 & $288.00 \odot$ & $288.0 \odot \odot$ & $1.000 \odot$ & $\odot .8044 \mathrm{E}+\odot 7$ \\
\hline 34 & $288 . \odot \odot \odot$ & $288 . \odot \odot \odot$ & $1.0 \odot \odot \odot$ & $\odot .8 \odot 44 \mathrm{E}+\odot 7$ \\
\hline 35 & $288.0 \odot \odot$ & 288.000 & 1.0000 & $\odot .8044 \mathrm{E}+\odot 7$ \\
\hline 36 & $288.00 \odot$ & $288.00 \odot$ & $1.000 \odot$ & $\odot .8044 \mathrm{E}+\odot 7$ \\
\hline 37 & $288 . \odot \odot \odot$ & $288 . \odot \odot \odot$ & $1.00 \odot \odot$ & $\odot .8 \odot 44 \mathrm{E}+\odot 7$ \\
\hline 38 & $288.00 \odot$ & $288.00 \odot$ & 1.0000 & $\odot .8044 \mathrm{E}+07$ \\
\hline 39 & $288.0 \odot \odot$ & $288.0 \odot \odot$ & 1.0000 & $\odot .8044 \mathrm{E}+07$ \\
\hline 40 & $288.0 \odot \odot$ & $288.00 \odot$ & $1.00 \odot \odot$ & $\odot .8 \odot 44 \mathrm{E}+\odot 7$ \\
\hline 41 & $288.0 \odot \odot$ & $288.00 \odot$ & 1. $\odot \odot \odot \odot$ & $\odot .8 \odot 44 \mathrm{E}+\odot 7$ \\
\hline 42 & $288.00 \odot$ & $288 . \odot \odot \odot$ & 1.0000 & $\odot .8 \odot 44 \mathrm{E}+\odot 7$ \\
\hline 43 & $288.0 \odot \odot$ & $288 . \odot \odot \odot$ & $1.000 \odot$ & $\odot .8 \odot 44 \mathrm{E}+\odot 7$ \\
\hline 44 & $288 . \odot \odot \odot$ & $288 . \odot \odot \odot$ & $1.0 \odot \odot \odot$ & $\odot .8 \odot 44 \mathrm{E}+\odot 7$ \\
\hline 45 & 288.000 & 288.000 & 1.0000 & $\odot .8044 \mathrm{E}+07$ \\
\hline 46 & $288.00 \odot$ & $288.00 \odot$ & $1.000 \odot$ & $\odot .8044 \mathrm{E}+\odot 7$ \\
\hline 47 & $288 . \odot \odot \odot$ & $288.00 \odot$ & $1.00 \odot \odot$ & $\odot .8 \odot 44 \mathrm{E}+\odot 7$ \\
\hline 48 & $288 . \odot \odot \odot$ & $288 . \odot \odot \odot$ & $1.0 \odot \odot \odot$ & $\odot .8 \odot 44 \mathrm{E}+\odot 7$ \\
\hline 49 & $288.00 \odot$ & $288.0 \odot \odot$ & $1.000 \odot$ & $\odot .8044 \mathrm{E}+\odot 7$ \\
\hline 50 & $288.00 \odot$ & $288.0 \odot \odot$ & $1.00 \odot \odot$ & $\odot .8 \odot 44 \mathrm{E}+\odot 7$ \\
\hline 51 & 288.000 & 288.000 & 1.0000 & $\odot .8044 \mathrm{E}+07$ \\
\hline 52 & $288.00 \odot$ & $288.00 \odot$ & 1.00 & $\odot .8 \odot 44 \mathrm{E}+07$ \\
\hline 53 & $288.00 \odot$ & $288.00 \odot$ & $1.00 \odot \odot$ & $\odot .8 \odot 44 \mathrm{E}+\odot 7$ \\
\hline 54 & $288.0 \odot \odot$ & $288.00 \odot$ & $1.000 \odot$ & $\odot .8 \odot 44 \mathrm{E}+\odot 7$ \\
\hline 55 & 288.000 & 288.000 & 1.0000 & $\odot .8044 \mathrm{E}+07$ \\
\hline 56 & $288.00 \odot$ & $288.00 \odot$ & $1.000 \odot$ & $\odot .8044 \mathrm{E}+\odot 7$ \\
\hline 57 & 288.000 & 288.000 & 1.0000 & $\odot .8044 \mathrm{E}+07$ \\
\hline 58 & $288 . \odot \odot \odot$ & $288.00 \odot$ & 1. $\odot \odot \odot \odot$ & $\odot .8 \odot 44 \mathrm{E}+\odot 7$ \\
\hline 59 & $288.0 \odot \odot$ & 288.000 & $1.000 \odot$ & $\odot .8044 \mathrm{E}+\odot 7$ \\
\hline 60 & $288.0 \odot \odot$ & $288 . \odot \odot \odot$ & $1.00 \odot \odot$ & $\odot .8 \odot 44 \mathrm{E}+\odot 7$ \\
\hline & & & -0 & 26522809 \\
\hline node & $\mathrm{T}(\mathrm{K})$ & Twall(K) & $\mathrm{x}$ & $P(P a)$ \\
\hline 1 & 132.116 & 186.993 & & $\odot .6764 \mathrm{E}+06$ \\
\hline
\end{tabular}




\begin{tabular}{|c|c|c|c|c|}
\hline 2 & 153.254 & 225.043 & $\odot .9877$ & $\odot .1248 \mathrm{E}+\odot 7$ \\
\hline 3 & 222.527 & 263.752 & 1.0000 & $\odot .5018 \mathrm{E}+07$ \\
\hline 4 & 234.229 & 275.180 & 1.0000 & $\odot .5796 \mathrm{E}+07$ \\
\hline 5 & 244.211 & 282.623 & 1.0000 & $\odot .6210 \mathrm{E}+\odot 7$ \\
\hline 6 & 265.479 & 286.976 & 1.0000 & $\odot .6899 \mathrm{E}+\odot 7$ \\
\hline 7 & 282.618 & $288 . \odot \odot \odot$ & 1.0000 & $\odot .7620 \mathrm{E}+\odot 7$ \\
\hline 8 & 287.590 & $288 . \odot \odot \odot$ & $1.000 \odot$ & $\odot .7966 \mathrm{E}+\odot 7$ \\
\hline 9 & $288.0 \odot \odot$ & $288.0 \odot \odot$ & 1.0000 & $\odot .8039 \mathrm{E}+\odot 7$ \\
\hline 10 & $288.0 \odot \odot$ & $288 . \odot \odot \odot$ & $1.000 \odot$ & $\odot .8 \odot 44 \mathrm{E}+\odot 7$ \\
\hline 11 & 288.000 & $288.0 \odot \odot$ & 1.0000 & $\odot .8044 \mathrm{E}+07$ \\
\hline 12 & $288 . \odot \odot \odot$ & $288 . \odot \odot \odot$ & 1.0000 & $\odot .8 \odot 44 \mathrm{E}+\odot 7$ \\
\hline 13 & $288.0 \odot \odot$ & 288.000 & 1.0000 & $\odot .8044 \mathrm{E}+\odot 7$ \\
\hline 14 & $288 . \odot \odot \odot$ & $288 . \odot \odot \odot$ & 1.0000 & $\odot .8 \odot 44 \mathrm{E}+\odot 7$ \\
\hline 15 & 288.000 & 288.000 & 1.0000 & $\odot .8 \odot 44 \mathrm{E}+\odot 7$ \\
\hline 16 & $288.0 \odot \odot$ & $288.0 \odot \odot$ & 1.0000 & $\odot .8044 \mathrm{E}+\odot 7$ \\
\hline 17 & 288.000 & 288.000 & 1.0000 & $\odot .8 \odot 44 \mathrm{E}+\odot 7$ \\
\hline 18 & $288 . \odot \odot \odot$ & $288.00 \odot$ & 1.0000 & $\odot .8 \odot 44 \mathrm{E}+\odot 7$ \\
\hline 19 & 288.000 & $288.00 \odot$ & 1.0000 & $\odot .8044 \mathrm{E}+07$ \\
\hline 20 & $288.0 \odot \odot$ & $288.0 \odot \odot$ & 1.0000 & $\odot .8044 \mathrm{E}+\odot 7$ \\
\hline 21 & $288 . \odot \odot \odot$ & $288 . \odot \odot \odot$ & 1.0000 & $\odot .8 \odot 44 \mathrm{E}+\odot 7$ \\
\hline 22 & $288.00 \odot$ & $288.0 \odot \odot$ & 1.0000 & $\odot .8044 \mathrm{E}+07$ \\
\hline 23 & $288.00 \odot$ & $288.0 \odot \odot$ & 1.0000 & $\odot .8044 \mathrm{E}+\odot 7$ \\
\hline 24 & $288.00 \odot$ & $288 . \odot \odot \odot$ & $1.000 \odot$ & $\odot .8044 \mathrm{E}+\odot 7$ \\
\hline 25 & $288 . \odot \odot \odot$ & $288 . \odot \odot \odot$ & 1.0000 & $\odot .8 \odot 44 \mathrm{E}+\odot 7$ \\
\hline 26 & 288.000 & $288.0 \odot \odot$ & 1.0000 & $\odot .8044 \mathrm{E}+07$ \\
\hline 27 & $288.0 \odot \odot$ & $288.0 \odot \odot$ & 1.0000 & $\odot .8044 \mathrm{E}+\odot 7$ \\
\hline 28 & $288.00 \odot$ & $288.0 \odot \odot$ & 1.0000 & $\odot .8044 \mathrm{E}+\odot 7$ \\
\hline 29 & $288.00 \odot$ & $288.0 \odot \odot$ & 1.0000 & $\odot .8044 \mathrm{E}+\odot 7$ \\
\hline 30 & $288.0 \odot \odot$ & $288.0 \odot \odot$ & 1.0000 & $\odot .8044 \mathrm{E}+\odot 7$ \\
\hline 31 & $288 . \odot \odot \odot$ & $288 . \odot \odot \odot$ & $1.000 \odot$ & $\odot .8 \odot 44 \mathrm{E}+\odot 7$ \\
\hline 32 & $288 . \odot \odot \odot$ & $288 . \odot \odot \odot$ & 1.0000 & $\odot .8044 \mathrm{E}+07$ \\
\hline 33 & 288.000 & $288.0 \odot \odot$ & 1.0000 & $\odot .8044 \mathrm{E}+07$ \\
\hline 34 & $288.00 \odot$ & $288.0 \odot \odot$ & 1.0000 & $\odot .8044 \mathrm{E}+07$ \\
\hline 35 & $288.0 \odot \odot$ & $288 . \odot \odot \odot$ & 1.0000 & $\odot .8 \odot 44 \mathrm{E}+\odot 7$ \\
\hline 36 & $288.00 \odot$ & $288.0 \odot \odot$ & 1.0000 & $\odot .8044 \mathrm{E}+07$ \\
\hline 37 & $288.0 \odot \odot$ & $288 . \odot \odot \odot$ & 1.0000 & $\odot .8 \odot 44 \mathrm{E}+\odot 7$ \\
\hline 38 & 288.000 & 288.000 & 1.0000 & $\odot .8 \odot 44 \mathrm{E}+\odot 7$ \\
\hline 39 & $288.00 \odot$ & $288.0 \odot \odot$ & 1.0000 & $\odot .8044 \mathrm{E}+07$ \\
\hline 40 & 288.000 & $288 . \odot \odot \odot$ & 1.0000 & $\odot .8044 \mathrm{E}+\odot 7$ \\
\hline 41 & $288 . \odot \odot \odot$ & $288 . \odot \odot \odot$ & 1.0000 & $\odot .8 \odot 44 \mathrm{E}+\odot 7$ \\
\hline 42 & $288 . \odot \odot \odot$ & $288 . \odot \odot \odot$ & 1.0000 & $\odot .8 \odot 44 \mathrm{E}+\odot 7$ \\
\hline 43 & $288.0 \odot \odot$ & $288.0 \odot \odot$ & 1.0000 & $\odot .8044 \mathrm{E}+\odot 7$ \\
\hline 44 & $288.0 \odot \odot$ & $288 . \odot \odot \odot$ & 1.0000 & $\odot .8 \odot 44 \mathrm{E}+\odot 7$ \\
\hline 45 & $288.00 \odot$ & $288.0 \odot \odot$ & 1.0000 & $\odot .8044 \mathrm{E}+07$ \\
\hline 46 & $288.0 \odot \odot$ & $288.0 \odot \odot$ & 1.0000 & $\odot .8044 \mathrm{E}+\odot 7$ \\
\hline 47 & $288 . \odot \odot \odot$ & $288 . \odot \odot \odot$ & 1.0000 & $\odot .8 \odot 44 \mathrm{E}+\odot 7$ \\
\hline 48 & $288 . \odot \odot \odot$ & $288 . \odot \odot \odot$ & 1.0000 & $\odot .8 \odot 44 \mathrm{E}+\odot 7$ \\
\hline 49 & 288.000 & $288.0 \odot \odot$ & 1.0000 & $\odot .8044 \mathrm{E}+07$ \\
\hline 50 & $288.0 \odot \odot$ & $288.0 \odot \odot$ & 1.0000 & $\odot .8044 \mathrm{E}+\odot 7$ \\
\hline 51 & 288.000 & $288.0 \odot \odot$ & 1.0000 & $\odot .8044 \mathrm{E}+07$ \\
\hline 52 & $288.00 \odot$ & $288.0 \odot \odot$ & 1.0000 & $\odot .8044 \mathrm{E}+07$ \\
\hline 53 & $288.0 \odot \odot$ & $288.0 \odot \odot$ & 1.0000 & $\odot .8044 \mathrm{E}+\odot 7$ \\
\hline 54 & $288 . \odot \odot \odot$ & $288 . \odot \odot \odot$ & 1.0000 & $\odot .8 \odot 44 \mathrm{E}+\odot 7$ \\
\hline 55 & $288.00 \odot$ & $288.0 \odot \odot$ & 1.0000 & $\odot .8044 \mathrm{E}+07$ \\
\hline 56 & $288.0 \odot \odot$ & $288.0 \odot \odot$ & 1.0000 & $\odot .8044 \mathrm{E}+\odot 7$ \\
\hline 57 & $288.0 \odot \odot$ & $288.0 \odot \odot$ & 1.0000 & $\odot .8044 \mathrm{E}+\odot 7$ \\
\hline 58 & $288.0 \odot \odot$ & $288.0 \odot \odot$ & 1.0000 & $\odot .8044 \mathrm{E}+\odot 7$ \\
\hline
\end{tabular}




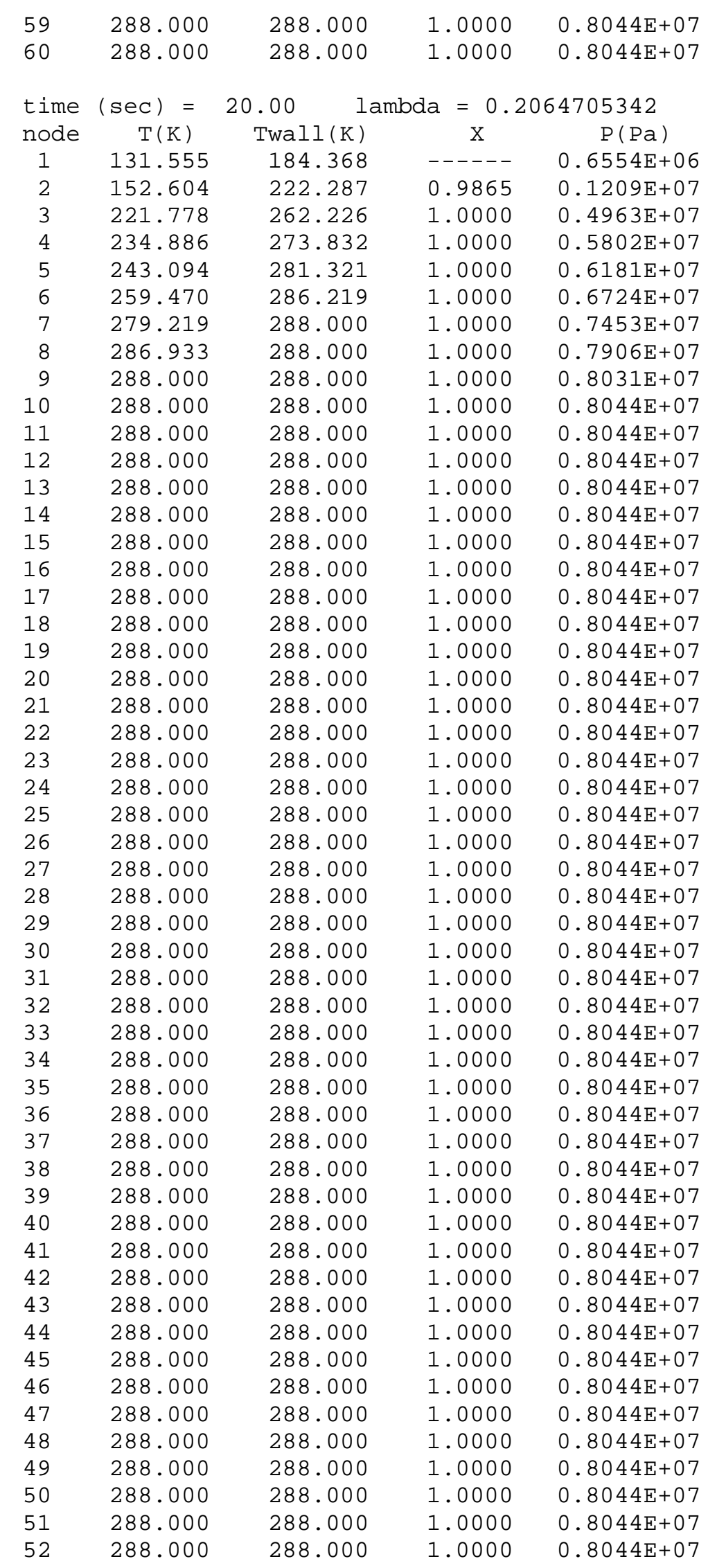




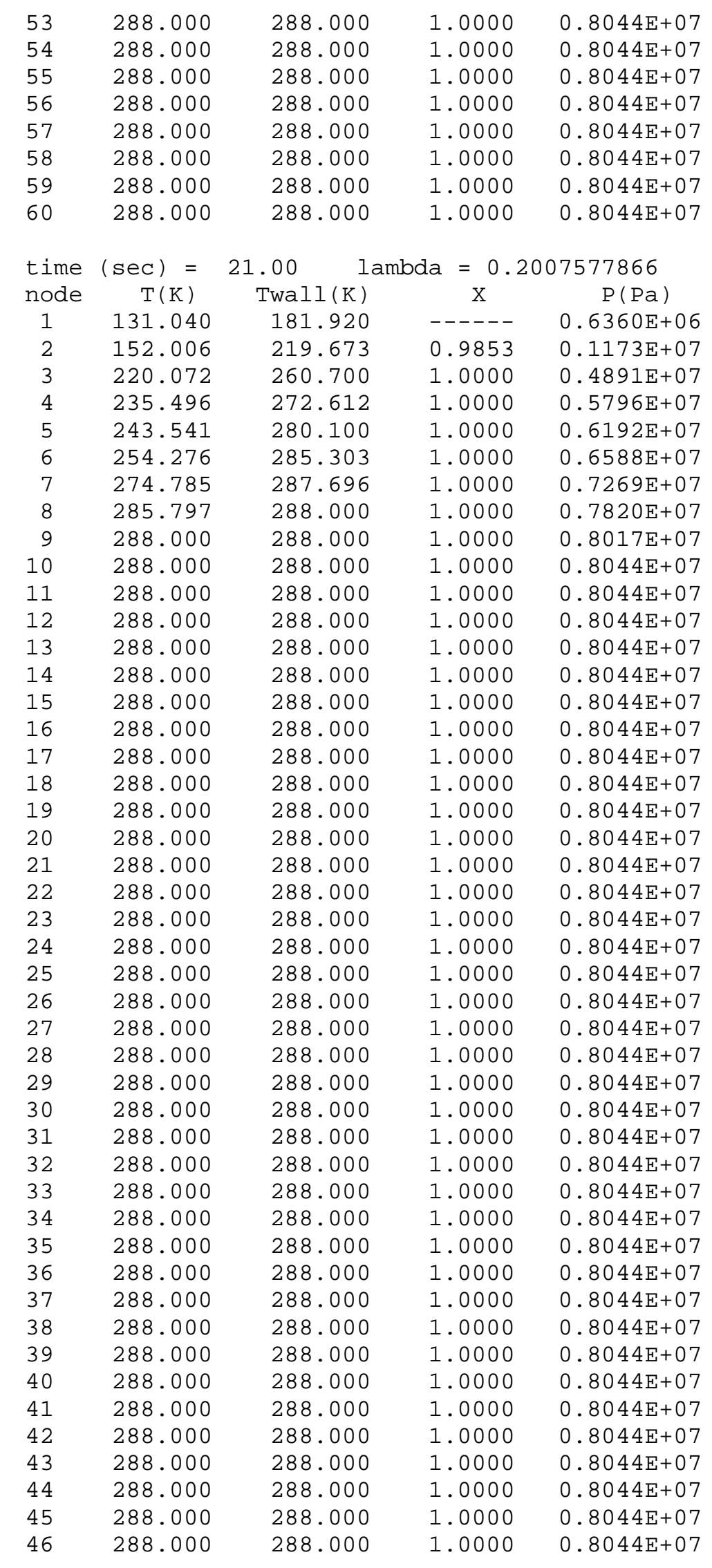




\begin{tabular}{|c|c|c|c|c|}
\hline 47 & $288.0 \odot \odot$ & $288.00 \odot$ & 1.0000 & $\odot .8 \odot 44 \mathrm{E}+\odot 7$ \\
\hline 48 & $288.0 \odot \odot$ & 288.000 & 1.0000 & $0.8044 \mathrm{E}+07$ \\
\hline 49 & $288 . \odot \odot \odot$ & $288 . \odot \odot \odot$ & $1.000 \odot$ & $\odot .8 \odot 44 \mathrm{E}+\odot 7$ \\
\hline 50 & $288 . \odot \odot \odot$ & $288.00 \odot$ & $1.00 \odot \odot$ & $\odot .8 \odot 44 \mathrm{E}+\odot 7$ \\
\hline 51 & 288.000 & 288.000 & 1.0000 & $\odot .8044 \mathrm{E}+07$ \\
\hline 52 & $288 . \odot \odot \odot$ & $288.0 \odot \odot$ & $1.000 \odot$ & $\odot .8044 \mathrm{E}+\odot 7$ \\
\hline 53 & $288.0 \odot \odot$ & $288.00 \odot$ & $1.000 \odot$ & $\odot .8044 \mathrm{E}+07$ \\
\hline 54 & $288.0 \odot \odot$ & 288.000 & 1.0000 & $0.8044 \mathrm{E}+07$ \\
\hline 55 & $288.0 \odot \odot$ & $288.00 \odot$ & $1.000 \odot$ & $\odot .8044 \mathrm{E}+07$ \\
\hline 56 & $288 . \odot \odot \odot$ & $288.0 \odot \odot$ & $1.000 \odot$ & $0.8044 \mathrm{E}+07$ \\
\hline 57 & $288 . \odot \odot \odot$ & $288.00 \odot$ & $1.00 \odot \odot$ & $\odot .8044 \mathrm{E}+07$ \\
\hline 58 & $288.0 \odot \odot$ & 288.000 & 1.0000 & $\odot .8044 \mathrm{E}+\odot 7$ \\
\hline 59 & $288 . \odot \odot \odot$ & $288.0 \odot \odot$ & $1.000 \odot$ & $0.8044 \mathrm{E}+07$ \\
\hline 60 & $288.00 \odot$ & 288.000 & 1.0000 & $0.8044 \mathrm{E}+07$ \\
\hline & & & & 4200566 \\
\hline O & $T(K)$ & Twall(K) & $x$ & $\mathrm{P}(\mathrm{Pa})$ \\
\hline 1 & 130.560 & 179.631 & $-\ldots$ & $\odot .6179 \mathrm{E}+\odot 6$ \\
\hline 2 & 151.449 & 217.193 & $\odot .9845$ & $0.1140 \mathrm{E}+07$ \\
\hline 3 & 217.618 & 259.174 & $1.000 \odot$ & $\odot .4808 \mathrm{E}+07$ \\
\hline 4 & 236.009 & 271.391 & $1.000 \odot$ & $\odot .5780 \mathrm{E}+\odot 7$ \\
\hline 5 & 244.030 & 278.879 & 1.0000 & $0.6205 \mathrm{E}+07$ \\
\hline 6 & 251.213 & 284.388 & $1.000 \odot$ & $\odot .6509 \mathrm{E}+07$ \\
\hline 7 & 69.554 & 287.375 & $1.00 \odot \odot$ & $\odot .7090 \mathrm{E}+\odot 7$ \\
\hline 8 & 283.947 & 288.000 & 1.0000 & $0.7706 \mathrm{E}+07$ \\
\hline 9 & 287.732 & $288.00 \odot$ & 1.0000 & $\odot .7986 \mathrm{E}+\odot 7$ \\
\hline 10 & $288.0 \odot \odot$ & $288.00 \odot$ & $1.00 \odot \odot$ & $\odot .8041 \mathrm{E}+\odot 7$ \\
\hline 11 & 88.000 & $288.0 \odot \odot$ & $1.00 \odot \odot$ & $\odot .8 \odot 44 \mathrm{E}+\odot 7$ \\
\hline 12 & $288.0 \odot \odot$ & $288.0 \odot \odot$ & $1.00 \odot \odot$ & $\odot .8 \odot 44 \mathrm{E}+\odot 7$ \\
\hline 13 & 88.000 & $288.00 \odot$ & 1.0000 & $\odot .8044 \mathrm{E}+07$ \\
\hline 14 & 288.000 & 288.000 & 1.0000 & $\odot .8044 \mathrm{E}+07$ \\
\hline 15 & $288.0 \odot \odot$ & $288.0 \odot \odot$ & 1.0000 & $\odot .8 \odot 44 \mathrm{E}+\odot 7$ \\
\hline 16 & $288.00 \odot$ & $288.0 \odot \odot$ & 1.0000 & $\odot .8044 \mathrm{E}+07$ \\
\hline 17 & 88.000 & $288.00 \odot$ & 1.0000 & $0.8044 \mathrm{E}+07$ \\
\hline 18 & $88.00 \odot$ & $288.00 \odot$ & 1.0000 & $\odot .8 \odot 44 \mathrm{E}+07$ \\
\hline 19 & $288.00 \odot$ & $288.0 \odot \odot$ & 1.0000 & $0.8044 \mathrm{E}+07$ \\
\hline 20 & $288 . \odot \odot \odot$ & $288.00 \odot$ & 1.0000 & $\odot .8044 \mathrm{E}+\odot 7$ \\
\hline 21 & $288.0 \odot \odot$ & $288.00 \odot$ & 1.0000 & $\odot .8044 \mathrm{E}+\odot 7$ \\
\hline 22 & $288.00 \odot$ & $288.00 \odot$ & 1.0000 & $\odot .8044 \mathrm{E}+07$ \\
\hline 23 & $288.00 \odot$ & $288 . \odot \odot \odot$ & 1.0000 & $\odot .8044 \mathrm{E}+\odot 7$ \\
\hline 24 & $88 . \odot \odot \odot$ & $288.0 \odot \odot$ & 1.0000 & $\odot .8044 \mathrm{E}+\odot 7$ \\
\hline 25 & 288.000 & $288.0 \odot \odot$ & 1.0000 & $\odot .8044 \mathrm{E}+07$ \\
\hline 26 & $288.00 \odot$ & $288.0 \odot \odot$ & 1.0000 & $\odot .8044 \mathrm{E}+07$ \\
\hline 27 & $288.00 \odot$ & $288.00 \odot$ & 1.0000 & $\odot .8044 \mathrm{E}+07$ \\
\hline 28 & 88. & 288.000 & 1.0000 & $\odot .8044 \mathrm{E}+07$ \\
\hline 29 & $288.00 \odot$ & $288 . \odot \odot \odot$ & 1.0000 & $0.8044 \mathrm{E}+07$ \\
\hline 30 & 88.000 & $288.0 \odot \odot$ & 1.0000 & $0.8044 \mathrm{E}+07$ \\
\hline 31 & 288.000 & 288.000 & 1.0000 & $0.8044 \mathrm{E}+07$ \\
\hline 32 & $288.00 \odot$ & $288.0 \odot \odot$ & 1.0000 & $0.8044 \mathrm{E}+07$ \\
\hline 33 & $288.00 \odot$ & $288 . \odot \odot \odot$ & 1.0000 & $0.8044 \mathrm{E}+07$ \\
\hline 34 & 288.000 & $288.00 \odot$ & 1.0000 & $0.8044 \mathrm{E}+07$ \\
\hline 35 & $288.00 \odot$ & 288.000 & 1.0000 & $\odot .8044 \mathrm{E}+\odot 7$ \\
\hline 36 & $288.00 \odot$ & $288 . \odot \odot \odot$ & $1.000 \odot$ & $\odot .8044 \mathrm{E}+\odot 7$ \\
\hline 37 & 288.000 & 288.000 & 1.0000 & $\odot .8044 \mathrm{E}+07$ \\
\hline 38 & 288.000 & $288.00 \odot$ & 1.0000 & $0.8044 \mathrm{E}+07$ \\
\hline 39 & 288.000 & $288.00 \odot$ & 1.0000 & $\odot .8044 \mathrm{E}+07$ \\
\hline $4 \odot$ & $288.00 \odot$ & $288.00 \odot$ & $1.0 \odot \odot \odot$ & $\odot .8044 \mathrm{E}+07$ \\
\hline
\end{tabular}




\begin{tabular}{|c|c|c|c|c|}
\hline 41 & $288.0 \odot \odot$ & 288.000 & $1.000 \odot$ & $\odot .8 \odot 44 \mathrm{E}+\odot 7$ \\
\hline 42 & 288.000 & 288.000 & 1.0000 & $0.8044 \mathrm{E}+07$ \\
\hline 43 & 288.000 & $288.0 \odot \odot$ & $1.000 \odot$ & $\odot .8 \odot 44 \mathrm{E}+\odot 7$ \\
\hline 44 & $288.00 \odot$ & $288.00 \odot$ & $1.00 \odot \odot$ & $\odot .8 \odot 44 \mathrm{E}+\odot 7$ \\
\hline 45 & 288.000 & $288.00 \odot$ & 1.0000 & $\odot .8044 \mathrm{E}+07$ \\
\hline 46 & 288.000 & $288 . \odot \odot \odot$ & $1.000 \odot$ & $\odot .8044 \mathrm{E}+\odot 7$ \\
\hline 47 & $288.00 \odot$ & $288.00 \odot$ & $1.000 \odot$ & $\odot .8044 \mathrm{E}+07$ \\
\hline 48 & 288.000 & $288.00 \odot$ & 1.0000 & $0.8044 \mathrm{E}+07$ \\
\hline 49 & $288.00 \odot$ & $288.00 \odot$ & $1.000 \odot$ & $\odot .8044 \mathrm{E}+07$ \\
\hline 50 & 288.000 & $288 . \odot \odot \odot$ & $1.000 \odot$ & $0.8044 \mathrm{E}+07$ \\
\hline 51 & $288.00 \odot$ & $288 . \odot \odot \odot$ & $1.00 \odot \odot$ & $\odot .8044 \mathrm{E}+07$ \\
\hline 52 & 288.000 & 288.000 & 1.0000 & $0.8044 \mathrm{E}+07$ \\
\hline 53 & 288.000 & $288.0 \odot \odot$ & $1.000 \odot$ & $0.8044 \mathrm{E}+07$ \\
\hline 54 & 288.000 & $288.00 \odot$ & 1.0000 & $0.8044 \mathrm{E}+07$ \\
\hline 55 & 288.000 & $288.00 \odot$ & 1.0000 & $0.8044 \mathrm{E}+07$ \\
\hline 56 & $288.00 \odot$ & $288.00 \odot$ & 1.0000 & $\odot .8044 \mathrm{E}+\odot 7$ \\
\hline 57 & 288.000 & $288 . \odot \odot \odot$ & $1.000 \odot$ & $\odot .8044 \mathrm{E}+\odot 7$ \\
\hline 58 & $288.0 \odot \odot$ & $288 . \odot \odot \odot$ & 1.0000 & $\odot .8 \odot 44 \mathrm{E}+\odot 7$ \\
\hline 59 & 288.000 & $288.00 \odot$ & 1.0000 & $0.8044 \mathrm{E}+07$ \\
\hline 60 & 288.000 & 288.000 & 1.0000 & $0.8044 \mathrm{E}+07$ \\
\hline time & & & & 03892905 \\
\hline node & $T(K)$ & Twall(K) & $x$ & $\mathrm{P}(\mathrm{Pa})$ \\
\hline 1 & 130.109 & 177.492 & - . - _ & $0.6010 \mathrm{E}+06$ \\
\hline 2 & 150.926 & 214.834 & 0.9839 & $0.1109 \mathrm{E}+07$ \\
\hline 3 & 214.628 & $257.6 \odot 2$ & 1.0000 & $0.4721 \mathrm{E}+07$ \\
\hline 4 & 236.081 & 270.359 & 1.0000 & $\odot .5747 \mathrm{E}+\odot 7$ \\
\hline 5 & 244.462 & 277.901 & 1.0000 & $0.6214 \mathrm{E}+07$ \\
\hline 6 & 250.297 & 283.472 & 1.0000 & $\odot .6485 \mathrm{E}+\odot 7$ \\
\hline 7 & 264.061 & 286.764 & 1.0000 & $\odot .6931 \mathrm{E}+07$ \\
\hline 8 & 281.169 & 288.000 & 1.0000 & $\odot .7560 \mathrm{E}+\odot 7$ \\
\hline 9 & 287.279 & 288.000 & 1.0000 & $\odot .7940 \mathrm{E}+07$ \\
\hline 10 & 288.000 & 288.000 & 1.0000 & $\odot .8036 \mathrm{E}+07$ \\
\hline 11 & $288.00 \odot$ & $288.00 \odot$ & 1.0000 & $0.8044 \mathrm{E}+07$ \\
\hline 12 & $288 . \odot \odot \odot$ & $288.00 \odot$ & 1.0000 & $\odot .8 \odot 44 \mathrm{E}+07$ \\
\hline 13 & $288.00 \odot$ & $288 . \odot \odot \odot$ & 1.0000 & $0.8044 \mathrm{E}+07$ \\
\hline 14 & $288.00 \odot$ & $288 . \odot \odot \odot$ & 1.0000 & $\odot .8044 \mathrm{E}+\odot 7$ \\
\hline 15 & $288.00 \odot$ & $288.00 \odot$ & 1.0000 & $\odot .8044 \mathrm{E}+\odot 7$ \\
\hline 16 & $288.00 \odot$ & $288.0 \odot \odot$ & 1.0000 & $\odot .8044 \mathrm{E}+07$ \\
\hline 17 & $288.00 \odot$ & $288.0 \odot \odot$ & 1.0000 & $\odot .8044 \mathrm{E}+\odot 7$ \\
\hline 18 & $288.00 \odot$ & $288.00 \odot$ & 1.0000 & $\odot .8044 \mathrm{E}+\odot 7$ \\
\hline 19 & 288.000 & $288.00 \odot$ & 1.0000 & $\odot .8044 \mathrm{E}+07$ \\
\hline 20 & 288.000 & $288.0 \odot \odot$ & 1.0000 & $\odot .8044 \mathrm{E}+07$ \\
\hline 21 & $288.00 \odot$ & $288.00 \odot$ & 1.0000 & $\odot .8044 \mathrm{E}+07$ \\
\hline 22 & $288 . c$ & 288.000 & 1.0000 & $\odot .8044 \mathrm{E}+07$ \\
\hline 23 & 288.000 & $288.0 \odot \odot$ & 1.0000 & $0.8044 \mathrm{E}+07$ \\
\hline 24 & 288.000 & $288.0 \odot \odot$ & 1.0000 & $0.8044 \mathrm{E}+07$ \\
\hline 25 & 288.000 & 288.000 & 1.0000 & $0.8044 \mathrm{E}+07$ \\
\hline 26 & 288.000 & $288.00 \odot$ & 1.0000 & $0.8044 \mathrm{E}+07$ \\
\hline 27 & 288.000 & $288.0 \odot \odot$ & 1.0000 & $0.8044 \mathrm{E}+07$ \\
\hline 28 & 288.000 & 288.000 & 1.0000 & $0.8044 \mathrm{E}+07$ \\
\hline 29 & 288.000 & 288.000 & 1.0000 & $\odot .8044 \mathrm{E}+\odot 7$ \\
\hline 30 & 288.000 & $288 . \odot \odot \odot$ & 1.0000 & $\odot .8044 \mathrm{E}+\odot 7$ \\
\hline 31 & 288.000 & $288.00 \odot$ & 1.0000 & $\odot .8044 \mathrm{E}+07$ \\
\hline 32 & 288.000 & $288.00 \odot$ & 1.0000 & $0.8044 \mathrm{E}+07$ \\
\hline 33 & 288.000 & 288.000 & 1.0000 & $\odot .8044 \mathrm{E}+07$ \\
\hline 34 & $288.00 \odot$ & $288.00 \odot$ & 1.0000 & $\odot .8044 \mathrm{E}+07$ \\
\hline
\end{tabular}




\begin{tabular}{|c|c|c|c|c|}
\hline 35 & 288.000 & 288.000 & 1.0000 & $\odot .8044 \mathrm{E}+07$ \\
\hline 36 & 288.000 & 288.000 & 1.0000 & $0.8044 \mathrm{E}+07$ \\
\hline 37 & $288.00 \odot$ & $288 . \odot \odot \odot$ & $1.00 \odot \odot$ & $\odot .8 \odot 44 \mathrm{E}+\odot 7$ \\
\hline 38 & $288.0 \odot \odot$ & $288.00 \odot$ & $1.00 \odot \odot$ & $\odot .8044 \mathrm{E}+\odot 7$ \\
\hline 39 & $288.00 \odot$ & $288.0 \odot \odot$ & 1. $0 \odot \odot \odot$ & $\odot .8044 \mathrm{E}+07$ \\
\hline 40 & $288.00 \odot$ & $288.0 \odot \odot$ & $1.0 \odot \odot \odot$ & $\odot .8 \odot 44 \mathrm{E}+\odot 7$ \\
\hline 41 & 288.000 & $288.00 \odot$ & $1.00 \odot \odot$ & $\odot .8044 \mathrm{E}+07$ \\
\hline 42 & 288.000 & 288.000 & 1.0000 & $0.8044 \mathrm{E}+07$ \\
\hline 43 & 288.000 & $288.00 \odot$ & $1.000 \odot$ & $\odot .8044 \mathrm{E}+07$ \\
\hline 44 & $288.00 \odot$ & $288.0 \odot \odot$ & $1.00 \odot \odot$ & $0.8044 \mathrm{E}+07$ \\
\hline 45 & $288.0 \odot \odot$ & $288.00 \odot$ & $1.00 \odot \odot$ & $\odot .8044 \mathrm{E}+07$ \\
\hline 46 & 288.000 & 288.000 & $1.000 \odot$ & $0.8044 \mathrm{E}+07$ \\
\hline 47 & $288.00 \odot$ & $288.0 \odot \odot$ & $1.00 \odot \odot$ & $0.8044 \mathrm{E}+07$ \\
\hline 48 & 288.000 & $288.00 \odot$ & $1.00 \odot \odot$ & $\odot .8044 \mathrm{E}+07$ \\
\hline 49 & $288 . \odot \odot \odot$ & $288.0 \odot \odot$ & 1. $\odot \odot \odot \odot$ & $\odot .8044 \mathrm{E}+07$ \\
\hline 50 & $288.00 \odot$ & 288.000 & $1.000 \odot$ & $\odot .8044 \mathrm{E}+\odot 7$ \\
\hline 51 & $288.00 \odot$ & $288 . \odot \odot \odot$ & $1.000 \odot$ & $\odot .8044 \mathrm{E}+\odot 7$ \\
\hline 52 & $288.0 \odot \odot$ & $288.0 \odot \odot$ & $1.00 \odot \odot$ & $\odot .8 \odot 44 \mathrm{E}+\odot 7$ \\
\hline 53 & 288.000 & $288.00 \odot$ & 1.0000 & $0.8044 \mathrm{E}+07$ \\
\hline 54 & $288.00 \odot$ & $288.0 \odot \odot$ & $1.000 \odot$ & $\odot .8044 \mathrm{E}+07$ \\
\hline 55 & $288.00 \odot$ & $288.0 \odot \odot$ & $1.000 \odot$ & $\odot .8 \odot 44 \mathrm{E}+07$ \\
\hline 56 & $288.00 \odot$ & $288.0 \odot \odot$ & $1.00 \odot \odot$ & $\odot .8 \odot 44 \mathrm{E}+07$ \\
\hline 57 & 288.000 & 288.000 & 1.0000 & $\odot .8044 \mathrm{E}+07$ \\
\hline 58 & $288.0 \odot \odot$ & $288.00 \odot$ & $1.00 \odot \odot$ & $0.8044 \mathrm{E}+07$ \\
\hline 59 & $288.00 \odot$ & $288.00 \odot$ & 1.0000 & $\odot .8 \odot 44 \mathrm{E}+\odot 7$ \\
\hline 60 & $288.00 \odot$ & 288.000 & 1.0000 & $0.8044 \mathrm{E}+07$ \\
\hline & & 24.00 & \multicolumn{2}{|c|}{ lambda $=0.1856184751$} \\
\hline node & $\mathrm{T}^{\prime}(\mathrm{K})$ & Twall(K) & $\mathrm{x}$ & $P(P a)$ \\
\hline 1 & 129.683 & 175.484 & $-\ldots$ & $\odot .5850 \mathrm{E}+06$ \\
\hline 2 & 150.432 & 212.574 & $\odot .9834$ & $\odot .1079 \mathrm{E}+07$ \\
\hline 3 & 211.335 & 255.771 & $1.00 \odot \odot$ & $\odot .4634 \mathrm{E}+07$ \\
\hline 4 & 235.585 & 269.444 & 1.0000 & $\odot .5695 \mathrm{E}+\odot 7$ \\
\hline 5 & 244.767 & 276.986 & $1.00 \odot \odot$ & $0.6214 \mathrm{E}+07$ \\
\hline 6 & $25 \odot .60 \odot$ & 282.556 & $1.000 \odot$ & $0.6493 \mathrm{E}+07$ \\
\hline 7 & 259.320 & 286.040 & 1.0000 & $0.6806 \mathrm{E}+07$ \\
\hline 8 & $277.4 \odot 2$ & 287.869 & $1.00 \odot \odot$ & $\odot .7398 \mathrm{E}+07$ \\
\hline 9 & 286.435 & $288.00 \odot$ & $1.000 \odot$ & $\odot .7870 \mathrm{E}+\odot 7$ \\
\hline 10 & $288.00 \odot$ & 288.000 & $1.000 \odot$ & $\odot .8025 \mathrm{E}+07$ \\
\hline 11 & $288.00 \odot$ & $288.00 \odot$ & 1.0000 & $\odot .8044 \mathrm{E}+07$ \\
\hline 12 & 88.000 & $288.00 \odot$ & $1.00 \odot \odot$ & $\odot .8044 \mathrm{E}+\odot 7$ \\
\hline 13 & 288.000 & $288.00 \odot$ & 1.0000 & $\odot .8044 \mathrm{E}+07$ \\
\hline 14 & $288.00 \odot$ & $288.0 \odot \odot$ & $1.000 \odot$ & $\odot .8044 \mathrm{E}+07$ \\
\hline 15 & $288.00 \odot$ & $288.00 \odot$ & $1.00 \odot \odot$ & $\odot .8044 \mathrm{E}+07$ \\
\hline 16 & 288.000 & 288.000 & 1.0000 & $0.8044 \mathrm{E}+07$ \\
\hline 17 & $288.00 \odot$ & $288 . \odot \odot \odot$ & 1.0000 & $\odot .8044 \mathrm{E}+07$ \\
\hline 18 & $288.00 \odot$ & $288.0 \odot \odot$ & 1.0000 & $\odot .8044 \mathrm{E}+07$ \\
\hline 19 & 288.000 & 288.000 & 1.0000 & $0.8044 \mathrm{E}+07$ \\
\hline 20 & $288.00 \odot$ & $288.0 \odot \odot$ & 1.0000 & $\odot .8044 \mathrm{E}+07$ \\
\hline 21 & $288.00 \odot$ & $288.0 \odot \odot$ & 1.0000 & $\odot .8044 \mathrm{E}+07$ \\
\hline 22 & $288 . \odot \odot \odot$ & $288.0 \odot \odot$ & 1.0000 & $\odot .8 \odot 44 \mathrm{E}+\odot 7$ \\
\hline 23 & $288.00 \odot$ & $288.00 \odot$ & 1.0000 & $\odot .8044 \mathrm{E}+\odot 7$ \\
\hline 24 & $288.00 \odot$ & $288 . \odot \odot \odot$ & $1.000 \odot$ & $\odot .8044 \mathrm{E}+\odot 7$ \\
\hline 25 & 288.000 & 288.000 & 1.0000 & $\odot .8044 \mathrm{E}+07$ \\
\hline 26 & 288.000 & $288.00 \odot$ & 1.0000 & $\odot .8044 \mathrm{E}+07$ \\
\hline 27 & 288.000 & $288.00 \odot$ & 1.0000 & $\odot .8044 \mathrm{E}+07$ \\
\hline 28 & $288.00 \odot$ & $288.00 \odot$ & 1.0000 & $\odot .8 \odot 44 \mathrm{E}+\odot 7$ \\
\hline
\end{tabular}




\begin{tabular}{|c|c|c|c|c|}
\hline 29 & $288.00 \odot$ & $288.00 \odot$ & 1. $0 \odot \odot \odot$ & $0.8044 \mathrm{E}+\odot 7$ \\
\hline 30 & 288.000 & 288.000 & 1.0000 & $0.8044 \mathrm{E}+07$ \\
\hline 31 & 288.000 & $288.0 \odot \odot$ & $1.00 \odot \odot$ & $\odot .8 \odot 44 \mathrm{E}+\odot 7$ \\
\hline 32 & $288.00 \odot$ & $288 . \odot \odot \odot$ & $1.00 \odot \odot$ & $\odot .8044 \mathrm{E}+\odot 7$ \\
\hline 33 & $288.00 \odot$ & $288.0 \odot \odot$ & 1. $0 \odot \odot \odot$ & $\odot .8044 \mathrm{E}+07$ \\
\hline 34 & $288.00 \odot$ & $288 . \odot \odot \odot$ & $1.0 \odot \odot \odot$ & $\odot .8 \odot 44 \mathrm{E}+\odot 7$ \\
\hline 35 & $288.00 \odot$ & $288.00 \odot$ & $1.00 \odot \odot$ & $\odot .8044 \mathrm{E}+07$ \\
\hline 36 & 288.000 & $288.00 \odot$ & 1.0000 & $0.8044 \mathrm{E}+07$ \\
\hline 37 & $288.00 \odot$ & $288.00 \odot$ & $1.000 \odot$ & $\odot .8044 \mathrm{E}+07$ \\
\hline 38 & $288.00 \odot$ & 288.000 & 1.0000 & $0.8044 \mathrm{E}+07$ \\
\hline 39 & $288.00 \odot$ & $288 . \odot \odot \odot$ & $1.00 \odot \odot$ & $\odot .8044 \mathrm{E}+07$ \\
\hline 40 & 288.000 & $288.00 \odot$ & 1.0000 & $\odot .8044 \mathrm{E}+\odot 7$ \\
\hline 41 & 288.000 & $288.0 \odot \odot$ & $1.00 \odot \odot$ & $0.8044 \mathrm{E}+07$ \\
\hline 42 & $288.00 \odot$ & $288.00 \odot$ & $1.00 \odot \odot$ & $\odot .8044 \mathrm{E}+07$ \\
\hline 43 & $288.0 \odot \odot$ & $288 . \odot \odot \odot$ & 1. $\odot \odot \odot \odot$ & $\odot .8044 \mathrm{E}+07$ \\
\hline 44 & $288.00 \odot$ & $288.0 \odot \odot$ & $1.00 \odot \odot$ & $\odot .8044 \mathrm{E}+07$ \\
\hline 45 & 288.000 & $288 . \odot \odot \odot$ & $1.000 \odot$ & $\odot .8044 \mathrm{E}+\odot 7$ \\
\hline 46 & $288.0 \odot \odot$ & $288 . \odot \odot \odot$ & $1.00 \odot \odot$ & $\odot .8 \odot 44 \mathrm{E}+\odot 7$ \\
\hline 47 & 288.000 & $288.00 \odot$ & 1.0000 & $0.8044 \mathrm{E}+07$ \\
\hline 48 & 288.000 & $288 . \odot \odot \odot$ & $1.000 \odot$ & $\odot .8044 \mathrm{E}+07$ \\
\hline 49 & $288.00 \odot$ & $288.0 \odot \odot$ & $1.000 \odot$ & $\odot .8 \odot 44 \mathrm{E}+07$ \\
\hline 50 & $288.0 \odot \odot$ & $288 . \odot \odot \odot$ & $1.00 \odot \odot$ & $\odot .8 \odot 44 \mathrm{E}+07$ \\
\hline 51 & 288.000 & $288.00 \odot$ & 1.0000 & $\odot .8044 \mathrm{E}+07$ \\
\hline 52 & $288.00 \odot$ & $288 . \odot \odot \odot$ & $1.00 \odot \odot$ & $0.8044 \mathrm{E}+07$ \\
\hline 53 & 288.000 & 288.000 & 1.0000 & $\odot .8044 \mathrm{E}+07$ \\
\hline 54 & 288.000 & $288.00 \odot$ & 1.0000 & $0.8044 \mathrm{E}+07$ \\
\hline 55 & 288.000 & $288.0 \odot \odot$ & 1.0000 & $\odot .8044 \mathrm{E}+\odot 7$ \\
\hline 56 & $288 . \odot \odot \odot$ & $288 . \odot \odot \odot$ & $1.00 \odot \odot$ & $\odot .8 \odot 44 \mathrm{E}+\odot 7$ \\
\hline 57 & $288.0 \odot \odot$ & $288 . \odot \odot \odot$ & $1.00 \odot \odot$ & $\odot .8 \odot 44 \mathrm{E}+\odot 7$ \\
\hline 58 & $288.00 \odot$ & $288.00 \odot$ & 1.0000 & $\odot .8044 \mathrm{E}+07$ \\
\hline 59 & 288.000 & 288.000 & 1.0000 & $\odot .8044 \mathrm{E}+07$ \\
\hline 60 & 288.000 & 288.000 & 1.0000 & $\odot .8044 \mathrm{E}+07$ \\
\hline time & & $25.0 \odot$ & \multicolumn{2}{|c|}{ lambda $=0.1810748130$} \\
\hline node & $\mathrm{T}^{\prime}(\mathrm{K})$ & Twall(K) & $\mathrm{x}$ & $P(P a)$ \\
\hline 1 & 129.278 & 173.591 & $-\ldots$ & $0.5698 \mathrm{E}+\odot 6$ \\
\hline 2 & 149.962 & 210.437 & $\odot .9828$ & $0.1051 \mathrm{E}+07$ \\
\hline 3 & 207.994 & 253.891 & $1.000 \odot$ & $\odot .4552 \mathrm{E}+\odot 7$ \\
\hline 4 & 234.557 & 268.528 & 1.0000 & $\odot .5630 \mathrm{E}+\odot 7$ \\
\hline 5 & 245.073 & 276.070 & 1.0000 & $0.6208 \mathrm{E}+07$ \\
\hline 6 & 50.902 & 281.641 & $1.00 \odot \odot$ & $\odot .6501 \mathrm{E}+07$ \\
\hline 7 & 256.569 & 285.124 & 1.0000 & $0.6734 \mathrm{E}+07$ \\
\hline 8 & 272.783 & 287.564 & $1.000 \odot$ & $\odot .7239 \mathrm{E}+\odot 7$ \\
\hline 9 & 284.973 & $288.0 \odot \odot$ & $1.00 \odot \odot$ & $\odot .7772 \mathrm{E}+\odot 7$ \\
\hline 10 & $287 . \varepsilon$ & 288.000 & 1.0000 & $0.8003 \mathrm{E}+07$ \\
\hline 11 & 288.000 & $288.00 \odot$ & 1.0000 & $\odot .8044 \mathrm{E}+07$ \\
\hline 12 & 288.000 & $288.00 \odot$ & 1.0000 & $\odot .8044 \mathrm{E}+07$ \\
\hline 13 & 288.000 & 288.000 & 1.0000 & $0.8044 \mathrm{E}+07$ \\
\hline 14 & 288.000 & 288.000 & 1.0000 & $\odot .8044 \mathrm{E}+07$ \\
\hline 15 & 288.000 & $288 . \odot \odot \odot$ & 1.0000 & $\odot .8044 \mathrm{E}+07$ \\
\hline 16 & $288 . \odot \odot \odot$ & $288.00 \odot$ & 1.0000 & $\odot .8 \odot 44 \mathrm{E}+\odot 7$ \\
\hline 17 & 288.000 & 288.000 & 1.0000 & $\odot .8044 \mathrm{E}+\odot 7$ \\
\hline 18 & 288.000 & $288 . \odot \odot \odot$ & $1.000 \odot$ & $\odot .8044 \mathrm{E}+\odot 7$ \\
\hline 19 & 288.000 & $288.00 \odot$ & 1.0000 & $\odot .8044 \mathrm{E}+07$ \\
\hline 20 & 288.000 & $288.00 \odot$ & 1.0000 & $\odot .8044 \mathrm{E}+07$ \\
\hline 21 & 288.000 & 288.000 & 1.0000 & $\odot .8044 \mathrm{E}+07$ \\
\hline 22 & $288.00 \odot$ & $288.00 \odot$ & 1.0000 & $\odot .8 \odot 44 \mathrm{E}+\odot 7$ \\
\hline
\end{tabular}




\begin{tabular}{|c|c|c|c|c|}
\hline 23 & 288.000 & 288.000 & 1.0000 & $\odot .8044 \mathrm{E}+07$ \\
\hline 24 & $288.0 \odot \odot$ & 288.000 & 1.0000 & $0.8044 \mathrm{E}+07$ \\
\hline 25 & $288.00 \odot$ & 288.000 & 1.0000 & $0.8044 \mathrm{E}+07$ \\
\hline 26 & $288 . \odot \odot \odot$ & $288 . \odot \odot \odot$ & $1.000 \odot$ & $\odot .8044 \mathrm{E}+\odot 7$ \\
\hline 27 & $288 . \odot \odot \odot$ & $288.00 \odot$ & 1.0000 & $0.8044 \mathrm{E}+07$ \\
\hline 28 & $288.0 \odot \odot$ & $288.00 \odot$ & 1.0000 & $\odot .8044 \mathrm{E}+07$ \\
\hline 29 & $288.0 \odot \odot$ & $288.0 \odot \odot$ & 1.0000 & $0.8044 \mathrm{E}+07$ \\
\hline 30 & $288.00 \odot$ & $288.00 \odot$ & 1.0000 & $0.8044 \mathrm{E}+07$ \\
\hline 31 & $288.0 \odot \odot$ & $288.00 \odot$ & 1.0000 & $0.8044 \mathrm{E}+07$ \\
\hline 32 & $288 . \odot 0 \odot$ & $288 . \odot \odot \odot$ & $1.000 \odot$ & $0.8044 \mathrm{E}+07$ \\
\hline 33 & $288 . \odot \odot \odot$ & $288 . \odot \odot \odot$ & $1.000 \odot$ & $0.8044 \mathrm{E}+\odot 7$ \\
\hline 34 & $288.00 \odot$ & $288.00 \odot$ & 1.0000 & $0.8044 \mathrm{E}+07$ \\
\hline 35 & $288.0 \odot \odot$ & $288.00 \odot$ & 1.0000 & $0.8044 \mathrm{E}+07$ \\
\hline 36 & $288.00 \odot$ & $288.00 \odot$ & 1.0000 & $0.8044 \mathrm{E}+07$ \\
\hline 37 & $288 . \odot \odot \odot$ & $288 . \odot \odot \odot$ & $1.000 \odot$ & $\odot .8044 \mathrm{E}+\odot 7$ \\
\hline 38 & 88.000 & 288.000 & 1.0000 & $\odot .8044 \mathrm{E}+07$ \\
\hline 39 & $288 . \odot \odot \odot$ & $288 . \odot \odot \odot$ & $1.000 \odot$ & $\odot .8044 \mathrm{E}+07$ \\
\hline 40 & $288 . \odot \odot \odot$ & $288 . \odot \odot \odot$ & $1.000 \odot$ & $\odot .8044 \mathrm{E}+\odot 7$ \\
\hline 41 & $288.0 \odot \odot$ & $288.0 \odot \odot$ & 1.0000 & $0.8044 \mathrm{E}+07$ \\
\hline 42 & 288.000 & 288.000 & 1.0000 & $0.8044 \mathrm{E}+07$ \\
\hline 43 & $288 . \odot \odot \odot$ & $288.0 \odot \odot$ & $1.000 \odot$ & $\odot .8044 \mathrm{E}+\odot 7$ \\
\hline 44 & $288 . \odot \odot \odot$ & $288.0 \odot \odot$ & $1.000 \odot$ & $\odot .8044 \mathrm{E}+\odot 7$ \\
\hline 45 & 288.000 & 288.000 & 1.0000 & $0.8044 \mathrm{E}+07$ \\
\hline 46 & $288 . \odot \odot \odot$ & $288 . \odot \odot \odot$ & $1.000 \odot$ & $0.8044 \mathrm{E}+\odot 7$ \\
\hline 47 & $288 . \odot \odot \odot$ & 288.000 & 1.0000 & $0.8044 \mathrm{E}+07$ \\
\hline 48 & $88.00 \odot$ & $288.00 \odot$ & 1.0000 & $0.8044 \mathrm{E}+07$ \\
\hline 49 & $288 . \odot \odot \odot$ & $288 . \odot \odot \odot$ & $1.000 \odot$ & $\odot .8044 \mathrm{E}+\odot 7$ \\
\hline 50 & $288 . \odot \odot \odot$ & $288.00 \odot$ & $1.000 \odot$ & $\odot .8044 \mathrm{E}+\odot 7$ \\
\hline 51 & $288.00 \odot$ & 288.000 & 1.0000 & $\odot .8044 \mathrm{E}+07$ \\
\hline 52 & $288.00 \odot$ & $288.0 \odot \odot$ & 1.0000 & $0.8044 \mathrm{E}+07$ \\
\hline 53 & $288.0 \odot \odot$ & 288.000 & 1.0000 & $\odot .8044 \mathrm{E}+07$ \\
\hline 54 & $288 . \odot \odot \odot$ & $288.0 \odot \odot$ & $1.000 \odot$ & $\odot .8044 \mathrm{E}+\odot 7$ \\
\hline 55 & $288 . \odot \odot \odot$ & $288.0 \odot \odot$ & 1.0000 & $0.8044 \mathrm{E}+07$ \\
\hline 56 & $288 . \odot \odot \odot$ & $288 . \odot \odot \odot$ & 1.0000 & $0.8044 \mathrm{E}+\odot 7$ \\
\hline 57 & $288 . \odot \odot \odot$ & $288.00 \odot$ & 1.0000 & $0.8044 \mathrm{E}+\odot 7$ \\
\hline 58 & 288.000 & 288.000 & 1.0000 & $0.8044 \mathrm{E}+07$ \\
\hline 59 & 288.000 & 288.000 & 1.0000 & $0.8044 \mathrm{E}+07$ \\
\hline 60 & $288.00 \odot$ & 288.000 & 1.0000 & $0.8044 \mathrm{E}+07$ \\
\hline tim & & 26.00 & \multicolumn{2}{|c|}{ lambda $=0.1767742932$} \\
\hline node & $\mathrm{T}(\mathrm{K})$ & Twall(K) & $\mathrm{x}$ & $\mathrm{P}(\mathrm{Pa})$ \\
\hline 1 & 128.896 & 171.789 & 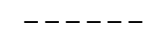 & $0.5554 \mathrm{E}+06$ \\
\hline 2 & 149.519 & 208.407 & 0.9822 & $0.1024 \mathrm{E}+07$ \\
\hline 3 & 204.874 & 251.907 & 1.0000 & $\odot .4480 \mathrm{E}+\odot 7$ \\
\hline 4 & & 267.339 & 1.0000 & $0.5553 \mathrm{E}+07$ \\
\hline 5 & 245.178 & 275.155 & 1.0000 & $0.6191 \mathrm{E}+07$ \\
\hline 6 & 251.055 & 280.725 & 1.0000 & $0.6505 \mathrm{E}+07$ \\
\hline 7 & 255.737 & 284.209 & 1.0000 & $0.6712 \mathrm{E}+07$ \\
\hline 8 & 267.680 & 287.146 & $1.00 \odot \odot$ & $0.7090 \mathrm{E}+07$ \\
\hline 9 & 282.724 & $288.00 \odot$ & 1.0000 & $0.7647 \mathrm{E}+07$ \\
\hline 10 & 574 & $288.00 \odot$ & $1.000 \odot$ & $\odot .7967 \mathrm{E}+\odot 7$ \\
\hline 11 & 288.000 & 288.000 & 1.0000 & $\odot .8039 \mathrm{E}+07$ \\
\hline 12 & $288.00 \odot$ & $288 . \odot \odot \odot$ & $1.000 \odot$ & $\odot .8044 \mathrm{E}+\odot 7$ \\
\hline 13 & 288.000 & $288.00 \odot$ & 1.0000 & $\odot .8044 \mathrm{E}+07$ \\
\hline 14 & $288 . \odot \odot \odot$ & $288 . \odot \odot \odot$ & 1.0000 & $\odot .8044 \mathrm{E}+07$ \\
\hline 15 & 288.000 & 288.000 & 1.0000 & $\odot .8044 \mathrm{E}+07$ \\
\hline 16 & 88.000 & $288.0 \odot \odot$ & 1.0000 & $\odot .8044 \mathrm{E}+07$ \\
\hline
\end{tabular}




\begin{tabular}{|c|c|c|c|c|}
\hline 17 & $288 . \odot \odot \odot$ & $288.00 \odot$ & $1.0 \odot \odot \odot$ & $0.8044 \mathrm{E}+\odot 7$ \\
\hline 18 & 288.000 & 288.000 & 1.0000 & $0.8044 \mathrm{E}+07$ \\
\hline 19 & $288.00 \odot$ & $288.0 \odot \odot$ & $1.000 \odot$ & $0.8044 \mathrm{E}+07$ \\
\hline 20 & $288.0 \odot \odot$ & $288 . \odot \odot \odot$ & $1.00 \odot \odot$ & $\odot .8044 \mathrm{E}+\odot 7$ \\
\hline 21 & $288.00 \odot$ & $288.0 \odot \odot$ & $1.000 \odot$ & $\odot .8044 \mathrm{E}+07$ \\
\hline 22 & $288.00 \odot$ & $288.00 \odot$ & 1.0000 & $\odot .8 \odot 44 \mathrm{E}+\odot 7$ \\
\hline 23 & 288.000 & $288.00 \odot$ & $1.000 \odot$ & $\odot .8044 \mathrm{E}+07$ \\
\hline 24 & 288.000 & $288.00 \odot$ & 1.0000 & $0.8044 \mathrm{E}+07$ \\
\hline 25 & 288.000 & $288.00 \odot$ & $1.000 \odot$ & $\odot .8044 \mathrm{E}+07$ \\
\hline 26 & 288.000 & 288.000 & 1.0000 & $0.8044 \mathrm{E}+07$ \\
\hline 27 & $288.0 \odot \odot$ & $288 . \odot \odot \odot$ & $1.00 \odot \odot$ & $\odot .8044 \mathrm{E}+07$ \\
\hline 28 & $288.00 \odot$ & 288.000 & 1.0000 & $0.8044 \mathrm{E}+07$ \\
\hline 29 & $288.00 \odot$ & $288.0 \odot \odot$ & $1.000 \odot$ & $0.8044 \mathrm{E}+07$ \\
\hline 30 & 288.000 & $288.00 \odot$ & $1.000 \odot$ & $\odot .8044 \mathrm{E}+07$ \\
\hline 31 & $288 . \odot \odot \odot$ & $288 . \odot \odot \odot$ & $1.00 \odot \odot$ & $\odot .8044 \mathrm{E}+07$ \\
\hline 32 & $288.00 \odot$ & $288.0 \odot \odot$ & $1.000 \odot$ & $\odot .8044 \mathrm{E}+07$ \\
\hline 33 & $288.00 \odot$ & $288 . \odot \odot \odot$ & $1.000 \odot$ & $\odot .8044 \mathrm{E}+\odot 7$ \\
\hline 34 & $288.0 \odot \odot$ & $288.0 \odot \odot$ & $1.00 \odot \odot$ & $\odot .8 \odot 44 \mathrm{E}+\odot 7$ \\
\hline 35 & 288.000 & $288.00 \odot$ & 1.0000 & $0.8044 \mathrm{E}+07$ \\
\hline 36 & $288.00 \odot$ & $288.0 \odot \odot$ & $1.000 \odot$ & $\odot .8044 \mathrm{E}+07$ \\
\hline 37 & $288.00 \odot$ & $288.0 \odot \odot$ & $1.000 \odot$ & $\odot .8 \odot 44 \mathrm{E}+07$ \\
\hline 38 & $288.00 \odot$ & $288.0 \odot \odot$ & $1.00 \odot \odot$ & $\odot .8044 \mathrm{E}+07$ \\
\hline 39 & 288.000 & $288.00 \odot$ & 1.0000 & $\odot .8044 \mathrm{E}+07$ \\
\hline 40 & $288.0 \odot \odot$ & $288.00 \odot$ & $1.00 \odot \odot$ & $0.8044 \mathrm{E}+07$ \\
\hline 41 & $288.00 \odot$ & 288.000 & 1.0000 & $0.8044 \mathrm{E}+07$ \\
\hline 42 & $288.00 \odot$ & $288.0 \odot \odot$ & 1.0000 & $0.8044 \mathrm{E}+07$ \\
\hline 43 & $288 . \odot \odot \odot$ & $288.0 \odot \odot$ & 1.0000 & $\odot .8044 \mathrm{E}+\odot 7$ \\
\hline 44 & $288 . \odot \odot \odot$ & $288.0 \odot \odot$ & $1.00 \odot \odot$ & $\odot .8 \odot 44 \mathrm{E}+\odot 7$ \\
\hline 45 & $288.0 \odot \odot$ & $288.0 \odot \odot$ & $1.00 \odot \odot$ & $\odot .8 \odot 44 \mathrm{E}+\odot 7$ \\
\hline 46 & 288.000 & $288.00 \odot$ & $1.00 \odot \odot$ & $\odot .8044 \mathrm{E}+07$ \\
\hline 47 & $288.0 \odot \odot$ & $288.0 \odot \odot$ & $1 . \odot \odot \odot \odot$ & $\odot .8044 \mathrm{E}+\odot 7$ \\
\hline 48 & $288.0 \odot \odot$ & $288.0 \odot \odot$ & $1 . \odot \odot \odot \odot$ & $\odot .8 \odot 44 \mathrm{E}+\odot 7$ \\
\hline 49 & 288.000 & $288.00 \odot$ & $1.000 \odot$ & $\odot .8044 \mathrm{E}+07$ \\
\hline 50 & $288.00 \odot$ & $288.00 \odot$ & $1.0 \odot \odot \odot$ & $0.8044 \mathrm{E}+07$ \\
\hline 51 & $288 . \odot \odot \odot$ & $288.00 \odot$ & $1 . \odot \odot \odot \odot$ & $\odot .8 \odot 44 \mathrm{E}+07$ \\
\hline 52 & $288.00 \odot$ & $288.0 \odot \odot$ & $1.000 \odot$ & $0.8044 \mathrm{E}+07$ \\
\hline 53 & $288 . \odot \odot \odot$ & $288.00 \odot$ & $1.0 \odot \odot \odot$ & $\odot .8044 \mathrm{E}+\odot 7$ \\
\hline 54 & $288.0 \odot \odot$ & $288.00 \odot$ & $1.0 \odot \odot \odot$ & $\odot .8044 \mathrm{E}+\odot 7$ \\
\hline 55 & $288.0 \odot \odot$ & $288 . \odot \odot \odot$ & $1.00 \odot \odot$ & $\odot .8 \odot 44 \mathrm{E}+\odot 7$ \\
\hline 56 & $288.00 \odot$ & $288.0 \odot \odot$ & $1.000 \odot$ & $\odot .8044 \mathrm{E}+\odot 7$ \\
\hline 57 & $288.0 \odot \odot$ & $288.0 \odot \odot$ & $1.0 \odot \odot \odot$ & $\odot .8044 \mathrm{E}+\odot 7$ \\
\hline 58 & 288.000 & $288.0 \odot \odot$ & 1.0000 & $\odot .8044 \mathrm{E}+07$ \\
\hline 59 & $288.00 \odot$ & $288.0 \odot \odot$ & $1.000 \odot$ & $\odot .8044 \mathrm{E}+07$ \\
\hline 60 & 288.000 & 288.000 & 1.0000 & $\odot .8044 \mathrm{E}+07$ \\
\hline time & & $27 . \odot \odot$ & lambda & 26806164 \\
\hline node & $\mathrm{T}(\mathrm{K})$ & Twall(K) & $x$ & $\mathrm{P}(\mathrm{Pa})$ \\
\hline 1 & 128.527 & 170.111 & - - - - & $0.5418 \mathrm{E}+06$ \\
\hline 2 & 149.091 & 206.445 & $\odot .9816$ & $\odot .9993 \mathrm{E}+06$ \\
\hline 3 & 202.150 & 249.824 & $1.000 \odot$ & $0.4421 \mathrm{E}+07$ \\
\hline 4 & 231.019 & 266.118 & $1.0 \odot \odot \odot$ & $\odot .5470 \mathrm{E}+\odot 7$ \\
\hline 5 & 244.943 & 274.239 & 1.0000 & $\odot .6160 \mathrm{E}+07$ \\
\hline 6 & 251.207 & 279.905 & $1.00 \odot \odot$ & $\odot .6504 \mathrm{E}+\odot 7$ \\
\hline 7 & 255.992 & 283.517 & $1.00 \odot \odot$ & $\odot .6719 \mathrm{E}+\odot 7$ \\
\hline 8 & 263.215 & 286.536 & $1 . \odot \odot \odot \odot$ & $\odot .6972 \mathrm{E}+\odot 7$ \\
\hline 9 & 279.545 & 288.000 & 1.0000 & $\odot .7505 \mathrm{E}+\odot 7$ \\
\hline 10 & 286.915 & $288 . \odot \odot \odot$ & $1.0 \odot \odot \odot$ & $\odot .7909 \mathrm{E}+07$ \\
\hline
\end{tabular}




\begin{tabular}{|c|c|c|c|c|}
\hline 11 & $288.0 \odot \odot$ & $288.00 \odot$ & 1.0000 & $\odot .8031 \mathrm{E}+07$ \\
\hline 12 & $288 . \odot \odot \odot$ & 288.000 & $1.000 \odot$ & $\odot .8044 \mathrm{E}+\odot 7$ \\
\hline 13 & $288.0 \odot \odot$ & $288.00 \odot$ & 1.0000 & $\odot .8044 \mathrm{E}+07$ \\
\hline 14 & $288.0 \odot \odot$ & $288.00 \odot$ & 1.0000 & $\odot .8044 \mathrm{E}+\odot 7$ \\
\hline 15 & $288.0 \odot \odot$ & 288.000 & 1.0000 & $0.8044 \mathrm{E}+07$ \\
\hline 16 & $288 . \odot \odot \odot$ & 288.000 & $1.000 \odot$ & $\odot .8044 \mathrm{E}+07$ \\
\hline 17 & $288.0 \odot \odot$ & $288.00 \odot$ & 1.0000 & $\odot .8044 \mathrm{E}+07$ \\
\hline 18 & $288.0 \odot \odot$ & 288.000 & 1.0000 & $\odot .8044 \mathrm{E}+\odot 7$ \\
\hline 19 & $288.0 \odot \odot$ & $288.00 \odot$ & 1.0000 & $\odot .8044 \mathrm{E}+07$ \\
\hline 20 & $288 . \odot \odot \odot$ & $288.0 \odot \odot$ & $1.000 \odot$ & $\odot .8044 \mathrm{E}+07$ \\
\hline 21 & $288.0 \odot \odot$ & 288.000 & 1.0000 & $\odot .8044 \mathrm{E}+07$ \\
\hline 22 & $288 . \odot \odot \odot$ & $288.0 \odot \odot$ & $1.000 \odot$ & $\odot .8044 \mathrm{E}+07$ \\
\hline 23 & $288.0 \odot \odot$ & 288.000 & 1.0000 & $\odot .8044 \mathrm{E}+07$ \\
\hline 24 & $288.0 \odot \odot$ & $288.0 \odot \odot$ & 1.0000 & $\odot .8044 \mathrm{E}+07$ \\
\hline 25 & $288.00 \odot$ & 288.000 & 1.0000 & $0.8044 \mathrm{E}+07$ \\
\hline 26 & $288 . \odot \odot \odot$ & $288.00 \odot$ & $1.000 \odot$ & $\odot .8044 \mathrm{E}+07$ \\
\hline 27 & $288 . \odot \odot \odot$ & $288.0 \odot \odot$ & $1.000 \odot$ & $\odot .8044 \mathrm{E}+\odot 7$ \\
\hline 28 & $288.00 \odot$ & 288.000 & 1.0000 & $0.8044 \mathrm{E}+07$ \\
\hline 29 & $288.0 \odot \odot$ & 288.000 & 1.0000 & $\odot .8044 \mathrm{E}+07$ \\
\hline 30 & $288.0 \odot \odot$ & 288.000 & 1.0000 & $0.8044 \mathrm{E}+07$ \\
\hline 31 & $288.00 \odot$ & 288.000 & 1.0000 & $0.8044 \mathrm{E}+07$ \\
\hline 32 & $288 . \odot \odot \odot$ & $288.0 \odot \odot$ & $1.000 \odot$ & $\odot .8044 \mathrm{E}+\odot 7$ \\
\hline 33 & $288 . \odot \odot \odot$ & 288.000 & 1.0000 & $0.8044 \mathrm{E}+07$ \\
\hline 34 & $288.0 \odot \odot$ & 288.000 & 1.0000 & $0.8044 \mathrm{E}+07$ \\
\hline 35 & $288.00 \odot$ & 288.000 & 1.0000 & $0.8044 \mathrm{E}+07$ \\
\hline 36 & $288.00 \odot$ & 288.000 & 1.0000 & $0.8044 \mathrm{E}+07$ \\
\hline 37 & 88.000 & 288.000 & 1.0000 & $0.8044 \mathrm{E}+07$ \\
\hline 38 & $288.0 \odot \odot$ & 288.000 & 1.0000 & $0.8044 \mathrm{E}+07$ \\
\hline 39 & $288 . \odot \odot \odot$ & $288.0 \odot \odot$ & $1.000 \odot$ & $\odot .8044 \mathrm{E}+\odot 7$ \\
\hline 40 & $288.0 \odot \odot$ & 288.000 & 1.0000 & $\odot .8044 \mathrm{E}+07$ \\
\hline 41 & $288.0 \odot \odot$ & 288.000 & 1.0000 & $0.8044 \mathrm{E}+07$ \\
\hline 42 & $288.00 \odot$ & 288.000 & 1.0000 & $0.8044 \mathrm{E}+07$ \\
\hline 43 & $88.00 \odot$ & $288.0 \odot \odot$ & $1.000 \odot$ & $\odot .8044 \mathrm{E}+\odot 7$ \\
\hline 44 & 88.000 & 288.000 & 1.0000 & $0.8044 \mathrm{E}+07$ \\
\hline 45 & $288 . \odot 0 \odot$ & 288.000 & 1.0000 & $0.8044 \mathrm{E}+07$ \\
\hline 46 & $288 . \odot \odot \odot$ & $288.00 \odot$ & $1.000 \odot$ & $0.8044 \mathrm{E}+\odot 7$ \\
\hline 47 & $288.00 \odot$ & 288.000 & 1.0000 & $0.8044 \mathrm{E}+07$ \\
\hline 48 & $288.00 \odot$ & 288.000 & 1.0000 & $0.8044 \mathrm{E}+07$ \\
\hline 49 & $288 . \odot \odot \odot$ & $288.0 \odot \odot$ & $1.000 \odot$ & $\odot .8044 \mathrm{E}+\odot 7$ \\
\hline 50 & $\odot \odot$ & $288.00 \odot$ & 1.00 & $\odot .8044 \mathrm{E}+\odot 7$ \\
\hline 51 & $288.00 \odot$ & $288.00 \odot$ & 1.0000 & $\odot .8044 \mathrm{E}+07$ \\
\hline 52 & $288 . \odot \odot \odot$ & $288.0 \odot \odot$ & $1.00 \odot \odot$ & $\odot .8044 \mathrm{E}+\odot 7$ \\
\hline 53 & $288.00 \odot$ & 288.000 & 1.0000 & $0.8044 \mathrm{E}+07$ \\
\hline 54 & $288.00 \odot$ & 288.000 & 1.0000 & $0.8044 \mathrm{E}+07$ \\
\hline 55 & $288 . \odot \odot \odot$ & $288.0 \odot \odot$ & $1.000 \odot$ & $\odot .8044 \mathrm{E}+\odot 7$ \\
\hline 56 & $288 . c$ & $288 . \odot$ & 1.00 & $0.8044 \mathrm{E}+07$ \\
\hline 57 & 288.000 & 288.000 & 1.0000 & $\odot .8044 \mathrm{E}+07$ \\
\hline 58 & 288.000 & 288.000 & 1.0000 & $\odot .8044 \mathrm{E}+07$ \\
\hline 59 & 288.000 & 288.000 & 1.0000 & $0.8044 \mathrm{E}+07$ \\
\hline 60 & $288 . \odot \odot \odot$ & $288.0 \odot \odot$ & $1.000 \odot$ & $\odot .8044 \mathrm{E}+\odot 7$ \\
\hline time & & \multicolumn{3}{|c|}{$28.00 \quad$ lambda } \\
\hline & & Twall(K) & $x$ & $P(P a)$ \\
\hline 1 & 127.918 & 168.519 & $-\ldots$ & $0.5279 \mathrm{E}+06$ \\
\hline 2 & 148.385 & 204.614 & 0.9810 & $0.9737 \mathrm{E}+06$ \\
\hline 3 & 199.940 & 247.688 & 1.0000 & $0.4372 \mathrm{E}+07$ \\
\hline 4 & & & 1.0000 & $\odot .5384 \mathrm{E}+\odot 7$ \\
\hline
\end{tabular}




\begin{tabular}{|c|c|c|c|c|}
\hline 5 & 244.373 & 273.324 & 1.0000 & $0.6117 \mathrm{E}+07$ \\
\hline 6 & 251.360 & 279.295 & 1.0000 & $\odot .6500 \mathrm{E}+07$ \\
\hline 7 & 256.263 & 282.906 & 1.0000 & $0.6726 \mathrm{E}+07$ \\
\hline 8 & 260.554 & 285.925 & 1.0000 & $\odot .6901 \mathrm{E}+\odot 7$ \\
\hline 9 & $275.5 \odot 4$ & 287.733 & $1.000 \odot$ & $\odot .7361 \mathrm{E}+07$ \\
\hline 10 & 285.799 & $288 . \odot \odot \odot$ & $1.000 \odot$ & $\odot .7828 \mathrm{E}+\odot 7$ \\
\hline 11 & $288.0 \odot \odot$ & $288 . \odot \odot \odot$ & $1.000 \odot$ & $\odot .8017 \mathrm{E}+07$ \\
\hline 12 & $288.0 \odot \odot$ & $288.0 \odot \odot$ & $1.000 \odot$ & $\odot .8044 \mathrm{E}+07$ \\
\hline 13 & $288.0 \odot \odot$ & $288 . \odot \odot \odot$ & $1.000 \odot$ & $\odot .8044 \mathrm{E}+07$ \\
\hline 14 & $288.0 \odot \odot$ & 288.000 & 1.0000 & $\odot .8044 \mathrm{E}+07$ \\
\hline 15 & $288 . \odot \odot \odot$ & $288 . \odot \odot \odot$ & 1.0000 & $\odot .8044 \mathrm{E}+07$ \\
\hline 16 & $288.0 \odot \odot$ & 288.000 & 1.0000 & $0.8044 \mathrm{E}+07$ \\
\hline 17 & $288 . \odot \odot \odot$ & $288.00 \odot$ & 1.0000 & $\odot .8044 \mathrm{E}+07$ \\
\hline 18 & $288.0 \odot \odot$ & $288 . \odot \odot \odot$ & $1.000 \odot$ & $\odot .8044 \mathrm{E}+07$ \\
\hline 19 & $288.0 \odot \odot$ & $288.0 \odot \odot$ & 1.0000 & $0.8044 \mathrm{E}+07$ \\
\hline 20 & 288.000 & $288.0 \odot \odot$ & 1.0000 & $\odot .8044 \mathrm{E}+07$ \\
\hline 21 & $288 . \odot \odot \odot$ & $288.00 \odot$ & 1.0000 & $\odot .8044 \mathrm{E}+\odot 7$ \\
\hline 22 & 288.000 & $288.00 \odot$ & 1.0000 & $0.8044 \mathrm{E}+07$ \\
\hline 23 & $288.0 \odot \odot$ & $288.00 \odot$ & 1.0000 & $\odot .8044 \mathrm{E}+07$ \\
\hline 24 & $288 . \odot \odot \odot$ & $288 . \odot \odot \odot$ & 1.0000 & $\odot .8044 \mathrm{E}+07$ \\
\hline 25 & $288.00 \odot$ & $288.0 \odot \odot$ & 1.0000 & $0.8044 \mathrm{E}+07$ \\
\hline 26 & $288.0 \odot \odot$ & $288.0 \odot \odot$ & 1.0000 & $0.8044 \mathrm{E}+07$ \\
\hline 27 & $288.00 \odot$ & 288.000 & $1.000 \odot$ & $\odot .8044 \mathrm{E}+07$ \\
\hline 28 & $288.00 \odot$ & 288.000 & 1.0000 & $0.8044 \mathrm{E}+07$ \\
\hline 29 & 288.000 & 288.000 & 1.0000 & $0.8044 \mathrm{E}+07$ \\
\hline 30 & $288.0 \odot \odot$ & $288.0 \odot \odot$ & 1.0000 & $0.8044 \mathrm{E}+07$ \\
\hline 31 & $288.00 \odot$ & $288.0 \odot \odot$ & 1.0000 & $0.8044 \mathrm{E}+07$ \\
\hline 32 & $288.00 \odot$ & $288.0 \odot \odot$ & 1.0000 & $\odot .8044 \mathrm{E}+07$ \\
\hline 33 & $288.0 \odot \odot$ & $288.0 \odot \odot$ & 1.0000 & $\odot .8044 \mathrm{E}+07$ \\
\hline 34 & $288 . \odot \odot \odot$ & $288 . \odot \odot \odot$ & $1.000 \odot$ & $\odot .8044 \mathrm{E}+07$ \\
\hline 35 & $288.00 \odot$ & $288.0 \odot \odot$ & 1.0000 & $\odot .8044 \mathrm{E}+07$ \\
\hline 36 & 288.000 & $288.0 \odot \odot$ & 1.0000 & $0.8044 \mathrm{E}+07$ \\
\hline 37 & $288.00 \odot$ & $288.0 \odot \odot$ & 1.0000 & $0.8044 \mathrm{E}+07$ \\
\hline 38 & 288.000 & $288.0 \odot \odot$ & 1.0000 & $0.8044 \mathrm{E}+07$ \\
\hline 39 & $288.00 \odot$ & $288.0 \odot \odot$ & 1.0000 & $0.8044 \mathrm{E}+07$ \\
\hline 40 & $288 . \odot \odot \odot$ & $288 . \odot \odot \odot$ & 1.0000 & $0.8044 \mathrm{E}+07$ \\
\hline 41 & $288.00 \odot$ & $288.00 \odot$ & 1.0000 & $0.8044 \mathrm{E}+07$ \\
\hline 42 & $288.00 \odot$ & $288.0 \odot \odot$ & 1.0000 & $0.8044 \mathrm{E}+07$ \\
\hline 43 & $288.00 \odot$ & $288 . \odot \odot \odot$ & 1.0000 & $0.8044 \mathrm{E}+07$ \\
\hline 44 & $288 . \odot \odot \odot$ & $288 . \odot \odot \odot$ & 1.0000 & $\odot .8044 \mathrm{E}+\odot 7$ \\
\hline 45 & 288.000 & $288.0 \odot \odot$ & 1.0000 & $0.8044 \mathrm{E}+07$ \\
\hline 46 & $288.0 \odot \odot$ & $288.0 \odot \odot$ & 1.0000 & $\odot .8044 \mathrm{E}+07$ \\
\hline 47 & 288.000 & 288.000 & 1.0000 & $\odot .8044 \mathrm{E}+07$ \\
\hline 48 & $288.00 \odot$ & $288.0 \odot \odot$ & 1.0000 & $0.8044 \mathrm{E}+07$ \\
\hline 49 & $288.00 \odot$ & $288 . \odot \odot \odot$ & 1.0000 & $\odot .8044 \mathrm{E}+\odot 7$ \\
\hline 50 & $288 . \odot \odot \odot$ & $288 . \odot \odot \odot$ & 1.0000 & $\odot .8044 \mathrm{E}+07$ \\
\hline 51 & $288.00 \odot$ & $288.00 \odot$ & 1.0000 & $0.8044 \mathrm{E}+07$ \\
\hline 52 & 288.000 & $288.0 \odot \odot$ & 1.0000 & $0.8044 \mathrm{E}+07$ \\
\hline 53 & $288.0 \odot \odot$ & 288.000 & 1.0000 & $\odot .8044 \mathrm{E}+07$ \\
\hline 54 & 288.000 & $288.0 \odot \odot$ & 1.0000 & $0.8044 \mathrm{E}+07$ \\
\hline 55 & 288.000 & $288.0 \odot \odot$ & 1.0000 & $\odot .8044 \mathrm{E}+07$ \\
\hline 56 & $288.0 \odot \odot$ & $288.0 \odot \odot$ & 1.0000 & $\odot .8044 \mathrm{E}+07$ \\
\hline 57 & $288 . \odot \odot \odot$ & $288 . \odot \odot \odot$ & 1.0000 & $\odot .8044 \mathrm{E}+\odot 7$ \\
\hline 58 & $288.00 \odot$ & $288.0 \odot \odot$ & 1.0000 & $\odot .8044 \mathrm{E}+07$ \\
\hline 59 & $288 . \odot \odot \odot$ & $288 . \odot \odot \odot$ & 1.0000 & $\odot .8044 \mathrm{E}+\odot 7$ \\
\hline 60 & $288.0 \odot \odot$ & 288.000 & 1.0000 & $\odot .8044 \mathrm{E}+07$ \\
\hline
\end{tabular}




\begin{tabular}{|c|c|c|c|c|}
\hline time & $(\sec )=$ & $29.00 \quad l$ & $d a=0$. & $\begin{array}{r}9131775 \\
P(\mathrm{~Pa})\end{array}$ \\
\hline 1 & 127.354 & $\begin{array}{r}166.993 \\
161+(n)\end{array}$ & _ _ . . & $\odot .5150 E+\odot 6$ \\
\hline 2 & 147.731 & 202.783 & $\odot .9804$ & $\odot .950 \odot E+\odot 6$ \\
\hline 3 & 198.404 & 245.640 & 1.0000 & $\odot .4339 \mathrm{E}+07$ \\
\hline 4 & 226.165 & 263.677 & $1.000 \odot$ & $\odot .5300 \mathrm{E}+\odot 7$ \\
\hline 5 & 243.471 & 272.408 & $1.000 \odot$ & $\odot .6062 \mathrm{E}+07$ \\
\hline 6 & 251.347 & 278.685 & $1.000 \odot$ & $\odot .6488 \mathrm{E}+\odot 7$ \\
\hline 7 & 256.263 & 282.296 & 1.0000 & $0.6726 \mathrm{E}+07$ \\
\hline 8 & 259.689 & 285.315 & $1.000 \odot$ & $0.6878 \mathrm{E}+07$ \\
\hline 9 & 270.870 & 287.428 & 1.0000 & $0.7224 \mathrm{E}+07$ \\
\hline 10 & 283.989 & 288.000 & 1. $\odot \odot \odot \odot$ & $\odot .7721 E+07$ \\
\hline 11 & 287.725 & $288.0 \odot \odot$ & $1.000 \odot$ & $\odot .7987 \mathrm{E}+\odot 7$ \\
\hline 12 & 288.000 & 288.000 & 1.0000 & $0.8041 \mathrm{E}+07$ \\
\hline 13 & $288.00 \odot$ & $288 . \odot \odot \odot$ & $1.000 \odot$ & $\odot .8044 \mathrm{E}+\odot 7$ \\
\hline 14 & $288.00 \odot$ & 288.000 & 1.0000 & $\odot .8044 \mathrm{E}+\odot 7$ \\
\hline 15 & 288.000 & 288.000 & 1.0000 & $0.8044 \mathrm{E}+07$ \\
\hline 16 & $288.0 \odot \odot$ & $288.0 \odot \odot$ & 1. $0 \odot \odot \odot$ & $\odot .8 \odot 44 \mathrm{E}+\odot 7$ \\
\hline 17 & 288.000 & $288.00 \odot$ & 1.0000 & $0.8044 \mathrm{E}+07$ \\
\hline 18 & 288.000 & 288.000 & 1.0000 & $0.8044 \mathrm{E}+07$ \\
\hline 19 & 288.000 & 288.000 & 1.0000 & $0.8044 \mathrm{E}+07$ \\
\hline 20 & 288.000 & $288.00 \odot$ & 1.0000 & $0.8044 \mathrm{E}+07$ \\
\hline 21 & 288.000 & $288 . \odot \odot \odot$ & 1.0000 & $\odot .8044 \mathrm{E}+07$ \\
\hline 22 & $288.00 \odot$ & 288.000 & $1.000 \odot$ & $0.8044 \mathrm{E}+07$ \\
\hline 23 & 288.000 & 288.000 & 1.0000 & $0.8044 \mathrm{E}+07$ \\
\hline 24 & 288.000 & 288.000 & 1.0000 & $0.8044 \mathrm{E}+07$ \\
\hline 25 & 288.000 & 288.000 & 1.0000 & $0.8044 \mathrm{E}+07$ \\
\hline 26 & 288.000 & 288.000 & 1.0000 & $\odot .8044 \mathrm{E}+07$ \\
\hline 27 & $288.0 \odot \odot$ & $288.0 \odot \odot$ & $1.00 \odot \odot$ & $\odot .8 \odot 44 \mathrm{E}+\odot 7$ \\
\hline 28 & $288.00 \odot$ & 288.000 & $1.000 \odot$ & $\odot .8044 \mathrm{E}+07$ \\
\hline 29 & 288.000 & 288.000 & $1.000 \odot$ & $\odot .8044 \mathrm{E}+\odot 7$ \\
\hline 30 & $288.00 \odot$ & $288.0 \odot \odot$ & $1.000 \odot$ & $\odot .8044 \mathrm{E}+\odot 7$ \\
\hline 31 & 288.000 & 288.000 & 1.0000 & $\odot .8044 \mathrm{E}+07$ \\
\hline 32 & $288.00 \odot$ & $288 . \odot \odot \odot$ & $1.00 \odot \odot$ & $\odot .8044 \mathrm{E}+\odot 7$ \\
\hline 33 & $288.00 \odot$ & $288.00 \odot$ & 1. $00 \odot \odot$ & $\odot .8 \odot 44 \mathrm{E}+07$ \\
\hline 34 & $288 . \odot \odot \odot$ & $288.0 \odot \odot$ & $1.000 \odot$ & $0.8044 \mathrm{E}+07$ \\
\hline 35 & 288.000 & 288.000 & 1.0000 & $0.8044 \mathrm{E}+07$ \\
\hline 36 & 288.000 & 288.000 & 1.0000 & $\odot .8044 \mathrm{E}+\odot 7$ \\
\hline 37 & $288.00 \odot$ & 288.000 & $1.000 \odot$ & $\odot .8044 \mathrm{E}+07$ \\
\hline 38 & 288.000 & 288.000 & 1.0000 & $0.8044 \mathrm{E}+07$ \\
\hline 39 & $288.00 \odot$ & $288.00 \odot$ & $1.000 \odot$ & $\odot .8044 \mathrm{E}+\odot 7$ \\
\hline 40 & 288.000 & $288.00 \odot$ & 1.0000 & $\odot .8044 \mathrm{E}+07$ \\
\hline 41 & 288.000 & 288.000 & 1.0000 & $0.8044 \mathrm{E}+07$ \\
\hline 42 & 288.000 & 288.000 & 1.0000 & $0.8044 \mathrm{E}+07$ \\
\hline 43 & 288.000 & $288.00 \odot$ & 1.0000 & $\odot .8044 \mathrm{E}+07$ \\
\hline 44 & 288.000 & $288 . \odot \odot \odot$ & 1.0000 & $\odot .8044 \mathrm{E}+07$ \\
\hline 45 & $288 . \odot \odot \odot$ & $288.0 \odot \odot$ & $1.000 \odot$ & $0.8044 \mathrm{E}+07$ \\
\hline 46 & 288.000 & 288.000 & 1.0000 & $0.8044 \mathrm{E}+07$ \\
\hline 47 & 288.000 & 288.000 & $1.000 \odot$ & $0.8044 \mathrm{E}+07$ \\
\hline 48 & 288.000 & 288.000 & 1.0000 & $\odot .8044 \mathrm{E}+07$ \\
\hline 49 & 288.000 & 288.000 & 1.0000 & $0.8044 \mathrm{E}+07$ \\
\hline 50 & $288.00 \odot$ & $288.00 \odot$ & 1.0000 & $\odot .8044 \mathrm{E}+07$ \\
\hline 51 & 288.000 & $288.0 \odot \odot$ & $1.000 \odot$ & $\odot .8044 \mathrm{E}+\odot 7$ \\
\hline 52 & 288.000 & 288.000 & $1.00 \odot \odot$ & $\odot .8 \odot 44 \mathrm{E}+\odot 7$ \\
\hline 53 & $288.00 \odot$ & $288.0 \odot \odot$ & $1.000 \odot$ & $\odot .8044 \mathrm{E}+\odot 7$ \\
\hline 54 & 288.000 & $288.00 \odot$ & 1. $\odot \odot \odot \odot$ & $\odot .8 \odot 44 \mathrm{E}+\odot 7$ \\
\hline 55 & 288.000 & 288.000 & 1.0000 & $0.8044 \mathrm{E}+07$ \\
\hline
\end{tabular}




$\begin{array}{lllll}56 & 288.00 \odot & 288.00 \odot & 1.00 \odot \odot & \odot .8 \odot 44 \mathrm{E}+\odot 7 \\ 57 & 288.00 \odot & 288.00 \odot & 1.00 \odot \odot & \odot .8044 \mathrm{E}+\odot 7 \\ 58 & 288.00 \odot & 288.00 \odot & 1.000 \odot & \odot .8044 \mathrm{E}+\odot 7 \\ 59 & 288.00 \odot & 288.00 \odot & 1.000 \odot & \odot .8044 \mathrm{E}+\odot 7 \\ 6 \odot & 288.00 \odot & 288.00 \odot & 1.000 \odot & \odot .8044 \mathrm{E}+\odot 7\end{array}$

\begin{tabular}{|c|c|c|c|c|}
\hline time & $(\sec )=$ & 30.00 la & $=0$ & $P(P a)$ \\
\hline $\begin{array}{c}\text { node } \\
1\end{array}$ & $\begin{array}{c}T(K) \\
126.836\end{array}$ & $\begin{array}{r}\text { Twall(K) } \\
165.555\end{array}$ & $\begin{array}{c}x \\
-\ldots-\ldots\end{array}$ & $\begin{array}{c}\mathrm{P}(\mathrm{Pa}) \\
0.5032 \mathrm{E}+06\end{array}$ \\
\hline 2 & 147.130 & 200.952 & $\odot .9798$ & $\odot .9282 E+\odot 6$ \\
\hline 3 & 197.549 & 243.657 & 1.0000 & $\odot .4320 \mathrm{E}+07$ \\
\hline 4 & 223.643 & 262.401 & 1.0000 & $\odot .5222 \mathrm{E}+07$ \\
\hline 5 & 242.233 & 271.493 & $1.000 \odot$ & $\odot .5998 \mathrm{E}+\odot 7$ \\
\hline 6 & 251.062 & 278.074 & 1.0000 & $\odot .6465 \mathrm{E}+07$ \\
\hline 7 & 256.263 & 281.685 & 1.0000 & $0.6721 \mathrm{E}+07$ \\
\hline 8 & 259.957 & 284.705 & 1.0000 & $\odot .6886 \mathrm{E}+07$ \\
\hline 9 & 266.636 & 286.954 & 1.0000 & $\odot .7111 \mathrm{E}+\odot 7$ \\
\hline 10 & 281.337 & $288.00 \odot$ & 1.0000 & $\odot .7595 \mathrm{E}+07$ \\
\hline 11 & 287.273 & $288 . \odot \odot \odot$ & 1.0000 & $\odot .7941 \mathrm{E}+07$ \\
\hline 12 & 288.000 & $288.0 \odot \odot$ & 1.0000 & $\odot .8036 \mathrm{E}+07$ \\
\hline 13 & $288.00 \odot$ & $288 . \odot \odot \odot$ & 1.0000 & $\odot .8044 \mathrm{E}+07$ \\
\hline 14 & 288.000 & $288.00 \odot$ & 1. $\odot \odot \odot \odot$ & $\odot .8044 \mathrm{E}+\odot 7$ \\
\hline 15 & 288.000 & 288.000 & 1.0000 & $\odot .8044 \mathrm{E}+07$ \\
\hline 16 & $288.0 \odot \odot$ & $288.00 \odot$ & 1.0000 & $\odot .8 \odot 44 \mathrm{E}+07$ \\
\hline 17 & 288.000 & $288.00 \odot$ & 1.0000 & $\odot .8044 \mathrm{E}+\odot 7$ \\
\hline 18 & 288.000 & 288.000 & 1.0000 & $\odot .8044 \mathrm{E}+07$ \\
\hline 19 & $288.00 \odot$ & $288 . \odot \odot \odot$ & $1.000 \odot$ & $\odot .8 \odot 44 \mathrm{E}+07$ \\
\hline 20 & 288.000 & $288.00 \odot$ & $1.00 \odot \odot$ & $\odot .8044 \mathrm{E}+\odot 7$ \\
\hline 21 & 288.000 & $288.00 \odot$ & 1.0000 & $\odot .8044 \mathrm{E}+07$ \\
\hline 22 & 288.000 & $288.00 \odot$ & $1.000 \odot$ & $\odot .8044 \mathrm{E}+\odot 7$ \\
\hline 23 & 288.000 & 288.000 & 1.0000 & $\odot .8044 \mathrm{E}+07$ \\
\hline 24 & 288.000 & $288.00 \odot$ & $1.000 \odot$ & $\odot .8044 \mathrm{E}+07$ \\
\hline 25 & 288.000 & 288.000 & 1.0000 & $\odot .8044 \mathrm{E}+\odot 7$ \\
\hline 26 & 288.000 & 288.000 & $1.0 \odot \odot \odot$ & $\odot .8044 \mathrm{E}+\odot 7$ \\
\hline 27 & 288.000 & 288.000 & 1.0000 & $\odot .8 \odot 44 \mathrm{E}+07$ \\
\hline 28 & 288.000 & 288.000 & 1.0000 & $\odot .8 \odot 44 \mathrm{E}+\odot 7$ \\
\hline 29 & 288.000 & 288.000 & 1.0000 & $\odot .8044 \mathrm{E}+07$ \\
\hline 30 & $288.00 \odot$ & $288 . \odot \odot \odot$ & $1.000 \odot$ & $\odot .8 \odot 44 \mathrm{E}+07$ \\
\hline 31 & 288.000 & $288.00 \odot$ & 1.0000 & $\odot .8044 \mathrm{E}+\odot 7$ \\
\hline 32 & 288.000 & 288.000 & 1.0000 & $\odot .8044 \mathrm{E}+\odot 7$ \\
\hline 33 & $288.00 \odot$ & $288 . \odot \odot \odot$ & 1.0000 & $\odot .8 \odot 44 \mathrm{E}+07$ \\
\hline 34 & $288.00 \odot$ & $288 . \odot \odot \odot$ & 1.0000 & $\odot .8 \odot 44 \mathrm{E}+\odot 7$ \\
\hline 35 & 288.000 & $288.0 \odot \odot$ & 1.0000 & $\odot .8044 \mathrm{E}+07$ \\
\hline 36 & $288.00 \odot$ & $288.00 \odot$ & $1.000 \odot$ & $\odot .8 \odot 44 \mathrm{E}+\odot 7$ \\
\hline 37 & 288.000 & $288.00 \odot$ & 1.0000 & $\odot .8044 \mathrm{E}+07$ \\
\hline 38 & $288.00 \odot$ & $288.0 \odot \odot$ & 1.0000 & $\odot .8 \odot 44 \mathrm{E}+\odot 7$ \\
\hline 39 & $288.0 \odot \odot$ & $288 . \odot \odot \odot$ & 1.0000 & $\odot .8 \odot 44 \mathrm{E}+07$ \\
\hline 40 & 288.000 & 288.000 & 1.0000 & $\odot .8044 \mathrm{E}+\odot 7$ \\
\hline 41 & 288.000 & 288.000 & 1.0000 & $\odot .8044 \mathrm{E}+07$ \\
\hline 42 & $288.0 \odot \odot$ & $288 . \odot \odot \odot$ & 1.0000 & $\odot .8 \odot 44 \mathrm{E}+\odot 7$ \\
\hline 43 & 288.000 & $288.00 \odot$ & 1.0000 & $\odot .8044 \mathrm{E}+\odot 7$ \\
\hline 44 & 288.000 & $288.00 \odot$ & 1.0000 & $\odot .8044 \mathrm{E}+07$ \\
\hline 45 & 288.000 & 288.000 & 1.0000 & $\odot .8044 \mathrm{E}+\odot 7$ \\
\hline 46 & 288.000 & 288.000 & 1.0000 & $\odot .8044 \mathrm{E}+07$ \\
\hline 47 & 288.000 & $288.00 \odot$ & 1.0000 & $\odot .8044 \mathrm{E}+07$ \\
\hline 48 & $288.00 \odot$ & $288 . \odot \odot \odot$ & 1.0000 & $\odot .8044 \mathrm{E}+07$ \\
\hline 49 & 288.000 & 288.000 & 1.0000 & $\odot .8044 \mathrm{E}+\odot 7$ \\
\hline
\end{tabular}




\begin{tabular}{|c|c|c|c|c|}
\hline 50 & 288.000 & 288.000 & 1.0000 & $\odot .8044 \mathrm{E}+\odot 7$ \\
\hline 51 & 288.000 & 288.000 & 1.0000 & $\odot .8044 \mathrm{E}+07$ \\
\hline 52 & $288 . \odot \odot \odot$ & $288.0 \odot \odot$ & 1.0000 & $\odot .8044 \mathrm{E}+\odot 7$ \\
\hline 53 & $288.00 \odot$ & $288.00 \odot$ & 1.0000 & $\odot .8044 \mathrm{E}+\mathrm{C}$ \\
\hline 54 & $288.0 \odot \odot$ & $288.0 \odot \odot$ & 1.0000 & $\odot .8 \odot 44 \mathrm{E}+\odot$ \\
\hline 55 & $288.0 \odot \odot$ & $288.0 \odot \odot$ & $1.000 \odot$ & $\odot .8 \odot 44 \mathrm{E}+\odot 7$ \\
\hline 56 & 288.000 & 288.000 & 1.0000 & $\odot .8044 \mathrm{E}+07$ \\
\hline 57 & $288.00 \odot$ & 288.000 & 1.0000 & $\odot .8044 \mathrm{E}+\odot 7$ \\
\hline 58 & $288.00 \odot$ & 288.000 & 1.0000 & $\odot .8044 \mathrm{E}+\mathrm{C}$ \\
\hline 59 & $288.0 \odot \odot$ & $288.00 \odot$ & 1.0000 & $0.8044 \mathrm{E}+\odot$ \\
\hline $6 \odot$ & 288.000 & $288.0 \odot \odot$ & 1.0000 & $0.8044 \mathrm{E}+\Theta$ \\
\hline
\end{tabular}

lambda_avg $=0.319210589$ 
Compressor station distance $=60$ miles, Pressure $=2100$ psi, 60 axial node elements

\begin{tabular}{|c|c|c|c|c|}
\hline \multicolumn{5}{|c|}{$1.00 \quad$ lambda $=0.8513534665$} \\
\hline node & $\mathrm{T}^{\prime}(\mathrm{K})$ & Twall(K) & $x$ & $P(P a)$ \\
\hline 1 & 247.451 & 277.435 & - - - - - & $\odot .6669 \mathrm{E}+07$ \\
\hline 2 & 287.043 & $288.00 \odot$ & $1.000 \odot$ & $\odot .1230 E+08$ \\
\hline 3 & 288.000 & 288.000 & $1.000 \odot$ & $\odot .1445 \mathrm{E}+08$ \\
\hline 4 & 288.000 & 288.000 & 1.0000 & $0.1448 \mathrm{E}+08$ \\
\hline 5 & 288.000 & $288.00 \odot$ & $1.000 \odot$ & $\odot .1448 \mathrm{E}+\odot 8$ \\
\hline 6 & $288.00 \odot$ & 288.000 & $1.000 \odot$ & $\odot .1448 \mathrm{E}+\odot 8$ \\
\hline 7 & 288.000 & 288.000 & $1.000 \odot$ & $\odot .1448 \mathrm{E}+08$ \\
\hline 8 & $288.00 \odot$ & $288.00 \odot$ & $1.000 \odot$ & $\odot .1448 \mathrm{E}+08$ \\
\hline 9 & 288.000 & 288.000 & 1.0000 & $\odot .1448 \mathrm{E}+08$ \\
\hline 10 & 288.000 & 288.000 & 1.0000 & $\odot .1448 \mathrm{E}+08$ \\
\hline 11 & $288.0 \odot \odot$ & $288.00 \odot$ & $1.00 \odot \odot$ & $\odot .1448 \mathrm{E}+08$ \\
\hline 12 & $288.00 \odot$ & $288.00 \odot$ & 1.0000 & $\odot .1448 \mathrm{E}+08$ \\
\hline 13 & 288.000 & 288.000 & 1.0000 & $\odot .1448 \mathrm{E}+\odot 8$ \\
\hline 14 & $288.00 \odot$ & $288.00 \odot$ & $1.000 \odot$ & $\odot .1448 \mathrm{E}+08$ \\
\hline 15 & $288.00 \odot$ & $288.00 \odot$ & 1.0000 & $\odot .1448 \mathrm{E}+08$ \\
\hline 16 & 288.000 & 288.000 & 1.0000 & $\odot .1448 \mathrm{E}+08$ \\
\hline 17 & 288.000 & 288.000 & 1.0000 & $0.1448 \mathrm{E}+08$ \\
\hline 18 & 288.000 & $288.00 \odot$ & 1.0000 & $\odot .1448 \mathrm{E}+08$ \\
\hline 19 & $288.00 \odot$ & $288.00 \odot$ & 1.0000 & $\odot .1448 \mathrm{E}+08$ \\
\hline 20 & $288.00 \odot$ & $288.0 \odot \odot$ & $1.00 \odot \odot$ & $\odot .1448 \mathrm{E}+\odot 8$ \\
\hline 21 & 288.000 & 288.000 & 1.0000 & $\odot .1448 \mathrm{E}+\odot 8$ \\
\hline 22 & $288.00 \odot$ & $288.00 \odot$ & 1.0000 & $\odot .1448 \mathrm{E}+08$ \\
\hline 23 & 288.000 & 288.000 & 1.0000 & $\odot .1448 \mathrm{E}+08$ \\
\hline 24 & $288.00 \odot$ & $288.0 \odot \odot$ & $1.000 \odot$ & $\odot .1448 \mathrm{E}+08$ \\
\hline 25 & 288.000 & 288.000 & 1.0000 & $\odot .1448 \mathrm{E}+08$ \\
\hline 26 & $288.00 \odot$ & $288.00 \odot$ & $1.00 \odot \odot$ & $\odot .1448 \mathrm{E}+\odot 8$ \\
\hline 27 & 288.000 & 288.000 & 1.0000 & $\odot .1448 \mathrm{E}+08$ \\
\hline 28 & $288.00 \odot$ & $288 . \odot \odot \odot$ & $1.000 \odot$ & $0.1448 \mathrm{E}+08$ \\
\hline 29 & 288.000 & 288.000 & 1.0000 & $\odot .1448 \mathrm{E}+08$ \\
\hline 30 & $288.00 \odot$ & $288.00 \odot$ & $1.0 \odot \odot \odot$ & $\odot .1448 \mathrm{E}+08$ \\
\hline 31 & $288.00 \odot$ & $288.0 \odot \odot$ & $1.000 \odot$ & $\odot .1448 \mathrm{E}+08$ \\
\hline 32 & 288.000 & 288.000 & 1.0000 & $\odot .1448 \mathrm{E}+08$ \\
\hline 33 & 288.000 & 288.000 & 1.0000 & $\odot .1448 \mathrm{E}+08$ \\
\hline 34 & $288.00 \odot$ & 288.000 & 1.0000 & $\odot .1448 \mathrm{E}+08$ \\
\hline 35 & $288.00 \odot$ & $288.00 \odot$ & 1.0000 & $\odot .1448 \mathrm{E}+08$ \\
\hline 36 & $288.00 \odot$ & $288.0 \odot \odot$ & $1.0 \odot \odot \odot$ & $\odot .1448 \mathrm{E}+\odot 8$ \\
\hline 37 & $288.00 \odot$ & $288.0 \odot \odot$ & $1.00 \odot \odot$ & $\odot .1448 \mathrm{E}+08$ \\
\hline 38 & $288.00 \odot$ & 288.000 & 1.0000 & $\odot .1448 \mathrm{E}+08$ \\
\hline 39 & 288.000 & 288.000 & 1.0000 & $\odot .1448 \mathrm{E}+08$ \\
\hline 40 & 288.000 & 288.000 & 1.0000 & $\odot .1448 \mathrm{E}+08$ \\
\hline 41 & 288.000 & 288.000 & 1.0000 & $\odot .1448 \mathrm{E}+08$ \\
\hline 42 & $288.00 \odot$ & $288.00 \odot$ & $1.000 \odot$ & $0.1448 \mathrm{E}+08$ \\
\hline 43 & $288.00 \odot$ & $288 . \odot \odot \odot$ & $1.0 \odot \odot \odot$ & $\odot .1448 \mathrm{E}+\odot 8$ \\
\hline 44 & $288.00 \odot$ & $288.0 \odot \odot$ & $1.000 \odot$ & $\odot .1448 \mathrm{E}+08$ \\
\hline 45 & 288.000 & $288.00 \odot$ & 1.0000 & $\odot .1448 \mathrm{E}+08$ \\
\hline 46 & 288.000 & 288.000 & 1.0000 & $\odot .1448 \mathrm{E}+08$ \\
\hline 47 & $288.00 \odot$ & $288.00 \odot$ & $1.00 \odot \odot$ & $\odot .1448 \mathrm{E}+08$ \\
\hline 48 & 288.000 & $288.00 \odot$ & 1.0000 & $\odot .1448 \mathrm{E}+08$ \\
\hline 49 & $288.00 \odot$ & 288.000 & $1.000 \odot$ & $\odot .1448 \mathrm{E}+08$ \\
\hline 50 & 288.000 & $288.00 \odot$ & $1.000 \odot$ & $\odot .1448 \mathrm{E}+08$ \\
\hline 51 & $288.0 \odot \odot$ & 288.000 & 1.0000 & $\odot .1448 \mathrm{E}+\odot 8$ \\
\hline 52 & 288.000 & $288.00 \odot$ & 1.0000 & $\odot .1448 \mathrm{E}+08$ \\
\hline
\end{tabular}




\begin{tabular}{|c|c|c|c|c|}
\hline 53 & $288.00 \odot$ & $288.00 \odot$ & 1.0000 & $0.1448 \mathrm{E}+08$ \\
\hline 54 & 288.000 & 288.000 & 1.0000 & $\odot .1448 \mathrm{E}+08$ \\
\hline 55 & $288 . \odot \odot \odot$ & $288 . \odot \odot \odot$ & $1.000 \odot$ & $\odot .1448 \mathrm{E}+\odot 8$ \\
\hline 56 & $288.0 \odot \odot$ & 288.000 & 1.0000 & $\odot .1448 \mathrm{E}+08$ \\
\hline 57 & $288 . \odot \odot \odot$ & $288.00 \odot$ & $1.00 \odot \odot$ & $\odot .1448 \mathrm{E}+08$ \\
\hline 58 & $288.00 \odot$ & $288.00 \odot$ & 1.0000 & $\odot .1448 \mathrm{E}+08$ \\
\hline 59 & $288 . \odot \odot \odot$ & $288.0 \odot \odot$ & $1.000 \odot$ & $\odot .1448 \mathrm{E}+08$ \\
\hline 60 & $288 . \odot \odot \odot$ & $288.00 \odot$ & $1.00 \odot \odot$ & $\odot .1448 \mathrm{E}+\odot 8$ \\
\hline
\end{tabular}

\begin{tabular}{|c|c|c|c|c|}
\hline \multicolumn{5}{|c|}{$.00 \quad$ lambda $=0.7250236869$} \\
\hline node & $\mathrm{T}(\mathrm{K})$ & Twall(K) & $x$ & $\mathrm{P}(\mathrm{Pa})$ \\
\hline 1 & 241.665 & 269.991 & - - _ - _ n & $\odot .5613 \mathrm{E}+07$ \\
\hline 2 & 280.330 & 287.886 & 1.0000 & $\odot .1035 \mathrm{E}+\odot 8$ \\
\hline 3 & 287.973 & 288.000 & $1.000 \odot$ & $\odot .1433 \mathrm{E}+\odot 8$ \\
\hline 4 & $288.0 \odot \odot$ & $288 . \odot \odot \odot$ & 1. $0 \odot \odot \odot$ & $\odot .1448 E+\odot 8$ \\
\hline 5 & $288.00 \odot$ & 288.000 & 1.0000 & $\odot .1448 \mathrm{E}+\odot 8$ \\
\hline 6 & 288.000 & 288.000 & 1.0000 & $\odot .1448 \mathrm{E}+08$ \\
\hline 7 & 288.000 & 288.000 & 1.0000 & $\odot .1448 \mathrm{E}+\odot 8$ \\
\hline 8 & 288.000 & 288.000 & 1.0000 & $\odot .1448 \mathrm{E}+\odot 8$ \\
\hline 9 & $288.00 \odot$ & $288.0 \odot \odot$ & 1.0000 & $\odot .1448 \mathrm{E}+\odot 8$ \\
\hline 10 & 288.000 & 288.000 & 1.0000 & $\odot .1448 \mathrm{E}+08$ \\
\hline 11 & 288.000 & $288.00 \odot$ & 1.0000 & $\odot .1448 \mathrm{E}+\odot 8$ \\
\hline 12 & 288.000 & 288.000 & $1.000 \odot$ & $\odot .1448 \mathrm{E}+08$ \\
\hline 13 & 288.000 & 288.000 & 1.0000 & $0.1448 \mathrm{E}+08$ \\
\hline 14 & 288.000 & 288.000 & $1.00 \odot \odot$ & $\odot .1448 \mathrm{E}+\odot 8$ \\
\hline 15 & 288.000 & $288.0 \odot \odot$ & 1.0000 & $\odot .1448 \mathrm{E}+\odot 8$ \\
\hline 16 & 288.000 & 288.000 & 1.0000 & $\odot .1448 \mathrm{E}+\odot 8$ \\
\hline 17 & 288.000 & 288.000 & 1.0000 & $\odot .1448 \mathrm{E}+08$ \\
\hline 18 & 288.000 & 288.000 & 1.0000 & $\odot .1448 \mathrm{E}+\odot 8$ \\
\hline 19 & 288.000 & 288.000 & 1.0000 & $\odot .1448 \mathrm{E}+08$ \\
\hline 20 & 288.000 & 288.000 & 1.0000 & $\odot .1448 \mathrm{E}+\odot 8$ \\
\hline 21 & 288.000 & 288.000 & 1.0000 & $\odot .1448 \mathrm{E}+\odot 8$ \\
\hline 22 & 288.000 & 288.000 & 1.0000 & $\odot .1448 \mathrm{E}+08$ \\
\hline 23 & 288.000 & 288.000 & 1.0000 & $\odot .1448 \mathrm{E}+\odot 8$ \\
\hline 24 & $288.0 \odot \odot$ & 288.000 & 1.0000 & $0.1448 \mathrm{E}+08$ \\
\hline 25 & 288.000 & 288.000 & 1.0000 & $\odot .1448 \mathrm{E}+\odot 8$ \\
\hline 26 & $288.00 \odot$ & $288.0 \odot \odot$ & 1.0000 & $0.1448 \mathrm{E}+08$ \\
\hline 27 & 288.000 & 288.000 & 1.0000 & $\odot .1448 \mathrm{E}+08$ \\
\hline 28 & 288.000 & 288.000 & 1.0000 & $0.1448 \mathrm{E}+08$ \\
\hline 29 & 288.000 & 288.000 & 1.0000 & $\odot .1448 \mathrm{E}+08$ \\
\hline 30 & 288.000 & $288.0 \odot \odot$ & 1.0000 & $\odot .1448 \mathrm{E}+\odot 8$ \\
\hline 31 & 288.000 & 288.000 & 1.0000 & $\odot .1448 \mathrm{E}+08$ \\
\hline 32 & 288.000 & 288.000 & 1.0000 & $\odot .1448 \mathrm{E}+08$ \\
\hline 33 & 288.000 & 288.000 & 1.0000 & $\odot .1448 \mathrm{E}+08$ \\
\hline 34 & 288.000 & $288.00 \odot$ & 1.0000 & $\odot .1448 \mathrm{E}+\odot 8$ \\
\hline 35 & 288.000 & 288.000 & 1.0000 & $0.1448 \mathrm{E}+08$ \\
\hline 36 & $288.00 \odot$ & 288.000 & 1.0000 & $\odot .1448 \mathrm{E}+\odot 8$ \\
\hline 37 & $288.0 \odot \odot$ & $288.0 \odot \odot$ & 1.0000 & $\odot .1448 \mathrm{E}+08$ \\
\hline 38 & 288.000 & 288.000 & 1.0000 & $0.1448 \mathrm{E}+08$ \\
\hline 39 & 288.000 & 288.000 & 1.0000 & $\odot .1448 \mathrm{E}+08$ \\
\hline 40 & 288.000 & 288.000 & 1.0000 & $\odot .1448 \mathrm{E}+08$ \\
\hline 41 & $288.00 \odot$ & 288.000 & 1.0000 & $\odot .1448 \mathrm{E}+\odot 8$ \\
\hline 42 & 288.000 & 288.000 & 1.0000 & $\odot .1448 \mathrm{E}+08$ \\
\hline 43 & $288.00 \odot$ & $288.00 \odot$ & $1.000 \odot$ & $\odot .1448 \mathrm{E}+\odot 8$ \\
\hline 44 & $288.0 \odot \odot$ & 288.000 & 1.0000 & $\odot .1448 \mathrm{E}+\odot 8$ \\
\hline 45 & 288.000 & 288.000 & 1.0000 & $0.1448 \mathrm{E}+08$ \\
\hline 46 & 288.000 & 288.000 & 1.0000 & $\odot .1448 \mathrm{E}+\odot 8$ \\
\hline
\end{tabular}




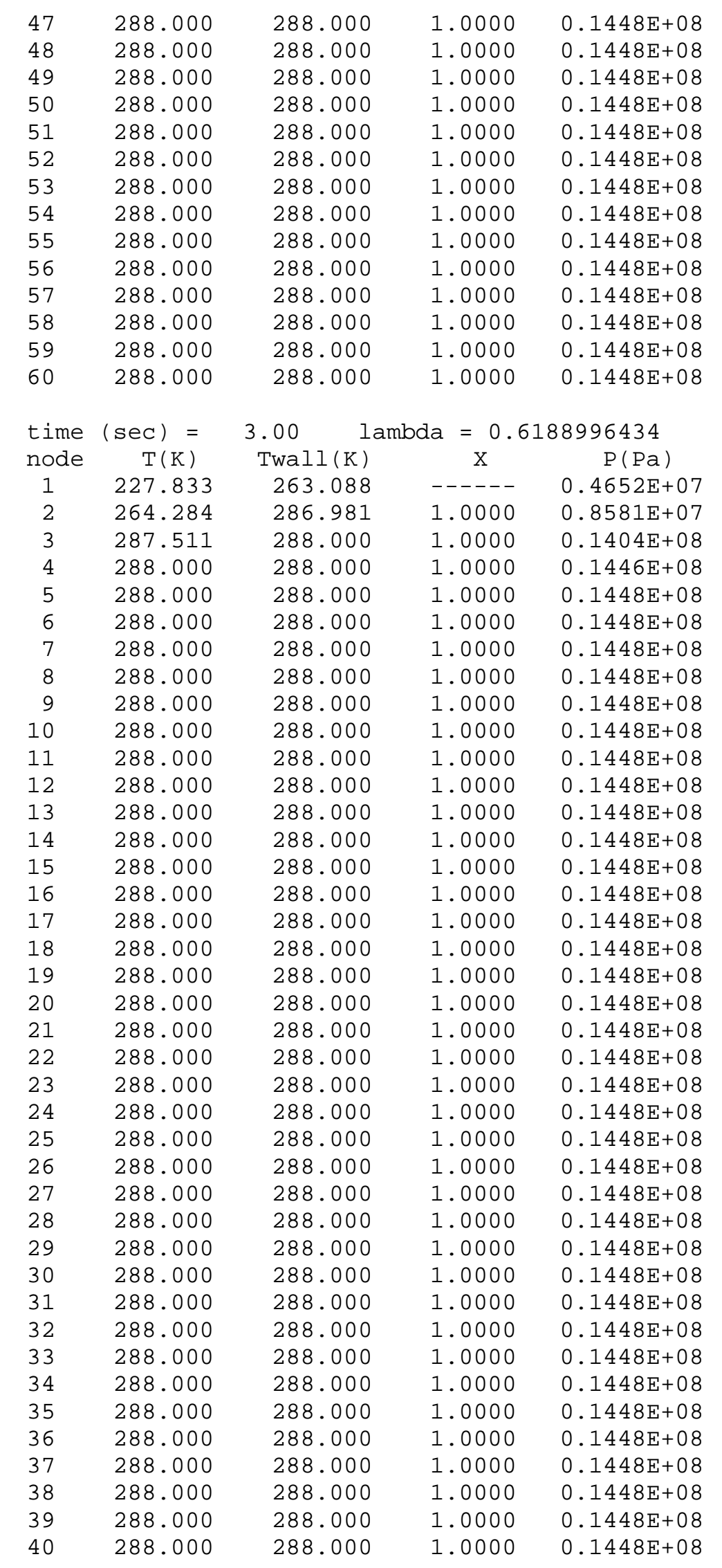




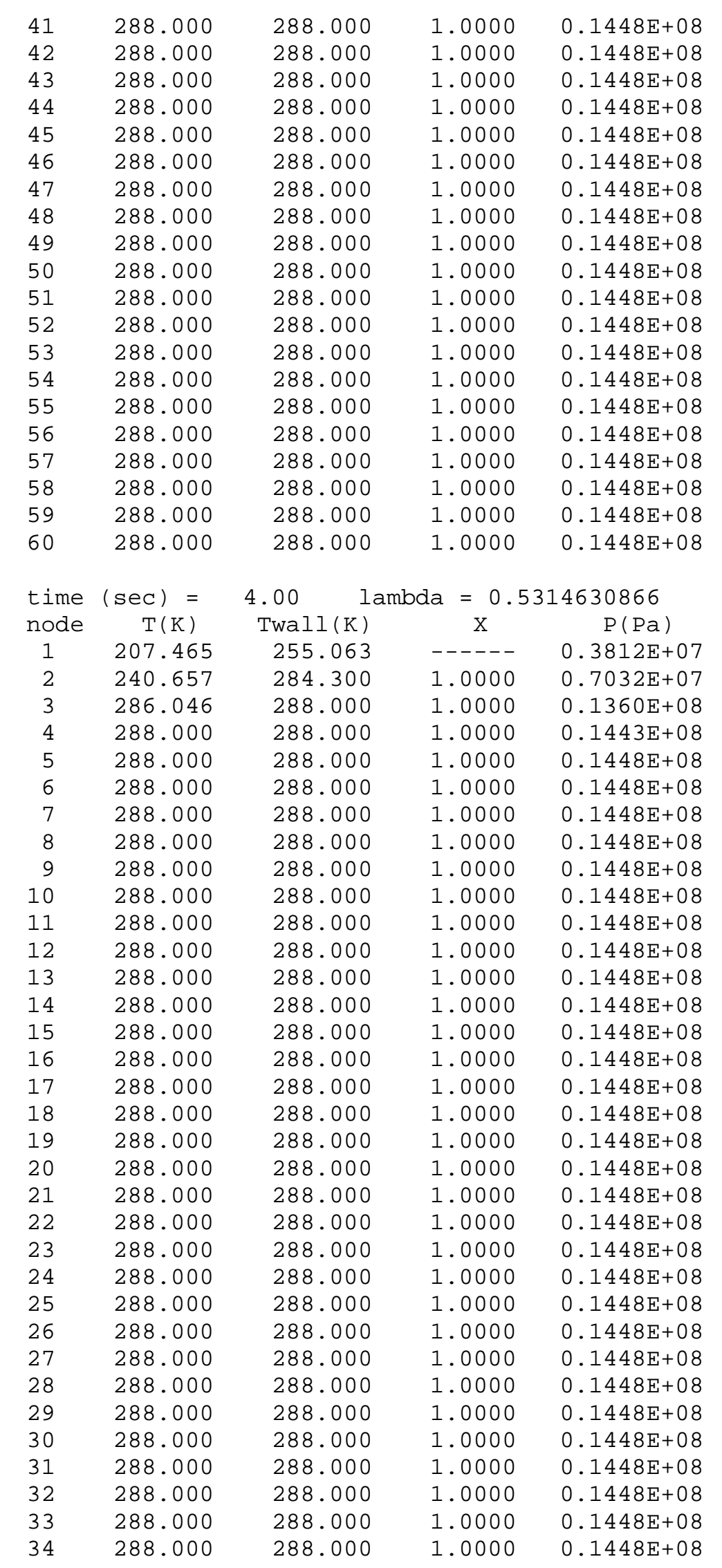




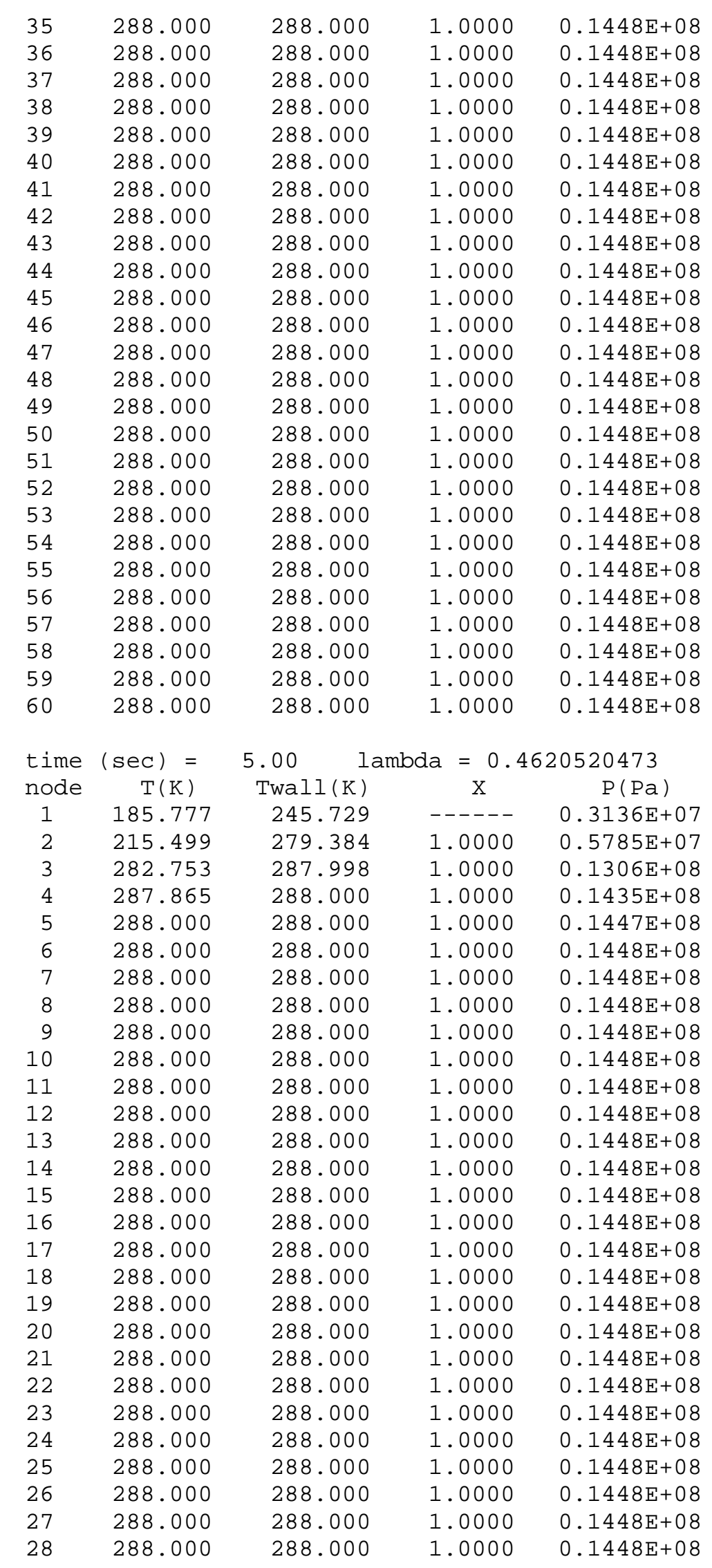




\begin{tabular}{|c|c|c|c|c|}
\hline 29 & 288.000 & 288.000 & 1.0000 & $\odot .1448 \mathrm{E}+08$ \\
\hline 30 & 288.000 & $288.00 \odot$ & 1.0000 & $0.1448 \mathrm{E}+08$ \\
\hline 31 & $288.00 \odot$ & $288 . \odot \odot \odot$ & $1.00 \odot \odot$ & $0.1448 \mathrm{E}+\odot 8$ \\
\hline 32 & 288.000 & 288.000 & 1.0000 & $0.1448 \mathrm{E}+08$ \\
\hline 33 & 288.000 & 288.000 & 1.0000 & $0.1448 E+08$ \\
\hline 34 & 288.000 & $288.00 \odot$ & 1.0000 & $0.1448 \mathrm{E}+08$ \\
\hline 35 & 288.000 & 288.000 & 1.0000 & $0.1448 \mathrm{E}+08$ \\
\hline 36 & 288.000 & 288.000 & 1.0000 & $0.1448 \mathrm{E}+08$ \\
\hline 37 & 288.000 & $288 . \odot \odot \odot$ & $1.000 \odot$ & $\odot .1448 \mathrm{E}+\odot 8$ \\
\hline 38 & 288.000 & 288.000 & 1.0000 & $0.1448 \mathrm{E}+08$ \\
\hline 39 & 288.000 & 288.000 & 1.0000 & $0.1448 \mathrm{E}+08$ \\
\hline 40 & 288.000 & 288.000 & 1.0000 & $0.1448 \mathrm{E}+08$ \\
\hline 41 & 288.000 & $288.00 \odot$ & 1.0000 & $0.1448 \mathrm{E}+08$ \\
\hline 42 & 288.000 & 288.000 & 1.0000 & $0.1448 \mathrm{E}+08$ \\
\hline 43 & 288.000 & $288.00 \odot$ & 1.0000 & $0.1448 \mathrm{E}+08$ \\
\hline 44 & 288.000 & 288.000 & 1.0000 & $\odot .1448 \mathrm{E}+08$ \\
\hline 45 & 288.000 & $288.00 \odot$ & 1.0000 & $0.1448 \mathrm{E}+08$ \\
\hline 46 & 288.000 & 288.000 & 1.0000 & $\odot .1448 \mathrm{E}+08$ \\
\hline 47 & 288.000 & $288 . \odot \odot \odot$ & $1.00 \odot \odot$ & $0.1448 \mathrm{E}+\odot 8$ \\
\hline 48 & 288.000 & $288.00 \odot$ & 1.0000 & $0.1448 \mathrm{E}+08$ \\
\hline 49 & 288.000 & 288.000 & 1.0000 & $0.1448 \mathrm{E}+08$ \\
\hline 50 & 288.000 & $288.00 \odot$ & 1.0000 & $0.1448 \mathrm{E}+08$ \\
\hline 51 & 288.000 & 288.000 & 1.0000 & $\odot .1448 \mathrm{E}+08$ \\
\hline 52 & 288.000 & 288.000 & 1.0000 & $0.1448 \mathrm{E}+08$ \\
\hline 53 & 288.000 & $288.00 \odot$ & 1.0000 & $0.1448 \mathrm{E}+08$ \\
\hline 54 & 288.000 & $288 . \odot \odot \odot$ & $1.000 \odot$ & $0.1448 \mathrm{E}+08$ \\
\hline 55 & 288.000 & 288.000 & 1.0000 & $0.1448 \mathrm{E}+08$ \\
\hline 56 & $288.00 \odot$ & $288.00 \odot$ & $1.000 \odot$ & $0.1448 \mathrm{E}+08$ \\
\hline 57 & 288.000 & $288.00 \odot$ & 1.0000 & $\odot .1448 \mathrm{E}+08$ \\
\hline 58 & 288.000 & 288.000 & 1.0000 & $0.1448 \mathrm{E}+08$ \\
\hline 59 & 288.000 & 288.000 & 1.0000 & $0.1448 \mathrm{E}+08$ \\
\hline 60 & 288.000 & 288.000 & 1.0000 & $0.1448 \mathrm{E}+08$ \\
\hline time & $(\mathrm{sec})=$ & \multicolumn{3}{|c|}{$6.00 \quad$ lambda $=0.4094181657$} \\
\hline node & $\mathrm{T}(\mathrm{K})$ & Twall(K) & $\mathrm{x}$ & $P(P a)$ \\
\hline 1 & 167.340 & 235.695 & 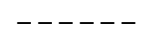 & $0.2637 \mathrm{E}+07$ \\
\hline 2 & 194.113 & 272.270 & 1.0000 & $0.4865 \mathrm{E}+07$ \\
\hline 3 & 277.016 & 287.690 & 1.0000 & $0.1245 E+08$ \\
\hline 4 & 287.421 & 288.000 & 1.0000 & $0.1420 \mathrm{E}+08$ \\
\hline 5 & 88.0 & 288.000 & 1.0000 & $0.1446 \mathrm{E}+08$ \\
\hline 6 & 288.0 & 288.000 & 1.0000 & $0.1448 \mathrm{E}+08$ \\
\hline 7 & 288.000 & 288.000 & 1.0000 & $0.1448 \mathrm{E}+08$ \\
\hline 8 & 288.000 & 288.000 & 1.0000 & $0.1448 \mathrm{E}+08$ \\
\hline 9 & 288.000 & 288.000 & 1.0000 & $0.1448 \mathrm{E}+08$ \\
\hline 10 & 288.000 & 288.000 & 1.0000 & $0.1448 \mathrm{E}+08$ \\
\hline 11 & 88.6 & 288.000 & 1.0000 & $0.1448 \mathrm{E}+08$ \\
\hline 12 & $288.00 \odot$ & $288.00 \odot$ & 1.0000 & $0.1448 \mathrm{E}+08$ \\
\hline 13 & 288.000 & 288.000 & 1.0000 & $0.1448 \mathrm{E}+08$ \\
\hline 14 & 288.000 & $288 . \odot \odot \odot$ & $1.000 \odot$ & $0.1448 \mathrm{E}+\odot 8$ \\
\hline 15 & 288.000 & $288.00 \odot$ & 1.0000 & $0.1448 \mathrm{E}+08$ \\
\hline 16 & $288.00 \odot$ & $288.00 \odot$ & $1.000 \odot$ & $0.1448 \mathrm{E}+08$ \\
\hline 17 & 288.000 & 288.000 & 1.0000 & $0.1448 \mathrm{E}+08$ \\
\hline 18 & 288.000 & 288.000 & 1.0000 & $0.1448 \mathrm{E}+08$ \\
\hline 19 & $288.00 \odot$ & $288.00 \odot$ & $1.000 \odot$ & $\odot .1448 \mathrm{E}+08$ \\
\hline 20 & 288.000 & $288.00 \odot$ & 1.0000 & $0.1448 \mathrm{E}+08$ \\
\hline 21 & 288.000 & 288.000 & 1.0000 & $\odot .1448 E+\odot 8$ \\
\hline 22 & 288.000 & 88.000 & $1.000 \odot$ & $\odot .1448 \mathrm{E}+\odot 8$ \\
\hline
\end{tabular}




\begin{tabular}{|c|c|c|c|c|}
\hline 23 & 288.000 & 288.000 & 1.0000 & $0.1448 \mathrm{E}+08$ \\
\hline 24 & 288.000 & $288.00 \odot$ & 1.0000 & $0.1448 E+08$ \\
\hline 25 & $288.00 \odot$ & $288 . \odot \odot \odot$ & $1.00 \odot \odot$ & $\odot .1448 E+08$ \\
\hline 26 & 288.000 & 288.000 & 1.0000 & $0.1448 \mathrm{E}+08$ \\
\hline 27 & 288.000 & 288.000 & 1.0000 & $0.1448 E+08$ \\
\hline 28 & 288.000 & $288.00 \odot$ & 1.0000 & $\odot .1448 \mathrm{E}+08$ \\
\hline 29 & $288.00 \odot$ & $288 . \odot \odot \odot$ & $1.0 \odot \odot \odot$ & $\odot .1448 E+08$ \\
\hline 30 & 288.000 & 288.000 & 1.0000 & $0.1448 \mathrm{E}+08$ \\
\hline 31 & 288.000 & $288 . \odot \odot \odot$ & $1.000 \odot$ & $\odot .1448 E+\odot 8$ \\
\hline 32 & 288.000 & 288.000 & 1.0000 & $0.1448 \mathrm{E}+08$ \\
\hline 33 & 288.000 & 288.000 & 1.0000 & $0.1448 \mathrm{E}+08$ \\
\hline 34 & 288.000 & 288.000 & 1.0000 & $\odot .1448 \mathrm{E}+08$ \\
\hline 35 & 288.000 & $288 . \odot \odot \odot$ & $1.000 \odot$ & $\odot .1448 E+\odot 8$ \\
\hline 36 & 288.000 & 288.000 & 1.0000 & $0.1448 \mathrm{E}+08$ \\
\hline 37 & 288.000 & $288.00 \odot$ & 1.0000 & $0.1448 \mathrm{E}+08$ \\
\hline 38 & 288.000 & 288.000 & 1.0000 & $\odot .1448 \mathrm{E}+\odot 8$ \\
\hline 39 & 288.000 & $288.00 \odot$ & 1.0000 & $0.1448 \mathrm{E}+08$ \\
\hline 40 & 288.000 & 288.000 & 1.0000 & $\odot .1448 \mathrm{E}+\odot 8$ \\
\hline 41 & 288.000 & $288 . \odot \odot \odot$ & $1.00 \odot \odot$ & $0.1448 \mathrm{E}+\odot 8$ \\
\hline 42 & $288.00 \odot$ & $288.00 \odot$ & 1.0000 & $\odot .1448 \mathrm{E}+08$ \\
\hline 43 & 288.000 & 288.000 & 1.0000 & $0.1448 \mathrm{E}+08$ \\
\hline 44 & 288.000 & $288.00 \odot$ & 1.0000 & $0.1448 \mathrm{E}+08$ \\
\hline 45 & 288.000 & 288.000 & 1.0000 & $\odot .1448 \mathrm{E}+08$ \\
\hline 46 & 288.000 & 288.000 & 1.0000 & $0.1448 \mathrm{E}+08$ \\
\hline 47 & 288.000 & $288.00 \odot$ & 1.0000 & $0.1448 \mathrm{E}+08$ \\
\hline 48 & 288.000 & $288 . \odot \odot \odot$ & $1.000 \odot$ & $0.1448 \mathrm{E}+08$ \\
\hline 49 & 288.000 & 288.000 & 1.0000 & $0.1448 \mathrm{E}+08$ \\
\hline 50 & 288.000 & 288.000 & 1.0000 & $0.1448 \mathrm{E}+08$ \\
\hline 51 & 288.000 & $288.00 \odot$ & 1.0000 & $\odot .1448 \mathrm{E}+08$ \\
\hline 52 & 288.000 & 288.000 & 1.0000 & $0.1448 \mathrm{E}+08$ \\
\hline 53 & 288.000 & 288.000 & 1.0000 & $0.1448 \mathrm{E}+08$ \\
\hline 54 & 288.000 & 288.000 & 1.0000 & $0.1448 \mathrm{E}+08$ \\
\hline 55 & 288.000 & 288.000 & 1.0000 & $0.1448 \mathrm{E}+08$ \\
\hline 56 & 288.000 & 288.000 & 1.0000 & $0.1448 \mathrm{E}+08$ \\
\hline 57 & $288.00 \odot$ & 288.000 & 1.0000 & $0.1448 \mathrm{E}+08$ \\
\hline 58 & 288.000 & 288.000 & 1.0000 & $0.1448 \mathrm{E}+08$ \\
\hline 59 & 288.000 & 288.000 & 1.0000 & $0.1448 \mathrm{E}+08$ \\
\hline 60 & 288.000 & 288.000 & 1.0000 & $0.1448 E+08$ \\
\hline time & $(\sec )=$ & \multicolumn{3}{|c|}{ lambda $=0.3815290630$} \\
\hline node & $\mathrm{T}(\mathrm{K})$ & Twall(K) & $\mathrm{x}$ & $\mathrm{P}(\mathrm{Pa})$ \\
\hline 1 & 163.687 & 225.921 & $-\ldots$ & $\odot .2431 E+07$ \\
\hline 2 & 189.877 & 264.893 & 0.6659 & $0.4484 \mathrm{E}+07$ \\
\hline 3 & 268.844 & 286.955 & 1.0000 & $0.1181 \mathrm{E}+08$ \\
\hline 4 & 286.366 & 288.000 & 1.0000 & $0.1397 \mathrm{E}+08$ \\
\hline 5 & 88.000 & 288.000 & 1.0000 & $0.1444 \mathrm{E}+08$ \\
\hline 6 & $288.00 \odot$ & $288.00 \odot$ & $1.000 \odot$ & $0.1448 \mathrm{E}+08$ \\
\hline 7 & 288.000 & 288.000 & 1.0000 & $0.1448 \mathrm{E}+08$ \\
\hline 8 & 288.000 & 288.000 & 1.0000 & $0.1448 \mathrm{E}+08$ \\
\hline 9 & 288.000 & 288.000 & 1.0000 & $0.1448 \mathrm{E}+08$ \\
\hline 10 & $288.00 \odot$ & $288.00 \odot$ & $1.000 \odot$ & $0.1448 \mathrm{E}+08$ \\
\hline 11 & 288.000 & 288.000 & 1.0000 & $0.1448 \mathrm{E}+08$ \\
\hline 12 & 288.000 & 288.000 & 1.0000 & $0.1448 \mathrm{E}+08$ \\
\hline 13 & 288.000 & 288.000 & 1.0000 & $0.1448 E+08$ \\
\hline 14 & 288.000 & $288.00 \odot$ & 1.0000 & $0.1448 \mathrm{E}+08$ \\
\hline 15 & 288.000 & 288.000 & 1.0000 & $\odot .1448 \mathrm{E}+08$ \\
\hline 16 & & 88.000 & $1.000 \odot$ & $0.1448 E+08$ \\
\hline
\end{tabular}




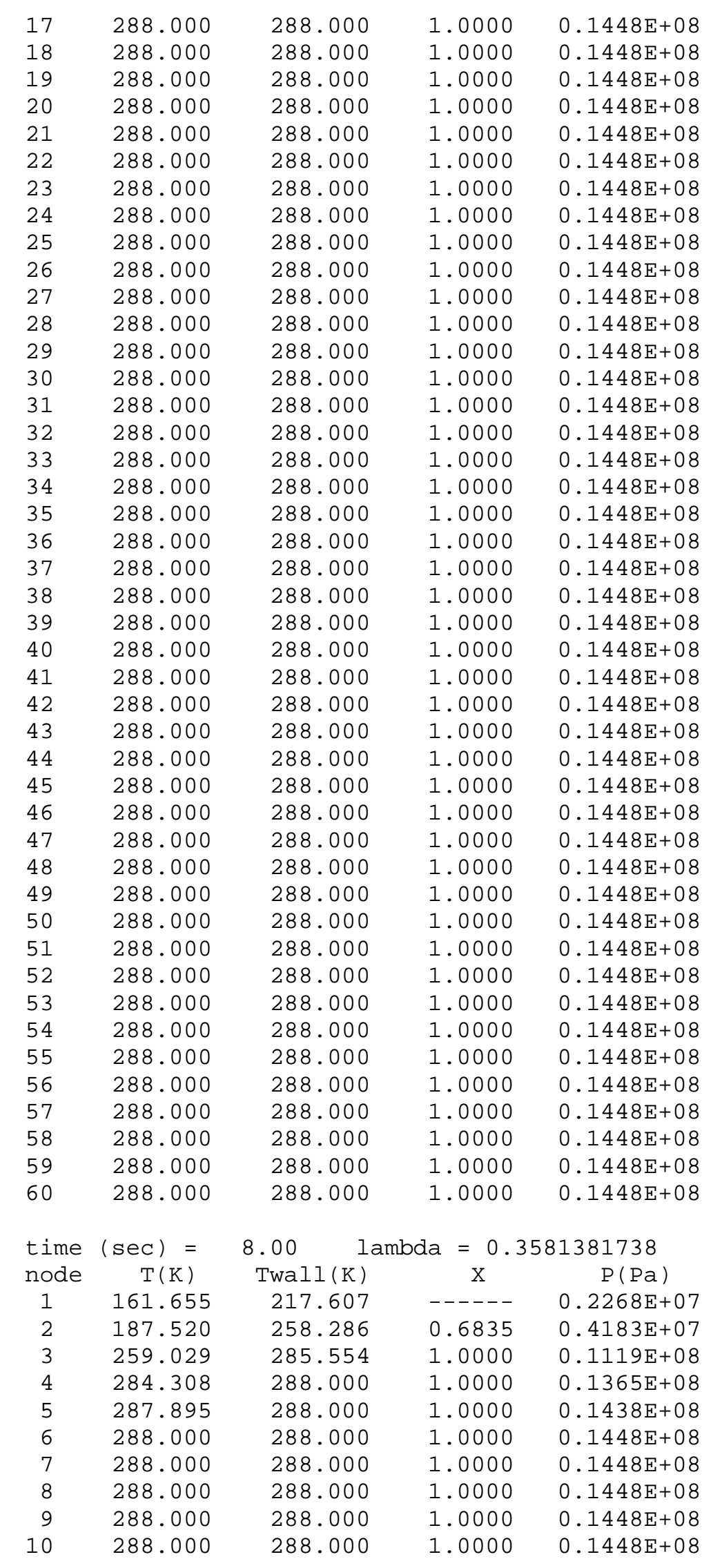




\begin{tabular}{|c|c|c|c|c|}
\hline 11 & 288.000 & $288.00 \odot$ & 1.0000 & $\odot .1448 \mathrm{E}+08$ \\
\hline 12 & $288.00 \odot$ & 288.000 & $1.000 \odot$ & $0.1448 E+\odot 8$ \\
\hline 13 & 288.000 & $288.00 \odot$ & 1.0000 & $0.1448 E+08$ \\
\hline 14 & 288.000 & 288.000 & 1.0000 & $\odot .1448 \mathrm{E}+\odot 8$ \\
\hline 15 & 288.000 & 288.000 & 1.0000 & $0.1448 \mathrm{E}+08$ \\
\hline 16 & $288.00 \odot$ & $288.00 \odot$ & $1.000 \odot$ & $\odot .1448 E+08$ \\
\hline 17 & 288.000 & $288.00 \odot$ & 1.0000 & $0.1448 E+08$ \\
\hline 18 & 288.000 & 288.000 & 1.0000 & $\odot .1448 \mathrm{E}+08$ \\
\hline 19 & 288.000 & 288.000 & 1.0000 & $0.1448 \mathrm{E}+08$ \\
\hline 20 & 288.000 & $288.00 \odot$ & $1.000 \odot$ & $0.1448 E+08$ \\
\hline 21 & 288.000 & 288.000 & 1.0000 & $\odot .1448 \mathrm{E}+08$ \\
\hline 22 & 288.000 & 288.000 & $1.000 \odot$ & $\odot .1448 \mathrm{E}+08$ \\
\hline 23 & 288.000 & 288.000 & 1.0000 & $0.1448 \mathrm{E}+08$ \\
\hline 24 & 288.000 & $288.00 \odot$ & 1.0000 & $0.1448 E+08$ \\
\hline 25 & 288.000 & $288.00 \odot$ & 1.0000 & $0.1448 \mathrm{E}+08$ \\
\hline 26 & 288.000 & 288.000 & 1.0000 & $0.1448 \mathrm{E}+08$ \\
\hline 27 & $288.00 \odot$ & $288.00 \odot$ & $1.000 \odot$ & $\odot .1448 \mathrm{E}+08$ \\
\hline 28 & 288.000 & $288.00 \odot$ & 1.0000 & $0.1448 \mathrm{E}+08$ \\
\hline 29 & 288.000 & 288.000 & 1.0000 & $0.1448 \mathrm{E}+08$ \\
\hline 30 & 288.000 & 288.000 & 1.0000 & $0.1448 E+08$ \\
\hline 31 & 288.000 & $288.00 \odot$ & 1.0000 & $0.1448 \mathrm{E}+08$ \\
\hline 32 & 288.000 & 288.000 & 1.0000 & $0.1448 \mathrm{E}+08$ \\
\hline 33 & 288.000 & 288.000 & 1.0000 & $0.1448 E+08$ \\
\hline 34 & 288.000 & 288.000 & 1.0000 & $0.1448 \mathrm{E}+08$ \\
\hline 35 & 288.000 & $288.00 \odot$ & 1.0000 & $0.1448 \mathrm{E}+08$ \\
\hline 36 & 288.000 & 288.000 & 1.0000 & $0.1448 \mathrm{E}+08$ \\
\hline 37 & 88.000 & $288.00 \odot$ & 1.0000 & $0.1448 \mathrm{E}+08$ \\
\hline 38 & 288.000 & 288.000 & 1.0000 & $0.1448 \mathrm{E}+08$ \\
\hline 39 & $288.00 \odot$ & $288.00 \odot$ & $1.000 \odot$ & $0.1448 \mathrm{E}+08$ \\
\hline 40 & 288.000 & $288.00 \odot$ & 1.0000 & $\odot .1448 \mathrm{E}+08$ \\
\hline 41 & 288.000 & $288.00 \odot$ & 1.0000 & $0.1448 \mathrm{E}+08$ \\
\hline 42 & 288.000 & $288.00 \odot$ & 1.0000 & $0.1448 \mathrm{E}+08$ \\
\hline 43 & 88.000 & 288.000 & 1.0000 & $0.1448 \mathrm{E}+08$ \\
\hline 44 & 88.000 & 288.000 & 1.0000 & $0.1448 \mathrm{E}+08$ \\
\hline 45 & $288.00 \odot$ & $288.00 \odot$ & 1.0000 & $0.1448 \mathrm{E}+08$ \\
\hline 46 & $288.00 \odot$ & 288.000 & 1.0000 & $0.1448 \mathrm{E}+08$ \\
\hline 47 & 288.000 & 288.000 & 1.0000 & $0.1448 \mathrm{E}+08$ \\
\hline 48 & 288.000 & 288.000 & 1.0000 & $\odot .1448 \mathrm{E}+08$ \\
\hline 49 & $288.00 \odot$ & $288.00 \odot$ & $1.000 \odot$ & $0.1448 \mathrm{E}+08$ \\
\hline 50 & $\odot \odot$ & 288.000 & 1.00 & $0.1448 \mathrm{E}+08$ \\
\hline 51 & 288.000 & 288.000 & 1.0000 & $0.1448 \mathrm{E}+08$ \\
\hline 52 & $288.00 \odot$ & $288.00 \odot$ & $1.000 \odot$ & $0.1448 \mathrm{E}+08$ \\
\hline 53 & 288.000 & 288.000 & 1.0000 & $0.1448 E+08$ \\
\hline 54 & 288.000 & 288.000 & 1.0000 & $0.1448 \mathrm{E}+08$ \\
\hline 55 & 288.000 & $288.00 \odot$ & 1.0000 & $0.1448 \mathrm{E}+08$ \\
\hline 56 & 288.0 & $288 . \odot$ & 1.00 & $0.1448 \mathrm{E}+08$ \\
\hline 57 & 288.000 & 288.000 & 1.0000 & $\odot .1448 \mathrm{E}+08$ \\
\hline 58 & 288.000 & 288.000 & 1.0000 & $0.1448 E+08$ \\
\hline 59 & 288.000 & 288.000 & 1.0000 & $0.1448 \mathrm{E}+08$ \\
\hline 60 & $288.00 \odot$ & $288.00 \odot$ & $1.000 \odot$ & $0.1448 \mathrm{E}+08$ \\
\hline time & $(\sec )=$ & \multicolumn{3}{|c|}{ lambda $=0.3367696702$} \\
\hline & $\mathrm{T}(\mathrm{K})$ & Twall(K) & & $P(P a)$ \\
\hline 1 & 159.734 & 210.369 & $-\ldots$ & $\odot .2120 \mathrm{E}+\odot 7$ \\
\hline 2 & 185.292 & 252.383 & 0.7027 & $\odot .3910 \mathrm{E}+07$ \\
\hline 3 & 48.826 & 283.343 & 1.0000 & $0.1063 \mathrm{E}+08$ \\
\hline 4 & & 87.888 & 1.0000 & $\odot .1325 \mathrm{E}+\odot$ \\
\hline
\end{tabular}




\begin{tabular}{|c|c|c|c|c|}
\hline 5 & 287.542 & $288.00 \odot$ & 1.0000 & $\odot .1429 \mathrm{E}+08$ \\
\hline 6 & 288.000 & $288 . \odot \odot \odot$ & $1.000 \odot$ & $\odot .1446 \mathrm{E}+\odot 8$ \\
\hline 7 & $288.00 \odot$ & $288 . \odot \odot \odot$ & 1.0000 & $\odot .1448 \mathrm{E}+08$ \\
\hline 8 & $288.00 \odot$ & $288 . \odot \odot \odot$ & 1.0000 & $\odot .1448 \mathrm{E}+\odot 8$ \\
\hline 9 & 288.000 & $288.0 \odot \odot$ & 1.0000 & $\odot .1448 \mathrm{E}+\odot 8$ \\
\hline 10 & $288.00 \odot$ & 288.000 & 1.0000 & $\odot .1448 \mathrm{E}+08$ \\
\hline 11 & $288.00 \odot$ & $288.0 \odot \odot$ & 1.0000 & $\odot .1448 \mathrm{E}+\odot 8$ \\
\hline 12 & 288.000 & 288.000 & 1.0000 & $\odot .1448 \mathrm{E}+08$ \\
\hline 13 & 288.000 & 288.000 & 1.0000 & $\odot .1448 \mathrm{E}+\odot 8$ \\
\hline 14 & $288.00 \odot$ & $288.0 \odot \odot$ & 1.0000 & $0.1448 \mathrm{E}+08$ \\
\hline 15 & 288.000 & 288.000 & 1.0000 & $\odot .1448 \mathrm{E}+08$ \\
\hline 16 & $288.00 \odot$ & 288.000 & 1.0000 & $\odot .1448 \mathrm{E}+\odot 8$ \\
\hline 17 & $288.00 \odot$ & $288.00 \odot$ & 1.0000 & $\odot .1448 \mathrm{E}+\odot 8$ \\
\hline 18 & $288.00 \odot$ & $288.00 \odot$ & 1.0000 & $\odot .1448 \mathrm{E}+08$ \\
\hline 19 & 288.000 & 288.000 & 1.0000 & $\odot .1448 \mathrm{E}+08$ \\
\hline 20 & 288.000 & 288.000 & 1.0000 & $\odot .1448 \mathrm{E}+\odot 8$ \\
\hline 21 & $288.00 \odot$ & $288.00 \odot$ & 1.0000 & $\odot .1448 \mathrm{E}+08$ \\
\hline 22 & 288.000 & 288.000 & 1.0000 & $\odot .1448 \mathrm{E}+08$ \\
\hline 23 & $288.00 \odot$ & $288.00 \odot$ & 1.0000 & $\odot .1448 \mathrm{E}+\odot 8$ \\
\hline 24 & 288.000 & $288.00 \odot$ & $1.000 \odot$ & $\odot .1448 \mathrm{E}+\odot 8$ \\
\hline 25 & 288.000 & 288.000 & $1.000 \odot$ & $\odot .1448 \mathrm{E}+\odot 8$ \\
\hline 26 & $288.00 \odot$ & $288.00 \odot$ & 1.0000 & $0.1448 \mathrm{E}+08$ \\
\hline 27 & $288.00 \odot$ & 288.000 & 1.0000 & $\odot .1448 E+\odot 8$ \\
\hline 28 & 288.000 & 288.000 & 1.0000 & $\odot .1448 \mathrm{E}+08$ \\
\hline 29 & $288.00 \odot$ & $288.00 \odot$ & $1.000 \odot$ & $\odot .1448 \mathrm{E}+\odot 8$ \\
\hline 30 & $288.00 \odot$ & 288.000 & 1.0000 & $\odot .1448 \mathrm{E}+\odot 8$ \\
\hline 31 & 288.000 & 288.000 & 1.0000 & $\odot .1448 \mathrm{E}+08$ \\
\hline 32 & $288.0 \odot \odot$ & $288 . \odot \odot \odot$ & 1.0000 & $\odot .1448 \mathrm{E}+08$ \\
\hline 33 & 288.000 & $288.0 \odot \odot$ & 1.0000 & $\odot .1448 \mathrm{E}+\odot 8$ \\
\hline 34 & 288.000 & 288.000 & 1.0000 & $\odot .1448 \mathrm{E}+08$ \\
\hline 35 & $288.0 \odot \odot$ & $288 . \odot \odot \odot$ & 1.0000 & $\odot .1448 \mathrm{E}+\odot 8$ \\
\hline 36 & 288.000 & 288.000 & 1.0000 & $\odot .1448 \mathrm{E}+08$ \\
\hline 37 & $288.00 \odot$ & $288.00 \odot$ & 1.0000 & $0.1448 \mathrm{E}+08$ \\
\hline 38 & $288.0 \odot \odot$ & $288 . \odot \odot \odot$ & $1.000 \odot$ & $\odot .1448 \mathrm{E}+\odot 8$ \\
\hline 39 & 288.000 & $288.0 \odot \odot$ & 1.0000 & $0.1448 \mathrm{E}+08$ \\
\hline 40 & $288.0 \odot \odot$ & $288.0 \odot \odot$ & 1..$\odot \odot \odot$ & $\odot .1448 E+\odot 8$ \\
\hline 41 & $288.00 \odot$ & $288.00 \odot$ & 1.0000 & $\odot .1448 \mathrm{E}+\odot 8$ \\
\hline 42 & 288.000 & $288.0 \odot \odot$ & 1.0000 & $\odot .1448 \mathrm{E}+\odot 8$ \\
\hline 43 & 288.000 & $288 . \odot \odot \odot$ & 1.0000 & $0.1448 \mathrm{E}+08$ \\
\hline 44 & $288.00 \odot$ & $288.0 \odot \odot$ & 1.0000 & $\odot .1448 \mathrm{E}+08$ \\
\hline 45 & 288.000 & $288.00 \odot$ & 1.0000 & $\odot .1448 \mathrm{E}+08$ \\
\hline 46 & $288.00 \odot$ & $288.00 \odot$ & 1.0000 & $\odot .1448 \mathrm{E}+\odot 8$ \\
\hline 47 & 288.000 & $288.0 \odot \odot$ & $1.000 \odot$ & $\odot .1448 \mathrm{E}+\odot 8$ \\
\hline 48 & 288.000 & $288.0 \odot \odot$ & 1.0000 & $\odot .1448 \mathrm{E}+08$ \\
\hline 49 & $288.00 \odot$ & $288 . \odot \odot \odot$ & 1.0000 & $\odot .1448 \mathrm{E}+08$ \\
\hline 50 & 288.000 & $288.0 \odot \odot$ & 1.0000 & $\odot .1448 \mathrm{E}+\odot 8$ \\
\hline 51 & 288.000 & $288.0 \odot \odot$ & 1.0000 & $\odot .1448 \mathrm{E}+\odot 8$ \\
\hline 52 & 288.000 & $288 . \odot \odot \odot$ & $1.000 \odot$ & $\odot .1448 \mathrm{E}+\odot 8$ \\
\hline 53 & 288.000 & 288.000 & 1.0000 & $\odot .1448 \mathrm{E}+\odot 8$ \\
\hline 54 & 288.000 & $288 . \odot \odot \odot$ & 1.0000 & $0.1448 \mathrm{E}+08$ \\
\hline 55 & 288.000 & $288.0 \odot \odot$ & 1.0000 & $\odot .1448 \mathrm{E}+08$ \\
\hline 56 & 288.000 & $288.0 \odot \odot$ & 1.0000 & $\odot .1448 \mathrm{E}+\odot 8$ \\
\hline 57 & 288.000 & $288 . \odot \odot \odot$ & $1.00 \odot \odot$ & $\odot .1448 \mathrm{E}+08$ \\
\hline 58 & $288.0 \odot \odot$ & $288 . \odot \odot \odot$ & 1.0000 & $\odot .1448 \mathrm{E}+\odot 8$ \\
\hline 59 & 288.000 & $288.0 \odot \odot$ & 1.0000 & $0.1448 \mathrm{E}+08$ \\
\hline 60 & 288.000 & 288.000 & 1.0000 & $\odot .1448 E+\odot 8$ \\
\hline
\end{tabular}




\begin{tabular}{|c|c|c|c|c|}
\hline \multicolumn{5}{|c|}{ lambda $=0.3171127141$} \\
\hline noue & $\mathrm{T}(\mathrm{K})$ & Twall(K) & $\mathrm{x}$ & $P(P a)$ \\
\hline 1 & 157.827 & 204.071 & $-\ldots$ & $\odot .1984 \mathrm{E}+07$ \\
\hline 2 & 183.079 & 247.059 & 0.7219 & $\odot .3659 \mathrm{E}+07$ \\
\hline 3 & 239.833 & 280.323 & 1.0000 & $\odot .1018 \mathrm{E}+\odot 8$ \\
\hline 4 & 275.810 & 287.560 & $1.000 \odot$ & $\odot .1279 \mathrm{E}+\odot 8$ \\
\hline 5 & 286.787 & 288.000 & 1.0000 & $0.1413 \mathrm{E}+08$ \\
\hline 6 & 288.000 & 288.000 & 1.0000 & $\odot .1445 \mathrm{E}+08$ \\
\hline 7 & 288.000 & 288.000 & 1.0000 & $\odot .1448 \mathrm{E}+08$ \\
\hline 8 & 288.000 & 288.000 & 1.0000 & $0.1448 \mathrm{E}+08$ \\
\hline 9 & $288.00 \odot$ & $288.00 \odot$ & $1.000 \odot$ & $\odot .1448 \mathrm{E}+\odot 8$ \\
\hline 10 & $288.00 \odot$ & 288.000 & 1.0000 & $\odot .1448 \mathrm{E}+08$ \\
\hline 11 & 288.000 & 288.000 & 1.0000 & $0.1448 \mathrm{E}+08$ \\
\hline 12 & 288.000 & 288.000 & 1.0000 & $0.1448 \mathrm{E}+08$ \\
\hline 13 & $288.00 \odot$ & $288.00 \odot$ & $1.000 \odot$ & $0.1448 \mathrm{E}+08$ \\
\hline 14 & 288.000 & 288.000 & 1.0000 & $\odot .1448 \mathrm{E}+\odot 8$ \\
\hline 15 & 288.000 & 288.000 & $1.000 \odot$ & $\odot .1448 \mathrm{E}+\odot 8$ \\
\hline 16 & 288.000 & 288.000 & 1.0000 & $\odot .1448 \mathrm{E}+\odot 8$ \\
\hline 17 & 288.000 & 288.000 & $1.000 \odot$ & $\odot .1448 \mathrm{E}+08$ \\
\hline 18 & 288.000 & 288.000 & 1.0000 & $\odot .1448 \mathrm{E}+\odot 8$ \\
\hline 19 & $288 . \odot \odot \odot$ & $288.00 \odot$ & $1.00 \odot \odot$ & $0.1448 \mathrm{E}+08$ \\
\hline 20 & $288.0 \odot \odot$ & $288.00 \odot$ & $1.000 \odot$ & $0.1448 \mathrm{E}+08$ \\
\hline 21 & $288.00 \odot$ & $288.00 \odot$ & $1.000 \odot$ & $\odot .1448 \mathrm{E}+08$ \\
\hline 22 & 288.000 & 288.000 & 1.0000 & $0.1448 \mathrm{E}+08$ \\
\hline 23 & 288.000 & 288.000 & 1.0000 & $\odot .1448 \mathrm{E}+\odot 8$ \\
\hline 24 & $288.00 \odot$ & $288.00 \odot$ & $1.000 \odot$ & $\odot .1448 \mathrm{E}+08$ \\
\hline 25 & 288.000 & 288.000 & 1.0000 & $\odot .1448 \mathrm{E}+08$ \\
\hline 26 & $288 . \odot \odot \odot$ & $288.00 \odot$ & $1.000 \odot$ & $0.1448 \mathrm{E}+08$ \\
\hline 27 & $288.00 \odot$ & $288.00 \odot$ & 1.0000 & $\odot .1448 \mathrm{E}+08$ \\
\hline 28 & $288.0 \odot \odot$ & 288.000 & 1.0000 & $\odot .1448 \mathrm{E}+08$ \\
\hline 29 & $288.0 \odot \odot$ & 288.000 & $1.000 \odot$ & $0.1448 \mathrm{E}+\odot 8$ \\
\hline 30 & $288 . \odot \odot \odot$ & $288.00 \odot$ & $1.00 \odot \odot$ & $0.1448 \mathrm{E}+08$ \\
\hline 31 & $288.0 \odot \odot$ & 288.000 & 1.0000 & $0.1448 \mathrm{E}+08$ \\
\hline 32 & $288.00 \odot$ & $288.0 \odot \odot$ & $1.00 \odot \odot$ & $\odot .1448 \mathrm{E}+\odot 8$ \\
\hline 33 & $288.00 \odot$ & 288.000 & 1.0000 & $0.1448 \mathrm{E}+\odot 8$ \\
\hline 34 & 288.000 & 288.000 & 1.0000 & $\odot .1448 \mathrm{E}+\odot 8$ \\
\hline 35 & $288.0 \odot \odot$ & 288.000 & 1.0000 & $0.1448 \mathrm{E}+08$ \\
\hline 36 & $288.00 \odot$ & $288.00 \odot$ & $1.000 \odot$ & $\odot .1448 \mathrm{E}+\odot 8$ \\
\hline 37 & $288.00 \odot$ & $288.00 \odot$ & $1.000 \odot$ & $\odot .1448 \mathrm{E}+08$ \\
\hline 38 & $288.00 \odot$ & 288.000 & 1.0000 & $0.1448 \mathrm{E}+08$ \\
\hline 39 & 288.000 & 288.000 & 1.0000 & $\odot .1448 \mathrm{E}+08$ \\
\hline 40 & $288.0 \odot \odot$ & $288.00 \odot$ & $1.000 \odot$ & $\odot .1448 \mathrm{E}+\odot 8$ \\
\hline 41 & 288.000 & 288.000 & 1.0000 & $\odot .1448 \mathrm{E}+\odot 8$ \\
\hline 42 & $288 . \odot \odot \odot$ & $288.0 \odot \odot$ & $1.00 \odot \odot$ & $\odot .1448 \mathrm{E}+\odot 8$ \\
\hline 43 & $288.00 \odot$ & $288.00 \odot$ & 1.0000 & $\odot .1448 \mathrm{E}+08$ \\
\hline 44 & $288.00 \odot$ & $288.00 \odot$ & $1.000 \odot$ & $0.1448 E+08$ \\
\hline 45 & $288.0 \odot \odot$ & 288.000 & 1.0000 & $0.1448 \mathrm{E}+08$ \\
\hline 46 & $288 . \odot \odot \odot$ & $288.00 \odot$ & $1.000 \odot$ & $0.1448 \mathrm{E}+08$ \\
\hline 47 & $288.00 \odot$ & $288.00 \odot$ & $1.000 \odot$ & $0.1448 \mathrm{E}+08$ \\
\hline 48 & $288 . \odot \odot \odot$ & $288.00 \odot$ & $1.000 \odot$ & $0.1448 \mathrm{E}+08$ \\
\hline 49 & $288.00 \odot$ & $288 . \odot \odot \odot$ & $1.000 \odot$ & $0.1448 \mathrm{E}+08$ \\
\hline 50 & $288.00 \odot$ & 288.000 & 1.0000 & $\odot .1448 \mathrm{E}+\odot 8$ \\
\hline 51 & $288.0 \odot \odot$ & 288.000 & $1.000 \odot$ & $\odot .1448 \mathrm{E}+\odot 8$ \\
\hline 52 & $288.00 \odot$ & $288.0 \odot \odot$ & $1.000 \odot$ & $\odot .1448 \mathrm{E}+\odot 8$ \\
\hline 53 & $288 . \odot \odot \odot$ & $288 . \odot \odot \odot$ & $1.000 \odot$ & $0.1448 \mathrm{E}+08$ \\
\hline 54 & 288.000 & 288.000 & 1.0000 & $\odot .1448 \mathrm{E}+08$ \\
\hline 55 & 288.000 & 288.000 & 1.0000 & $\odot .1448 \mathrm{E}+08$ \\
\hline
\end{tabular}




$\begin{array}{lllll}56 & 288.00 \odot & 288.00 \odot & 1.000 \odot & \odot .1448 \mathrm{E}+\odot 8 \\ 57 & 288.00 \odot & 288.00 \odot & 1.000 \odot & \odot .1448 \mathrm{E}+08 \\ 58 & 288.00 \odot & 288.00 \odot & 1.00 \odot \odot & \odot .1448 \mathrm{E}+08 \\ 59 & 288.00 \odot & 288.00 \odot & 1.000 \odot & \odot .1448 \mathrm{E}+08 \\ 6 \odot & 288.000 & 288.00 \odot & 1.000 \odot & \odot .1448 \mathrm{E}+08\end{array}$

\begin{tabular}{|c|c|c|c|c|}
\hline time & $(\sec )=$ & $11.00 \quad 1$ & $=0$ & $\begin{array}{l}P(P a) \\
P(P a)\end{array}$ \\
\hline $\begin{array}{l}\text { node } \\
1\end{array}$ & 156.114 & $\begin{array}{r}\text { TwaLI(K) } \\
198.651\end{array}$ & $\begin{array}{c}\lambda \\
---\ldots\end{array}$ & $0.1862 \mathrm{E}+07$ \\
\hline 2 & 181.092 & 242.201 & $\odot .7401$ & $\odot .3435 \mathrm{E}+07$ \\
\hline 3 & 233.062 & 276.763 & 1.0000 & $\odot .9857 \mathrm{E}+07$ \\
\hline 4 & 269.330 & 286.861 & 1.0000 & $\odot .1231 \mathrm{E}+08$ \\
\hline 5 & 285.347 & 288.000 & $1.000 \odot$ & $\odot .1391 \mathrm{E}+\odot 8$ \\
\hline 6 & 287.989 & 288.000 & 1.0000 & $0.1441 \mathrm{E}+08$ \\
\hline 7 & 288.000 & 288.000 & 1.0000 & $0.1448 \mathrm{E}+08$ \\
\hline 8 & 288.000 & 288.000 & 1.0000 & $\odot .1448 E+08$ \\
\hline 9 & 288.000 & 288.000 & 1.0000 & $\odot .1448 \mathrm{E}+\odot 8$ \\
\hline 10 & 288.000 & 288.000 & 1.0000 & $\odot .1448 \mathrm{E}+08$ \\
\hline 11 & $288.00 \odot$ & $288.0 \odot \odot$ & 1.0000 & $\odot .1448 \mathrm{E}+\odot 8$ \\
\hline 12 & 288.000 & 288.000 & 1.0000 & $\odot .1448 \mathrm{E}+\odot 8$ \\
\hline 13 & $288.00 \odot$ & $288.0 \odot \odot$ & 1.0000 & $\odot .1448 \mathrm{E}+08$ \\
\hline 14 & 288.000 & 288.000 & 1. $\odot \odot \odot \odot$ & $\odot .1448 E+\odot 8$ \\
\hline 15 & 288.000 & 288.000 & 1.0000 & $\odot .1448 \mathrm{E}+08$ \\
\hline 16 & $288.0 \odot \odot$ & $288.0 \odot \odot$ & 1.0000 & $\odot .1448 \mathrm{E}+08$ \\
\hline 17 & 288.000 & $288.0 \odot \odot$ & 1.0000 & $\odot .1448 \mathrm{E}+\odot 8$ \\
\hline 18 & 288.000 & 288.000 & 1.0000 & $\odot .1448 \mathrm{E}+08$ \\
\hline 19 & $288.00 \odot$ & $288 . \odot \odot \odot$ & $1.000 \odot$ & $0.1448 \mathrm{E}+08$ \\
\hline 20 & 288.000 & $288.00 \odot$ & $1.00 \odot \odot$ & $\odot .1448 E+\odot 8$ \\
\hline 21 & 288.000 & 288.000 & 1.0000 & $\odot .1448 \mathrm{E}+08$ \\
\hline 22 & 288.000 & 288.000 & $1.000 \odot$ & $\odot .1448 \mathrm{E}+\odot 8$ \\
\hline 23 & 288.000 & 288.000 & 1.0000 & $\odot .1448 \mathrm{E}+\odot 8$ \\
\hline 24 & 288.000 & 288.000 & $1.000 \odot$ & $0.1448 \mathrm{E}+08$ \\
\hline 25 & 288.000 & 288.000 & 1.0000 & $\odot .1448 E+08$ \\
\hline 26 & 288.000 & 288.000 & 1.0000 & $\odot .1448 \mathrm{E}+08$ \\
\hline 27 & 288.000 & 288.000 & 1.0000 & $\odot .1448 \mathrm{E}+\odot 8$ \\
\hline 28 & 288.000 & 288.000 & 1.0000 & $\odot .1448 \mathrm{E}+\odot 8$ \\
\hline 29 & 288.000 & 288.000 & 1.0000 & $\odot .1448 \mathrm{E}+08$ \\
\hline 30 & $288.00 \odot$ & $288 . \odot \odot \odot$ & $1.000 \odot$ & $0.1448 \mathrm{E}+08$ \\
\hline 31 & 288.000 & 288.000 & 1.0000 & $\odot .1448 \mathrm{E}+\odot 8$ \\
\hline 32 & 288.000 & 288.000 & 1.0000 & $\odot .1448 \mathrm{E}+\odot 8$ \\
\hline 33 & 288.000 & 288.000 & $1.00 \odot \odot$ & $\odot .1448 E+08$ \\
\hline 34 & $288.00 \odot$ & $288.0 \odot \odot$ & 1.0000 & $\odot .1448 \mathrm{E}+\odot 8$ \\
\hline 35 & 288.000 & 288.000 & 1.0000 & $\odot .1448 \mathrm{E}+08$ \\
\hline 36 & $288.00 \odot$ & $288.00 \odot$ & $1.000 \odot$ & $\odot .1448 \mathrm{E}+\odot 8$ \\
\hline 37 & 288.000 & 288.000 & 1.0000 & $0.1448 \mathrm{E}+08$ \\
\hline 38 & 288.000 & 288.000 & 1.0000 & $\odot .1448 \mathrm{E}+08$ \\
\hline 39 & 288.000 & 288.000 & 1.0000 & $\odot .1448 \mathrm{E}+08$ \\
\hline 40 & 288.000 & $288.00 \odot$ & 1.0000 & $\odot .1448 \mathrm{E}+\odot 8$ \\
\hline 41 & 288.000 & 288.000 & 1.0000 & $\odot .1448 E+08$ \\
\hline 42 & $288.0 \odot \odot$ & $288 . \odot \odot \odot$ & 1.0000 & $0.1448 \mathrm{E}+08$ \\
\hline 43 & 288.000 & 288.000 & 1.0000 & $\odot .1448 E+08$ \\
\hline 44 & 288.000 & 288.000 & 1.0000 & $\odot .1448 \mathrm{E}+08$ \\
\hline 45 & $288.00 \odot$ & $288.0 \odot \odot$ & 1.0000 & $\odot .1448 \mathrm{E}+\odot 8$ \\
\hline 46 & 288.000 & 288.000 & 1.0000 & $\odot .1448 \mathrm{E}+\odot 8$ \\
\hline 47 & 288.000 & 288.000 & 1.0000 & $\odot .1448 \mathrm{E}+08$ \\
\hline 48 & $288.00 \odot$ & $288.00 \odot$ & 1.0000 & $\odot .1448 \mathrm{E}+08$ \\
\hline 49 & 288.000 & 288.000 & 1.0000 & $\odot .1448 \mathrm{E}+08$ \\
\hline
\end{tabular}




\begin{tabular}{|c|c|c|c|c|}
\hline 50 & 288.000 & $288.0 \odot \odot$ & 1.0000 & $\odot .1448 \mathrm{E}+\odot 8$ \\
\hline 51 & $288.00 \odot$ & $288 . \odot \odot \odot$ & 1.0000 & $\odot .1448 \mathrm{E}+08$ \\
\hline 52 & 288.000 & 288.000 & 1.0000 & $\odot .1448 E+08$ \\
\hline 53 & $288.0 \odot \odot$ & $288.0 \odot \odot$ & $1.000 \odot$ & $\odot .1448 \mathrm{E}+\odot 8$ \\
\hline 54 & $288.00 \odot$ & $288.0 \odot \odot$ & $1.00 \odot \odot$ & $\odot .1448 \mathrm{E}+08$ \\
\hline 55 & $288.0 \odot \odot$ & $288.0 \odot \odot$ & 1.0000 & $\odot .1448 \mathrm{E}+\odot 8$ \\
\hline 56 & $288.0 \odot \odot$ & $288 . \odot \odot \odot$ & $1.000 \odot$ & $\odot .1448 \mathrm{E}+\odot 8$ \\
\hline 57 & $288.00 \odot$ & $288.00 \odot$ & $1.00 \odot \odot$ & $\odot .1448 \mathrm{E}+08$ \\
\hline 58 & $288.00 \odot$ & $288 . \odot \odot \odot$ & 1.0000 & $\odot .1448 \mathrm{E}+\odot 8$ \\
\hline 59 & 288.000 & 288.000 & 1.0000 & $0.1448 \mathrm{E}+08$ \\
\hline 60 & 288.000 & 288.000 & $1.000 \odot$ & $\odot .1448 \mathrm{E}+\odot 8$ \\
\hline
\end{tabular}

\begin{tabular}{|c|c|c|c|c|}
\hline $\begin{array}{l}\text { time } \\
\text { node }\end{array}$ & $\begin{array}{r}(\mathrm{sec})= \\
\mathrm{T}(\mathrm{K})\end{array}$ & $.00 \quad 1$ & $a=0$ & $P(P a)$ \\
\hline 1 & 154.582 & 193.969 & $\ldots$ & $0.1753 \mathrm{E}+07$ \\
\hline 2 & 179.315 & 237.735 & 0.7574 & $\odot .3234 \mathrm{E}+\odot 7$ \\
\hline 3 & 228.743 & 273.101 & $1.00 \odot \odot$ & $\odot .9654 \mathrm{E}+\odot 7$ \\
\hline 4 & 262.007 & 285.718 & $1.00 \odot \odot$ & $\odot .1185 E+\odot 8$ \\
\hline 5 & 282.957 & 288.000 & 1.0000 & $\odot .1362 E+\odot 8$ \\
\hline 6 & 287.683 & 288.000 & 1.0000 & $\odot .1434 \mathrm{E}+08$ \\
\hline 7 & 288.000 & 288.000 & 1.0000 & $\odot .1447 \mathrm{E}+\odot 8$ \\
\hline 8 & 288.000 & 288.000 & 1.0000 & $\odot .1448 \mathrm{E}+08$ \\
\hline 9 & 288.000 & $288.00 \odot$ & 1.0000 & $\odot .1448 \mathrm{E}+\odot 8$ \\
\hline 10 & 288.000 & $288.00 \odot$ & $1.000 \odot$ & $\odot .1448 \mathrm{E}+\odot 8$ \\
\hline 11 & 288.000 & 288.000 & 1.0000 & $\odot .1448 \mathrm{E}+08$ \\
\hline 12 & 288.000 & 288.000 & 1.0000 & $\odot .1448 \mathrm{E}+\odot 8$ \\
\hline 13 & 288.000 & $288.00 \odot$ & 1.0000 & $\odot .1448 \mathrm{E}+08$ \\
\hline 14 & 288.000 & 288.000 & 1.0000 & $\odot .1448 \mathrm{E}+\odot 8$ \\
\hline 15 & 288.000 & 288.000 & 1.0000 & $\odot .1448 \mathrm{E}+08$ \\
\hline 16 & $288.00 \odot$ & $288.0 \odot \odot$ & $1.00 \odot \odot$ & $\odot .1448 \mathrm{E}+\odot 8$ \\
\hline 17 & 288.000 & 288.000 & 1.0000 & $\odot .1448 \mathrm{E}+08$ \\
\hline 18 & 288.000 & 288.000 & 1.0000 & $\odot .1448 \mathrm{E}+08$ \\
\hline 19 & 288.000 & 288.000 & 1.0000 & $\odot .1448 \mathrm{E}+08$ \\
\hline 20 & 288.000 & $288.0 \odot \odot$ & $1.00 \odot \odot$ & $\odot .1448 \mathrm{E}+\odot 8$ \\
\hline 21 & $288.00 \odot$ & $288.00 \odot$ & $1.00 \odot \odot$ & $\odot .1448 \mathrm{E}+\odot 8$ \\
\hline 22 & 288.000 & $288.00 \odot$ & 1.0000 & $\odot .1448 \mathrm{E}+\odot 8$ \\
\hline 23 & 288.000 & 288.000 & 1.0000 & $\odot .1448 \mathrm{E}+08$ \\
\hline 24 & 288.000 & 288.000 & 1.0000 & $0.1448 \mathrm{E}+08$ \\
\hline 25 & 288.000 & $288.0 \odot \odot$ & 1.0000 & $\odot .1448 \mathrm{E}+08$ \\
\hline 26 & $288.00 \odot$ & $288.0 \odot \odot$ & $1.00 \odot \odot$ & $\odot .1448 \mathrm{E}+\odot 8$ \\
\hline 27 & $288.00 \odot$ & $288 . \odot \odot \odot$ & $1.00 \odot \odot$ & $\odot .1448 \mathrm{E}+\odot 8$ \\
\hline 28 & 288.000 & 288.000 & 1.0000 & $\odot .1448 \mathrm{E}+\odot 8$ \\
\hline 29 & 288.000 & 288.000 & 1.0000 & $\odot .1448 \mathrm{E}+08$ \\
\hline 30 & 288.000 & 288.000 & 1.0000 & $\odot .1448 \mathrm{E}+08$ \\
\hline 31 & 288.000 & 288.000 & 1.0000 & $\odot .1448 \mathrm{E}+08$ \\
\hline 32 & 288.000 & $288.00 \odot$ & 1.0000 & $\odot .1448 \mathrm{E}+08$ \\
\hline 33 & $288.00 \odot$ & $288.0 \odot \odot$ & $1.00 \odot \odot$ & $\odot .1448 \mathrm{E}+\odot 8$ \\
\hline 34 & 288.000 & 288.000 & 1.0000 & $0.1448 \mathrm{E}+08$ \\
\hline 35 & $288.00 \odot$ & $288.0 \odot \odot$ & $1.000 \odot$ & $\odot .1448 \mathrm{E}+08$ \\
\hline 36 & 288.000 & 288.000 & $1.000 \odot$ & $\odot .1448 \mathrm{E}+08$ \\
\hline 37 & 288.000 & 288.000 & 1.0000 & $\odot .1448 \mathrm{E}+\odot 8$ \\
\hline 38 & 288.000 & 288.000 & 1.0000 & $\odot .1448 \mathrm{E}+08$ \\
\hline 39 & 288.000 & $288.0 \odot \odot$ & 1.0000 & $\odot .1448 \mathrm{E}+08$ \\
\hline 40 & 288.000 & 288.000 & 1.0000 & $\odot .1448 \mathrm{E}+08$ \\
\hline 41 & 288.000 & $288.00 \odot$ & $1.000 \odot$ & $\odot .1448 \mathrm{E}+08$ \\
\hline 42 & $288.00 \odot$ & $288.0 \odot \odot$ & $1.000 \odot$ & $\odot .1448 \mathrm{E}+08$ \\
\hline 43 & 288.000 & 288.000 & 1.0000 & $\odot .1448 \mathrm{E}+\odot 8$ \\
\hline
\end{tabular}




\begin{tabular}{|c|c|c|c|c|}
\hline 44 & $288.0 \odot \odot$ & $288.00 \odot$ & $1.000 \odot$ & $\odot .1448 E+\odot 8$ \\
\hline 45 & $288.0 \odot \odot$ & 288.000 & 1.0000 & $0.1448 E+08$ \\
\hline 46 & $288 . \odot \odot \odot$ & $288 . \odot \odot \odot$ & $1.000 \odot$ & $0.1448 \mathrm{E}+08$ \\
\hline 47 & $288 . \odot \odot \odot$ & $288.00 \odot$ & $1.00 \odot \odot$ & $0.1448 \mathrm{E}+\odot 8$ \\
\hline 48 & 288.000 & 288.000 & 1.0000 & $0.1448 \mathrm{E}+08$ \\
\hline 49 & $288 . \odot \odot \odot$ & $288.0 \odot \odot$ & $1.000 \odot$ & $\odot .1448 \mathrm{E}+\odot 8$ \\
\hline 50 & $288.0 \odot \odot$ & $288.00 \odot$ & $1.000 \odot$ & $\odot .1448 \mathrm{E}+08$ \\
\hline 51 & $288.0 \odot \odot$ & $288.00 \odot$ & 1.0000 & $0.1448 \mathrm{E}+08$ \\
\hline 52 & $288.0 \odot \odot$ & $288.00 \odot$ & $1.000 \odot$ & $\odot .1448 E+\odot 8$ \\
\hline 53 & $288 . \odot \odot \odot$ & $288.0 \odot \odot$ & $1.000 \odot$ & $0.1448 \mathrm{E}+\odot 8$ \\
\hline 54 & $288 . \odot \odot \odot$ & $288.00 \odot$ & $1.00 \odot \odot$ & $0.1448 E+\odot 8$ \\
\hline 55 & $288.0 \odot \odot$ & 288.000 & 1.0000 & $0.1448 \mathrm{E}+08$ \\
\hline 56 & $288 . \odot \odot \odot$ & $288.0 \odot \odot$ & $1.000 \odot$ & $0.1448 \mathrm{E}+\odot 8$ \\
\hline 57 & $288.00 \odot$ & 288.000 & 1.0000 & $0.1448 \mathrm{E}+08$ \\
\hline 58 & $288.0 \odot \odot$ & $288.0 \odot \odot$ & 1.0000 & $0.1448 \mathrm{E}+08$ \\
\hline 59 & $288.0 \odot \odot$ & 288.000 & 1.0000 & $\odot .1448 E+\odot 8$ \\
\hline 60 & 288.000 & 288.000 & 1.0000 & $\odot .1448 E+08$ \\
\hline time & $(\sec )=$ & \multicolumn{3}{|l|}{13.00} \\
\hline node & $\mathrm{T}(\mathrm{K})$ & Twall(K) & $\mathrm{X}$ & $P(P a)$ \\
\hline 1 & 153.223 & 189.897 & - - - - - & $\odot .1656 \mathrm{E}+07$ \\
\hline 2 & 177.739 & 233.562 & $\odot .7738$ & $\odot .3055 \mathrm{E}+07$ \\
\hline 3 & 226.663 & 269.451 & $1.000 \odot$ & $\odot .9546 \mathrm{E}+\odot 7$ \\
\hline 4 & 254.659 & 284.032 & $1.00 \odot \odot$ & $0.1143 E+08$ \\
\hline 5 & 279.388 & 287.782 & 1.0000 & $0.1328 \mathrm{E}+08$ \\
\hline 6 & 287.153 & $288.00 \odot$ & 1.0000 & $0.1423 E+08$ \\
\hline 7 & $288.0 \odot \odot$ & $288.00 \odot$ & $1.00 \odot \odot$ & $0.1446 \mathrm{E}+\odot 8$ \\
\hline 8 & $288 . \odot \odot \odot$ & $288.0 \odot \odot$ & $1.00 \odot \odot$ & $\odot .1448 E+\odot 8$ \\
\hline 9 & $288.0 \odot \odot$ & $288.0 \odot \odot$ & $1.00 \odot \odot$ & $\odot .1448 E+\odot 8$ \\
\hline 10 & 288.000 & $288.00 \odot$ & 1.0000 & $\odot .1448 \mathrm{E}+08$ \\
\hline 11 & 288.000 & 288.000 & 1.0000 & $0.1448 \mathrm{E}+08$ \\
\hline 12 & $288.0 \odot \odot$ & $288.0 \odot \odot$ & $1.0 \odot \odot \odot$ & $\odot .1448 E+\odot 8$ \\
\hline 13 & $288.00 \odot$ & $288.00 \odot$ & 1.0000 & $\odot .1448 \mathrm{E}+08$ \\
\hline 14 & $288.00 \odot$ & $288.0 \odot \odot$ & $1.0 \odot \odot \odot$ & $0.1448 E+\odot 8$ \\
\hline 15 & $288 . \odot \odot \odot$ & $288.00 \odot$ & $1.00 \odot \odot$ & $0.1448 E+\odot 8$ \\
\hline 16 & $288.00 \odot$ & $288.0 \odot \odot$ & $1.000 \odot$ & $0.1448 \mathrm{E}+\odot 8$ \\
\hline 17 & $288 . \odot \odot \odot$ & $288.00 \odot$ & $1.00 \odot \odot$ & $0.1448 E+\odot 8$ \\
\hline 18 & $288.0 \odot \odot$ & $288.00 \odot$ & $1.00 \odot \odot$ & $0.1448 \mathrm{E}+\odot 8$ \\
\hline 19 & $288.00 \odot$ & $288 . \odot \odot \odot$ & 1.0000 & $\odot .1448 \mathrm{E}+08$ \\
\hline 20 & $288.00 \odot$ & $288 . \odot \odot \odot$ & $1.000 \odot$ & $\odot .1448 \mathrm{E}+\odot 8$ \\
\hline 21 & $288.0 \odot \odot$ & $288.0 \odot \odot$ & $1.0 \odot \odot \odot$ & $\odot .1448 E+\odot 8$ \\
\hline 22 & 288.000 & $288.0 \odot \odot$ & 1.0000 & $0.1448 \mathrm{E}+08$ \\
\hline 23 & $288.00 \odot$ & $288 . \odot \odot \odot$ & $1.00 \odot \odot$ & $0.1448 \mathrm{E}+\odot 8$ \\
\hline 24 & $288.00 \odot$ & $288.00 \odot$ & $1.00 \odot \odot$ & $0.1448 \mathrm{E}+\odot 8$ \\
\hline 25 & $288 . \odot$ & 288.000 & 1.0000 & $0.1448 \mathrm{E}+08$ \\
\hline 26 & $288.00 \odot$ & $288 . \odot \odot \odot$ & $1.000 \odot$ & $0.1448 \mathrm{E}+\odot 8$ \\
\hline 27 & $288.00 \odot$ & $288.0 \odot \odot$ & $1.000 \odot$ & $0.1448 \mathrm{E}+\odot 8$ \\
\hline 28 & 288.000 & 288.000 & 1.0000 & $0.1448 E+08$ \\
\hline 29 & $288.00 \odot$ & $288.00 \odot$ & $1.000 \odot$ & $0.1448 E+\odot 8$ \\
\hline 30 & $288.00 \odot$ & $288 . \odot \odot \odot$ & $1.000 \odot$ & $0.1448 \mathrm{E}+08$ \\
\hline 31 & 288.000 & $288.00 \odot$ & 1.0000 & $0.1448 \mathrm{E}+08$ \\
\hline 32 & 288.000 & 288.000 & $1.000 \odot$ & $\odot .1448 E+08$ \\
\hline 33 & $288.00 \odot$ & $288 . \odot \odot \odot$ & $1.000 \odot$ & $\odot .1448 \mathrm{E}+\odot 8$ \\
\hline 34 & 288.000 & 288.000 & 1.0000 & $0.1448 \mathrm{E}+08$ \\
\hline 35 & $288 . \odot \odot \odot$ & $288.00 \odot$ & $1.00 \odot \odot$ & $\odot .1448 E+\odot 8$ \\
\hline 36 & 288.000 & $288.00 \odot$ & 1.0000 & $0.1448 \mathrm{E}+08$ \\
\hline 37 & $288.00 \odot$ & $288.00 \odot$ & $1.00 \odot \odot$ & $\odot .1448 E+08$ \\
\hline
\end{tabular}




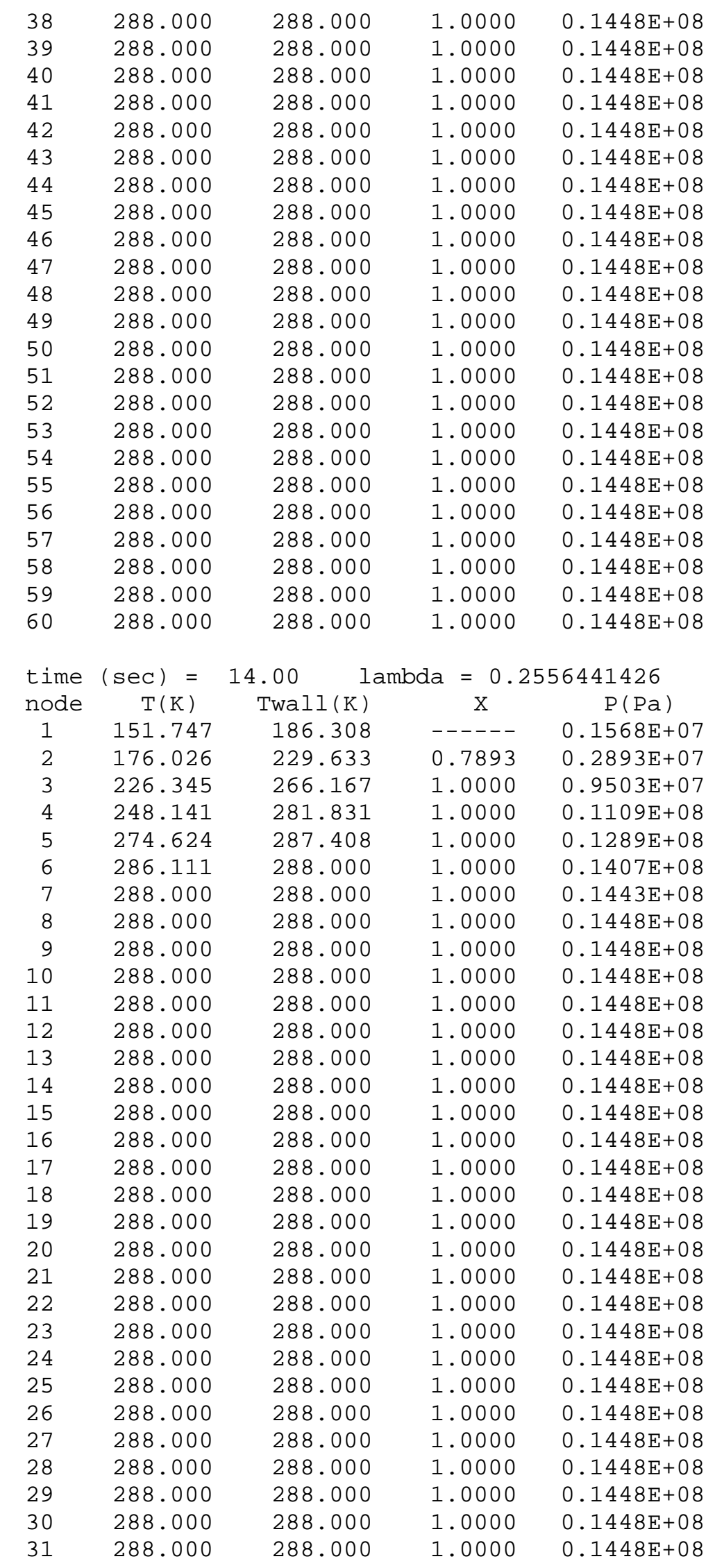




\begin{tabular}{|c|c|c|c|c|}
\hline 32 & 288.000 & 288.000 & 1.0000 & $\odot .1448 \mathrm{E}+08$ \\
\hline 33 & 288.000 & $288.00 \odot$ & 1.0000 & $0.1448 \mathrm{E}+08$ \\
\hline 34 & $288.00 \odot$ & $288.00 \odot$ & 1.0000 & $0.1448 \mathrm{E}+\odot 8$ \\
\hline 35 & 288.000 & 288.000 & 1.0000 & $0.1448 \mathrm{E}+08$ \\
\hline 36 & 288.000 & 288.000 & 1.0000 & $0.1448 E+08$ \\
\hline 37 & 288.000 & 288.000 & 1.0000 & $0.1448 \mathrm{E}+08$ \\
\hline 38 & 288.000 & 288.000 & 1.0000 & $0.1448 \mathrm{E}+08$ \\
\hline 39 & 288.000 & 288.000 & 1.0000 & $0.1448 \mathrm{E}+08$ \\
\hline 40 & 288.000 & $288.0 \odot \odot$ & 1.0000 & $\odot .1448 \mathrm{E}+\odot 8$ \\
\hline 41 & 288.000 & 288.000 & 1.0000 & $0.1448 \mathrm{E}+08$ \\
\hline 42 & 288.000 & 288.000 & 1.0000 & $0.1448 \mathrm{E}+08$ \\
\hline 43 & 288.000 & 288.000 & $1.000 \odot$ & $0.1448 \mathrm{E}+08$ \\
\hline 44 & 288.000 & 288.000 & 1.0000 & $0.1448 \mathrm{E}+08$ \\
\hline 45 & 288.000 & 288.000 & 1.0000 & $0.1448 \mathrm{E}+08$ \\
\hline 46 & 288.000 & $288.0 \odot \odot$ & 1.0000 & $0.1448 \mathrm{E}+08$ \\
\hline 47 & 288.000 & 288.000 & 1.0000 & $\odot .1448 \mathrm{E}+08$ \\
\hline 48 & 288.000 & 288.000 & 1.0000 & $0.1448 \mathrm{E}+08$ \\
\hline 49 & 288.000 & $288.00 \odot$ & $1.000 \odot$ & $\odot .1448 E+\odot 8$ \\
\hline 50 & 288.000 & $288.0 \odot \odot$ & 1.0000 & $0.1448 \mathrm{E}+\odot 8$ \\
\hline 51 & 288.000 & 288.000 & 1.0000 & $0.1448 \mathrm{E}+08$ \\
\hline 52 & 288.000 & $288.00 \odot$ & 1.0000 & $0.1448 \mathrm{E}+08$ \\
\hline 53 & 288.000 & 288.000 & $1.000 \odot$ & $0.1448 \mathrm{E}+08$ \\
\hline 54 & 288.000 & 288.000 & 1.0000 & $\odot .1448 \mathrm{E}+08$ \\
\hline 55 & 288.000 & 288.000 & 1.0000 & $0.1448 \mathrm{E}+08$ \\
\hline 56 & 288.000 & $288.0 \odot \odot$ & 1.0000 & $0.1448 \mathrm{E}+08$ \\
\hline 57 & 288.000 & $288.0 \odot \odot$ & 1.0000 & $0.1448 \mathrm{E}+08$ \\
\hline 58 & 288.000 & 288.000 & 1.0000 & $0.1448 \mathrm{E}+08$ \\
\hline 59 & $288.00 \odot$ & $288.0 \odot \odot$ & $1.000 \odot$ & $0.1448 \mathrm{E}+08$ \\
\hline 60 & $288.00 \odot$ & $288.00 \odot$ & 1.0000 & $\odot .1448 \mathrm{E}+08$ \\
\hline time & $(\mathrm{sec})$ & \multicolumn{3}{|c|}{ lambda $=0.2440384477$} \\
\hline node & $\mathrm{T}(\mathrm{K})$ & Twall(K) & $\mathrm{x}$ & $\mathrm{P}(\mathrm{Pa})$ \\
\hline 1 & 150.328 & 183.105 & 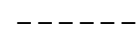 & $0.1490 \mathrm{E}+07$ \\
\hline 2 & 174.380 & 225.877 & 0.8023 & $0.2748 \mathrm{E}+07$ \\
\hline 3 & 227.110 & 263.277 & 1.0000 & $\odot .9492 \mathrm{E}+\odot 7$ \\
\hline 4 & 243.187 & 279.311 & 1.0000 & $0.1086 \mathrm{E}+08$ \\
\hline 5 & 268.916 & 286.704 & 1.0000 & $0.1250 \mathrm{E}+08$ \\
\hline 6 & 284.342 & 288.000 & 1.0000 & $0.1384 \mathrm{E}+08$ \\
\hline 7 & 287.810 & 288.000 & 1.0000 & $0.1438 \mathrm{E}+08$ \\
\hline 8 & $38 . \odot$ & 288.000 & 1.0000 & $0.1447 \mathrm{E}+08$ \\
\hline 9 & 288.0 & 288.000 & $1.000 \odot$ & $0.1448 \mathrm{E}+08$ \\
\hline 10 & 288.000 & 288.000 & $1.000 \odot$ & $0.1448 \mathrm{E}+08$ \\
\hline 11 & 288.000 & 288.000 & 1.0000 & $0.1448 \mathrm{E}+08$ \\
\hline 12 & 288.000 & 288.000 & 1.0000 & $0.1448 \mathrm{E}+08$ \\
\hline 13 & 288.000 & 288.000 & $1.000 \odot$ & $0.1448 \mathrm{E}+08$ \\
\hline 14 & 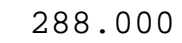 & 288.000 & 1.0000 & $0.1448 \mathrm{E}+08$ \\
\hline 15 & $288.00 \odot$ & $288.00 \odot$ & 1.0000 & $0.1448 \mathrm{E}+08$ \\
\hline 16 & 288.000 & 288.000 & 1.0000 & $0.1448 \mathrm{E}+08$ \\
\hline 17 & 288.000 & $288.00 \odot$ & 1.0000 & $0.1448 \mathrm{E}+\odot 8$ \\
\hline 18 & 288.000 & 288.000 & 1.0000 & $0.1448 \mathrm{E}+08$ \\
\hline 19 & $288.00 \odot$ & $288 . \odot \odot \odot$ & 1.0000 & $0.1448 \mathrm{E}+08$ \\
\hline 20 & 288.000 & $288.00 \odot$ & $1.000 \odot$ & $0.1448 \mathrm{E}+08$ \\
\hline 21 & 288.000 & $288.00 \odot$ & 1.0000 & $0.1448 \mathrm{E}+08$ \\
\hline 22 & $288.00 \odot$ & $288.0 \odot \odot$ & $1.000 \odot$ & $\odot .1448 \mathrm{E}+08$ \\
\hline 23 & 288.000 & $288.00 \odot$ & 1.0000 & $0.1448 \mathrm{E}+08$ \\
\hline 24 & 288.000 & 288.000 & 1.0000 & $\odot .1448 E+\odot 8$ \\
\hline 25 & 288.000 & 88.000 & $1.000 \odot$ & $\odot .1448 \mathrm{E}+\odot 8$ \\
\hline
\end{tabular}




\begin{tabular}{|c|c|c|c|c|}
\hline 26 & $288.00 \odot$ & 288.000 & 1.0000 & $\odot .1448 \mathrm{E}+08$ \\
\hline 27 & 288.000 & $288 . \odot \odot \odot$ & $1.000 \odot$ & $\odot .1448 \mathrm{E}+\odot 8$ \\
\hline 28 & $288.00 \odot$ & 288.000 & 1.0000 & $\odot .1448 E+08$ \\
\hline 29 & $288.00 \odot$ & $288.0 \odot \odot$ & 1.0000 & $\odot .1448 \mathrm{E}+08$ \\
\hline 30 & $288.00 \odot$ & $288.0 \odot \odot$ & $1.000 \odot$ & $\odot .1448 \mathrm{E}+08$ \\
\hline 31 & $288.00 \odot$ & $288.00 \odot$ & 1.0000 & $\odot .1448 \mathrm{E}+08$ \\
\hline 32 & $288 . \odot \odot \odot$ & $288 . \odot \odot \odot$ & $1.0 \odot \odot \odot$ & $\odot .1448 \mathrm{E}+\odot 8$ \\
\hline 33 & $288.00 \odot$ & $288.0 \odot \odot$ & 1.0000 & $\odot .1448 \mathrm{E}+08$ \\
\hline 34 & $288 . \odot \odot \odot$ & $288 . \odot \odot \odot$ & $1.000 \odot$ & $\odot .1448 \mathrm{E}+\odot 8$ \\
\hline 35 & $288.00 \odot$ & $288.00 \odot$ & 1.0000 & $\odot .1448 \mathrm{E}+08$ \\
\hline 36 & $288.00 \odot$ & $288.00 \odot$ & 1.0000 & $0.1448 \mathrm{E}+08$ \\
\hline 37 & $288 . \odot \odot \odot$ & $288 . \odot \odot \odot$ & 1.0000 & $\odot .1448 \mathrm{E}+\odot 8$ \\
\hline 38 & $288 . \odot \odot \odot$ & $288 . \odot \odot \odot$ & $1.000 \odot$ & $\odot .1448 \mathrm{E}+\odot 8$ \\
\hline 39 & 288.000 & $288.0 \odot \odot$ & 1.0000 & $0.1448 \mathrm{E}+08$ \\
\hline 40 & $288 . \odot \odot \odot$ & $288.0 \odot \odot$ & 1.0000 & $0.1448 \mathrm{E}+08$ \\
\hline 41 & 288.000 & 288.000 & 1.0000 & $\odot .1448 E+08$ \\
\hline 42 & $288.00 \odot$ & $288.00 \odot$ & 1.0000 & $\odot .1448 \mathrm{E}+08$ \\
\hline 43 & $288.00 \odot$ & 288.000 & $1.000 \odot$ & $\odot .1448 \mathrm{E}+\odot 8$ \\
\hline 44 & $288 . \odot \odot \odot$ & $288 . \odot \odot \odot$ & $1.000 \odot$ & $\odot .1448 \mathrm{E}+\odot 8$ \\
\hline 45 & $288 . \odot \odot \odot$ & $288 . \odot \odot \odot$ & $1.0 \odot \odot \odot$ & $\odot .1448 \mathrm{E}+08$ \\
\hline 46 & $288.00 \odot$ & $288.0 \odot \odot$ & 1.0000 & $0.1448 \mathrm{E}+08$ \\
\hline 47 & $288.00 \odot$ & $288.0 \odot \odot$ & 1.0000 & $0.1448 \mathrm{E}+08$ \\
\hline 48 & 288.000 & $288.0 \odot \odot$ & 1.0000 & $\odot .1448 \mathrm{E}+08$ \\
\hline 49 & $288.00 \odot$ & $288.00 \odot$ & 1.0000 & $0.1448 \mathrm{E}+08$ \\
\hline 50 & $288.00 \odot$ & $288.0 \odot \odot$ & 1.0000 & $\odot .1448 \mathrm{E}+\odot 8$ \\
\hline 51 & $288 . \odot \odot \odot$ & $288 . \odot \odot \odot$ & $1.000 \odot$ & $\odot .1448 \mathrm{E}+\odot 8$ \\
\hline 52 & $288.00 \odot$ & $288.00 \odot$ & 1.0000 & $\odot .1448 \mathrm{E}+08$ \\
\hline 53 & $288.00 \odot$ & $288.0 \odot \odot$ & 1.0000 & $\odot .1448 \mathrm{E}+\odot 8$ \\
\hline 54 & $288.0 \odot \odot$ & $288 . \odot \odot \odot$ & $1.000 \odot$ & $\odot .1448 \mathrm{E}+\odot 8$ \\
\hline 55 & 288. & $288 . \odot \odot \odot$ & $1.0 \odot \odot \odot$ & $\odot .1448 \mathrm{E}+\odot 8$ \\
\hline 56 & $288.00 \odot$ & $288.00 \odot$ & 1.0000 & $\odot .1448 \mathrm{E}+08$ \\
\hline 57 & $288.00 \odot$ & $288.0 \odot \odot$ & 1.0000 & $\odot .1448 \mathrm{E}+\odot 8$ \\
\hline 58 & 288.000 & $288.0 \odot \odot$ & 1.0000 & $0.1448 \mathrm{E}+08$ \\
\hline 59 & $288.00 \odot$ & $288.0 \odot \odot$ & 1.0000 & $0.1448 \mathrm{E}+08$ \\
\hline 60 & $288.00 \odot$ & $288.0 \odot \odot$ & 1.0000 & $0.1448 \mathrm{E}+08$ \\
\hline tim & ec) $=$ & & $\Theta$. & 38320762 \\
\hline node & $\mathrm{T}(\mathrm{K})$ & Twall(K) & $x$ & $\mathrm{P}(\mathrm{Pa})$ \\
\hline 1 & 149.090 & 180.218 & - - - - - & $\odot .1422 \mathrm{E}+07$ \\
\hline 2 & 172.944 & 222.299 & ๑. 8162 & $\odot .2623 \mathrm{E}+07$ \\
\hline 3 & 228.393 & 260.799 & 1.0000 & $\odot .9488 \mathrm{E}+07$ \\
\hline 4 & $24 \odot .073$ & 276.565 & $1.00 \odot \odot$ & $\odot .1072 \mathrm{E}+\odot 8$ \\
\hline 5 & 262.812 & 285.625 & 1.0000 & $\odot .1212 \mathrm{E}+\odot 8$ \\
\hline 6 & 281.663 & 287.974 & 1.0000 & $\odot .1357 \mathrm{E}+08$ \\
\hline 7 & 287.425 & $288 . \odot \odot \odot$ & $1.000 \odot$ & $\odot .1430 E+\odot 8$ \\
\hline 8 & $288.00 \odot$ & 288.0 & 1.00 & $0.1446 \mathrm{E}+08$ \\
\hline 9 & 288.0 & 288.0 & $1.000 \odot$ & $\odot .1448 \mathrm{E}+08$ \\
\hline 10 & $288.0 \odot \odot$ & $288.0 \odot \odot$ & 1.0000 & $0.1448 \mathrm{E}+08$ \\
\hline 11 & $288 . \odot \odot \odot$ & $288 . \odot \odot \odot$ & $1.000 \odot$ & $\odot .1448 \mathrm{E}+\odot 8$ \\
\hline 12 & 288.000 & 288.000 & 1.0000 & $\odot .1448 E+08$ \\
\hline 13 & $288 . \odot \odot \odot$ & $288.0 \odot \odot$ & $1.000 \odot$ & $\odot .1448 \mathrm{E}+\odot 8$ \\
\hline 14 & $288 . \odot \odot \odot$ & $288 . \odot \odot \odot$ & $1.000 \odot$ & $\odot .1448 \mathrm{E}+\odot 8$ \\
\hline 15 & $288.00 \odot$ & $288.00 \odot$ & 1.0000 & $0.1448 \mathrm{E}+08$ \\
\hline 16 & $288.00 \odot$ & $288.0 \odot \odot$ & $1.000 \odot$ & $0.1448 E+08$ \\
\hline 17 & $288.00 \odot$ & $288.0 \odot \odot$ & 1.0000 & $0.1448 \mathrm{E}+08$ \\
\hline 18 & 288.000 & 288.000 & 1.0000 & $\odot .1448 \mathrm{E}+08$ \\
\hline 19 & $288.00 \odot$ & $288.0 \odot \odot$ & 1.0000 & $0.1448 \mathrm{E}+08$ \\
\hline
\end{tabular}




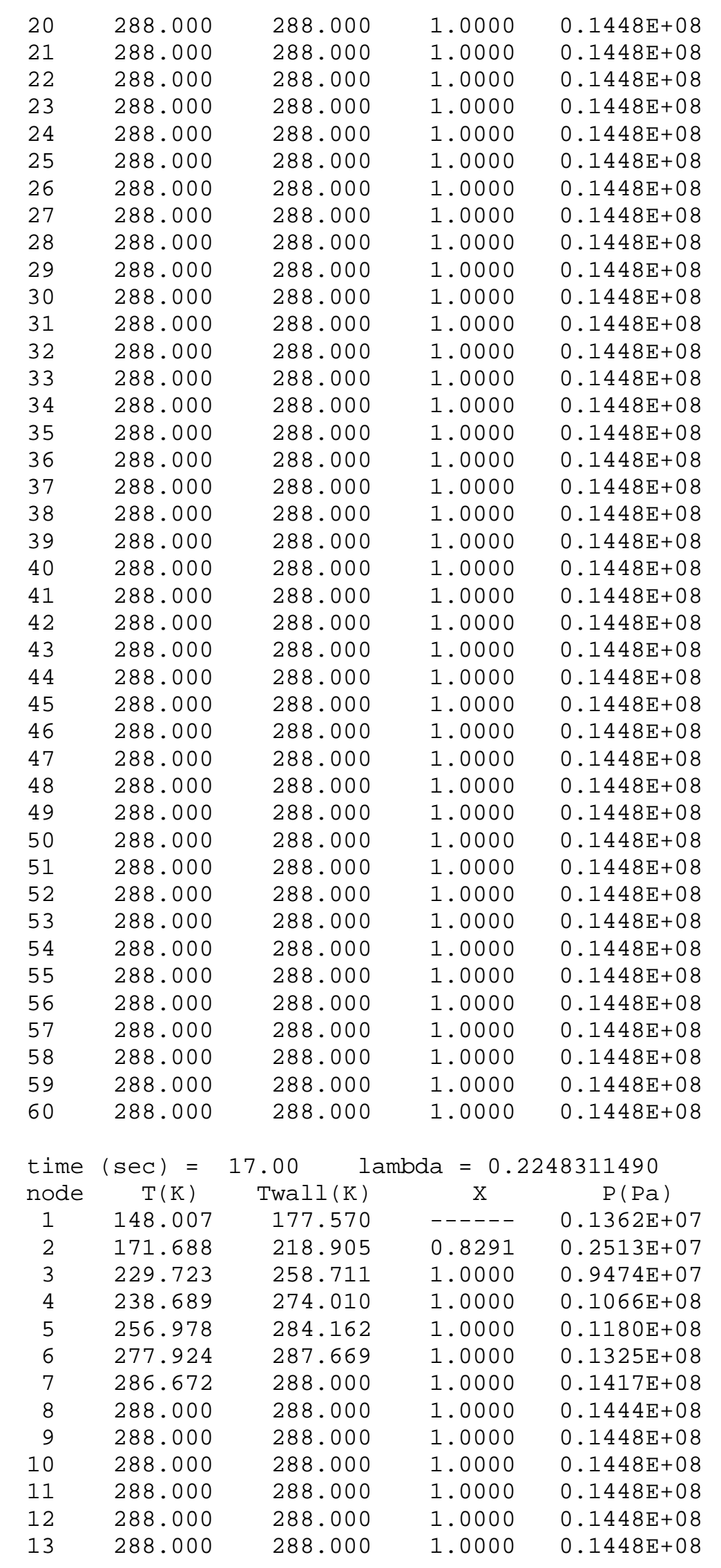




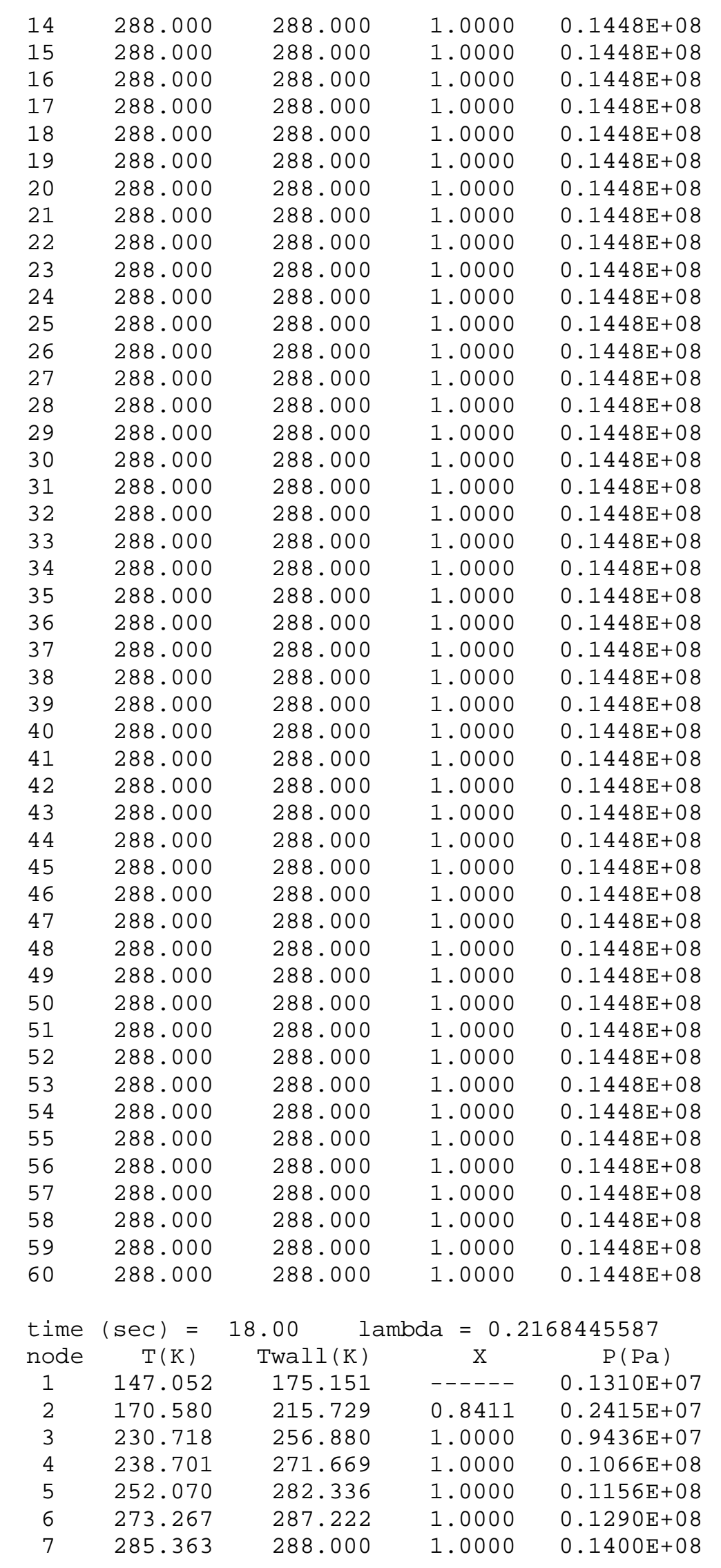




\begin{tabular}{|c|c|c|c|c|}
\hline 8 & 287.953 & 288.000 & $1.0 \odot \odot \odot$ & $\odot .1441 \mathrm{E}+\odot 8$ \\
\hline 9 & $288.0 \odot \odot$ & 288.000 & 1.0000 & $\odot .1448 \mathrm{E}+\odot 8$ \\
\hline 10 & $288 . \odot \odot \odot$ & $288.00 \odot$ & $1.000 \odot$ & $\odot .1448 \mathrm{E}+08$ \\
\hline 11 & $288 . \odot \odot \odot$ & $288.00 \odot$ & $1.00 \odot \odot$ & $\odot .1448 \mathrm{E}+\odot 8$ \\
\hline 12 & 288.000 & 288.000 & 1.0000 & $\odot .1448 \mathrm{E}+08$ \\
\hline 13 & $288.0 \odot \odot$ & $288.00 \odot$ & $1.00 \odot \odot$ & $\odot .1448 \mathrm{E}+\odot 8$ \\
\hline 14 & $288 . \odot \odot \odot$ & $288.00 \odot$ & $1.00 \odot \odot$ & $\odot .1448 \mathrm{E}+\odot 8$ \\
\hline 15 & $288.0 \odot \odot$ & 288.000 & 1.0000 & $\odot .1448 \mathrm{E}+08$ \\
\hline 16 & $288.0 \odot \odot$ & $288.00 \odot$ & $1.000 \odot$ & $\odot .1448 \mathrm{E}+\odot 8$ \\
\hline 17 & $288.00 \odot$ & 288.000 & 1.0000 & $\odot .1448 \mathrm{E}+08$ \\
\hline 18 & $288 . \odot \odot \odot$ & $288.00 \odot$ & $1.00 \odot \odot$ & $\odot .1448 \mathrm{E}+\odot 8$ \\
\hline 19 & $288.0 \odot \odot$ & 288.000 & $1.000 \odot$ & $\odot .1448 \mathrm{E}+\odot 8$ \\
\hline 20 & $288.0 \odot \odot$ & $288.00 \odot$ & $1.00 \odot \odot$ & $\odot .1448 \mathrm{E}+\odot 8$ \\
\hline 21 & 288.000 & 288.000 & 1.0000 & $\odot .1448 \mathrm{E}+\odot 8$ \\
\hline 22 & $288.00 \odot$ & 288.000 & $1.000 \odot$ & $\odot .1448 \mathrm{E}+\odot 8$ \\
\hline 23 & $288.0 \odot \odot$ & 288.000 & 1.0000 & $\odot .1448 \mathrm{E}+08$ \\
\hline 24 & $288.0 \odot \odot$ & $288.00 \odot$ & $1.00 \odot \odot$ & $\odot .1448 \mathrm{E}+\odot 8$ \\
\hline 25 & $288.0 \odot \odot$ & $288.00 \odot$ & 1.0000 & $\odot .1448 \mathrm{E}+\odot 8$ \\
\hline 26 & $288.00 \odot$ & $288.00 \odot$ & $1.000 \odot$ & $\odot .1448 \mathrm{E}+\odot 8$ \\
\hline 27 & $288.0 \odot \odot$ & $288.00 \odot$ & $1.00 \odot \odot$ & $\odot .1448 \mathrm{E}+\odot 8$ \\
\hline 28 & $288.0 \odot \odot$ & $288.00 \odot$ & 1.0000 & $\odot .1448 \mathrm{E}+08$ \\
\hline 29 & $288 . \odot \odot \odot$ & $288 . \odot \odot \odot$ & $1.000 \odot$ & $\odot .1448 \mathrm{E}+08$ \\
\hline 30 & $288.0 \odot \odot$ & 288.000 & $1.000 \odot$ & $\odot .1448 \mathrm{E}+\odot 8$ \\
\hline 31 & $288.00 \odot$ & $288.0 \odot \odot$ & $1.000 \odot$ & $\odot .1448 \mathrm{E}+\odot 8$ \\
\hline 32 & $288 . \odot \odot \odot$ & $288.0 \odot \odot$ & 1.0000 & $\odot .1448 \mathrm{E}+\odot 8$ \\
\hline 33 & $288.00 \odot$ & $288.0 \odot \odot$ & $1.000 \odot$ & $\odot .1448 \mathrm{E}+\odot 8$ \\
\hline 34 & $288 . \odot \odot \odot$ & $288 . \odot \odot \odot$ & $1.0 \odot \odot \odot$ & $\odot .1448 \mathrm{E}+\odot 8$ \\
\hline 35 & $288.0 \odot \odot$ & 288.000 & 1.0000 & $\odot .1448 \mathrm{E}+08$ \\
\hline 36 & $288.00 \odot$ & $288.00 \odot$ & $1.000 \odot$ & $\odot .1448 \mathrm{E}+\odot 8$ \\
\hline 37 & $288 . \odot \odot \odot$ & $288.00 \odot$ & $1.00 \odot \odot$ & $\odot .1448 \mathrm{E}+\odot 8$ \\
\hline 38 & $288.00 \odot$ & $288.00 \odot$ & 1.0000 & $0.1448 \mathrm{E}+08$ \\
\hline 39 & $288.0 \odot \odot$ & $288.0 \odot \odot$ & 1.0000 & $\odot .1448 \mathrm{E}+\odot 8$ \\
\hline 40 & $288.0 \odot \odot$ & $288.00 \odot$ & $1.00 \odot \odot$ & $\odot .1448 \mathrm{E}+\odot 8$ \\
\hline 41 & $288.0 \odot \odot$ & $288.00 \odot$ & 1. $\odot \odot \odot \odot$ & $\odot .1448 \mathrm{E}+\odot 8$ \\
\hline 42 & $288 . \odot \odot \odot$ & $288.00 \odot$ & $1.0 \odot \odot \odot$ & $\odot .1448 \mathrm{E}+\odot 8$ \\
\hline 43 & $288.0 \odot \odot$ & $288 . \odot \odot \odot$ & $1.000 \odot$ & $\odot .1448 \mathrm{E}+\odot 8$ \\
\hline 44 & $288 . \odot \odot \odot$ & $288 . \odot \odot \odot$ & $1.0 \odot \odot \odot$ & $\odot .1448 \mathrm{E}+\odot 8$ \\
\hline 45 & $288.0 \odot \odot$ & $288 . \odot \odot \odot$ & $1.0 \odot \odot \odot$ & $\odot .1448 \mathrm{E}+\odot 8$ \\
\hline 46 & $288.00 \odot$ & $288.00 \odot$ & $1.000 \odot$ & $\odot .1448 \mathrm{E}+08$ \\
\hline 47 & $288.00 \odot$ & $288.00 \odot$ & $1.00 \odot \odot$ & $\odot .1448 \mathrm{E}+\odot 8$ \\
\hline 48 & $288 . \odot \odot \odot$ & $288 . \odot \odot \odot$ & $1.0 \odot \odot \odot$ & $\odot .1448 \mathrm{E}+\odot 8$ \\
\hline 49 & $288.00 \odot$ & $288.00 \odot$ & $1.000 \odot$ & $\odot .1448 \mathrm{E}+\odot 8$ \\
\hline 50 & $288.00 \odot$ & $288.0 \odot \odot$ & $1.00 \odot \odot$ & $\odot .1448 \mathrm{E}+\odot 8$ \\
\hline 51 & $288.0 \odot \odot$ & $288.00 \odot$ & $1.0 \odot \odot \odot$ & $\odot .1448 \mathrm{E}+\odot 8$ \\
\hline 52 & $288.00 \odot$ & 288.000 & 1.0000 & $\odot .1448 E+08$ \\
\hline 53 & $288.00 \odot$ & $288.00 \odot$ & $1.00 \odot \odot$ & $\odot .1448 \mathrm{E}+\odot 8$ \\
\hline 54 & $288.00 \odot$ & $288.00 \odot$ & $1.000 \odot$ & $\odot .1448 \mathrm{E}+\odot 8$ \\
\hline 55 & 288.000 & 288.000 & 1.0000 & $\odot .1448 \mathrm{E}+\odot 8$ \\
\hline 56 & $288.00 \odot$ & $288.00 \odot$ & $1.000 \odot$ & $\odot .1448 \mathrm{E}+\odot 8$ \\
\hline 57 & $288.0 \odot \odot$ & $288.00 \odot$ & $1.00 \odot \odot$ & $\odot .1448 \mathrm{E}+08$ \\
\hline 58 & 288.000 & 288.000 & 1.0000 & $\odot .1448 \mathrm{E}+08$ \\
\hline 59 & $288.0 \odot \odot$ & 288.000 & $1.000 \odot$ & $\odot .1448 \mathrm{E}+\odot 8$ \\
\hline $6 \odot$ & $288.0 \odot \odot$ & $288.00 \odot$ & $1.00 \odot \odot$ & $\odot .1448 \mathrm{E}+\odot 8$ \\
\hline & & & $\mathrm{la}=0$. & 96893489 \\
\hline node & $\mathrm{T}(\mathrm{K})$ & Twall(K) & $x$ & $P(P a)$ \\
\hline 1 & 146.201 & 172.909 & & $\odot .1263 \mathrm{E}+\odot 7$ \\
\hline
\end{tabular}




\begin{tabular}{|c|c|c|c|c|}
\hline 2 & 169.593 & 212.745 & $\odot .8522$ & $\odot .2329 \mathrm{E}+07$ \\
\hline 3 & 231.051 & 255.314 & $1.000 \odot$ & $\odot .9370 \mathrm{E}+07$ \\
\hline 4 & 239.527 & 269.642 & 1.0000 & $\odot .1070 \mathrm{E}+\odot 8$ \\
\hline 5 & 248.677 & 280.249 & 1.0000 & $\odot .1140 \mathrm{E}+08$ \\
\hline 6 & 267.971 & 286.477 & 1.0000 & $\odot .1256 \mathrm{E}+\odot 8$ \\
\hline 7 & 283.272 & $288.00 \odot$ & 1.0000 & $\odot .1377 \mathrm{E}+08$ \\
\hline 8 & 287.648 & $288.0 \odot \odot$ & 1.0000 & $\odot .1435 \mathrm{E}+\odot 8$ \\
\hline 9 & 288.000 & $288.0 \odot \odot$ & 1.0000 & $\odot .1447 \mathrm{E}+08$ \\
\hline 10 & $288.00 \odot$ & $288.00 \odot$ & 1.0000 & $\odot .1448 \mathrm{E}+\odot 8$ \\
\hline 11 & 288.000 & 288.000 & 1.0000 & $\odot .1448 \mathrm{E}+08$ \\
\hline 12 & $288 . \odot \odot \odot$ & $288.0 \odot \odot$ & 1.0000 & $\odot .1448 \mathrm{E}+\odot 8$ \\
\hline 13 & $288.00 \odot$ & 288.000 & 1.0000 & $0.1448 \mathrm{E}+08$ \\
\hline 14 & $288.00 \odot$ & 288.000 & 1.0000 & $\odot .1448 \mathrm{E}+08$ \\
\hline 15 & $288.0 \odot \odot$ & 288.000 & 1.0000 & $\odot .1448 \mathrm{E}+\odot 8$ \\
\hline 16 & $288.00 \odot$ & 288.000 & 1.0000 & $\odot .1448 \mathrm{E}+\odot 8$ \\
\hline 17 & $288.0 \odot \odot$ & 288.000 & 1.0000 & $\odot .1448 \mathrm{E}+\odot 8$ \\
\hline 18 & $288 . \odot \odot \odot$ & $288.0 \odot \odot$ & 1.0000 & $\odot .1448 \mathrm{E}+08$ \\
\hline 19 & 288.000 & $288.00 \odot$ & 1.0000 & $\odot .1448 \mathrm{E}+\odot 8$ \\
\hline 20 & $288.0 \odot \odot$ & $288.00 \odot$ & 1.0000 & $\odot .1448 \mathrm{E}+08$ \\
\hline 21 & $288 . \odot \odot \odot$ & $288.00 \odot$ & $1.000 \odot$ & $\odot .1448 \mathrm{E}+\odot 8$ \\
\hline 22 & $288.00 \odot$ & 288.000 & 1.0000 & $\odot .1448 \mathrm{E}+\odot 8$ \\
\hline 23 & $288.00 \odot$ & $288.00 \odot$ & 1.0000 & $0.1448 \mathrm{E}+08$ \\
\hline 24 & $288.00 \odot$ & $288.00 \odot$ & 1.0000 & $0.1448 \mathrm{E}+08$ \\
\hline 25 & $288.0 \odot \odot$ & 288.000 & 1.0000 & $0.1448 \mathrm{E}+08$ \\
\hline 26 & $288.00 \odot$ & 288.000 & 1.0000 & $\odot .1448 \mathrm{E}+\odot 8$ \\
\hline 27 & $288.0 \odot \odot$ & $288.00 \odot$ & 1.0000 & $\odot .1448 \mathrm{E}+08$ \\
\hline 28 & 288.000 & 288.000 & 1.0000 & $\odot .1448 \mathrm{E}+08$ \\
\hline 29 & $288 . \odot \odot \odot$ & $288.00 \odot$ & 1.0000 & $\odot .1448 \mathrm{E}+08$ \\
\hline 30 & 288.000 & $288.0 \odot \odot$ & 1.0000 & $\odot .1448 \mathrm{E}+\odot 8$ \\
\hline 31 & 288.000 & 288.000 & 1.0000 & $\odot .1448 \mathrm{E}+08$ \\
\hline 32 & $288.0 \odot \odot$ & $288 . \odot \odot \odot$ & 1.0000 & $\odot .1448 E+08$ \\
\hline 33 & $288.00 \odot$ & 288.000 & 1.0000 & $\odot .1448 \mathrm{E}+\odot 8$ \\
\hline 34 & 288.000 & $288.0 \odot \odot$ & 1.0000 & $\odot .1448 \mathrm{E}+\odot 8$ \\
\hline 35 & $288.0 \odot \odot$ & $288.0 \odot \odot$ & $1.000 \odot$ & $\odot .1448 \mathrm{E}+08$ \\
\hline 36 & $288 . \odot \odot \odot$ & $288 . \odot \odot \odot$ & 1.0000 & $\odot .1448 \mathrm{E}+08$ \\
\hline 37 & $288.00 \odot$ & 288.000 & 1.0000 & $\odot .1448 \mathrm{E}+08$ \\
\hline 38 & 288.000 & 288.000 & 1.0000 & $\odot .1448 \mathrm{E}+\odot 8$ \\
\hline 39 & $288.0 \odot \odot$ & 288.000 & 1.0000 & $\odot .1448 \mathrm{E}+\odot 8$ \\
\hline 40 & 288.000 & $288.00 \odot$ & 1.0000 & $\odot .1448 \mathrm{E}+\odot 8$ \\
\hline 41 & $288 . \odot \odot \odot$ & $288 . \odot \odot \odot$ & 1.0000 & $\odot .1448 \mathrm{E}+08$ \\
\hline 42 & 288.000 & $288.0 \odot \odot$ & 1.0000 & $\odot .1448 \mathrm{E}+08$ \\
\hline 43 & $288.00 \odot$ & $288.00 \odot$ & 1.0000 & $\odot .1448 \mathrm{E}+08$ \\
\hline 44 & $288 . \odot \odot \odot$ & $288 . \odot \odot \odot$ & $1.000 \odot$ & $\odot .1448 \mathrm{E}+\odot 8$ \\
\hline 45 & 288.000 & $288.0 \odot \odot$ & 1.0000 & $\odot .1448 \mathrm{E}+08$ \\
\hline 46 & $288.00 \odot$ & $288.0 \odot \odot$ & 1.0000 & $0.1448 \mathrm{E}+08$ \\
\hline 47 & 288.000 & $288 . \odot \odot \odot$ & 1.0000 & $0.1448 \mathrm{E}+08$ \\
\hline 48 & 288.000 & $288.0 \odot \odot$ & 1.0000 & $\odot .1448 \mathrm{E}+\odot 8$ \\
\hline 49 & $288.00 \odot$ & $288.00 \odot$ & 1.0000 & $0.1448 \mathrm{E}+08$ \\
\hline 50 & $288.0 \odot \odot$ & $288 . \odot \odot \odot$ & 1.0000 & $\odot .1448 \mathrm{E}+\odot 8$ \\
\hline 51 & 288.000 & 288.000 & 1.0000 & $\odot .1448 E+08$ \\
\hline 52 & $288 . \odot \odot \odot$ & $288 . \odot \odot \odot$ & $1.000 \odot$ & $0.1448 \mathrm{E}+08$ \\
\hline 53 & 288.000 & 288.000 & 1.0000 & $\odot .1448 \mathrm{E}+08$ \\
\hline 54 & $288.00 \odot$ & $288.00 \odot$ & 1.0000 & $\odot .1448 \mathrm{E}+08$ \\
\hline 55 & 288.000 & 288.000 & 1.0000 & $\odot .1448 E+08$ \\
\hline 56 & $288.00 \odot$ & $288.0 \odot \odot$ & 1.0000 & $\odot .1448 \mathrm{E}+\odot 8$ \\
\hline 57 & 288.000 & $288.0 \odot \odot$ & 1.0000 & $\odot .1448 \mathrm{E}+\odot 8$ \\
\hline 58 & 288.000 & $288.0 \odot \odot$ & 1.0000 & $\odot .1448 E+08$ \\
\hline
\end{tabular}




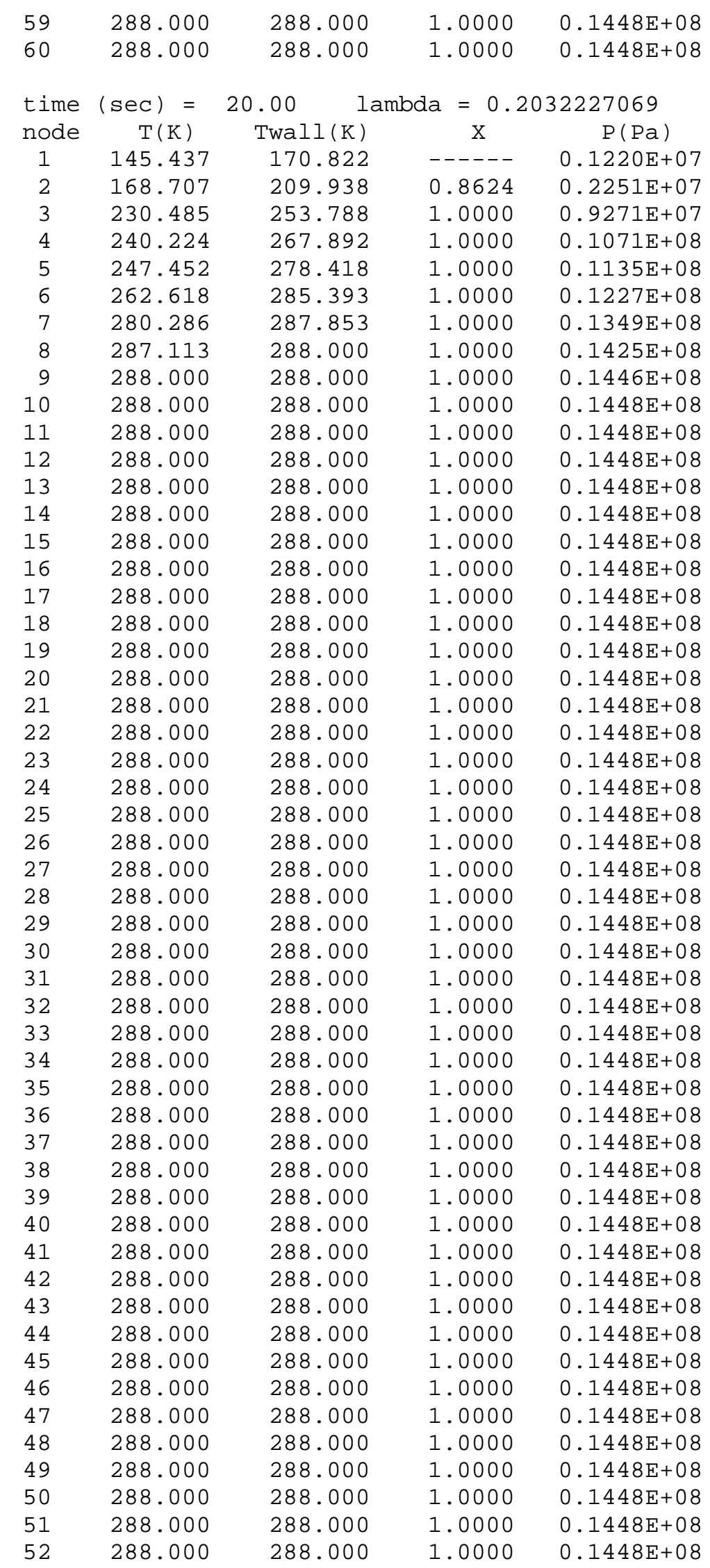




\begin{tabular}{|c|c|c|c|c|}
\hline 53 & $288.00 \odot$ & $288.00 \odot$ & 1.0000 & $0.1448 \mathrm{E}+08$ \\
\hline 54 & 288.000 & 288.000 & 1.0000 & $\odot .1448 \mathrm{E}+08$ \\
\hline 55 & $288 . \odot \odot \odot$ & $288 . \odot \odot \odot$ & $1.000 \odot$ & $\odot .1448 \mathrm{E}+\odot 8$ \\
\hline 56 & $288.0 \odot \odot$ & 288.000 & 1.0000 & $\odot .1448 \mathrm{E}+08$ \\
\hline 57 & $288 . \odot \odot \odot$ & $288.00 \odot$ & $1.00 \odot \odot$ & $\odot .1448 \mathrm{E}+08$ \\
\hline 58 & $288.00 \odot$ & $288.00 \odot$ & 1.0000 & $\odot .1448 \mathrm{E}+08$ \\
\hline 59 & $288 . \odot \odot \odot$ & $288.0 \odot \odot$ & $1.000 \odot$ & $\odot .1448 \mathrm{E}+08$ \\
\hline 60 & $288 . \odot \odot \odot$ & $288.00 \odot$ & $1.00 \odot \odot$ & $\odot .1448 \mathrm{E}+\odot 8$ \\
\hline
\end{tabular}

\begin{tabular}{|c|c|c|c|c|}
\hline $\begin{array}{l}\text { time } \\
\text { node }\end{array}$ & $\begin{array}{l}(\mathrm{sec})= \\
T(\mathrm{~K})\end{array}$ & $\begin{array}{l}21.00 \text { la } \\
\text { Twall(K) }\end{array}$ & $x=0$. & $\begin{array}{l}-92851 \\
P(P a)\end{array}$ \\
\hline $\begin{array}{l}\text { node } \\
1\end{array}$ & 144.740 & 168.871 & $\ldots$ & $\odot .1182 \mathrm{E}+07$ \\
\hline 2 & 167.898 & 207.298 & $\odot .8718$ & $\odot .2180 \mathrm{E}+\odot 7$ \\
\hline 3 & 229.044 & 252.262 & 1.0000 & $\odot .9147 \mathrm{E}+\odot 7$ \\
\hline 4 & 240.834 & 266.366 & 1.0000 & $\odot .1070 \mathrm{E}+\odot 8$ \\
\hline 5 & 247.740 & 276.657 & 1.0000 & $\odot .1136 \mathrm{E}+\odot 8$ \\
\hline 6 & 257.910 & 283.987 & 1.0000 & $\odot .1205 \mathrm{E}+08$ \\
\hline 7 & 276.376 & 287.548 & $1.000 \odot$ & $\odot .1319 \mathrm{E}+08$ \\
\hline 8 & 286.114 & $288.0 \odot \odot$ & 1.0000 & $\odot .1411 \mathrm{E}+\odot 8$ \\
\hline 9 & 288.000 & 288.000 & 1.0000 & $\odot .1443 \mathrm{E}+08$ \\
\hline 10 & 288.000 & $288.00 \odot$ & 1.0000 & $\odot .1448 E+\odot 8$ \\
\hline 11 & 288.000 & $288.0 \odot \odot$ & 1.0000 & $\odot .1448 \mathrm{E}+\odot 8$ \\
\hline 12 & $288.00 \odot$ & $288.00 \odot$ & $1.000 \odot$ & $\odot .1448 \mathrm{E}+08$ \\
\hline 13 & 288.000 & 288.000 & 1.0000 & $\odot .1448 \mathrm{E}+08$ \\
\hline 14 & 288.000 & 288.000 & 1.0000 & $\odot .1448 \mathrm{E}+\odot 8$ \\
\hline 15 & $288.00 \odot$ & $288.00 \odot$ & $1.000 \odot$ & $\odot .1448 \mathrm{E}+08$ \\
\hline 16 & $288.00 \odot$ & $288 . \odot \odot \odot$ & 1.0000 & $\odot .1448 \mathrm{E}+\odot 8$ \\
\hline 17 & 288.000 & $288.00 \odot$ & 1.0000 & $\odot .1448 \mathrm{E}+\odot 8$ \\
\hline 18 & 288.000 & $288.00 \odot$ & $1.000 \odot$ & $\odot .1448 \mathrm{E}+08$ \\
\hline 19 & 288.000 & $288.00 \odot$ & 1.0000 & $\odot .1448 \mathrm{E}+\odot 8$ \\
\hline 20 & 288.000 & $288.00 \odot$ & 1.0000 & $\odot .1448 \mathrm{E}+08$ \\
\hline 21 & $288.00 \odot$ & $288 . \odot \odot \odot$ & $1.000 \odot$ & $\odot .1448 \mathrm{E}+08$ \\
\hline 22 & $288.00 \odot$ & $288 . \odot \odot \odot$ & 1.0000 & $\odot .1448 \mathrm{E}+\odot 8$ \\
\hline 23 & 288.000 & $288.0 \odot \odot$ & 1.0000 & $\odot .1448 \mathrm{E}+\odot 8$ \\
\hline 24 & $288.0 \odot \odot$ & $288 . \odot \odot \odot$ & $1.000 \odot$ & $\odot .1448 \mathrm{E}+08$ \\
\hline 25 & 288.000 & 288.000 & 1.0000 & $\odot .1448 \mathrm{E}+08$ \\
\hline 26 & 288.000 & $288.00 \odot$ & 1.0000 & $\odot .1448 \mathrm{E}+\odot 8$ \\
\hline 27 & 288.000 & $288 . \odot \odot \odot$ & 1.0000 & $\odot .1448 E+08$ \\
\hline 28 & 288.000 & $288.0 \odot \odot$ & 1.0000 & $\odot .1448 \mathrm{E}+\odot 8$ \\
\hline 29 & 288.000 & $288.00 \odot$ & 1.0000 & $\odot .1448 \mathrm{E}+\odot 8$ \\
\hline 30 & $288.0 \odot \odot$ & $288 . \odot \odot \odot$ & $1.000 \odot$ & $\odot .1448 \mathrm{E}+\odot 8$ \\
\hline 31 & 288.000 & $288.00 \odot$ & 1.0000 & $0.1448 \mathrm{E}+08$ \\
\hline 32 & 288.000 & 288.000 & 1.0000 & $\odot .1448 \mathrm{E}+08$ \\
\hline 33 & 288.000 & $288.00 \odot$ & 1.0000 & $\odot .1448 \mathrm{E}+\odot 8$ \\
\hline 34 & 288.000 & $288.0 \odot \odot$ & 1.0000 & $\odot .1448 \mathrm{E}+\odot 8$ \\
\hline 35 & 288.000 & $288.00 \odot$ & 1.0000 & $\odot .1448 \mathrm{E}+08$ \\
\hline 36 & $288.00 \odot$ & $288 . \odot \odot \odot$ & $1.000 \odot$ & $\odot .1448 \mathrm{E}+08$ \\
\hline 37 & 288.000 & 288.000 & 1.0000 & $0.1448 \mathrm{E}+08$ \\
\hline 38 & 288.000 & $288.00 \odot$ & $1.000 \odot$ & $\odot .1448 \mathrm{E}+08$ \\
\hline 39 & $288.00 \odot$ & $288 . \odot \odot \odot$ & 1.0000 & $\odot .1448 \mathrm{E}+\odot 8$ \\
\hline 40 & 288.000 & $288.00 \odot$ & 1.0000 & $0.1448 \mathrm{E}+08$ \\
\hline 41 & $288.00 \odot$ & 288.000 & 1.0000 & $\odot .1448 \mathrm{E}+08$ \\
\hline 42 & $288.00 \odot$ & $288 . \odot \odot \odot$ & 1.0000 & $\odot .1448 \mathrm{E}+08$ \\
\hline 43 & $288.00 \odot$ & $288.0 \odot \odot$ & $1.000 \odot$ & $\odot .1448 \mathrm{E}+08$ \\
\hline 44 & $288.00 \odot$ & $288 . \odot \odot \odot$ & 1.0000 & $\odot .1448 \mathrm{E}+08$ \\
\hline 45 & $288.00 \odot$ & $288.00 \odot$ & 1.0000 & $\odot .1448 \mathrm{E}+\odot 8$ \\
\hline 46 & 288.000 & $288.0 \odot \odot$ & 1.0000 & $\odot .1448 \mathrm{E}+\odot 8$ \\
\hline
\end{tabular}




\begin{tabular}{|c|c|c|c|c|}
\hline 47 & $288 . \odot \odot \odot$ & $288.0 \odot \odot$ & $1.00 \odot \odot$ & $\odot .1448 \mathrm{E}+08$ \\
\hline 48 & $288.0 \odot \odot$ & $288.00 \odot$ & 1.0000 & $\odot .1448 E+08$ \\
\hline 49 & $288.0 \odot \odot$ & $288.00 \odot$ & 1.0000 & $\odot .1448 \mathrm{E}+\odot 8$ \\
\hline 50 & $288.00 \odot$ & $288.00 \odot$ & 1.0000 & $\odot .1448 \mathrm{E}+\odot 8$ \\
\hline 51 & 288.000 & $288.0 \odot \odot$ & 1.0000 & $\odot .1448 \mathrm{E}+08$ \\
\hline 52 & $288 . \odot \odot \odot$ & $288 . \odot \odot \odot$ & $1.000 \odot$ & $\odot .1448 \mathrm{E}+08$ \\
\hline 53 & 288.000 & 288.000 & 1.0000 & $\odot .1448 E+08$ \\
\hline 54 & $288.00 \odot$ & $288.00 \odot$ & 1.0000 & $\odot .1448 \mathrm{E}+\odot 8$ \\
\hline 55 & $288.00 \odot$ & $288.0 \odot \odot$ & 1.0000 & $\odot .1448 \mathrm{E}+\odot 8$ \\
\hline 56 & $288.00 \odot$ & $288 . \odot \odot \odot$ & $1.000 \odot$ & $\odot .1448 \mathrm{E}+08$ \\
\hline 57 & 288.000 & 288.000 & 1.0000 & $\odot .1448 \mathrm{E}+08$ \\
\hline 58 & $288 . \odot \odot \odot$ & $288 . \odot \odot \odot$ & $1.000 \odot$ & $\odot .1448 \mathrm{E}+\odot 8$ \\
\hline 59 & $288.00 \odot$ & 288.000 & 1.0000 & $\odot .1448 \mathrm{E}+\odot 8$ \\
\hline 60 & $288.0 \odot \odot$ & $288 . \odot \odot \odot$ & 1.0000 & $\odot .1448 \mathrm{E}+\odot 8$ \\
\hline
\end{tabular}

\begin{tabular}{|c|c|c|c|c|}
\hline $\begin{array}{l}\text { time } \\
\text { node }\end{array}$ & $\begin{array}{c}(\mathrm{sec})= \\
T(K)\end{array}$ & $\begin{array}{l}2.00 \text { la } \\
\text { Twall(K) }\end{array}$ & $={ }_{x}^{0}$. & $\begin{array}{l}44822 \\
\mathrm{P}(\mathrm{Pa})\end{array}$ \\
\hline 1 & 144.097 & 167.040 & - _ _ & $0.1147 \mathrm{E}+07$ \\
\hline 2 & 167.153 & 204.813 & $\odot .8804$ & $\odot .2115 \mathrm{E}+\odot 7$ \\
\hline 3 & 226.929 & 250.734 & $1.000 \odot$ & $\odot .90 \odot 7 \mathrm{E}+\odot 7$ \\
\hline 4 & 241.387 & 265.051 & $1.00 \odot \odot$ & $\odot .1067 \mathrm{E}+\odot 8$ \\
\hline 5 & 248.194 & 275.131 & $1.000 \odot$ & $\odot .1138 \mathrm{E}+\odot 8$ \\
\hline 6 & 254.953 & 282.461 & 1.0000 & $\odot .1191 \mathrm{E}+08$ \\
\hline 7 & 271.756 & 287.008 & 1.0000 & $\odot .1289 \mathrm{E}+\odot 8$ \\
\hline 8 & 284.511 & $288.0 \odot \odot$ & 1.0000 & $\odot .1392 \mathrm{E}+\odot 8$ \\
\hline 9 & 287.801 & $288 . \odot \odot \odot$ & 1.0000 & $\odot .1438 \mathrm{E}+08$ \\
\hline 10 & 288.000 & 288.000 & 1.0000 & $\odot .1447 \mathrm{E}+08$ \\
\hline 11 & 288.000 & $288.00 \odot$ & 1.0000 & $\odot .1448 \mathrm{E}+\odot 8$ \\
\hline 12 & $288.00 \odot$ & $288.00 \odot$ & 1.0000 & $\odot .1448 \mathrm{E}+08$ \\
\hline 13 & 288.000 & $288.00 \odot$ & 1.0000 & $\odot .1448 \mathrm{E}+08$ \\
\hline 14 & 288.000 & 288.000 & 1.0000 & $\odot .1448 \mathrm{E}+\odot 8$ \\
\hline 15 & 288.000 & 288.000 & 1.0000 & $\odot .1448 \mathrm{E}+\odot 8$ \\
\hline 16 & $288.00 \odot$ & $288 . \odot \odot \odot$ & 1.0000 & $\odot .1448 \mathrm{E}+\odot 8$ \\
\hline 17 & 288.000 & 288.000 & 1.0000 & $\odot .1448 \mathrm{E}+08$ \\
\hline 18 & 288.000 & 288.000 & 1.0000 & $\odot .1448 \mathrm{E}+\odot 8$ \\
\hline 19 & 288.000 & 288.000 & $1.000 \odot$ & $\odot .1448 \mathrm{E}+\odot 8$ \\
\hline 20 & 288.000 & 288.000 & 1.0000 & $\odot .1448 \mathrm{E}+08$ \\
\hline 21 & 288.000 & 288.000 & 1.0000 & $\odot .1448 \mathrm{E}+\odot 8$ \\
\hline 22 & $288.00 \odot$ & $288 . \odot \odot \odot$ & 1.0000 & $\odot .1448 \mathrm{E}+08$ \\
\hline 23 & 288.000 & 288.000 & 1.0000 & $\odot .1448 \mathrm{E}+\odot 8$ \\
\hline 24 & $288.00 \odot$ & $288 . \odot \odot \odot$ & 1.0000 & $\odot .1448 \mathrm{E}+08$ \\
\hline 25 & $288.00 \odot$ & $288 . \odot \odot \odot$ & 1.0000 & $\odot .1448 \mathrm{E}+\odot 8$ \\
\hline 26 & 288.000 & $288.0 \odot \odot$ & 1.0000 & $\odot .1448 \mathrm{E}+08$ \\
\hline 27 & 288.000 & 288.000 & 1.0000 & $\odot .1448 \mathrm{E}+08$ \\
\hline 28 & 288.000 & $288.00 \odot$ & $1.000 \odot$ & $\odot .1448 \mathrm{E}+\odot 8$ \\
\hline 29 & 288.000 & $288.0 \odot \odot$ & 1.0000 & $\odot .1448 \mathrm{E}+08$ \\
\hline 30 & 288.000 & 288.000 & 1.0000 & $\odot .1448 \mathrm{E}+\odot 8$ \\
\hline 31 & 288.000 & 288.000 & 1.0000 & $\odot .1448 \mathrm{E}+\odot 8$ \\
\hline 32 & 288.000 & 288.000 & 1.0000 & $\odot .1448 \mathrm{E}+\odot 8$ \\
\hline 33 & $288.0 \odot \odot$ & $288 . \odot \odot \odot$ & 1.0000 & $\odot .1448 \mathrm{E}+\odot 8$ \\
\hline 34 & 288.000 & $288.00 \odot$ & 1.0000 & $\odot .1448 \mathrm{E}+\odot 8$ \\
\hline 35 & $288.00 \odot$ & $288.00 \odot$ & 1.0000 & $\odot .1448 \mathrm{E}+08$ \\
\hline 36 & 288.000 & $288.0 \odot \odot$ & 1.0000 & $\odot .1448 \mathrm{E}+08$ \\
\hline 37 & 288.000 & 288.000 & 1.0000 & $\odot .1448 \mathrm{E}+\odot 8$ \\
\hline 38 & 288.000 & $288.00 \odot$ & 1. $\odot \odot \odot \odot$ & $\odot .1448 E+\odot 8$ \\
\hline 39 & $288.00 \odot$ & $288 . \odot \odot \odot$ & 1.0000 & $\odot .1448 \mathrm{E}+\odot 8$ \\
\hline 40 & 288.000 & $288.00 \odot$ & 1.0000 & $\odot .1448 \mathrm{E}+08$ \\
\hline
\end{tabular}




\begin{tabular}{|c|c|c|c|c|}
\hline 41 & $288.00 \odot$ & 288.000 & 1.0000 & $\odot .1448 \mathrm{E}+08$ \\
\hline 42 & $288 . \odot \odot \odot$ & $288.00 \odot$ & $1 . \odot \odot \odot \odot$ & $\odot .1448 \mathrm{E}+\odot 8$ \\
\hline 43 & $288.00 \odot$ & $288 . \odot \odot \odot$ & 1.0000 & $\odot .1448 E+08$ \\
\hline 44 & $288.00 \odot$ & 288.000 & 1.0000 & $\odot .1448 \mathrm{E}+08$ \\
\hline 45 & $288.00 \odot$ & 288.000 & $1.000 \odot$ & $\odot .1448 \mathrm{E}+08$ \\
\hline 46 & $288.00 \odot$ & $288.0 \odot \odot$ & 1.0000 & $\odot .1448 \mathrm{E}+08$ \\
\hline 47 & 288.000 & 288.000 & 1.0000 & $\odot .1448 \mathrm{E}+08$ \\
\hline 48 & $288.00 \odot$ & $288.00 \odot$ & 1.0000 & $\odot .1448 \mathrm{E}+08$ \\
\hline 49 & $288 . \odot \odot \odot$ & $288 . \odot \odot \odot$ & $1.000 \odot$ & $\odot .1448 \mathrm{E}+\odot 8$ \\
\hline 50 & 288.000 & $288.00 \odot$ & 1.0000 & $\odot .1448 \mathrm{E}+08$ \\
\hline 51 & $288.00 \odot$ & 288.000 & 1.0000 & $0.1448 \mathrm{E}+08$ \\
\hline 52 & $288.00 \odot$ & $288.00 \odot$ & 1.0000 & $\odot .1448 \mathrm{E}+08$ \\
\hline 53 & $288 . \odot \odot \odot$ & $288.0 \odot \odot$ & $1.000 \odot$ & $\odot .1448 \mathrm{E}+\odot 8$ \\
\hline 54 & 288.000 & 288.000 & 1.0000 & $\odot .1448 E+08$ \\
\hline 55 & $288 . \odot \odot \odot$ & $288.00 \odot$ & 1.0000 & $\odot .1448 \mathrm{E}+\odot 8$ \\
\hline 56 & 288.000 & 288.000 & 1.0000 & $\odot .1448 E+08$ \\
\hline 57 & $288.00 \odot$ & $288.0 \odot \odot$ & 1.0000 & $\odot .1448 \mathrm{E}+08$ \\
\hline 58 & $288.00 \odot$ & $288.00 \odot$ & 1.0000 & $\odot .1448 \mathrm{E}+\odot 8$ \\
\hline 59 & $288 . \odot \odot \odot$ & $288 . \odot \odot \odot$ & $1.000 \odot$ & $0.1448 \mathrm{E}+\odot 8$ \\
\hline 60 & $288 . \odot \odot \odot$ & $288.00 \odot$ & $1.0 \odot \odot \odot$ & $\odot .1448 \mathrm{E}+08$ \\
\hline time & $(\sec )=$ & $23.00 \quad 1$ & $a=0$. & 67003143 \\
\hline node & $\mathrm{T}(\mathrm{K})$ & Twall(K) & $\mathrm{x}$ & $\mathrm{P}(\mathrm{Pa})$ \\
\hline 1 & 143.501 & 165.357 & - - - - - & $\odot .1114 \mathrm{E}+07$ \\
\hline 2 & 166.461 & 202.470 & $\odot .8882$ & $\odot .2054 \mathrm{E}+\odot 7$ \\
\hline 3 & 224.343 & 249.055 & $1.00 \odot \odot$ & $\odot .8862 \mathrm{E}+\odot 7$ \\
\hline 4 & 241.588 & 263.830 & 1.0000 & $\odot .1062 \mathrm{E}+08$ \\
\hline 5 & 248.499 & 273.750 & 1.0000 & $0.1139 \mathrm{E}+08$ \\
\hline 6 & 253.894 & 280.935 & 1.0000 & $0.1186 \mathrm{E}+08$ \\
\hline 7 & 266.873 & 286.198 & 1..$\odot \odot \odot$ & $\odot .1262 E+\odot 8$ \\
\hline 8 & 282.093 & 288.000 & 1.0000 & $0.1368 \mathrm{E}+08$ \\
\hline 9 & 287.425 & 288.000 & 1.0000 & $\odot .1431 \mathrm{E}+08$ \\
\hline 10 & 288.000 & $288.0 \odot \odot$ & 1.0000 & $0.1446 \mathrm{E}+08$ \\
\hline 11 & $288.00 \odot$ & 288.000 & 1.0000 & $0.1448 \mathrm{E}+08$ \\
\hline 12 & $288 . \odot \odot \odot$ & $288.00 \odot$ & $1.000 \odot$ & $\odot .1448 \mathrm{E}+08$ \\
\hline 13 & 288.000 & 288.000 & 1.0000 & $0.1448 \mathrm{E}+08$ \\
\hline 14 & $288.00 \odot$ & 288.000 & 1.0000 & $0.1448 \mathrm{E}+08$ \\
\hline 15 & $288.00 \odot$ & 288.000 & 1.0000 & $0.1448 \mathrm{E}+08$ \\
\hline 16 & $288.00 \odot$ & $288.00 \odot$ & 1.0000 & $\odot .1448 \mathrm{E}+08$ \\
\hline 17 & $288.00 \odot$ & 288.000 & 1.0000 & $\odot .1448 \mathrm{E}+08$ \\
\hline 18 & $288.00 \odot$ & $288.00 \odot$ & 1.0000 & $\odot .1448 \mathrm{E}+08$ \\
\hline 19 & $288 . \odot \odot \odot$ & $288 . \odot \odot \odot$ & $1.000 \odot$ & $\odot .1448 \mathrm{E}+\odot 8$ \\
\hline 20 & $288.00 \odot$ & $288.00 \odot$ & 1.0000 & $\odot .1448 \mathrm{E}+08$ \\
\hline 21 & $288.00 \odot$ & $288.00 \odot$ & 1.0000 & $\odot .1448 \mathrm{E}+08$ \\
\hline 22 & 288.000 & $288.00 \odot$ & $1.000 \odot$ & $\odot .1448 E+\odot 8$ \\
\hline 23 & 288.000 & 288.0 & 1.00 & $0.1448 \mathrm{E}+08$ \\
\hline 24 & $288 . \odot$ & $288 . \odot$ & 1.0000 & $0.1448 \mathrm{E}+08$ \\
\hline 25 & $288.00 \odot$ & 288.000 & 1.0000 & $0.1448 \mathrm{E}+08$ \\
\hline 26 & $288.0 \odot \odot$ & $288.0 \odot \odot$ & $1.000 \odot$ & $\odot .1448 \mathrm{E}+\odot 8$ \\
\hline 27 & 288.000 & $288.0 \odot \odot$ & 1.0000 & $\odot .1448 E+08$ \\
\hline 28 & $288 . \odot \odot \odot$ & $288.00 \odot$ & $1.000 \odot$ & $\odot .1448 \mathrm{E}+\odot 8$ \\
\hline 29 & $288.00 \odot$ & $288.00 \odot$ & 1.0000 & $\odot .1448 \mathrm{E}+08$ \\
\hline 30 & $288.00 \odot$ & $288.0 \odot \odot$ & 1.0000 & $0.1448 \mathrm{E}+08$ \\
\hline 31 & $288.00 \odot$ & 288.000 & $1.000 \odot$ & $0.1448 E+08$ \\
\hline 32 & $288.00 \odot$ & $288.00 \odot$ & 1.0000 & $0.1448 \mathrm{E}+08$ \\
\hline 33 & 288.000 & 288.000 & 1.0000 & $\odot .1448 \mathrm{E}+08$ \\
\hline 34 & $288.00 \odot$ & $288.00 \odot$ & 1.0000 & $0.1448 \mathrm{E}+08$ \\
\hline
\end{tabular}




\begin{tabular}{|c|c|c|c|c|}
\hline 35 & 288.000 & 288.000 & 1.0000 & $\odot .1448 \mathrm{E}+08$ \\
\hline 36 & 288.000 & $288.00 \odot$ & 1. $\odot \odot \odot \odot$ & $\odot .1448 \mathrm{E}+\odot 8$ \\
\hline 37 & $288.0 \odot \odot$ & $288 . \odot \odot \odot$ & $1.00 \odot \odot$ & $\odot .1448 \mathrm{E}+\odot 8$ \\
\hline 38 & 288.000 & 288.000 & 1.0000 & $\odot .1448 \mathrm{E}+08$ \\
\hline 39 & 288.000 & 288.000 & 1.0000 & $\odot .1448 \mathrm{E}+08$ \\
\hline 40 & 288.000 & $288.0 \odot \odot$ & 1.0000 & $\odot .1448 \mathrm{E}+08$ \\
\hline 41 & 288.000 & 288.000 & 1.0000 & $\odot .1448 \mathrm{E}+08$ \\
\hline 42 & 288.000 & $288.00 \odot$ & 1.0000 & $\odot .1448 \mathrm{E}+08$ \\
\hline 43 & $288.00 \odot$ & $288 . \odot \odot \odot$ & $1.000 \odot$ & $\odot .1448 \mathrm{E}+\odot 8$ \\
\hline 44 & 288.000 & $288.00 \odot$ & 1.0000 & $\odot .1448 \mathrm{E}+08$ \\
\hline 45 & 288.000 & 288.000 & 1.0000 & $0.1448 \mathrm{E}+08$ \\
\hline 46 & $288.00 \odot$ & 288.000 & 1.0000 & $\odot .1448 \mathrm{E}+08$ \\
\hline 47 & $288.00 \odot$ & $288.0 \odot \odot$ & $1.00 \odot \odot$ & $\odot .1448 \mathrm{E}+\odot 8$ \\
\hline 48 & 288.000 & 288.000 & 1.0000 & $0.1448 \mathrm{E}+08$ \\
\hline 49 & $288.00 \odot$ & $288.00 \odot$ & 1.0000 & $\odot .1448 \mathrm{E}+\odot 8$ \\
\hline 50 & 288.000 & 288.000 & 1.0000 & $\odot .1448 \mathrm{E}+\odot 8$ \\
\hline 51 & 288.000 & $288.0 \odot \odot$ & 1.0000 & $\odot .1448 \mathrm{E}+08$ \\
\hline 52 & 288.000 & $288.00 \odot$ & $1.000 \odot$ & $\odot .1448 \mathrm{E}+\odot 8$ \\
\hline 53 & $288.00 \odot$ & $288 . \odot \odot \odot$ & $1.00 \odot \odot$ & $\odot .1448 \mathrm{E}+\odot 8$ \\
\hline 54 & 288.000 & $288.0 \odot \odot$ & 1.0000 & $\odot .1448 \mathrm{E}+08$ \\
\hline 55 & 288.000 & 288.000 & 1.0000 & $\odot .1448 \mathrm{E}+\odot 8$ \\
\hline 56 & $288.00 \odot$ & $288.00 \odot$ & $1.000 \odot$ & $0.1448 \mathrm{E}+08$ \\
\hline 57 & 288.000 & 288.000 & 1.0000 & $\odot .1448 \mathrm{E}+08$ \\
\hline 58 & 288.000 & 288.000 & 1.0000 & $0.1448 \mathrm{E}+08$ \\
\hline 59 & 288.000 & $288.00 \odot$ & $1.000 \odot$ & $\odot .1448 \mathrm{E}+\odot 8$ \\
\hline 60 & $288.00 \odot$ & $288 . \odot \odot \odot$ & $1.000 \odot$ & $\odot .1448 \mathrm{E}+\odot 8$ \\
\hline time & ec) $=$ & $4.00 \quad 16$ & $a=0$. & 9036007 \\
\hline node & $\mathrm{T}(\mathrm{K})$ & Twall(K) & $\mathrm{X}$ & $P(P a)$ \\
\hline 1 & 142.931 & 163.762 & - - - - - & $\odot .1083 \mathrm{E}+07$ \\
\hline 2 & 165.800 & 200.249 & $\odot .8950$ & $\odot .1998 \mathrm{E}+07$ \\
\hline 3 & 221.511 & 247.289 & 1.0000 & $\odot .8720 \mathrm{E}+\odot 7$ \\
\hline 4 & 241.320 & 262.609 & 1.0000 & $\odot .1054 \mathrm{E}+08$ \\
\hline 5 & 248.804 & 272.529 & 1.0000 & $\odot .1139 \mathrm{E}+08$ \\
\hline 6 & 254.106 & 279.629 & $1.000 \odot$ & $\odot .1187 \mathrm{E}+08$ \\
\hline 7 & 262.460 & 285.099 & 1.0000 & $0.1241 \mathrm{E}+08$ \\
\hline 8 & 278.806 & 287.727 & 1.0000 & $0.1341 \mathrm{E}+08$ \\
\hline 9 & 286.693 & 288.000 & 1.0000 & $\odot .1419 \mathrm{E}+08$ \\
\hline 10 & 288.000 & $288.00 \odot$ & 1.0000 & $0.1444 \mathrm{E}+08$ \\
\hline 11 & 288. & $288 . \odot$ & 1.0 & $\odot .1448 \mathrm{E}+08$ \\
\hline 12 & $288.0 \odot \odot$ & $288.00 \odot$ & $1.000 \odot$ & $\odot .1448 \mathrm{E}+08$ \\
\hline 13 & $288.00 \odot$ & $288.00 \odot$ & 1.0000 & $\odot .1448 \mathrm{E}+08$ \\
\hline 14 & 288.000 & $288.0 \odot \odot$ & 1.0000 & $\odot .1448 \mathrm{E}+08$ \\
\hline 15 & 288.000 & $288.00 \odot$ & 1.0000 & $\odot .1448 \mathrm{E}+08$ \\
\hline 16 & $288.00 \odot$ & $288.00 \odot$ & 1.0000 & $0.1448 \mathrm{E}+08$ \\
\hline 17 & 288.000 & $288.00 \odot$ & 1.00 & $\odot .1448 \mathrm{E}+08$ \\
\hline 18 & $288.00 \odot$ & $288.00 \odot$ & $1.00 \mathrm{C}$ & $\odot .1448 E+08$ \\
\hline 19 & $288.00 \odot$ & $288.00 \odot$ & 1.0000 & $0.1448 \mathrm{E}+08$ \\
\hline 20 & $288.00 \odot$ & $288.00 \odot$ & $1.000 \odot$ & $0.1448 \mathrm{E}+08$ \\
\hline 21 & 288.000 & $288.00 \odot$ & 1.0000 & $\odot .1448 \mathrm{E}+08$ \\
\hline 22 & $288.00 \odot$ & $288 . \odot \odot \odot$ & $1.000 \odot$ & $0.1448 \mathrm{E}+08$ \\
\hline 23 & $288.00 \odot$ & $288.00 \odot$ & 1.0000 & $\odot .1448 \mathrm{E}+08$ \\
\hline 24 & 288.000 & $288.0 \odot \odot$ & 1.0000 & $\odot .1448 \mathrm{E}+08$ \\
\hline 25 & 288.000 & 288.000 & $1.0 \odot \odot \odot$ & $\odot .1448 \mathrm{E}+08$ \\
\hline 26 & 288.000 & $288.00 \odot$ & $1.000 \odot$ & $\odot .1448 \mathrm{E}+\odot 8$ \\
\hline 27 & 288.000 & 288.000 & 1.0000 & $\odot .1448 E+08$ \\
\hline 28 & 288.000 & $288.00 \odot$ & 1.0000 & $\odot .1448 \mathrm{E}+\odot$ \\
\hline
\end{tabular}




\begin{tabular}{|c|c|c|c|c|}
\hline 29 & 288.000 & 288.000 & 1.0000 & $\odot .1448 \mathrm{E}+08$ \\
\hline 30 & 288.000 & $288.00 \odot$ & 1.0000 & $0.1448 \mathrm{E}+08$ \\
\hline 31 & $288.00 \odot$ & $288.00 \odot$ & $1.00 \odot \odot$ & $0.1448 \mathrm{E}+\odot 8$ \\
\hline 32 & 288.000 & 288.000 & 1.0000 & $0.1448 \mathrm{E}+08$ \\
\hline 33 & 288.000 & 288.000 & 1.0000 & $0.1448 E+08$ \\
\hline 34 & 288.000 & 288.000 & 1.0000 & $\odot .1448 \mathrm{E}+08$ \\
\hline 35 & 288.000 & 288.000 & 1.0000 & $0.1448 \mathrm{E}+08$ \\
\hline 36 & 288.000 & 288.000 & 1.0000 & $0.1448 \mathrm{E}+08$ \\
\hline 37 & 288.000 & $288.0 \odot \odot$ & $1.000 \odot$ & $\odot .1448 \mathrm{E}+\odot 8$ \\
\hline 38 & 288.000 & 288.000 & 1.0000 & $0.1448 \mathrm{E}+08$ \\
\hline 39 & 288.000 & 288.000 & 1.0000 & $0.1448 \mathrm{E}+08$ \\
\hline 40 & 288.000 & 288.000 & 1.0000 & $0.1448 \mathrm{E}+08$ \\
\hline 41 & 288.000 & 288.000 & 1.0000 & $0.1448 \mathrm{E}+08$ \\
\hline 42 & 288.000 & 288.000 & 1.0000 & $0.1448 \mathrm{E}+08$ \\
\hline 43 & 288.000 & $288.0 \odot \odot$ & 1.0000 & $0.1448 \mathrm{E}+08$ \\
\hline 44 & 288.000 & 288.000 & 1.0000 & $\odot .1448 \mathrm{E}+08$ \\
\hline 45 & 288.000 & 288.000 & 1.0000 & $0.1448 \mathrm{E}+08$ \\
\hline 46 & 288.000 & 288.000 & 1.0000 & $\odot .1448 \mathrm{E}+08$ \\
\hline 47 & 288.000 & $288.0 \odot \odot$ & $1.00 \odot \odot$ & $0.1448 \mathrm{E}+\odot 8$ \\
\hline 48 & 288.000 & 288.000 & 1.0000 & $0.1448 \mathrm{E}+08$ \\
\hline 49 & 288.000 & $288.00 \odot$ & 1.0000 & $0.1448 \mathrm{E}+08$ \\
\hline 50 & 288.000 & 288.000 & 1.0000 & $0.1448 \mathrm{E}+08$ \\
\hline 51 & 288.000 & 288.000 & 1.0000 & $\odot .1448 \mathrm{E}+08$ \\
\hline 52 & 288.000 & 288.000 & 1.0000 & $0.1448 \mathrm{E}+08$ \\
\hline 53 & 288.000 & $288.0 \odot \odot$ & 1.0000 & $0.1448 \mathrm{E}+08$ \\
\hline 54 & 288.000 & $288.0 \odot \odot$ & $1.000 \odot$ & $0.1448 \mathrm{E}+08$ \\
\hline 55 & 288.000 & 288.000 & 1.0000 & $0.1448 \mathrm{E}+08$ \\
\hline 56 & $288.00 \odot$ & $288.0 \odot \odot$ & $1.000 \odot$ & $0.1448 \mathrm{E}+08$ \\
\hline 57 & 288.000 & 288.000 & 1.0000 & $\odot .1448 \mathrm{E}+08$ \\
\hline 58 & 288.000 & 288.000 & 1.0000 & $0.1448 \mathrm{E}+08$ \\
\hline 59 & 288.000 & 288.000 & 1.0000 & $0.1448 \mathrm{E}+08$ \\
\hline 60 & 288.000 & $288.00 \odot$ & 1.0000 & $0.1448 \mathrm{E}+08$ \\
\hline time & $(\mathrm{sec})$ & \multicolumn{3}{|l|}{25.00} \\
\hline node & $\mathrm{T}(\mathrm{K})$ & Twall(K) & $\mathrm{X}$ & $P(P a)$ \\
\hline 1 & 142.109 & 162.236 & - - - - & $\odot .1052 \mathrm{E}+07$ \\
\hline 2 & 164.847 & 198.112 & 0.9017 & $\odot .1941 \mathrm{E}+07$ \\
\hline 3 & 218.676 & 245.455 & 1.0000 & $\odot .8588 \mathrm{E}+07$ \\
\hline 4 & 240.589 & 261.389 & 1.0000 & $0.1043 \mathrm{E}+08$ \\
\hline 5 & 49.098 & 271.308 & 1.0000 & $0.1139 \mathrm{E}+08$ \\
\hline 6 & 254.399 & 278.409 & 1.0000 & $0.1188 \mathrm{E}+08$ \\
\hline 7 & 259.720 & 283.878 & 1.0000 & $\odot .1228 \mathrm{E}+08$ \\
\hline 8 & 274.757 & 287.388 & 1.0000 & $0.1314 \mathrm{E}+08$ \\
\hline 9 & 285.454 & 288.000 & 1.0000 & $0.1403 \mathrm{E}+08$ \\
\hline 10 & 287.960 & 288.000 & 1.0000 & $0.1441 \mathrm{E}+08$ \\
\hline 11 & 88.000 & 288.000 & 1.0000 & $0.1448 \mathrm{E}+08$ \\
\hline 12 & $288.0 \odot \odot$ & $288.00 \odot$ & $1.000 \odot$ & $0.1448 \mathrm{E}+08$ \\
\hline 13 & 288.000 & 288.000 & 1.0000 & $0.1448 \mathrm{E}+08$ \\
\hline 14 & 288.000 & $288.0 \odot \odot$ & $1.000 \odot$ & $0.1448 \mathrm{E}+\odot 8$ \\
\hline 15 & 288.000 & 288.000 & 1.0000 & $0.1448 \mathrm{E}+08$ \\
\hline 16 & $288.00 \odot$ & $288 . \odot \odot \odot$ & $1.000 \odot$ & $0.1448 \mathrm{E}+08$ \\
\hline 17 & 288.000 & 288.000 & 1.0000 & $0.1448 \mathrm{E}+08$ \\
\hline 18 & 288.000 & 288.000 & 1.0000 & $0.1448 \mathrm{E}+08$ \\
\hline 19 & $288.00 \odot$ & $288.0 \odot \odot$ & $1.000 \odot$ & $\odot .1448 \mathrm{E}+08$ \\
\hline 20 & 288.000 & $288.00 \odot$ & 1.0000 & $0.1448 \mathrm{E}+08$ \\
\hline 21 & 288.000 & 288.000 & 1.0000 & $\odot .1448 E+\odot 8$ \\
\hline 22 & 288.000 & 88.000 & $1.000 \odot$ & $\odot .1448 \mathrm{E}+\odot 8$ \\
\hline
\end{tabular}




\begin{tabular}{|c|c|c|c|c|}
\hline 23 & 288.000 & $288.00 \odot$ & 1.0000 & $0.1448 \mathrm{E}+08$ \\
\hline 24 & $288.00 \odot$ & $288.00 \odot$ & 1.0000 & $0.1448 \mathrm{E}+08$ \\
\hline 25 & $288.0 \odot \odot$ & $288.00 \odot$ & $1.00 \odot \odot$ & $0.1448 \mathrm{E}+\odot 8$ \\
\hline 26 & 288.000 & 288.000 & 1.0000 & $0.1448 \mathrm{E}+08$ \\
\hline 27 & 288.000 & 288.000 & 1.0000 & $0.1448 E+08$ \\
\hline 28 & 288.000 & 288.000 & 1.0000 & $0.1448 \mathrm{E}+08$ \\
\hline 29 & $288.0 \odot \odot$ & $288.0 \odot \odot$ & $1.0 \odot \odot \odot$ & $0.1448 \mathrm{E}+\odot 8$ \\
\hline 30 & 288.000 & 288.000 & 1.0000 & $0.1448 \mathrm{E}+08$ \\
\hline 31 & $288.00 \odot$ & $288.0 \odot \odot$ & $1.000 \odot$ & $\odot .1448 \mathrm{E}+\odot 8$ \\
\hline 32 & 288.000 & 288.000 & 1.0000 & $0.1448 \mathrm{E}+08$ \\
\hline 33 & 288.000 & 288.000 & 1.0000 & $0.1448 \mathrm{E}+08$ \\
\hline 34 & 288.000 & 288.000 & 1.0000 & $0.1448 \mathrm{E}+08$ \\
\hline 35 & $288.00 \odot$ & $288.0 \odot \odot$ & $1.000 \odot$ & $0.1448 \mathrm{E}+\odot 8$ \\
\hline 36 & 288.000 & 288.000 & 1.0000 & $0.1448 \mathrm{E}+08$ \\
\hline 37 & $288.00 \odot$ & $288.0 \odot \odot$ & 1.0000 & $0.1448 \mathrm{E}+08$ \\
\hline 38 & 288.000 & 288.000 & 1.0000 & $\odot .1448 \mathrm{E}+\odot 8$ \\
\hline 39 & 288.000 & 288.000 & 1.0000 & $0.1448 \mathrm{E}+08$ \\
\hline 40 & 288.000 & 288.000 & 1.0000 & $\odot .1448 \mathrm{E}+\odot 8$ \\
\hline 41 & $288.00 \odot$ & $288.0 \odot \odot$ & $1.00 \odot \odot$ & $0.1448 \mathrm{E}+\odot 8$ \\
\hline 42 & $288.00 \odot$ & $288.00 \odot$ & 1.0000 & $\odot .1448 \mathrm{E}+08$ \\
\hline 43 & 288.000 & $288.00 \odot$ & 1.0000 & $0.1448 \mathrm{E}+08$ \\
\hline 44 & 288.000 & $288.00 \odot$ & 1.0000 & $0.1448 \mathrm{E}+08$ \\
\hline 45 & 288.000 & 288.000 & 1.0000 & $\odot .1448 \mathrm{E}+08$ \\
\hline 46 & 288.000 & 288.000 & 1.0000 & $0.1448 \mathrm{E}+08$ \\
\hline 47 & $288.00 \odot$ & $288.0 \odot \odot$ & 1.0000 & $0.1448 \mathrm{E}+08$ \\
\hline 48 & $288.00 \odot$ & $288.0 \odot \odot$ & $1.000 \odot$ & $0.1448 \mathrm{E}+08$ \\
\hline 49 & 288.000 & 288.000 & 1.0000 & $0.1448 \mathrm{E}+08$ \\
\hline 50 & $288.00 \odot$ & $288.00 \odot$ & 1.0000 & $0.1448 \mathrm{E}+08$ \\
\hline 51 & 288.000 & 288.000 & 1.0000 & $\odot .1448 \mathrm{E}+08$ \\
\hline 52 & 288.000 & 288.000 & 1.0000 & $0.1448 \mathrm{E}+08$ \\
\hline 53 & 288.000 & 288.000 & 1.0000 & $0.1448 \mathrm{E}+08$ \\
\hline 54 & 288.000 & $288.00 \odot$ & 1.0000 & $0.1448 \mathrm{E}+08$ \\
\hline 55 & 288.000 & 288.000 & 1.0000 & $0.1448 \mathrm{E}+08$ \\
\hline 56 & 288.000 & 288.000 & 1.0000 & $0.1448 \mathrm{E}+08$ \\
\hline 57 & $288.0 \odot \odot$ & $288.0 \odot \odot$ & $1.000 \odot$ & $0.1448 \mathrm{E}+08$ \\
\hline 58 & 288.000 & 288.000 & 1.0000 & $0.1448 \mathrm{E}+08$ \\
\hline 59 & 288.000 & 288.000 & 1.0000 & $0.1448 \mathrm{E}+08$ \\
\hline 60 & 288.000 & 288.000 & 1.0000 & $0.1448 E+08$ \\
\hline tim & $(\sec )=$ & $26 . \odot \odot$ & $\mathrm{a} a=0$. & 28569865 \\
\hline & $\mathrm{T}(\mathrm{K})$ & Twall(K) & $x$ & $\mathrm{P}(\mathrm{Pa})$ \\
\hline 1 & 141.346 & 160.783 & - - - - - & $0.1023 \mathrm{E}+07$ \\
\hline 2 & 163.962 & 196.089 & 0.9078 & $\odot .1888 \mathrm{E}+07$ \\
\hline 3 & 216.056 & 243.471 & 1.0000 & $\odot .8474 \mathrm{E}+07$ \\
\hline 4 & 239.426 & 260.168 & 1.0000 & $0.1031 \mathrm{E}+08$ \\
\hline 5 & 49.179 & 270.377 & 1.0000 & $0.1136 \mathrm{E}+08$ \\
\hline 6 & 254.552 & 277.232 & 1.0000 & $\odot .1189 \mathrm{E}+08$ \\
\hline 7 & 258.703 & 282.657 & 1.0000 & $0.1224 \mathrm{E}+08$ \\
\hline 8 & 270.247 & 286.778 & 1.0000 & $0.1290 \mathrm{E}+08$ \\
\hline 9 & 83.531 & 288.000 & 1.0000 & $0.1383 \mathrm{E}+08$ \\
\hline 10 & 287.655 & $288 . \odot \odot \odot$ & $1.000 \odot$ & $0.1435 \mathrm{E}+08$ \\
\hline 11 & 288.000 & 288.000 & 1.0000 & $0.1447 \mathrm{E}+08$ \\
\hline 12 & 288.000 & $288.00 \odot$ & 1.0000 & $0.1448 \mathrm{E}+08$ \\
\hline 13 & 288.000 & 288.000 & 1.0000 & $0.1448 E+08$ \\
\hline 14 & $288.00 \odot$ & $288.00 \odot$ & 1.0000 & $0.1448 \mathrm{E}+08$ \\
\hline 15 & 288.000 & 288.000 & 1.0000 & $\odot .1448 \mathrm{E}+08$ \\
\hline 16 & & 88.000 & $1.000 \odot$ & $0.1448 E+08$ \\
\hline
\end{tabular}




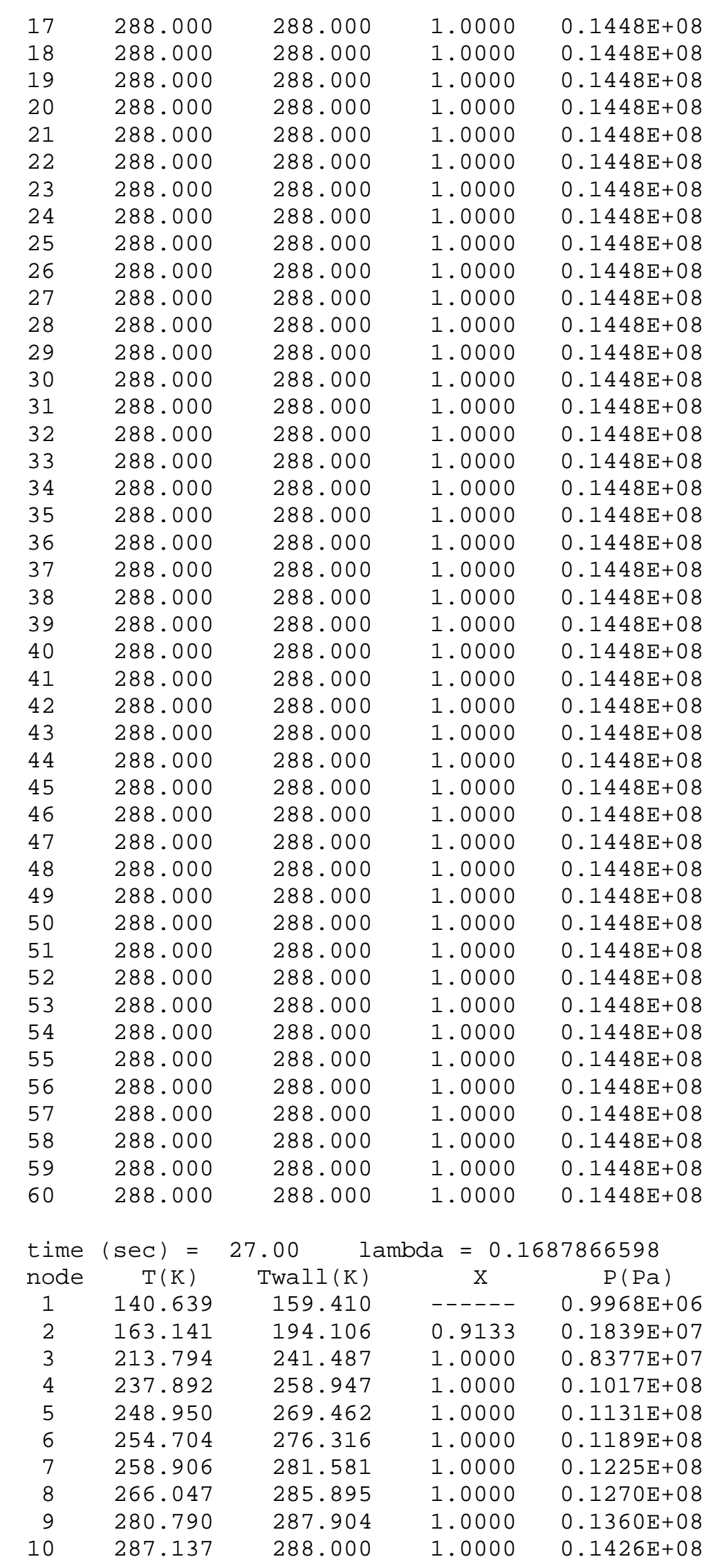




\begin{tabular}{|c|c|c|c|c|}
\hline 11 & $288.0 \odot \odot$ & $288.00 \odot$ & 1.0000 & $\odot .1446 \mathrm{E}+08$ \\
\hline 12 & $288 . \odot \odot \odot$ & 288.000 & $1.000 \odot$ & $0.1448 E+\odot 8$ \\
\hline 13 & $288.0 \odot \odot$ & $288.00 \odot$ & 1.0000 & $0.1448 E+08$ \\
\hline 14 & $288.0 \odot \odot$ & $288.00 \odot$ & 1.0000 & $\odot .1448 \mathrm{E}+\odot 8$ \\
\hline 15 & $288.0 \odot \odot$ & 288.000 & 1.0000 & $0.1448 \mathrm{E}+08$ \\
\hline 16 & $288 . \odot \odot \odot$ & $288.0 \odot \odot$ & $1.000 \odot$ & $\odot .1448 E+08$ \\
\hline 17 & $288.0 \odot \odot$ & $288.00 \odot$ & 1.0000 & $0.1448 E+08$ \\
\hline 18 & $288.0 \odot \odot$ & 288.000 & 1.0000 & $\odot .1448 \mathrm{E}+08$ \\
\hline 19 & $288.0 \odot \odot$ & $288.00 \odot$ & 1.0000 & $0.1448 \mathrm{E}+08$ \\
\hline 20 & $288.00 \odot$ & $288.0 \odot \odot$ & $1.000 \odot$ & $0.1448 E+08$ \\
\hline 21 & $288.0 \odot \odot$ & 288.000 & 1.0000 & $\odot .1448 \mathrm{E}+08$ \\
\hline 22 & 288.000 & 288.000 & $1.000 \odot$ & $\odot .1448 \mathrm{E}+08$ \\
\hline 23 & $288.0 \odot \odot$ & 288.000 & 1.0000 & $0.1448 \mathrm{E}+08$ \\
\hline 24 & $288.0 \odot \odot$ & $288.0 \odot \odot$ & 1.0000 & $0.1448 E+08$ \\
\hline 25 & $288.00 \odot$ & 288.000 & 1.0000 & $0.1448 \mathrm{E}+08$ \\
\hline 26 & $288.0 \odot \odot$ & 288.000 & 1.0000 & $0.1448 \mathrm{E}+08$ \\
\hline 27 & $288 . \odot \odot \odot$ & $288.0 \odot \odot$ & $1.000 \odot$ & $\odot .1448 \mathrm{E}+08$ \\
\hline 28 & $288.00 \odot$ & 288.000 & 1.0000 & $0.1448 \mathrm{E}+08$ \\
\hline 29 & $288.0 \odot \odot$ & 288.000 & 1.0000 & $0.1448 \mathrm{E}+08$ \\
\hline 30 & $288.0 \odot \odot$ & 288.000 & 1.0000 & $0.1448 E+08$ \\
\hline 31 & $288.00 \odot$ & 288.000 & 1.0000 & $0.1448 \mathrm{E}+08$ \\
\hline 32 & $288.00 \odot$ & 288.000 & 1.0000 & $0.1448 \mathrm{E}+08$ \\
\hline 33 & $288 . \odot \odot \odot$ & 288.000 & 1.0000 & $0.1448 E+08$ \\
\hline 34 & $288.0 \odot \odot$ & 288.000 & 1.0000 & $0.1448 \mathrm{E}+08$ \\
\hline 35 & $288.00 \odot$ & 288.000 & 1.0000 & $0.1448 \mathrm{E}+08$ \\
\hline 36 & $288.00 \odot$ & 288.000 & 1.0000 & $0.1448 \mathrm{E}+08$ \\
\hline 37 & 88.000 & 288.000 & 1.0000 & $0.1448 \mathrm{E}+08$ \\
\hline 38 & $288.0 \odot \odot$ & 288.000 & 1.0000 & $0.1448 \mathrm{E}+08$ \\
\hline 39 & $288 . \odot \odot \odot$ & $288.0 \odot \odot$ & $1.000 \odot$ & $0.1448 \mathrm{E}+08$ \\
\hline 40 & $288.0 \odot \odot$ & 288.000 & 1.0000 & $\odot .1448 \mathrm{E}+08$ \\
\hline 41 & $288.0 \odot \odot$ & 288.000 & 1.0000 & $0.1448 \mathrm{E}+08$ \\
\hline 42 & $288.00 \odot$ & 288.000 & 1.0000 & $0.1448 \mathrm{E}+08$ \\
\hline 43 & $88.00 \odot$ & $288.0 \odot \odot$ & $1.000 \odot$ & $0.1448 \mathrm{E}+\odot 8$ \\
\hline 44 & 88.000 & 288.000 & 1.0000 & $0.1448 \mathrm{E}+08$ \\
\hline 45 & $288 . \odot 0 \odot$ & 288.000 & 1.0000 & $0.1448 \mathrm{E}+08$ \\
\hline 46 & $288 . \odot \odot \odot$ & $288.00 \odot$ & $1.000 \odot$ & $0.1448 \mathrm{E}+08$ \\
\hline 47 & $288.00 \odot$ & 288.000 & 1.0000 & $0.1448 \mathrm{E}+08$ \\
\hline 48 & $288.00 \odot$ & 288.000 & 1.0000 & $\odot .1448 \mathrm{E}+08$ \\
\hline 49 & $288 . \odot \odot \odot$ & $288.0 \odot \odot$ & $1.000 \odot$ & $0.1448 \mathrm{E}+08$ \\
\hline 50 & $\odot \odot$ & $288.00 \odot$ & 1.00 & $0.1448 \mathrm{E}+\odot 8$ \\
\hline 51 & $288.00 \odot$ & $288.00 \odot$ & 1.0000 & $\odot .1448 \mathrm{E}+08$ \\
\hline 52 & $288 . \odot \odot \odot$ & $288.0 \odot \odot$ & $1.00 \odot \odot$ & $0.1448 \mathrm{E}+08$ \\
\hline 53 & $288.00 \odot$ & 288.000 & 1.0000 & $0.1448 E+08$ \\
\hline 54 & $288.00 \odot$ & 288.000 & 1.0000 & $0.1448 \mathrm{E}+08$ \\
\hline 55 & $288 . \odot \odot \odot$ & $288.0 \odot \odot$ & $1.000 \odot$ & $0.1448 \mathrm{E}+08$ \\
\hline 56 & 288.0 & $288 . \odot$ & 1.00 & $0.1448 \mathrm{E}+08$ \\
\hline 57 & 288.000 & 288.000 & 1.0000 & $\odot .1448 \mathrm{E}+08$ \\
\hline 58 & $288.00 \odot$ & 288.000 & 1.0000 & $\odot .1448 E+08$ \\
\hline 59 & $288.00 \odot$ & 288.000 & 1.0000 & $0.1448 \mathrm{E}+08$ \\
\hline 60 & $288 . \odot \odot \odot$ & $288.0 \odot \odot$ & $1.000 \odot$ & $0.1448 \mathrm{E}+08$ \\
\hline time & & \multicolumn{3}{|c|}{$28.00 \quad$ lambda } \\
\hline & & Twall(K) & & $\mathrm{P}(\mathrm{Pa})$ \\
\hline 1 & 139.987 & 158.048 & $-\ldots$ & $0.9723 \mathrm{E}+06$ \\
\hline 2 & 162.385 & 192.256 & 0.9184 & $0.1794 \mathrm{E}+07$ \\
\hline 3 & 11.979 & 239.504 & 1.0000 & $0.8304 \mathrm{E}+07$ \\
\hline 4 & & 57.727 & 1.0000 & $0.1004 \mathrm{E}+\odot$ \\
\hline
\end{tabular}




\begin{tabular}{|c|c|c|c|c|}
\hline 5 & 248.417 & 268.546 & 1.0000 & $0.1124 \mathrm{E}+\odot 8$ \\
\hline 6 & 254.857 & 275.401 & 1.0000 & $0.1188 \mathrm{E}+08$ \\
\hline 7 & 259.198 & 280.665 & 1.0000 & $0.1226 \mathrm{E}+\odot 8$ \\
\hline 8 & 263.389 & 284.979 & 1.0000 & $\odot .1257 \mathrm{E}+\odot 8$ \\
\hline 9 & 277.272 & 287.598 & $1.000 \odot$ & $\odot .1336 \mathrm{E}+\odot 8$ \\
\hline 10 & 286.194 & $288.00 \odot$ & 1.0000 & $\odot .1413 E+\odot 8$ \\
\hline 11 & 288.000 & $288.00 \odot$ & $1.000 \odot$ & $\odot .1443 E+08$ \\
\hline 12 & 288.000 & $288.00 \odot$ & $1.000 \odot$ & $\odot .1448 \mathrm{E}+\odot 8$ \\
\hline 13 & 288.000 & $288.00 \odot$ & $1.000 \odot$ & $\odot .1448 E+08$ \\
\hline 14 & 288.000 & 288.000 & 1.0000 & $0.1448 E+\odot 8$ \\
\hline 15 & $288.00 \odot$ & $288.00 \odot$ & 1.0000 & $0.1448 E+\odot 8$ \\
\hline 16 & $288.00 \odot$ & 288.000 & 1.0000 & $0.1448 \mathrm{E}+08$ \\
\hline 17 & $288.00 \odot$ & $288.00 \odot$ & 1.0000 & $0.1448 \mathrm{E}+\odot 8$ \\
\hline 18 & $288.00 \odot$ & 288.000 & $1.000 \odot$ & $\odot .1448 \mathrm{E}+08$ \\
\hline 19 & $288.00 \odot$ & 288.000 & 1.0000 & $0.1448 \mathrm{E}+08$ \\
\hline 20 & 288.000 & $288.00 \odot$ & 1.0000 & $\odot .1448 \mathrm{E}+\odot 8$ \\
\hline 21 & $288.00 \odot$ & $288.00 \odot$ & 1.0000 & $\odot .1448 \mathrm{E}+\odot 8$ \\
\hline 22 & 288.000 & $288.00 \odot$ & 1.0000 & $0.1448 E+08$ \\
\hline 23 & $288.00 \odot$ & $288.00 \odot$ & 1.0000 & $0.1448 \mathrm{E}+08$ \\
\hline 24 & $288.00 \odot$ & $288.00 \odot$ & 1.0000 & $\odot .1448 E+\odot 8$ \\
\hline 25 & 288.000 & 288.000 & 1.0000 & $0.1448 \mathrm{E}+08$ \\
\hline 26 & 288.000 & 288.000 & 1.0000 & $0.1448 \mathrm{E}+08$ \\
\hline 27 & 288.000 & 288.000 & $1.000 \odot$ & $\odot .1448 E+08$ \\
\hline 28 & 288.000 & 288.000 & 1.0000 & $0.1448 \mathrm{E}+08$ \\
\hline 29 & 288.000 & 288.000 & 1.0000 & $0.1448 E+08$ \\
\hline 30 & $288.00 \odot$ & 288.000 & 1.0000 & $0.1448 \mathrm{E}+08$ \\
\hline 31 & 288.000 & 288.000 & 1.0000 & $0.1448 \mathrm{E}+08$ \\
\hline 32 & 288.000 & 288.000 & 1.0000 & $0.1448 \mathrm{E}+08$ \\
\hline 33 & $288.00 \odot$ & $288.00 \odot$ & 1.0000 & $0.1448 \mathrm{E}+08$ \\
\hline 34 & $288.00 \odot$ & $288.00 \odot$ & $1.000 \odot$ & $\odot .1448 \mathrm{E}+08$ \\
\hline 35 & 288.000 & 288.000 & 1.0000 & $0.1448 \mathrm{E}+08$ \\
\hline 36 & 288.000 & 288.000 & 1.0000 & $0.1448 E+08$ \\
\hline 37 & 288.000 & 288.000 & 1.0000 & $0.1448 \mathrm{E}+08$ \\
\hline 38 & 288.000 & 288.000 & 1.0000 & $0.1448 \mathrm{E}+08$ \\
\hline 39 & 288.000 & 288.000 & 1.0000 & $0.1448 \mathrm{E}+08$ \\
\hline 40 & $288.00 \odot$ & $288.00 \odot$ & 1.0000 & $0.1448 \mathrm{E}+\odot 8$ \\
\hline 41 & 288.000 & 288.000 & 1.0000 & $0.1448 \mathrm{E}+08$ \\
\hline 42 & 288.000 & 288.000 & 1.0000 & $0.1448 \mathrm{E}+08$ \\
\hline 43 & $288.00 \odot$ & $288.00 \odot$ & 1.0000 & $0.1448 \mathrm{E}+08$ \\
\hline 44 & $288.00 \odot$ & $288.00 \odot$ & 1.0000 & $\odot .1448 \mathrm{E}+\odot 8$ \\
\hline 45 & 288.000 & 288.000 & 1.0000 & $0.1448 \mathrm{E}+08$ \\
\hline 46 & $288.00 \odot$ & $288.00 \odot$ & 1.0000 & $0.1448 \mathrm{E}+08$ \\
\hline 47 & 288.000 & 288.000 & 1.0000 & $\odot .1448 \mathrm{E}+08$ \\
\hline 48 & 288.000 & 288.000 & 1.0000 & $0.1448 \mathrm{E}+08$ \\
\hline 49 & $288.00 \odot$ & $288 . \odot \odot \odot$ & $1.000 \odot$ & $\odot .1448 E+08$ \\
\hline 50 & $288.00 \odot$ & $288.00 \odot$ & $1.000 \odot$ & $0.1448 E+\odot 8$ \\
\hline 51 & 288.000 & 288.000 & 1.0000 & $0.1448 \mathrm{E}+08$ \\
\hline 52 & 288.000 & 288.000 & 1.0000 & $0.1448 E+08$ \\
\hline 53 & 288.000 & $288.00 \odot$ & 1.0000 & $0.1448 \mathrm{E}+08$ \\
\hline 54 & 288.000 & 288.000 & 1.0000 & $0.1448 \mathrm{E}+08$ \\
\hline 55 & 288.000 & 288.000 & 1.0000 & $0.1448 \mathrm{E}+08$ \\
\hline 56 & $288.00 \odot$ & 288.000 & 1.0000 & $0.1448 \mathrm{E}+08$ \\
\hline 57 & $288.00 \odot$ & $288.00 \odot$ & 1.0000 & $\odot .1448 \mathrm{E}+\odot 8$ \\
\hline 58 & 288.000 & 288.000 & 1.0000 & $0.1448 \mathrm{E}+08$ \\
\hline 59 & $288.0 \odot \odot$ & $288 . \odot \odot \odot$ & 1.0000 & $0.1448 \mathrm{E}+08$ \\
\hline 60 & 288.000 & $288.00 \odot$ & 1.0000 & $\odot .1448 E+08$ \\
\hline
\end{tabular}




\begin{tabular}{|c|c|c|c|c|}
\hline \multicolumn{5}{|c|}{ lambda $=0.1615453064$} \\
\hline noue & $\mathrm{T}(\mathrm{K})$ & Twall(K) & $\mathrm{x}$ & $P(P a)$ \\
\hline 1 & 139.388 & 156.828 & $-\ldots$ & $0.9498 \mathrm{E}+06$ \\
\hline 2 & 161.690 & 190.425 & $\odot .9230$ & $\odot .1752 \mathrm{E}+07$ \\
\hline 3 & 210.633 & 237.597 & 1.0000 & $\odot .8252 E+07$ \\
\hline 4 & 234.171 & 256.457 & $1.000 \odot$ & $\odot .9906 \mathrm{E}+\odot 7$ \\
\hline 5 & 247.588 & 267.631 & 1.0000 & $\odot .1115 \mathrm{E}+08$ \\
\hline 6 & 254.909 & 274.485 & 1.0000 & $\odot .1186 \mathrm{E}+08$ \\
\hline 7 & 259.198 & 279.749 & 1.0000 & $\odot .1226 \mathrm{E}+\odot 8$ \\
\hline 8 & 262.316 & 284.063 & 1.0000 & $\odot .1252 \mathrm{E}+08$ \\
\hline 9 & 273.189 & 287.156 & 1.0000 & $\odot .1313 E+\odot 8$ \\
\hline 10 & 284.673 & 288.000 & 1.0000 & $\odot .1396 \mathrm{E}+08$ \\
\hline 11 & 287.817 & 288.000 & 1.0000 & $0.1439 E+08$ \\
\hline 12 & 288.000 & 288.000 & 1.0000 & $0.1447 \mathrm{E}+08$ \\
\hline 13 & $288.00 \odot$ & $288.00 \odot$ & $1.000 \odot$ & $0.1448 \mathrm{E}+08$ \\
\hline 14 & 288.000 & 288.000 & 1.0000 & $\odot .1448 \mathrm{E}+\odot 8$ \\
\hline 15 & $288.00 \odot$ & $288 . \odot \odot \odot$ & $1.000 \odot$ & $\odot .1448 \mathrm{E}+08$ \\
\hline 16 & 288.000 & 288.000 & 1.0000 & $\odot .1448 \mathrm{E}+\odot 8$ \\
\hline 17 & 288.000 & 288.000 & 1.0000 & $\odot .1448 \mathrm{E}+08$ \\
\hline 18 & 288.000 & 288.000 & 1.0000 & $\odot .1448 \mathrm{E}+\odot 8$ \\
\hline 19 & $288.00 \odot$ & $288.00 \odot$ & $1.000 \odot$ & $0.1448 \mathrm{E}+08$ \\
\hline 20 & $288.0 \odot \odot$ & $288.0 \odot \odot$ & $1.000 \odot$ & $0.1448 \mathrm{E}+08$ \\
\hline 21 & $288.00 \odot$ & 288.000 & 1.0000 & $\odot .1448 \mathrm{E}+08$ \\
\hline 22 & $288.00 \odot$ & 288.000 & 1.0000 & $0.1448 \mathrm{E}+08$ \\
\hline 23 & 288.000 & 288.000 & 1.0000 & $\odot .1448 \mathrm{E}+08$ \\
\hline 24 & $288.00 \odot$ & 288.000 & $1.000 \odot$ & $\odot .1448 \mathrm{E}+08$ \\
\hline 25 & 288.000 & 288.000 & 1.0000 & $\odot .1448 \mathrm{E}+08$ \\
\hline 26 & $288 . \odot \odot \odot$ & $288.00 \odot$ & $1.000 \odot$ & $0.1448 \mathrm{E}+08$ \\
\hline 27 & $288.00 \odot$ & 288.000 & 1.0000 & $\odot .1448 \mathrm{E}+08$ \\
\hline 28 & $288.0 \odot \odot$ & 288.000 & 1.0000 & $\odot .1448 \mathrm{E}+08$ \\
\hline 29 & $288.0 \odot \odot$ & 288.000 & $1.000 \odot$ & $0.1448 \mathrm{E}+\odot 8$ \\
\hline 30 & $288 . \odot \odot \odot$ & $288 . \odot \odot \odot$ & $1.00 \odot \odot$ & $0.1448 \mathrm{E}+08$ \\
\hline 31 & $288.0 \odot \odot$ & 288.000 & 1.0000 & $0.1448 \mathrm{E}+08$ \\
\hline 32 & $288 . \odot \odot \odot$ & $288 . \odot \odot \odot$ & $1.00 \odot \odot$ & $\odot .1448 \mathrm{E}+\odot 8$ \\
\hline 33 & $288.00 \odot$ & 288.000 & 1.0000 & $0.1448 \mathrm{E}+\odot 8$ \\
\hline 34 & 288.000 & 288.000 & 1.0000 & $\odot .1448 \mathrm{E}+\odot 8$ \\
\hline 35 & $288.0 \odot \odot$ & 288.000 & 1.0000 & $0.1448 \mathrm{E}+08$ \\
\hline 36 & $288.00 \odot$ & $288.00 \odot$ & $1.000 \odot$ & $\odot .1448 \mathrm{E}+\odot 8$ \\
\hline 37 & $288.00 \odot$ & 288.000 & $1.000 \odot$ & $\odot .1448 \mathrm{E}+08$ \\
\hline 38 & $288.00 \odot$ & 288.000 & 1.0000 & $0.1448 \mathrm{E}+08$ \\
\hline 39 & 288.000 & 288.000 & 1.0000 & $\odot .1448 \mathrm{E}+08$ \\
\hline 40 & $288.0 \odot \odot$ & $288.00 \odot$ & $1.000 \odot$ & $\odot .1448 \mathrm{E}+\odot 8$ \\
\hline 41 & 288.000 & 288.000 & 1.0000 & $\odot .1448 \mathrm{E}+\odot 8$ \\
\hline 42 & $288 . \odot \odot \odot$ & $288 . \odot \odot \odot$ & $1.00 \odot \odot$ & $\odot .1448 \mathrm{E}+\odot 8$ \\
\hline 43 & $288.00 \odot$ & 288.000 & 1.0000 & $\odot .1448 \mathrm{E}+08$ \\
\hline 44 & $288.00 \odot$ & $288.00 \odot$ & $1.000 \odot$ & $0.1448 E+08$ \\
\hline 45 & $288.0 \odot \odot$ & 288.000 & 1.0000 & $0.1448 \mathrm{E}+08$ \\
\hline 46 & $288 . \odot \odot \odot$ & $288.00 \odot$ & $1.000 \odot$ & $0.1448 \mathrm{E}+08$ \\
\hline 47 & $288.00 \odot$ & $288.00 \odot$ & $1.000 \odot$ & $0.1448 \mathrm{E}+08$ \\
\hline 48 & $288 . \odot \odot \odot$ & $288 . \odot \odot \odot$ & $1.000 \odot$ & $0.1448 \mathrm{E}+08$ \\
\hline 49 & $288.00 \odot$ & $288.00 \odot$ & $1.000 \odot$ & $0.1448 \mathrm{E}+08$ \\
\hline 50 & $288.00 \odot$ & 288.000 & 1.0000 & $\odot .1448 \mathrm{E}+\odot 8$ \\
\hline 51 & $288.0 \odot \odot$ & $288.00 \odot$ & 1.0000 & $\odot .1448 \mathrm{E}+\odot 8$ \\
\hline 52 & $288.00 \odot$ & $288.00 \odot$ & $1.000 \odot$ & $\odot .1448 \mathrm{E}+\odot 8$ \\
\hline 53 & $288 . \odot \odot \odot$ & $288 . \odot \odot \odot$ & $1.000 \odot$ & $0.1448 \mathrm{E}+08$ \\
\hline 54 & 288.000 & 288.000 & 1.0000 & $\odot .1448 \mathrm{E}+08$ \\
\hline 55 & 288.000 & 288.000 & 1.0000 & $\odot .1448 \mathrm{E}+08$ \\
\hline
\end{tabular}




$\begin{array}{lllll}56 & 288.00 \odot & 288.00 \odot & 1.000 \odot & \odot .1448 \mathrm{E}+08 \\ 57 & 288.00 \odot & 288.00 \odot & 1.000 \odot & \odot .1448 \mathrm{E}+08 \\ 58 & 288.00 \odot & 288.00 \odot & 1.000 \odot & \odot .1448 \mathrm{E}+08 \\ 59 & 288.00 \odot & 288.00 \odot & 1.000 \odot & \odot .1448 \mathrm{E}+08 \\ 6 \odot & 288.00 \odot & 288.00 \odot & 1.000 \odot & \odot .1448 \mathrm{E}+08\end{array}$

\begin{tabular}{|c|c|c|c|c|}
\hline time & $(\sec )=$ & $\begin{array}{ll}30.00 \quad 1 \\
\text { Twall(K) }\end{array}$ & $a=0$ & $\begin{array}{r}393603 \\
P(P a)\end{array}$ \\
\hline $\begin{array}{l}\text { node } \\
1\end{array}$ & 138.837 & $\begin{array}{r}\text { Iwa } \perp(K) \\
155.608\end{array}$ & $\begin{array}{c}\lambda \\
---\ldots\end{array}$ & $\begin{array}{c}P(\mathrm{~Pa}) \\
0.9291 \mathrm{E}+06\end{array}$ \\
\hline 2 & 161.051 & 188.740 & $\odot .9272$ & $\odot .1714 \mathrm{E}+\odot 7$ \\
\hline 3 & 209.740 & 235.766 & 1.0000 & $\odot .8213 \mathrm{E}+07$ \\
\hline 4 & 232.266 & 255.038 & 1.0000 & $\odot .9786 \mathrm{E}+07$ \\
\hline 5 & 246.468 & 266.715 & $1.000 \odot$ & $\odot .1104 \mathrm{E}+\odot 8$ \\
\hline 6 & 254.695 & 273.569 & 1.0000 & $\odot .1182 \mathrm{E}+08$ \\
\hline 7 & 259.198 & 278.834 & $1.000 \odot$ & $0.1225 \mathrm{E}+08$ \\
\hline 8 & 262.478 & 283.148 & 1.0000 & $\odot .1253 E+\odot 8$ \\
\hline 9 & 269.199 & 286.545 & 1.0000 & $\odot .1294 \mathrm{E}+\odot 8$ \\
\hline 10 & 282.431 & 288.000 & 1.0000 & $0.1375 \mathrm{E}+08$ \\
\hline 11 & 287.464 & $288.0 \odot \odot$ & 1.0000 & $\odot .1432 \mathrm{E}+\odot 8$ \\
\hline 12 & 288.000 & 288.000 & 1.0000 & $\odot .1446 \mathrm{E}+\odot 8$ \\
\hline 13 & $288.00 \odot$ & $288.0 \odot \odot$ & 1.0000 & $\odot .1448 \mathrm{E}+08$ \\
\hline 14 & 288.000 & 288.000 & 1. $\odot \odot \odot \odot$ & $\odot .1448 E+\odot 8$ \\
\hline 15 & 288.000 & 288.000 & 1.0000 & $\odot .1448 \mathrm{E}+08$ \\
\hline 16 & $288.0 \odot \odot$ & $288.0 \odot \odot$ & 1.0000 & $\odot .1448 \mathrm{E}+08$ \\
\hline 17 & 288.000 & $288.0 \odot \odot$ & 1.0000 & $\odot .1448 \mathrm{E}+\odot 8$ \\
\hline 18 & 288.000 & 288.000 & 1.0000 & $\odot .1448 \mathrm{E}+08$ \\
\hline 19 & $288.00 \odot$ & $288 . \odot \odot \odot$ & $1.000 \odot$ & $0.1448 \mathrm{E}+08$ \\
\hline 20 & 288.000 & $288.00 \odot$ & 1.0000 & $\odot .1448 \mathrm{E}+\odot 8$ \\
\hline 21 & 288.000 & 288.000 & 1.0000 & $\odot .1448 \mathrm{E}+08$ \\
\hline 22 & 288.000 & 288.000 & $1.000 \odot$ & $\odot .1448 \mathrm{E}+\odot 8$ \\
\hline 23 & 288.000 & 288.000 & 1.0000 & $\odot .1448 \mathrm{E}+\odot 8$ \\
\hline 24 & 288.000 & 288.000 & $1.000 \odot$ & $0.1448 \mathrm{E}+08$ \\
\hline 25 & 288.000 & 288.000 & 1.0000 & $\odot .1448 \mathrm{E}+\odot 8$ \\
\hline 26 & 288.000 & 288.000 & $1.0 \odot \odot \odot$ & $\odot .1448 E+\odot 8$ \\
\hline 27 & 288.000 & 288.000 & 1.0000 & $\odot .1448 \mathrm{E}+\odot 8$ \\
\hline 28 & 288.000 & 288.000 & 1.0000 & $\odot .1448 \mathrm{E}+\odot 8$ \\
\hline 29 & 288.000 & 288.000 & 1.0000 & $\odot .1448 \mathrm{E}+08$ \\
\hline 30 & $288.00 \odot$ & $288 . \odot \odot \odot$ & $1.000 \odot$ & $0.1448 \mathrm{E}+08$ \\
\hline 31 & 288.000 & $288.0 \odot \odot$ & 1.0000 & $\odot .1448 \mathrm{E}+\odot 8$ \\
\hline 32 & 288.000 & 288.000 & 1.0000 & $\odot .1448 \mathrm{E}+\odot 8$ \\
\hline 33 & 288.000 & 288.000 & $1.00 \odot \odot$ & $\odot .1448 E+08$ \\
\hline 34 & $288.00 \odot$ & $288.0 \odot \odot$ & 1.0000 & $\odot .1448 \mathrm{E}+\odot 8$ \\
\hline 35 & 288.000 & 288.000 & 1.0000 & $\odot .1448 \mathrm{E}+08$ \\
\hline 36 & $288.00 \odot$ & $288.00 \odot$ & $1.000 \odot$ & $\odot .1448 \mathrm{E}+\odot 8$ \\
\hline 37 & 288.000 & 288.000 & 1.0000 & $0.1448 \mathrm{E}+08$ \\
\hline 38 & 288.000 & 288.000 & 1.0000 & $\odot .1448 \mathrm{E}+08$ \\
\hline 39 & $288.0 \odot \odot$ & $288.0 \odot \odot$ & 1.0000 & $0.1448 \mathrm{E}+08$ \\
\hline 40 & 288.000 & $288.00 \odot$ & 1.0000 & $\odot .1448 \mathrm{E}+\odot 8$ \\
\hline 41 & 288.000 & 288.000 & 1.0000 & $\odot .1448 E+08$ \\
\hline 42 & $288.0 \odot \odot$ & $288 . \odot \odot \odot$ & 1.0000 & $0.1448 \mathrm{E}+08$ \\
\hline 43 & 288.000 & $288.0 \odot \odot$ & 1.0000 & $\odot .1448 \mathrm{E}+\odot 8$ \\
\hline 44 & 288.000 & 288.000 & 1.0000 & $\odot .1448 \mathrm{E}+08$ \\
\hline 45 & $288.00 \odot$ & $288.0 \odot \odot$ & 1.0000 & $\odot .1448 \mathrm{E}+\odot 8$ \\
\hline 46 & 288.000 & 288.000 & 1.0000 & $\odot .1448 \mathrm{E}+\odot 8$ \\
\hline 47 & 288.000 & 288.000 & 1.0000 & $\odot .1448 \mathrm{E}+08$ \\
\hline 48 & $288.00 \odot$ & $288.00 \odot$ & 1.0000 & $\odot .1448 \mathrm{E}+08$ \\
\hline 49 & 288.000 & 288.000 & 1.0000 & $\odot .1448 \mathrm{E}+08$ \\
\hline
\end{tabular}




\begin{tabular}{|c|c|c|c|c|}
\hline 50 & 288.000 & 288.000 & 1.0000 & $\odot .1448 E+\odot 8$ \\
\hline 51 & 288.000 & 288.000 & 1.0000 & $0.1448 \mathrm{E}+0$ \\
\hline 52 & $288.00 \odot$ & $288 . \odot \odot \odot$ & 1.0000 & $0.1448 \mathrm{E}+\mathrm{C}$ \\
\hline 53 & $288.00 \odot$ & $288.0 \odot \odot$ & 1.0000 & $\odot .1448 \mathrm{E}+\mathrm{C}$ \\
\hline 54 & $288 . \odot \odot \odot$ & $288.0 \odot \odot$ & 1.0000 & $\odot .1448 \mathrm{E}+$ \\
\hline 55 & $288.0 \odot \odot$ & $288.0 \odot \odot$ & $1.000 \odot$ & $\odot .1448 \mathrm{E}+$ \\
\hline 56 & $288.00 \odot$ & 288.000 & $1.000 \odot$ & $\odot .1448 \mathrm{E}+$ \\
\hline 57 & $288.0 \odot \odot$ & $288.00 \odot$ & 1.0000 & $\odot .1448 \mathrm{E}+\mathrm{C}$ \\
\hline 58 & $288 . \odot \odot \odot$ & $288.00 \odot$ & $1.000 \odot$ & $\odot .1448 \mathrm{E}+$ \\
\hline 59 & $288 . \odot \odot \odot$ & $288.00 \odot$ & 1.0000 & $0.1448 \mathrm{E}+$ \\
\hline 60 & 288.000 & $288.0 \odot \odot$ & 1.0000 & $\odot .1448 \mathrm{E}+\mathrm{C}$ \\
\hline
\end{tabular}

lambda_avg $=0.320041120$ 

ORNL/TM-2006/579

\section{INTERNAL DISTRIBUTION}
1. D. M. Hetrick
2. J. E. Lake
3. R. H. Morris
4. R. W. Murphy

\author{
5-6. C. B. Oland \\ 7-9. C. D. Sulfredge \\ 10. B. A. Worley \\ 11. ORNL Office of Technical \\ Information and Classification
}

\section{EXTERNAL DISTRIBUTION}

12-13. E. Skalnek, U.S. Dept. of Transportation, Pipeline and Hazardous Materials Safety Administration, Room 2103, 400 Seventh St., S.W., Washington, DC 20590 